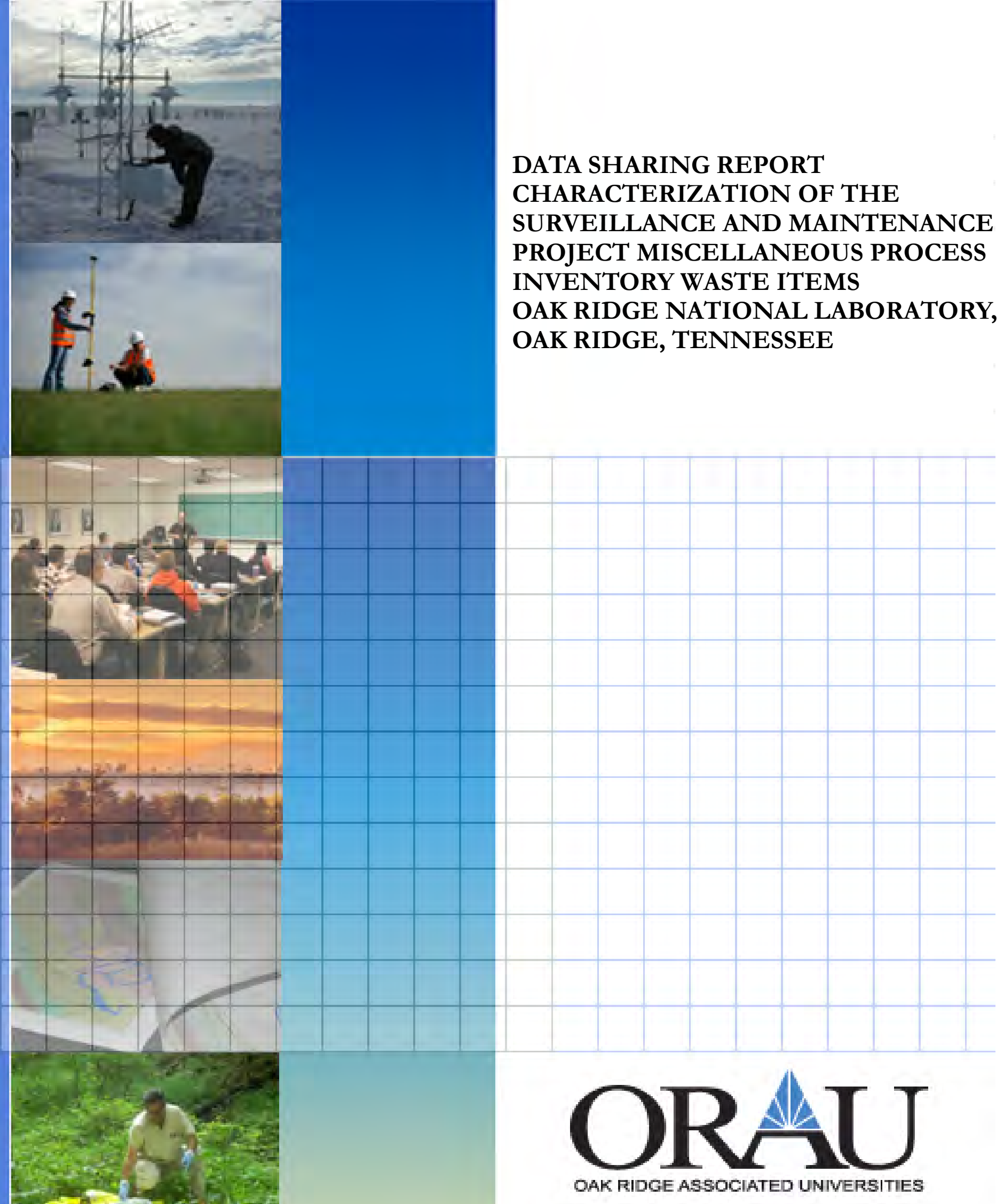

This document is approved for public release per review by: December 11, 2013 
ORAU provides innovative scientific and technical solutions to advance research and education, protect public health and the environment, and strengthen national security. Through specialized teams of experts, unique laboratory capabilities and access to a consortium of more than 100 major Ph.D.-granting institutions, ORAU works with federal, state, local and commercial customers to advance national priorities and serve the public interest. A 501(c)(3) nonprofit corporation and federal contractor, ORAU manages the Oak Ridge Institute for Science and Education (ORISE) for the U.S. Department of Energy (DOE). Learn more about ORAU at www.orau.org.

\section{NOTICES}

The opinions expressed herein do not necessarily reflect the opinions of the sponsoring institutions of Oak Ridge Associated Universities.

This report was prepared as an account of work sponsored by the United States Government. Neither the United States Government nor the U.S. Department of Energy, nor any of their employees, makes any warranty, expressed or implied, or assumes any legal liability or responsibility for the accuracy, completeness, or usefulness of any information, apparatus, product, or process disclosed, or represents that its use would not infringe on privately owned rights. Reference herein to any specific commercial product, process, or service by trade name, mark, manufacturer, or otherwise, does not necessarily constitute or imply its endorsement or recommendation, or favor by the U.S. Government or any agency thereof. The views and opinions of authors expressed herein do not necessarily state or reflect those of the U.S. Government or any agency thereof. 


\title{
DATA SHARING REPORT CHARACTERIZATION OF THE SURVEILLANCE AND MAINTENANCE PROJECT MISCELLANEOUS PROCESS INVENTORY WASTE ITEMS OAK RIDGE NATIONAL LABORATORY, OAK RIDGE, TENNESSEE
}

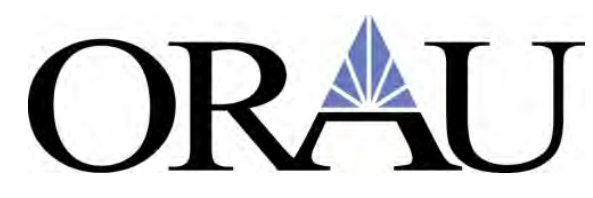

\author{
Prepared by \\ Phyllis C. Weaver \\ Independent Environmental Assessment and Verification Program \\ Oak Ridge Associated Universities
}

December 2013

Prepared for the

U.S. Department of Energy

Prepared by Oak Ridge Associated Universities under the Oak Ridge Institute for Science and Education contract, number DE-AC05-06OR23100, with the U.S. Department of Energy. 


\section{DATA SHARING REPORT CHARACTERIZATION OF THE \\ SURVEILLANCE AND MAINTENANCE PROJECT MISCELLANEOUS PROCESS INVENTORY WASTE ITEMS OAK RIDGE NATIONAL LABORATORY, OAK RIDGE, TENNESSEE}

Prepared by:

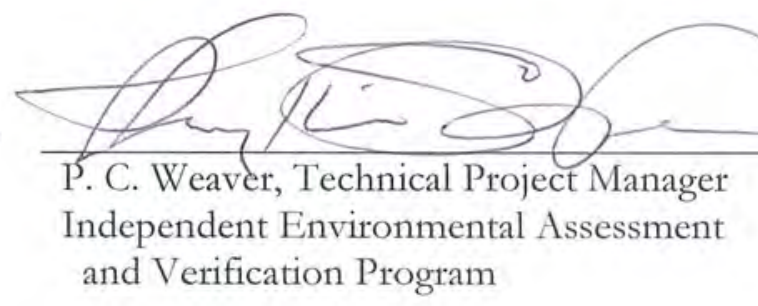

Date:

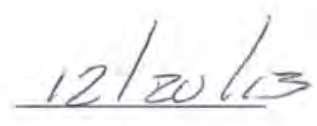

Reviewed by:

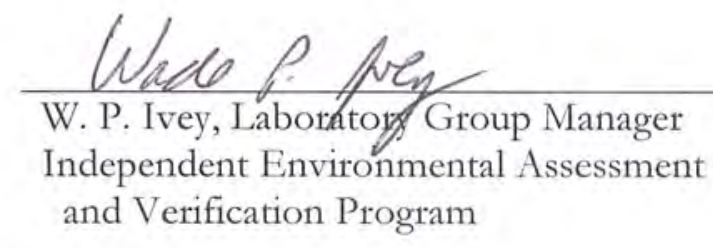

Date:

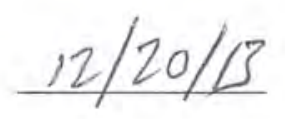

Reviewed by:

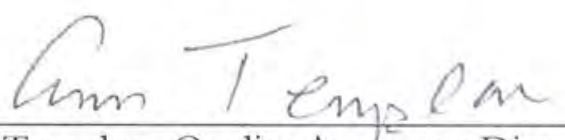

Date:

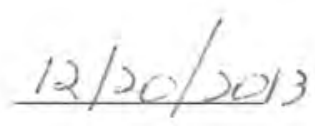

F. A. Templon, Quality Assurance Director

Independent Environmental Assessment and Verification Program

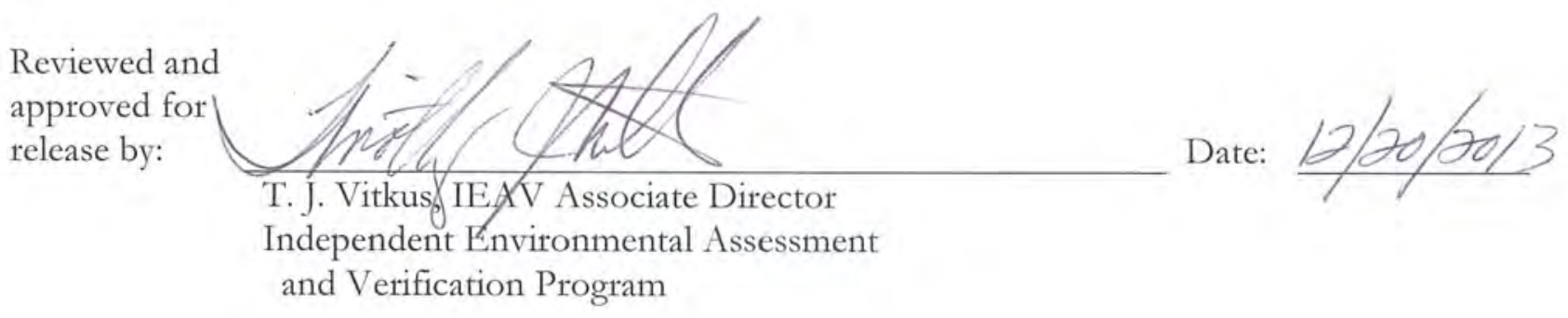

DECEMBER 2013 
CONTENTS

TABLES iv

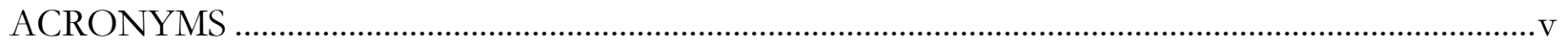

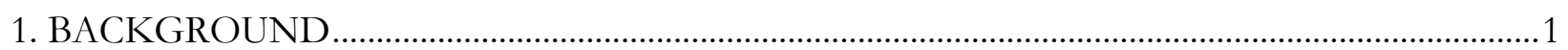

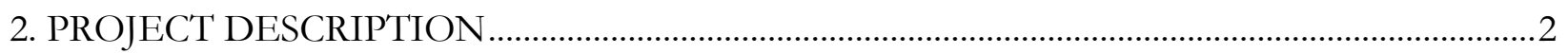

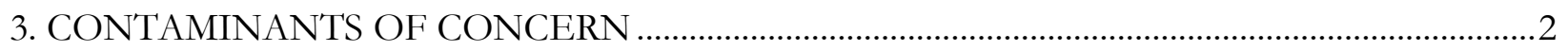

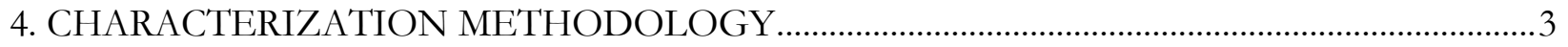

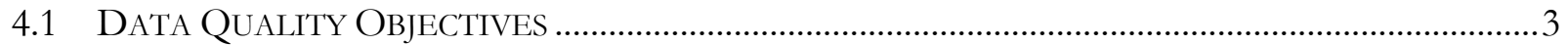

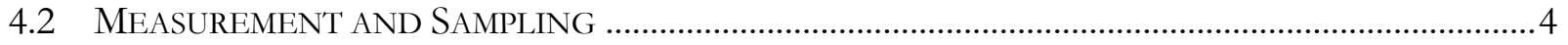

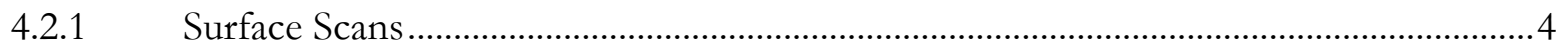

4.2.2 Direct Measurements ..................................................................................................

4.2.3 Sample Collection........................................................................................... 5

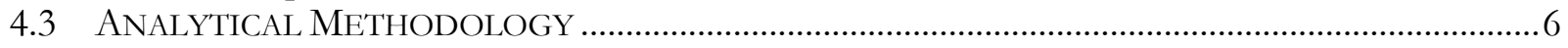

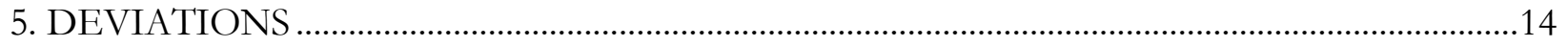

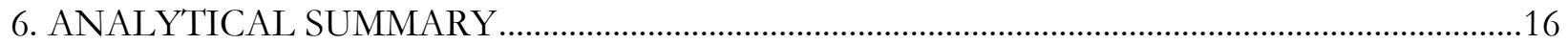

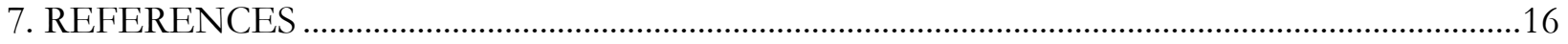

APPENDIX A PROCESS KNOWLEDGE SUMMARIES FOR FACILITIES ASSOCIATED WITH OAK RIDGE NATIONAL LABORATORY SURVEILLANCE AND MAINTENANCE PROJECT WASTE ITEMS

APPENDIX B POPULATIONS 4, 5, 6, 7, AND 8 SUBSET OF MISCELLANEOUS WASTE ITEMS IDENTIFIED FOR CHARACTERIZATION

APPENDIX C TOTAL AND REMOVABLE SURFACE ACTIVITY MEASUREMENTS APPENDIX D SAMPLE PHOTOGRAPH AND IDENTIFICATION APPENDIX E DATA SUMMARY STATISTICS 


\section{TABLES}

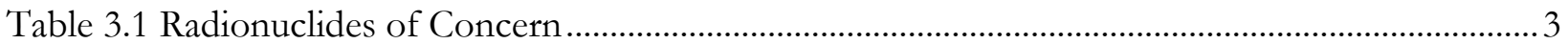

Table 4.1. Surface Radiation Guidelines and Action Levels................................................................... 6

Table 4.2. Analytical Methods and Target Detection Limits for Radiological SRCs ............................ 7

Table 4.3. Analytical Methods and Target Detection Limits for Non-radiological SRCs...................... 8

Table 5.1 Process Inventory Waste SAP 3 Deviations Records Form ...................................................15 


\section{ACRONYMS}

ACM

AL

ARRA

COC

DAW

DOE

DQO

EM-OR

EMWMF

EPA

NNSS

ORAU

ORISE

ORNL

PCB

PK

POP

QC

S\&M

SAP

SLF

$\mathrm{SMO}$

SRC

SVOC

TCLP

TSDF

UCOR

VOC

WAC

WHP

WL asbestos-containing material

action level

American Recovery and Reinvestment Act

contaminant of concern

dry active waste

U.S. Department of Energy

data quality objective

Oak Ridge Office of Environmental Management

Environmental Management Waste Management Facility

U.S. Environmental Protection Agency

Nevada National Security Site

Oak Ridge Associated Universities

Oak Ridge Institute for Science and Education

Oak Ridge National Laboratory

polychlorinated biphenyl

process knowledge

population

quality control

surveillance and maintenance

sampling and analysis plan

sanitary landfill

Sample Management Office

site-related contaminant

semi-volatile organic compound

toxicity characteristic leaching procedure

treatment, storage, and disposal facility

URS | CH2M Oak Ridge, LLC

volatile organic compound

waste acceptance criteria

waste handling plan

waste lot 


\section{DATA SHARING REPORT \\ CHARACTERIZATION OF THE \\ SURVEILLANCE AND MAINTENANCE PROJECT \\ MISCELLANEOUS PROCESS INVENTORY WASTE ITEMS \\ OAK RIDGE NATIONAL LABORATORY, \\ OAK RIDGE, TENNESSEE}

\section{BACKGROUND}

The U.S. Department of Energy (DOE) Oak Ridge Office of Environmental Management (EM-OR) requested Oak Ridge Associated Universities (ORAU), working under the Oak Ridge Institute for Science and Education (ORISE) contract, to provide technical and independent waste management planning support under the American Recovery and Reinvestment Act (ARRA). Specifically, DOE EM-OR requested ORAU to plan and implement a sampling and analysis campaign to target certain items associated with URS|CH2M Oak Ridge, LLC (UCOR) surveillance and maintenance $(\mathrm{S} \& \mathrm{M})$ process inventory waste.

Eight populations of historical and reoccurring S\&M waste at the Oak Ridge National Laboratory (ORNL) have been identified in the Waste Handling Plan for Surveillance and Maintenance Activities at the Oak Ridge National Laboratory, DOE/OR/01-2565\&D2 (WHP) (DOE 2012) for evaluation and processing for final disposal. This waste was generated during processing, surveillance, and maintenance activities associated with the facilities identified in the process knowledge (PK) provided in Appendix A. A list of items for sampling and analysis were generated from a subset of materials identified in the WHP populations (POPs) 4, 5, 6, 7, and 8, plus a small number of items not explicitly addressed by the WHP. Specifically, UCOR S\&M project personnel identified 62 miscellaneous waste items that would require some level of evaluation to identify the appropriate pathway for disposal. These items are highly diverse, relative to origin; composition; physical description; contamination level; data requirements; and the presumed treatment, storage, and disposal facility (TSDF). Because of this diversity, ORAU developed a structured approach to address item-specific data requirements necessary for acceptance in a presumed TSDF that includes the Environmental Management Waste Management Facility (EMWMF)—using the approved Waste Lot (WL) 108.1 profile-the Y-12 Sanitary Landfill (SLF) if appropriate; EnergySolutions Clive; and the Nevada National Security Site (NNSS) (ORAU 2013b). Finally, the evaluation of these wastes was more suited to a judgmental sampling approach rather than a statistical design, 
meaning data were collected for each individual item, thereby providing information for item-byitem disposition decisions.

ORAU prepared a sampling and analysis plan (SAP) that outlined data collection strategies, methodologies, and analytical guidelines and requirements necessary for characterizing targeted items (ORAU 2013b). The SAP described an approach to collect samples that allowed evaluation as to whether or not the waste would be eligible for disposal at the EMWMF. If the waste was determined not to be eligible for EMWMF disposal, then there would be adequate information collected that would allow the waste to be profiled for one of the alternate TSDFs listed above.

\section{PROJECT DESCRIPTION}

The ORNL S\&M Project is responsible for a large, highly diverse, and inhomogeneous inventory of waste items that required characterization to complete disposition planning. Items included, but were not limited to: B-25 containers and drums containing lead pieces, electronic equipment, filter media, metal specimens, dry active wastes (DAW), personal protective equipment, etc., as presented in Appendix B. The information presented in Appendix B includes the container type, assigned identification number as applicable, a brief physical description of the item, facility of origin if known, associated site-related contaminants (SRCs), anticipated waste volume, whether sampled, and presumed TSDF. The SAP outlined the requirements for obtaining radiation activity measurements, collecting surface smears for the determination of removable activity, and collecting grab volumetric samples for analytical laboratory analysis to fill data gaps necessary to demonstrate compliance with the TSDF specific waste acceptance criteria (WAC), specifically, EMWMF as appropriate.

\section{CONTAMINANTS OF CONCERN}

A variety of diverse research and development processes were performed at Isotope Row involving both chemical and radiological constituents. Based on historical site use, general survey and scoping data, and information from operations personnel, the probable list of chemical contaminants of concern (COC) included asbestos-containing material (ACM), polychlorinated biphenyls (PCBs), semi-volatile organic compounds (SVOCs), volatile organic compounds (VOCs), herbicides, 
pesticides, and various heavy metals (e.g., lead). Radionuclides of concern are listed in Table 3.1. Knowledge of the chemical and radiological constituent concentrations is required to ultimately satisfy the waste acceptance criteria for a specified treatment, storage, disposal, or recycling (TSDR) facility considering the waste populations for these facilities.

\begin{tabular}{|l|l|l|l|}
\hline \multicolumn{5}{|c|}{$\begin{array}{c}\text { Table 3.1 Radionuclides of Concern } \\
\text { Oak Ridge National Laboratory }\end{array}$} \\
\hline Am-241 & H-3 & Ra-226 & U-233/234 \\
\hline C-14 & I-129 & Sr-90 & U-235/236 \\
\hline Cs-137 & Np-237 & Tc-99 & U-238 \\
\hline Co-60 & Pu-238 & Th-230 & Total U \\
\hline Cm-243/244 & Pu-239/240 & Th-232 & \\
\hline
\end{tabular}

\section{CHARACTERIZATION METHODOLOGY}

\subsection{Data Quality OBJectives}

A graded approach to the data quality objective (DQO) process was used to develop the SAP to satisfy the data gaps associated with the 62 miscellaneous ORNL S\&M project waste items. In accordance with the Guidance on Systematic Planning Using the Data Quality Objectives Process, issued by the U.S. Environmental Protection Agency (EPA), DQOs are the qualitative and quantitative statements that specify the quality of data required to support decisions during investigation and remedial response activities (EPA 2006). The DQO process defines the purpose for which data will be used and sets guidelines for designing a data collection program that will meet regulatory objectives (ORAU 2013b).

The principal study question and alternative actions decisions for evaluating these wastes for WL 108.1 is:

Determine through process knowledge, evaluation of radiation survey, and laboratory analytical data, whether the miscellaneous waste items satisfy the WAC for the Y-12 SLF, Energy Solutions Clive, NNSS, or EMWMF under WL 108.1. 
The SAP outlined the conceptual model for selecting the appropriate TSDF. Hazardous waste is specifically targeted for disposal at EnergySolutions Clive, Utah, and highly contaminated waste or contaminated waste that lacks PK is targeted for disposal at NNSS. Items that satisfy the Y-12 SLF WAC will be shipped to that facility, and the balance is presumed to satisfy the EMWMF WAC and WL 108.1 requirements (ORAU 2013b).

The question considers a graded approach in the "level of effort" necessary for evaluating the level of survey effort to design a classification process that determines the disposition pathway for the S\&M wastes. The initial assessment was to determine if the waste was non-impacted or impacted, with impacted having the highest potential to exceed the action levels and therefore requiring the greatest level of effort. Process knowledge, as well as physical attributes, was used in making the assessment of the level of sample effort required.

\subsection{MEASUREMENT AND SAMPLING}

Of the original 62 listed items, 40 were accessible for radiological surface scans and or sampling. Item \#29 appears to be a duplicate of item \#28, nine items could not be located, and 12 items were omitted to avoid and prevent personnel exposure to biological hazards or high radiation levels. An additional container (X10C0401770) and a pressure washer gun were added to the list of items to assess at the request of the UCOR site representative. Appendix B identifies the items that were assessed.

\subsubsection{Surface Scans}

Radiation scans were performed on accessible surfaces of a container or item. The scan results were then used to identify items that had measurable radioactivity. These items were set aside (or tagged) for volumetric sampling. Scans were performed for both alpha and beta radiation using $100 \mathrm{~cm}^{2}$ alpha and beta scintillation detectors. A 2-inch $\times 2$-inch sodium iodide $(\mathrm{NaI})$ scintillation detector was used for identifying gamma radiation. All detectors were connected to Ludlum Model 2221 ratemeter/scalers with data logging capabilities.

To ensure validity/reliability of the radiation measurement data and to successfully meet the DQOs, the appropriate quality control (QC) data were obtained. These data included media/surface-specific background detector response data and surface efficiencies, radiation-specific instrument efficiencies 
determined using National Institute of Standards and Technology-traceable sources, operational checkout requirements for background and check source responses. Specific requirements are described in the Survey Procedures Manual for the Independent Environmental Assessment and Verification Program (ORAU/ORISE 2013a) and are not repeated in this report.

\subsubsection{Direct Measurements}

Two fixed-point/static measurements for both alpha-radiation and beta-radiation were collected on most of the items. Measurement locations were selected based on scan results. One location was representative of the maximum radiation level detected by the scan and the other of the general area radiation levels determined for the item.

\subsubsection{Sample Collection}

A smear sample for removable radioactivity was collected at the selected fixed-point/static measurement location. Seventy-four smears on 41 items were counted for gross alpha and beta activity. Removable radioactivity results are provided in Appendix C. Two additional smears were collected and analyzed for gross alpha and beta radiation at the request of UCOR on items not associated with the waste populations of this project. Results of those smears, 5219R0071 and 5219R0072, were provided to UCOR but are not included in this report.

A sample or no sample decision was based on a fixed-point or static measurement above the action levels (ALs) (Table 4.1), the type or function of the item, process knowledge, limited scan as a result of physical design, or surveyor judgment. Thirty-nine volumetric samples and two duplicates were collected for analytical determination of radiological and chemical contaminant concentrations relative to the EMWMF WAC. Sample photos are provided in Appendix D. Waste packages inside of a container were sampled, unless the container was empty. An empty container was sampled, if feasible to do so or if approved by UCOR. Individual items such as hoods, equipment, scrap metal, etc. were sampled as found. No items that contained lead such as lead pigs, bricks, or sheets were volumetrically sampled. However, a smear sample was collected from the surface area representing the lowest and highest radiation level detected. A data summary of each sample analyte concentration in tabulated and graphical form is provided in Appendix E. 


\subsection{ANALYTICAL Methodology}

The performance and acceptance criteria for radiation survey data are provided in Table 4.1 representing the isotope-specific, element-specific, or radiation-emission-specific acceptance criteria for the Y-12 SLF. To ensure validity/reliability of the radiation measurement data and to successfully meet the DQOs, the ALs are established at $80 \%$ of the guideline values to account for the variability of radiation measurements data associated with background response estimates, efficiency inputs, surveyor technique and other factors. The appropriate QC data were obtained, including media/surface-specific background detector response data and efficiencies, radiationspecific efficiencies, and operational checkout requirements for background..

\section{Table 4.1. Surface Radiation Guidelines and Action Levels}

\section{Residual Surface Contamination Limits}

\begin{tabular}{|c|c|c|c|c|c|c|}
\hline \multirow[t]{2}{*}{ Radionuclides } & \multicolumn{2}{|c|}{$\begin{array}{c}\text { Average } \\
\text { (fixed }+ \\
\text { removable) } \\
\left(\mathrm{dpm} / 100 \mathrm{~cm}^{2}\right)\end{array}$} & \multicolumn{2}{|c|}{$\begin{array}{c}\text { Total } \\
(\text { fixed }+ \\
\text { removable }) \\
\left(\mathrm{dpm} / 100 \mathrm{~cm}^{2}\right)\end{array}$} & \multicolumn{2}{|c|}{$\begin{array}{c}\text { Removable } \\
(\mathrm{dpm} / 100 \\
\left.\mathrm{cm}^{2}\right)\end{array}$} \\
\hline & GV & AL & GV & AL & GV & AL \\
\hline $\begin{array}{c}\text { Transuranics, I-125, I-129, Th-228, } \\
\text { Th-230, Pa-231 }\end{array}$ & 100 & 80 & 300 & 240 & 20 & 16 \\
\hline $\begin{array}{c}\text { Th-natural, Sr-90, I-126, I-131, I-133, } \\
\text { Ra-223, Ra-224, U-232, Th-232 }\end{array}$ & 1,000 & 800 & 3,000 & 2,400 & 200 & 160 \\
\hline $\begin{array}{l}\text { Beta-gamma emitters except as } \\
\text { otherwise noted }\end{array}$ & 5,000 & 4,000 & 15,000 & 12,000 & 1,000 & 800 \\
\hline $\begin{array}{l}\text { U-natural, U-235, U-238, and associated } \\
\text { decay products, alpha emitters }\end{array}$ & 5,000 & 4,000 & 15,000 & 12,000 & 1,000 & 800 \\
\hline
\end{tabular}

All media samples collected were returned to the ORAU/ORISE Radiological and Environmental Analytical Laboratory in Oak Ridge, Tennessee and prepared for either analyses or for shipment to another Oak Ridge Sample Management Office (SMO) approved laboratory. Requirements and methods for radiological and chemical analysis were specified in the SAP and are provided in Tables 4.2 and 4.3 respectively. Target detection limits were intended to be at or below waste profile limits. Actual detection limits may be sample-specific, but the data measurement objective was to obtain data with detection limits adequate to satisfy these levels. All analyses and QC requirements 
were performed in accordance with the SAP, the ORAU/ORISE laboratory procedures

(ORAU/ORISE 2013b), and the SMO laboratory statement of work, as appropriate.

In the event that an item sampled did not have the necessary minimum weight requirement to complete the stated chemical analyses, the recommended hierarchy of analyses as outlined in the SAP was implemented. With a low sample weight, the result could potentially fail to meet the analytical detection limit levels specified in the SAP and could only be used for qualified analytical results reporting (ORAU 2013b).

The parameters of interest for volumetric (laboratory analytical) data are concentrations in picocuries per gram (pCi/g), milligrams per kilograms $(\mathrm{mg} / \mathrm{kg})$, and milligrams per liter $(\mathrm{mg} / \mathrm{L})$. The parameter of interest for radiation measurement data collected on surface contaminated items is concentration in counts per minute (cpm) converted to disintegrations per minute per $100 \mathrm{~cm}^{2}$ $\left(\mathrm{dpm} / 100 \mathrm{~cm}^{2}\right)$.

\begin{tabular}{|c|c|c|c|c|c|}
\hline Analyte & Method & Units & Reference level & $\begin{array}{c}\text { Target detection } \\
\text { limit }\end{array}$ & $\begin{array}{c}\text { Method } \\
\text { detection limit }\end{array}$ \\
\hline H-3 & liquid scint. & $\mathrm{pCi} / \mathrm{g}$ & $15,000^{a}$ & 1,500 & 5 \\
\hline C-14 & liquid scint. & $\mathrm{pCi} / \mathrm{g}$ & $165^{a}$ & 16.5 & 3 \\
\hline $\mathrm{Co}-60$ & gamma spec. & $\mathrm{pCi} / \mathrm{g}$ & $2^{\mathrm{b}} / 6,900,000^{\mathrm{c}}$ & 0.2 & 0.1 \\
\hline Sr-90 & GPC & $\mathrm{pCi} / \mathrm{g}$ & $30^{b} / 400,000^{c}$ & 3 & 0.4 \\
\hline Tc-99 & liquid scint. & $\mathrm{pCi} / \mathrm{g}$ & $40^{d} / 172^{c}$ & 4 & 0.3 \\
\hline I-129 & gamma spec. & $\mathrm{pCi} / \mathrm{g}$ & $13^{a}$ & 1.3 & 0.2 \\
\hline Cs-137 & gamma spec. & $\mathrm{pCi} / \mathrm{g}$ & $10^{\mathrm{b}} / 1,500,000^{\mathrm{c}}$ & 1 & 0.1 \\
\hline Ra-226 & gamma spec. & $\mathrm{pCi} / \mathrm{g}$ & $3^{\mathrm{b}} / 300,000^{c}$ & 0.3 & 0.8 \\
\hline Th-230 & alpha spec. & $\mathrm{pCi} / \mathrm{g}$ & $3^{\mathrm{d}} / 15,000^{c}$ & 0.3 & 0.2 \\
\hline Th-232 & alpha spec. & $\mathrm{pCi} / \mathrm{g}$ & $3^{d} / 2,500^{c}$ & 0.3 & 0.2 \\
\hline U-233/U-234 & alpha spec. & $\mathrm{pCi} / \mathrm{g}$ & $35^{\mathrm{d}} / 1,700^{a}$ & 3.5 & 0.1 \\
\hline U-235 & alpha spec. & $\mathrm{pCi} / \mathrm{g}$ & $35^{\mathrm{d}} / 1,500^{a}$ & 3.5 & 0.1 \\
\hline $\mathrm{U}-238$ & alpha spec. & $\mathrm{pCi} / \mathrm{g}$ & $35^{\mathrm{d}} / 1,200^{a}$ & 3.5 & 0.1 \\
\hline Total U & alpha spec. & $\mathrm{pCi} / \mathrm{g}$ & $35^{d}$ & 3.5 & 0.1 \\
\hline $\mathrm{Cm}-243 / \mathrm{Cm}-244$ & alpha spec. & $\mathrm{pCi} / \mathrm{g}$ & $20,000^{a} / 26,000^{a}$ & $2,000 / 2,600$ & $0.4 / 0.4$ \\
\hline $\mathrm{Np}-237$ & gamma spec. & $\mathrm{pCi} / \mathrm{g}$ & $3^{\mathrm{d}} / 320^{a}$ & 0.3 & 0.1 \\
\hline $\mathrm{Pu}-238$ & alpha spec. & $\mathrm{pCi} / \mathrm{g}$ & $3^{\mathrm{d}} / 15,000^{c}$ & 0.3 & 0.1 \\
\hline Pu-239 & alpha spec. & $\mathrm{pCi} / \mathrm{g}$ & $3 \mathrm{~d} / 720^{a}$ & 0.3 & 0.1 \\
\hline Am-241 & alpha spec. & $\mathrm{pCi} / \mathrm{g}$ & $3^{\mathrm{d}} / 13,000^{c}$ & 0.3 & 0.1 \\
\hline
\end{tabular}

*Table 4.2 notes on next page 
${ }^{a}$ EMWMF analytic waste acceptance criterion (DOE/OR/01-1909\&D3)

bDose-based screening criterion for the Y-12 sanitary landfill, Master Profile S-040

'EMWMF auditable safety analysis-derived WAC (DOE/OR/01-1909\&D3 and the 2008 revised Table A.1)

dAdministrative limit for the Y-12 sanitary landfill, Master Profile S-040

Reference levels taken from the WHP (DOE/OR/01-2565\&D2), when available; else from DOE/OR/01$1909 \& D 3$

GPC = gas proportional counting

SRCs $=$ site related contaminants

Table 4.3. Analytical Methods and Target Detection Limits for Non-radiological SRCs

\begin{tabular}{|c|c|c|c|c|}
\hline $\begin{array}{c}\text { Analysis } \\
\text { type }\end{array}$ & Parameter & CAS number & Analytical method & $\begin{array}{l}\text { Project reporting level } \\
\text { (MDA or MDL) } \\
\text { (total metals }=\mathrm{mg} / \mathrm{kg} \\
\text { total organics }=\mu \mathrm{g} / \mathrm{kg} \\
\text { TCLP }=\mathrm{mg} / \mathrm{L} \text { ) }\end{array}$ \\
\hline SVOC & 1-Hexanol & $111-27-3$ & SW-846-8270D/3550C & 666 \\
\hline SVOC & 2,3,4,6-Tetrachlorophenol & $58-90-2$ & SW-846-8270D/3550C & 330 \\
\hline SVOC & 2,4,5-Trichlorophenol & $95-65-4$ & SW-846-8270D/3550C & 330 \\
\hline SVOC & 2,4,6-Trichlorophenol & $88-06-2$ & SW-846-8270D/3550C & 330 \\
\hline SVOC & 2,4-Dichlorophenol & $120-83-2$ & SW-846-8270D/3550C & 330 \\
\hline SVOC & 2,4-Dimethylphenol & $105-67-9$ & SW-846-8270D/3550C & 330 \\
\hline SVOC & 2,4-Dinitrophenol & $51-28-5$ & SW-846-8270D/3550C & 1,650 \\
\hline SVOC & 2,4-Dinitrotoluene & $121-14-2$ & SW-846-8270D/3550C & 330 \\
\hline SVOC & 2,6-Dinitrotoluene & $606-20-2$ & SW-846-8270D/3550C & 330 \\
\hline SVOC & 2-Chloronaphthalene & $91-58-7$ & SW-846-8270D/3550C & 330 \\
\hline SVOC & 2-Chlorophenol & $95-57-8$ & SW-846-8270D/3550C & 330 \\
\hline SVOC & 2-Methylnaphthalene & $91-57-6$ & SW-846-8270D/3550C & 330 \\
\hline SVOC & 2-Methylphenol & $95-48-7$ & SW-846-8270D/3550C & 330 \\
\hline SVOC & 2-Nitroaniline & 88-74-4 & SW-846-8270D/3550C & 1,650 \\
\hline SVOC & 2-Nitrophenol & $88-75-5$ & SW-846-8270D/3550C & 330 \\
\hline SVOC & 3,3'-Dichlorobenzidine & $91-94-1$ & SW-846-8270D/3550C & 660 \\
\hline SVOC & 3-Methylphenol ${ }^{a}$ & $108-39-4$ & SW-846-8270D/3550C & 330 \\
\hline SVOC & 3-Nitroaniline & $99-09-2$ & SW-846-8270D/3550C & 1,650 \\
\hline SVOC & 4,6,-Dinitro-2-methylphenol & $534-52-1$ & SW-846-8270D/3550C & 1,650 \\
\hline SVOC & 4-Bromophenylphenyl ether & $101-55-3$ & SW-846-8270D/3550C & 330 \\
\hline SVOC & 4-Chloro-3-methylphenol & $59-50-7$ & SW-846-8270D/3550C & 330 \\
\hline SVOC & 4-Chloroaniline & $106-47-8$ & SW-846-8270D/3550C & 330 \\
\hline SVOC & 4-Chlorophenyl phenyl ether & $7005-72-3$ & SW-846-8270D/3550C & 1,650 \\
\hline SVOC & 4-Methylphenola & $106-44-5$ & SW-846-8270D/3550C & 330 \\
\hline SVOC & 4-Nitroaniline & 100-01-6 & SW-846-8270D/3550C & 1,650 \\
\hline SVOC & 4-Nitrophenol & $100-02-7$ & SW-846-8270D/3550C & 1,650 \\
\hline SVOC & Acenaphthene & 83-32-9 & SW-846-8270D/3550C & 330 \\
\hline SVOC & Acenaphthylene & $208-96-8$ & SW-846-8270D/3550C & 330 \\
\hline SVOC & Acetophenone & $98-86-2$ & SW-846-8270D/3550C & 330 \\
\hline SVOC & Anthracene & $120-12-7$ & SW-846-8270D/3550C & 330 \\
\hline SVOC & Benzidine & $92-87-5$ & SW-846-8270D/3550C & 330 \\
\hline SVOC & Benzo(a)anthracene & $56-55-3$ & SW-846-8270D/3550C & 330 \\
\hline SVOC & Benzo(a)pyrene & $50-32-8$ & SW-846-8270D/3550C & 330 \\
\hline
\end{tabular}


Table 4.3. Analytical Methods and Target Detection Limits for Non-radiological SRCs

\begin{tabular}{|c|c|c|c|c|}
\hline $\begin{array}{c}\text { Analysis } \\
\text { type }\end{array}$ & Parameter & CAS number & Analytical method & $\begin{array}{l}\text { Project reporting level } \\
\text { (MDA or MDL) } \\
\text { (total metals }=\mathrm{mg} / \mathrm{kg} \\
\text { total organics }=\mu \mathrm{g} / \mathrm{kg} \\
\text { TCLP }=\mathrm{mg} / \mathrm{L} \text { ) } \\
\end{array}$ \\
\hline SVOC & Benzo(b)fluoranthene & $205-99-2$ & SW-846-8270D/3550C & 330 \\
\hline SVOC & Benzo(g,h,i)perylene & $191-24-2$ & SW-846-8270D/3550C & 330 \\
\hline SVOC & Benzo $(\mathrm{k})$ fluoranthene & 207-08-9 & SW-846-8270D/3550C & 330 \\
\hline SVOC & Benzoic acid & $65-85-0$ & SW-846-8270D/3550C & 1,650 \\
\hline SVOC & Benzyl Alcohol & $100-51-6$ & SW-846-8270D/3550C & 330 \\
\hline SVOC & bis(2-chloroethoxy) methane & $111-91-1$ & SW-846-8270D/3550C & 330 \\
\hline SVOC & bis(2-chloroethyl) ether & $111-44-4$ & SW-846-8270D/3550C & 330 \\
\hline SVOC & bis(2-chloroisopropyl) ether & $108-60-1$ & SW-846-8270D/3550C & 330 \\
\hline SVOC & bis(2-ethylhexyl) ether & $117-81-7$ & SW-846-8270D/3550C & 330 \\
\hline SVOC & Butyl benzyl phthalate & $85-68-7$ & SW-846-8270D/3550C & 330 \\
\hline SVOC & Carbazole & $86-74-8$ & SW-846-8270D/3550C & 330 \\
\hline SVOC & Carbofuran & $1563-66-2$ & SW-846-8270D/3550C & 666 \\
\hline SVOC & Chrysene & 218-01-9 & SW-846-8270D/3550C & 330 \\
\hline SVOC & Di-n-butylphthalate & $84-74-2$ & SW-846-8270D/3550C & 330 \\
\hline SVOC & Di-n-octylphthalate & $117-84-0$ & SW-846-8270D/3550C & 330 \\
\hline SVOC & Dibenzo(a,h)anthracene & $53-70-3$ & SW-846-8270D/3550C & 330 \\
\hline SVOC & Dibenzofuran & $132-64-9$ & SW-846-8270D/3550C & 330 \\
\hline SVOC & Diethylphthalate & 84-66-2 & SW-846-8270D/3550C & 330 \\
\hline SVOC & Dimethylphthalate & $131-11-3$ & SW-846-8270D/3550C & 330 \\
\hline SVOC & Fluoranthene & $206-44-0$ & SW-846-8270D/3550C & 330 \\
\hline SVOC & Fluorene & $86-73-7$ & SW-846-8270D/3550C & 330 \\
\hline SVOC & Hexachlorobenzene & 118-74-1 & SW-846-8270D/3550C & 330 \\
\hline SVOC & Hexachlorobutadiene & $87-68-3$ & SW-846-8270D/3550C & 330 \\
\hline SVOC & Hexachlorocyclopentadiene & $77-47-4$ & SW-846-8270D/3550C & 330 \\
\hline SVOC & Indeno(1,2,3-c,d)pyrene & $193-39-5$ & SW-846-8270D/3550C & 330 \\
\hline SVOC & Isophorone & $78-59-1$ & SW-846-8270D/3550C & 330 \\
\hline SVOC & N-Nitrosodi-n-propylamine & $621-64-7$ & SW-846-8270D/3550C & 330 \\
\hline SVOC & N-Nitrosodiphenylamine & $86-30-6$ & SW-846-8270D/3550C & 330 \\
\hline SVOC & Naphthalene & $91-20-3$ & SW-846-8270D/3550C & 330 \\
\hline SVOC & Nitrobenzene & $98-95-3$ & SW-846-8270D/3550C & 330 \\
\hline SVOC & Pentachlorophenol & $87-86-5$ & SW-846-8270D/3550C & 1,650 \\
\hline SVOC & Phenanthrene & $85-01-8$ & SW-846-8270D/3550C & 330 \\
\hline SVOC & Phenol & $108-95-2$ & SW-846-8270D/3550C & 330 \\
\hline SVOC & Propylene Glycol ${ }^{\mathrm{b}}$ & $57-55-6$ & SW-846-8015C /3550C & 330 \\
\hline SVOC & Pyridine & $110-86-1$ & SW-846-8270D/3550C & 330 \\
\hline VOC & 1,1,1,2-Tetrachloroethane & 630-20-6 & SW-846-8260B/5035 & 10 \\
\hline VOC & 1,1,1-Trichloroethane & $71-55-6$ & SW-846-8260B/5035 & 10 \\
\hline VOC & 1,1,2,2-Tetrachloroethane & $79-34-5$ & SW-846-8260B/5035 & 10 \\
\hline VOC & 1,1,2-Trichloroethane & $79-00-5$ & SW-846-8260B/5035 & 10 \\
\hline VOC & 1,1-Dichloroethane & $75-34-3$ & SW-846-8260B/5035 & 10 \\
\hline VOC & 1,1-Dichloroethene & $75-35-4$ & SW-846-8260B/5035 & 10 \\
\hline VOC & 1,1-Dichloropropene & 563-58-6 & SW-846-8260B/5035 & 10 \\
\hline VOC & 1,2,3-Trichlorobenzene & $87-61-6$ & SW-846-8260B/5035 & 10 \\
\hline
\end{tabular}


Table 4.3. Analytical Methods and Target Detection Limits for Non-radiological SRCs

\begin{tabular}{|c|c|c|c|c|}
\hline $\begin{array}{c}\text { Analysis } \\
\text { type }\end{array}$ & Parameter & CAS number & Analytical method & $\begin{array}{l}\text { Project reporting level } \\
\text { (MDA or MDL) } \\
\text { (total metals }=\mathrm{mg} / \mathrm{kg} \\
\text { total organics }=\mu \mathrm{g} / \mathrm{kg} \\
\text { TCLP }=\mathrm{mg} / \mathrm{L} \text { ) } \\
\end{array}$ \\
\hline VOC & 1,2,3-Trichloropropane & $96-18-4$ & SW-846-8260B/5035 & 10 \\
\hline VOC & 1,2,4-Trichlorobenzene & $120-82-1$ & SW-846-8260B/5035 & 10 \\
\hline VOC & 1,2,4-Trimethylbenzene & $95-63-6$ & SW-846-8260B/5035 & 10 \\
\hline VOC & 1,2-Dibromo-3-chloropropane & $96-12-8$ & SW-846-8260B/5035 & 10 \\
\hline VOC & 1,2-Dibromoethane & $106-93-4$ & SW-846-8260B/5035 & 10 \\
\hline VOC & 1,2-Dichlorobenzene & $95-50-1$ & SW-846-8260B/5035 & 10 \\
\hline VOC & 1,3-Dichlorobenzene (total) & $541-73-1$ & SW-846-8260B/5035 & 10 \\
\hline VOC & 1,4-Dichlorobenzene & $106-46-7$ & SW-846-8260B/5035 & 10 \\
\hline VOC & 1,2-Dichloroethane & $107-06-2$ & SW-846-8260B/5035 & 10 \\
\hline VOC & 1,2-Dichloroethene (total) & $540-59-0$ & SW-846-8260B/5035 & 10 \\
\hline VOC & 1,2-Dichloropropane & $78-87-5$ & SW-846-8260B/5035 & 10 \\
\hline VOC & 1,3,5-Trimethylbenzene & $108-67-8$ & SW-846-8260B/5035 & 10 \\
\hline VOC & 1,3-Dichloropropane & $142-28-9$ & SW-846-8260B/5035 & 10 \\
\hline VOC & 2,2-Dichloropropane & $594-20-7$ & SW-846-8260B/5035 & 10 \\
\hline VOC & 2-Butanone & $78-93-3$ & SW-846-8260B/5035 & 10 \\
\hline VOC & 2-Chlorotoluene & $95-49-8$ & SW-846-8260B/5035 & 10 \\
\hline VOC & 2-Hexanone & $591-78-6$ & SW-846-8260B/5035 & 10 \\
\hline VOC & 4-Chlorotoluene & $106-43-4$ & SW-846-8260B/5035 & 10 \\
\hline VOC & p-Isopropyltoluene & $99-87-6$ & SW-846-8260B/5035 & 10 \\
\hline VOC & 4-Methyl-2-pentanone & $108-10-1$ & SW-846-8260B/5035 & 10 \\
\hline VOC & Acetone & $67-64-17$ & SW-846-8260B/5035 & 10 \\
\hline VOC & Acetonitrile & $75-05-8$ & SW-846-8260B/5035 & 10 \\
\hline VOC & Acrolein & $107-02-8$ & SW-846-8260B/5035 & 10 \\
\hline VOC & Acrylonitrile & $107-13-1$ & SW-846-8260B/5035 & 10 \\
\hline VOC & Benzene & $71-43-2$ & SW-846-8260B/5035 & 10 \\
\hline VOC & Bromobenzene & $108-86-1$ & SW-846-8260B/5035 & 10 \\
\hline VOC & Bromochloromethane & $74-97-5$ & SW-846-8260B/5035 & 10 \\
\hline VOC & Bromodichloromethane & $75-27-4$ & SW-846-8260B/5035 & 10 \\
\hline VOC & Bromoform & $75-25-2$ & SW-846-8260B/5035 & 10 \\
\hline VOC & Bromomethane & 74-83-9 & SW-846-8260B/5035 & 10 \\
\hline VOC & n-Butylbenzene & $104-51-8$ & SW-846-8260B/5035 & 10 \\
\hline VOC & Carbon disulfide & $75-15-0$ & SW-846-8260B/5035 & 10 \\
\hline VOC & Carbon tetrachloride & $56-23-5$ & SW-846-8260B/5035 & 10 \\
\hline VOC & Chlorobenzene & $108-90-7$ & SW-846-8260B/5035 & 10 \\
\hline VOC & Chloroethane & $75-00-3$ & SW-846-8260B/5035 & 10 \\
\hline VOC & Chloroform & $67-66-3$ & SW-846-8260B/5035 & 10 \\
\hline VOC & Chloromethane & $74-87-3$ & SW-846-8260B/5035 & 10 \\
\hline VOC & cis-1,2-Dichloroethene & $156-59-2$ & SW-846-8260B/5035 & 10 \\
\hline VOC & cis-1,3-Dichloropropene & 10061-01-5 & SW-846-8260B/5035 & 10 \\
\hline VOC & Dibromochloromethane & $124-48-1$ & SW-846-8260B/5035 & 10 \\
\hline VOC & Dibromomethane & $74-95-3$ & SW-846-8260B/5035 & 10 \\
\hline VOC & Dichlorodifluoromethane & $75-71-8$ & SW-846-8260B/5035 & 10 \\
\hline
\end{tabular}


Table 4.3. Analytical Methods and Target Detection Limits for Non-radiological SRCs

\begin{tabular}{|c|c|c|c|c|}
\hline $\begin{array}{c}\text { Analysis } \\
\text { type }\end{array}$ & Parameter & CAS number & Analytical method & $\begin{array}{l}\text { Project reporting level } \\
\text { (MDA or MDL) } \\
\text { (total metals }=\mathrm{mg} / \mathrm{kg} \\
\text { total organics }=\mu \mathrm{g} / \mathrm{kg} \\
\text { TCLP }=\mathrm{mg} / \mathrm{L} \text { ) } \\
\end{array}$ \\
\hline VOC & Ethyl benzene & $100-41-4$ & SW-846-8260B/5035 & 10 \\
\hline VOC & Hexachloroethane & $67-72-1$ & SW-846-8260B/5035 & 10 \\
\hline VOC & Isopropylbenzene & $98-82-8$ & SW-846-8260B/5035 & 10 \\
\hline VOC & m,p-Xylene & NS831 & SW-846-8260B/5035 & 10 \\
\hline VOC & Methanolc $^{c}$ & $67-56-1$ & SW-846- 8015C / 5035 & 50 \\
\hline VOC & Methyl Methacrylate & $80-62-6$ & SW-846-8260B/5035 & 10 \\
\hline VOC & Methylcyclohexane & $108-87-2$ & SW-846-8260B/5035 & 10 \\
\hline VOC & Methylene chloride & $75-09-2$ & SW-846-8260B/5035 & 10 \\
\hline VOC & $\mathrm{n}-\mathrm{Hexane}^{\mathrm{a}}$ & $110-54-3$ & $\begin{array}{c}\text { SW-846-8260B or } 8015 \mathrm{C} \\
/ 5035\end{array}$ & 20 \\
\hline VOC & n-Propylbenzene & $103-65-1$ & SW-846-8260B/5035 & 10 \\
\hline VOC & o-Xylene & $95-47-6$ & SW-846-8260B/5035 & 10 \\
\hline VOC & sec-Butylbenzene & $135-98-8$ & SW-846-8260B/5035 & 10 \\
\hline VOC & Styrene & $100-42-5$ & SW-846-8260B/5035 & 10 \\
\hline VOC & tert-butylbenzene & 98-06-6 & SW-846-8260B/5035 & 10 \\
\hline VOC & Tetrachloroethene & $127-18-4$ & SW-846-8260B/5035 & 10 \\
\hline VOC & Toluene & $108-88-3$ & SW-846-8260B/5035 & 10 \\
\hline VOC & trans-1,2-Dichloroethene & $156-60-5$ & SW-846-8260B/5035 & 10 \\
\hline VOC & trans-1,3-Dichloropropene & $10061-02-6$ & SW-846-8260B/5035 & 10 \\
\hline VOC & Trichloroethene & 79-01-6 & SW-846-8260B/5035 & 10 \\
\hline VOC & Trichlorofluoromethane & $75-69-4$ & SW-846-8260B/5035 & 10 \\
\hline VOC & Vinyl chloride & $75-01-4$ & SW-846-8260B/5035 & 10 \\
\hline VOC & Xylenes (total) & $1330-2-7$ & SW-846-8260B/5035 & 10 \\
\hline PEST & $4,4^{\prime}-\mathrm{DDD}$ & $72-54-8$ & SW-846-8081B & 1.7 \\
\hline PEST & 4,4'-DDE & $72-55-9$ & SW-846-8081B & 1.7 \\
\hline PEST & 4,4'-DDT & $50-29-3$ & SW-846-8081B & 1.7 \\
\hline PEST & Aldrin & $309-00-2$ & SW-846-8081B & 1.7 \\
\hline PEST & alpha-BHC & $319-84-6$ & SW-846-8081B & 1.7 \\
\hline PEST & alpha-Chlordane & 5103-71-9 & SW-846-8081B & 1.7 \\
\hline PEST & beta-BHC & $319-85-7$ & SW-846-8081B & 1.7 \\
\hline PEST & delta-BHC & $319-86-8$ & SW-846-8081B & 1.7 \\
\hline PEST & Dieldrin & $60-57-1$ & SW-846-8081B & 1.7 \\
\hline PEST & Endosulfan I & 959-98-8 & SW-846-8081B & 1.7 \\
\hline PEST & Endosulfan II & $33213-65-9$ & SW-846-8081B & 1.7 \\
\hline PEST & Endosulfan sulfate & $1031-07-8$ & SW-846-8081B & 1.7 \\
\hline PEST & Endrin & $72-20-8$ & SW-846-8081B & 1.7 \\
\hline PEST & Endrin aldehyde & $7421-93-4$ & SW-846-8081B & 1.7 \\
\hline PEST & Endrin ketone & $53494-70-5$ & SW-846-8081B & 1.7 \\
\hline PEST & gamma-BHC (Lindane) & 58-89-9 & SW-846-8081B & 1.7 \\
\hline PEST & gamma-Chlordane & $5103-74-2$ & SW-846-8081B & 1.7 \\
\hline PEST & Heptachlor & $76-44-8$ & SW-846-8081B & 1.7 \\
\hline PEST & Heptachlor epoxide & $1024-57-3$ & SW-846-8081B & 1.7 \\
\hline PEST & Methoxychlor & $72-43-5$ & SW-846-8081B & 17 \\
\hline
\end{tabular}


Table 4.3. Analytical Methods and Target Detection Limits for Non-radiological SRCs

\begin{tabular}{|c|c|c|c|c|}
\hline $\begin{array}{c}\text { Analysis } \\
\text { type }\end{array}$ & Parameter & CAS number & Analytical method & $\begin{array}{l}\text { Project reporting level } \\
\text { (MDA or MDL) } \\
\text { (total metals }=\mathrm{mg} / \mathrm{kg} \\
\text { total organics }=\mu \mathrm{g} / \mathrm{kg} \\
\text { TCLP }=\mathrm{mg} / \mathrm{L} \text { ) }\end{array}$ \\
\hline PEST & Toxaphene & $8001-35-2$ & SW-846-8081B & 170 \\
\hline HERB & $2,4-\mathrm{D}$ & $94-75-7$ & SW-846-8051A & 1.7 \\
\hline HERB & 2,4,5-TP (Silvex) & $93-72-1$ & SW-846-8051A & 1.7 \\
\hline PCB & Arochlor-1016 & $12674-11-2$ & SW-846-8082A & 33 \\
\hline PCB & Arochlor-1221 & $11104-28-2$ & SW-846-8082A & 33 \\
\hline PCB & Arochlor-1232 & $11141-16-5$ & SW-846-8082A & 33 \\
\hline PCB & Arochlor-1242 & 53469-21-9 & SW-846-8082A & 33 \\
\hline PCB & Arochlor-1248 & $12672-29-6$ & SW-846-8082A & 33 \\
\hline PCB & Arochlor-1254 & 11097-69-1 & SW-846-8082A & 33 \\
\hline PCB & Arochlor-1260 & $11096-82-5$ & SW-846-8082A & 33 \\
\hline PCB & Arochlor-1262 & $11096-82-5$ & SW-846-8082A & 33 \\
\hline PCB & Arochlor-1268 & $11096-82-5$ & SW-846-8082A & 33 \\
\hline PCB & Total PCBs & Total PCBs & SW-846-8082A TPCB & 33 \\
\hline METAL & Aluminum & 7429-90-5 & SW-846-6010 & 4 \\
\hline METAL & Antimony & $7440-36-0$ & SW-846-6010 & 1.5 \\
\hline METAL & Arsenic & $7440-38-2$ & SW-846-6010 & 0.4 \\
\hline METAL & Barium & $7440-39-3$ & SW-846-6010 & 0.2 \\
\hline METAL & Beryllium & $7440-41-7$ & SW-846-6010 & 0.1 \\
\hline METAL & Boron & $7440-42-8$ & SW-846-6010 & 0.1 \\
\hline METAL & Cadmium & 7440-43-9 & SW-846-6010 & 0.2 \\
\hline METAL & Calcium & $7440-70-2$ & SW-846-6010 & 10 \\
\hline METAL & Chromium & $7440-47-3$ & SW-846-6010 & 0.5 \\
\hline METAL & Cobalt & $7440-48-4$ & SW-846-6010 & 0.2 \\
\hline METAL & Copper & $7440-50-8$ & SW-846-6010 & 0.2 \\
\hline METAL & Iron & $7439-89-6$ & SW-846-6010 & 2 \\
\hline METAL & Lead & 7439-92-1 & SW-846-6010 & 1 \\
\hline METAL & Lithium & $7439-93-2$ & SW-846-6010 & 1 \\
\hline METAL & Magnesium & 7439-95-4 & SW-846-6010 & 5 \\
\hline METAL & Manganese & $7439-96-5$ & SW-846-6010 & 0.2 \\
\hline METAL & Mercury & 7439-97-6 & SW-846-7471A & 0.04 \\
\hline METAL & Molybdenum & 7439-98-7 & SW-846-6010 & 1 \\
\hline METAL & Nickel & $7440-02-0$ & SW-846-6010 & 0.2 \\
\hline METAL & Potassium & $7440-09-7$ & SW-846-6010 & 10 \\
\hline METAL & Selenium & $7782-49-2$ & SW-846-6010 & 0.5 \\
\hline METAL & Silver & $7440-22-4$ & SW-846-6010 & 0.4 \\
\hline METAL & Sodium & $7440-23-5$ & SW-846-6010 & 25 \\
\hline METAL & Strontium & $7440-24-6$ & SW-846-6010 & 1 \\
\hline METAL & Thallium & $7440-28-0$ & SW-846-6010 & 0.5 \\
\hline METAL & Tin & $7440-31-5$ & SW-846-6010 & 1 \\
\hline METAL & Vanadium & $7440-62-2$ & SW-846-6010 & 1 \\
\hline METAL & Zinc & $7440-66-6$ & SW-846-6010 & 0.5 \\
\hline TCLPMET & Arsenic & $744-38-2$ & SW-846-1311/6020 & See note c \\
\hline TCLPMET & Barium & $7440-39-3$ & SW-846-1311/6020 & See note c \\
\hline
\end{tabular}


Table 4.3. Analytical Methods and Target Detection Limits for Non-radiological SRCs

\begin{tabular}{|c|c|c|c|c|}
\hline $\begin{array}{c}\text { Analysis } \\
\text { type }\end{array}$ & Parameter & CAS number & Analytical method & $\begin{array}{l}\text { Project reporting level } \\
\text { (MDA or MDL) } \\
\text { (total metals }=\mathrm{mg} / \mathrm{kg} \\
\text { total organics }=\mu \mathrm{g} / \mathrm{kg} \\
\text { TCLP }=\mathrm{mg} / \mathrm{L})\end{array}$ \\
\hline TCLPMET & Cadmium & $7440-43-9$ & SW-846-1311/6020 & See note $\mathrm{c}$ \\
\hline TCLPMET & Chromium & $7440-47-3$ & SW-846-1311/6020 & See note c \\
\hline TCLPMET & Lead & $7439-92-1$ & SW-846-1311/6020 & See note c \\
\hline TCLPMET & Mercury & 7439-97-6 & SW-846-1311/7470A & See note c \\
\hline TCLPMET & Selenium & $7782-49-2$ & SW-846-1311/6020 & See note c \\
\hline TCLPMET & Silver & $7440-22-4$ & SW-846-1311/6020 & See note c \\
\hline TCLPVOC & Benzene & $71-43-2$ & SW-846-1311/8260B & See note c \\
\hline TCLPVOC & Carbon tetrachloride & $56-23-5$ & SW-846-1311/8260B & See note c \\
\hline TCLPVOC & Chlorobenzene & $108-90-7$ & SW-846-1311/8260B & See note c \\
\hline TCLPVOC & Chloroform & $67-66-3$ & SW-846-1311/8260B & See note c \\
\hline TCLPVOC & 1,4-Dichlorobenzene & $106-46-7$ & SW-846-1311/8260B & See note c \\
\hline TCLPVOC & 1,2-Dichloroethane & $107-06-2$ & SW-846-1311/8260B & See note c \\
\hline TCLPVOC & 1,1-Dichloroethylene & $75-35-4$ & SW-846-1311/8260B & See note c \\
\hline TCLPVOC & Methyl ethyl ketone & $78-93-3$ & SW-846-1311/8260B & See note c \\
\hline TCLPVOC & Tetrachloroethylene & $127-18-4$ & SW-846-1311/8260B & See note c \\
\hline TCLPVOC & Trichloroethylene & 79-01-6 & SW-846-1311/8260B & See note c \\
\hline TCLPVOC & Vinyl chloride & $75-01-4$ & SW-846-1311/8260B & See note c \\
\hline TCLPSVOC & 2,4-Dinitrotoluene & $121-14-2$ & SW-846-1311/8270D & See note c \\
\hline TCLPSVOC & Hexachlorobenzene & $118-74-1$ & SW-846-1311/8270D & See note c \\
\hline TCLPSVOC & Hexachlorobutadiene & $87-68-3$ & SW-846-1311/8270D & See note c \\
\hline TCLPSVOC & Hexachloroethane & $67-72-1$ & SW-846-1311/8270D & See note c \\
\hline TCLPSVOC & Nitrobenzene & $98-95-3$ & SW-846-1311/8270D & See note c \\
\hline TCLPSVOC & o-Cresol & $95-48-7$ & SW-846-1311/8270D & See note c \\
\hline TCLPSVOC & $\mathrm{m}-\mathrm{Cresol}^{\mathrm{a}}$ & $108-39-4$ & SW-846-1311/8270D & See note c \\
\hline TCLPSVOC & p-Cresola & $106-44-5$ & SW-846-1311/8270D & See note c \\
\hline TCLPSVOC & Pyridine & $110-86-1$ & SW-846-1311/8270D & See note c \\
\hline TCLPSVOC & Pentachlorophenol & $87-86-5$ & SW-846-1311/8270D & See note c \\
\hline TCLPSVOC & 2,4,5-Trichlorophenol & $95-95-4$ & SW-846-1311/8270D & See note c \\
\hline TCLPSVOC & 2,4,6-Trichlorophenol & $88-06-2$ & SW-846-1311/8270D & See note c \\
\hline TCLPPEST & Chlordane & $57-74-9$ & SW-846-1311/8081B & See note c \\
\hline TCLPPEST & Endrin & $72-20-8$ & SW-846-1311/8081B & See note c \\
\hline TCLPPEST & Heptachlor (and its epoxide) & $76-44-8$ & SW-846-1311/8081B & See note c \\
\hline TCLPPEST & Lindane & 58-89-9 & SW-846-1311/8081B & See note c \\
\hline TCLPPEST & Methoxychlor & $72-43-5$ & SW-846-1311/8081B & See note c \\
\hline TCLPPEST & Toxaphene & $8001-35-2$ & SW-846-1311/8081B & See note c \\
\hline TCLPHERB & $2,4-\mathrm{D}$ & $94-75-7$ & SW-846-1311/8051A & See note c \\
\hline TCLPHERB & 2,4,5-TP (Silvex) & $93-72-1$ & SW-846-1311/8051A & See note c \\
\hline
\end{tabular}

a3- and 4- Methylphenols were reported together.

b'The laboratory indicated that this analysis had to be run by $8015 \mathrm{C}$.

'Reporting levels for TCLP metals and TCLP organics are one-tenth the regulatory limit where possible, and at least

$<$ the regulatory limit.

CAS $=$ Chemical Abstracts Service registry number

HERB $=$ herbicide 
$\mathrm{MDA}=$ minimum detectable activity

MDL $=$ method detection limit

$\mathrm{PCB}=$ polychlorinated biphenyls

$\mathrm{PEST}=$ pesticide

SVOC $=$ volatile organic compound

TCLP = toxicity characteristic leaching procedure

VOC $=$ volatile organic compound

\section{DEVIATIONS}

Departures from the procedures or sampling design as specified by the SAP were documented in the deviation log provided in Table 5.1. This included any change in the methodology, requirements, or commitments that would potentially impact sample or data quality. Deviations were also required if employee and/or environmental safety could have been negatively or significantly impacted.

Deviations that were identified required and received authorization by the ORAU Project Manager or designee. The ORAU Project Manager was required per the SAP to notify EM-OR of deviations and outcome. 


\section{ORA}

Table 5.1 Process Inventory Waste SAP 3 Deviations Records Form

\begin{tabular}{|c|c|c|c|c|c|c|c|}
\hline No. & Description & $\begin{array}{c}\text { Date } \\
\text { Reported }\end{array}$ & Facility & $\begin{array}{c}\text { Priority } \\
(\mathrm{H} / \mathrm{M} / \mathrm{L})\end{array}$ & Reported by & Resolution/Comment & $\begin{array}{c}\text { ORAU } \\
\text { Concurrence }\end{array}$ \\
\hline 1 & $\begin{array}{l}\text { SAP } 3 \text { items planned to be } \\
\text { surveyed but comprised of lead } \\
\text { were not sampled. }\end{array}$ & $8 / 19 / 2013$ & $\begin{array}{l}3001,3010 \\
\text { Annex, 3042, } \\
\text { 7500, W- } \\
19 / 20,3597 \\
4507,3026-C\end{array}$ & M & E. Harpenau & $\begin{array}{l}\text { Items were surveyed and smeared } \\
\text { for removable contamination. }\end{array}$ & P. Weaver \\
\hline 2 & $\begin{array}{l}\text { HEPA filters located inside a } \\
\text { Radiation Area. ESH controls } \\
\text { prevented field team from } \\
\text { working in RAs and HRAs. }\end{array}$ & $9 / 4 / 2013$ & 3029 & $\mathrm{H}$ & E. Harpenau & $\begin{array}{l}\text { Items were not surveyed or } \\
\text { sampled. Contractor reported } \\
\text { having nondestructive assay data on } \\
\text { the filter houses. }\end{array}$ & D. King \\
\hline 3 & $\begin{array}{l}\text { Container X10C0 } 401770 \text { added } \\
\text { to the list of items at the request } \\
\text { of UCOR. }\end{array}$ & $9 / 18 / 2013$ & $3505 \mathrm{Pad}$ & $\mathrm{L}$ & E. Harpenau & $\begin{array}{l}\text { Item contained absorbent material. } \\
\text { Surveyed and smeared but not } \\
\text { sampled. }\end{array}$ & P. Weaver \\
\hline 4 & $\begin{array}{l}\text { Only total metals results for the } \\
8 \text { RCRA metals were requested } \\
\text { by SMO instead of the full list of } \\
\text { metals requested for analysis. It } \\
\text { appears that an artifact from a } \\
\text { previous sample submittal was } \\
\text { used by the SMO in the } \\
\text { instructions to the contracted } \\
\text { laboratory. }\end{array}$ & $11 / 12 / 2013$ & $\mathrm{~N} / \mathrm{A}$ & $\mathrm{H}$ & J. Lee & $\begin{array}{l}\text { The SMO was requested to provide } \\
\text { the analytical results for the } \\
\text { remaining total metals. The } \\
\text { contracted laboratory indicated that } \\
\text { they would need to charge a } \\
\text { reprocessing fee to pull the } \\
\text { additional data. ORAU determined } \\
\text { that funding would not support this } \\
\text { effort and requested the analytical } \\
\text { laboratory not provide the } \\
\text { additional total metals data. }\end{array}$ & D. Maldonado \\
\hline 5 & $\begin{array}{l}\text { Lithium analysis was not } \\
\text { conducted for any of the } 51 \\
\text { samples provided to the SMO } \\
\text { approved laboratory. }\end{array}$ & & & & & $\begin{array}{l}\text { ORAU instructed the SMO to not } \\
\text { perform additional analysis for } \\
\text { lithium. }\end{array}$ & \\
\hline
\end{tabular}




\section{ANALYTICAL SUMMARY}

The analytical summary results reports are provided in Appendix E. Characterization activities included the following data collection modes: visual inspection, radiological survey, direct measurement, and removable activity and volumetric sampling. All analytical results are available in the Project Environmental Measurements System (PEMS) under the project name "ORNL ISO," are managed and maintained by the SMO, and have been provided electronically to key UCOR S\&M Project representatives. The following data files are provided:

- Graphical representation of summary statistics of analyte concentrations, Appendix E.

- Sample Data Graphs: Data are represented by sample number. Herbicides are not shown by separate graphs as there are only two compounds that were identified.

- Data Summary Statistics: The summary statistics are represented by container per analyte.

\section{REFERENCES}

DOE 2012. Waste Handling Plan for Surveillance and Maintenance Activities at the Oak Ridge National Laboratory, Oak Ridge, Tennessee. DOE/OR/01-2565\&D2. U.S. Department of Energy, Office of Environmental Management. Oak Ridge, Tennessee.

EPA 2006. Guidance on Systematic Planning Using the Data Quality Objectives Process. EPA QA/G-4, EPA/240/B-06/001. U.S. Environmental Protection Agency. Washington, D.C.

ORAU 2013a. Quality Program Manual for the Independent Environmental Assessment and Verification Program. Prepared by Oak Ridge Associated Universities under the Oak Ridge Institute for Science and Education contract. Oak Ridge, Tennessee. August 15.

ORAU 2013b. Sampling and Analysis Plan for Characterizing Miscellaneous Process Inventory Waste Items From Oak Ridge National Laboratory Surveillance And Maintenance Project, Oak Ridge, Tennessee. Prepared by Oak Ridge Associated Universities under the Oak Ridge Institute for Science and Education contract. August 15.

ORAU/ORISE 2013a. Survey Procedures Manual for the Independent Environmental Assessment and Verification Program. Prepared by Oak Ridge Associated Universities under the Oak Ridge Institute for Science and Education contract. Oak Ridge, Tennessee. January 18.

ORAU/ORISE 2013b. Laboratory Procedures Manual for the Independent Environmental Assessment and Verification Program. Prepared by Oak Ridge Associated Universities under the Oak Ridge Institute for Science and Education contract. Oak Ridge, Tennessee. August 15. 
APPENDIX A

PROCESS KNOWLEDGE SUMMARIES FOR FACILITIES ASSOCIATED WITH OAK RIDGE NATIONAL LABORATORY SURVEILLANCE AND MAINTENANCE PROJECT WASTE ITEMS 


\section{PROCESS KNOWLEDGE SUMMARIES FOR FACILITIES ASSOCIATED WITH OAK RIDGE NATIONAL LABORATORY SURVEILLANCE AND MAINTENANCE PROJECT WASTE ITEMS}

The following presents a brief overview of how each facility is tied to the target subpopulation, plus the facility-specific site related contaminants (SRCs), when available, which will be used to build the analyte list for this investigation as well as to prepare a Listed Waste Determination for the anticipated waste stream.

Bldg. 2026 - Radioactive Materials Analytical Laboratory. Building 2026, constructed in 1964 (with additions in 1966 and 1985), provides laboratory and office space dedicated to the application of general analytical chemistry of radioactive materials. The building has special containment and ventilation systems to handle radioactive materials with high gamma dose rates (hot cells) and high levels of alpha radiation-bearing material (glovebox system). It houses facilities designed to segregate low and high levels of radioactivity. In addition, it includes a radioactive liquid waste system that meets current regulatory requirements. It is equipped to handle the packaging and disposal of radioactive solid waste. A personnel contamination incident occurred in Bldg. 2026 on October 6, 2003, when a waste drain overflowed due to a clogged line (ORAU 2006). The broad suite of radiological SRCs is assumed including fission products, uranium, thorium, and transuranics.

Bldg. 3001 (formerly Bldg. 105) - Graphite Reactor Building (Clinton Pile). Building 3001 operated from November 4, 1943 to November 1963. The reactor was designed and built as a pilot plant to test the control and operating procedures of proposed larger production reactors and to provide needed quantities of plutonium for the Manhattan Engineer District for testing. Natural uranium fuel slugs were inserted in the reactor through holes in the front face into horizontal channels in the graphite matrix. The ORNL plutonium production mission was accomplished by the end of 1944. Ruptured fuel slugs, allowed to oxidize in the transfer canal, were the major source of contamination and dose in the reactor building. Irradiated fuel slugs were moved underwater to the separations building (currently Bldg. 3019, originally 205) after a cooling period to allow decay of short-lived radioisotopes. Elevated levels of Co-60 and Cs-137 were reported in the canal waters in 1954. Researchers later discovered that particulate contamination became elevated in the airborne effluent stream when fuel slugs pushed out the back of the reactor would fall and strike the mattress plates prior to being submerged underwater. The impact would sometimes breach or further breach 
the aluminum cladding of the slugs, resulting in a release of particulate matter to the air. The large building ventilation fans would entrain these particles and exhaust them out the stack. Therefore, concentrations of gaseous wastes such as noble gases (Ar-41 and several xenon/krypton isotopes) and radioiodines were not filtered or quantified (ORAU 2006).

Bldg. 3010 - Bulk Shielding Reactor Facility (BSR). The BSR was a pool-type reactor originally designed to operate at a power level of $10 \mathrm{~kW}$ for conducting radiation shielding experiments for the Aircraft Nuclear Propulsion (ANP) Project. After the ANP Project, the facility was used by the Space Program to determine the effects of radiation on space capsules for the Mercury Project. In 1960, the Low-Temperature Irradiation Facility was installed to test the effects of radiation on metals and other materials at low temperatures. It also was used by a number of organizations for isotope production, materials irradiation, radiation shielding experiments, and training for reactor operators and students. It served as a model for university, industry, and government pool reactors. It began operating in 1951 for shielding experiments. In 1963, it was made available to the site as a general research reactor due to the shutdown of the Graphite Reactor. The BSR was permanently shut down on September 10,1991. Modifications to the reactor allowed it to run at a thermal power level of $2 \mathrm{MW}$ when it was designated a general research reactor. The aluminum-clad, uraniumaluminum alloy fuel appears to have been highly enriched, similar to that used in the Materials Test Reactor (ORAU 2006).

The Pool Critical Assembly (PCA) is in the northwest corner of the same pool as the BSR. It was designed as a low-power reactor (up to $10 \mathrm{~kW}$ ) to supplement experiments that took place at other site reactors. The reactor controls were similar to those for the BSR. The PCA was used to train nuclear engineering students from around the country (ORAU 2006).

The BSR pool previously contained 73 irradiated fuel elements; 32 were uranium silicide with lowenriched uranium. The other 41 elements were highly-enriched uranium with either uranium-dioxide or uranium-aluminum alloy fuel. Three radioactive sealed sources (Co-60, Ra-226, and Sb-124) were suspended in the pool using wires but were all removed by 2003 (BJC 2007). The primary SRCs are assumed to be Co-60, Sr-90, Cs-137, U-235, U-238, and Am-241.

\section{Bldg. 3026-C and 3026-D (formerly Bldgs. 70-C and 706-D) - By-Product Processing and} Chemical Separations Laboratory. In mid-1942, radiological research was directed at small-scale 
studies of different radioisotopes in the Metallurgical Laboratory at the University of Chicago. While most of the research focused on the production of fissionable material for use in weapons manufacturing, some was on other radioisotopes (i.e., activation and fission products) to determine their potential value to the war effort. While the Graphite Reactor was still being conceptualized, special attention was given to barium, strontium, and lanthanum separation chemistry. Although initially purified Ba-140 had little gamma activity, La-140, which grew into the purified Ba-140, was of great interest due to its $1.6-\mathrm{MeV}$ gamma ray and high specific activity. The program for the separation and production of radioactive lanthanum was called the radioactive lanthanum (RaLa) Project. In the fall of 1943, much of the Metallurgical Laboratory moved to ORNL to continue the research on fission products. This research began in Bldg. 3550 (706-A); on October 21, 1943, there was a request to construct a larger production facility. Building 3026-C (706-C) was constructed with larger containers and equipment in 1944, and the new facility was placed in operation; its initial production run began on September 10, 1944 (ORAU 2006).

The 3026-C facility was designed to handle 1- to 10-Ci amounts of radioactivity but, due to time constraints and unusual circumstances, at startup it was producing greater than $100 \mathrm{Ci}$ amounts. The facility processed irradiated slugs from the Graphite Reactor. In addition, nine production runs of RaLa were completed for shipment to Los Alamos, for a total of 3852 Ci. (Multiple batches of irradiated slugs were dissolved to make up a completed run.) The last run in Bldg. 3026-C was on May 28, 1945. During the initial lower activity production runs, spills, plugged lines, low chemical yields (requiring dissolution of additional batches of slugs), and failed equipment affected the completion of runs (ORAU 2006).

After the second successful RaLa run in Bldg. 3026-C, discussions indicated the need to increase production rates further. Although the use of existing facilities in the Pilot Plant (Bldg. 3019) was discussed and evaluated, it was determined that a new facility annexed to the current facility would be needed. Building 3026-D (706-D), which was designed to process much larger (up to 1,000 Ci) amounts of radioactivity, was attached to the east end of the 3026-C facility. As with the construction of Bldg. 3026-C, time constraints caused design and development work to coincide with facility construction. The first production run of materials occurred on May 26, 1945, as the ninth and final run of RaLa was completed in Bldg. 3026-C. Building 3026-D processed much higher activities than those designed for the facility (ORAU 2006). 
Around 1948, the AEC decided to use irradiated slugs from Hanford production reactors as feed for RaLa. The higher power levels of the Hanford reactors (in relation to those of the Graphite Reactor) meant production of much larger quantities of RaLa in a shorter time, even with shipping the slugs across the country. A total of 68 RaLa runs had been completed at ORNL when operations ended in October 1956. The RaLa program was transferred to the Idaho Chemical Processing Plant (ORAU 2006).

Due to the relatively short half-life of the parent Ba-140, the freshly irradiated fuel slugs used in the RaLa process contained relatively large quantities of short-lived fission products, most notably noble gases and radioiodines (ORAU 2006).

Much of the iodine volatilized during the slug dissolving process was effectively removed from the dissolver off-gas stream by the reflux condenser and chemical scrubber in line before the gaseous waste went to the stack. Dissolver off-gas from RaLa production was vented to the 3020 stack from 1944 to 1950 and to the 3039 stack from 1950 to 1956. From 1944 to 1950, other airborne wastes were withdrawn from the building's two process cells to a 30-ft stack adjacent to the building until this stream was routed to the 3039 stack. In the later part of 1948, operations in Bldg. 3026-D were a major contributor of airborne particles on the site. Air filter houses were installed to reduce particulate material in the effluent stream before release from the building. Liquid wastes from RaLa operations went to the local tank farms (ORAU 2006).

Table A-1 presents a summary of facility operations from construction to the end of programmatic activities in the mid-1990s. Most of the building was demolished in 2010, through the substructure and hot cells remain under a weather-proof coating.

The following isotopes would have been in the Bldg. 3026-C and -D complex: I-129, I-131, Se-79, Ru/Rh-106, Pd-107, Pm-147, Cs-137, Ba/La-140, Sr-90, Kr-85, W-188, Os-191, Xe-133, actinides, uranium, Tc-99, Co-60, plutonium, and H-3 (ORAU 2006). 


\begin{tabular}{|c|l|l|}
\hline \multicolumn{3}{|c|}{ Table A-1. Summary of Bldg. 3026 Historical Operations } \\
\hline Time Period ${ }^{a}$ & \multicolumn{1}{|c|}{ Bldg. 3026-C } & \multicolumn{1}{c|}{ Bldg. 3026-C } \\
\hline $1940 s$ & $\begin{array}{l}\text { Facility constructed; processed irradiated } \\
\text { reactor targets for research isotopes }\end{array}$ & $\begin{array}{l}\text { Facility constructed; processed irradiated } \\
\text { reactor targets for research isotopes }\end{array}$ \\
\hline 1950 s & $\begin{array}{l}\text { Fission product recovery of liquid } \\
\text { wastes }\end{array}$ & Fission product recovery of liquid wastes \\
\hline $1960 s$ & $\begin{array}{l}\text { Segmenting of reactor fuel; hot } \\
\text { metallographic work }\end{array}$ & $\begin{array}{l}\text { Segmenting of reactor fuel; hot metallographic } \\
\text { work }\end{array}$ \\
\hline 1970 s and 1980s & $\begin{array}{l}\text { Tritium facility packaged, stored and } \\
\text { tested radio-luminescent lights }\end{array}$ & $\begin{array}{l}\text { Tritium facility packaged, stored and tested } \\
\text { radio-luminescent lights }\end{array}$ \\
\hline $1990-1995$ & Facility inactive & Facility inactive \\
\hline 2010 & Demolition & Demolition \\
\hline
\end{tabular}

Bldg. 3029 (formerly Bldg. 909) Source Development Laboratory. Building 3029 was constructed in 1952 to support the Isotopes Program. A small manipulator cell (now called Cell 4) and a system of remotely operated barricades supported source fabrication of Ir-192. In addition, small Co-60 sources were fabricated. In 1955 and 1956, an additional cell (Cell 1) was built to handle large amounts of Co-60 for source fabrication. The amount of Co-60 sources fabricated in the facility diminished in the late 1950s. Between 1960 and 1962, Cell 3 was built for Cs-137 source fabrication. Cell 2, which is between Cells 1 and 3, handled waste and served as a pass-through between the two cells. Major operations in Bldg. 3029 included Co-60, Cs-137, and Sr-90 source fabrication and Ir-192 processing. A Co-60 storage and irradiation facility, called the Co-60 Garden, is below the floor of the east wing. The exact dates of operation of this facility are unknown, but the program ended in the late 1980s (ORAU 2006).

\section{Bldg. 3038 (formerly Bldg. 902) - Isotope Research Materials Laboratory, Radioisotope} Analytical and Packing Building. Building 3038 was used from 1949 until 1990 for packaging, inspection, and shipping activities involving radioisotopes. It contained five hot cells shielded by water-filled steel tanks. The operating face of each cell consisted of three feet of shielding, a viewing window, and manipulator ports. Each cell was 10 feet deep. From 1968 until 1990, the western portion, referred to as the Alpha Handling Facility (AHF), was used for the fabrication of targets. The center portion (referred to as 3038-M) contained radioactive isotope shipping operations. The east end (3038-E) contained analytical chemistry laboratories that supported isotope shipping 
activities. This area was converted to an isotope production and development facility and was used in the late 1970s and early 1980s to support research activities involving plutonium alloys and compounds. In the mid-1980s, gloveboxes in this area were used for research on Pm-147 doped crystals. The northwest corner (3038-W) contained a storage area and an isotope technology low-level laboratory. Isotopes formerly stored, used, or produced at the facility include the following (ORAU 2006):

- 3038-AHF: Cf-252, Cm-244, Am-241, Pu-238, U-234, Np-237, Pa-231, and others

- 3038-E: transuranics (specifically including, but not necessarily limited to, Cm-244 and Am-241), Y-90, U-235, Pm-147, and Sr-90

- 3038-M: I-129, Cs-137, Co-60, Sr-90, Tc-99, Mn-89, Ru-106, and Cl-36

Bldg. 3042 - Oak Ridge Research Reactor (ORR). Construction of the ORR was completed near the end of 1957 and criticality was attained in March 1958. The reactor was a light-watermoderated and cooled, beryllium- and water-reflected research reactor using highly enriched uranium-aluminum alloy plate fuel. The reactor was designed to produce high neutron fluxes for basic research in the fields of physics and chemistry, as well as to test materials and potential fuels for power-producing reactors. When operational, the ORR produced greater quantities of radioisotopes for research, medical, and industrial use than any reactor in the world. It contained the most advanced safety devices, including filters and scrubbers to minimize airborne releases, though reports indicate several releases from 1959 to 1961 (ORAU 2006).

Bldg. 3044 - West Complex Field Shop. Building 3044 was constructed as a materials machine shop in 1955 to support ORNL research activities. The facility was known as the "hot" machine shop, was used to make fuel elements, and was known to support work with uranium, thorium, beryllium, fiberglass, and large quantities of graphite (QC et al. 2004). The facility once had extensive radiological contamination in the ceiling and ductwork. The assumed primary contaminants are Th-232, Cs-137, and Sr-90. The facility was demolished to the slab in 2011.

Bldg. 3517 - Fission Products Pilot Plant (FPDL). Constructed in 1958, FPDL is a two-story, concrete block structure with about $14,400 \mathrm{ft}^{2}$ of floor space. The operating area consisted of nine process cells, ten manipulator and service cells, four tank farm cells, a ventilation filter pit, filtration 
housings, and several service/pipe tunnels. Two cell ventilation facilities (Bldgs. 3547 and 3548) and a storage building (Bldg. 3542) are associated with Bldg. 3517.

The FPDL was originally designed and constructed to separate kilocurie quantities of Cs-137, Sr-90, Ce-144, and Pm-147 from REDOX- and PUREX-type (liquid) waste streams. The facility was modified in 1963 to allow production of megacurie amounts of Cs-137, Sr-90, and Ce-144, primarily for use in the U.S. Atomic Energy Commission's Systems for Nuclear Auxiliary Power program. At the conclusion of the program in 1975, the facility was placed in standby, and initial decontamination efforts undertaken. Large quantities of $\mathrm{Cm}-244$ were stored in the building. A memo dated August 30, 1965, describes a contamination incident that occurred during unloading of a carrier containing strontium titanate pellets. The incident occurred on July 7, 1965, so strontium titanate was handled in Bldg. 3517 circa mid-1965. Isotopes formerly stored, used, or produced at the facility include Sr-90, Cs-137, Ce-144, Pm-147, Ru-106, Tc-99, Co-60, Ir-192, U-235, Eu-152,154, Cm-244, and Am-241 (ORAU 2006).

Bldg. 3597 - Hot Storage Garden. Building 3597, which is an annex to Bldg. 3503, was used to store fuel assemblies and other irradiated materials. Spent fuel rods from Bldg. 4607 were stored at the facility and the primary SRCs are U-232, Pa-231, Am-241, Cm-242, Cm-243, Cm-244, and isotopic plutonium (McCrotty 2013).

Bldg. 4507 - High-Level Chemical Development Facility. Building 4507 was constructed in 1957 and once housed chemical separations research on highly irradiated nuclear fuels. Small sections of irradiated fuel or prototype fuel elements would be dissolved for laboratory-scale process development research. The building consists of hot cell banks and associated charging and operating areas. Following its work with irradiated nuclear fuels, Bldg. 4507 became the Curium Recovery Facility, which tested TRU processes at high activity levels. Curium-242 was recovered from ${ }^{241} \mathrm{AmO}_{2}-\mathrm{Al}$ targets irradiated in the Materials Test Reactor and ORR. Curium Recovery Facility operations resulted in the successful isolation of about $35 \mathrm{~g}$ of Am-243 and Cm-244 and $25 \mathrm{~g}$ of Cm-242. Primary SRCs are U-232, Pa-231, Am-241, Cm-242, Cm-243, Cm-244, and isotopic plutonium (McCrotty 2013).

Bldg. 4556 - Filter Pit for Bldg. 4507. (See Bldg. 4507 summary.) Primary SRCs are U-232, Pa-231, Am-241, Cm-242, Cm-243, Cm-244, and isotopic plutonium (McCrotty 2013). 
Bldg. 7500 - Homogeneous Reactor Experiment (HRE). Building 7500 was constructed to conduct testing of homogeneous (fluidized fuel) reactors. The building initially contained the first experiment (HRE-1). A second reactor (HRE-2) took its place in 1953. The HRE-1 was an aqueous homogeneous fuel reactor. Its purpose was to prove the theory that a homogeneous reactor could generate electricity. The initial test run was completed in October 1952 and the designed power level of $1 \mathrm{MW}$ was achieved on February 24, 1953. Many experiments were performed with the HRE-1 before its shutdown and dismantling in 1954 (ORAU 2006).

The HRE-2 was built between 1953 and 1956. HRE-2, sometimes referred to as the Homogeneous Reactor Test, was a homogeneous reactor that could produce electricity and act as a breeder reactor, irradiating Th-232 source material to create U-233 for use as reactor fuel. The reactor consisted of the core and the thorium blanket. It reached criticality in 1957 and operated at normal full power of $5 \mathrm{MW}$. After 16,295 MW-hr of operation, the reactor shut down in 1961 due to a hole in the core tank, which allowed mixing of the fuel and blanket regions (ORAU 2006). The primary SRCs are assumed to be Sr-90, Cs-137, U-233, and Th-232.

Hill Cut Test Facility (HCTE). Facility 7822E, the Hill Cut Text Facility, is located in Solid Waste Storage Area 6 at ORNL. The HCTF is a waste disposal demonstration project that was started in 1981 and discontinued the following year before any wastes were placed for disposal at the site. In 1985, the project was reactivated to evaluate the use of hillside cuts as possible sites for future engineered waste disposal facilities. The objective of the project was to assess the degree of hydrologic isolation for this type of design. The facility consists of a concrete pad constructed in a cut from the side slope of a hill, 27 concrete boxes of low-level waste placed on the pad and covered with soil, and a runoff collection system. Water can reach the concrete boxes by infiltration of precipitation and shallow storm water flow. The runoff collection system collects drainage from both the pad and the gravel area surrounding the pad. Wastes were placed at the facility in August and September of 1986 and monitoring began.

Melton Valley Decontamination and Decommissioning Office Trailers. These trailers were used as office space during and after the Melton Valley hydraulic isolation and other remedial action effort around 2005. There is no history of contamination or use/storage of radiological materials at the facility, thus there are no known SRCs. 
Low-Level Waste Storage Tanks W-19 and W-20. Tanks W-19 and W-20 are 2250 gallon stainless steel tanks located north of Bldg. 3517 and east of Bldg. 3505. They were installed in 1955 to serve the Metal Recovery Facility (Bldg. 3505) and were used to collect waste produced during recovery and reprocessing of uranium and other nuclear materials associated with Bldgs. 3019 and 3517. The tanks were removed from service in 1960 and a 1998 sampling event identified a nearly dry hard heal at the bottom of each tank. Primary contaminants are isotopic uranium including U-233, Am-241, isotopic plutonium, isotopic neptunium, Cs-137, and S-90 (DOE 1999; Simpson and Emery 1981).

\section{REFERENCES}

BJC 2007. Preliminary Hazard Screening for Buildings 3010, 3009, \& 3119, Oak Ridge National Laboratory, Oak Ridge, Tennessee. PHS-OR-3010-0840. Rev. 2. July.

DOE 1999. Action Memorandum for the Bethel Valley Main Plant Inactive Liquid Low-Level Radioactive Waste Tanks, Oak Ridge National Laboratory, Oak Ridge, Tennessee. DOE/OR-01-1813\&01, April.

McCrotty 2013. Attachment in e-mail correspondence from Stephen McCrotty (Alliant) to David King (ORAU). E-mail subject: "Re: SAP 3 SRCS.” E-mail attachment: excerpt from BJC Surveillance and Maintenance RWA, Rev. 1.; a document concerning Bechtel Jacobs Corporation's surveillance and maintenance activities at Oak Ridge National Laboratory. Oak Ridge, Tennessee. June 24.

ORAU 2006. Oak Ridge National Laboratory - Site Description. ORAUT-TKBS-0012-2 Rev. 01. ORAU TEAM Dose Reconstruction Project for NIOSH. Oak Ridge, Tennessee. August 30.

D. K. Simpson, and J. F. Emery 1981 (exact year unknown), Radiological Assessment of the Decontamination and Decommissioning of a Small-Scale Fuel-Reprocessing Plant, Oak Ridge National Laboratory, Oak Ridge, Tennessee. 
APPENDIX B

POPULATIONS 4, 5, 6, 7, AND 8 SUBSET OF MISCELLANEOUS WASTE ITEMS IDENTIFIED FOR CHARACTERIZATION 


\begin{tabular}{|c|c|c|c|c|c|c|c|c|c|c|c|}
\hline 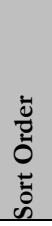 & Container & ID & Description & Origin & 5400.5 SRCs & 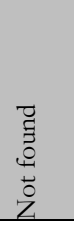 & 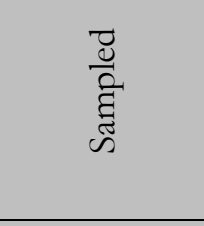 & 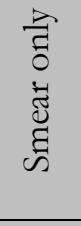 & 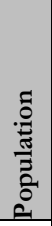 & $\mathrm{ft}^{3}$ & 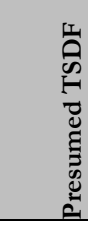 \\
\hline 1 & None & X10C0404228 & Chiller & 3517 & $\begin{array}{l}\text { None or unknown; assume } \\
\text { transuranics and beta-gamma } \\
\text { emitters }\end{array}$ & $\mathrm{X}$ & & & 6 & 25 & Y-12 \\
\hline 2 & Dumpster & UT-B\# 01-0127 & $\begin{array}{l}\text { Sanitary office } \\
\text { trash }\end{array}$ & $\begin{array}{l}\text { MV D\&D } \\
\text { office trailers }\end{array}$ & $\begin{array}{c}\text { None or unknown; assume } \\
\text { transuranics and beta-gamma } \\
\text { emitters }\end{array}$ & & No & No & 6 & 270 & Y-12 \\
\hline 3 & Dumpster & X1011999991 & $\begin{array}{l}\text { Sanitary office } \\
\text { trash }\end{array}$ & Unknown & $\begin{array}{c}\text { None or unknown; assume } \\
\text { transuranics and beta-gamma } \\
\text { emitters }\end{array}$ & & No & No & 6 & 150 & Y-12 \\
\hline 4 & B-12 & X10C0404151 & Empty B-12 & Unknown & $\begin{array}{c}\text { None or unknown; assume } \\
\text { transuranics and beta-gamma } \\
\text { emitters }\end{array}$ & & 5219M0005 & & 6 & 45 & $\mathrm{Y}-12$ \\
\hline 5 & Self & X10C0700128 & $\begin{array}{l}\text { Metal framing } \\
\quad \text { (sanitary) }\end{array}$ & Unknown & $\begin{array}{c}\text { None or unknown; assume } \\
\text { transuranics and beta-gamma } \\
\text { emitters }\end{array}$ & $\mathrm{X}$ & & & 6 & 192 & $\mathrm{Y}-12$ \\
\hline 6 & Unpackaged & K25C1100766 & $\begin{array}{l}\text { Honda pressure } \\
\text { washer }\end{array}$ & Unknown & $\begin{array}{c}\text { None or unknown; assume } \\
\text { transuranics and beta-gamma } \\
\text { emitters }\end{array}$ & & 5219M0001 & & 6 & TBD & Y-12 \\
\hline 7 & 55-gal & X10C1101241 & $\begin{array}{l}\text { Empty } 55 \text {-gal } \\
\text { drum }\end{array}$ & Unknown & $\begin{array}{c}\text { None or unknown; assume } \\
\text { transuranics and beta-gamma } \\
\text { emitters }\end{array}$ & & & Yes & 6 & TBD & $\mathrm{Y}-12$ \\
\hline 8 & Self & K25C1101517 & Toolbox & Unknown & $\begin{array}{c}\text { None or unknown; assume } \\
\text { transuranics and beta-gamma } \\
\text { emitters }\end{array}$ & & $\begin{array}{l}5219 \mathrm{M} 0002 \\
5219 \mathrm{M} 0003\end{array}$ & & 6 & TBD & Y-12 \\
\hline 9 & 55-gal & X10C1101242 & $\begin{array}{l}\text { Empty } 55 \text {-gal } \\
\text { drum }\end{array}$ & Unknown & $\begin{array}{c}\text { None or unknown; assume } \\
\text { transuranics and beta-gamma } \\
\text { emitters }\end{array}$ & & 5219M0004 & & 6 & TBD & Y-12 \\
\hline
\end{tabular}




\begin{tabular}{|c|c|c|c|c|c|c|c|c|c|c|c|}
\hline 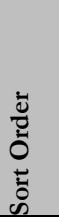 & Container & ID & Description & Origin & 5400.5 SRCs & 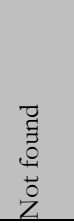 & 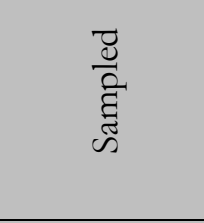 & 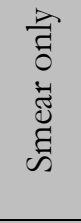 & 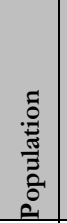 & $\mathrm{ft}^{3}$ & 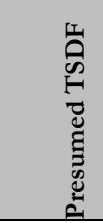 \\
\hline 10 & 55-gal & X10C0301534 & $\begin{array}{l}\text { Empty } 55 \text {-gal } \\
\text { drum }\end{array}$ & Unknown & $\begin{array}{c}\text { None or unknown; assume } \\
\text { transuranics and beta-gamma } \\
\text { emitters }\end{array}$ & $\mathrm{X}$ & & & 6 & TBD & $\mathrm{Y}-12$ \\
\hline 11 & Wood box & X10I0700529 & Cryostatic trap & 3001 & $\begin{array}{c}\text { None or unknown; assume } \\
\text { transuranics and beta-gamma } \\
\text { emitters }\end{array}$ & & & Yes & 8 & 2 & $\mathrm{Y}-12$ \\
\hline 12 & Unpackaged & None & $\begin{array}{l}\text { Duoseal vacuum } \\
\text { pump }\end{array}$ & 3001 & $\begin{array}{c}\text { None or unknown; assume } \\
\text { transuranics and beta-gamma } \\
\text { emitters }\end{array}$ & & 5219M0021 & & 8 & 2 & $\mathrm{Y}-12$ \\
\hline 13 & Unpackaged & $\mathrm{Id} \# 1411$ & Fire extinguisher & 3001 & $\begin{array}{c}\text { None or unknown; assume } \\
\text { transuranics and beta-gamma } \\
\text { emitters }\end{array}$ & & & Yes & 8 & 1 & $\mathrm{Y}-12$ \\
\hline 14 & Unpackaged & None & 10-ft Rubber hose & 3001 & $\begin{array}{c}\text { None or unknown; assume } \\
\text { transuranics and beta-gamma } \\
\text { emitters }\end{array}$ & $\mathrm{X}$ & & & 8 & 1 & $\mathrm{Y}-12$ \\
\hline 15 & Unpackaged & $\mathrm{X} 10 \mathrm{I} 0700523$ & Toledo scale & Unknown & $\begin{array}{l}\text { None; assume transuranics } \\
\text { and beta-gamma emitters }\end{array}$ & & & Yes & 8 & 3 & $\mathrm{Y}-12$ \\
\hline 16 & Unpackaged & $3042-1994-01$ & $\begin{array}{l}\text { Particle board } \\
\text { (Ceiling tile) }\end{array}$ & 3042 & $\begin{array}{c}\text { Transuranics; beta-gamma } \\
\text { emitters }\end{array}$ & & 5219M0038 & & 8 & 10 & $\mathrm{Y}-12$ \\
\hline 17 & 55-gal PL & X10C0404157 & $\begin{array}{c}\text { SS tubing and } \\
\text { glass trap } \\
\text { (Computer boards } \\
\text { found })^{\mathrm{a}}\end{array}$ & 3038 & $\begin{array}{l}\text { Th-228, Th-230, Th-232; U- } \\
\text { natural, U-235, U-238, and } \\
\text { associated decay products, } \\
\text { alpha emitters; transuranics; } \\
\text { beta-gamma emitters }\end{array}$ & & & Yes & 8 & 6 & $\mathrm{Y}-12$ \\
\hline
\end{tabular}




\begin{tabular}{|c|c|c|c|c|c|c|c|c|c|c|c|}
\hline 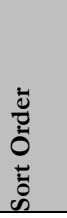 & Container & ID & Description & Origin & 5400.5 SRCs & 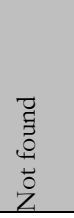 & 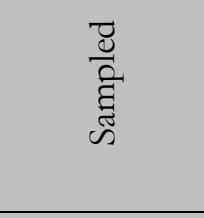 & 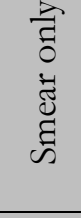 & 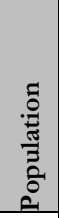 & $\mathrm{ft}^{3}$ & 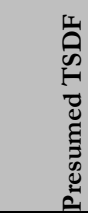 \\
\hline 18 & Wood box & 3001-Debris-03 & Backflow Device? & 3044 & $\begin{array}{c}\text { Th-natural; U-natural, U-235, } \\
\text { U-238, and associated decay } \\
\text { products, alpha emitters; } \\
\text { transuranics; beta-gamma } \\
\text { emitters }\end{array}$ & $\mathrm{X}$ & & & 8 & 20 & $\mathrm{Y}-12$ \\
\hline 19 & Pallet & None & $\begin{array}{c}\text { Lead } \\
\text { sheet/bricks }{ }^{\mathrm{a}} \\
\text { (identified3 } \\
\text { pallets: Lead } \\
\text { sheet, metal grate, } \\
\text { and concrete } \\
\text { block) }\end{array}$ & 3010 annex & $\begin{array}{l}\text { Th-natural; U-natural, U-235, } \\
\text { U-238, and associated decay } \\
\text { products, alpha emitters; } \\
\text { transurnanics; beta-gamma } \\
\text { emitters }\end{array}$ & & 5219M0037 & Yes & $\mathrm{X}$ & TBD & ES \\
\hline 20 & Loose & None & $\begin{array}{c}15 \text { lead brick } \\
\text { pieces }\end{array}$ & 3042 & $\begin{array}{c}\text { Transuranics, beta-gamma } \\
\text { emitters }\end{array}$ & & & Yes & $\mathrm{X}$ & TBD & ES \\
\hline 21 & 55-gal & X10I020315 & Lead chunks & 3042 & $\begin{array}{c}\text { Transuranics, beta-gamma } \\
\text { emitters }\end{array}$ & & & Yes & $\mathrm{X}$ & TBD & ES \\
\hline 22 & 5-gal & X10C0404164 & Lead waste & 3042 & $\begin{array}{c}\text { Transuranics, beta-gamma } \\
\text { emitters }\end{array}$ & & & Yes & $\mathrm{X}$ & TBD & ES \\
\hline 23 & 55-gal & X10C0404152 & Lead chunks & 7500 & $\begin{array}{c}\text { Th-232; U-natural, U-235, U- } \\
\text { 238, and associated decay } \\
\text { products, alpha emitters; } \\
\text { transuranics; beta-gamma } \\
\text { emitters }\end{array}$ & & & Yes & 8 & 6 & $\mathrm{ES}$ \\
\hline 24 & 55 -gal & X10C0404154 & $\begin{array}{l}\text { Contaminated } \\
\text { lead }\end{array}$ & 7500 & $\begin{array}{l}\text { Th-232; U-natural, U-235, U- } \\
\text { 238, and associated decay } \\
\text { products, alpha emitters; } \\
\text { transuranics; beta-gamma } \\
\text { emitters }\end{array}$ & & & Yes & 8 & 6 & $\mathrm{ES}$ \\
\hline
\end{tabular}




\begin{tabular}{|c|c|c|c|c|c|c|c|c|c|c|c|}
\hline 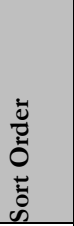 & Container & ID & Description & Origin & 5400.5 SRCs & 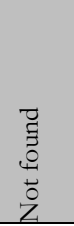 & 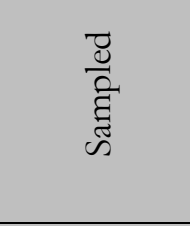 & $\begin{array}{l}\text { ते } \\
\text { ठ } \\
\text { है } \\
\text { घ̆ }\end{array}$ & 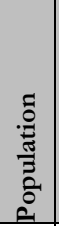 & $\mathrm{ft}^{3}$ & 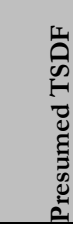 \\
\hline 25 & 30-gal & X10C0404156 & Lead pigs, carriers & 3042 & $\begin{array}{c}\text { Transuranics, beta-gamma } \\
\text { emitters }\end{array}$ & & & Yes & 8 & 3 & ES \\
\hline 26 & 85-gal & X10C0404158 & $\begin{array}{l}\text { Electrical } \\
\text { equipment } \\
\text { (Identified } \\
\text { cardboard } \\
\text { cylinder and } \\
\text { electronics) }\end{array}$ & 3038 & $\begin{array}{c}\text { Th-232; U-natural, U-235, U- } \\
\text { 238, and associated decay } \\
\text { products, alpha emitters; } \\
\text { transuranics; beta-gamma } \\
\text { emitters }\end{array}$ & & & Yes & 8 & 10 & ES \\
\hline 27 & 30-gal & X10C0404159 & $\begin{array}{l}\text { Electrical } \\
\text { equipment(Identif } \\
\text { ied cardboard } \\
\text { cylinder with } \\
\text { PPE) }\end{array}$ & 3038 & $\begin{array}{c}\text { Th-232; U-natural, U-235, U- } \\
\text { 238, and associated decay } \\
\text { products, alpha emitters; } \\
\text { transuranics; beta-gamma } \\
\text { emitters }\end{array}$ & & 5219M0019 & & 8 & 3 & $\mathrm{ES}$ \\
\hline 28 & 5-gal PL & X10C0404155 & $\begin{array}{l}\text { Electrical } \\
\text { equipment }\end{array}$ & 3038 & $\begin{array}{c}\text { Th-232; U-natural, U-235, U- } \\
\text { 238, and associated decay } \\
\text { products, alpha emitters; } \\
\text { transuranics; beta-gamma } \\
\text { emitters }\end{array}$ & $\mathrm{X}$ & & & 8 & 1 & ES \\
\hline $29^{\mathrm{b}}$ & 5-gal PL & X10C0404155 & $\begin{array}{l}\text { Electrical } \\
\text { equipment }\end{array}$ & 3038 & $\begin{array}{c}\text { Th-232; U-natural, U-235, U- } \\
\text { 238, and associated decay } \\
\text { products, alpha emitters; } \\
\text { transuranics; beta-gamma } \\
\text { emitters }\end{array}$ & & & & 8 & 20 & ES \\
\hline 30 & 30-gal & X10C0300011 & $\begin{array}{l}\text { Bagged paint } \\
\text { chips }\end{array}$ & Various & $\begin{array}{c}\text { None or unknown; assume } \\
\text { transuranics and beta-gamma } \\
\text { emitters }\end{array}$ & $\mathrm{X}$ & & & 8 & 1 & ES \\
\hline 31 & 30-gal & X10C9401325 & $\begin{array}{l}\text { Bagged paint } \\
\text { chips }\end{array}$ & Various & $\begin{array}{c}\text { None or unknown; assume } \\
\text { transuranics and beta-gamma } \\
\text { emitters }\end{array}$ & $\mathrm{X}$ & & & 8 & 1 & ES \\
\hline
\end{tabular}




\begin{tabular}{|c|c|c|c|c|c|c|c|c|c|c|c|}
\hline 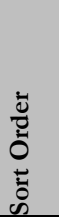 & Container & ID & Description & Origin & 5400.5 SRCs & 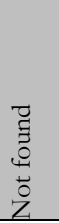 & 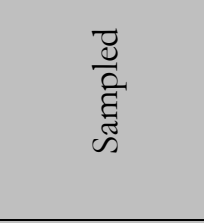 & 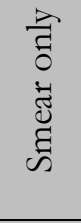 & 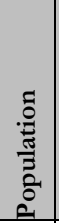 & $\mathrm{ft}^{3}$ & 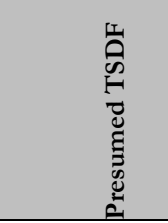 \\
\hline 32 & None & None & Lead pieces & 3001 Room 305 & $\begin{array}{l}\text { None or unknown; assume } \\
\text { transuranics and beta-gamma } \\
\text { emitters }\end{array}$ & & & Yes & 8 & TBD & $\mathrm{ES}$ \\
\hline 33 & None & None & Lead sheet & 3001 Room 220 & $\begin{array}{l}\text { None or unknown; assume } \\
\text { transuranics and beta-gamma } \\
\text { emitters }\end{array}$ & & & Yes & 8 & TBD & ES \\
\hline 34 & 5 -gal & X10I0700534 & $\begin{array}{c}\text { Electronics } \\
\text { (identified an air } \\
\text { dryer) }\end{array}$ & 3001 cage & $\begin{array}{c}\text { None or unknown; assume } \\
\text { transuranics and beta-gamma } \\
\text { emitters }\end{array}$ & & 5219M0020 & & 8 & TBD & ES \\
\hline 35 & 5 -gal & $\mathrm{X} 10 \mathrm{I} 0700535$ & $\begin{array}{l}\text { Electronics } \\
\text { (capacitors) }\end{array}$ & 3001 cage & $\begin{array}{c}\text { None or unknown; assume } \\
\text { transuranics and beta-gamma } \\
\text { emitters }\end{array}$ & & & Yes & 8 & TBD & ES \\
\hline 36 & B-12 & X10C0012632 & Various lead items & $\begin{array}{c}\text { W-19/20,3597, } \\
4507\end{array}$ & $\begin{array}{l}\text { Th-232; U-235, U-238, and } \\
\text { associated decay products, } \\
\text { alpha emitters; transuranics; } \\
\text { beta-gamma emitters }\end{array}$ & & & Yes & 7 & 45 & ES \\
\hline 37 & B-12 & X10C0505935 & $\begin{array}{l}\text { ACM, paint chips, } \\
\text { PPE, HEPA }\end{array}$ & $\begin{array}{l}\text { 3026-D Metals } \\
\text { Examination } \\
\text { Facility }\end{array}$ & $\begin{array}{l}\text { U-235, U-238, alpha emitters; } \\
\text { beta-gamma emitters }\end{array}$ & & & Yes & 7 & 45 & ES \\
\hline 38 & B-25 & X10C0506720 & $\begin{array}{l}\text { Pu-contaminated } \\
\text { lead sink }\end{array}$ & 3026-C Hot Lab & Transuranics & & & Yes & 7 & 90 & ES \\
\hline 39 & 55-gal & X10C0404149 & $\begin{array}{c}\text { Plastic hose, scrap } \\
\text { metal }\end{array}$ & $\begin{array}{l}\text { Hill Cut Test } \\
\text { Facility }\end{array}$ & $\begin{array}{c}\text { Transuranics, beta-gamma } \\
\text { emitters }\end{array}$ & & 5219M0039 & & 7 & 90 & EMWMF \\
\hline 40 & B-25 & X10C0301464 & Unknown & 7500 & $\begin{array}{l}\text { Th-232; U-235, U-238, and } \\
\text { associated decay products, } \\
\text { alpha emitters; transuranics; } \\
\text { beta-gamma emitters }\end{array}$ & & & Yes & 7 & 90 & EMWMF \\
\hline
\end{tabular}




\begin{tabular}{|c|c|c|c|c|c|c|c|c|c|c|c|}
\hline 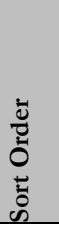 & Container & ID & Description & Origin & 5400.5 SRCs & 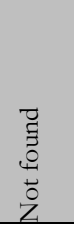 & 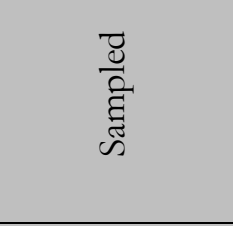 & $\begin{array}{l}\text { ते } \\
\text { है } \\
\text { हूँ } \\
\text { घू }\end{array}$ & 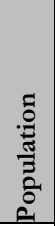 & $\mathrm{ft}^{3}$ & 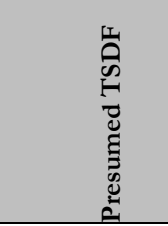 \\
\hline 41 & None & K25C1100740 & $\begin{array}{c}\text { Cryogenic } \mathrm{CO}_{2} \\
\text { system }\end{array}$ & HRE Pond & $\begin{array}{l}\text { Th-natural; U-235, U-238, } \\
\text { and associated decay } \\
\text { products, beta-gamma } \\
\text { emitters }\end{array}$ & & $\begin{array}{c}\text { 5219M0031 } \\
\text { 5219M0031D } \\
\text { 5219M0032 } \\
\text { 5219M0033 }\end{array}$ & & 6 & 350 & EMWMF \\
\hline 42 & None & $\mathrm{X} 10 \mathrm{I} 0200455$ & HVAC unit & Unknown & $\begin{array}{l}\text { Th-232; U-235, U-238, and } \\
\text { associated decay products; } \\
\text { transuranics; beta-gamma } \\
\text { emitters }\end{array}$ & $\mathrm{X}$ & & & 6 & 50 & EMWMF \\
\hline 43 & Dumpster & $\begin{array}{c}\text { 7500-DUMP-02 } \\
\text { (X19C0700324) }\end{array}$ & Scrap metal & $\begin{array}{l}\text { Unknown } \\
\text { (located at } \\
7500)\end{array}$ & $\begin{array}{l}\text { Th-232; U-235, U-238, and } \\
\text { associated decay products; } \\
\text { transuranics; beta-gamma } \\
\text { emitters }\end{array}$ & & $\begin{array}{l}5219 \mathrm{M} 0027 \\
5219 \mathrm{M} 0028 \\
5219 \mathrm{M} 0029 \\
5219 \mathrm{M} 0030\end{array}$ & & 6 & 150 & EMWMF \\
\hline 44 & Dumpster & $\begin{array}{l}\text { X10110200445 } \\
\text { (actual\#- } \\
\text { X10I1020445) }\end{array}$ & Scrap metal & Unknown & $\begin{array}{c}\text { None or unknown; assume } \\
\text { transuranics and beta-gamma } \\
\text { emitters }\end{array}$ & & $\begin{array}{c}\text { 5219M0022 } \\
5219 \mathrm{M} 0022 \mathrm{D} \\
5219 \mathrm{M} 0023 \\
5219 \mathrm{M} 0024 \\
5219 \mathrm{M} 0025\end{array}$ & & 6 & 150 & EMWMF \\
\hline 45 & None & K25C1101518 & $\begin{array}{l}\text { JLG, Model } \\
\text { 60GX }\end{array}$ & Unknown & $\begin{array}{l}\text { None or unknown; assume } \\
\text { transuranics and beta-gamma } \\
\text { emitters }\end{array}$ & & 5219M0016 & & 6 & 700 & EMWMF \\
\hline 46 & None & $68-7165$ & $\begin{array}{c}\text { Drilling HEPA } \\
\text { cart }\end{array}$ & $\begin{array}{l}\text { HRE Ponds, } \\
\text { Water Quality } \\
\text { Projects }\end{array}$ & $\begin{array}{l}\text { Th-natural; U-235, U-238, } \\
\text { and associated decay } \\
\text { products; transuranics; beta- } \\
\text { gamma emitters }\end{array}$ & & 5219M0034 & & 6 & 500 & EMWMF \\
\hline
\end{tabular}




\begin{tabular}{|c|c|c|c|c|c|c|c|c|c|c|c|}
\hline 苞 & Container & ID & Description & Origin & $5400.5 \mathrm{SRCs}$ & 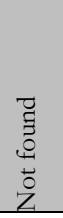 & $\frac{\vec{d}}{\frac{0}{n}}$ & 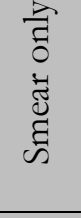 & 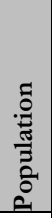 & $\mathrm{ft}^{3}$ & 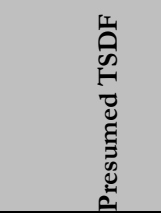 \\
\hline 47 & None & $68-7166$ & $\begin{array}{l}\text { Drilling HEPA } \\
\text { Cart }\end{array}$ & $\begin{array}{l}\text { HRE Ponds, } \\
\text { Water Quality } \\
\text { Projects }\end{array}$ & $\begin{array}{l}\text { Th-natural; U-235, U-238, } \\
\text { and associated decay } \\
\text { products; transuranics; beta- } \\
\text { gamma emitters }\end{array}$ & & 5219M0035 & & 6 & 500 & EMWMF \\
\hline 48 & None & $68-7167$ & $\begin{array}{c}\text { Drilling HEPA } \\
\text { cart }\end{array}$ & $\begin{array}{l}\text { HRE Ponds, } \\
\text { Water Quality } \\
\text { Projects }\end{array}$ & $\begin{array}{l}\text { Th-natural; U-235, U-238, } \\
\text { and associated decay } \\
\text { products; transuranics; beta- } \\
\text { gamma emitters }\end{array}$ & & & Yes & 6 & 500 & EMWMF \\
\hline 49 & None & X10C0700129 & Exhaust hood & Unknown & $\begin{array}{l}\text { None or unknown; assume } \\
\text { transuranics and beta-gamma } \\
\text { emitters }\end{array}$ & & $\begin{array}{l}\text { 5219M0017 } \\
\text { 5219M0018 }\end{array}$ & & 6 & 45 & EMWMF \\
\hline 50 & Fiber box & X10I1020381 & $\begin{array}{l}\text { HEPA filters } \\
24 " \times 24 " \times 11.5 "\end{array}$ & $\begin{array}{c}3029 \text { Filter } \\
\text { House FH-209 }\end{array}$ & Beta-gamma emitters & & No & No & 4 & 4 & NNSS \\
\hline 51 & Fiber box & X10I1020383 & $\begin{array}{c}\text { HEPA filters } \\
24 " \times 24^{\prime \prime} \times 11.5 "\end{array}$ & $\begin{array}{c}3029 \text { Filter } \\
\text { House FH-209 }\end{array}$ & Beta-gamma emitters & & No & No & 4 & 4 & NNSS \\
\hline 52 & $\begin{array}{l}\text { Concrete } \\
\text { cask }\end{array}$ & 1 & $\begin{array}{l}\text { Irradiated metal } \\
\text { specimens }\end{array}$ & $\begin{array}{l}\text { 3026-D Metals } \\
\text { Examination } \\
\text { Facility }\end{array}$ & Beta-gamma emitters & & No & No & 5 & 27 & NNSS \\
\hline 53 & $\begin{array}{l}\text { Concrete } \\
\text { cask }\end{array}$ & 2 & $\begin{array}{l}\text { Irradiated metal } \\
\text { specimens, 4Ts, } \\
\text { bucket, scales, in } \\
\text { cell light }\end{array}$ & $\begin{array}{l}\text { 3026-D Metals } \\
\text { Examination } \\
\text { Facility }\end{array}$ & Beta-gamma emitters & & No & No & 5 & 27 & NNSS \\
\hline
\end{tabular}




\begin{tabular}{|c|c|c|c|c|c|c|c|c|c|c|c|}
\hline 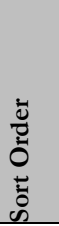 & Container & ID & Description & Origin & 5400.5 SRCs & 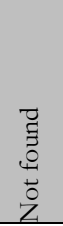 & 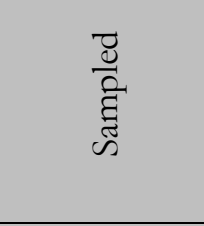 & $\begin{array}{l}\text { ते } \\
\text { है } \\
\text { हूँ } \\
\text { घू }\end{array}$ & 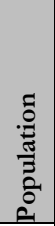 & $\mathrm{ft}^{3}$ & 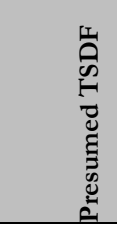 \\
\hline 54 & $\begin{array}{c}\text { Concrete } \\
\text { cask }\end{array}$ & 3 & $\begin{array}{c}\text { Irradiated metal } \\
\text { specimens, hot } \\
\text { plate, light source, } \\
\text { vacuum, } \\
\text { calibration tools, } \\
\text { mirror cell tools, } \\
\text { scrap metal, lead } \\
\text { pig }\end{array}$ & $\begin{array}{l}\text { 3026-D Metals } \\
\text { Examination } \\
\text { Facility }\end{array}$ & Beta-gamma emitters & & No & No & 5 & 27 & NNSS \\
\hline 55 & $\begin{array}{c}\text { Concrete } \\
\text { cask }\end{array}$ & 4 & $\begin{array}{l}\text { Irradiated metal } \\
\text { specimens in cans }\end{array}$ & $\begin{array}{c}\text { 3026-D Metals } \\
\text { Examination } \\
\text { Facility }\end{array}$ & Beta-gamma emitters & & $\mathrm{No}$ & $\mathrm{No}$ & 5 & 27 & NNSS \\
\hline 56 & $\begin{array}{c}\text { Concrete } \\
\text { cask }\end{array}$ & 5 & $\begin{array}{l}\text { Irradiated metal } \\
\text { specimens }\end{array}$ & $\begin{array}{c}\text { 3026-D Metals } \\
\text { Examination } \\
\text { Facility }\end{array}$ & Beta-gamma emitters & & $\mathrm{No}$ & $\mathrm{No}$ & 5 & 27 & NNSS \\
\hline 57 & $\begin{array}{c}\text { Concrete } \\
\text { cask }\end{array}$ & 6 & $\begin{array}{l}\text { Irradiated metal } \\
\text { specimens }\end{array}$ & $\begin{array}{c}\text { 3026-D Metals } \\
\text { Examination } \\
\text { Facility }\end{array}$ & Beta-gamma emitters & & $\mathrm{No}$ & No & 5 & 27 & NNSS \\
\hline 58 & Box & $\mathrm{X} 10 \mathrm{C} 0400325$ & $\begin{array}{l}\text { PPE, sump pump, } \\
\text { pvc pipe, g-flex, } \\
\text { paper, metal }\end{array}$ & $\begin{array}{l}\text { 4507 Hot Cells } \\
1-4,4556 \text { Filter } \\
\text { Pit }\end{array}$ & $\begin{array}{c}\text { Tranuranics, beta-gamma } \\
\text { emitters }\end{array}$ & & $\begin{array}{l}\text { 5219M0011 } \\
5219 \mathrm{M} 0012 \\
5219 \mathrm{M} 0013 \\
5219 \mathrm{M} 0014 \\
5219 \mathrm{M} 0015\end{array}$ & & 7 & 90 & NNSS \\
\hline 59 & Box & X10C0500911 & $\begin{array}{c}\text { PPE, Plastic, } \\
\text { insulation, sand } \\
\text { bag, rope, vacuum } \\
\text { HEPA filter, } \\
\text { metal }\end{array}$ & 3029 & Beta-gamma emitters & & $\begin{array}{l}\text { 5219M0006 } \\
5219 \mathrm{M} 0007 \\
5219 \mathrm{M} 0008 \\
5219 \mathrm{M} 0009 \\
5219 \mathrm{M} 0010\end{array}$ & & 7 & 90 & NNSS \\
\hline
\end{tabular}




\begin{tabular}{|c|c|c|c|c|c|c|c|c|c|c|c|}
\hline 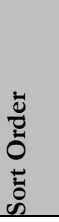 & Container & ID & Description & Origin & $5400.5 \mathrm{SRCs}$ & 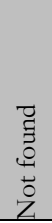 & 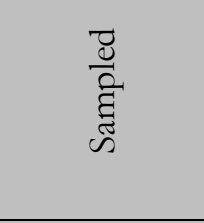 & 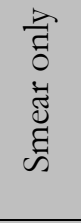 & 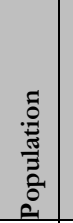 & $\mathrm{ft}^{3}$ & 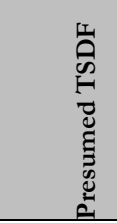 \\
\hline 60 & B-25 & X10C9601883 & $\begin{array}{l}\text { Electrical J-boxes, } \\
\text { conduit, wiring, } \\
\text { air controllers, } \\
\text { insulation wrap, } \\
\text { two drums }\end{array}$ & $\begin{array}{c}2026 \text { Drain } \\
\text { Mod, MK } \\
\text { Ferguson }\end{array}$ & Beta-gamma emitters & & 5219M0036 & & 7 & 90 & NNSS \\
\hline 61 & B-25 & X10C9600488 & Soil, rock & $\begin{array}{l}2026 \text { Drain } \\
\text { Mod, MK } \\
\text { Ferguson }\end{array}$ & Beta-gamma emitters & & No & No & 7 & 90 & NNSS \\
\hline 62 & B-25 & X10C9600535 & $\begin{array}{l}\text { Shielded concrete } \\
\text { cores }\end{array}$ & $\begin{array}{c}\text { 2026 Drain } \\
\text { Mod, MK } \\
\text { Ferguson }\end{array}$ & Beta-gamma emitters & & No & No & 7 & 90 & NNSS \\
\hline & 35 gal drum & X10C0401770 & $\begin{array}{l}\text { Individual bagged } \\
\text { items }\end{array}$ & $-^{c}$ & - & & & Yes & 一 & - & - \\
\hline & & $\begin{array}{l}\text { US-DOE-SN- } \\
\text { X134120 }\end{array}$ & $\begin{array}{l}\text { Water Pressure } \\
\text { gun }\end{array}$ & - & - & & 5219M0026 & & 一 & - & - \\
\hline
\end{tabular}

aItems containing lead including computer boards with lead solder not sampled. Smears only.

bItem 29 is a duplicate entry from original list.

c_ = Not applicable; information unknown. 
APPENDIX C

TOTAL AND REMOVABLE SURFACE ACTIVITY MEASUREMENTS 


\begin{tabular}{|c|c|c|c|c|c|c|c|c|}
\hline \multicolumn{9}{|c|}{ Direct Measurements and Removable Activity } \\
\hline Location ID & Container No. & Surface & $\begin{array}{l}\text { Alpha } \\
(\mathrm{cpm})\end{array}$ & $\begin{array}{c}\text { Alpha } \\
\left(\mathrm{dpm} / 100 \mathrm{~cm}^{2}\right)\end{array}$ & Beta (cpm) & $\begin{array}{c}\text { Beta } \\
(\mathrm{dpm} / 100 \\
\left.\mathrm{cm}^{2}\right)\end{array}$ & $\begin{array}{l}\text { Removable Alpha } \\
\left(\mathrm{dpm} / 100 \mathrm{~cm}^{2}\right)\end{array}$ & $\begin{array}{c}\text { Removable Beta } \\
\left(\mathrm{dpm} / 100 \mathrm{~cm}^{2}\right)\end{array}$ \\
\hline 5219R0001 & \multirow{2}{*}{ K25C1101517 } & \multirow{2}{*}{$\begin{array}{l}\text { Tool box - steel cables, rigging straps, } \\
\text { hemp rope, wire brush, rigging softeners }\end{array}$} & 6 & 10 & 330 & -130 & -1 & 2 \\
\hline 5219R0002 & & & 3 & -21 & 300 & -240 & -1 & 2 \\
\hline 5219R0003 & \multirow{2}{*}{ K25C1100766 } & \multirow{2}{*}{ Unpackaged Honda pressure washer } & 4 & -10 & 420 & 150 & -1 & 0 \\
\hline 5219R0004 & & & 12 & 72 & 370 & -3 & -1 & 5 \\
\hline 5219R0005 & \multirow{2}{*}{ K25C1101518 } & \multirow{2}{*}{ JLG Model 60GX } & 78 & 750 & 550 & 550 & 8 & 10 \\
\hline 5219R0006 & & & 8 & 31 & 280 & -310 & -1 & 0 \\
\hline 5219R0007 & \multirow{2}{*}{ X10C1101242 } & \multirow{2}{*}{ Empty 55-gal drum } & 4 & -10 & 340 & -110 & -1 & 1 \\
\hline 5219R0008 & & & 9 & 41 & 320 & -170 & 6 & 10 \\
\hline 5219R0009 & \multirow{2}{*}{ X10C1101241 } & \multirow{2}{*}{ Empty stainless 55-gal drum } & 3 & -21 & 260 & -360 & -1 & 6 \\
\hline 5219R0010 & & & 4 & -10 & 340 & -110 & -1 & 3 \\
\hline 5219R0011 & \multirow{2}{*}{ X10C0404151 } & \multirow{2}{*}{ Empty B-12 } & 55 & 510 & 1700 & 4,000 & 64 & 430 \\
\hline 5219R0012 & & & 140 & 1,400 & 370 & -16 & 220 & 2300 \\
\hline 5219R0013 & \multirow{2}{*}{ X10C0301464 } & \multirow{2}{*}{ B-25 } & 4 & -10 & 280 & -290 & -1 & -1 \\
\hline 5219R0014 & & & 5 & 0 & 270 & -330 & -1 & 2 \\
\hline 5219R0015 & \multirow{2}{*}{ X10C0700129 } & \multirow{2}{*}{ Exhaust hood } & 21 & 160 & 1,300 & 2,800 & -1 & 2 \\
\hline 5219R0016 & & & 52 & 480 & 800 & 1,300 & 4 & -1 \\
\hline 5219R0017 & \multirow{2}{*}{ X10C0404152 } & \multirow{2}{*}{ 55-gal drum full of lead chunks } & 480 & 4,900 & 8600 & 26,000 & 8 & 38 \\
\hline 5219R0018 & & & 5 & $\bar{\sigma}$ & 470 & 320 & -1 & 12 \\
\hline 5219R0019 & \multirow{2}{*}{ X10C0404154 } & \multirow{2}{*}{ 55-gal drum full of lead chunks } & 6 & 10 & 510 & 420 & -1 & 6 \\
\hline 5219R0020 & & & 5 & 0 & 410 & 110 & -1 & 3 \\
\hline 5219R0021 & \multirow{2}{*}{ X10C0404156 } & \multirow{2}{*}{ 30-gal drum full of lead chunks } & 4 & -10 & 24,000 & 73,000 & 210 & 7200 \\
\hline 5219R0022 & & & 7 & 21 & 370 & 3 & 1 & 22 \\
\hline 5219R0023 & \multirow{2}{*}{ X10C0400325 } & \multirow{2}{*}{ B-25 } & 11 & 62 & 730,000 & $2,300,000$ & -1 & 2 \\
\hline 5219R0024 & & & 7 & 21 & 1,100 & 2,300 & -1 & -2 \\
\hline 5219R0025 & \multirow{2}{*}{ X10I0700529 } & $C$ & 2 & -31 & 320 & -180 & -1 & 0 \\
\hline 5219R0026 & & Cryostatic trap & 4 & -10 & 340 & -91 & -1 & 3 \\
\hline 5219R0027 & Y1010700523 & Toledo cole & 13 & 82 & 310 & -210 & 1 & 0 \\
\hline 5219R0028 & X1010/00523 & 10 ledo scale & 6 & 10 & 370 & -25 & -1 & 2 \\
\hline 5219R0029 & & & 3 & -21 & 270 & -330 & -1 & 4 \\
\hline 5219R0030 & 1411 & Fire extinguisher & 4 & -10 & 390 & 38 & -1 & 4 \\
\hline 5219R0031 & Y1010700524 & & 8 & 31 & 280 & -280 & -1 & 1 \\
\hline 5219R0032 & X1010700534 & Air dryer & 3 & -21 & 320 & -180 & -1 & 6 \\
\hline 5219R0033 & & & 4 & -10 & 280 & -280 & 1 & -1 \\
\hline 5219R0034 & X10C0700535 & Capacitors & 0 & -51 & 280 & -310 & 4 & -3 \\
\hline 5219R0035 & $\mathrm{Y} 10 \mathrm{C} 0500011$ & B 25 & 4 & -10 & $1,000,000^{2}$ & $3,100,000$ & 4 & 1 \\
\hline 5219R0036 & X10C0500911 & B-25 & 5 & 0 & 880 & 1,600 & 4 & 9 \\
\hline
\end{tabular}




\begin{tabular}{|c|c|c|c|c|c|c|c|c|}
\hline \multicolumn{9}{|c|}{ Direct Measurements and Removable Activity } \\
\hline Location ID & Container No. & Surface & $\begin{array}{l}\text { Alpha } \\
\text { (cpm) }\end{array}$ & $\begin{array}{c}\text { Alpha } \\
\left(\mathrm{dpm} / 100 \mathrm{~cm}^{2}\right)\end{array}$ & Beta (cpm) & $\begin{array}{c}\text { Beta } \\
(\mathrm{dpm} / 100 \\
\left.\mathrm{cm}^{2}\right)\end{array}$ & $\begin{array}{l}\text { Removable Alpha } \\
\left(\mathrm{dpm} / 100 \mathrm{~cm}^{2}\right)\end{array}$ & $\begin{array}{l}\text { Removable Beta } \\
\left(\mathrm{dpm} / 100 \mathrm{~cm}^{2}\right)\end{array}$ \\
\hline 5219R0037 & \multirow{2}{*}{ X10C0404157 } & \multirow{2}{*}{ 55-gal drum full of computer items } & 6 & 10 & 270 & -310 & -1 & 2 \\
\hline 5219R0038 & & & 0 & -51 & 300 & -220 & -1 & 2 \\
\hline 5219R0039 & \multirow{2}{*}{ X10C0404158 } & \multirow{2}{*}{ 85-gal drum full of electrical equipment } & 3 & -21 & 290 & -260 & 1 & 2 \\
\hline 5219R0040 & & & 8 & 31 & 300 & -240 & -1 & -1 \\
\hline 5219R0041 & \multirow{3}{*}{ X10C0404159 } & \multirow{3}{*}{ 30-gal drum full of electrical equipment } & 150 & 1,400 & 650 & 870 & 110 & 33 \\
\hline 5219R0042 & & & 12 & 72 & 290 & -260 & 6 & 1 \\
\hline 5219R0043 & & & 150 & 1,500 & 760 & 1,200 & -1 & 29 \\
\hline 5219R0044 & Pallet & Lead pieces from $\mathrm{Rm} \# 305$ & 11 & 62 & 8,600 & 26,000 & -1 & 29 \\
\hline 5219R0045 & Pallet & Lead pieces from $\mathrm{Rm} \# 305$ & 12 & 72 & 1800 & 4,600 & -1 & 5 \\
\hline 5219R0046 & Pallet & Lead pieces from $\mathrm{Rm} \# 220$ & 7 & 21 & 230 & -460 & -1 & 4 \\
\hline 5219R0047 & Pallet & Lead pieces from $\mathrm{Rm} \# 220$ & 8 & 31 & 270 & -330 & -1 & -2 \\
\hline 5219R0048 & On floor & Lead brick & 0 & -51 & 0 & $-1,200$ & -1 & 2 \\
\hline 5219R0049 & On floor & Stack of metal & 0 & -51 & 0 & $-1,200$ & -1 & -2 \\
\hline 5219R0050 & \multirow{2}{*}{ 68-7165 } & \multirow{2}{*}{ Drilling HEPA cart } & 11 & 62 & 430 & 180 & 1 & 2 \\
\hline 5219R0051 & & & 6 & 10 & 1,900 & 4,900 & 6 & 104 \\
\hline 5219R0052 & \multirow{2}{*}{$68-7166$} & \multirow{2}{*}{ Drilling HEPA cart } & 4 & -10 & 360 & -50 & -1 & 2 \\
\hline 5219R0053 & & & 5 & 0 & 1,700 & 4,100 & -1 & 5 \\
\hline 5219R0054 & \multirow{2}{*}{$68-7167$} & \multirow{2}{*}{ Drilling HEPA cart } & 1 & -41 & 440 & 210 & -1 & 3 \\
\hline 5219R0055 & & & 10 & 51 & 300 & -190 & 1 & -1 \\
\hline 5219R0056 & \multirow{2}{*}{ X10C0505935 } & \multirow{2}{*}{ B-12 with two bags of waste } & 8 & 31 & 890 & 1,600 & -1 & -2 \\
\hline 5219R0057 & & & 9 & 41 & 1,600 & 3,800 & 4 & 5 \\
\hline 5219R0058 & \multirow{2}{*}{ X10C0506720 } & \multirow{2}{*}{ B-25 with Pu-contaminated lead } & 5 & 0 & 5,200 & 15,000 & -1 & 2 \\
\hline 5219R0059 & & & 10 & 51 & 3700 & 10,400 & -1 & -1 \\
\hline 5219R0060 & \multirow{2}{*}{ X10C9601883 } & \multirow{2}{*}{ B-25 with 2 drums } & 12 & 72 & 11,000 & 34,000 & -1 & 3 \\
\hline 5219R0061 & & & 11 & 62 & 5,700 & 17,000 & -1 & -2 \\
\hline 5219R0062 & \multirow{2}{*}{ X10C0404149 } & \multirow{2}{*}{ 55-gal drum } & 15 & 100 & 2,200 & 5,800 & 1 & 0 \\
\hline 5219R0063 & & & 9 & 41 & 4,200 & 12,000 & 1 & 1 \\
\hline 5219R0064 & \multirow{2}{*}{ Pallet } & \multirow{2}{*}{$\begin{array}{l}\text { Pallet - metal grating, lead sheets, } \\
\text { concrete blocks }\end{array}$} & 6 & 10 & 320 & -170 & -1 & -2 \\
\hline 5219R0065 & & & 3 & -21 & 310 & -210 & 1 & 1 \\
\hline 5219R0066 & \multirow{2}{*}{ Pallet } & \multirow{2}{*}{$\begin{array}{l}\text { Loose bag of lead pigs at Rm 202, } \\
\text { cardboard drum }\end{array}$} & 6 & 10 & 730 & 1,100 & -1 & 9 \\
\hline 5219R0067 & & & 9 & 41 & 290 & -250 & -1 & 3 \\
\hline 5219R0068 & \multirow{2}{*}{ X10C0404164 } & 55-oal drum full of lead & 5 & 0 & 1,700 & 4,100 & -1 & -2 \\
\hline 5219R0069 & & 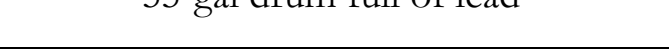 & 6 & 10 & 810 & 1,400 & -1 & 0 \\
\hline 5219R0070 & Pallet & Loose lead bricks & 11 & 62 & 190 & -590 & -1 & -1 \\
\hline 5219R0073 & X10C0404149 & 55-gal drum with plastic hose & 9 & 41 & 6,900 & 20,400 & -1 & 2 \\
\hline 5219R0074 & X10C0012632 & B-12 with lead pieces & 17 & 120 & 3,000 & 8,100 & 21 & 760 \\
\hline
\end{tabular}


Direct Measurements and Removable Activity

\begin{tabular}{|c|c|c|c|c|c|c|c|c|}
\hline Location ID & Container No. & Surface & $\begin{array}{l}\text { Alpha } \\
\text { (cpm) }\end{array}$ & $\begin{array}{c}\text { Alpha } \\
\left(\mathrm{dpm} / 100 \mathrm{~cm}^{2}\right)\end{array}$ & Beta (cpm) & $\begin{array}{c}\text { Beta } \\
(\mathrm{dpm} / 100 \\
\left.\mathrm{cm}^{2}\right)\end{array}$ & $\begin{array}{l}\text { Removable Alpha } \\
\left(\mathrm{dpm} / 100 \mathrm{~cm}^{2}\right)\end{array}$ & $\begin{array}{c}\text { Removable Beta } \\
\left(\mathrm{dpm} / 100 \mathrm{~cm}^{2}\right)\end{array}$ \\
\hline 5219R0075 & \multirow{2}{*}{ X10C0401770 } & \multirow{2}{*}{ Rusty 55-gal drum } & 11 & 62 & 1,200 & 2,400 & -1 & 3 \\
\hline 5219R0076 & & & 5 & 0 & 1,400 & 3,100 & -1 & 3 \\
\hline
\end{tabular}

Beta measurement on this item exceeded the capacity of the instrumentation to record exact value. 
APPENDIX D

SAMPLE PHOTOGRAPH AND IDENTIFICATION 


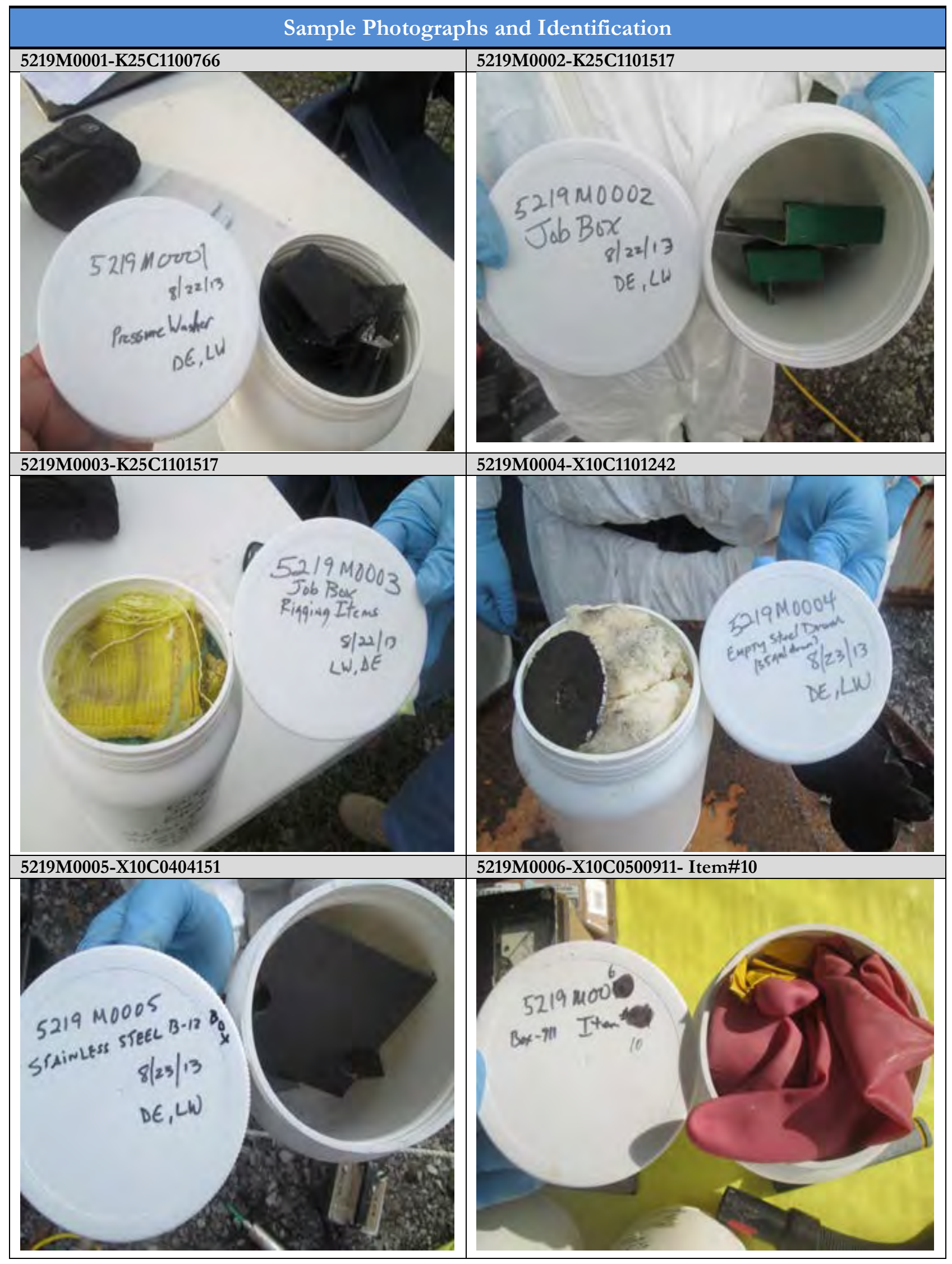




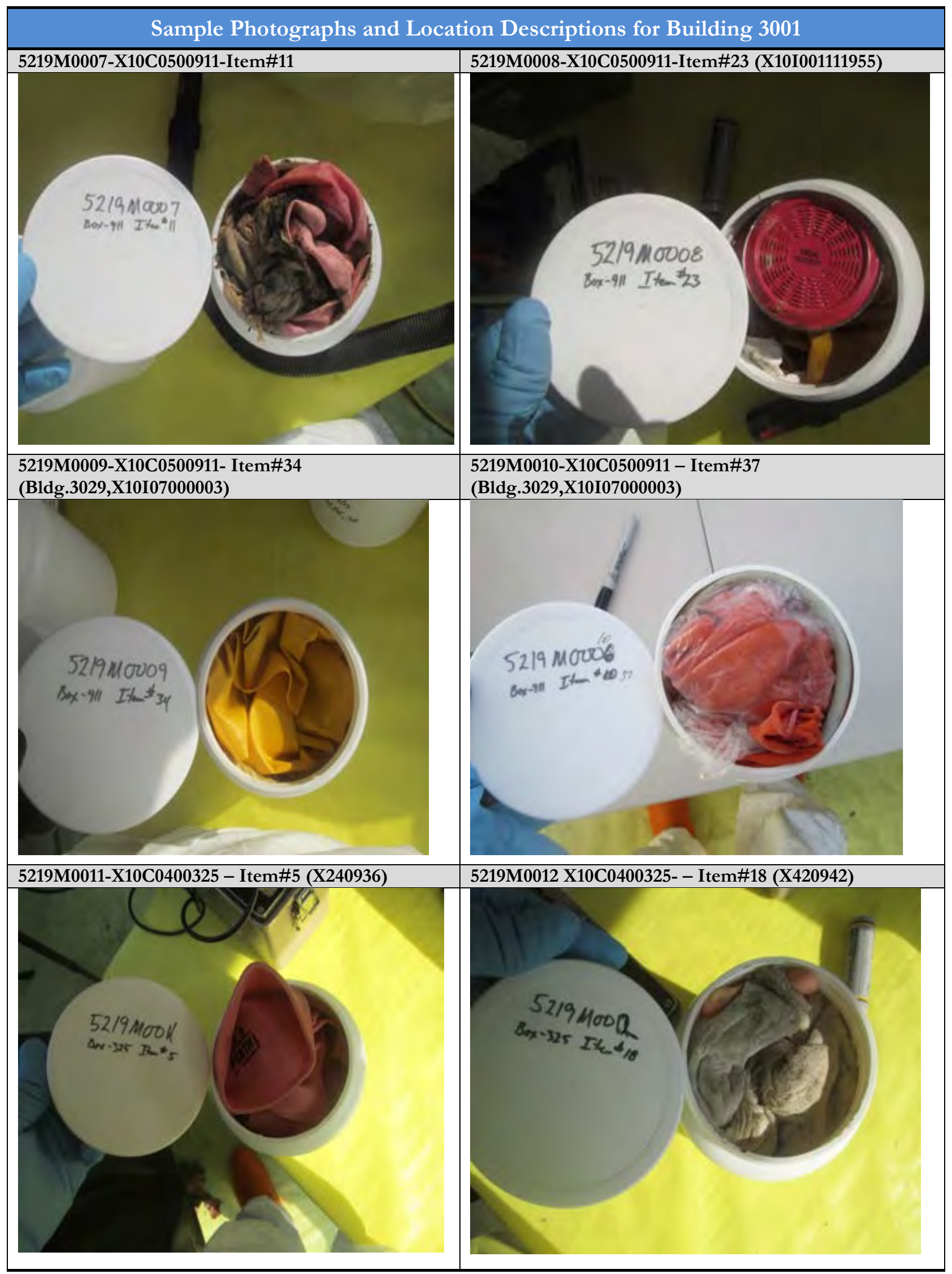




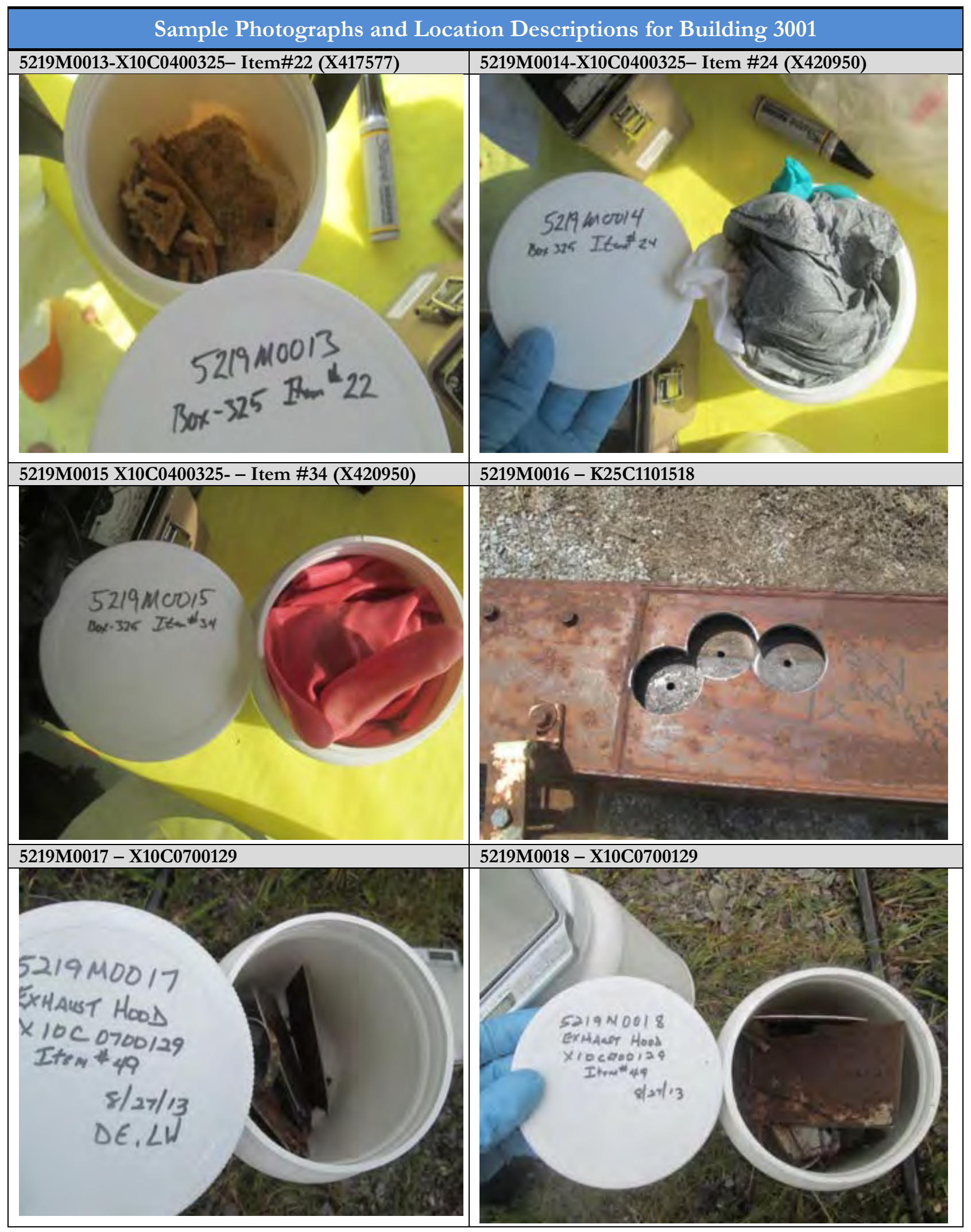




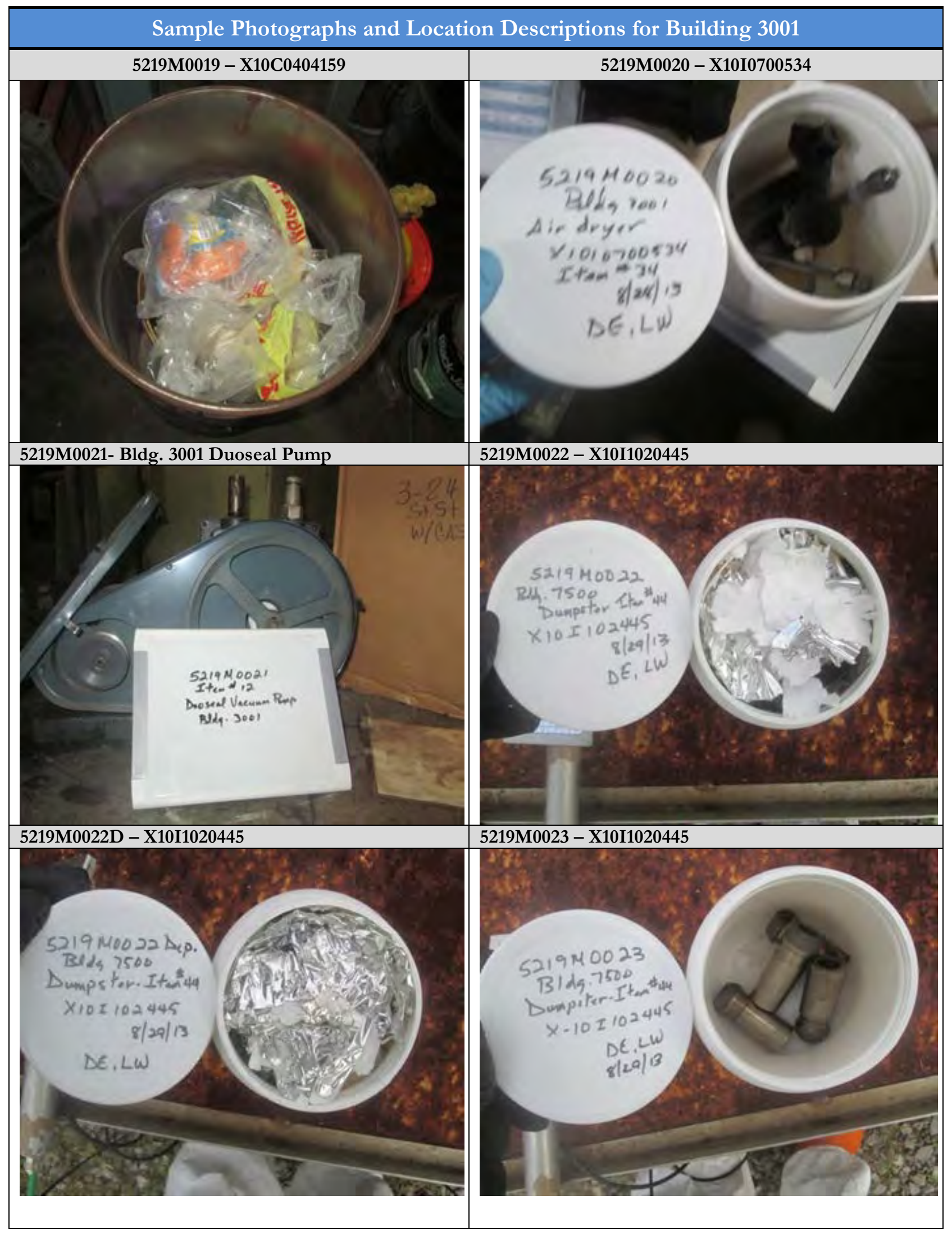




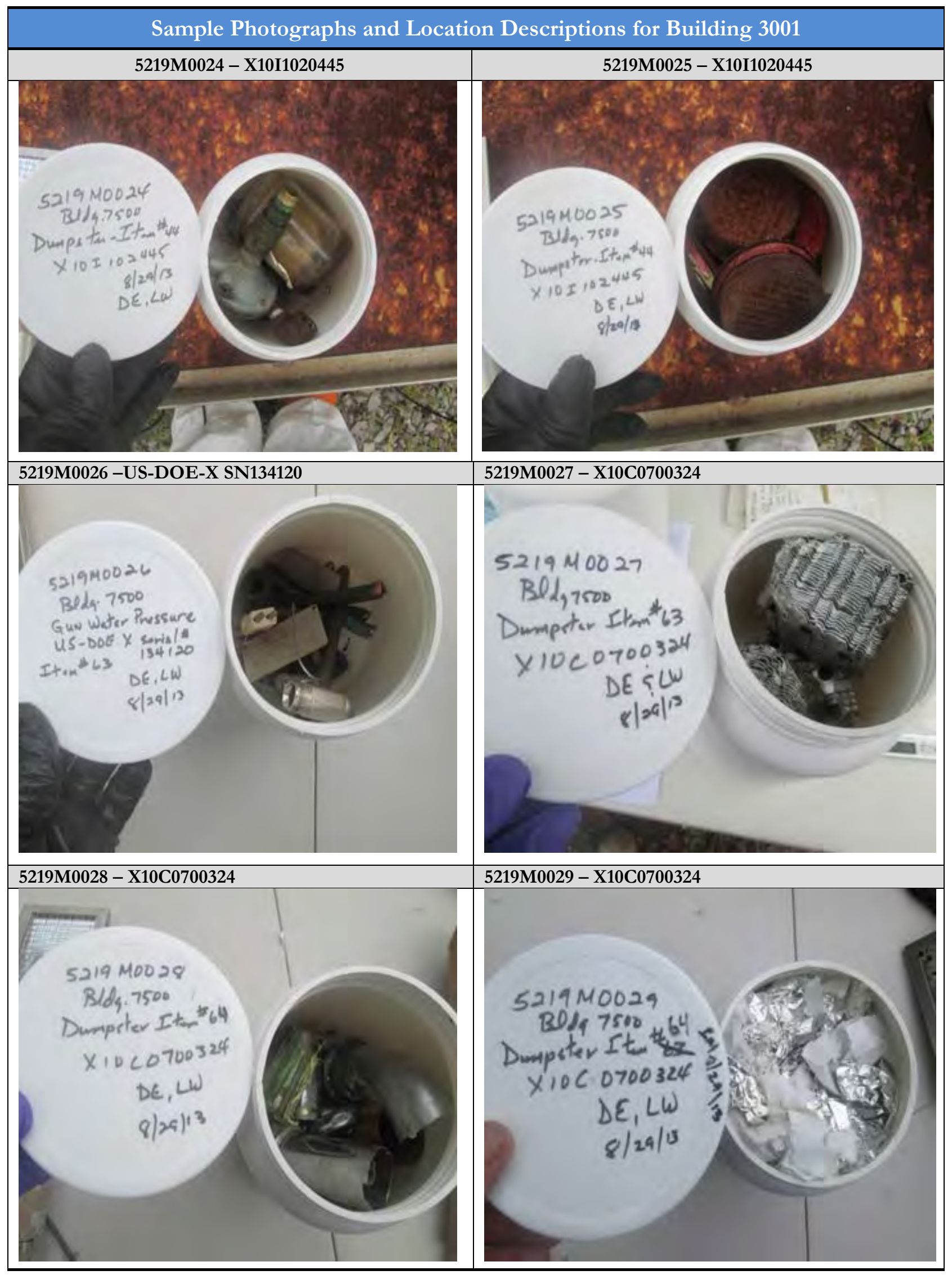




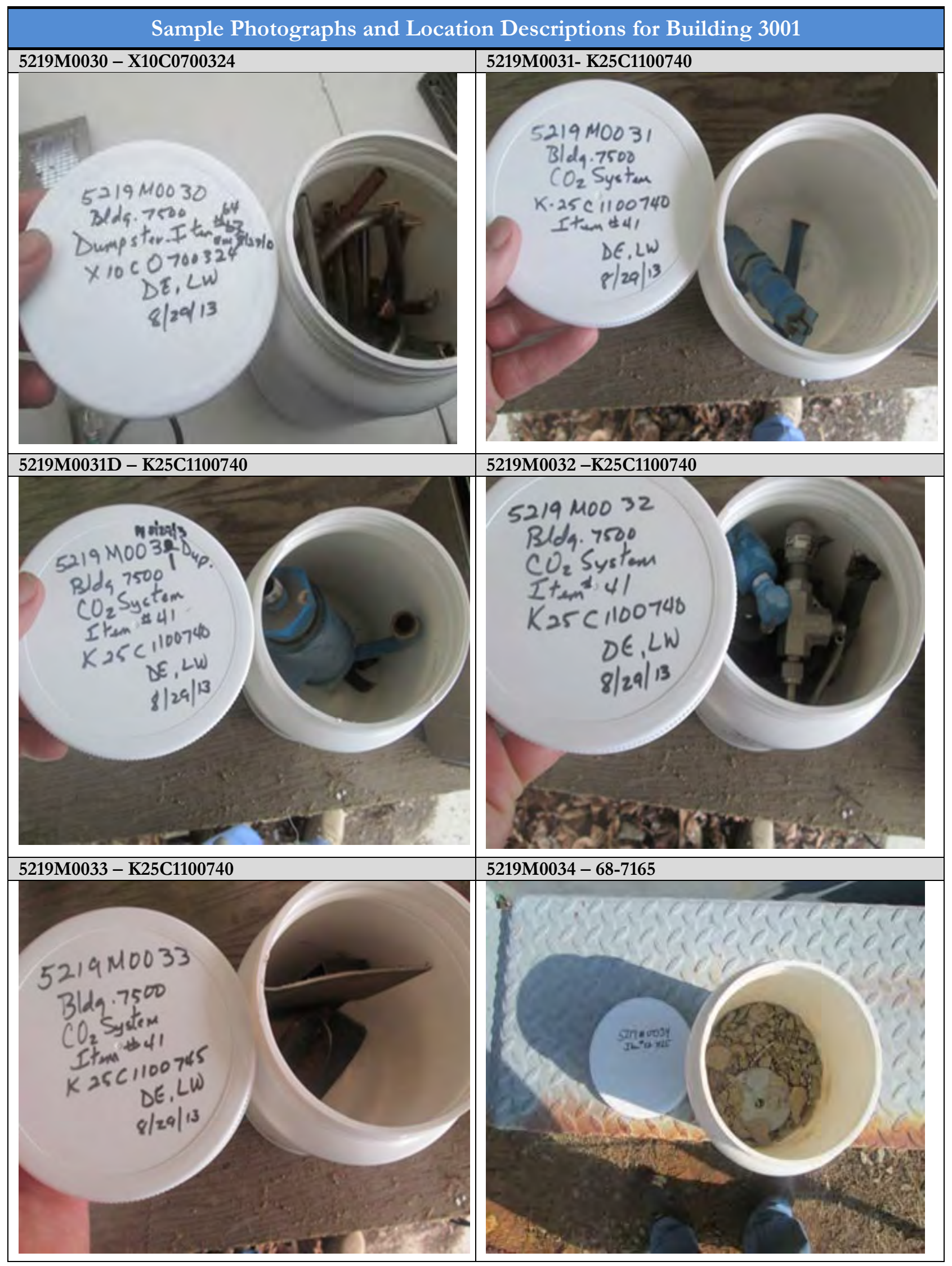




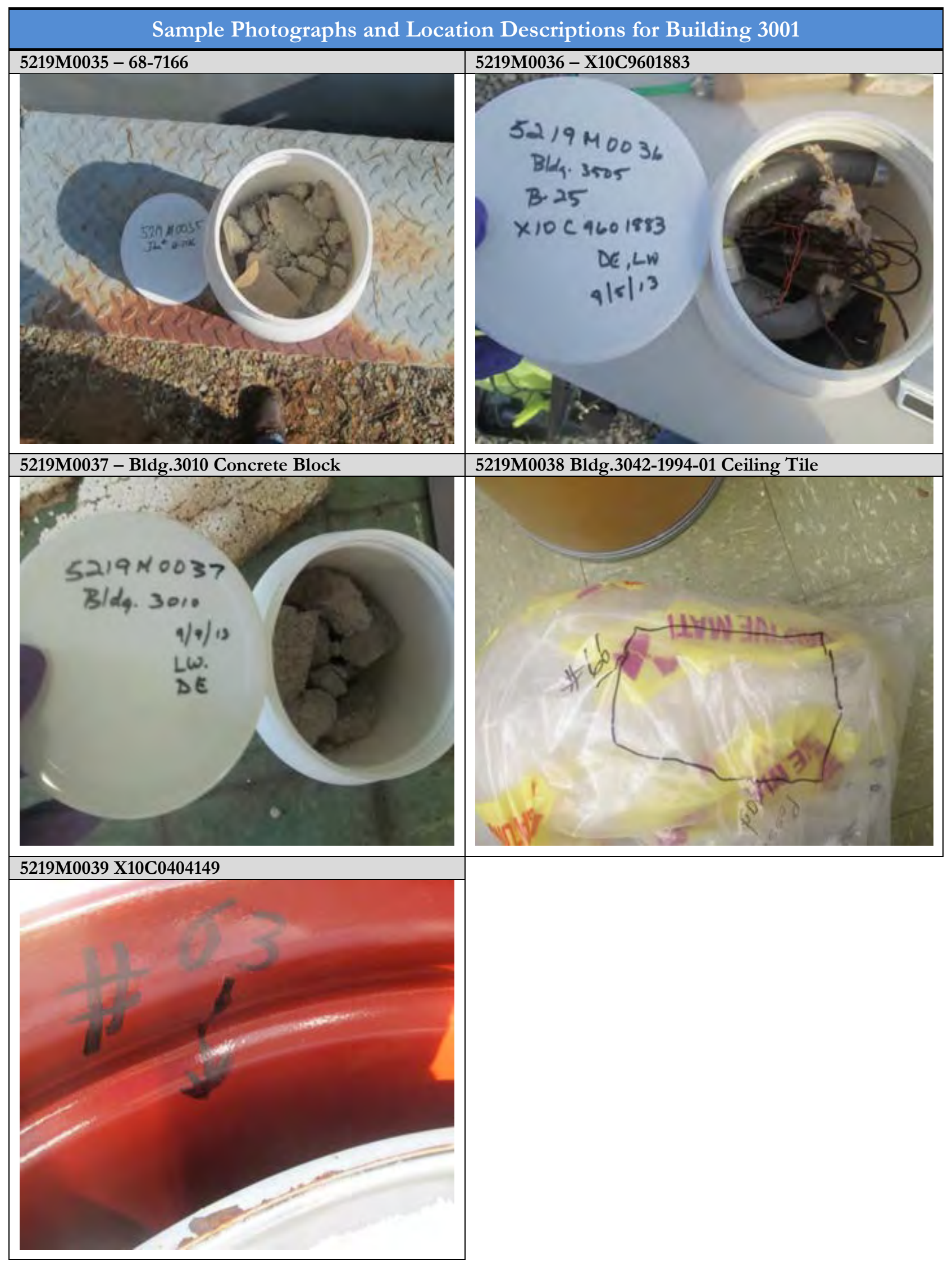


APPENDIX E

DATA SUMMARY STATISTICS 


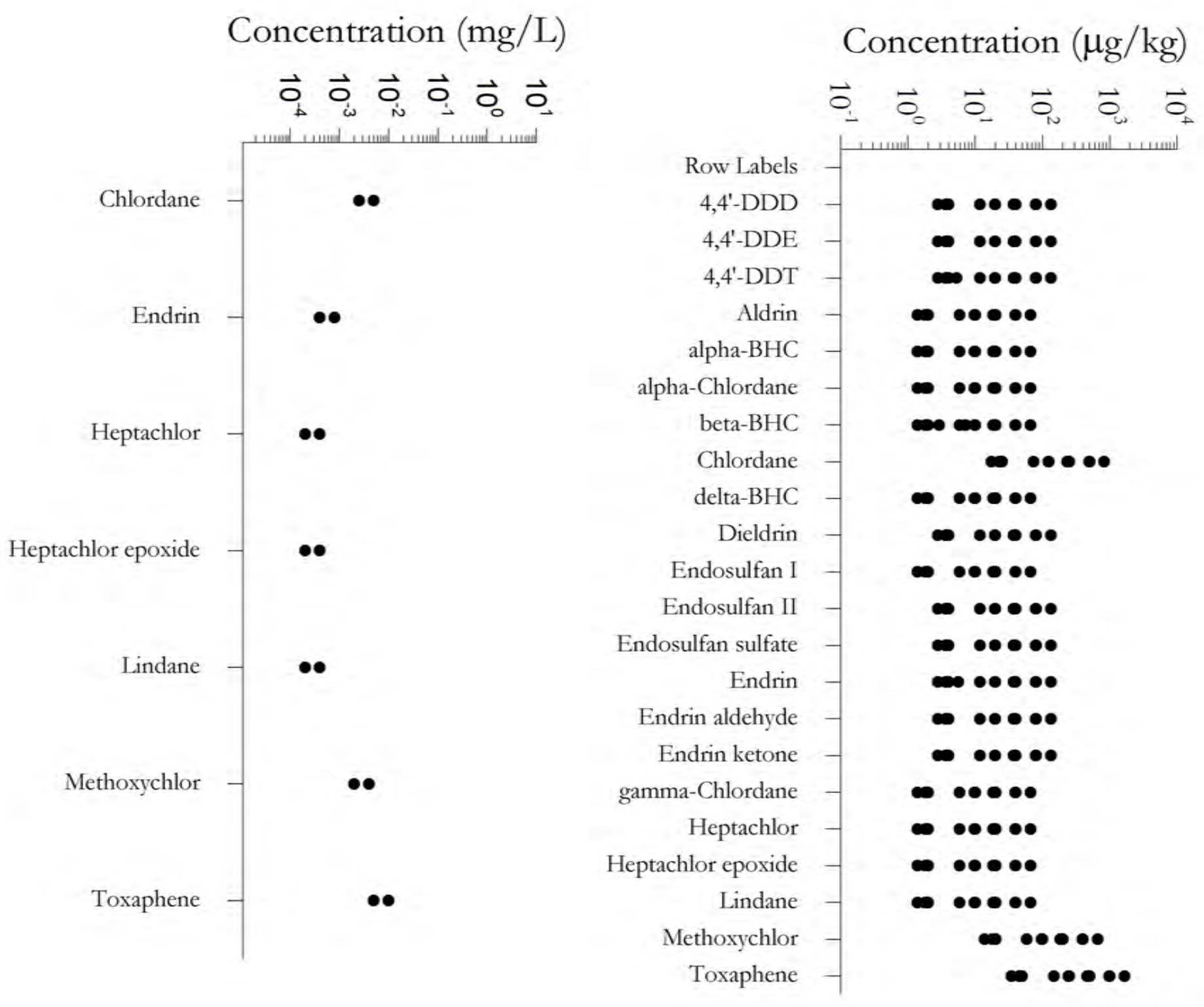




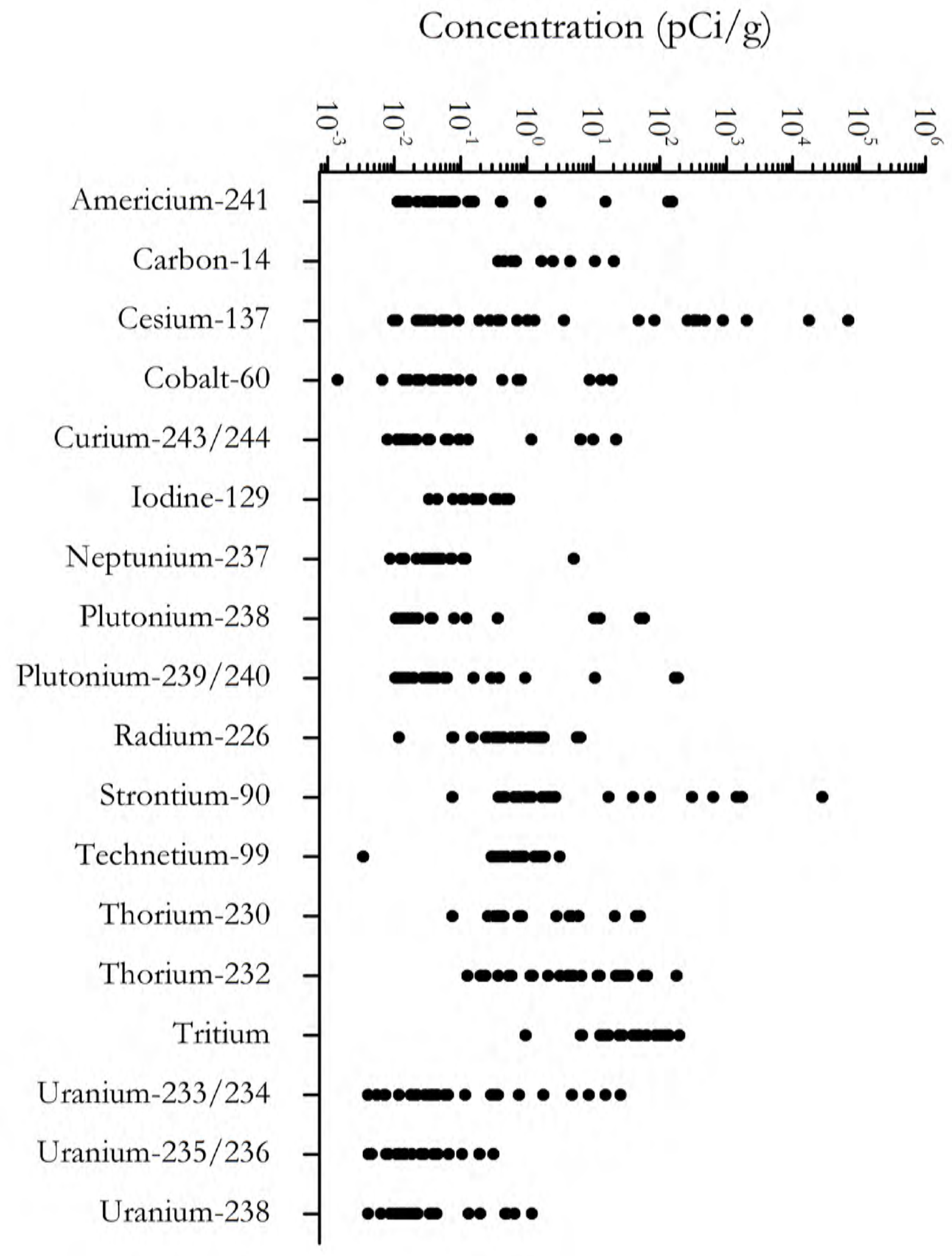




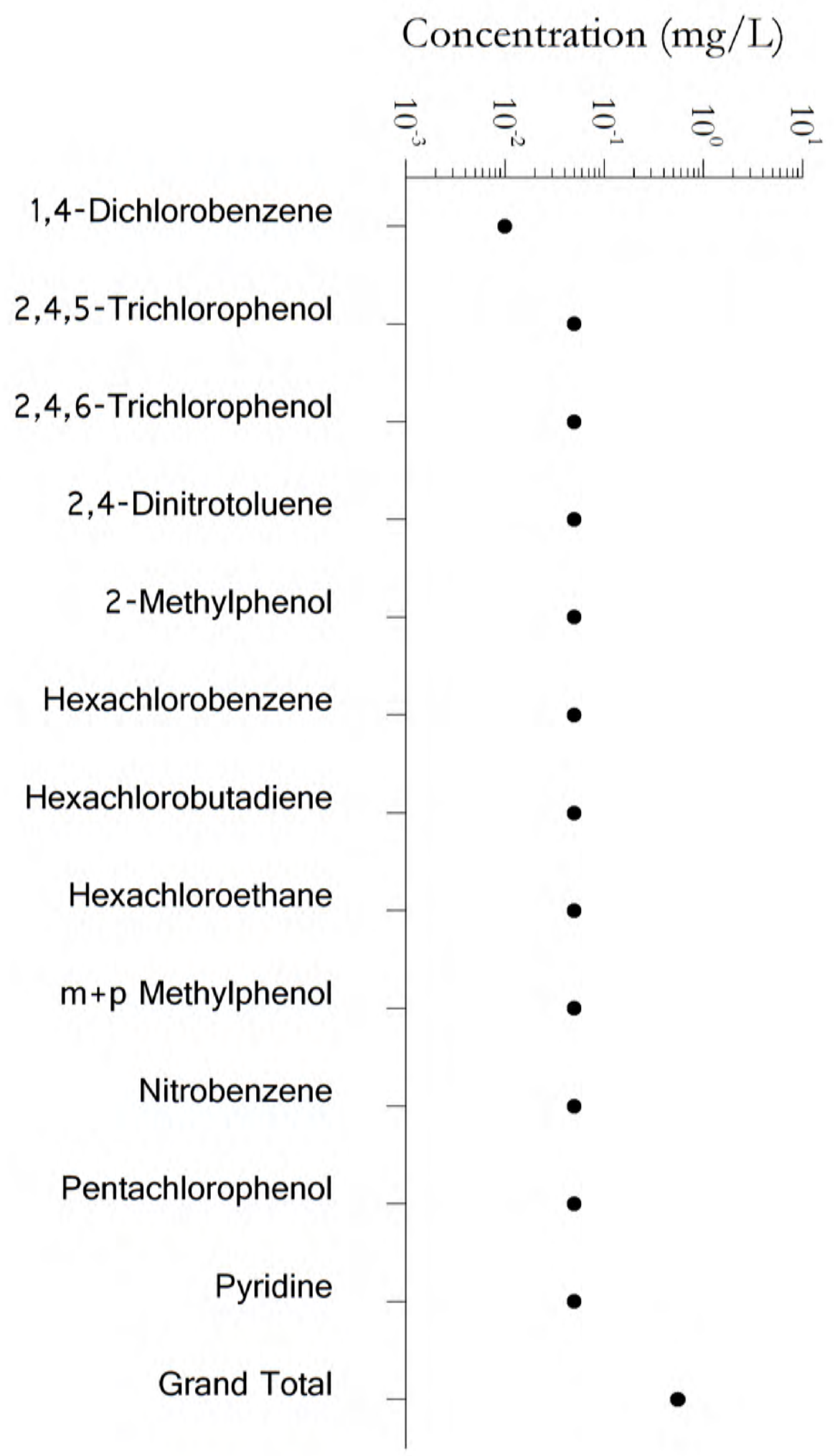




\section{Concentration $(\mu \mathrm{g} / \mathrm{kg})$}

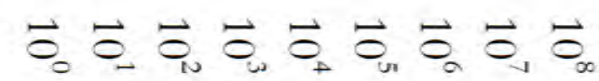

Benzo(a)pyrene

Benzo(b)fluoranthene

Benzo(ghi)perylene

$\operatorname{Benzo}(\mathrm{k})$ fluoranthene

Benzoic acid

Bis(2-chloroethoxy)methane

Bis(2-chloroethyl) ether

Bis(2-ethylhexyl)phthalate

Butyl benzyl phthalate

Carbazole

Chrysene

Dibenz(a,h)anthracene

Dibenzofuran

Diethyl phthalate

Dimethyl phthalate

Di-n-butyl phthalate

Di-n-octylphthalate

Diphenylamine

Fluoranthene

Fluorene

Hexachlorobenzene

Hexachlorobutadiene

Hexachlorocyclopentadiene

Hexachloroethane

Indeno(1,2,3-cd)pyrene Isophorone

$\mathrm{m}+\mathrm{p}$ Methylphenol

Naphthalene

Nitrobenzene

N-Nitroso-di-n-propylamine

Pentachlorophenol

Phenanthrene

Phenol

Pyridine

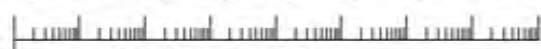

00

-

-

-

•

๑

-

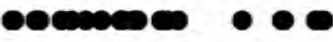

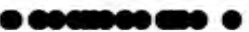

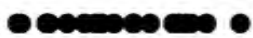

๑ ๑

-

•

๑ •

- ๑

๑

-

๑

๑ด ๑

-

๑ •

๑

๑

๑

๑

-

-

-

๑

- •

•

$\bullet$

$\bullet$

๑
Concentration $(\mu g / \mathrm{kg})$

$\vec{\sigma}_{0} \vec{\sigma}_{1} \vec{\sigma}_{w} \vec{\sigma}_{f} \vec{\sigma}_{n} \vec{\sigma}_{a} \vec{\sigma}_{N} \vec{\sigma}_{\infty}$

1,2,4-Trichlorobenzene

1,2-Dichlorobenzene

1,3-Dichlorobenzene

1,4-Dichlorobenzene

2,2'-Dichlorodiisopropyl ether

2,3,4,6-Tetrachlorophenol

2,4,5-Trichlorophenol

2,4,6-Trichlorophenol

2,4-Dichlorophenol

2,4-Dimethylphenol

2,4-Dinitrophenol

2,4-Dinitrotoluene

2,6-Dinitrotoluene

2-Chloronaphthalene 2-Chlorophenol

2-Methyl-4,6-dinitrophenol

2-Methylnaphthalene 2-Methylphenol

2-Nitrobenzenamine 2-Nitrophenol

3,3'-Dichlorobenzidine

3-Nitrobenzenamine

4-Bromophenyl phenyl ether 4-Chloro-3-methylphenol

4-Chlorobenzenamine

4-Chlorophenyl phenyl ether

4-Nitrobenzenamine 4-Nitrophenol

Acenaphthene Acenaphthylene Acetophenone Anthracene

Benz(a)anthracene

Benzenemethanol Benzidine 


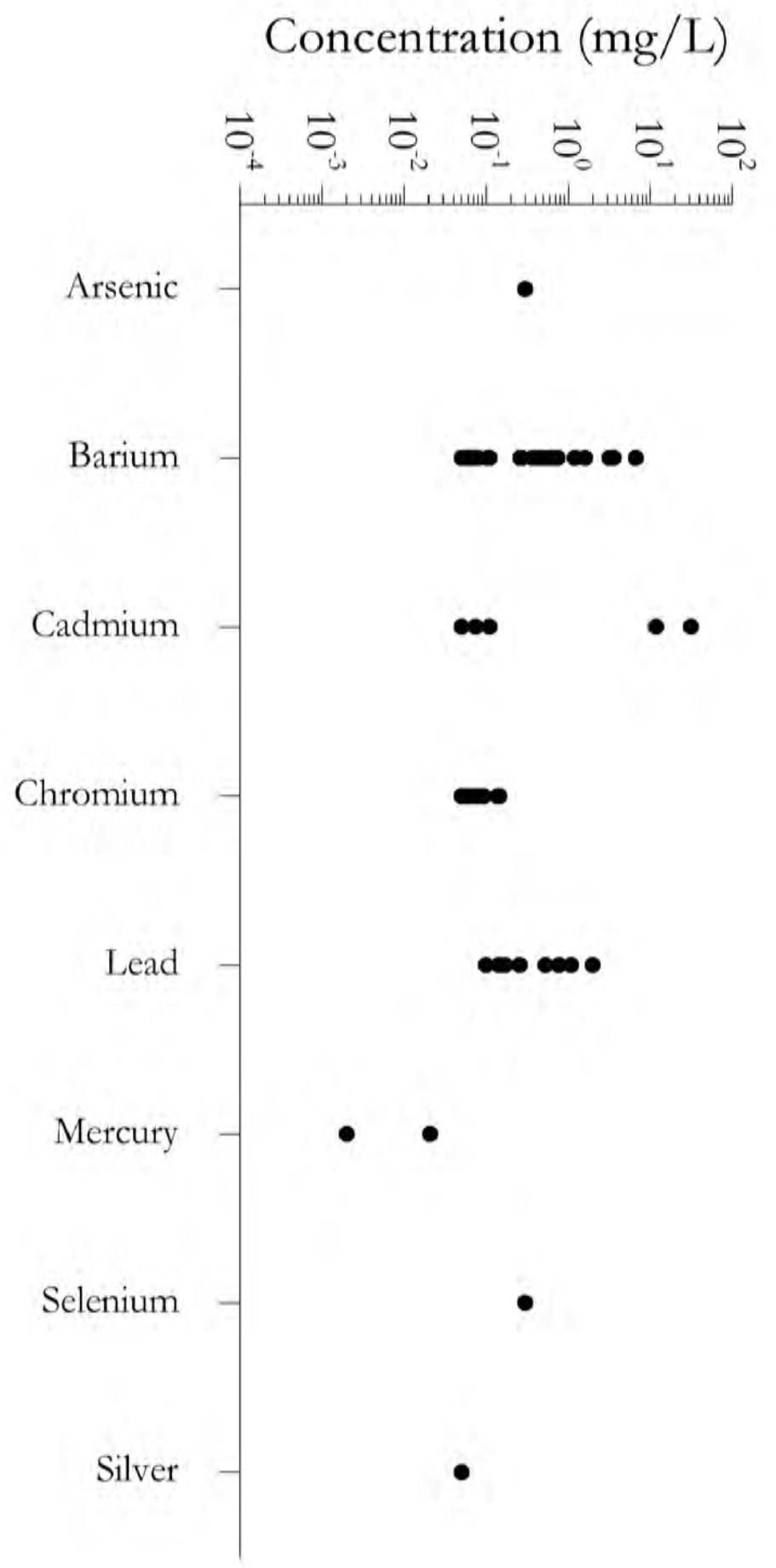




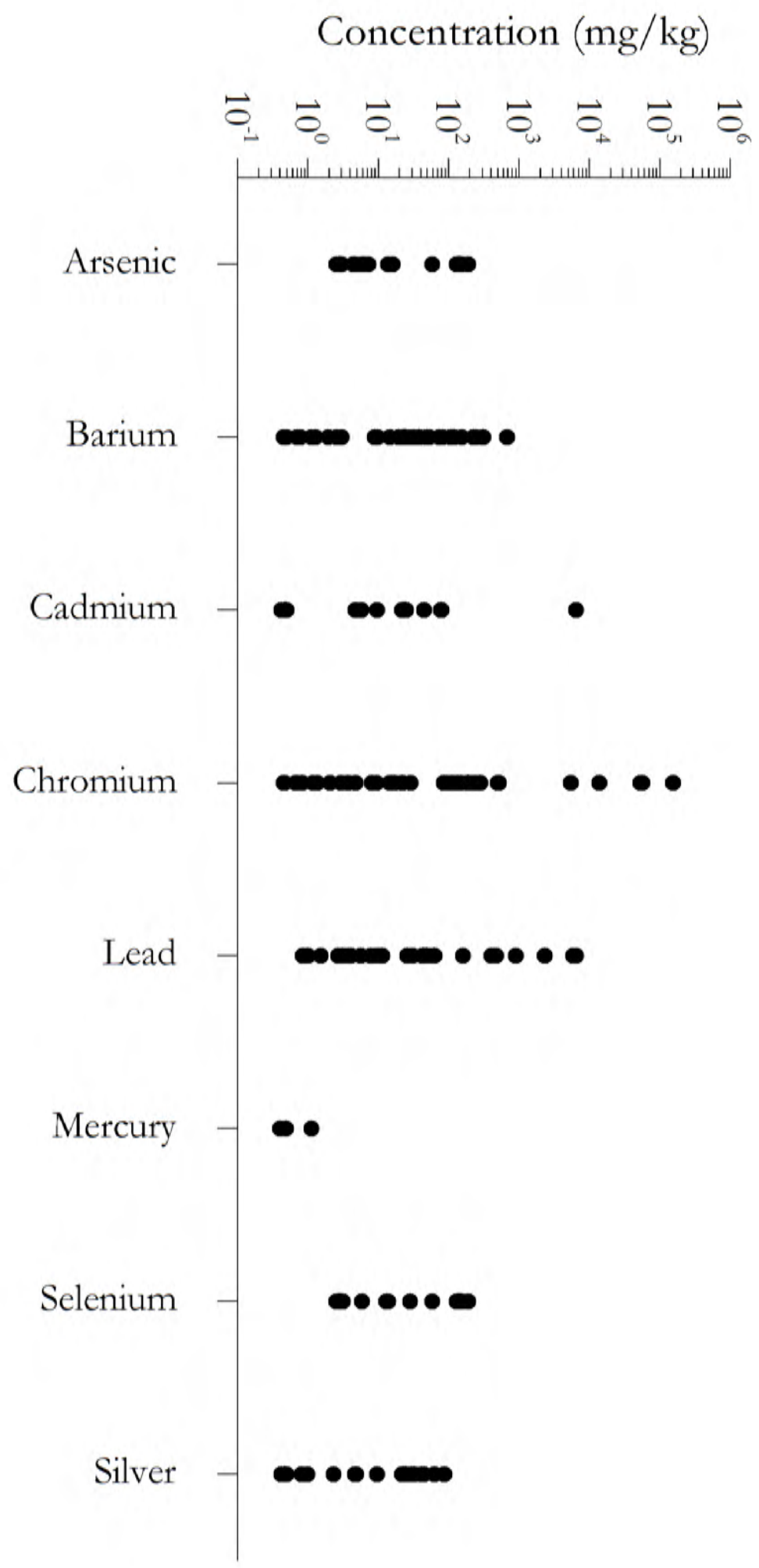




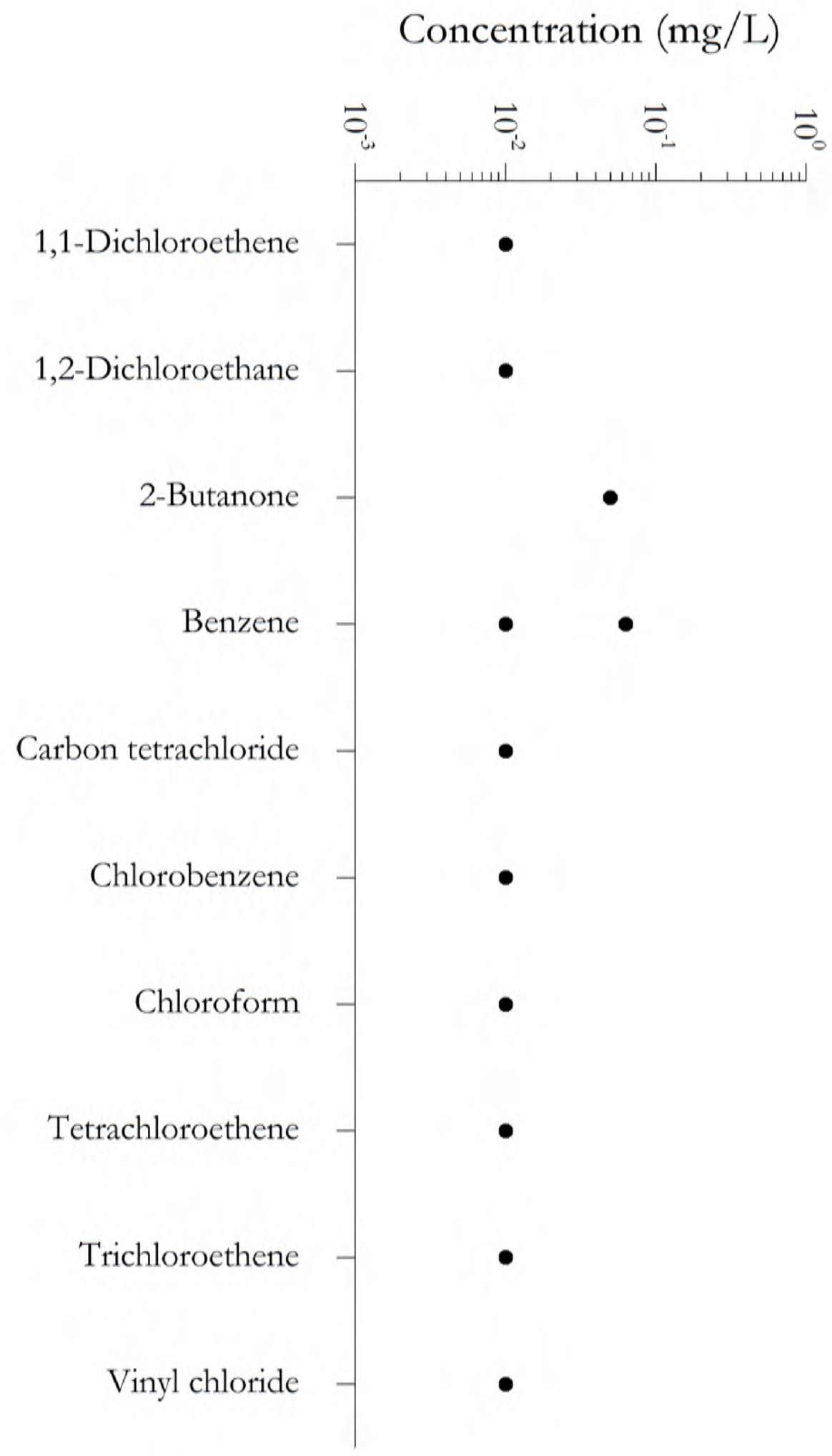


Concentration $(\mu \mathrm{g} / \mathrm{kg})$

(1,1-Dimethylethyl)benzene

(1-Methylpropyl)benzene

1,1,1,2-Tetrachloroethane

1,1,1-Trichloroethane

1,1,2,2-Tetrachloroethane

1,1,2-Trichloroethane

1,1-Dichloroethane

1,1-Dichloroethene

1,1-Dichloropropene

1,2,3-Trichlorobenzene

1,2,3-Trichloropropane

1,2,4-Trimethylbenzene

1,2-Dibromo-3-chloropropane

1,2-Dibromoethane

1,2-Dichloroethane

1,2-Dichloroethene

1,2-Dichloropropane

12-Dimethylbenzene

1,3,5-Trimethylbenzene

1,3-Dichloropropane

1-Chloro-4-methylbenzene

1-Methyl-4-(1-methylethyl)benzene

2,2-Dichloropropane 2-Butanone 2-Hexanone

4-Methyl-2-pentanone Acetone Acetonitrile Acrolein Acrylonitrile Benzene

Bromobenzene

Bromochloromethane Bromodichloromethane Bromoform

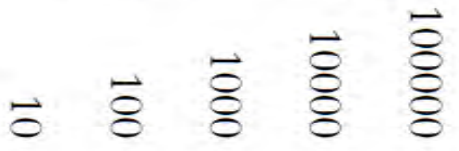

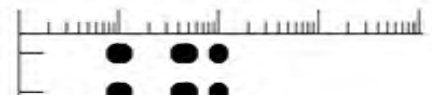

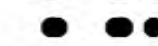

-

-

0

-

-

-

-

-

- $\bullet$

-

-

-

-

-

คอด

-

-

-

-

-

-

-

-

- 0

-

-

-

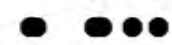

- • •

- $\bullet$

- ๑

- $\bullet$ $\infty 6$
Concentration $(\mu \mathrm{g} / \mathrm{kg})$

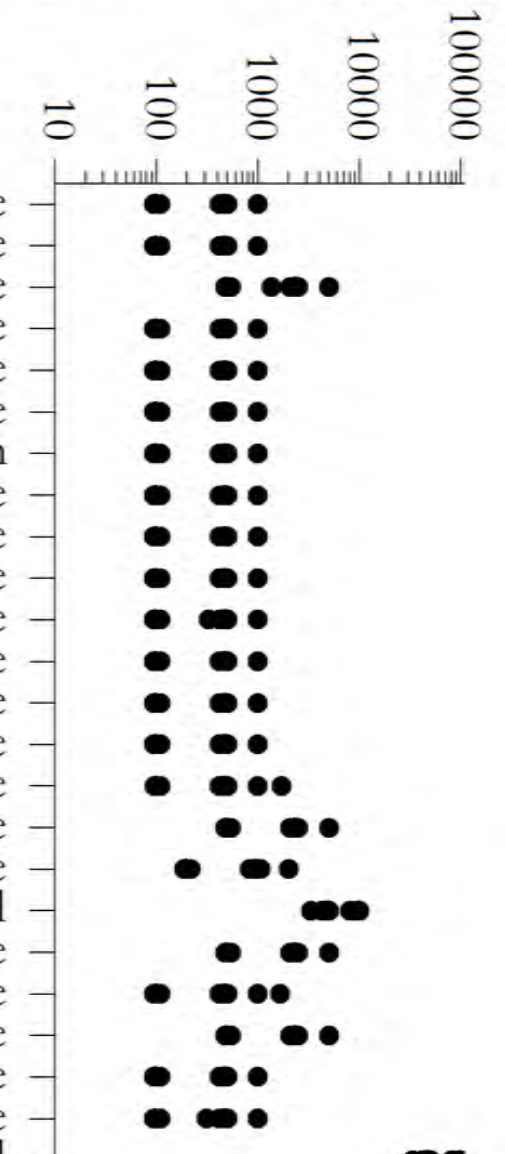

cis-1,2-Dichloroethene cis-1,3-Dichloropropene Cumene

Dibromochloromethane

Dibromomethane

Dichlorodifluoromethane

Ethylbenzene Hexane

$\mathrm{M}+\mathrm{P}$ Xylene Methanol

Methyl methacrylate

Methylcyclohexane

Methylene chloride

o-Chlorotoluene

Propylbenzene

Propylene glycol Styrene

Tetrachloroethene Toluene

Total Xylene

trans-1,2-Dichloroethene trans-1,3-Dichloropropene

Trichloroethene

Trichlorofluoromethane

Vinyl chloride 


\begin{tabular}{|c|c|c|c|c|c|c|c|c|c|c|}
\hline Container/ Item ID & $\begin{array}{c}\text { Analysis } \\
\text { Type }\end{array}$ & Chemical Name & $\begin{array}{l}\text { Sample } \\
\text { Count }\end{array}$ & $\begin{array}{c}\text { Num } \\
\text { Detects }\end{array}$ & Units & All Min & All Max & All Ave & $\begin{array}{c}\text { All } \\
\text { Median }\end{array}$ & $\begin{array}{c}\text { All } \\
\text { StdDev }\end{array}$ \\
\hline 3042-1994-01 & HERB & $2,4-\mathrm{D}$ & 2 & 0 & $\mathrm{ug} / \mathrm{kg}$ & 0.05 & 24.7 & 12.375 & 12.375 & 17.430182 \\
\hline 3042-1994-01 & HERB & Silvex & 2 & 0 & $\mathrm{ug} / \mathrm{kg}$ & 0.05 & 24.7 & 12.375 & 12.375 & 17.430182 \\
\hline 3042-1994-01 & METAL & Arsenic & 2 & 0 & $\mathrm{mg} / \mathrm{L}$ & 0.3 & 2.77 & 1.535 & 1.535 & 1.7465537 \\
\hline 3042-1994-01 & METAL & Barium & 1 & 1 & $\mathrm{mg} / \mathrm{L}$ & 0.0614 & 0.0614 & 0.0614 & 0.0614 & \\
\hline 3042-1994-01 & METAL & Cadmium & 2 & 0 & $\mathrm{mg} / \mathrm{L}$ & 0.05 & 0.462 & 0.256 & 0.256 & 0.291328 \\
\hline 3042-1994-01 & METAL & Chromium & 2 & 1 & $\mathrm{mg} / \mathrm{L}$ & 0.05 & 28.9 & 14.475 & 14.475 & 20.400031 \\
\hline 3042-1994-01 & METAL & Lead & 2 & 0 & $\mathrm{mg} / \mathrm{L}$ & 0.1 & 9.24 & 4.67 & 4.67 & 6.462956 \\
\hline 3042-1994-01 & METAL & Mercury & 2 & 0 & $\mathrm{mg} / \mathrm{L}$ & 0.0206 & 1.13 & 0.5753 & 0.5753 & 0.7844643 \\
\hline 3042-1994-01 & METAL & Selenium & 2 & 1 & $\mathrm{mg} / \mathrm{L}$ & 0.3 & 3.11 & 1.705 & 1.705 & 1.9869701 \\
\hline 3042-1994-01 & METAL & Silver & 2 & 0 & $\mathrm{mg} / \mathrm{L}$ & 0.05 & 4.62 & 2.335 & 2.335 & 3.231478 \\
\hline 3042-1994-01 & METAL & Total Uranium & 1 & 0 & $\mathrm{pCi} / \mathrm{g}$ & 1.31 & 1.31 & 1.31 & & \\
\hline 3042-1994-01 & PPCB & 4,4'-DDD & 1 & 0 & $\mathrm{ug} / \mathrm{kg}$ & 40 & 40 & 40 & & \\
\hline 3042-1994-01 & РPCB & 4,4'-DDE & 1 & 0 & $\mathrm{ug} / \mathrm{kg}$ & 40 & 40 & 40 & & \\
\hline 3042-1994-01 & РPCB & 4,4'-DDT & 1 & 0 & $\mathrm{ug} / \mathrm{kg}$ & 40 & 40 & 40 & & \\
\hline 3042-1994-01 & РPCB & Aldrin & 1 & 0 & $\mathrm{ug} / \mathrm{kg}$ & 20 & 20 & 20 & & \\
\hline 3042-1994-01 & PPCB & alpha-BHC & 1 & 0 & $\mathrm{ug} / \mathrm{kg}$ & 20 & 20 & 20 & & \\
\hline 3042-1994-01 & РPCB & alpha-Chlordane & 1 & 0 & $\mathrm{ug} / \mathrm{kg}$ & 20 & 20 & 20 & & \\
\hline 3042-1994-01 & РPCB & beta-BHC & 1 & 0 & $\mathrm{ug} / \mathrm{kg}$ & 20 & 20 & 20 & & \\
\hline 3042-1994-01 & РРCB & Chlordane & 2 & 0 & $\mathrm{ug} / \mathrm{kg}$ & 0.0025 & 250 & 125.00125 & 125.00125 & 176.77493 \\
\hline 3042-1994-01 & РPCB & delta-BHC & 1 & 0 & $\mathrm{ug} / \mathrm{kg}$ & 20 & 20 & 20 & & \\
\hline 3042-1994-01 & PPCB & Dieldrin & 1 & 0 & $\mathrm{ug} / \mathrm{kg}$ & 40 & 40 & 40 & & \\
\hline 3042-1994-01 & РPCB & Endosulfan I & 1 & 0 & $\mathrm{ug} / \mathrm{kg}$ & 20 & 20 & 20 & & \\
\hline 3042-1994-01 & РPCB & Endosulfan II & 1 & 0 & $\mathrm{ug} / \mathrm{kg}$ & 40 & 40 & 40 & & \\
\hline 3042-1994-01 & РPCB & Endosulfan sulfate & 1 & 0 & $\mathrm{ug} / \mathrm{kg}$ & 40 & 40 & 40 & & \\
\hline 3042-1994-01 & РРCB & Endrin & 2 & 0 & $\mathrm{ug} / \mathrm{kg}$ & 0.0004 & 40 & 20.0002 & 20.0002 & 28.283988 \\
\hline 3042-1994-01 & PPCB & Endrin aldehyde & 1 & 0 & $\mathrm{ug} / \mathrm{kg}$ & 40 & 40 & 40 & & \\
\hline 3042-1994-01 & PPCB & Endrin ketone & 1 & 0 & $\mathrm{ug} / \mathrm{kg}$ & 40 & 40 & 40 & & \\
\hline 3042-1994-01 & РPCB & gamma-Chlordane & 1 & 0 & $\mathrm{ug} / \mathrm{kg}$ & 20 & 20 & 20 & & \\
\hline 3042-1994-01 & РPCB & Heptachlor & 2 & 0 & $\mathrm{ug} / \mathrm{kg}$ & 0.0002 & 20 & 10.0001 & 10.0001 & 14.141994 \\
\hline 3042-1994-01 & РPCB & Heptachlor epoxide & 2 & 0 & $\mathrm{ug} / \mathrm{kg}$ & 0.0002 & 20 & 10.0001 & 10.0001 & 14.141994 \\
\hline 3042-1994-01 & РPCB & Lindane & 2 & 0 & $\mathrm{ug} / \mathrm{kg}$ & 0.0002 & 20 & 10.0001 & 10.0001 & 14.141994 \\
\hline 3042-1994-01 & PPCB & Methoxychlor & 2 & 0 & $\mathrm{ug} / \mathrm{kg}$ & 0.002 & 200 & 100.001 & 100.001 & 141.41994 \\
\hline 3042-1994-01 & РPCB & Toxaphene & 2 & 0 & $\mathrm{ug} / \mathrm{kg}$ & 0.005 & 500 & 250.0025 & 250.0025 & 353.54986 \\
\hline 3042-1994-01 & RADS & Americium-241 & 1 & 0 & $\mathrm{pCi} / \mathrm{g}$ & 0 & 0 & 0 & & \\
\hline 3042-1994-01 & RADS & Carbon-14 & 1 & 0 & $\mathrm{pCi} / \mathrm{g}$ & -4.78 & -4.78 & -4.78 & & \\
\hline 3042-1994-01 & RADS & Cesium-137 & 1 & 0 & $\mathrm{pCi} / \mathrm{g}$ & 0.0932 & 0.0932 & 0.0932 & & \\
\hline 3042-1994-01 & RADS & Cobalt- 60 & 1 & 0 & $\mathrm{pCi} / \mathrm{g}$ & 0.142 & 0.142 & 0.142 & & \\
\hline 3042-1994-01 & RADS & Curium-243/244 & 1 & 0 & $\mathrm{pCi} / \mathrm{g}$ & -0.021 & -0.021 & -0.021 & & \\
\hline 3042-1994-01 & RADS & Iodine-129 & 1 & 0 & $\mathrm{pCi} / \mathrm{g}$ & -0.127 & -0.127 & -0.127 & & \\
\hline 3042-1994-01 & RADS & Neptunium-237 & 1 & 0 & $\mathrm{pCi} / \mathrm{g}$ & -0.131 & -0.131 & -0.131 & & \\
\hline 3042-1994-01 & RADS & Plutonium-238 & 1 & 0 & $\mathrm{pCi} / \mathrm{g}$ & -0.00476 & -0.00476 & -0.00476 & & \\
\hline 3042-1994-01 & RADS & Plutonium-239/240 & 1 & 0 & $\mathrm{pCi} / \mathrm{g}$ & 0 & 0 & 0 & & \\
\hline 3042-1994-01 & RADS & Radium-226 & 1 & 0 & $\mathrm{pCi} / \mathrm{g}$ & 0.437 & 0.437 & 0.437 & & \\
\hline 3042-1994-01 & RADS & Strontium-90 & 1 & 0 & $\mathrm{pCi} / \mathrm{g}$ & -0.387 & -0.387 & -0.387 & & \\
\hline 3042-1994-01 & RADS & Technetium-99 & 1 & 0 & $\mathrm{pCi} / \mathrm{g}$ & -1.64 & -1.64 & -1.64 & & \\
\hline 3042-1994-01 & RADS & Thorium-230 & 1 & 0 & $\mathrm{pCi} / \mathrm{g}$ & 0.437 & 0.437 & 0.437 & & \\
\hline 3042-1994-01 & RADS & Thorium-232 & 1 & 0 & $\mathrm{pCi} / \mathrm{g}$ & 1.12 & 1.12 & 1.12 & & \\
\hline 3042-1994-01 & RADS & Tritium & 1 & 0 & $\mathrm{pCi} / \mathrm{g}$ & 0.939 & 0.939 & 0.939 & & \\
\hline 3042-1994-01 & RADS & Uranium-232 & 1 & 1 & $\%$ & 78.8 & 78.8 & 78.8 & 78.8 & \\
\hline 3042-1994-01 & RADS & Uranium-233/234 & 1 & 1 & $\mathrm{pCi} / \mathrm{g}$ & 0.749 & 0.749 & 0.749 & 0.749 & \\
\hline 3042-1994-01 & RADS & Uranium-235/236 & 1 & 0 & $\mathrm{pCi} / \mathrm{g}$ & 0.066 & 0.066 & 0.066 & & \\
\hline 3042-1994-01 & RADS & Uranium-238 & 1 & 0 & $\mathrm{pCi} / \mathrm{g}$ & 0.495 & 0.495 & 0.495 & & \\
\hline 3042-1994-01 & SVOA & 1,2,4-Trichlorobenzene & 1 & 0 & $\mathrm{ug} / \mathrm{kg}$ & 500 & 500 & 500 & & \\
\hline 3042-1994-01 & SVOA & 1,2-Dichlorobenzene & 1 & 0 & $\mathrm{ug} / \mathrm{kg}$ & 500 & 500 & 500 & & \\
\hline 3042-1994-01 & SVOA & 1,3-Dichlorobenzene & 1 & 0 & $\mathrm{ug} / \mathrm{kg}$ & 500 & 500 & 500 & & \\
\hline 3042-1994-01 & SVOA & 1,4-Dichlorobenzene & 2 & 0 & $\mathrm{ug} / \mathrm{kg}$ & 0.01 & 500 & 250.005 & 250.005 & 353.54632 \\
\hline 3042-1994-01 & SVOA & 2,2'-Dichlorodiisopropyl ether & 1 & 0 & $\mathrm{ug} / \mathrm{kg}$ & 999 & 999 & 999 & & \\
\hline 3042-1994-01 & SVOA & 2,3,4,6-Tetrachlorophenol & 1 & 0 & $\mathrm{ug} / \mathrm{kg}$ & 999 & 999 & 999 & & \\
\hline 3042-1994-01 & SVOA & 2,4,5-Trichlorophenol & 2 & 0 & $\mathrm{ug} / \mathrm{kg}$ & 0.05 & 999 & 499.525 & 499.525 & 706.36432 \\
\hline 3042-1994-01 & SVOA & 2,4,6-Trichlorophenol & 2 & 0 & $\mathrm{ug} / \mathrm{kg}$ & 0.05 & 999 & 499.525 & 499.525 & 706.36432 \\
\hline 3042-1994-01 & SVOA & 2,4-Dichlorophenol & 1 & 0 & $\mathrm{ug} / \mathrm{kg}$ & 999 & 999 & 999 & & \\
\hline 3042-1994-01 & SVOA & 2,4-Dimethylphenol & 1 & 0 & $\mathrm{ug} / \mathrm{kg}$ & 999 & 999 & 999 & & \\
\hline 3042-1994-01 & SVOA & 2,4-Dinitrophenol & 1 & 0 & $\mathrm{ug} / \mathrm{kg}$ & 2000 & 2000 & 2000 & & \\
\hline 3042-1994-01 & SVOA & 2,4-Dinitrotoluene & 2 & 0 & $\mathrm{ug} / \mathrm{kg}$ & 0.05 & 999 & 499.525 & 499.525 & 706.36432 \\
\hline 3042-1994-01 & SVOA & 2,6-Dinitrotoluene & 1 & 0 & $\mathrm{ug} / \mathrm{kg}$ & 999 & 999 & 999 & & \\
\hline 3042-1994-01 & SVOA & 2-Chloronaphthalene & 1 & 0 & $\mathrm{ug} / \mathrm{kg}$ & 99.9 & 99.9 & 99.9 & & \\
\hline 3042-1994-01 & SVOA & 2-Chlorophenol & 1 & 0 & $\mathrm{ug} / \mathrm{kg}$ & 999 & 999 & 999 & & \\
\hline 3042-1994-01 & SVOA & 2-Methyl-4,6-dinitrophenol & 1 & 0 & $\mathrm{ug} / \mathrm{kg}$ & 999 & 999 & 999 & & \\
\hline
\end{tabular}




\begin{tabular}{|c|c|c|c|c|c|c|c|c|c|c|}
\hline 3042-1994-01 & SVOA & 2-Methylnaphthalene & 1 & 0 & $\mathrm{ug} / \mathrm{kg}$ & 99.9 & 99.9 & 99.9 & & \\
\hline 3042-1994-01 & SVOA & 2-Methylphenol & 2 & 0 & $\mathrm{ug} / \mathrm{kg}$ & 0.05 & 999 & 499.525 & 499.525 & 706.36432 \\
\hline 3042-1994-01 & SVOA & 2-Nitrobenzenamine & 1 & 0 & $\mathrm{ug} / \mathrm{kg}$ & 999 & 999 & 999 & & \\
\hline 3042-1994-01 & SVOA & 2-Nitrophenol & 1 & 0 & $\mathrm{ug} / \mathrm{kg}$ & 999 & 999 & 999 & & \\
\hline 3042-1994-01 & SVOA & 3,3'-Dichlorobenzidine & 1 & 0 & $\mathrm{ug} / \mathrm{kg}$ & 999 & 999 & 999 & & \\
\hline 3042-1994-01 & SVOA & 3-Nitrobenzenamine & 1 & 0 & $\mathrm{ug} / \mathrm{kg}$ & 999 & 999 & 999 & & \\
\hline 3042-1994-01 & SVOA & 4-Bromophenyl phenyl ether & 1 & 0 & $\mathrm{ug} / \mathrm{kg}$ & 999 & 999 & 999 & & \\
\hline 3042-1994-01 & SVOA & 4-Chloro-3-methylphenol & 1 & 0 & $\mathrm{ug} / \mathrm{kg}$ & 999 & 999 & 999 & & \\
\hline 3042-1994-01 & SVOA & 4-Chlorobenzenamine & 1 & 0 & $\mathrm{ug} / \mathrm{kg}$ & 999 & 999 & 999 & & \\
\hline 3042-1994-01 & SVOA & 4-Chlorophenyl phenyl ether & 1 & 0 & $\mathrm{ug} / \mathrm{kg}$ & 999 & 999 & 999 & & \\
\hline 3042-1994-01 & SVOA & 4-Nitrobenzenamine & 1 & 0 & $\mathrm{ug} / \mathrm{kg}$ & 999 & 999 & 999 & & \\
\hline 3042-1994-01 & SVOA & 4-Nitrophenol & 1 & 0 & $\mathrm{ug} / \mathrm{kg}$ & 999 & 999 & 999 & & \\
\hline 3042-1994-01 & SVOA & Acenaphthene & 1 & 0 & $\mathrm{ug} / \mathrm{kg}$ & 99.9 & 99.9 & 99.9 & & \\
\hline 3042-1994-01 & SVOA & Acenaphthylene & 1 & 0 & $\mathrm{ug} / \mathrm{kg}$ & 99.9 & 99.9 & 99.9 & & \\
\hline 3042-1994-01 & SVOA & Acetophenone & 1 & 0 & $\mathrm{ug} / \mathrm{kg}$ & 999 & 999 & 999 & & \\
\hline 3042-1994-01 & SVOA & Anthracene & 1 & 0 & $\mathrm{ug} / \mathrm{kg}$ & 99.9 & 99.9 & 99.9 & & \\
\hline 3042-1994-01 & SVOA & Benz(a)anthracene & 1 & 0 & $\mathrm{ug} / \mathrm{kg}$ & 99.9 & 99.9 & 99.9 & & \\
\hline 3042-1994-01 & SVOA & Benzenemethanol & 1 & 0 & $\mathrm{ug} / \mathrm{kg}$ & 999 & 999 & 999 & & \\
\hline 3042-1994-01 & SVOA & Benzidine & 1 & 0 & $\mathrm{ug} / \mathrm{kg}$ & 999 & 999 & 999 & & \\
\hline 3042-1994-01 & SVOA & Benzo(a)pyrene & 1 & 0 & $\mathrm{ug} / \mathrm{kg}$ & 99.9 & 99.9 & 99.9 & & \\
\hline 3042-1994-01 & SVOA & Benzo(b)fluoranthene & 1 & 0 & $\mathrm{ug} / \mathrm{kg}$ & 99.9 & 99.9 & 99.9 & & \\
\hline 3042-1994-01 & SVOA & Benzo(ghi)perylene & 1 & 0 & $\mathrm{ug} / \mathrm{kg}$ & 99.9 & 99.9 & 99.9 & & \\
\hline 3042-1994-01 & SVOA & Benzo(k)fluoranthene & 1 & 0 & $\mathrm{ug} / \mathrm{kg}$ & 99.9 & 99.9 & 99.9 & & \\
\hline 3042-1994-01 & SVOA & Benzoic acid & 1 & 0 & $\mathrm{ug} / \mathrm{kg}$ & 2000 & 2000 & 2000 & & \\
\hline 3042-1994-01 & SVOA & Bis(2-chloroethoxy)methane & 1 & 0 & $\mathrm{ug} / \mathrm{kg}$ & 999 & 999 & 999 & & \\
\hline 3042-1994-01 & SVOA & Bis(2-chloroethyl) ether & 1 & 0 & $\mathrm{ug} / \mathrm{kg}$ & 999 & 999 & 999 & & \\
\hline 3042-1994-01 & SVOA & Bis(2-ethylhexyl)phthalate & 1 & 1 & $\mathrm{ug} / \mathrm{kg}$ & 1110 & 1110 & 1110 & 1110 & \\
\hline 3042-1994-01 & SVOA & Butyl benzyl phthalate & 1 & 1 & $\mathrm{ug} / \mathrm{kg}$ & 1710 & 1710 & 1710 & 1710 & \\
\hline 3042-1994-01 & SVOA & Carbazole & 1 & 0 & $\mathrm{ug} / \mathrm{kg}$ & 99.9 & 99.9 & 99.9 & & \\
\hline 3042-1994-01 & SVOA & Chrysene & 1 & 0 & $\mathrm{ug} / \mathrm{kg}$ & 99.9 & 99.9 & 99.9 & & \\
\hline 3042-1994-01 & SVOA & Dibenz $(\mathrm{a}, \mathrm{h})$ anthracene & 1 & 0 & $\mathrm{ug} / \mathrm{kg}$ & 99.9 & 99.9 & 99.9 & & \\
\hline 3042-1994-01 & SVOA & Dibenzofuran & 1 & 0 & $\mathrm{ug} / \mathrm{kg}$ & 999 & 999 & 999 & & \\
\hline 3042-1994-01 & SVOA & Diethyl phthalate & 1 & 0 & $\mathrm{ug} / \mathrm{kg}$ & 999 & 999 & 999 & & \\
\hline 3042-1994-01 & SVOA & Dimethyl phthalate & 1 & 0 & $\mathrm{ug} / \mathrm{kg}$ & 999 & 999 & 999 & & \\
\hline 3042-1994-01 & SVOA & Di-n-butyl phthalate & 1 & 0 & $\mathrm{ug} / \mathrm{kg}$ & 999 & 999 & 999 & & \\
\hline 3042-1994-01 & SVOA & Di-n-octylphthalate & 1 & 0 & $\mathrm{ug} / \mathrm{kg}$ & 999 & 999 & 999 & & \\
\hline 3042-1994-01 & SVOA & Diphenylamine & 1 & 0 & $\mathrm{ug} / \mathrm{kg}$ & 999 & 999 & 999 & & \\
\hline 3042-1994-01 & SVOA & Fluoranthene & 1 & 0 & $\mathrm{ug} / \mathrm{kg}$ & 99.9 & 99.9 & 99.9 & & \\
\hline 3042-1994-01 & SVOA & Fluorene & 1 & 0 & $\mathrm{ug} / \mathrm{kg}$ & 99.9 & 99.9 & 99.9 & & \\
\hline 3042-1994-01 & SVOA & Hexachlorobenzene & 2 & 0 & $\mathrm{ug} / \mathrm{kg}$ & 0.05 & 999 & 499.525 & 499.525 & 706.36432 \\
\hline 3042-1994-01 & SVOA & Hexachlorobutadiene & 2 & 0 & $\mathrm{ug} / \mathrm{kg}$ & 0.05 & 999 & 499.525 & 499.525 & 706.36432 \\
\hline 3042-1994-01 & SVOA & Hexachlorocyclopentadiene & 1 & 0 & $\mathrm{ug} / \mathrm{kg}$ & 999 & 999 & 999 & & \\
\hline 3042-1994-01 & SVOA & Hexachloroethane & 2 & 0 & $\mathrm{ug} / \mathrm{kg}$ & 0.05 & 999 & 499.525 & 499.525 & 706.36432 \\
\hline 3042-1994-01 & SVOA & Indeno(1,2,3-cd)pyrene & 1 & 0 & $\mathrm{ug} / \mathrm{kg}$ & 99.9 & 99.9 & 99.9 & & \\
\hline 3042-1994-01 & SVOA & Isophorone & 1 & 0 & $\mathrm{ug} / \mathrm{kg}$ & 999 & 999 & 999 & & \\
\hline 3042-1994-01 & SVOA & $\mathrm{m}+\mathrm{p}$ Methylphenol & 2 & 0 & $\mathrm{ug} / \mathrm{kg}$ & 0.05 & 999 & 499.525 & 499.525 & 706.36432 \\
\hline 3042-1994-01 & SVOA & Naphthalene & 1 & 0 & $\mathrm{ug} / \mathrm{kg}$ & 99.9 & 99.9 & 99.9 & & \\
\hline 3042-1994-01 & SVOA & Nitrobenzene & 2 & 0 & $\mathrm{ug} / \mathrm{kg}$ & 0.05 & 999 & 499.525 & 499.525 & 706.36432 \\
\hline 3042-1994-01 & SVOA & N-Nitroso-di-n-propylamine & 1 & 0 & $\mathrm{ug} / \mathrm{kg}$ & 999 & 999 & 999 & & \\
\hline 3042-1994-01 & SVOA & Pentachlorophenol & 2 & 0 & $\mathrm{ug} / \mathrm{kg}$ & 0.05 & 999 & 499.525 & 499.525 & 706.36432 \\
\hline 3042-1994-01 & SVOA & Phenanthrene & 1 & 0 & $\mathrm{ug} / \mathrm{kg}$ & 99.9 & 99.9 & 99.9 & & \\
\hline 3042-1994-01 & SVOA & Phenol & 1 & 0 & $\mathrm{ug} / \mathrm{kg}$ & 999 & 999 & 999 & & \\
\hline 3042-1994-01 & SVOA & Pyridine & 2 & 0 & $\mathrm{ug} / \mathrm{kg}$ & 0.05 & 999 & 499.525 & 499.525 & 706.36432 \\
\hline 3042-1994-01 & VOA & (1,1-Dimethylethyl)benzene & 1 & 0 & $\mathrm{ug} / \mathrm{kg}$ & 500 & 500 & 500 & & \\
\hline 3042-1994-01 & VOA & (1-Methylpropyl)benzene & 1 & 0 & $\mathrm{ug} / \mathrm{kg}$ & 500 & 500 & 500 & & \\
\hline 3042-1994-01 & VOA & 1,1,1,2-Tetrachloroethane & 1 & 0 & $\mathrm{ug} / \mathrm{kg}$ & 500 & 500 & 500 & & \\
\hline 3042-1994-01 & VOA & 1,1,1-Trichloroethane & 1 & 0 & $\mathrm{ug} / \mathrm{kg}$ & 500 & 500 & 500 & & \\
\hline 3042-1994-01 & VOA & 1,1,2,2-Tetrachloroethane & 1 & 0 & $\mathrm{ug} / \mathrm{kg}$ & 500 & 500 & 500 & & \\
\hline 3042-1994-01 & VOA & 1,1,2-Trichloroethane & 1 & 0 & $\mathrm{ug} / \mathrm{kg}$ & 500 & 500 & 500 & & \\
\hline 3042-1994-01 & VOA & 1,1-Dichloroethane & 1 & 0 & $\mathrm{ug} / \mathrm{kg}$ & 500 & 500 & 500 & & \\
\hline 3042-1994-01 & VOA & 1,1-Dichloroethene & 2 & 0 & $\mathrm{ug} / \mathrm{kg}$ & 0.01 & 500 & 250.005 & 250.005 & 353.54632 \\
\hline 3042-1994-01 & VOA & 1,1-Dichloropropene & 1 & 0 & $\mathrm{ug} / \mathrm{kg}$ & 500 & 500 & 500 & & \\
\hline 3042-1994-01 & VOA & 1,2,3-Trichlorobenzene & 1 & 0 & $\mathrm{ug} / \mathrm{kg}$ & 500 & 500 & 500 & & \\
\hline 3042-1994-01 & VOA & 1,2,3-Trichloropropane & 1 & 0 & $\mathrm{ug} / \mathrm{kg}$ & 500 & 500 & 500 & & \\
\hline 3042-1994-01 & VOA & 1,2,4-Trimethylbenzene & 1 & 0 & $\mathrm{ug} / \mathrm{kg}$ & 500 & 500 & 500 & & \\
\hline 3042-1994-01 & VOA & 1,2-Dibromo-3-chloropropane & 1 & 0 & $\mathrm{ug} / \mathrm{kg}$ & 500 & 500 & 500 & & \\
\hline 3042-1994-01 & VOA & 1,2-Dibromoethane & 1 & 0 & $\mathrm{ug} / \mathrm{kg}$ & 500 & 500 & 500 & & \\
\hline 3042-1994-01 & VOA & 1,2-Dichloroethane & 2 & 0 & $\mathrm{ug} / \mathrm{kg}$ & 0.01 & 500 & 250.005 & 250.005 & 353.54632 \\
\hline 3042-1994-01 & VOA & 1,2-Dichloroethene & 1 & 0 & $\mathrm{ug} / \mathrm{kg}$ & 1000 & 1000 & 1000 & & \\
\hline 3042-1994-01 & VOA & 1,2-Dichloropropane & 1 & 0 & $\mathrm{ug} / \mathrm{kg}$ & 500 & 500 & 500 & & \\
\hline
\end{tabular}




\begin{tabular}{|c|c|c|c|c|c|c|c|c|c|c|}
\hline 3042-1994-01 & VOA & 1,2-Dimethylbenzene & 1 & 0 & $\mathrm{ug} / \mathrm{kg}$ & 500 & 500 & 500 & & \\
\hline 3042-1994-01 & VOA & 1,3,5-Trimethylbenzene & 1 & 0 & $\mathrm{ug} / \mathrm{kg}$ & 500 & 500 & 500 & & \\
\hline 3042-1994-01 & VOA & 1,3-Dichloropropane & 1 & 0 & $\mathrm{ug} / \mathrm{kg}$ & 500 & 500 & 500 & & \\
\hline 3042-1994-01 & VOA & 1-Chloro-4-methylbenzene & 1 & 0 & $\mathrm{ug} / \mathrm{kg}$ & 500 & 500 & 500 & & \\
\hline 3042-1994-01 & VOA & 1-Methyl-4-(1-methylethyl)benzene & 1 & 0 & $\mathrm{ug} / \mathrm{kg}$ & 500 & 500 & 500 & & \\
\hline 3042-1994-01 & VOA & 2,2-Dichloropropane & 1 & 0 & $\mathrm{ug} / \mathrm{kg}$ & 500 & 500 & 500 & & \\
\hline 3042-1994-01 & VOA & 2-Butanone & 2 & 0 & $\mathrm{ug} / \mathrm{kg}$ & 0.05 & 2500 & 1250.025 & 1250.025 & 1767.7316 \\
\hline 3042-1994-01 & VOA & 2-Hexanone & 1 & 0 & $\mathrm{ug} / \mathrm{kg}$ & 2500 & 2500 & 2500 & & \\
\hline 3042-1994-01 & VOA & 4-Methyl-2-pentanone & 1 & 0 & $\mathrm{ug} / \mathrm{kg}$ & 2500 & 2500 & 2500 & & \\
\hline 3042-1994-01 & VOA & Acetone & 1 & 0 & $\mathrm{ug} / \mathrm{kg}$ & 2500 & 2500 & 2500 & & \\
\hline 3042-1994-01 & VOA & Acetonitrile & 1 & 0 & $\mathrm{ug} / \mathrm{kg}$ & 12500 & 12500 & 12500 & & \\
\hline 3042-1994-01 & VOA & Acrolein & 1 & 0 & $\mathrm{ug} / \mathrm{kg}$ & 2500 & 2500 & 2500 & & \\
\hline 3042-1994-01 & VOA & Acrylonitrile & 1 & 0 & $\mathrm{ug} / \mathrm{kg}$ & 2500 & 2500 & 2500 & & \\
\hline 3042-1994-01 & VOA & Benzene & 2 & 0 & $\mathrm{ug} / \mathrm{kg}$ & 0.01 & 500 & 250.005 & 250.005 & 353.54632 \\
\hline 3042-1994-01 & VOA & Bromobenzene & 1 & 0 & $\mathrm{ug} / \mathrm{kg}$ & 500 & 500 & 500 & & \\
\hline 3042-1994-01 & VOA & Bromochloromethane & 1 & 0 & $\mathrm{ug} / \mathrm{kg}$ & 500 & 500 & 500 & & \\
\hline 3042-1994-01 & VOA & Bromodichloromethane & 1 & 0 & $\mathrm{ug} / \mathrm{kg}$ & 500 & 500 & 500 & & \\
\hline 3042-1994-01 & VOA & Bromoform & 1 & 0 & $\mathrm{ug} / \mathrm{kg}$ & 500 & 500 & 500 & & \\
\hline 3042-1994-01 & VOA & Bromomethane & 1 & 0 & $\mathrm{ug} / \mathrm{kg}$ & 500 & 500 & 500 & & \\
\hline 3042-1994-01 & VOA & Butylbenzene & 1 & 0 & $\mathrm{ug} / \mathrm{kg}$ & 500 & 500 & 500 & & \\
\hline 3042-1994-01 & VOA & Carbon disulfide & 1 & 0 & $\mathrm{ug} / \mathrm{kg}$ & 2500 & 2500 & 2500 & & \\
\hline 3042-1994-01 & VOA & Carbon tetrachloride & 2 & 0 & $\mathrm{ug} / \mathrm{kg}$ & 0.01 & 500 & 250.005 & 250.005 & 353.54632 \\
\hline 3042-1994-01 & VOA & Chlorobenzene & 2 & 0 & $\mathrm{ug} / \mathrm{kg}$ & 0.01 & 500 & 250.005 & 250.005 & 353.54632 \\
\hline 3042-1994-01 & VOA & Chloroethane & 1 & 0 & $\mathrm{ug} / \mathrm{kg}$ & 500 & 500 & 500 & & \\
\hline 3042-1994-01 & VOA & Chloroform & 2 & 0 & $\mathrm{ug} / \mathrm{kg}$ & 0.01 & 500 & 250.005 & 250.005 & 353.54632 \\
\hline 3042-1994-01 & VOA & Chloromethane & 1 & 0 & $\mathrm{ug} / \mathrm{kg}$ & 500 & 500 & 500 & & \\
\hline 3042-1994-01 & VOA & cis-1,2-Dichloroethene & 1 & 0 & $\mathrm{ug} / \mathrm{kg}$ & 500 & 500 & 500 & & \\
\hline 3042-1994-01 & VOA & cis-1,3-Dichloropropene & 1 & 0 & $\mathrm{ug} / \mathrm{kg}$ & 500 & 500 & 500 & & \\
\hline 3042-1994-01 & VOA & Cumene & 1 & 0 & $\mathrm{ug} / \mathrm{kg}$ & 500 & 500 & 500 & & \\
\hline 3042-1994-01 & VOA & Dibromochloromethane & 1 & 0 & $\mathrm{ug} / \mathrm{kg}$ & 500 & 500 & 500 & & \\
\hline 3042-1994-01 & VOA & Dibromomethane & 1 & 0 & $\mathrm{ug} / \mathrm{kg}$ & 500 & 500 & 500 & & \\
\hline 3042-1994-01 & VOA & Dichlorodifluoromethane & 1 & 0 & $\mathrm{ug} / \mathrm{kg}$ & 500 & 500 & 500 & & \\
\hline 3042-1994-01 & VOA & Ethylbenzene & 1 & 0 & $\mathrm{ug} / \mathrm{kg}$ & 500 & 500 & 500 & & \\
\hline 3042-1994-01 & VOA & Hexane & 1 & 0 & $\mathrm{ug} / \mathrm{kg}$ & 2500 & 2500 & 2500 & & \\
\hline 3042-1994-01 & VOA & M + P Xylene & 1 & 0 & $\mathrm{ug} / \mathrm{kg}$ & 1000 & 1000 & 1000 & & \\
\hline 3042-1994-01 & VOA & Methanol & 1 & 0 & $\mathrm{ug} / \mathrm{kg}$ & 5000 & 5000 & 5000 & & \\
\hline 3042-1994-01 & VOA & Methyl methacrylate & 1 & 0 & $\mathrm{ug} / \mathrm{kg}$ & 2500 & 2500 & 2500 & & \\
\hline 3042-1994-01 & VOA & Methylcyclohexane & 1 & 0 & $\mathrm{ug} / \mathrm{kg}$ & 500 & 500 & 500 & & \\
\hline 3042-1994-01 & VOA & Methylene chloride & 1 & 0 & $\mathrm{ug} / \mathrm{kg}$ & 2500 & 2500 & 2500 & & \\
\hline 3042-1994-01 & VOA & o-Chlorotoluene & 1 & 0 & $\mathrm{ug} / \mathrm{kg}$ & 500 & 500 & 500 & & \\
\hline 3042-1994-01 & VOA & Propylbenzene & 1 & 0 & $\mathrm{ug} / \mathrm{kg}$ & 500 & 500 & 500 & & \\
\hline 3042-1994-01 & VOA & Propylene glycol & 1 & 0 & $\mathrm{ug} / \mathrm{kg}$ & 50000 & 50000 & 50000 & & \\
\hline 3042-1994-01 & VOA & Styrene & 1 & 0 & $\mathrm{ug} / \mathrm{kg}$ & 500 & 500 & 500 & & \\
\hline 3042-1994-01 & VOA & Tetrachloroethene & 2 & 0 & $\mathrm{ug} / \mathrm{kg}$ & 0.01 & 500 & 250.005 & 250.005 & 353.54632 \\
\hline 3042-1994-01 & VOA & Toluene & 1 & 0 & $\mathrm{ug} / \mathrm{kg}$ & 500 & 500 & 500 & & \\
\hline 3042-1994-01 & VOA & Total Xylene & 1 & 0 & $\mathrm{ug} / \mathrm{kg}$ & 1500 & 1500 & 1500 & & \\
\hline 3042-1994-01 & VOA & trans-1,2-Dichloroethene & 1 & 0 & $\mathrm{ug} / \mathrm{kg}$ & 500 & 500 & 500 & & \\
\hline 3042-1994-01 & VOA & trans-1,3-Dichloropropene & 1 & 0 & $\mathrm{ug} / \mathrm{kg}$ & 500 & 500 & 500 & & \\
\hline 3042-1994-01 & VOA & Trichloroethene & 2 & 0 & $\mathrm{ug} / \mathrm{kg}$ & 0.01 & 500 & 250.005 & 250.005 & 353.54632 \\
\hline 3042-1994-01 & VOA & Trichlorofluoromethane & 1 & 0 & $\mathrm{ug} / \mathrm{kg}$ & 500 & 500 & 500 & & \\
\hline 3042-1994-01 & VOA & Vinyl chloride & 2 & 0 & $\mathrm{ug} / \mathrm{kg}$ & 0.01 & 500 & 250.005 & 250.005 & 353.54632 \\
\hline $68-7165$ & HERB & $2,4-\mathrm{D}$ & 2 & 0 & $\mathrm{ug} / \mathrm{kg}$ & 0.05 & 24.7 & 12.375 & 12.375 & 17.430182 \\
\hline $68-7165$ & HERB & Silvex & 2 & 0 & $\mathrm{ug} / \mathrm{kg}$ & 0.05 & 24.7 & 12.375 & 12.375 & 17.430182 \\
\hline $68-7165$ & METAL & Arsenic & 2 & 0 & $\mathrm{mg} / \mathrm{L}$ & 0.3 & 57.8 & 29.05 & 29.05 & 40.65864 \\
\hline $68-7165$ & METAL & Barium & 2 & 2 & $\mathrm{mg} / \mathrm{L}$ & 0.74 & 673 & 336.87 & 336.87 & 475.3596 \\
\hline $68-7165$ & METAL & Cadmium & 2 & 0 & $\mathrm{mg} / \mathrm{L}$ & 0.05 & 9.63 & 4.84 & 4.84 & 6.774083 \\
\hline $68-7165$ & METAL & Chromium & 2 & 1 & $\mathrm{mg} / \mathrm{L}$ & 0.05 & 85.7 & 42.875 & 42.875 & 60.563696 \\
\hline $68-7165$ & METAL & Lead & 2 & 1 & $\mathrm{mg} / \mathrm{L}$ & 0.1 & 11.4 & 5.75 & 5.75 & 7.9903066 \\
\hline $68-7165$ & METAL & Mercury & 2 & 0 & $\mathrm{mg} / \mathrm{L}$ & 0.002 & 0.4 & 0.201 & 0.201 & 0.2814285 \\
\hline $68-7165$ & METAL & Selenium & 2 & 0 & $\mathrm{mg} / \mathrm{L}$ & 0.3 & 57.8 & 29.05 & 29.05 & 40.65864 \\
\hline $68-7165$ & METAL & Silver & 2 & 0 & $\mathrm{mg} / \mathrm{L}$ & 0.05 & 9.63 & 4.84 & 4.84 & 6.774083 \\
\hline $68-7165$ & METAL & Total Uranium & 1 & 0 & $\mathrm{pCi} / \mathrm{g}$ & 26.1 & 26.1 & 26.1 & & \\
\hline $68-7165$ & PPCB & 4,4'-DDD & 1 & 0 & $\mathrm{ug} / \mathrm{kg}$ & 3.87 & 3.87 & 3.87 & & \\
\hline $68-7165$ & РPCB & 4,4'-DDE & 1 & 0 & $\mathrm{ug} / \mathrm{kg}$ & 3.87 & 3.87 & 3.87 & & \\
\hline $68-7165$ & РРCB & 4,4'-DDT & 1 & 0 & $\mathrm{ug} / \mathrm{kg}$ & 5.31 & 5.31 & 5.31 & & \\
\hline $68-7165$ & РPCB & Aldrin & 1 & 0 & $\mathrm{ug} / \mathrm{kg}$ & 1.94 & 1.94 & 1.94 & & \\
\hline $68-7165$ & РPCB & alpha-BHC & 1 & 0 & $\mathrm{ug} / \mathrm{kg}$ & 1.94 & 1.94 & 1.94 & & \\
\hline $68-7165$ & РPCB & alpha-Chlordane & 1 & 0 & $\mathrm{ug} / \mathrm{kg}$ & 1.94 & 1.94 & 1.94 & & \\
\hline $68-7165$ & РPCB & beta-BHC & 1 & 0 & $\mathrm{ug} / \mathrm{kg}$ & 1.94 & 1.94 & 1.94 & & \\
\hline $68-7165$ & РPCB & Chlordane & 2 & 0 & $\mathrm{ug} / \mathrm{kg}$ & 0.0025 & 24.2 & 12.10125 & 12.10125 & 17.110216 \\
\hline
\end{tabular}




\begin{tabular}{|c|c|c|c|c|c|c|c|c|c|c|}
\hline $68-7165$ & РPCB & delta-BHC & 1 & 0 & $\mathrm{ug} / \mathrm{kg}$ & 1.94 & 1.94 & 1.94 & & \\
\hline $68-7165$ & РPCB & Dieldrin & 1 & 0 & $\mathrm{ug} / \mathrm{kg}$ & 3.87 & 3.87 & 3.87 & & \\
\hline $68-7165$ & РPCB & Endosulfan I & 1 & 0 & $\mathrm{ug} / \mathrm{kg}$ & 1.94 & 1.94 & 1.94 & & \\
\hline $68-7165$ & РPCB & Endosulfan II & 1 & 0 & $\mathrm{ug} / \mathrm{kg}$ & 3.87 & 3.87 & 3.87 & & \\
\hline $68-7165$ & PPCB & Endosulfan sulfate & 1 & 0 & $\mathrm{ug} / \mathrm{kg}$ & 3.87 & 3.87 & 3.87 & & \\
\hline $68-7165$ & PPCB & Endrin & 2 & 0 & $\mathrm{ug} / \mathrm{kg}$ & 0.0004 & 5.59 & 2.7952 & 2.7952 & 3.9524441 \\
\hline $68-7165$ & PPCB & Endrin aldehyde & 1 & 0 & $\mathrm{ug} / \mathrm{kg}$ & 3.87 & 3.87 & 3.87 & & \\
\hline $68-7165$ & PPCB & Endrin ketone & 1 & 0 & $\mathrm{ug} / \mathrm{kg}$ & 3.87 & 3.87 & 3.87 & & \\
\hline $68-7165$ & РPCB & gamma-Chlordane & 1 & 0 & $\mathrm{ug} / \mathrm{kg}$ & 1.94 & 1.94 & 1.94 & & \\
\hline $68-7165$ & PPCB & Heptachlor & 2 & 0 & $\mathrm{ug} / \mathrm{kg}$ & 0.0002 & 1.94 & 0.9701 & 0.9701 & 1.3716457 \\
\hline $68-7165$ & PPCB & Heptachlor epoxide & 2 & 0 & $\mathrm{ug} / \mathrm{kg}$ & 0.0002 & 1.94 & 0.9701 & 0.9701 & 1.3716457 \\
\hline $68-7165$ & РPCB & Lindane & 2 & 0 & $\mathrm{ug} / \mathrm{kg}$ & 0.0002 & 1.94 & 0.9701 & 0.9701 & 1.3716457 \\
\hline $68-7165$ & РPCB & Methoxychlor & 2 & 0 & $\mathrm{ug} / \mathrm{kg}$ & 0.002 & 19.4 & 9.701 & 9.701 & 13.716457 \\
\hline $68-7165$ & РPCB & Toxaphene & 2 & 0 & $\mathrm{ug} / \mathrm{kg}$ & 0.005 & 48.4 & 24.2025 & 24.2025 & 34.220433 \\
\hline $68-7165$ & RADS & Americium-241 & 1 & 0 & $\mathrm{pCi} / \mathrm{g}$ & 0.0319 & 0.0319 & 0.0319 & & \\
\hline $68-7165$ & RADS & Carbon-14 & 1 & 0 & $\mathrm{pCi} / \mathrm{g}$ & -5.65 & -5.65 & -5.65 & & \\
\hline $68-7165$ & RADS & Cesium-137 & 1 & 1 & $\mathrm{pCi} / \mathrm{g}$ & 47.6 & 47.6 & 47.6 & 47.6 & \\
\hline $68-7165$ & RADS & Cobalt-60 & 1 & 0 & $\mathrm{pCi} / \mathrm{g}$ & -0.0165 & -0.0165 & -0.0165 & & \\
\hline $68-7165$ & RADS & Curium-243/244 & 1 & 0 & $\mathrm{pCi} / \mathrm{g}$ & 0.0315 & 0.0315 & 0.0315 & & \\
\hline $68-7165$ & RADS & Iodine-129 & 1 & 0 & $\mathrm{pCi} / \mathrm{g}$ & -0.000552 & -0.000552 & -0.000552 & & \\
\hline $68-7165$ & RADS & Neptunium-237 & 1 & 0 & $\mathrm{pCi} / \mathrm{g}$ & 0.00858 & 0.00858 & 0.00858 & & \\
\hline $68-7165$ & RADS & Plutonium-238 & 1 & 0 & $\mathrm{pCi} / \mathrm{g}$ & -0.0127 & -0.0127 & -0.0127 & & \\
\hline $68-7165$ & RADS & Plutonium-239/240 & 1 & 0 & $\mathrm{pCi} / \mathrm{g}$ & 0.0274 & 0.0274 & 0.0274 & & \\
\hline $68-7165$ & RADS & Radium-226 & 1 & 1 & $\mathrm{pCi} / \mathrm{g}$ & 0.826 & 0.826 & 0.826 & 0.826 & \\
\hline $68-7165$ & RADS & Strontium-90 & 1 & 1 & $\mathrm{pCi} / \mathrm{g}$ & 302 & 302 & 302 & 302 & \\
\hline $68-7165$ & RADS & Technetium-99 & 1 & 0 & $\mathrm{pCi} / \mathrm{g}$ & 0.29 & 0.29 & 0.29 & & \\
\hline $68-7165$ & RADS & Thorium-230 & 1 & 1 & $\mathrm{pCi} / \mathrm{g}$ & 0.826 & 0.826 & 0.826 & 0.826 & \\
\hline $68-7165$ & RADS & Thorium-232 & 1 & 1 & $\mathrm{pCi} / \mathrm{g}$ & 1.2 & 1.2 & 1.2 & 1.2 & \\
\hline $68-7165$ & RADS & Tritium & 1 & 0 & $\mathrm{pCi} / \mathrm{g}$ & 118 & 118 & 118 & & \\
\hline $68-7165$ & RADS & Uranium-232 & 1 & 1 & $\%$ & 99.9 & 99.9 & 99.9 & 99.9 & \\
\hline $68-7165$ & RADS & Uranium-233/234 & 1 & 1 & $\mathrm{pCi} / \mathrm{g}$ & 25.2 & 25.2 & 25.2 & 25.2 & \\
\hline $68-7165$ & RADS & Uranium-235/236 & 1 & 1 & $\mathrm{pCi} / \mathrm{g}$ & 0.308 & 0.308 & 0.308 & 0.308 & \\
\hline $68-7165$ & RADS & Uranium-238 & 1 & 1 & $\mathrm{pCi} / \mathrm{g}$ & 0.643 & 0.643 & 0.643 & 0.643 & \\
\hline $68-7165$ & SVOA & 1,2,4-Trichlorobenzene & 1 & 0 & $\mathrm{ug} / \mathrm{kg}$ & 98 & 98 & 98 & & \\
\hline $68-7165$ & SVOA & 1,2-Dichlorobenzene & 1 & 0 & $\mathrm{ug} / \mathrm{kg}$ & 98 & 98 & 98 & & \\
\hline $68-7165$ & SVOA & 1,3-Dichlorobenzene & 1 & 0 & $\mathrm{ug} / \mathrm{kg}$ & 98 & 98 & 98 & & \\
\hline $68-7165$ & SVOA & 1,4-Dichlorobenzene & 2 & 0 & $\mathrm{ug} / \mathrm{kg}$ & 0.01 & 98 & 49.005 & 49.005 & 69.289393 \\
\hline $68-7165$ & SVOA & 2,2'-Dichlorodiisopropyl ether & 1 & 0 & $\mathrm{ug} / \mathrm{kg}$ & 995 & 995 & 995 & & \\
\hline $68-7165$ & SVOA & 2,3,4,6-Tetrachlorophenol & 1 & 0 & $\mathrm{ug} / \mathrm{kg}$ & 995 & 995 & 995 & & \\
\hline $68-7165$ & SVOA & 2,4,5-Trichlorophenol & 2 & 0 & $\mathrm{ug} / \mathrm{kg}$ & 0.05 & 995 & 497.525 & 497.525 & 703.53589 \\
\hline $68-7165$ & SVOA & 2,4,6-Trichlorophenol & 2 & 0 & $\mathrm{ug} / \mathrm{kg}$ & 0.05 & 995 & 497.525 & 497.525 & 703.53589 \\
\hline $68-7165$ & SVOA & 2,4-Dichlorophenol & 1 & 0 & $\mathrm{ug} / \mathrm{kg}$ & 995 & 995 & 995 & & \\
\hline $68-7165$ & SVOA & 2,4-Dimethylphenol & 1 & 0 & $\mathrm{ug} / \mathrm{kg}$ & 995 & 995 & 995 & & \\
\hline $68-7165$ & SVOA & 2,4-Dinitrophenol & 1 & 0 & $\mathrm{ug} / \mathrm{kg}$ & 1990 & 1990 & 1990 & & \\
\hline $68-7165$ & SVOA & 2,4-Dinitrotoluene & 2 & 0 & $\mathrm{ug} / \mathrm{kg}$ & 0.05 & 995 & 497.525 & 497.525 & 703.53589 \\
\hline $68-7165$ & SVOA & 2,6-Dinitrotoluene & 1 & 0 & $\mathrm{ug} / \mathrm{kg}$ & 995 & 995 & 995 & & \\
\hline $68-7165$ & SVOA & 2-Chloronaphthalene & 1 & 0 & $\mathrm{ug} / \mathrm{kg}$ & 99.5 & 99.5 & 99.5 & & \\
\hline $68-7165$ & SVOA & 2-Chlorophenol & 1 & 0 & $\mathrm{ug} / \mathrm{kg}$ & 995 & 995 & 995 & & \\
\hline $68-7165$ & SVOA & 2-Methyl-4,6-dinitrophenol & 1 & 0 & $\mathrm{ug} / \mathrm{kg}$ & 995 & 995 & 995 & & \\
\hline $68-7165$ & SVOA & 2-Methylnaphthalene & 1 & 0 & $\mathrm{ug} / \mathrm{kg}$ & 99.5 & 99.5 & 99.5 & & \\
\hline $68-7165$ & SVOA & 2-Methylphenol & 2 & 0 & $\mathrm{ug} / \mathrm{kg}$ & 0.05 & 995 & 497.525 & 497.525 & 703.53589 \\
\hline $68-7165$ & SVOA & 2-Nitrobenzenamine & 1 & 0 & $\mathrm{ug} / \mathrm{kg}$ & 995 & 995 & 995 & & \\
\hline $68-7165$ & SVOA & 2-Nitrophenol & 1 & 0 & $\mathrm{ug} / \mathrm{kg}$ & 995 & 995 & 995 & & \\
\hline $68-7165$ & SVOA & 3,3'-Dichlorobenzidine & 1 & 0 & $\mathrm{ug} / \mathrm{kg}$ & 995 & 995 & 995 & & \\
\hline $68-7165$ & SVOA & 3-Nitrobenzenamine & 1 & 0 & $\mathrm{ug} / \mathrm{kg}$ & 995 & 995 & 995 & & \\
\hline $68-7165$ & SVOA & 4-Bromophenyl phenyl ether & 1 & 0 & $\mathrm{ug} / \mathrm{kg}$ & 995 & 995 & 995 & & \\
\hline $68-7165$ & SVOA & 4-Chloro-3-methylphenol & 1 & 0 & $\mathrm{ug} / \mathrm{kg}$ & 995 & 995 & 995 & & \\
\hline $68-7165$ & SVOA & 4-Chlorobenzenamine & 1 & 0 & $\mathrm{ug} / \mathrm{kg}$ & 995 & 995 & 995 & & \\
\hline $68-7165$ & SVOA & 4-Chlorophenyl phenyl ether & 1 & 0 & $\mathrm{ug} / \mathrm{kg}$ & 995 & 995 & 995 & & \\
\hline $68-7165$ & SVOA & 4-Nitrobenzenamine & 1 & 0 & $\mathrm{ug} / \mathrm{kg}$ & 995 & 995 & 995 & & \\
\hline $68-7165$ & SVOA & 4-Nitrophenol & 1 & 0 & $\mathrm{ug} / \mathrm{kg}$ & 995 & 995 & 995 & & \\
\hline $68-7165$ & SVOA & Acenaphthene & 1 & 0 & $\mathrm{ug} / \mathrm{kg}$ & 99.5 & 99.5 & 99.5 & & \\
\hline $68-7165$ & SVOA & Acenaphthylene & 1 & 0 & $\mathrm{ug} / \mathrm{kg}$ & 99.5 & 99.5 & 99.5 & & \\
\hline $68-7165$ & SVOA & Acetophenone & 1 & 0 & $\mathrm{ug} / \mathrm{kg}$ & 995 & 995 & 995 & & \\
\hline $68-7165$ & SVOA & Anthracene & 1 & 1 & $\mathrm{ug} / \mathrm{kg}$ & 176 & 176 & 176 & 176 & \\
\hline $68-7165$ & SVOA & Benz(a)anthracene & 1 & 1 & $\mathrm{ug} / \mathrm{kg}$ & 768 & 768 & 768 & 768 & \\
\hline $68-7165$ & SVOA & Benzenemethanol & 1 & 0 & $\mathrm{ug} / \mathrm{kg}$ & 995 & 995 & 995 & & \\
\hline $68-7165$ & SVOA & Benzidine & 1 & 0 & $\mathrm{ug} / \mathrm{kg}$ & 995 & 995 & 995 & & \\
\hline $68-7165$ & SVOA & Benzo(a)pyrene & 1 & 1 & $\mathrm{ug} / \mathrm{kg}$ & 681 & 681 & 681 & 681 & \\
\hline $68-7165$ & SVOA & Benzo(b)fluoranthene & 1 & 1 & $\mathrm{ug} / \mathrm{kg}$ & 1150 & 1150 & 1150 & 1150 & \\
\hline
\end{tabular}




\begin{tabular}{|c|c|c|c|c|c|c|c|c|c|c|}
\hline $68-7165$ & SVOA & Benzo(ghi)perylene & 1 & 1 & $\mathrm{ug} / \mathrm{kg}$ & 425 & 425 & 425 & 425 & \\
\hline $68-7165$ & SVOA & Benzo(k)fluoranthene & 1 & 1 & $\mathrm{ug} / \mathrm{kg}$ & 373 & 373 & 373 & 373 & \\
\hline $68-7165$ & SVOA & Benzoic acid & 1 & 0 & $\mathrm{ug} / \mathrm{kg}$ & 1990 & 1990 & 1990 & & \\
\hline $68-7165$ & SVOA & Bis(2-chloroethoxy)methane & 1 & 0 & $\mathrm{ug} / \mathrm{kg}$ & 995 & 995 & 995 & & \\
\hline $68-7165$ & SVOA & Bis(2-chloroethyl) ether & 1 & 0 & $\mathrm{ug} / \mathrm{kg}$ & 995 & 995 & 995 & & \\
\hline $68-7165$ & SVOA & Bis(2-ethylhexyl)phthalate & 1 & 0 & $\mathrm{ug} / \mathrm{kg}$ & 995 & 995 & 995 & & \\
\hline $68-7165$ & SVOA & Butyl benzyl phthalate & 1 & 0 & $\mathrm{ug} / \mathrm{kg}$ & 995 & 995 & 995 & & \\
\hline $68-7165$ & SVOA & Carbazole & 1 & 1 & $\mathrm{ug} / \mathrm{kg}$ & 192 & 192 & 192 & 192 & \\
\hline $68-7165$ & SVOA & Chrysene & 1 & 1 & $\mathrm{ug} / \mathrm{kg}$ & 1060 & 1060 & 1060 & 1060 & \\
\hline $68-7165$ & SVOA & Dibenz(a,h)anthracene & 1 & 1 & $\mathrm{ug} / \mathrm{kg}$ & 104 & 104 & 104 & 104 & \\
\hline $68-7165$ & SVOA & Dibenzofuran & 1 & 0 & $\mathrm{ug} / \mathrm{kg}$ & 995 & 995 & 995 & & \\
\hline $68-7165$ & SVOA & Diethyl phthalate & 1 & 0 & $\mathrm{ug} / \mathrm{kg}$ & 995 & 995 & 995 & & \\
\hline $68-7165$ & SVOA & Dimethyl phthalate & 1 & 0 & $\mathrm{ug} / \mathrm{kg}$ & 995 & 995 & 995 & & \\
\hline $68-7165$ & SVOA & Di-n-butyl phthalate & 1 & 0 & $\mathrm{ug} / \mathrm{kg}$ & 995 & 995 & 995 & & \\
\hline $68-7165$ & SVOA & Di-n-octylphthalate & 1 & 0 & $\mathrm{ug} / \mathrm{kg}$ & 995 & 995 & 995 & & \\
\hline $68-7165$ & SVOA & Diphenylamine & 1 & 0 & $\mathrm{ug} / \mathrm{kg}$ & 995 & 995 & 995 & & \\
\hline $68-7165$ & SVOA & Fluoranthene & 1 & 1 & $\mathrm{ug} / \mathrm{kg}$ & 2130 & 2130 & 2130 & 2130 & \\
\hline $68-7165$ & SVOA & Fluorene & 1 & 1 & $\mathrm{ug} / \mathrm{kg}$ & 101 & 101 & 101 & 101 & \\
\hline $68-7165$ & SVOA & Hexachlorobenzene & 2 & 0 & $\mathrm{ug} / \mathrm{kg}$ & 0.05 & 995 & 497.525 & 497.525 & 703.53589 \\
\hline $68-7165$ & SVOA & Hexachlorobutadiene & 2 & 0 & $\mathrm{ug} / \mathrm{kg}$ & 0.05 & 995 & 497.525 & 497.525 & 703.53589 \\
\hline $68-7165$ & SVOA & Hexachlorocyclopentadiene & 1 & 0 & $\mathrm{ug} / \mathrm{kg}$ & 995 & 995 & 995 & & \\
\hline $68-7165$ & SVOA & Hexachloroethane & 2 & 0 & $\mathrm{ug} / \mathrm{kg}$ & 0.05 & 995 & 497.525 & 497.525 & 703.53589 \\
\hline $68-7165$ & SVOA & Indeno(1,2,3-cd)pyrene & 1 & 1 & $\mathrm{ug} / \mathrm{kg}$ & 486 & 486 & 486 & 486 & \\
\hline $68-7165$ & SVOA & Isophorone & 1 & 0 & $\mathrm{ug} / \mathrm{kg}$ & 995 & 995 & 995 & & \\
\hline $68-7165$ & SVOA & $\mathrm{m}+\mathrm{p}$ Methylphenol & 2 & 0 & $\mathrm{ug} / \mathrm{kg}$ & 0.05 & 995 & 497.525 & 497.525 & 703.53589 \\
\hline $68-7165$ & SVOA & Naphthalene & 1 & 0 & $\mathrm{ug} / \mathrm{kg}$ & 99.5 & 99.5 & 99.5 & & \\
\hline $68-7165$ & SVOA & Nitrobenzene & 2 & 0 & $\mathrm{ug} / \mathrm{kg}$ & 0.05 & 995 & 497.525 & 497.525 & 703.53589 \\
\hline $68-7165$ & SVOA & N-Nitroso-di-n-propylamine & 1 & 0 & $\mathrm{ug} / \mathrm{kg}$ & 995 & 995 & 995 & & \\
\hline $68-7165$ & SVOA & Pentachlorophenol & 2 & 0 & $\mathrm{ug} / \mathrm{kg}$ & 0.05 & 995 & 497.525 & 497.525 & 703.53589 \\
\hline $68-7165$ & SVOA & Phenanthrene & 1 & 1 & $\mathrm{ug} / \mathrm{kg}$ & 1820 & 1820 & 1820 & 1820 & \\
\hline $68-7165$ & SVOA & Phenol & 1 & 0 & $\mathrm{ug} / \mathrm{kg}$ & 995 & 995 & 995 & & \\
\hline $68-7165$ & SVOA & Pyridine & 2 & 0 & $\mathrm{ug} / \mathrm{kg}$ & 0.05 & 995 & 497.525 & 497.525 & 703.53589 \\
\hline $68-7165$ & $\mathrm{VOA}$ & (1,1-Dimethylethyl)benzene & 1 & 0 & $\mathrm{ug} / \mathrm{kg}$ & 98 & 98 & 98 & & \\
\hline $68-7165$ & VOA & (1-Methylpropyl)benzene & 1 & 0 & $\mathrm{ug} / \mathrm{kg}$ & 98 & 98 & 98 & & \\
\hline $68-7165$ & VOA & 1,1,1,2-Tetrachloroethane & 1 & 0 & $\mathrm{ug} / \mathrm{kg}$ & 98 & 98 & 98 & & \\
\hline $68-7165$ & VOA & 1,1,1-Trichloroethane & 1 & 0 & $\mathrm{ug} / \mathrm{kg}$ & 98 & 98 & 98 & & \\
\hline $68-7165$ & VOA & 1,1,2,2-Tetrachloroethane & 1 & 0 & $\mathrm{ug} / \mathrm{kg}$ & 98 & 98 & 98 & & \\
\hline $68-7165$ & VOA & 1,1,2-Trichloroethane & 1 & 0 & $\mathrm{ug} / \mathrm{kg}$ & 98 & 98 & 98 & & \\
\hline $68-7165$ & VOA & 1,1-Dichloroethane & 1 & 0 & $\mathrm{ug} / \mathrm{kg}$ & 98 & 98 & 98 & & \\
\hline $68-7165$ & VOA & 1,1-Dichloroethene & 2 & 0 & $\mathrm{ug} / \mathrm{kg}$ & 0.01 & 98 & 49.005 & 49.005 & 69.289393 \\
\hline $68-7165$ & VOA & 1,1-Dichloropropene & 1 & 0 & $\mathrm{ug} / \mathrm{kg}$ & 98 & 98 & 98 & & \\
\hline $68-7165$ & VOA & 1,2,3-Trichlorobenzene & 1 & 0 & $\mathrm{ug} / \mathrm{kg}$ & 98 & 98 & 98 & & \\
\hline $68-7165$ & VOA & 1,2,3-Trichloropropane & 1 & 0 & $\mathrm{ug} / \mathrm{kg}$ & 98 & 98 & 98 & & \\
\hline $68-7165$ & VOA & 1,2,4-Trimethylbenzene & 1 & 0 & $\mathrm{ug} / \mathrm{kg}$ & 98 & 98 & 98 & & \\
\hline $68-7165$ & VOA & 1,2-Dibromo-3-chloropropane & 1 & 0 & $\mathrm{ug} / \mathrm{kg}$ & 98 & 98 & 98 & & \\
\hline $68-7165$ & VOA & 1,2-Dibromoethane & 1 & 0 & $\mathrm{ug} / \mathrm{kg}$ & 98 & 98 & 98 & & \\
\hline $68-7165$ & VOA & 1,2-Dichloroethane & 2 & 0 & $\mathrm{ug} / \mathrm{kg}$ & 0.01 & 98 & 49.005 & 49.005 & 69.289393 \\
\hline $68-7165$ & VOA & 1,2-Dichloroethene & 1 & 0 & $\mathrm{ug} / \mathrm{kg}$ & 196 & 196 & 196 & & \\
\hline $68-7165$ & $\mathrm{VOA}$ & 1,2-Dichloropropane & 1 & 0 & $\mathrm{ug} / \mathrm{kg}$ & 98 & 98 & 98 & & \\
\hline $68-7165$ & VOA & 1,2-Dimethylbenzene & 1 & 0 & $\mathrm{ug} / \mathrm{kg}$ & 98 & 98 & 98 & & \\
\hline $68-7165$ & VOA & 1,3,5-Trimethylbenzene & 1 & 0 & $\mathrm{ug} / \mathrm{kg}$ & 98 & 98 & 98 & & \\
\hline $68-7165$ & VOA & 1,3-Dichloropropane & 1 & 0 & $\mathrm{ug} / \mathrm{kg}$ & 98 & 98 & 98 & & \\
\hline $68-7165$ & VOA & 1-Chloro-4-methylbenzene & 1 & 0 & $\mathrm{ug} / \mathrm{kg}$ & 98 & 98 & 98 & & \\
\hline $68-7165$ & VOA & 1-Methyl-4-(1-methylethyl)benzene & 1 & 0 & $\mathrm{ug} / \mathrm{kg}$ & 98 & 98 & 98 & & \\
\hline $68-7165$ & VOA & 2,2-Dichloropropane & 1 & 0 & $\mathrm{ug} / \mathrm{kg}$ & 98 & 98 & 98 & & \\
\hline $68-7165$ & VOA & 2-Butanone & 2 & 0 & $\mathrm{ug} / \mathrm{kg}$ & 0.05 & 490 & 245.025 & 245.025 & 346.44697 \\
\hline $68-7165$ & VOA & 2-Hexanone & 1 & 0 & $\mathrm{ug} / \mathrm{kg}$ & 490 & 490 & 490 & & \\
\hline $68-7165$ & $\mathrm{VOA}$ & 4-Methyl-2-pentanone & 1 & 0 & $\mathrm{ug} / \mathrm{kg}$ & 490 & 490 & 490 & & \\
\hline $68-7165$ & VOA & Acetone & 1 & 0 & $\mathrm{ug} / \mathrm{kg}$ & 490 & 490 & 490 & & \\
\hline $68-7165$ & VOA & Acetonitrile & 1 & 0 & $\mathrm{ug} / \mathrm{kg}$ & 2450 & 2450 & 2450 & & \\
\hline $68-7165$ & VOA & Acrolein & 1 & 0 & $\mathrm{ug} / \mathrm{kg}$ & 490 & 490 & 490 & & \\
\hline $68-7165$ & VOA & Acrylonitrile & 1 & 0 & $\mathrm{ug} / \mathrm{kg}$ & 490 & 490 & 490 & & \\
\hline $68-7165$ & VOA & Benzene & 2 & 0 & $\mathrm{ug} / \mathrm{kg}$ & 0.01 & 98 & 49.005 & 49.005 & 69.289393 \\
\hline $68-7165$ & VOA & Bromobenzene & 1 & 0 & $\mathrm{ug} / \mathrm{kg}$ & 98 & 98 & 98 & & \\
\hline $68-7165$ & VOA & Bromochloromethane & 1 & 0 & $\mathrm{ug} / \mathrm{kg}$ & 98 & 98 & 98 & & \\
\hline $68-7165$ & VOA & Bromodichloromethane & 1 & 0 & $\mathrm{ug} / \mathrm{kg}$ & 98 & 98 & 98 & & \\
\hline $68-7165$ & $\mathrm{VOA}$ & Bromoform & 1 & 0 & $\mathrm{ug} / \mathrm{kg}$ & 98 & 98 & 98 & & \\
\hline $68-7165$ & VOA & Bromomethane & 1 & 0 & $\mathrm{ug} / \mathrm{kg}$ & 98 & 98 & 98 & & \\
\hline $68-7165$ & VOA & Butylbenzene & 1 & 0 & $\mathrm{ug} / \mathrm{kg}$ & 98 & 98 & 98 & & \\
\hline $68-7165$ & VOA & Carbon disulfide & 1 & 0 & $\mathrm{ug} / \mathrm{kg}$ & 490 & 490 & 490 & & \\
\hline
\end{tabular}




\begin{tabular}{|c|c|c|c|c|c|c|c|c|c|c|}
\hline $68-7165$ & VOA & Carbon tetrachloride & 2 & 0 & $\mathrm{ug} / \mathrm{kg}$ & 0.01 & 98 & 49.005 & 49.005 & 69.289393 \\
\hline $68-7165$ & $\mathrm{VOA}$ & Chlorobenzene & 2 & 0 & $\mathrm{ug} / \mathrm{kg}$ & 0.01 & 98 & 49.005 & 49.005 & 69.289393 \\
\hline $68-7165$ & VOA & Chloroethane & 1 & 0 & $\mathrm{ug} / \mathrm{kg}$ & 98 & 98 & 98 & & \\
\hline $68-7165$ & VOA & Chloroform & 2 & 0 & $\mathrm{ug} / \mathrm{kg}$ & 0.01 & 98 & 49.005 & 49.005 & 69.289393 \\
\hline $68-7165$ & VOA & Chloromethane & 1 & 0 & $\mathrm{ug} / \mathrm{kg}$ & 98 & 98 & 98 & & \\
\hline $68-7165$ & VOA & cis-1,2-Dichloroethene & 1 & 0 & $\mathrm{ug} / \mathrm{kg}$ & 98 & 98 & 98 & & \\
\hline $68-7165$ & VOA & cis-1,3-Dichloropropene & 1 & 0 & $\mathrm{ug} / \mathrm{kg}$ & 98 & 98 & 98 & & \\
\hline $68-7165$ & VOA & Cumene & 1 & 0 & $\mathrm{ug} / \mathrm{kg}$ & 98 & 98 & 98 & & \\
\hline $68-7165$ & VOA & Dibromochloromethane & 1 & 0 & $\mathrm{ug} / \mathrm{kg}$ & 98 & 98 & 98 & & \\
\hline $68-7165$ & VOA & Dibromomethane & 1 & 0 & $\mathrm{ug} / \mathrm{kg}$ & 98 & 98 & 98 & & \\
\hline $68-7165$ & VOA & Dichlorodifluoromethane & 1 & 0 & $\mathrm{ug} / \mathrm{kg}$ & 98 & 98 & 98 & & \\
\hline $68-7165$ & VOA & Ethylbenzene & 1 & 0 & $\mathrm{ug} / \mathrm{kg}$ & 98 & 98 & 98 & & \\
\hline $68-7165$ & VOA & Hexane & 1 & 0 & $\mathrm{ug} / \mathrm{kg}$ & 490 & 490 & 490 & & \\
\hline $68-7165$ & VOA & M + P Xylene & 1 & 0 & $\mathrm{ug} / \mathrm{kg}$ & 196 & 196 & 196 & & \\
\hline $68-7165$ & VOA & Methanol & 1 & 0 & $\mathrm{ug} / \mathrm{kg}$ & 4810 & 4810 & 4810 & & \\
\hline $68-7165$ & VOA & Methyl methacrylate & 1 & 0 & $\mathrm{ug} / \mathrm{kg}$ & 490 & 490 & 490 & & \\
\hline $68-7165$ & VOA & Methylcyclohexane & 1 & 0 & $\mathrm{ug} / \mathrm{kg}$ & 98 & 98 & 98 & & \\
\hline $68-7165$ & VOA & Methylene chloride & 1 & 0 & $\mathrm{ug} / \mathrm{kg}$ & 490 & 490 & 490 & & \\
\hline $68-7165$ & VOA & o-Chlorotoluene & 1 & 0 & $\mathrm{ug} / \mathrm{kg}$ & 98 & 98 & 98 & & \\
\hline $68-7165$ & VOA & Propylbenzene & 1 & 0 & $\mathrm{ug} / \mathrm{kg}$ & 98 & 98 & 98 & & \\
\hline $68-7165$ & VOA & Propylene glycol & 1 & 0 & $\mathrm{ug} / \mathrm{kg}$ & 48100 & 48100 & 48100 & & \\
\hline $68-7165$ & VOA & Styrene & 1 & 0 & $\mathrm{ug} / \mathrm{kg}$ & 98 & 98 & 98 & & \\
\hline $68-7165$ & VOA & Tetrachloroethene & 2 & 0 & $\mathrm{ug} / \mathrm{kg}$ & 0.01 & 98 & 49.005 & 49.005 & 69.289393 \\
\hline $68-7165$ & VOA & Toluene & 1 & 0 & $\mathrm{ug} / \mathrm{kg}$ & 98 & 98 & 98 & & \\
\hline $68-7165$ & VOA & Total Xylene & 1 & 0 & $\mathrm{ug} / \mathrm{kg}$ & 294 & 294 & 294 & & \\
\hline $68-7165$ & VOA & trans-1,2-Dichloroethene & 1 & 0 & $\mathrm{ug} / \mathrm{kg}$ & 98 & 98 & 98 & & \\
\hline $68-7165$ & VOA & trans-1,3-Dichloropropene & 1 & 0 & $\mathrm{ug} / \mathrm{kg}$ & 98 & 98 & 98 & & \\
\hline $68-7165$ & VOA & Trichloroethene & 2 & 0 & $\mathrm{ug} / \mathrm{kg}$ & 0.01 & 98 & 49.005 & 49.005 & 69.289393 \\
\hline $68-7165$ & VOA & Trichlorofluoromethane & 1 & 0 & $\mathrm{ug} / \mathrm{kg}$ & 98 & 98 & 98 & & \\
\hline $68-7165$ & $\mathrm{VOA}$ & Vinyl chloride & 2 & 0 & $\mathrm{ug} / \mathrm{kg}$ & 0.01 & 98 & 49.005 & 49.005 & 69.289393 \\
\hline $68-7166$ & HERB & $2,4-\mathrm{D}$ & 2 & 0 & $\mathrm{ug} / \mathrm{kg}$ & 0.05 & 24.9 & 12.475 & 12.475 & 17.571604 \\
\hline 68-7166 & HERB & Silvex & 2 & 0 & $\mathrm{ug} / \mathrm{kg}$ & 0.05 & 24.9 & 12.475 & 12.475 & 17.571604 \\
\hline $68-7166$ & METAL & Arsenic & 2 & 0 & $\mathrm{mg} / \mathrm{L}$ & 0.3 & 2.77 & 1.535 & 1.535 & 1.7465537 \\
\hline $68-7166$ & METAL & Barium & 2 & 2 & $\mathrm{mg} / \mathrm{L}$ & 0.631 & 82.5 & 41.5655 & 41.5655 & 57.890125 \\
\hline $68-7166$ & METAL & Cadmium & 2 & 0 & $\mathrm{mg} / \mathrm{L}$ & 0.05 & 0.462 & 0.256 & 0.256 & 0.291328 \\
\hline $68-7166$ & METAL & Chromium & 2 & 1 & $\mathrm{mg} / \mathrm{L}$ & 0.05 & 8.02 & 4.035 & 4.035 & 5.635641 \\
\hline $68-7166$ & METAL & Lead & 2 & 0 & $\mathrm{mg} / \mathrm{L}$ & 0.1 & 9.24 & 4.67 & 4.67 & 6.462956 \\
\hline $68-7166$ & METAL & Mercury & 2 & 0 & $\mathrm{mg} / \mathrm{L}$ & 0.002 & 0.4 & 0.201 & 0.201 & 0.2814285 \\
\hline $68-7166$ & METAL & Selenium & 2 & 0 & $\mathrm{mg} / \mathrm{L}$ & 0.3 & 2.77 & 1.535 & 1.535 & 1.7465537 \\
\hline $68-7166$ & METAL & Silver & 2 & 0 & $\mathrm{mg} / \mathrm{L}$ & 0.05 & 4.62 & 2.335 & 2.335 & 3.231478 \\
\hline $68-7166$ & METAL & Total Uranium & 1 & 0 & $\mathrm{pCi} / \mathrm{g}$ & 15.6 & 15.6 & 15.6 & & \\
\hline $68-7166$ & PPCB & 4,4 '-DDD & 1 & 0 & $\mathrm{ug} / \mathrm{kg}$ & 3.86 & 3.86 & 3.86 & & \\
\hline $68-7166$ & РPCB & 4,4'-DDE & 1 & 0 & $\mathrm{ug} / \mathrm{kg}$ & 3.86 & 3.86 & 3.86 & & \\
\hline $68-7166$ & PPCB & 4,4'-DDT & 1 & 1 & $\mathrm{ug} / \mathrm{kg}$ & 3.86 & 3.86 & 3.86 & 3.86 & \\
\hline $68-7166$ & PPCB & Aldrin & 1 & 0 & $\mathrm{ug} / \mathrm{kg}$ & 1.93 & 1.93 & 1.93 & & \\
\hline $68-7166$ & PPCB & alpha-BHC & 1 & 0 & $\mathrm{ug} / \mathrm{kg}$ & 1.93 & 1.93 & 1.93 & & \\
\hline $68-7166$ & PPCB & alpha-Chlordane & 1 & 0 & $\mathrm{ug} / \mathrm{kg}$ & 1.93 & 1.93 & 1.93 & & \\
\hline $68-7166$ & РPCB & beta-BHC & 1 & 0 & $\mathrm{ug} / \mathrm{kg}$ & 1.93 & 1.93 & 1.93 & & \\
\hline $68-7166$ & PPCB & Chlordane & 2 & 0 & $\mathrm{ug} / \mathrm{kg}$ & 0.0025 & 24.1 & 12.05125 & 12.05125 & 17.039506 \\
\hline $68-7166$ & PPCB & delta-BHC & 1 & 0 & $\mathrm{ug} / \mathrm{kg}$ & 1.93 & 1.93 & 1.93 & & \\
\hline $68-7166$ & РPCB & Dieldrin & 1 & 0 & $\mathrm{ug} / \mathrm{kg}$ & 3.86 & 3.86 & 3.86 & & \\
\hline $68-7166$ & PPCB & Endosulfan I & 1 & 0 & $\mathrm{ug} / \mathrm{kg}$ & 1.93 & 1.93 & 1.93 & & \\
\hline $68-7166$ & РPCB & Endosulfan II & 1 & 0 & $\mathrm{ug} / \mathrm{kg}$ & 3.86 & 3.86 & 3.86 & & \\
\hline $68-7166$ & PPCB & Endosulfan sulfate & 1 & 0 & $\mathrm{ug} / \mathrm{kg}$ & 3.86 & 3.86 & 3.86 & & \\
\hline $68-7166$ & PPCB & Endrin & 2 & 0 & $\mathrm{ug} / \mathrm{kg}$ & 0.0004 & 3.86 & 1.9302 & 1.9302 & 2.7291493 \\
\hline $68-7166$ & РPCB & Endrin aldehyde & 1 & 0 & $\mathrm{ug} / \mathrm{kg}$ & 3.86 & 3.86 & 3.86 & & \\
\hline $68-7166$ & РPCB & Endrin ketone & 1 & 0 & $\mathrm{ug} / \mathrm{kg}$ & 3.86 & 3.86 & 3.86 & & \\
\hline $68-7166$ & PPCB & gamma-Chlordane & 1 & 0 & $\mathrm{ug} / \mathrm{kg}$ & 1.93 & 1.93 & 1.93 & & \\
\hline $68-7166$ & PPCB & Heptachlor & 2 & 0 & $\mathrm{ug} / \mathrm{kg}$ & 0.0002 & 1.93 & 0.9651 & 0.9651 & 1.3645747 \\
\hline $68-7166$ & РPCB & Heptachlor epoxide & 2 & 0 & $\mathrm{ug} / \mathrm{kg}$ & 0.0002 & 1.93 & 0.9651 & 0.9651 & 1.3645747 \\
\hline $68-7166$ & PPCB & Lindane & 2 & 0 & $\mathrm{ug} / \mathrm{kg}$ & 0.0002 & 1.93 & 0.9651 & 0.9651 & 1.3645747 \\
\hline $68-7166$ & РPCB & Methoxychlor & 2 & 0 & $\mathrm{ug} / \mathrm{kg}$ & 0.002 & 19.3 & 9.651 & 9.651 & 13.645747 \\
\hline $68-7166$ & PPCB & Toxaphene & 2 & 0 & $\mathrm{ug} / \mathrm{kg}$ & 0.005 & 48.2 & 24.1025 & 24.1025 & 34.079011 \\
\hline $68-7166$ & RADS & Americium-241 & 1 & 0 & $\mathrm{pCi} / \mathrm{g}$ & -0.0109 & -0.0109 & -0.0109 & & \\
\hline $68-7166$ & RADS & Carbon-14 & 1 & 0 & $\mathrm{pCi} / \mathrm{g}$ & -2.63 & -2.63 & -2.63 & & \\
\hline $68-7166$ & RADS & Cesium-137 & 1 & 1 & $\mathrm{pCi} / \mathrm{g}$ & 0.37 & 0.37 & 0.37 & 0.37 & \\
\hline $68-7166$ & RADS & Cobalt- 60 & 1 & 0 & $\mathrm{pCi} / \mathrm{g}$ & 0.0217 & 0.0217 & 0.0217 & & \\
\hline $68-7166$ & RADS & Curium-243/244 & 1 & 0 & $\mathrm{pCi} / \mathrm{g}$ & -0.0108 & -0.0108 & -0.0108 & & \\
\hline $68-7166$ & RADS & Iodine-129 & 1 & 0 & $\mathrm{pCi} / \mathrm{g}$ & -0.0861 & -0.0861 & -0.0861 & & \\
\hline $68-7166$ & RADS & Neptunium-237 & 1 & 0 & $\mathrm{pCi} / \mathrm{g}$ & -0.0153 & -0.0153 & -0.0153 & & \\
\hline
\end{tabular}




\begin{tabular}{|c|c|c|c|c|c|c|c|c|c|c|}
\hline $68-7166$ & RADS & Plutonium-238 & 1 & 0 & $\mathrm{pCi} / \mathrm{g}$ & 0 & 0 & 0 & & \\
\hline $68-7166$ & RADS & Plutonium-239/240 & 1 & 0 & $\mathrm{pCi} / \mathrm{g}$ & 0.0161 & 0.0161 & 0.0161 & & \\
\hline $68-7166$ & RADS & Radium-226 & 1 & 1 & $\mathrm{pCi} / \mathrm{g}$ & 0.357 & 0.357 & 0.357 & 0.357 & \\
\hline $68-7166$ & RADS & Strontium-90 & 1 & 1 & $\mathrm{pCi} / \mathrm{g}$ & 71.1 & 71.1 & 71.1 & 71.1 & \\
\hline $68-7166$ & RADS & Technetium-99 & 1 & 0 & $\mathrm{pCi} / \mathrm{g}$ & 0.804 & 0.804 & 0.804 & & \\
\hline $68-7166$ & RADS & Thorium-230 & 1 & 1 & $\mathrm{pCi} / \mathrm{g}$ & 0.357 & 0.357 & 0.357 & 0.357 & \\
\hline $68-7166$ & RADS & Thorium-232 & 1 & 1 & $\mathrm{pCi} / \mathrm{g}$ & 0.53 & 0.53 & 0.53 & 0.53 & \\
\hline $68-7166$ & RADS & Tritium & 1 & 0 & $\mathrm{pCi} / \mathrm{g}$ & 84.4 & 84.4 & 84.4 & & \\
\hline $68-7166$ & RADS & Uranium-232 & 1 & 1 & $\%$ & 93.7 & 93.7 & 93.7 & 93.7 & \\
\hline $68-7166$ & RADS & Uranium-233/234 & 1 & 1 & $\mathrm{pCi} / \mathrm{g}$ & 15 & 15 & 15 & 15 & \\
\hline $68-7166$ & RADS & Uranium-235/236 & 1 & 1 & $\mathrm{pCi} / \mathrm{g}$ & 0.104 & 0.104 & 0.104 & 0.104 & \\
\hline $68-7166$ & RADS & Uranium-238 & 1 & 1 & $\mathrm{pCi} / \mathrm{g}$ & 0.469 & 0.469 & 0.469 & 0.469 & \\
\hline $68-7166$ & SVOA & 1,2,4-Trichlorobenzene & 1 & 0 & $\mathrm{ug} / \mathrm{kg}$ & 96.2 & 96.2 & 96.2 & & \\
\hline $68-7166$ & SVOA & 1,2-Dichlorobenzene & 1 & 0 & $\mathrm{ug} / \mathrm{kg}$ & 96.2 & 96.2 & 96.2 & & \\
\hline $68-7166$ & SVOA & 1,3-Dichlorobenzene & 1 & 0 & $\mathrm{ug} / \mathrm{kg}$ & 96.2 & 96.2 & 96.2 & & \\
\hline $68-7166$ & SVOA & 1,4-Dichlorobenzene & 2 & 0 & $\mathrm{ug} / \mathrm{kg}$ & 0.01 & 96.2 & 48.105 & 48.105 & 68.016601 \\
\hline $68-7166$ & SVOA & 2,2'-Dichlorodiisopropyl ether & 1 & 0 & $\mathrm{ug} / \mathrm{kg}$ & 994 & 994 & 994 & & \\
\hline $68-7166$ & SVOA & 2,3,4,6-Tetrachlorophenol & 1 & 0 & $\mathrm{ug} / \mathrm{kg}$ & 994 & 994 & 994 & & \\
\hline $68-7166$ & SVOA & 2,4,5-Trichlorophenol & 2 & 0 & $\mathrm{ug} / \mathrm{kg}$ & 0.05 & 994 & 497.025 & 497.025 & 702.82879 \\
\hline $68-7166$ & SVOA & 2,4,6-Trichlorophenol & 2 & 0 & $\mathrm{ug} / \mathrm{kg}$ & 0.05 & 994 & 497.025 & 497.025 & 702.82879 \\
\hline $68-7166$ & SVOA & 2,4-Dichlorophenol & 1 & 0 & $\mathrm{ug} / \mathrm{kg}$ & 994 & 994 & 994 & & \\
\hline $68-7166$ & SVOA & 2,4-Dimethylphenol & 1 & 0 & $\mathrm{ug} / \mathrm{kg}$ & 994 & 994 & 994 & & \\
\hline $68-7166$ & SVOA & 2,4-Dinitrophenol & 1 & 0 & $\mathrm{ug} / \mathrm{kg}$ & 1990 & 1990 & 1990 & & \\
\hline $68-7166$ & SVOA & 2,4-Dinitrotoluene & 2 & 0 & $\mathrm{ug} / \mathrm{kg}$ & 0.05 & 994 & 497.025 & 497.025 & 702.82879 \\
\hline $68-7166$ & SVOA & 2,6-Dinitrotoluene & 1 & 0 & $\mathrm{ug} / \mathrm{kg}$ & 994 & 994 & 994 & & \\
\hline $68-7166$ & SVOA & 2-Chloronaphthalene & 1 & 0 & $\mathrm{ug} / \mathrm{kg}$ & 99.4 & 99.4 & 99.4 & & \\
\hline $68-7166$ & SVOA & 2-Chlorophenol & 1 & 0 & $\mathrm{ug} / \mathrm{kg}$ & 994 & 994 & 994 & & \\
\hline $68-7166$ & SVOA & 2-Methyl-4,6-dinitrophenol & 1 & 0 & $\mathrm{ug} / \mathrm{kg}$ & 994 & 994 & 994 & & \\
\hline $68-7166$ & SVOA & 2-Methylnaphthalene & 1 & 0 & $\mathrm{ug} / \mathrm{kg}$ & 99.4 & 99.4 & 99.4 & & \\
\hline $68-7166$ & SVOA & 2-Methylphenol & 2 & 0 & $\mathrm{ug} / \mathrm{kg}$ & 0.05 & 994 & 497.025 & 497.025 & 702.82879 \\
\hline $68-7166$ & SVOA & 2-Nitrobenzenamine & 1 & 0 & $\mathrm{ug} / \mathrm{kg}$ & 994 & 994 & 994 & & \\
\hline $68-7166$ & SVOA & 2-Nitrophenol & 1 & 0 & $\mathrm{ug} / \mathrm{kg}$ & 994 & 994 & 994 & & \\
\hline $68-7166$ & SVOA & 3,3'-Dichlorobenzidine & 1 & 0 & $\mathrm{ug} / \mathrm{kg}$ & 994 & 994 & 994 & & \\
\hline $68-7166$ & SVOA & 3-Nitrobenzenamine & 1 & 0 & $\mathrm{ug} / \mathrm{kg}$ & 994 & 994 & 994 & & \\
\hline $68-7166$ & SVOA & 4-Bromophenyl phenyl ether & 1 & 0 & $\mathrm{ug} / \mathrm{kg}$ & 994 & 994 & 994 & & \\
\hline $68-7166$ & SVOA & 4-Chloro-3-methylphenol & 1 & 0 & $\mathrm{ug} / \mathrm{kg}$ & 994 & 994 & 994 & & \\
\hline $68-7166$ & SVOA & 4-Chlorobenzenamine & 1 & 0 & $\mathrm{ug} / \mathrm{kg}$ & 994 & 994 & 994 & & \\
\hline $68-7166$ & SVOA & 4-Chlorophenyl phenyl ether & 1 & 0 & $\mathrm{ug} / \mathrm{kg}$ & 994 & 994 & 994 & & \\
\hline $68-7166$ & SVOA & 4-Nitrobenzenamine & 1 & 0 & $\mathrm{ug} / \mathrm{kg}$ & 994 & 994 & 994 & & \\
\hline $68-7166$ & SVOA & 4-Nitrophenol & 1 & 0 & $\mathrm{ug} / \mathrm{kg}$ & 994 & 994 & 994 & & \\
\hline $68-7166$ & SVOA & Acenaphthene & 1 & 0 & $\mathrm{ug} / \mathrm{kg}$ & 99.4 & 99.4 & 99.4 & & \\
\hline $68-7166$ & SVOA & Acenaphthylene & 1 & 0 & $\mathrm{ug} / \mathrm{kg}$ & 99.4 & 99.4 & 99.4 & & \\
\hline $68-7166$ & SVOA & Acetophenone & 1 & 0 & $\mathrm{ug} / \mathrm{kg}$ & 994 & 994 & 994 & & \\
\hline $68-7166$ & SVOA & Anthracene & 1 & 0 & $\mathrm{ug} / \mathrm{kg}$ & 99.4 & 99.4 & 99.4 & & \\
\hline $68-7166$ & SVOA & Benz(a)anthracene & 1 & 0 & $\mathrm{ug} / \mathrm{kg}$ & 99.4 & 99.4 & 99.4 & & \\
\hline $68-7166$ & SVOA & Benzenemethanol & 1 & 0 & $\mathrm{ug} / \mathrm{kg}$ & 994 & 994 & 994 & & \\
\hline $68-7166$ & SVOA & Benzidine & 1 & 0 & $\mathrm{ug} / \mathrm{kg}$ & 994 & 994 & 994 & & \\
\hline $68-7166$ & SVOA & Benzo(a)pyrene & 1 & 0 & $\mathrm{ug} / \mathrm{kg}$ & 99.4 & 99.4 & 99.4 & & \\
\hline $68-7166$ & SVOA & Benzo(b)fluoranthene & 1 & 0 & $\mathrm{ug} / \mathrm{kg}$ & 99.4 & 99.4 & 99.4 & & \\
\hline $68-7166$ & SVOA & Benzo(ghi)perylene & 1 & 0 & $\mathrm{ug} / \mathrm{kg}$ & 99.4 & 99.4 & 99.4 & & \\
\hline $68-7166$ & SVOA & Benzo(k)fluoranthene & 1 & 0 & $\mathrm{ug} / \mathrm{kg}$ & 99.4 & 99.4 & 99.4 & & \\
\hline $68-7166$ & SVOA & Benzoic acid & 1 & 0 & $\mathrm{ug} / \mathrm{kg}$ & 1990 & 1990 & 1990 & & \\
\hline $68-7166$ & SVOA & Bis(2-chloroethoxy)methane & 1 & 0 & $\mathrm{ug} / \mathrm{kg}$ & 994 & 994 & 994 & & \\
\hline $68-7166$ & SVOA & Bis(2-chloroethyl) ether & 1 & 0 & $\mathrm{ug} / \mathrm{kg}$ & 994 & 994 & 994 & & \\
\hline $68-7166$ & SVOA & Bis(2-ethylhexyl)phthalate & 1 & 0 & $\mathrm{ug} / \mathrm{kg}$ & 994 & 994 & 994 & & \\
\hline $68-7166$ & SVOA & Butyl benzyl phthalate & 1 & 0 & $\mathrm{ug} / \mathrm{kg}$ & 994 & 994 & 994 & & \\
\hline $68-7166$ & SVOA & Carbazole & 1 & 0 & $\mathrm{ug} / \mathrm{kg}$ & 99.4 & 99.4 & 99.4 & & \\
\hline $68-7166$ & SVOA & Chrysene & 1 & 0 & $\mathrm{ug} / \mathrm{kg}$ & 99.4 & 99.4 & 99.4 & & \\
\hline $68-7166$ & SVOA & Dibenz(a,h)anthracene & 1 & 0 & $\mathrm{ug} / \mathrm{kg}$ & 99.4 & 99.4 & 99.4 & & \\
\hline $68-7166$ & SVOA & Dibenzofuran & 1 & 0 & $\mathrm{ug} / \mathrm{kg}$ & 994 & 994 & 994 & & \\
\hline $68-7166$ & SVOA & Diethyl phthalate & 1 & 0 & $\mathrm{ug} / \mathrm{kg}$ & 994 & 994 & 994 & & \\
\hline $68-7166$ & SVOA & Dimethyl phthalate & 1 & 0 & $\mathrm{ug} / \mathrm{kg}$ & 994 & 994 & 994 & & \\
\hline $68-7166$ & SVOA & Di-n-butyl phthalate & 1 & 0 & $\mathrm{ug} / \mathrm{kg}$ & 994 & 994 & 994 & & \\
\hline $68-7166$ & SVOA & Di-n-octylphthalate & 1 & 0 & $\mathrm{ug} / \mathrm{kg}$ & 994 & 994 & 994 & & \\
\hline $68-7166$ & SVOA & Diphenylamine & 1 & 0 & $\mathrm{ug} / \mathrm{kg}$ & 994 & 994 & 994 & & \\
\hline $68-7166$ & SVOA & Fluoranthene & 1 & 0 & $\mathrm{ug} / \mathrm{kg}$ & 99.4 & 99.4 & 99.4 & & \\
\hline $68-7166$ & SVOA & Fluorene & 1 & 0 & $\mathrm{ug} / \mathrm{kg}$ & 99.4 & 99.4 & 99.4 & & \\
\hline $68-7166$ & SVOA & Hexachlorobenzene & 2 & 0 & $\mathrm{ug} / \mathrm{kg}$ & 0.05 & 994 & 497.025 & 497.025 & 702.82879 \\
\hline $68-7166$ & SVOA & Hexachlorobutadiene & 2 & 0 & $\mathrm{ug} / \mathrm{kg}$ & 0.05 & 994 & 497.025 & 497.025 & 702.82879 \\
\hline $68-7166$ & SVOA & Hexachlorocyclopentadiene & 1 & 0 & $\mathrm{ug} / \mathrm{kg}$ & 994 & 994 & 994 & & \\
\hline
\end{tabular}




\begin{tabular}{|c|c|c|c|c|c|c|c|c|c|c|}
\hline $68-7166$ & SVOA & Hexachloroethane & 2 & 0 & $\mathrm{ug} / \mathrm{kg}$ & 0.05 & 994 & 497.025 & 497.025 & 702.82879 \\
\hline $68-7166$ & SVOA & Indeno(1,2,3-cd)pyrene & 1 & 0 & $\mathrm{ug} / \mathrm{kg}$ & 99.4 & 99.4 & 99.4 & & \\
\hline $68-7166$ & SVOA & Isophorone & 1 & 0 & $\mathrm{ug} / \mathrm{kg}$ & 994 & 994 & 994 & & \\
\hline $68-7166$ & SVOA & $\mathrm{m}+\mathrm{p}$ Methylphenol & 2 & 0 & $\mathrm{ug} / \mathrm{kg}$ & 0.05 & 994 & 497.025 & 497.025 & 702.82879 \\
\hline $68-7166$ & SVOA & Naphthalene & 1 & 0 & $\mathrm{ug} / \mathrm{kg}$ & 99.4 & 99.4 & 99.4 & & \\
\hline $68-7166$ & SVOA & Nitrobenzene & 2 & 0 & $\mathrm{ug} / \mathrm{kg}$ & 0.05 & 994 & 497.025 & 497.025 & 702.82879 \\
\hline $68-7166$ & SVOA & N-Nitroso-di-n-propylamine & 1 & 0 & $\mathrm{ug} / \mathrm{kg}$ & 994 & 994 & 994 & & \\
\hline $68-7166$ & SVOA & Pentachlorophenol & 2 & 0 & $\mathrm{ug} / \mathrm{kg}$ & 0.05 & 994 & 497.025 & 497.025 & 702.82879 \\
\hline $68-7166$ & SVOA & Phenanthrene & 1 & 0 & $\mathrm{ug} / \mathrm{kg}$ & 99.4 & 99.4 & 99.4 & & \\
\hline $68-7166$ & SVOA & Phenol & 1 & 0 & $\mathrm{ug} / \mathrm{kg}$ & 994 & 994 & 994 & & \\
\hline $68-7166$ & SVOA & Pyridine & 2 & 0 & $\mathrm{ug} / \mathrm{kg}$ & 0.05 & 994 & 497.025 & 497.025 & 702.82879 \\
\hline $68-7166$ & VOA & (1,1-Dimethylethyl)benzene & 1 & 0 & $\mathrm{ug} / \mathrm{kg}$ & 96.2 & 96.2 & 96.2 & & \\
\hline $68-7166$ & VOA & (1-Methylpropyl)benzene & 1 & 0 & $\mathrm{ug} / \mathrm{kg}$ & 96.2 & 96.2 & 96.2 & & \\
\hline $68-7166$ & VOA & 1,1,1,2-Tetrachloroethane & 1 & 0 & $\mathrm{ug} / \mathrm{kg}$ & 96.2 & 96.2 & 96.2 & & \\
\hline $68-7166$ & VOA & 1,1,1-Trichloroethane & 1 & 0 & $\mathrm{ug} / \mathrm{kg}$ & 96.2 & 96.2 & 96.2 & & \\
\hline $68-7166$ & VOA & 1,1,2,2-Tetrachloroethane & 1 & 0 & $\mathrm{ug} / \mathrm{kg}$ & 96.2 & 96.2 & 96.2 & & \\
\hline $68-7166$ & VOA & 1,1,2-Trichloroethane & 1 & 0 & $\mathrm{ug} / \mathrm{kg}$ & 96.2 & 96.2 & 96.2 & & \\
\hline $68-7166$ & VOA & 1,1-Dichloroethane & 1 & 0 & $\mathrm{ug} / \mathrm{kg}$ & 96.2 & 96.2 & 96.2 & & \\
\hline $68-7166$ & VOA & 1,1-Dichloroethene & 2 & 0 & $\mathrm{ug} / \mathrm{kg}$ & 0.01 & 96.2 & 48.105 & 48.105 & 68.016601 \\
\hline $68-7166$ & $\mathrm{VOA}$ & 1,1-Dichloropropene & 1 & 0 & $\mathrm{ug} / \mathrm{kg}$ & 96.2 & 96.2 & 96.2 & & \\
\hline $68-7166$ & VOA & 1,2,3-Trichlorobenzene & 1 & 0 & $\mathrm{ug} / \mathrm{kg}$ & 96.2 & 96.2 & 96.2 & & \\
\hline $68-7166$ & VOA & 1,2,3-Trichloropropane & 1 & 0 & $\mathrm{ug} / \mathrm{kg}$ & 96.2 & 96.2 & 96.2 & & \\
\hline $68-7166$ & VOA & 1,2,4-Trimethylbenzene & 1 & 0 & $\mathrm{ug} / \mathrm{kg}$ & 96.2 & 96.2 & 96.2 & & \\
\hline $68-7166$ & VOA & 1,2-Dibromo-3-chloropropane & 1 & 0 & $\mathrm{ug} / \mathrm{kg}$ & 96.2 & 96.2 & 96.2 & & \\
\hline $68-7166$ & VOA & 1,2-Dibromoethane & 1 & 0 & $\mathrm{ug} / \mathrm{kg}$ & 96.2 & 96.2 & 96.2 & & \\
\hline $68-7166$ & VOA & 1,2-Dichloroethane & 2 & 0 & $\mathrm{ug} / \mathrm{kg}$ & 0.01 & 96.2 & 48.105 & 48.105 & 68.016601 \\
\hline $68-7166$ & VOA & 1,2-Dichloroethene & 1 & 0 & $\mathrm{ug} / \mathrm{kg}$ & 192 & 192 & 192 & & \\
\hline $68-7166$ & VOA & 1,2-Dichloropropane & 1 & 0 & $\mathrm{ug} / \mathrm{kg}$ & 96.2 & 96.2 & 96.2 & & \\
\hline $68-7166$ & VOA & 1,2-Dimethylbenzene & 1 & 0 & $\mathrm{ug} / \mathrm{kg}$ & 96.2 & 96.2 & 96.2 & & \\
\hline $68-7166$ & VOA & 1,3,5-Trimethylbenzene & 1 & 0 & $\mathrm{ug} / \mathrm{kg}$ & 96.2 & 96.2 & 96.2 & & \\
\hline $68-7166$ & VOA & 1,3-Dichloropropane & 1 & 0 & $\mathrm{ug} / \mathrm{kg}$ & 96.2 & 96.2 & 96.2 & & \\
\hline $68-7166$ & VOA & 1-Chloro-4-methylbenzene & 1 & 0 & $\mathrm{ug} / \mathrm{kg}$ & 96.2 & 96.2 & 96.2 & & \\
\hline $68-7166$ & VOA & 1-Methyl-4-(1-methylethyl)benzene & 1 & 0 & $\mathrm{ug} / \mathrm{kg}$ & 96.2 & 96.2 & 96.2 & & \\
\hline $68-7166$ & VOA & 2,2-Dichloropropane & 1 & 0 & $\mathrm{ug} / \mathrm{kg}$ & 96.2 & 96.2 & 96.2 & & \\
\hline $68-7166$ & $\mathrm{VOA}$ & 2-Butanone & 2 & 0 & $\mathrm{ug} / \mathrm{kg}$ & 0.05 & 481 & 240.525 & 240.525 & 340.08301 \\
\hline $68-7166$ & VOA & 2-Hexanone & 1 & 0 & $\mathrm{ug} / \mathrm{kg}$ & 481 & 481 & 481 & & \\
\hline $68-7166$ & VOA & 4-Methyl-2-pentanone & 1 & 0 & $\mathrm{ug} / \mathrm{kg}$ & 481 & 481 & 481 & & \\
\hline $68-7166$ & VOA & Acetone & 1 & 0 & $\mathrm{ug} / \mathrm{kg}$ & 481 & 481 & 481 & & \\
\hline $68-7166$ & VOA & Acetonitrile & 1 & 0 & $\mathrm{ug} / \mathrm{kg}$ & 2400 & 2400 & 2400 & & \\
\hline $68-7166$ & VOA & Acrolein & 1 & 0 & $\mathrm{ug} / \mathrm{kg}$ & 481 & 481 & 481 & & \\
\hline $68-7166$ & VOA & Acrylonitrile & 1 & 0 & $\mathrm{ug} / \mathrm{kg}$ & 481 & 481 & 481 & & \\
\hline $68-7166$ & $\mathrm{VOA}$ & Benzene & 2 & 0 & $\mathrm{ug} / \mathrm{kg}$ & 0.01 & 96.2 & 48.105 & 48.105 & 68.016601 \\
\hline $68-7166$ & VOA & Bromobenzene & 1 & 0 & $\mathrm{ug} / \mathrm{kg}$ & 96.2 & 96.2 & 96.2 & & \\
\hline $68-7166$ & VOA & Bromochloromethane & 1 & 0 & $\mathrm{ug} / \mathrm{kg}$ & 96.2 & 96.2 & 96.2 & & \\
\hline $68-7166$ & VOA & Bromodichloromethane & 1 & 0 & $\mathrm{ug} / \mathrm{kg}$ & 96.2 & 96.2 & 96.2 & & \\
\hline $68-7166$ & VOA & Bromoform & 1 & 0 & $\mathrm{ug} / \mathrm{kg}$ & 96.2 & 96.2 & 96.2 & & \\
\hline $68-7166$ & VOA & Bromomethane & 1 & 0 & $\mathrm{ug} / \mathrm{kg}$ & 96.2 & 96.2 & 96.2 & & \\
\hline $68-7166$ & VOA & Butylbenzene & 1 & 0 & $\mathrm{ug} / \mathrm{kg}$ & 96.2 & 96.2 & 96.2 & & \\
\hline $68-7166$ & VOA & Carbon disulfide & 1 & 0 & $\mathrm{ug} / \mathrm{kg}$ & 481 & 481 & 481 & & \\
\hline $68-7166$ & VOA & Carbon tetrachloride & 2 & 0 & $\mathrm{ug} / \mathrm{kg}$ & 0.01 & 96.2 & 48.105 & 48.105 & 68.016601 \\
\hline $68-7166$ & VOA & Chlorobenzene & 2 & 0 & $\mathrm{ug} / \mathrm{kg}$ & 0.01 & 96.2 & 48.105 & 48.105 & 68.016601 \\
\hline $68-7166$ & VOA & Chloroethane & 1 & 0 & $\mathrm{ug} / \mathrm{kg}$ & 96.2 & 96.2 & 96.2 & & \\
\hline $68-7166$ & VOA & Chloroform & 2 & 0 & $\mathrm{ug} / \mathrm{kg}$ & 0.01 & 96.2 & 48.105 & 48.105 & 68.016601 \\
\hline $68-7166$ & VOA & Chloromethane & 1 & 0 & $\mathrm{ug} / \mathrm{kg}$ & 96.2 & 96.2 & 96.2 & & \\
\hline $68-7166$ & $\mathrm{VOA}$ & cis-1,2-Dichloroethene & 1 & 0 & $\mathrm{ug} / \mathrm{kg}$ & 96.2 & 96.2 & 96.2 & & \\
\hline $68-7166$ & VOA & cis-1,3-Dichloropropene & 1 & 0 & $\mathrm{ug} / \mathrm{kg}$ & 96.2 & 96.2 & 96.2 & & \\
\hline $68-7166$ & VOA & Cumene & 1 & 0 & $\mathrm{ug} / \mathrm{kg}$ & 96.2 & 96.2 & 96.2 & & \\
\hline $68-7166$ & VOA & Dibromochloromethane & 1 & 0 & $\mathrm{ug} / \mathrm{kg}$ & 96.2 & 96.2 & 96.2 & & \\
\hline $68-7166$ & VOA & Dibromomethane & 1 & 0 & $\mathrm{ug} / \mathrm{kg}$ & 96.2 & 96.2 & 96.2 & & \\
\hline $68-7166$ & VOA & Dichlorodifluoromethane & 1 & 0 & $\mathrm{ug} / \mathrm{kg}$ & 96.2 & 96.2 & 96.2 & & \\
\hline $68-7166$ & VOA & Ethylbenzene & 1 & 0 & $\mathrm{ug} / \mathrm{kg}$ & 96.2 & 96.2 & 96.2 & & \\
\hline $68-7166$ & VOA & Hexane & 1 & 0 & $\mathrm{ug} / \mathrm{kg}$ & 481 & 481 & 481 & & \\
\hline $68-7166$ & VOA & M + P Xylene & 1 & 0 & $\mathrm{ug} / \mathrm{kg}$ & 192 & 192 & 192 & & \\
\hline $68-7166$ & VOA & Methanol & 1 & 0 & $\mathrm{ug} / \mathrm{kg}$ & 3330 & 3330 & 3330 & & \\
\hline $68-7166$ & VOA & Methyl methacrylate & 1 & 0 & $\mathrm{ug} / \mathrm{kg}$ & 481 & 481 & 481 & & \\
\hline $68-7166$ & VOA & Methylcyclohexane & 1 & 0 & $\mathrm{ug} / \mathrm{kg}$ & 96.2 & 96.2 & 96.2 & & \\
\hline $68-7166$ & VOA & Methylene chloride & 1 & 0 & $\mathrm{ug} / \mathrm{kg}$ & 481 & 481 & 481 & & \\
\hline $68-7166$ & VOA & o-Chlorotoluene & 1 & 0 & $\mathrm{ug} / \mathrm{kg}$ & 96.2 & 96.2 & 96.2 & & \\
\hline $68-7166$ & VOA & Propylbenzene & 1 & 0 & $\mathrm{ug} / \mathrm{kg}$ & 96.2 & 96.2 & 96.2 & & \\
\hline $68-7166$ & VOA & Propylene glycol & 1 & 0 & $\mathrm{ug} / \mathrm{kg}$ & 33300 & 33300 & 33300 & & \\
\hline
\end{tabular}




\begin{tabular}{|c|c|c|c|c|c|c|c|c|c|c|}
\hline $68-7166$ & VOA & Styrene & 1 & 0 & $\mathrm{ug} / \mathrm{kg}$ & 96.2 & 96.2 & 96.2 & & \\
\hline $68-7166$ & VOA & Tetrachloroethene & 2 & 0 & $\mathrm{ug} / \mathrm{kg}$ & 0.01 & 96.2 & 48.105 & 48.105 & 68.016601 \\
\hline $68-7166$ & VOA & Toluene & 1 & 0 & $\mathrm{ug} / \mathrm{kg}$ & 96.2 & 96.2 & 96.2 & & \\
\hline $68-7166$ & VOA & Total Xylene & 1 & 0 & $\mathrm{ug} / \mathrm{kg}$ & 288 & 288 & 288 & & \\
\hline $68-7166$ & VOA & trans-1,2-Dichloroethene & 1 & 0 & $\mathrm{ug} / \mathrm{kg}$ & 96.2 & 96.2 & 96.2 & & \\
\hline $68-7166$ & VOA & trans-1,3-Dichloropropene & 1 & 0 & $\mathrm{ug} / \mathrm{kg}$ & 96.2 & 96.2 & 96.2 & & \\
\hline $68-7166$ & VOA & Trichloroethene & 2 & 0 & $\mathrm{ug} / \mathrm{kg}$ & 0.01 & 96.2 & 48.105 & 48.105 & 68.016601 \\
\hline $68-7166$ & VOA & Trichlorofluoromethane & 1 & 0 & $\mathrm{ug} / \mathrm{kg}$ & 96.2 & 96.2 & 96.2 & & \\
\hline $68-7166$ & VOA & Vinyl chloride & 2 & 0 & $\mathrm{ug} / \mathrm{kg}$ & 0.01 & 96.2 & 48.105 & 48.105 & 68.016601 \\
\hline Duoseal vacuum pump & HERB & $2,4-\mathrm{D}$ & 2 & 0 & $\mathrm{ug} / \mathrm{kg}$ & 0.05 & 122 & 61.025 & 61.025 & 86.231672 \\
\hline Duoseal vacuum pump & HERB & Silvex & 2 & 0 & $\mathrm{ug} / \mathrm{kg}$ & 0.05 & 122 & 61.025 & 61.025 & 86.231672 \\
\hline Duoseal vacuum pump & METAL & Arsenic & 2 & 0 & $\mathrm{mg} / \mathrm{L}$ & 0.3 & 5.91 & 3.105 & 3.105 & 3.966869 \\
\hline Duoseal vacuum pump & METAL & Barium & 2 & 2 & $\mathrm{mg} / \mathrm{L}$ & 0.11 & 53.4 & 26.755 & 26.755 & 37.68172 \\
\hline Duoseal vacuum pump & METAL & Cadmium & 2 & 2 & $\mathrm{mg} / \mathrm{L}$ & 31.7 & 79.1 & 55.4 & 55.4 & 33.516861 \\
\hline Duoseal vacuum pump & METAL & Chromium & 2 & 1 & $\mathrm{mg} / \mathrm{L}$ & 0.05 & 2.97 & 1.51 & 1.51 & 2.0647518 \\
\hline Duoseal vacuum pump & METAL & Lead & 2 & 2 & $\mathrm{mg} / \mathrm{L}$ & 0.143 & 61.7 & 30.9215 & 30.9215 & 43.527372 \\
\hline Duoseal vacuum pump & METAL & Mercury & 2 & 1 & $\mathrm{mg} / \mathrm{L}$ & 0.002 & 0.482 & 0.242 & 0.242 & 0.3394113 \\
\hline Duoseal vacuum pump & METAL & Selenium & 2 & 0 & $\mathrm{mg} / \mathrm{L}$ & 0.3 & 5.91 & 3.105 & 3.105 & 3.966869 \\
\hline Duoseal vacuum pump & METAL & Silver & 2 & 0 & $\mathrm{mg} / \mathrm{L}$ & 0.05 & 0.984 & 0.517 & 0.517 & 0.6604377 \\
\hline Duoseal vacuum pump & METAL & Total Uranium & 1 & 0 & $\mathrm{pCi} / \mathrm{g}$ & 0.0163 & 0.0163 & 0.0163 & & \\
\hline Duoseal vacuum pump & PPCB & 4,4'-DDD & 1 & 0 & $\mathrm{ug} / \mathrm{kg}$ & 39.8 & 39.8 & 39.8 & & \\
\hline Duoseal vacuum pump & PPCB & 4,4'-DDE & 1 & 0 & $\mathrm{ug} / \mathrm{kg}$ & 39.8 & 39.8 & 39.8 & & \\
\hline Duoseal vacuum pump & РPCB & 4,4'-DDT & 1 & 0 & $\mathrm{ug} / \mathrm{kg}$ & 39.8 & 39.8 & 39.8 & & \\
\hline Duoseal vacuum pump & РРCB & Aldrin & 1 & 0 & $\mathrm{ug} / \mathrm{kg}$ & 19.9 & 19.9 & 19.9 & & \\
\hline Duoseal vacuum pump & PPCB & alpha-BHC & 1 & 0 & $\mathrm{ug} / \mathrm{kg}$ & 19.9 & 19.9 & 19.9 & & \\
\hline Duoseal vacuum pump & PPCB & alpha-Chlordane & 1 & 0 & $\mathrm{ug} / \mathrm{kg}$ & 19.9 & 19.9 & 19.9 & & \\
\hline Duoseal vacuum pump & PPCB & beta-BHC & 1 & 0 & $\mathrm{ug} / \mathrm{kg}$ & 19.9 & 19.9 & 19.9 & & \\
\hline Duoseal vacuum pump & PPCB & Chlordane & 2 & 0 & $\mathrm{ug} / \mathrm{kg}$ & 0.0025 & 249 & 124.50125 & 124.50125 & 176.06782 \\
\hline Duoseal vacuum pump & PPCB & delta-BHC & 1 & 0 & $\mathrm{ug} / \mathrm{kg}$ & 19.9 & 19.9 & 19.9 & & \\
\hline Duoseal vacuum pump & PPCB & Dieldrin & 1 & 0 & $\mathrm{ug} / \mathrm{kg}$ & 39.8 & 39.8 & 39.8 & & \\
\hline Duoseal vacuum pump & РPCB & Endosulfan I & 1 & 0 & $\mathrm{ug} / \mathrm{kg}$ & 19.9 & 19.9 & 19.9 & & \\
\hline Duoseal vacuum pump & РPCB & Endosulfan II & 1 & 0 & $\mathrm{ug} / \mathrm{kg}$ & 39.8 & 39.8 & 39.8 & & \\
\hline Duoseal vacuum pump & РPCB & Endosulfan sulfate & 1 & 0 & $\mathrm{ug} / \mathrm{kg}$ & 39.8 & 39.8 & 39.8 & & \\
\hline Duoseal vacuum pump & РPCB & Endrin & 2 & 0 & $\mathrm{ug} / \mathrm{kg}$ & 0.0004 & 39.8 & 19.9002 & 19.9002 & 28.142567 \\
\hline Duoseal vacuum pump & РРСВ & Endrin aldehyde & 1 & 0 & $\mathrm{ug} / \mathrm{kg}$ & 39.8 & 39.8 & 39.8 & & \\
\hline Duoseal vacuum pump & РPCB & Endrin ketone & 1 & 0 & $\mathrm{ug} / \mathrm{kg}$ & 39.8 & 39.8 & 39.8 & & \\
\hline Duoseal vacuum pump & PPCB & gamma-Chlordane & 1 & 0 & $\mathrm{ug} / \mathrm{kg}$ & 19.9 & 19.9 & 19.9 & & \\
\hline Duoseal vacuum pump & РPCB & Heptachlor & 2 & 0 & $\mathrm{ug} / \mathrm{kg}$ & 0.0002 & 19.9 & 9.9501 & 9.9501 & 14.071284 \\
\hline Duoseal vacuum pump & РРCB & Heptachlor epoxide & 2 & 0 & $\mathrm{ug} / \mathrm{kg}$ & 0.0002 & 19.9 & 9.9501 & 9.9501 & 14.071284 \\
\hline Duoseal vacuum pump & PPCB & Lindane & 2 & 0 & $\mathrm{ug} / \mathrm{kg}$ & 0.0002 & 19.9 & 9.9501 & 9.9501 & 14.071284 \\
\hline Duoseal vacuum pump & PPCB & Methoxychlor & 2 & 0 & $\mathrm{ug} / \mathrm{kg}$ & 0.002 & 199 & 99.501 & 99.501 & 140.71284 \\
\hline Duoseal vacuum pump & РPCB & Toxaphene & 2 & 0 & $\mathrm{ug} / \mathrm{kg}$ & 0.005 & 497 & 248.5025 & 248.5025 & 351.42853 \\
\hline Duoseal vacuum pump & RADS & Americium-241 & 1 & 0 & $\mathrm{pCi} / \mathrm{g}$ & 0.0162 & 0.0162 & 0.0162 & & \\
\hline Duoseal vacuum pump & RADS & Carbon-14 & 1 & 0 & $\mathrm{pCi} / \mathrm{g}$ & -1.32 & -1.32 & -1.32 & & \\
\hline Duoseal vacuum pump & RADS & Cesium-137 & 1 & 0 & $\mathrm{pCi} / \mathrm{g}$ & 0.0599 & 0.0599 & 0.0599 & & \\
\hline Duoseal vacuum pump & RADS & Cobalt- 60 & 1 & 0 & $\mathrm{pCi} / \mathrm{g}$ & 0.0252 & 0.0252 & 0.0252 & & \\
\hline Duoseal vacuum pump & RADS & Curium-243/244 & 1 & 0 & $\mathrm{pCi} / \mathrm{g}$ & -0.0144 & -0.0144 & -0.0144 & & \\
\hline Duoseal vacuum pump & RADS & Iodine-129 & 1 & 0 & $\mathrm{pCi} / \mathrm{g}$ & 0.538 & 0.538 & 0.538 & & \\
\hline Duoseal vacuum pump & RADS & Neptunium-237 & 1 & 0 & $\mathrm{pCi} / \mathrm{g}$ & -0.0088 & -0.0088 & -0.0088 & & \\
\hline Duoseal vacuum pump & RADS & Plutonium-238 & 1 & 0 & $\mathrm{pCi} / \mathrm{g}$ & -0.0198 & -0.0198 & -0.0198 & & \\
\hline Duoseal vacuum pump & RADS & Plutonium-239/240 & 1 & 0 & $\mathrm{pCi} / \mathrm{g}$ & 0.0569 & 0.0569 & 0.0569 & & \\
\hline Duoseal vacuum pump & RADS & Radium-226 & 1 & 0 & $\mathrm{pCi} / \mathrm{g}$ & 0.144 & 0.144 & 0.144 & & \\
\hline Duoseal vacuum pump & RADS & Strontium-90 & 1 & 0 & $\mathrm{pCi} / \mathrm{g}$ & -0.325 & -0.325 & -0.325 & & \\
\hline Duoseal vacuum pump & RADS & Technetium-99 & 1 & 0 & $\mathrm{pCi} / \mathrm{g}$ & -1.79 & -1.79 & -1.79 & & \\
\hline Duoseal vacuum pump & RADS & Thorium-230 & 1 & 0 & $\mathrm{pCi} / \mathrm{g}$ & -4.38 & -4.38 & -4.38 & & \\
\hline Duoseal vacuum pump & RADS & Thorium-232 & 1 & 0 & $\mathrm{pCi} / \mathrm{g}$ & 2.06 & 2.06 & 2.06 & & \\
\hline Duoseal vacuum pump & RADS & Tritium & 1 & 0 & $\mathrm{pCi} / \mathrm{g}$ & 13.6 & 13.6 & 13.6 & & \\
\hline Duoseal vacuum pump & RADS & Uranium-233/234 & 1 & 0 & $\mathrm{pCi} / \mathrm{g}$ & 0.029 & 0.029 & 0.029 & & \\
\hline Duoseal vacuum pump & RADS & Uranium-235/236 & 1 & 0 & $\mathrm{pCi} / \mathrm{g}$ & -0.0224 & -0.0224 & -0.0224 & & \\
\hline Duoseal vacuum pump & RADS & Uranium-238 & 1 & 0 & $\mathrm{pCi} / \mathrm{g}$ & 0.00967 & 0.00967 & 0.00967 & & \\
\hline Duoseal vacuum pump & SVOA & 1,2,4-Trichlorobenzene & 1 & 0 & $\mathrm{ug} / \mathrm{kg}$ & 417 & 417 & 417 & & \\
\hline Duoseal vacuum pump & SVOA & 1,2-Dichlorobenzene & 1 & 0 & $\mathrm{ug} / \mathrm{kg}$ & 417 & 417 & 417 & & \\
\hline Duoseal vacuum pump & SVOA & 1,3-Dichlorobenzene & 1 & 0 & $\mathrm{ug} / \mathrm{kg}$ & 417 & 417 & 417 & & \\
\hline Duoseal vacuum pump & SVOA & 1,4-Dichlorobenzene & 2 & 0 & $\mathrm{ug} / \mathrm{kg}$ & 0.01 & 417 & 208.505 & 208.505 & 294.85646 \\
\hline Duoseal vacuum pump & SVOA & 2,2'-Dichlorodiisopropyl ether & 1 & 0 & $\mathrm{ug} / \mathrm{kg}$ & 9810 & 9810 & 9810 & & \\
\hline Duoseal vacuum pump & SVOA & 2,3,4,6-Tetrachlorophenol & 1 & 0 & $\mathrm{ug} / \mathrm{kg}$ & 9810 & 9810 & 9810 & & \\
\hline Duoseal vacuum pump & SVOA & 2,4,5-Trichlorophenol & 2 & 0 & $\mathrm{ug} / \mathrm{kg}$ & 0.05 & 9810 & 4905.025 & 4905.025 & 6936.6822 \\
\hline Duoseal vacuum pump & SVOA & 2,4,6-Trichlorophenol & 2 & 0 & $\mathrm{ug} / \mathrm{kg}$ & 0.05 & 9810 & 4905.025 & 4905.025 & 6936.6822 \\
\hline Duoseal vacuum pump & SVOA & 2,4-Dichlorophenol & 1 & 0 & $\mathrm{ug} / \mathrm{kg}$ & 9810 & 9810 & 9810 & & \\
\hline Duoseal vacuum pump & SVOA & 2,4-Dimethylphenol & 1 & 0 & $\mathrm{ug} / \mathrm{kg}$ & 9810 & 9810 & 9810 & & \\
\hline
\end{tabular}




\begin{tabular}{|c|c|c|c|c|c|c|c|c|c|c|}
\hline Duoseal vacuum pump & SVOA & 2,4-Dinitrophenol & 1 & 0 & $\mathrm{ug} / \mathrm{kg}$ & 19600 & 19600 & 19600 & & \\
\hline Duoseal vacuum pump & SVOA & 2,4-Dinitrotoluene & 2 & 0 & $\mathrm{ug} / \mathrm{kg}$ & 0.05 & 9810 & 4905.025 & 4905.025 & 6936.6822 \\
\hline Duoseal vacuum pump & SVOA & 2,6-Dinitrotoluene & 1 & 0 & $\mathrm{ug} / \mathrm{kg}$ & 9810 & 9810 & 9810 & & \\
\hline Duoseal vacuum pump & SVOA & 2-Chloronaphthalene & 1 & 0 & $\mathrm{ug} / \mathrm{kg}$ & 981 & 981 & 981 & & \\
\hline Duoseal vacuum pump & SVOA & 2-Chlorophenol & 1 & 0 & $\mathrm{ug} / \mathrm{kg}$ & 9810 & 9810 & 9810 & & \\
\hline Duoseal vacuum pump & SVOA & 2-Methyl-4,6-dinitrophenol & 1 & 0 & $\mathrm{ug} / \mathrm{kg}$ & 9810 & 9810 & 9810 & & \\
\hline Duoseal vacuum pump & SVOA & 2-Methylnaphthalene & 1 & 0 & $\mathrm{ug} / \mathrm{kg}$ & 981 & 981 & 981 & & \\
\hline Duoseal vacuum pump & SVOA & 2-Methylphenol & 2 & 0 & $\mathrm{ug} / \mathrm{kg}$ & 0.05 & 9810 & 4905.025 & 4905.025 & 6936.6822 \\
\hline Duoseal vacuum pump & SVOA & 2-Nitrobenzenamine & 1 & 0 & $\mathrm{ug} / \mathrm{kg}$ & 9810 & 9810 & 9810 & & \\
\hline Duoseal vacuum pump & SVOA & 2-Nitrophenol & 1 & 0 & $\mathrm{ug} / \mathrm{kg}$ & 9810 & 9810 & 9810 & & \\
\hline Duoseal vacuum pump & SVOA & 3,3'-Dichlorobenzidine & 1 & 0 & $\mathrm{ug} / \mathrm{kg}$ & 9810 & 9810 & 9810 & & \\
\hline Duoseal vacuum pump & SVOA & 3-Nitrobenzenamine & 1 & 0 & $\mathrm{ug} / \mathrm{kg}$ & 9810 & 9810 & 9810 & & \\
\hline Duoseal vacuum pump & SVOA & 4-Bromophenyl phenyl ether & 1 & 0 & $\mathrm{ug} / \mathrm{kg}$ & 9810 & 9810 & 9810 & & \\
\hline Duoseal vacuum pump & SVOA & 4-Chloro-3-methylphenol & 1 & 0 & $\mathrm{ug} / \mathrm{kg}$ & 9810 & 9810 & 9810 & & \\
\hline Duoseal vacuum pump & SVOA & 4-Chlorobenzenamine & 1 & 0 & $\mathrm{ug} / \mathrm{kg}$ & 9810 & 9810 & 9810 & & \\
\hline Duoseal vacuum pump & SVOA & 4-Chlorophenyl phenyl ether & 1 & 0 & $\mathrm{ug} / \mathrm{kg}$ & 9810 & 9810 & 9810 & & \\
\hline Duoseal vacuum pump & SVOA & 4-Nitrobenzenamine & 1 & 0 & $\mathrm{ug} / \mathrm{kg}$ & 9810 & 9810 & 9810 & & \\
\hline Duoseal vacuum pump & SVOA & 4-Nitrophenol & 1 & 0 & $\mathrm{ug} / \mathrm{kg}$ & 9810 & 9810 & 9810 & & \\
\hline Duoseal vacuum pump & SVOA & Acenaphthene & 1 & 0 & $\mathrm{ug} / \mathrm{kg}$ & 981 & 981 & 981 & & \\
\hline Duoseal vacuum pump & SVOA & Acenaphthylene & 1 & 0 & $\mathrm{ug} / \mathrm{kg}$ & 981 & 981 & 981 & & \\
\hline Duoseal vacuum pump & SVOA & Acetophenone & 1 & 0 & $\mathrm{ug} / \mathrm{kg}$ & 9810 & 9810 & 9810 & & \\
\hline Duoseal vacuum pump & SVOA & Anthracene & 1 & 0 & $\mathrm{ug} / \mathrm{kg}$ & 981 & 981 & 981 & & \\
\hline Duoseal vacuum pump & SVOA & Benz(a)anthracene & 1 & 0 & $\mathrm{ug} / \mathrm{kg}$ & 981 & 981 & 981 & & \\
\hline Duoseal vacuum pump & SVOA & Benzenemethanol & 1 & 0 & $\mathrm{ug} / \mathrm{kg}$ & 9810 & 9810 & 9810 & & \\
\hline Duoseal vacuum pump & SVOA & Benzidine & 1 & 0 & $\mathrm{ug} / \mathrm{kg}$ & 9810 & 9810 & 9810 & & \\
\hline Duoseal vacuum pump & SVOA & Benzo(a)pyrene & 1 & 1 & $\mathrm{ug} / \mathrm{kg}$ & 1170 & 1170 & 1170 & 1170 & \\
\hline Duoseal vacuum pump & SVOA & Benzo(b)fluoranthene & 1 & 0 & $\mathrm{ug} / \mathrm{kg}$ & 981 & 981 & 981 & & \\
\hline Duoseal vacuum pump & SVOA & Benzo(ghi)perylene & 1 & 1 & $\mathrm{ug} / \mathrm{kg}$ & 9910 & 9910 & 9910 & 9910 & \\
\hline Duoseal vacuum pump & SVOA & Benzo(k)fluoranthene & 1 & 0 & $\mathrm{ug} / \mathrm{kg}$ & 981 & 981 & 981 & & \\
\hline Duoseal vacuum pump & SVOA & Benzoic acid & 1 & 0 & $\mathrm{ug} / \mathrm{kg}$ & 19600 & 19600 & 19600 & & \\
\hline Duoseal vacuum pump & SVOA & Bis(2-chloroethoxy)methane & 1 & 0 & $\mathrm{ug} / \mathrm{kg}$ & 9810 & 9810 & 9810 & & \\
\hline Duoseal vacuum pump & SVOA & Bis(2-chloroethyl) ether & 1 & 0 & $\mathrm{ug} / \mathrm{kg}$ & 9810 & 9810 & 9810 & & \\
\hline Duoseal vacuum pump & SVOA & Bis(2-ethylhexyl)phthalate & 1 & 0 & $\mathrm{ug} / \mathrm{kg}$ & 9810 & 9810 & 9810 & & \\
\hline Duoseal vacuum pump & SVOA & Butyl benzyl phthalate & 1 & 0 & $\mathrm{ug} / \mathrm{kg}$ & 9810 & 9810 & 9810 & & \\
\hline Duoseal vacuum pump & SVOA & Carbazole & 1 & 0 & $\mathrm{ug} / \mathrm{kg}$ & 981 & 981 & 981 & & \\
\hline Duoseal vacuum pump & SVOA & Chrysene & 1 & 0 & $\mathrm{ug} / \mathrm{kg}$ & 981 & 981 & 981 & & \\
\hline Duoseal vacuum pump & SVOA & Dibenz(a,h)anthracene & 1 & 0 & $\mathrm{ug} / \mathrm{kg}$ & 981 & 981 & 981 & & \\
\hline Duoseal vacuum pump & SVOA & Dibenzofuran & 1 & 0 & $\mathrm{ug} / \mathrm{kg}$ & 9810 & 9810 & 9810 & & \\
\hline Duoseal vacuum pump & SVOA & Diethyl phthalate & 1 & 0 & $\mathrm{ug} / \mathrm{kg}$ & 9810 & 9810 & 9810 & & \\
\hline Duoseal vacuum pump & SVOA & Dimethyl phthalate & 1 & 0 & $\mathrm{ug} / \mathrm{kg}$ & 9810 & 9810 & 9810 & & \\
\hline Duoseal vacuum pump & SVOA & Di-n-butyl phthalate & 1 & 0 & $\mathrm{ug} / \mathrm{kg}$ & 9810 & 9810 & 9810 & & \\
\hline Duoseal vacuum pump & SVOA & Di-n-octylphthalate & 1 & 0 & $\mathrm{ug} / \mathrm{kg}$ & 9810 & 9810 & 9810 & & \\
\hline Duoseal vacuum pump & SVOA & Diphenylamine & 1 & 0 & $\mathrm{ug} / \mathrm{kg}$ & 9810 & 9810 & 9810 & & \\
\hline Duoseal vacuum pump & SVOA & Fluoranthene & 1 & 1 & $\mathrm{ug} / \mathrm{kg}$ & 1670 & 1670 & 1670 & 1670 & \\
\hline Duoseal vacuum pump & SVOA & Fluorene & 1 & 0 & $\mathrm{ug} / \mathrm{kg}$ & 981 & 981 & 981 & & \\
\hline Duoseal vacuum pump & SVOA & Hexachlorobenzene & 2 & 0 & $\mathrm{ug} / \mathrm{kg}$ & 0.05 & 9810 & 4905.025 & 4905.025 & 6936.6822 \\
\hline Duoseal vacuum pump & SVOA & Hexachlorobutadiene & 2 & 0 & $\mathrm{ug} / \mathrm{kg}$ & 0.05 & 9810 & 4905.025 & 4905.025 & 6936.6822 \\
\hline Duoseal vacuum pump & SVOA & Hexachlorocyclopentadiene & 1 & 0 & $\mathrm{ug} / \mathrm{kg}$ & 9810 & 9810 & 9810 & & \\
\hline Duoseal vacuum pump & SVOA & Hexachloroethane & 2 & 0 & $\mathrm{ug} / \mathrm{kg}$ & 0.05 & 9810 & 4905.025 & 4905.025 & 6936.6822 \\
\hline Duoseal vacuum pump & SVOA & Indeno(1,2,3-cd)pyrene & 1 & 0 & $\mathrm{ug} / \mathrm{kg}$ & 1550 & 1550 & 1550 & & \\
\hline Duoseal vacuum pump & SVOA & Isophorone & 1 & 0 & $\mathrm{ug} / \mathrm{kg}$ & 9810 & 9810 & 9810 & & \\
\hline Duoseal vacuum pump & SVOA & $\mathrm{m}+\mathrm{p}$ Methylphenol & 2 & 0 & $\mathrm{ug} / \mathrm{kg}$ & 0.05 & 9810 & 4905.025 & 4905.025 & 6936.6822 \\
\hline Duoseal vacuum pump & SVOA & Naphthalene & 1 & 0 & $\mathrm{ug} / \mathrm{kg}$ & 981 & 981 & 981 & & \\
\hline Duoseal vacuum pump & SVOA & Nitrobenzene & 2 & 0 & $\mathrm{ug} / \mathrm{kg}$ & 0.05 & 9810 & 4905.025 & 4905.025 & 6936.6822 \\
\hline Duoseal vacuum pump & SVOA & N-Nitroso-di-n-propylamine & 1 & 0 & $\mathrm{ug} / \mathrm{kg}$ & 9810 & 9810 & 9810 & & \\
\hline Duoseal vacuum pump & SVOA & Pentachlorophenol & 2 & 0 & $\mathrm{ug} / \mathrm{kg}$ & 0.05 & 9810 & 4905.025 & 4905.025 & 6936.6822 \\
\hline Duoseal vacuum pump & SVOA & Phenanthrene & 1 & 0 & $\mathrm{ug} / \mathrm{kg}$ & 981 & 981 & 981 & & \\
\hline Duoseal vacuum pump & SVOA & Phenol & 1 & 0 & $\mathrm{ug} / \mathrm{kg}$ & 9810 & 9810 & 9810 & & \\
\hline Duoseal vacuum pump & SVOA & Pyridine & 2 & 0 & $\mathrm{ug} / \mathrm{kg}$ & 0.05 & 9810 & 4905.025 & 4905.025 & 6936.6822 \\
\hline Duoseal vacuum pump & VOA & (1,1-Dimethylethyl)benzene & 1 & 0 & $\mathrm{ug} / \mathrm{kg}$ & 417 & 417 & 417 & & \\
\hline Duoseal vacuum pump & VOA & (1-Methylpropyl)benzene & 1 & 0 & $\mathrm{ug} / \mathrm{kg}$ & 417 & 417 & 417 & & \\
\hline Duoseal vacuum pump & VOA & 1,1,1,2-Tetrachloroethane & 1 & 0 & $\mathrm{ug} / \mathrm{kg}$ & 417 & 417 & 417 & & \\
\hline Duoseal vacuum pump & VOA & 1,1,1-Trichloroethane & 1 & 0 & $\mathrm{ug} / \mathrm{kg}$ & 417 & 417 & 417 & & \\
\hline Duoseal vacuum pump & VOA & 1,1,2,2-Tetrachloroethane & 1 & 0 & $\mathrm{ug} / \mathrm{kg}$ & 417 & 417 & 417 & & \\
\hline Duoseal vacuum pump & VOA & 1,1,2-Trichloroethane & 1 & 0 & $\mathrm{ug} / \mathrm{kg}$ & 417 & 417 & 417 & & \\
\hline Duoseal vacuum pump & VOA & 1,1-Dichloroethane & 1 & 0 & $\mathrm{ug} / \mathrm{kg}$ & 417 & 417 & 417 & & \\
\hline Duoseal vacuum pump & VOA & 1,1-Dichloroethene & 2 & 0 & $\mathrm{ug} / \mathrm{kg}$ & 0.01 & 417 & 208.505 & 208.505 & 294.85646 \\
\hline Duoseal vacuum pump & VOA & 1,1-Dichloropropene & 1 & 0 & $\mathrm{ug} / \mathrm{kg}$ & 417 & 417 & 417 & & \\
\hline Duoseal vacuum pump & VOA & 1,2,3-Trichlorobenzene & 1 & 0 & $\mathrm{ug} / \mathrm{kg}$ & 417 & 417 & 417 & & \\
\hline Duoseal vacuum pump & VOA & 1,2,3-Trichloropropane & 1 & 0 & $\mathrm{ug} / \mathrm{kg}$ & 417 & 417 & 417 & & \\
\hline
\end{tabular}




\begin{tabular}{|c|c|c|c|c|c|c|c|c|c|c|}
\hline Duoseal vacuum pump & VOA & 1,2,4-Trimethylbenzene & 1 & 0 & $\mathrm{ug} / \mathrm{kg}$ & 417 & 417 & 417 & & \\
\hline Duoseal vacuum pump & VOA & 1,2-Dibromo-3-chloropropane & 1 & 0 & $\mathrm{ug} / \mathrm{kg}$ & 417 & 417 & 417 & & \\
\hline Duoseal vacuum pump & VOA & 1,2-Dibromoethane & 1 & 0 & $\mathrm{ug} / \mathrm{kg}$ & 417 & 417 & 417 & & \\
\hline Duoseal vacuum pump & VOA & 1,2-Dichloroethane & 2 & 0 & $\mathrm{ug} / \mathrm{kg}$ & 0.01 & 417 & 208.505 & 208.505 & 294.85646 \\
\hline Duoseal vacuum pump & VOA & 1,2-Dichloroethene & 1 & 0 & $\mathrm{ug} / \mathrm{kg}$ & 833 & 833 & 833 & & \\
\hline Duoseal vacuum pump & VOA & 1,2-Dichloropropane & 1 & 0 & $\mathrm{ug} / \mathrm{kg}$ & 417 & 417 & 417 & & \\
\hline Duoseal vacuum pump & VOA & 1,2-Dimethylbenzene & 1 & 0 & $\mathrm{ug} / \mathrm{kg}$ & 417 & 417 & 417 & & \\
\hline Duoseal vacuum pump & VOA & 1,3,5-Trimethylbenzene & 1 & 0 & $\mathrm{ug} / \mathrm{kg}$ & 417 & 417 & 417 & & \\
\hline Duoseal vacuum pump & VOA & 1,3-Dichloropropane & 1 & 0 & $\mathrm{ug} / \mathrm{kg}$ & 417 & 417 & 417 & & \\
\hline Duoseal vacuum pump & VOA & 1-Chloro-4-methylbenzene & 1 & 0 & $\mathrm{ug} / \mathrm{kg}$ & 417 & 417 & 417 & & \\
\hline Duoseal vacuum pump & VOA & 1-Methyl-4-(1-methylethyl)benzene & 1 & 0 & $\mathrm{ug} / \mathrm{kg}$ & 417 & 417 & 417 & & \\
\hline Duoseal vacuum pump & VOA & 2,2-Dichloropropane & 1 & 0 & $\mathrm{ug} / \mathrm{kg}$ & 417 & 417 & 417 & & \\
\hline Duoseal vacuum pump & VOA & 2-Butanone & 2 & 0 & $\mathrm{ug} / \mathrm{kg}$ & 0.05 & 2080 & 1040.025 & 1040.025 & 1470.7467 \\
\hline Duoseal vacuum pump & VOA & 2-Hexanone & 1 & 0 & $\mathrm{ug} / \mathrm{kg}$ & 2080 & 2080 & 2080 & & \\
\hline Duoseal vacuum pump & VOA & 4-Methyl-2-pentanone & 1 & 0 & $\mathrm{ug} / \mathrm{kg}$ & 2080 & 2080 & 2080 & & \\
\hline Duoseal vacuum pump & VOA & Acetone & 1 & 0 & $\mathrm{ug} / \mathrm{kg}$ & 2080 & 2080 & 2080 & & \\
\hline Duoseal vacuum pump & VOA & Acetonitrile & 1 & 0 & $\mathrm{ug} / \mathrm{kg}$ & 10400 & 10400 & 10400 & & \\
\hline Duoseal vacuum pump & VOA & Acrolein & 1 & 0 & $\mathrm{ug} / \mathrm{kg}$ & 2080 & 2080 & 2080 & & \\
\hline Duoseal vacuum pump & VOA & Acrylonitrile & 1 & 0 & $\mathrm{ug} / \mathrm{kg}$ & 2080 & 2080 & 2080 & & \\
\hline Duoseal vacuum pump & VOA & Benzene & 2 & 0 & $\mathrm{ug} / \mathrm{kg}$ & 0.01 & 417 & 208.505 & 208.505 & 294.85646 \\
\hline Duoseal vacuum pump & VOA & Bromobenzene & 1 & 0 & $\mathrm{ug} / \mathrm{kg}$ & 417 & 417 & 417 & & \\
\hline Duoseal vacuum pump & VOA & Bromochloromethane & 1 & 0 & $\mathrm{ug} / \mathrm{kg}$ & 417 & 417 & 417 & & \\
\hline Duoseal vacuum pump & VOA & Bromodichloromethane & 1 & 0 & $\mathrm{ug} / \mathrm{kg}$ & 417 & 417 & 417 & & \\
\hline Duoseal vacuum pump & VOA & Bromoform & 1 & 0 & $\mathrm{ug} / \mathrm{kg}$ & 417 & 417 & 417 & & \\
\hline Duoseal vacuum pump & VOA & Bromomethane & 1 & 0 & $\mathrm{ug} / \mathrm{kg}$ & 417 & 417 & 417 & & \\
\hline Duoseal vacuum pump & VOA & Butylbenzene & 1 & 0 & $\mathrm{ug} / \mathrm{kg}$ & 417 & 417 & 417 & & \\
\hline Duoseal vacuum pump & VOA & Carbon disulfide & 1 & 0 & $\mathrm{ug} / \mathrm{kg}$ & 2080 & 2080 & 2080 & & \\
\hline Duoseal vacuum pump & VOA & Carbon tetrachloride & 2 & 0 & $\mathrm{ug} / \mathrm{kg}$ & 0.01 & 417 & 208.505 & 208.505 & 294.85646 \\
\hline Duoseal vacuum pump & VOA & Chlorobenzene & 2 & 0 & $\mathrm{ug} / \mathrm{kg}$ & 0.01 & 417 & 208.505 & 208.505 & 294.85646 \\
\hline Duoseal vacuum pump & VOA & Chloroethane & 1 & 0 & $\mathrm{ug} / \mathrm{kg}$ & 417 & 417 & 417 & & \\
\hline Duoseal vacuum pump & VOA & Chloroform & 2 & 0 & $\mathrm{ug} / \mathrm{kg}$ & 0.01 & 417 & 208.505 & 208.505 & 294.85646 \\
\hline Duoseal vacuum pump & VOA & Chloromethane & 1 & 0 & $\mathrm{ug} / \mathrm{kg}$ & 417 & 417 & 417 & & \\
\hline Duoseal vacuum pump & VOA & cis-1,2-Dichloroethene & 1 & 0 & $\mathrm{ug} / \mathrm{kg}$ & 417 & 417 & 417 & & \\
\hline Duoseal vacuum pump & VOA & cis-1,3-Dichloropropene & 1 & 0 & $\mathrm{ug} / \mathrm{kg}$ & 417 & 417 & 417 & & \\
\hline Duoseal vacuum pump & VOA & Cumene & 1 & 0 & $\mathrm{ug} / \mathrm{kg}$ & 417 & 417 & 417 & & \\
\hline Duoseal vacuum pump & VOA & Dibromochloromethane & 1 & 0 & $\mathrm{ug} / \mathrm{kg}$ & 417 & 417 & 417 & & \\
\hline Duoseal vacuum pump & VOA & Dibromomethane & 1 & 0 & $\mathrm{ug} / \mathrm{kg}$ & 417 & 417 & 417 & & \\
\hline Duoseal vacuum pump & VOA & Dichlorodifluoromethane & 1 & 0 & $\mathrm{ug} / \mathrm{kg}$ & 417 & 417 & 417 & & \\
\hline Duoseal vacuum pump & VOA & Ethylbenzene & 1 & 0 & $\mathrm{ug} / \mathrm{kg}$ & 417 & 417 & 417 & & \\
\hline Duoseal vacuum pump & VOA & Hexane & 1 & 0 & $\mathrm{ug} / \mathrm{kg}$ & 2080 & 2080 & 2080 & & \\
\hline Duoseal vacuum pump & VOA & $\mathrm{M}+\mathrm{P}$ Xylene & 1 & 0 & $\mathrm{ug} / \mathrm{kg}$ & 833 & 833 & 833 & & \\
\hline Duoseal vacuum pump & VOA & Methanol & 1 & 0 & $\mathrm{ug} / \mathrm{kg}$ & 4900 & 4900 & 4900 & & \\
\hline Duoseal vacuum pump & VOA & Methyl methacrylate & 1 & 0 & $\mathrm{ug} / \mathrm{kg}$ & 2080 & 2080 & 2080 & & \\
\hline Duoseal vacuum pump & VOA & Methylcyclohexane & 1 & 0 & $\mathrm{ug} / \mathrm{kg}$ & 417 & 417 & 417 & & \\
\hline Duoseal vacuum pump & VOA & Methylene chloride & 1 & 0 & $\mathrm{ug} / \mathrm{kg}$ & 2080 & 2080 & 2080 & & \\
\hline Duoseal vacuum pump & VOA & o-Chlorotoluene & 1 & 0 & $\mathrm{ug} / \mathrm{kg}$ & 417 & 417 & 417 & & \\
\hline Duoseal vacuum pump & VOA & Propylbenzene & 1 & 0 & $\mathrm{ug} / \mathrm{kg}$ & 417 & 417 & 417 & & \\
\hline Duoseal vacuum pump & VOA & Propylene glycol & 1 & 0 & $\mathrm{ug} / \mathrm{kg}$ & 49000 & 49000 & 49000 & & \\
\hline Duoseal vacuum pump & VOA & Styrene & 1 & 0 & $\mathrm{ug} / \mathrm{kg}$ & 417 & 417 & 417 & & \\
\hline Duoseal vacuum pump & VOA & Tetrachloroethene & 2 & 0 & $\mathrm{ug} / \mathrm{kg}$ & 0.01 & 417 & 208.505 & 208.505 & 294.85646 \\
\hline Duoseal vacuum pump & VOA & Toluene & 1 & 0 & $\mathrm{ug} / \mathrm{kg}$ & 417 & 417 & 417 & & \\
\hline Duoseal vacuum pump & VOA & Total Xylene & 1 & 0 & $\mathrm{ug} / \mathrm{kg}$ & 1250 & 1250 & 1250 & & \\
\hline Duoseal vacuum pump & VOA & trans-1,2-Dichloroethene & 1 & 0 & $\mathrm{ug} / \mathrm{kg}$ & 417 & 417 & 417 & & \\
\hline Duoseal vacuum pump & VOA & trans-1,3-Dichloropropene & 1 & 0 & $\mathrm{ug} / \mathrm{kg}$ & 417 & 417 & 417 & & \\
\hline Duoseal vacuum pump & VOA & Trichloroethene & 2 & 0 & $\mathrm{ug} / \mathrm{kg}$ & 0.01 & 417 & 208.505 & 208.505 & 294.85646 \\
\hline Duoseal vacuum pump & VOA & Trichlorofluoromethane & 1 & 0 & $\mathrm{ug} / \mathrm{kg}$ & 417 & 417 & 417 & & \\
\hline Duoseal vacuum pump & $\mathrm{VOA}$ & Vinyl chloride & 2 & 0 & $\mathrm{ug} / \mathrm{kg}$ & 0.01 & 417 & 208.505 & 208.505 & 294.85646 \\
\hline K25C1100740 & HERB & $2,4-\mathrm{D}$ & 6 & $\overline{0}$ & $\mathrm{ug} / \mathrm{kg}$ & 0.05 & 123 & 27.791667 & 9.675 & 47.870089 \\
\hline K25C1100740 & HERB & Silvex & 6 & 0 & $\mathrm{ug} / \mathrm{kg}$ & 0.05 & 123 & 27.791667 & 9.675 & 47.870089 \\
\hline K25C1100740 & METAL & Arsenic & 6 & 0 & $\mathrm{mg} / \mathrm{L}$ & 0.3 & 190 & 77.816667 & 64.65 & 87.198243 \\
\hline K25C1100740 & METAL & Barium & 6 & 4 & $\mathrm{mg} / \mathrm{L}$ & 0.05 & 155 & 49.019467 & 16.0055 & 66.41579 \\
\hline K25C1100740 & METAL & Cadmium & 6 & 3 & $\mathrm{mg} / \mathrm{L}$ & 0.05 & 6520 & 1096.3373 & 16.7 & 2657.0613 \\
\hline K25C1100740 & METAL & Chromium & 6 & 4 & $\mathrm{mg} / \mathrm{L}$ & 0.05 & 283 & 117.87417 & 94.5725 & 132.41534 \\
\hline K25C1100740 & METAL & Lead & 6 & 2 & $\mathrm{mg} / \mathrm{L}$ & 0.1 & 2340 & 408.095 & 22.535 & 946.80718 \\
\hline K25C1100740 & METAL & Mercury & 6 & 0 & $\mathrm{mg} / \mathrm{L}$ & 0.002 & 0.4 & 0.201 & 0.201 & 0.2179936 \\
\hline K25C1100740 & METAL & Selenium & 6 & 0 & $\mathrm{mg} / \mathrm{L}$ & 0.3 & 190 & 77.816667 & 64.65 & 87.198243 \\
\hline K25C1100740 & METAL & Silver & 6 & 0 & $\mathrm{mg} / \mathrm{L}$ & 0.05 & 31.6 & 12.975 & 10.825 & 14.527723 \\
\hline K25C1100740 & METAL & Total Uranium & 3 & 0 & $\mathrm{pCi} / \mathrm{g}$ & 0.0333 & 0.0893 & 0.0552333 & 0.0431 & 0.0299067 \\
\hline K25C1100740 & PPCB & $4,4^{\prime}-\mathrm{DDD}$ & 3 & 0 & $\mathrm{ug} / \mathrm{kg}$ & 4 & 19.5 & 11.733333 & 11.7 & 7.7500538 \\
\hline K25C1100740 & PPCB & 4,4'-DDE & 3 & 0 & $\mathrm{ug} / \mathrm{kg}$ & 4 & 19.5 & 11.733333 & 11.7 & 7.7500538 \\
\hline
\end{tabular}




\begin{tabular}{|c|c|c|c|c|c|c|c|c|c|c|}
\hline K25C1100740 & PPCB & 4,4'-DDT & 3 & 0 & $\mathrm{ug} / \mathrm{kg}$ & 4 & 19.5 & 11.733333 & 11.7 & 7.7500538 \\
\hline K25C1100740 & РPCB & Aldrin & 3 & 0 & $\mathrm{ug} / \mathrm{kg}$ & 2 & 9.74 & 5.87 & 5.87 & 3.87 \\
\hline K25C1100740 & РPCB & alpha-BHC & 3 & 0 & $\mathrm{ug} / \mathrm{kg}$ & 2 & 9.74 & 5.87 & 5.87 & 3.87 \\
\hline K25C1100740 & PPCB & alpha-Chlordane & 3 & 0 & $\mathrm{ug} / \mathrm{kg}$ & 2 & 9.74 & 5.87 & 5.87 & 3.87 \\
\hline K25C1100740 & PPCB & beta-BHC & 3 & 0 & $\mathrm{ug} / \mathrm{kg}$ & 2 & 9.74 & 5.87 & 5.87 & 3.87 \\
\hline K25C1100740 & PPCB & Chlordane & 6 & 0 & $\mathrm{ug} / \mathrm{kg}$ & 0.0025 & 122 & 36.717917 & 12.50125 & 50.581929 \\
\hline K25C1100740 & РPCB & delta-BHC & 3 & 0 & $\mathrm{ug} / \mathrm{kg}$ & 2 & 9.74 & 5.87 & 5.87 & 3.87 \\
\hline K25C1100740 & РPCB & Dieldrin & 3 & 0 & $\mathrm{ug} / \mathrm{kg}$ & 4 & 19.5 & 11.733333 & 11.7 & 7.7500538 \\
\hline K25C1100740 & PPCB & Endosulfan I & 3 & 0 & $\mathrm{ug} / \mathrm{kg}$ & 2 & 9.74 & 5.87 & 5.87 & 3.87 \\
\hline K25C1100740 & PPCB & Endosulfan II & 3 & 0 & $\mathrm{ug} / \mathrm{kg}$ & 4 & 19.5 & 11.733333 & 11.7 & 7.7500538 \\
\hline K25C1100740 & PPCB & Endosulfan sulfate & 3 & 0 & $\mathrm{ug} / \mathrm{kg}$ & 4 & 19.5 & 11.733333 & 11.7 & 7.7500538 \\
\hline K25C1100740 & PPCB & Endrin & 6 & 0 & $\mathrm{ug} / \mathrm{kg}$ & 0.0004 & 19.5 & 5.8668667 & 2.0002 & 8.0823172 \\
\hline K25C1100740 & РPCB & Endrin aldehyde & 3 & 0 & $\mathrm{ug} / \mathrm{kg}$ & 4 & 19.5 & 11.733333 & 11.7 & 7.7500538 \\
\hline K25C1100740 & РPCB & Endrin ketone & 3 & 0 & $\mathrm{ug} / \mathrm{kg}$ & 4 & 19.5 & 11.733333 & 11.7 & 7.7500538 \\
\hline K25C1100740 & PPCB & gamma-Chlordane & 3 & 0 & $\mathrm{ug} / \mathrm{kg}$ & 2 & 9.74 & 5.87 & 5.87 & 3.87 \\
\hline K25C1100740 & PPCB & Heptachlor & 6 & 0 & $\mathrm{ug} / \mathrm{kg}$ & 0.0002 & 9.74 & 2.9351 & 1.0001 & 4.0406838 \\
\hline K25C1100740 & PPCB & Heptachlor epoxide & 6 & 0 & $\mathrm{ug} / \mathrm{kg}$ & 0.0002 & 9.74 & 2.9351 & 1.0001 & 4.0406838 \\
\hline K25C1100740 & PPCB & Lindane & 6 & 0 & $\mathrm{ug} / \mathrm{kg}$ & 0.0002 & 9.74 & 2.9351 & 1.0001 & 4.0406838 \\
\hline K25C1100740 & PPCB & Methoxychlor & 6 & 0 & $\mathrm{ug} / \mathrm{kg}$ & 0.002 & 97.4 & 29.351 & 10.001 & 40.406838 \\
\hline K25C1100740 & PPCB & Toxaphene & 6 & 0 & $\mathrm{ug} / \mathrm{kg}$ & 0.005 & 243 & 73.335833 & 25.0025 & 100.88522 \\
\hline K25C1100740 & RADS & Americium-241 & 3 & 0 & $\mathrm{pCi} / \mathrm{g}$ & -0.00353 & 0.029 & 0.0125233 & 0.0121 & 0.0162691 \\
\hline K25C1100740 & RADS & Carbon-14 & 3 & 0 & $\mathrm{pCi} / \mathrm{g}$ & -3.92 & 0.368 & -1.697333 & -1.54 & 2.1483252 \\
\hline K25C1100740 & RADS & Cesium-137 & 3 & 1 & $\mathrm{pCi} / \mathrm{g}$ & 0.0326 & 0.274 & 0.1147667 & 0.0377 & 0.1379237 \\
\hline K25C1100740 & RADS & Cobalt- 60 & 3 & 0 & $\mathrm{pCi} / \mathrm{g}$ & -0.0245 & 0.0577 & 0.019 & 0.0238 & 0.0413097 \\
\hline K25C1100740 & RADS & Curium-243/244 & 3 & 0 & $\mathrm{pCi} / \mathrm{g}$ & -0.0111 & 0.011 & -0.001333 & -0.0039 & 0.0112714 \\
\hline K25C1100740 & RADS & Iodine-129 & 3 & 0 & $\mathrm{pCi} / \mathrm{g}$ & -0.0373 & 0.115 & 0.0549333 & 0.0871 & 0.0810854 \\
\hline K25C1100740 & RADS & Neptunium-237 & 3 & 0 & $\mathrm{pCi} / \mathrm{g}$ & -0.0431 & 0.0338 & 0.0081333 & 0.0337 & 0.0443694 \\
\hline K25C1100740 & RADS & Plutonium-238 & 3 & 0 & $\mathrm{pCi} / \mathrm{g}$ & -0.00862 & 0.0189 & 0.0069267 & 0.0105 & 0.0141037 \\
\hline K25C1100740 & RADS & Plutonium-239/240 & 3 & 0 & $\mathrm{pCi} / \mathrm{g}$ & 0.00622 & 0.155 & 0.0656067 & 0.0356 & 0.0787983 \\
\hline K25C1100740 & RADS & Radium-226 & 3 & 0 & $\mathrm{pCi} / \mathrm{g}$ & 0.28 & 1.12 & 0.734 & 0.802 & 0.4241085 \\
\hline K25C1100740 & RADS & Strontium-90 & 3 & 0 & $\mathrm{pCi} / \mathrm{g}$ & -0.126 & 0.46 & 0.0905 & -0.0625 & 0.3215676 \\
\hline K25C1100740 & RADS & Technetium-99 & 3 & 0 & $\mathrm{pCi} / \mathrm{g}$ & -0.351 & 0.00341 & -0.15953 & -0.131 & 0.1789192 \\
\hline K25C1100740 & RADS & Thorium-230 & 3 & 0 & $\mathrm{pCi} / \mathrm{g}$ & -5.52 & -4.68 & -5.236667 & -5.51 & 0.4821134 \\
\hline K25C1100740 & RADS & Thorium-232 & 3 & 0 & $\mathrm{pCi} / \mathrm{g}$ & -9.65 & 27.5 & 8.1233333 & 6.52 & 18.626826 \\
\hline K25C1100740 & RADS & Tritium & 3 & 0 & $\mathrm{pCi} / \mathrm{g}$ & 6.66 & 74 & 40.953333 & 42.2 & 33.687305 \\
\hline K25C1100740 & RADS & Uranium-233/234 & 3 & 0 & $\mathrm{pCi} / \mathrm{g}$ & 0.00727 & 0.0642 & 0.0330233 & 0.0276 & 0.0288499 \\
\hline K25C1100740 & RADS & Uranium-235/236 & 3 & 0 & $\mathrm{pCi} / \mathrm{g}$ & 0.011 & 0.0146 & 0.0133 & 0.0143 & 0.0019975 \\
\hline K25C1100740 & RADS & Uranium-238 & 3 & 0 & $\mathrm{pCi} / \mathrm{g}$ & -0.00526 & 0.0212 & 0.0089133 & 0.0108 & 0.0133305 \\
\hline K25C1100740 & SVOA & 1,2,4-Trichlorobenzene & 3 & 0 & $\mathrm{ug} / \mathrm{kg}$ & 100 & 500 & 233.33333 & 100 & 230.94011 \\
\hline K25C1100740 & SVOA & 1,2-Dichlorobenzene & 3 & 0 & $\mathrm{ug} / \mathrm{kg}$ & 100 & 500 & 233.33333 & 100 & 230.94011 \\
\hline K25C1100740 & SVOA & 1,3-Dichlorobenzene & 3 & 0 & $\mathrm{ug} / \mathrm{kg}$ & 100 & 500 & 233.33333 & 100 & 230.94011 \\
\hline K25C1100740 & SVOA & 1,4-Dichlorobenzene & 6 & 0 & $\mathrm{ug} / \mathrm{kg}$ & 0.01 & 500 & 116.67167 & 50.005 & 194.07541 \\
\hline K25C1100740 & SVOA & 2,2'-Dichlorodiisopropyl ether & 3 & 0 & $\mathrm{ug} / \mathrm{kg}$ & 332 & 4920 & 2079 & 985 & 2481.9474 \\
\hline K25C1100740 & SVOA & 2,3,4,6-Tetrachlorophenol & 3 & 0 & $\mathrm{ug} / \mathrm{kg}$ & 332 & 4920 & 2079 & 985 & 2481.9474 \\
\hline K25C1100740 & SVOA & 2,4,5-Trichlorophenol & 6 & 0 & $\mathrm{ug} / \mathrm{kg}$ & 0.05 & 4920 & 1039.525 & 166.025 & 1939.2357 \\
\hline K25C1100740 & SVOA & 2,4,6-Trichlorophenol & 6 & 0 & $\mathrm{ug} / \mathrm{kg}$ & 0.05 & 4920 & 1039.525 & 166.025 & 1939.2357 \\
\hline K25C1100740 & SVOA & 2,4-Dichlorophenol & 3 & 0 & $\mathrm{ug} / \mathrm{kg}$ & 332 & 4920 & 2079 & 985 & 2481.9474 \\
\hline K25C1100740 & SVOA & 2,4-Dimethylphenol & 3 & 0 & $\mathrm{ug} / \mathrm{kg}$ & 332 & 4920 & 2079 & 985 & 2481.9474 \\
\hline K25C1100740 & SVOA & 2,4-Dinitrophenol & 3 & 0 & $\mathrm{ug} / \mathrm{kg}$ & 665 & 9830 & 4155 & 1970 & 4957.8196 \\
\hline K25C1100740 & SVOA & 2,4-Dinitrotoluene & 6 & 0 & $\mathrm{ug} / \mathrm{kg}$ & 0.05 & 4920 & 1039.525 & 166.025 & 1939.2357 \\
\hline K25C1100740 & SVOA & 2,6-Dinitrotoluene & 3 & 0 & $\mathrm{ug} / \mathrm{kg}$ & 332 & 4920 & 2079 & 985 & 2481.9474 \\
\hline K25C1100740 & SVOA & 2-Chloronaphthalene & 3 & 0 & $\mathrm{ug} / \mathrm{kg}$ & 33.2 & 492 & 207.9 & 98.5 & 248.19474 \\
\hline K25C1100740 & SVOA & 2-Chlorophenol & 3 & 0 & $\mathrm{ug} / \mathrm{kg}$ & 332 & 4920 & 2079 & 985 & 2481.9474 \\
\hline K25C1100740 & SVOA & 2-Methyl-4,6-dinitrophenol & 3 & 0 & $\mathrm{ug} / \mathrm{kg}$ & 332 & 4920 & 2079 & 985 & 2481.9474 \\
\hline K25C1100740 & SVOA & 2-Methylnaphthalene & 3 & 0 & $\mathrm{ug} / \mathrm{kg}$ & 33.2 & 492 & 207.9 & 98.5 & 248.19474 \\
\hline K25C1100740 & SVOA & 2-Methylphenol & 6 & 0 & $\mathrm{ug} / \mathrm{kg}$ & 0.05 & 4920 & 1039.525 & 166.025 & 1939.2357 \\
\hline K25C1100740 & SVOA & 2-Nitrobenzenamine & 3 & 0 & $\mathrm{ug} / \mathrm{kg}$ & 332 & 4920 & 2079 & 985 & 2481.9474 \\
\hline K25C1100740 & SVOA & 2-Nitrophenol & 3 & 0 & $\mathrm{ug} / \mathrm{kg}$ & 332 & 4920 & 2079 & 985 & 2481.9474 \\
\hline K25C1100740 & SVOA & 3,3'-Dichlorobenzidine & 3 & 0 & $\mathrm{ug} / \mathrm{kg}$ & 332 & 4920 & 2079 & 985 & 2481.9474 \\
\hline K25C1100740 & SVOA & 3-Nitrobenzenamine & 3 & 0 & $\mathrm{ug} / \mathrm{kg}$ & 332 & 4920 & 2079 & 985 & 2481.9474 \\
\hline K25C1100740 & SVOA & 4-Bromophenyl phenyl ether & 3 & 0 & $\mathrm{ug} / \mathrm{kg}$ & 332 & 4920 & 2079 & 985 & 2481.9474 \\
\hline K25C1100740 & SVOA & 4-Chloro-3-methylphenol & 3 & 0 & $\mathrm{ug} / \mathrm{kg}$ & 332 & 4920 & 2079 & 985 & 2481.9474 \\
\hline K25C1100740 & SVOA & 4-Chlorobenzenamine & 3 & 0 & $\mathrm{ug} / \mathrm{kg}$ & 332 & 4920 & 2079 & 985 & 2481.9474 \\
\hline K25C1100740 & SVOA & 4-Chlorophenyl phenyl ether & 3 & 0 & $\mathrm{ug} / \mathrm{kg}$ & 332 & 4920 & 2079 & 985 & 2481.9474 \\
\hline K25C1100740 & SVOA & 4-Nitrobenzenamine & 3 & 0 & $\mathrm{ug} / \mathrm{kg}$ & 332 & 4920 & 2079 & 985 & 2481.9474 \\
\hline K25C1100740 & SVOA & 4-Nitrophenol & 3 & 0 & $\mathrm{ug} / \mathrm{kg}$ & 332 & 4920 & 2079 & 985 & 2481.9474 \\
\hline K25C1100740 & SVOA & Acenaphthene & 3 & 0 & $\mathrm{ug} / \mathrm{kg}$ & 33.2 & 492 & 207.9 & 98.5 & 248.19474 \\
\hline K25C1100740 & SVOA & Acenaphthylene & 3 & 0 & $\mathrm{ug} / \mathrm{kg}$ & 33.2 & 492 & 207.9 & 98.5 & 248.19474 \\
\hline K25C1100740 & SVOA & Acetophenone & 3 & 0 & $\mathrm{ug} / \mathrm{kg}$ & 332 & 4920 & 2079 & 985 & 2481.9474 \\
\hline K25C1100740 & SVOA & Anthracene & 3 & 0 & $\mathrm{ug} / \mathrm{kg}$ & 33.2 & 492 & 207.9 & 98.5 & 248.19474 \\
\hline
\end{tabular}




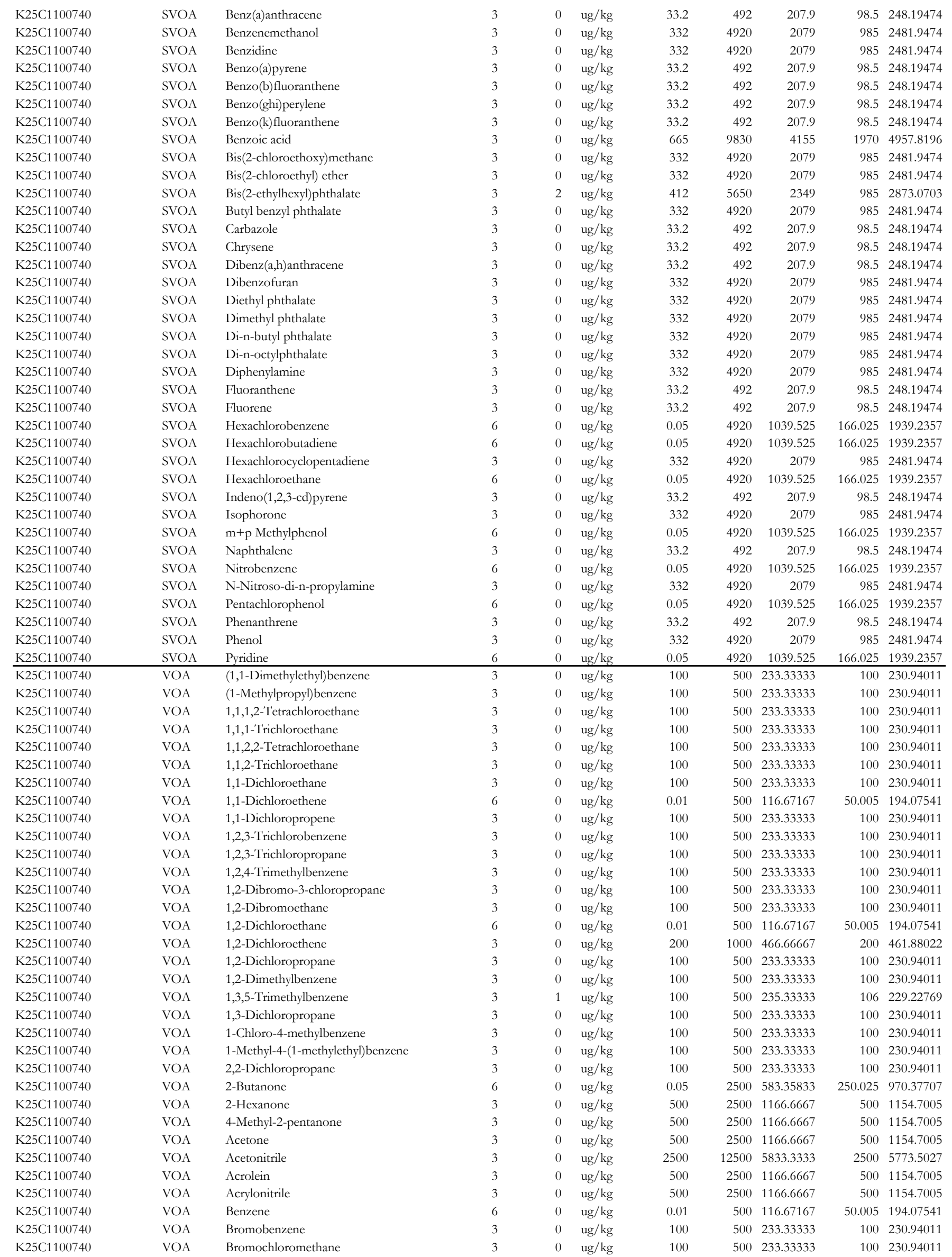




\begin{tabular}{|c|c|c|c|c|c|c|c|c|c|c|}
\hline K25C1100740 & VOA & Bromodichloromethane & 3 & 0 & $\mathrm{ug} / \mathrm{kg}$ & 100 & 500 & 233.33333 & 100 & 230.94011 \\
\hline K25C1100740 & VOA & Bromoform & 3 & 0 & $\mathrm{ug} / \mathrm{kg}$ & 100 & 500 & 233.33333 & 100 & 230.94011 \\
\hline K25C1100740 & VOA & Bromomethane & 3 & 0 & $\mathrm{ug} / \mathrm{kg}$ & 100 & 500 & 233.33333 & 100 & 230.94011 \\
\hline K25C1100740 & VOA & Butylbenzene & 3 & 0 & $\mathrm{ug} / \mathrm{kg}$ & 100 & 500 & 233.33333 & 100 & 230.94011 \\
\hline K25C1100740 & VOA & Carbon disulfide & 3 & 0 & $\mathrm{ug} / \mathrm{kg}$ & 500 & 2500 & 1166.6667 & 500 & 1154.7005 \\
\hline K25C1100740 & VOA & Carbon tetrachloride & 6 & 0 & $\mathrm{ug} / \mathrm{kg}$ & 0.01 & 500 & 116.67167 & 50.005 & 194.07541 \\
\hline K25C1100740 & VOA & Chlorobenzene & 6 & 0 & $\mathrm{ug} / \mathrm{kg}$ & 0.01 & 500 & 116.67167 & 50.005 & 194.07541 \\
\hline K25C1100740 & VOA & Chloroethane & 3 & 0 & $\mathrm{ug} / \mathrm{kg}$ & 100 & 500 & 233.33333 & 100 & 230.94011 \\
\hline K25C1100740 & VOA & Chloroform & 6 & 0 & $\mathrm{ug} / \mathrm{kg}$ & 0.01 & 500 & 116.67167 & 50.005 & 194.07541 \\
\hline K25C1100740 & VOA & Chloromethane & 3 & 0 & $\mathrm{ug} / \mathrm{kg}$ & 100 & 500 & 233.33333 & 100 & 230.94011 \\
\hline K25C1100740 & VOA & cis-1,2-Dichloroethene & 3 & 0 & $\mathrm{ug} / \mathrm{kg}$ & 100 & 500 & 233.33333 & 100 & 230.94011 \\
\hline K25C1100740 & VOA & cis-1,3-Dichloropropene & 3 & 0 & $\mathrm{ug} / \mathrm{kg}$ & 100 & 500 & 233.33333 & 100 & 230.94011 \\
\hline K25C1100740 & VOA & Cumene & 3 & 0 & $\mathrm{ug} / \mathrm{kg}$ & 100 & 500 & 233.33333 & 100 & 230.94011 \\
\hline K25C1100740 & VOA & Dibromochloromethane & 3 & 0 & $\mathrm{ug} / \mathrm{kg}$ & 100 & 500 & 233.33333 & 100 & 230.94011 \\
\hline K25C1100740 & VOA & Dibromomethane & 3 & 0 & $\mathrm{ug} / \mathrm{kg}$ & 100 & 500 & 233.33333 & 100 & 230.94011 \\
\hline K25C1100740 & VOA & Dichlorodifluoromethane & 3 & 0 & $\mathrm{ug} / \mathrm{kg}$ & 100 & 500 & 233.33333 & 100 & 230.94011 \\
\hline K25C1100740 & VOA & Ethylbenzene & 3 & 0 & $\mathrm{ug} / \mathrm{kg}$ & 100 & 500 & 233.33333 & 100 & 230.94011 \\
\hline K25C1100740 & VOA & Hexane & 3 & 0 & $\mathrm{ug} / \mathrm{kg}$ & 500 & 2500 & 1166.6667 & 500 & 1154.7005 \\
\hline K25C1100740 & VOA & M + P Xylene & 3 & 0 & $\mathrm{ug} / \mathrm{kg}$ & 200 & 1000 & 466.66667 & 200 & 461.88022 \\
\hline K25C1100740 & VOA & Methanol & 3 & 0 & $\mathrm{ug} / \mathrm{kg}$ & 4630 & 4950 & 4750 & 4670 & 174.35596 \\
\hline K25C1100740 & VOA & Methyl methacrylate & 3 & 0 & $\mathrm{ug} / \mathrm{kg}$ & 500 & 2500 & 1166.6667 & 500 & 1154.7005 \\
\hline K25C1100740 & VOA & Methylcyclohexane & 3 & 0 & $\mathrm{ug} / \mathrm{kg}$ & 100 & 500 & 233.33333 & 100 & 230.94011 \\
\hline K25C1100740 & VOA & Methylene chloride & 3 & 0 & $\mathrm{ug} / \mathrm{kg}$ & 500 & 2500 & 1166.6667 & 500 & 1154.7005 \\
\hline K25C1100740 & VOA & o-Chlorotoluene & 3 & 0 & $\mathrm{ug} / \mathrm{kg}$ & 100 & 500 & 233.33333 & 100 & 230.94011 \\
\hline K25C1100740 & VOA & Propylbenzene & 3 & 0 & $\mathrm{ug} / \mathrm{kg}$ & 100 & 500 & 233.33333 & 100 & 230.94011 \\
\hline K25C1100740 & VOA & Propylene glycol & 3 & 0 & $\mathrm{ug} / \mathrm{kg}$ & 46300 & 49500 & 47500 & 46700 & 1743.5596 \\
\hline K25C1100740 & VOA & Styrene & 3 & 0 & $\mathrm{ug} / \mathrm{kg}$ & 100 & 500 & 233.33333 & 100 & 230.94011 \\
\hline K25C1100740 & VOA & Tetrachloroethene & 6 & 0 & $\mathrm{ug} / \mathrm{kg}$ & 0.01 & 500 & 116.67167 & 50.005 & 194.07541 \\
\hline K25C1100740 & VOA & Toluene & 3 & 0 & $\mathrm{ug} / \mathrm{kg}$ & 100 & 500 & 233.33333 & 100 & 230.94011 \\
\hline K25C1100740 & $\mathrm{VOA}$ & Total Xylene & 3 & 0 & $\mathrm{ug} / \mathrm{kg}$ & 300 & 1500 & 700 & 300 & 692.82032 \\
\hline K25C1100740 & VOA & trans-1,2-Dichloroethene & 3 & 0 & $\mathrm{ug} / \mathrm{kg}$ & 100 & 500 & 233.33333 & 100 & 230.94011 \\
\hline K25C1100740 & VOA & trans-1,3-Dichloropropene & 3 & 0 & $\mathrm{ug} / \mathrm{kg}$ & 100 & 500 & 233.33333 & 100 & 230.94011 \\
\hline K25C1100740 & VOA & Trichloroethene & 6 & 0 & $\mathrm{ug} / \mathrm{kg}$ & 0.01 & 500 & 116.67167 & 50.005 & 194.07541 \\
\hline K25C1100740 & VOA & Trichlorofluoromethane & 3 & 0 & $\mathrm{ug} / \mathrm{kg}$ & 100 & 500 & 233.33333 & 100 & 230.94011 \\
\hline K25C1100740 & $\mathrm{VOA}$ & Vinyl chloride & 6 & 0 & $\mathrm{ug} / \mathrm{kg}$ & 0.01 & 500 & 116.67167 & 50.005 & 194.07541 \\
\hline K25C1100766 & HERB & $2,4-\mathrm{D}$ & 2 & 0 & $\mathrm{ug} / \mathrm{kg}$ & 0.05 & 24.7 & 12.375 & 12.375 & 17.430182 \\
\hline K25C1100766 & HERB & Silvex & 2 & 0 & $\mathrm{ug} / \mathrm{kg}$ & 0.05 & 24.7 & 12.375 & 12.375 & 17.430182 \\
\hline K25C1100766 & METAL & Arsenic & 2 & 0 & $\mathrm{mg} / \mathrm{L}$ & 0.3 & 2.86 & 1.58 & 1.58 & 1.8101934 \\
\hline K25C1100766 & METAL & Barium & 2 & 1 & $\mathrm{mg} / \mathrm{L}$ & 0.05 & 37.6 & 18.825 & 18.825 & 26.55186 \\
\hline K25C1100766 & METAL & Cadmium & 2 & 0 & $\mathrm{mg} / \mathrm{L}$ & 0.05 & 0.477 & 0.2635 & 0.2635 & 0.3019346 \\
\hline K25C1100766 & METAL & Chromium & 2 & 2 & $\mathrm{mg} / \mathrm{L}$ & 0.0866 & 9.45 & 4.7683 & 4.7683 & 6.6209236 \\
\hline K25C1100766 & METAL & Lead & 2 & 1 & $\mathrm{mg} / \mathrm{L}$ & 0.1 & 26 & 13.05 & 13.05 & 18.314066 \\
\hline K25C1100766 & METAL & Mercury & 2 & 0 & $\mathrm{mg} / \mathrm{L}$ & 0.002 & 0.4 & 0.201 & 0.201 & 0.2814285 \\
\hline K25C1100766 & METAL & Selenium & 2 & 0 & $\mathrm{mg} / \mathrm{L}$ & 0.3 & 2.86 & 1.58 & 1.58 & 1.8101934 \\
\hline K25C1100766 & METAL & Silver & 2 & 0 & $\mathrm{mg} / \mathrm{L}$ & 0.05 & 0.477 & 0.2635 & 0.2635 & 0.3019346 \\
\hline K25C1100766 & METAL & Total Uranium & 1 & 0 & $\mathrm{pCi} / \mathrm{g}$ & -0.00389 & -0.00389 & -0.00389 & & \\
\hline K25C1100766 & PPCB & $4,4^{\prime}-\mathrm{DDD}$ & 1 & 0 & $\mathrm{ug} / \mathrm{kg}$ & 3.96 & 3.96 & 3.96 & & \\
\hline K25C1100766 & РPCB & 4,4'-DDE & 1 & 0 & $\mathrm{ug} / \mathrm{kg}$ & 3.96 & 3.96 & 3.96 & & \\
\hline K25C1100766 & PPCB & 4,4'-DDT & 1 & 0 & $\mathrm{ug} / \mathrm{kg}$ & 3.96 & 3.96 & 3.96 & & \\
\hline K25C1100766 & PPCB & Aldrin & 1 & 0 & $\mathrm{ug} / \mathrm{kg}$ & 1.98 & 1.98 & 1.98 & & \\
\hline K25C1100766 & PPCB & alpha-BHC & 1 & 0 & $\mathrm{ug} / \mathrm{kg}$ & 1.98 & 1.98 & 1.98 & & \\
\hline K25C1100766 & PPCB & alpha-Chlordane & 1 & 0 & $\mathrm{ug} / \mathrm{kg}$ & 1.98 & 1.98 & 1.98 & & \\
\hline K25C1100766 & PPCB & beta-BHC & 1 & 0 & $\mathrm{ug} / \mathrm{kg}$ & 1.98 & 1.98 & 1.98 & & \\
\hline K25C1100766 & РPCB & Chlordane & 2 & 0 & $\mathrm{ug} / \mathrm{kg}$ & 0.0025 & 24.8 & 12.40125 & 12.40125 & 17.53448 \\
\hline K25C1100766 & РPCB & delta-BHC & 1 & 0 & $\mathrm{ug} / \mathrm{kg}$ & 1.98 & 1.98 & 1.98 & & \\
\hline K25C1100766 & РPCB & Dieldrin & 1 & 0 & $\mathrm{ug} / \mathrm{kg}$ & 3.96 & 3.96 & 3.96 & & \\
\hline K25C1100766 & PPCB & Endosulfan I & 1 & 0 & $\mathrm{ug} / \mathrm{kg}$ & 1.98 & 1.98 & 1.98 & & \\
\hline K25C1100766 & РPCB & Endosulfan II & 1 & 0 & $\mathrm{ug} / \mathrm{kg}$ & 3.96 & 3.96 & 3.96 & & \\
\hline K25C1100766 & РPCB & Endosulfan sulfate & 1 & 0 & $\mathrm{ug} / \mathrm{kg}$ & 3.96 & 3.96 & 3.96 & & \\
\hline K25C1100766 & PPCB & Endrin & 2 & 0 & $\mathrm{ug} / \mathrm{kg}$ & 0.0004 & 3.96 & 1.9802 & 1.9802 & 2.79986 \\
\hline K25C1100766 & РPCB & Endrin aldehyde & 1 & 0 & $\mathrm{ug} / \mathrm{kg}$ & 3.96 & 3.96 & 3.96 & & \\
\hline K25C1100766 & РPCB & Endrin ketone & 1 & 0 & $\mathrm{ug} / \mathrm{kg}$ & 3.96 & 3.96 & 3.96 & & \\
\hline K25C1100766 & РPCB & gamma-Chlordane & 1 & 0 & $\mathrm{ug} / \mathrm{kg}$ & 1.98 & 1.98 & 1.98 & & \\
\hline K25C1100766 & РPCB & Heptachlor & 2 & 0 & $\mathrm{ug} / \mathrm{kg}$ & 0.0002 & 1.98 & 0.9901 & 0.9901 & 1.39993 \\
\hline K25C1100766 & PPCB & Heptachlor epoxide & 2 & 0 & $\mathrm{ug} / \mathrm{kg}$ & 0.0002 & 1.98 & 0.9901 & 0.9901 & 1.39993 \\
\hline K25C1100766 & РPCB & Lindane & 2 & 0 & $\mathrm{ug} / \mathrm{kg}$ & 0.0002 & 1.98 & 0.9901 & 0.9901 & 1.39993 \\
\hline K25C1100766 & РPCB & Methoxychlor & 2 & 0 & $\mathrm{ug} / \mathrm{kg}$ & 0.002 & 19.8 & 9.901 & 9.901 & 13.9993 \\
\hline K25C1100766 & PPCB & Toxaphene & 2 & 0 & $\mathrm{ug} / \mathrm{kg}$ & 0.005 & 49.6 & 24.8025 & 24.8025 & 35.068961 \\
\hline K25C1100766 & RADS & Americium-241 & 1 & 0 & $\mathrm{pCi} / \mathrm{g}$ & 0.0397 & 0.0397 & 0.0397 & & \\
\hline K25C1100766 & RADS & Carbon-14 & 1 & 0 & $\mathrm{pCi} / \mathrm{g}$ & -1.67 & -1.67 & -1.67 & & \\
\hline
\end{tabular}




\begin{tabular}{|c|c|c|c|c|c|c|c|c|c|c|}
\hline K25C1100766 & RADS & Cesium-137 & 1 & 1 & $\mathrm{pCi} / \mathrm{g}$ & 0.71 & 0.71 & 0.71 & \multicolumn{2}{|l|}{0.71} \\
\hline K25C1100766 & RADS & Cobalt-60 & 1 & 0 & $\mathrm{pCi} / \mathrm{g}$ & -0.0487 & -0.0487 & -0.0487 & & \\
\hline K25C1100766 & RADS & Curium-243/244 & 1 & 0 & $\mathrm{pCi} / \mathrm{g}$ & 0.0344 & 0.0344 & 0.0344 & & \\
\hline K25C1100766 & RADS & Iodine-129 & 1 & 0 & $\mathrm{pCi} / \mathrm{g}$ & 0.184 & 0.184 & 0.184 & & \\
\hline K25C1100766 & RADS & Neptunium-237 & 1 & 0 & $\mathrm{pCi} / \mathrm{g}$ & 0.0284 & 0.0284 & 0.0284 & & \\
\hline K25C1100766 & RADS & Plutonium-238 & 1 & 0 & $\mathrm{pCi} / \mathrm{g}$ & -0.00886 & -0.00886 & -0.00886 & & \\
\hline K25C1100766 & RADS & Plutonium-239/240 & 1 & 0 & $\mathrm{pCi} / \mathrm{g}$ & 0.0103 & 0.0103 & 0.0103 & & \\
\hline K25C1100766 & RADS & Radium-226 & 1 & 0 & $\mathrm{pCi} / \mathrm{g}$ & -2.13 & -2.13 & -2.13 & & \\
\hline K25C1100766 & RADS & Strontium-90 & 1 & 0 & $\mathrm{pCi} / \mathrm{g}$ & 2.04 & 2.04 & 2.04 & & \\
\hline K25C1100766 & RADS & Technetium-99 & 1 & 0 & $\mathrm{pCi} / \mathrm{g}$ & -1.15 & -1.15 & -1.15 & & \\
\hline K25C1100766 & RADS & Thorium-230 & 1 & 0 & $\mathrm{pCi} / \mathrm{g}$ & -11 & -11 & -11 & & \\
\hline K25C1100766 & RADS & Thorium-232 & 1 & 0 & $\mathrm{pCi} / \mathrm{g}$ & 30 & 30 & 30 & & \\
\hline K25C1100766 & RADS & Tritium & 1 & 0 & $\mathrm{pCi} / \mathrm{g}$ & -49.4 & -49.4 & -49.4 & & \\
\hline K25C1100766 & RADS & Uranium-233/234 & 1 & 0 & $\mathrm{pCi} / \mathrm{g}$ & 0.00545 & 0.00545 & 0.00545 & & \\
\hline K25C1100766 & RADS & Uranium-235/236 & 1 & 0 & $\mathrm{pCi} / \mathrm{g}$ & 0 & 0 & 0 & & \\
\hline K25C1100766 & RADS & Uranium-238 & 1 & 0 & $\mathrm{pCi} / \mathrm{g}$ & -0.00934 & -0.00934 & -0.00934 & & \\
\hline K25C1100766 & SVOA & 1,2,4-Trichlorobenzene & 1 & 0 & $\mathrm{ug} / \mathrm{kg}$ & 463 & 463 & 463 & & \\
\hline K25C1100766 & SVOA & 1,2-Dichlorobenzene & 1 & 0 & $\mathrm{ug} / \mathrm{kg}$ & 463 & 463 & 463 & & \\
\hline K25C1100766 & SVOA & 1,3-Dichlorobenzene & 1 & 0 & $\mathrm{ug} / \mathrm{kg}$ & 463 & 463 & 463 & & \\
\hline K25C1100766 & SVOA & 1,4-Dichlorobenzene & 2 & 0 & $\mathrm{ug} / \mathrm{kg}$ & 0.01 & 463 & 231.505 & 231.505 & 327.38337 \\
\hline K25C1100766 & SVOA & 2,2'-Dichlorodiisopropyl ether & 1 & 0 & $\mathrm{ug} / \mathrm{kg}$ & 3450 & 3450 & 3450 & & \\
\hline K25C1100766 & SVOA & 2,3,4,6-Tetrachlorophenol & 1 & 0 & $\mathrm{ug} / \mathrm{kg}$ & 3450 & 3450 & 3450 & & \\
\hline K25C1100766 & SVOA & 2,4,5-Trichlorophenol & 2 & 0 & $\mathrm{ug} / \mathrm{kg}$ & 0.05 & 3450 & 1725.025 & 1725.025 & 2439.483 \\
\hline K25C1100766 & SVOA & 2,4,6-Trichlorophenol & 2 & 0 & $\mathrm{ug} / \mathrm{kg}$ & 0.05 & 3450 & 1725.025 & 1725.025 & 2439.483 \\
\hline K25C1100766 & SVOA & 2,4-Dichlorophenol & 1 & 0 & $\mathrm{ug} / \mathrm{kg}$ & 3450 & 3450 & 3450 & & \\
\hline K25C1100766 & SVOA & 2,4-Dimethylphenol & 1 & 0 & $\mathrm{ug} / \mathrm{kg}$ & 3450 & 3450 & 3450 & & \\
\hline K25C1100766 & SVOA & 2,4-Dinitrophenol & 1 & 0 & $\mathrm{ug} / \mathrm{kg}$ & 6910 & 6910 & 6910 & & \\
\hline K25C1100766 & SVOA & 2,4-Dinitrotoluene & 2 & 0 & $\mathrm{ug} / \mathrm{kg}$ & 0.05 & 3450 & 1725.025 & 1725.025 & 2439.483 \\
\hline K25C1100766 & SVOA & 2,6-Dinitrotoluene & 1 & 0 & $\mathrm{ug} / \mathrm{kg}$ & 3450 & 3450 & 3450 & & \\
\hline K25C1100766 & SVOA & 2-Chloronaphthalene & 1 & 0 & $\mathrm{ug} / \mathrm{kg}$ & 345 & 345 & 345 & & \\
\hline K25C1100766 & SVOA & 2-Chlorophenol & 1 & 0 & $\mathrm{ug} / \mathrm{kg}$ & 3450 & 3450 & 3450 & & \\
\hline K25C1100766 & SVOA & 2-Methyl-4,6-dinitrophenol & 1 & 0 & $\mathrm{ug} / \mathrm{kg}$ & 3450 & 3450 & 3450 & & \\
\hline K25C1100766 & SVOA & 2-Methylnaphthalene & 1 & 0 & $\mathrm{ug} / \mathrm{kg}$ & 345 & 345 & 345 & & \\
\hline K25C1100766 & SVOA & 2-Methylphenol & 2 & 0 & $\mathrm{ug} / \mathrm{kg}$ & 0.05 & 3450 & 1725.025 & 1725.025 & 2439.483 \\
\hline K25C1100766 & SVOA & 2-Nitrobenzenamine & 1 & 0 & $\mathrm{ug} / \mathrm{kg}$ & 3450 & 3450 & 3450 & & \\
\hline K25C1100766 & SVOA & 2-Nitrophenol & 1 & 0 & $\mathrm{ug} / \mathrm{kg}$ & 3450 & 3450 & 3450 & & \\
\hline K25C1100766 & SVOA & 3,3'-Dichlorobenzidine & 1 & 0 & $\mathrm{ug} / \mathrm{kg}$ & 3450 & 3450 & 3450 & & \\
\hline K25C1100766 & SVOA & 3-Nitrobenzenamine & 1 & 0 & $\mathrm{ug} / \mathrm{kg}$ & 3450 & 3450 & 3450 & & \\
\hline K25C1100766 & SVOA & 4-Bromophenyl phenyl ether & 1 & 0 & $\mathrm{ug} / \mathrm{kg}$ & 3450 & 3450 & 3450 & & \\
\hline K25C1100766 & SVOA & 4-Chloro-3-methylphenol & 1 & 0 & $\mathrm{ug} / \mathrm{kg}$ & 3450 & 3450 & 3450 & & \\
\hline K25C1100766 & SVOA & 4-Chlorobenzenamine & 1 & 0 & $\mathrm{ug} / \mathrm{kg}$ & 3450 & 3450 & 3450 & & \\
\hline K25C1100766 & SVOA & 4-Chlorophenyl phenyl ether & 1 & 0 & $\mathrm{ug} / \mathrm{kg}$ & 3450 & 3450 & 3450 & & \\
\hline K25C1100766 & SVOA & 4-Nitrobenzenamine & 1 & 0 & $\mathrm{ug} / \mathrm{kg}$ & 3450 & 3450 & 3450 & & \\
\hline K25C1100766 & SVOA & 4-Nitrophenol & 1 & 0 & $\mathrm{ug} / \mathrm{kg}$ & 3450 & 3450 & 3450 & & \\
\hline K25C1100766 & SVOA & Acenaphthene & 1 & 0 & $\mathrm{ug} / \mathrm{kg}$ & 345 & 345 & 345 & & \\
\hline K25C1100766 & SVOA & Acenaphthylene & 1 & 0 & $\mathrm{ug} / \mathrm{kg}$ & 345 & 345 & 345 & & \\
\hline K25C1100766 & SVOA & Acetophenone & 1 & 0 & $\mathrm{ug} / \mathrm{kg}$ & 3450 & 3450 & 3450 & & \\
\hline K25C1100766 & SVOA & Anthracene & 1 & 0 & $\mathrm{ug} / \mathrm{kg}$ & 345 & 345 & 345 & & \\
\hline K25C1100766 & SVOA & Benz(a)anthracene & 1 & 0 & $\mathrm{ug} / \mathrm{kg}$ & 345 & 345 & 345 & & \\
\hline K25C1100766 & SVOA & Benzenemethanol & 1 & 0 & $\mathrm{ug} / \mathrm{kg}$ & 3450 & 3450 & 3450 & & \\
\hline K25C1100766 & SVOA & Benzidine & 1 & 0 & $\mathrm{ug} / \mathrm{kg}$ & 3450 & 3450 & 3450 & & \\
\hline K25C1100766 & SVOA & Benzo(a)pyrene & 1 & 0 & $\mathrm{ug} / \mathrm{kg}$ & 345 & 345 & 345 & & \\
\hline K25C1100766 & SVOA & Benzo(b)fluoranthene & 1 & 0 & $\mathrm{ug} / \mathrm{kg}$ & 345 & 345 & 345 & & \\
\hline K25C1100766 & SVOA & Benzo(ghi)perylene & 1 & 0 & $\mathrm{ug} / \mathrm{kg}$ & 345 & 345 & 345 & & \\
\hline K25C1100766 & SVOA & Benzo(k)fluoranthene & 1 & 0 & $\mathrm{ug} / \mathrm{kg}$ & 345 & 345 & 345 & & \\
\hline K25C1100766 & SVOA & Benzoic acid & 1 & 0 & $\mathrm{ug} / \mathrm{kg}$ & 6910 & 6910 & 6910 & & \\
\hline K25C1100766 & SVOA & Bis(2-chloroethoxy)methane & 1 & 0 & $\mathrm{ug} / \mathrm{kg}$ & 3450 & 3450 & 3450 & & \\
\hline K25C1100766 & SVOA & Bis(2-chloroethyl) ether & 1 & 0 & $\mathrm{ug} / \mathrm{kg}$ & 3450 & 3450 & 3450 & & \\
\hline K25C1100766 & SVOA & Bis(2-ethylhexyl)phthalate & 1 & 0 & $\mathrm{ug} / \mathrm{kg}$ & 3450 & 3450 & 3450 & & \\
\hline K25C1100766 & SVOA & Butyl benzyl phthalate & 1 & 0 & $\mathrm{ug} / \mathrm{kg}$ & 3450 & 3450 & 3450 & & \\
\hline K25C1100766 & SVOA & Carbazole & 1 & 0 & $\mathrm{ug} / \mathrm{kg}$ & 345 & 345 & 345 & & \\
\hline K25C1100766 & SVOA & Chrysene & 1 & 0 & $\mathrm{ug} / \mathrm{kg}$ & 345 & 345 & 345 & & \\
\hline K25C1100766 & SVOA & Dibenz(a,h)anthracene & 1 & 0 & $\mathrm{ug} / \mathrm{kg}$ & 345 & 345 & 345 & & \\
\hline K25C1100766 & SVOA & Dibenzofuran & 1 & 0 & $\mathrm{ug} / \mathrm{kg}$ & 3450 & 3450 & 3450 & & \\
\hline K25C1100766 & SVOA & Diethyl phthalate & 1 & 0 & $\mathrm{ug} / \mathrm{kg}$ & 3450 & 3450 & 3450 & & \\
\hline K25C1100766 & SVOA & Dimethyl phthalate & 1 & 0 & $\mathrm{ug} / \mathrm{kg}$ & 3450 & 3450 & 3450 & & \\
\hline K25C1100766 & SVOA & Di-n-butyl phthalate & 1 & 0 & $\mathrm{ug} / \mathrm{kg}$ & 3450 & 3450 & 3450 & & \\
\hline K25C1100766 & SVOA & Di-n-octylphthalate & 1 & 0 & $\mathrm{ug} / \mathrm{kg}$ & 3450 & 3450 & 3450 & & \\
\hline K25C1100766 & SVOA & Diphenylamine & 1 & 0 & $\mathrm{ug} / \mathrm{kg}$ & 3450 & 3450 & 3450 & & \\
\hline K25C1100766 & SVOA & Fluoranthene & 1 & 0 & $\mathrm{ug} / \mathrm{kg}$ & 345 & 345 & 345 & & \\
\hline
\end{tabular}




\begin{tabular}{|c|c|c|c|c|c|c|c|c|c|c|}
\hline K25C1100766 & SVOA & Fluorene & 1 & 0 & $\mathrm{ug} / \mathrm{kg}$ & 345 & 345 & 345 & & \\
\hline K25C1100766 & SVOA & Hexachlorobenzene & 2 & 0 & $\mathrm{ug} / \mathrm{kg}$ & 0.05 & 3450 & 1725.025 & 1725.025 & 2439.483 \\
\hline K25C1100766 & SVOA & Hexachlorobutadiene & 2 & 0 & $\mathrm{ug} / \mathrm{kg}$ & 0.05 & 3450 & 1725.025 & 1725.025 & 2439.483 \\
\hline K25C1100766 & SVOA & Hexachlorocyclopentadiene & 1 & 0 & $\mathrm{ug} / \mathrm{kg}$ & 3450 & 3450 & 3450 & & \\
\hline K25C1100766 & SVOA & Hexachloroethane & 2 & 0 & $\mathrm{ug} / \mathrm{kg}$ & 0.05 & 3450 & 1725.025 & 1725.025 & 2439.483 \\
\hline K25C1100766 & SVOA & Indeno(1,2,3-cd)pyrene & 1 & 0 & $\mathrm{ug} / \mathrm{kg}$ & 345 & 345 & 345 & & \\
\hline K25C1100766 & SVOA & Isophorone & 1 & 0 & $\mathrm{ug} / \mathrm{kg}$ & 3450 & 3450 & 3450 & & \\
\hline K25C1100766 & SVOA & $\mathrm{m}+\mathrm{p}$ Methylphenol & 2 & 0 & $\mathrm{ug} / \mathrm{kg}$ & 0.05 & 3450 & 1725.025 & 1725.025 & 2439.483 \\
\hline K25C1100766 & SVOA & Naphthalene & 1 & 0 & $\mathrm{ug} / \mathrm{kg}$ & 345 & 345 & 345 & & \\
\hline K25C1100766 & SVOA & Nitrobenzene & 2 & 0 & $\mathrm{ug} / \mathrm{kg}$ & 0.05 & 3450 & 1725.025 & 1725.025 & 2439.483 \\
\hline K25C1100766 & SVOA & N-Nitroso-di-n-propylamine & 1 & 0 & $\mathrm{ug} / \mathrm{kg}$ & 3450 & 3450 & 3450 & & \\
\hline K25C1100766 & SVOA & Pentachlorophenol & 2 & 0 & $\mathrm{ug} / \mathrm{kg}$ & 0.05 & 3450 & 1725.025 & 1725.025 & 2439.483 \\
\hline K25C1100766 & SVOA & Phenanthrene & 1 & 0 & $\mathrm{ug} / \mathrm{kg}$ & 345 & 345 & 345 & & \\
\hline K25C1100766 & SVOA & Phenol & 1 & 0 & $\mathrm{ug} / \mathrm{kg}$ & 3450 & 3450 & 3450 & & \\
\hline K25C1100766 & SVOA & Pyridine & 2 & 0 & $\mathrm{ug} / \mathrm{kg}$ & 0.05 & 3450 & 1725.025 & 1725.025 & 2439.483 \\
\hline K25C1100766 & $\mathrm{VOA}$ & (1,1-Dimethylethyl)benzene & 1 & 0 & $\mathrm{ug} / \mathrm{kg}$ & 463 & 463 & 463 & & \\
\hline K25C1100766 & VOA & (1-Methylpropyl)benzene & 1 & 0 & $\mathrm{ug} / \mathrm{kg}$ & 463 & 463 & 463 & & \\
\hline K25C1100766 & $\mathrm{VOA}$ & 1,1,1,2-Tetrachloroethane & 1 & 0 & $\mathrm{ug} / \mathrm{kg}$ & 463 & 463 & 463 & & \\
\hline K25C1100766 & VOA & 1,1,1-Trichloroethane & 1 & 0 & $\mathrm{ug} / \mathrm{kg}$ & 463 & 463 & 463 & & \\
\hline K25C1100766 & VOA & 1,1,2,2-Tetrachloroethane & 1 & 0 & $\mathrm{ug} / \mathrm{kg}$ & 463 & 463 & 463 & & \\
\hline K25C1100766 & $\mathrm{VOA}$ & 1,1,2-Trichloroethane & 1 & 0 & $\mathrm{ug} / \mathrm{kg}$ & 463 & 463 & 463 & & \\
\hline K25C1100766 & VOA & 1,1-Dichloroethane & 1 & 0 & $\mathrm{ug} / \mathrm{kg}$ & 463 & 463 & 463 & & \\
\hline K25C1100766 & VOA & 1,1-Dichloroethene & 2 & 0 & $\mathrm{ug} / \mathrm{kg}$ & 0.01 & 463 & 231.505 & 231.505 & 327.38337 \\
\hline K25C1100766 & VOA & 1,1-Dichloropropene & 1 & 0 & $\mathrm{ug} / \mathrm{kg}$ & 463 & 463 & 463 & & \\
\hline K25C1100766 & $\mathrm{VOA}$ & 1,2,3-Trichlorobenzene & 1 & 0 & $\mathrm{ug} / \mathrm{kg}$ & 463 & 463 & 463 & & \\
\hline K25C1100766 & VOA & 1,2,3-Trichloropropane & 1 & 0 & $\mathrm{ug} / \mathrm{kg}$ & 463 & 463 & 463 & & \\
\hline K25C1100766 & VOA & 1,2,4-Trimethylbenzene & 1 & 0 & $\mathrm{ug} / \mathrm{kg}$ & 463 & 463 & 463 & & \\
\hline K25C1100766 & $\mathrm{VOA}$ & 1,2-Dibromo-3-chloropropane & 1 & 0 & $\mathrm{ug} / \mathrm{kg}$ & 463 & 463 & 463 & & \\
\hline K25C1100766 & VOA & 1,2-Dibromoethane & 1 & 0 & $\mathrm{ug} / \mathrm{kg}$ & 463 & 463 & 463 & & \\
\hline K25C1100766 & VOA & 1,2-Dichloroethane & 2 & 0 & $\mathrm{ug} / \mathrm{kg}$ & 0.01 & 463 & 231.505 & 231.505 & 327.38337 \\
\hline K25C1100766 & VOA & 1,2-Dichloroethene & 1 & 0 & $\mathrm{ug} / \mathrm{kg}$ & 926 & 926 & 926 & & \\
\hline K25C1100766 & VOA & 1,2-Dichloropropane & 1 & 0 & $\mathrm{ug} / \mathrm{kg}$ & 463 & 463 & 463 & & \\
\hline K25C1100766 & VOA & 1,2-Dimethylbenzene & 1 & 0 & $\mathrm{ug} / \mathrm{kg}$ & 463 & 463 & 463 & & \\
\hline K25C1100766 & VOA & 1,3,5-Trimethylbenzene & 1 & 0 & $\mathrm{ug} / \mathrm{kg}$ & 463 & 463 & 463 & & \\
\hline K25C1100766 & VOA & 1,3-Dichloropropane & 1 & 0 & $\mathrm{ug} / \mathrm{kg}$ & 463 & 463 & 463 & & \\
\hline K25C1100766 & VOA & 1-Chloro-4-methylbenzene & 1 & 0 & $\mathrm{ug} / \mathrm{kg}$ & 463 & 463 & 463 & & \\
\hline K25C1100766 & VOA & 1-Methyl-4-(1-methylethyl)benzene & 1 & 0 & $\mathrm{ug} / \mathrm{kg}$ & 463 & 463 & 463 & & \\
\hline K25C1100766 & VOA & 2,2-Dichloropropane & 1 & 0 & $\mathrm{ug} / \mathrm{kg}$ & 463 & 463 & 463 & & \\
\hline K25C1100766 & VOA & 2-Butanone & 2 & 0 & $\mathrm{ug} / \mathrm{kg}$ & 0.05 & 2310 & 1155.025 & 1155.025 & 1633.3813 \\
\hline K25C1100766 & VOA & 2-Hexanone & 1 & 0 & $\mathrm{ug} / \mathrm{kg}$ & 2310 & 2310 & 2310 & & \\
\hline K25C1100766 & VOA & 4-Methyl-2-pentanone & 1 & 0 & $\mathrm{ug} / \mathrm{kg}$ & 2310 & 2310 & 2310 & & \\
\hline K25C1100766 & VOA & Acetone & 1 & 0 & $\mathrm{ug} / \mathrm{kg}$ & 2310 & 2310 & 2310 & & \\
\hline K25C1100766 & $\mathrm{VOA}$ & Acetonitrile & 1 & 0 & $\mathrm{ug} / \mathrm{kg}$ & 11600 & 11600 & 11600 & & \\
\hline K25C1100766 & VOA & Acrolein & 1 & 0 & $\mathrm{ug} / \mathrm{kg}$ & 2310 & 2310 & 2310 & & \\
\hline K25C1100766 & VOA & Acrylonitrile & 1 & 0 & $\mathrm{ug} / \mathrm{kg}$ & 2310 & 2310 & 2310 & & \\
\hline K25C1100766 & VOA & Benzene & 2 & 0 & $\mathrm{ug} / \mathrm{kg}$ & 0.01 & 463 & 231.505 & 231.505 & 327.38337 \\
\hline K25C1100766 & VOA & Bromobenzene & 1 & 0 & $\mathrm{ug} / \mathrm{kg}$ & 463 & 463 & 463 & & \\
\hline K25C1100766 & VOA & Bromochloromethane & 1 & 0 & $\mathrm{ug} / \mathrm{kg}$ & 463 & 463 & 463 & & \\
\hline K25C1100766 & VOA & Bromodichloromethane & 1 & 0 & $\mathrm{ug} / \mathrm{kg}$ & 463 & 463 & 463 & & \\
\hline K25C1100766 & VOA & Bromoform & 1 & 0 & $\mathrm{ug} / \mathrm{kg}$ & 463 & 463 & 463 & & \\
\hline K25C1100766 & VOA & Bromomethane & 1 & 0 & $\mathrm{ug} / \mathrm{kg}$ & 463 & 463 & 463 & & \\
\hline K25C1100766 & VOA & Butylbenzene & 1 & 0 & $\mathrm{ug} / \mathrm{kg}$ & 463 & 463 & 463 & & \\
\hline K25C1100766 & VOA & Carbon disulfide & 1 & 0 & $\mathrm{ug} / \mathrm{kg}$ & 2310 & 2310 & 2310 & & \\
\hline K25C1100766 & VOA & Carbon tetrachloride & 2 & 0 & $\mathrm{ug} / \mathrm{kg}$ & 0.01 & 463 & 231.505 & 231.505 & 327.38337 \\
\hline K25C1100766 & VOA & Chlorobenzene & 2 & 0 & $\mathrm{ug} / \mathrm{kg}$ & 0.01 & 463 & 231.505 & 231.505 & 327.38337 \\
\hline K25C1100766 & $\mathrm{VOA}$ & Chloroethane & 1 & 0 & $\mathrm{ug} / \mathrm{kg}$ & 463 & 463 & 463 & & \\
\hline K25C1100766 & VOA & Chloroform & 2 & 0 & $\mathrm{ug} / \mathrm{kg}$ & 0.01 & 463 & 231.505 & 231.505 & 327.38337 \\
\hline K25C1100766 & VOA & Chloromethane & 1 & 0 & $\mathrm{ug} / \mathrm{kg}$ & 463 & 463 & 463 & & \\
\hline K25C1100766 & VOA & cis-1,2-Dichloroethene & 1 & 0 & $\mathrm{ug} / \mathrm{kg}$ & 463 & 463 & 463 & & \\
\hline K25C1100766 & VOA & cis-1,3-Dichloropropene & 1 & 0 & $\mathrm{ug} / \mathrm{kg}$ & 463 & 463 & 463 & & \\
\hline K25C1100766 & VOA & Cumene & 1 & 0 & $\mathrm{ug} / \mathrm{kg}$ & 463 & 463 & 463 & & \\
\hline K25C1100766 & $\mathrm{VOA}$ & Dibromochloromethane & 1 & 0 & $\mathrm{ug} / \mathrm{kg}$ & 463 & 463 & 463 & & \\
\hline K25C1100766 & VOA & Dibromomethane & 1 & 0 & $\mathrm{ug} / \mathrm{kg}$ & 463 & 463 & 463 & & \\
\hline K25C1100766 & VOA & Dichlorodifluoromethane & 1 & 0 & $\mathrm{ug} / \mathrm{kg}$ & 463 & 463 & 463 & & \\
\hline K25C1100766 & VOA & Ethylbenzene & 1 & 0 & $\mathrm{ug} / \mathrm{kg}$ & 463 & 463 & 463 & & \\
\hline K25C1100766 & VOA & Hexane & 1 & 0 & $\mathrm{ug} / \mathrm{kg}$ & 2310 & 2310 & 2310 & & \\
\hline K25C1100766 & VOA & $\mathrm{M}+\mathrm{P}$ Xylene & 1 & 0 & $\mathrm{ug} / \mathrm{kg}$ & 926 & 926 & 926 & & \\
\hline K25C1100766 & VOA & Methanol & 1 & 0 & $\mathrm{ug} / \mathrm{kg}$ & 4590 & 4590 & 4590 & & \\
\hline K25C1100766 & VOA & Methyl methacrylate & 1 & 0 & $\mathrm{ug} / \mathrm{kg}$ & 2310 & 2310 & 2310 & & \\
\hline K25C1100766 & VOA & Methylcyclohexane & 1 & 0 & $\mathrm{ug} / \mathrm{kg}$ & 463 & 463 & 463 & & \\
\hline
\end{tabular}




\begin{tabular}{|c|c|c|c|c|c|c|c|c|c|c|}
\hline K25C1100766 & VOA & Methylene chloride & 1 & 0 & $\mathrm{ug} / \mathrm{kg}$ & 2310 & 2310 & 2310 & & \\
\hline K25C1100766 & VOA & o-Chlorotoluene & 1 & 0 & $\mathrm{ug} / \mathrm{kg}$ & 463 & 463 & 463 & & \\
\hline K25C1100766 & VOA & Propylbenzene & 1 & 0 & $\mathrm{ug} / \mathrm{kg}$ & 463 & 463 & 463 & & \\
\hline K25C1100766 & VOA & Propylene glycol & 1 & 0 & $\mathrm{ug} / \mathrm{kg}$ & 45900 & 45900 & 45900 & & \\
\hline K25C1100766 & VOA & Styrene & 1 & 0 & $\mathrm{ug} / \mathrm{kg}$ & 463 & 463 & 463 & & \\
\hline K25C1100766 & VOA & Tetrachloroethene & 2 & 0 & $\mathrm{ug} / \mathrm{kg}$ & 0.01 & 463 & 231.505 & 231.505 & 327.38337 \\
\hline K25C1100766 & VOA & Toluene & 1 & 0 & $\mathrm{ug} / \mathrm{kg}$ & 463 & 463 & 463 & & \\
\hline K25C1100766 & VOA & Total Xylene & 1 & 0 & $\mathrm{ug} / \mathrm{kg}$ & 1390 & 1390 & 1390 & & \\
\hline K25C1100766 & VOA & trans-1,2-Dichloroethene & 1 & 0 & $\mathrm{ug} / \mathrm{kg}$ & 463 & 463 & 463 & & \\
\hline K25C1100766 & VOA & trans-1,3-Dichloropropene & 1 & 0 & $\mathrm{ug} / \mathrm{kg}$ & 463 & 463 & 463 & & \\
\hline K25C1100766 & VOA & Trichloroethene & 2 & 0 & $\mathrm{ug} / \mathrm{kg}$ & 0.01 & 463 & 231.505 & 231.505 & 327.38337 \\
\hline K25C1100766 & VOA & Trichlorofluoromethane & 1 & 0 & $\mathrm{ug} / \mathrm{kg}$ & 463 & 463 & 463 & & \\
\hline K25C1100766 & VOA & Vinyl chloride & 2 & 0 & $\mathrm{ug} / \mathrm{kg}$ & 0.01 & 463 & 231.505 & 231.505 & 327.38337 \\
\hline K25C1101517 & HERB & 2,4-D & 4 & 0 & $\mathrm{ug} / \mathrm{kg}$ & 0.05 & 24.4 & 8.4825 & 4.74 & 11.496067 \\
\hline K25C1101517 & HERB & Silvex & 4 & 0 & $\mathrm{ug} / \mathrm{kg}$ & 0.05 & 24.4 & 8.4825 & 4.74 & 11.496067 \\
\hline K25C1101517 & METAL & Arsenic & 4 & 0 & $\mathrm{mg} / \mathrm{L}$ & 0.3 & 144 & 36.8925 & 1.635 & 71.416092 \\
\hline K25C1101517 & METAL & Barium & 4 & 4 & $\mathrm{mg} / \mathrm{L}$ & 0.0595 & 302 & 78.847375 & 6.665 & 148.82218 \\
\hline K25C1101517 & METAL & Cadmium & 4 & 0 & $\mathrm{mg} / \mathrm{L}$ & 0.05 & 24 & 6.14875 & 0.2725 & 11.902682 \\
\hline K25C1101517 & METAL & Chromium & 4 & 3 & $\mathrm{mg} / \mathrm{L}$ & 0.05 & 193 & 49.21235 & 1.8997 & 95.874175 \\
\hline K25C1101517 & METAL & Lead & 4 & 1 & $\mathrm{mg} / \mathrm{L}$ & 0.1 & 48.1 & 14.015 & 3.93 & 23.008453 \\
\hline K25C1101517 & METAL & Mercury & 4 & 0 & $\mathrm{mg} / \mathrm{L}$ & 0.002 & 0.4 & 0.201 & 0.201 & 0.2297854 \\
\hline K25C1101517 & METAL & Selenium & 4 & 0 & $\mathrm{mg} / \mathrm{L}$ & 0.3 & 144 & 36.8925 & 1.635 & 71.416092 \\
\hline K25C1101517 & METAL & Silver & 4 & 0 & $\mathrm{mg} / \mathrm{L}$ & 0.05 & 24 & 6.14875 & 0.2725 & 11.902682 \\
\hline K25C1101517 & METAL & Total Uranium & 2 & 0 & $\mathrm{pCi} / \mathrm{g}$ & 0.025 & 0.0442 & 0.0346 & 0.0346 & 0.0135765 \\
\hline K25C1101517 & PPCB & 4,4'-DDD & 2 & 0 & $\mathrm{ug} / \mathrm{kg}$ & 3.86 & 3.93 & 3.895 & 3.895 & 0.0494975 \\
\hline K25C1101517 & РPCB & 4,4'-DDE & 2 & 0 & $\mathrm{ug} / \mathrm{kg}$ & 3.86 & 3.93 & 3.895 & 3.895 & 0.0494975 \\
\hline K25C1101517 & РPCB & 4,4'-DDT & 2 & 0 & $\mathrm{ug} / \mathrm{kg}$ & 3.86 & 3.93 & 3.895 & 3.895 & 0.0494975 \\
\hline K25C1101517 & PPCB & Aldrin & 2 & 0 & $\mathrm{ug} / \mathrm{kg}$ & 1.93 & 1.97 & 1.95 & 1.95 & 0.0282843 \\
\hline K25C1101517 & PPCB & alpha-BHC & 2 & 0 & $\mathrm{ug} / \mathrm{kg}$ & 1.93 & 1.97 & 1.95 & 1.95 & 0.0282843 \\
\hline K25C1101517 & PPCB & alpha-Chlordane & 2 & 0 & $\mathrm{ug} / \mathrm{kg}$ & 1.93 & 1.97 & 1.95 & 1.95 & 0.0282843 \\
\hline K25C1101517 & РPCB & beta-BHC & 2 & 0 & $\mathrm{ug} / \mathrm{kg}$ & 1.93 & 7.21 & 4.57 & 4.57 & 3.7335238 \\
\hline K25C1101517 & РPCB & Chlordane & 4 & 0 & $\mathrm{ug} / \mathrm{kg}$ & 0.0025 & 24.6 & 12.20125 & 12.10125 & 14.08685 \\
\hline K25C1101517 & PPCB & delta-BHC & 2 & 0 & $\mathrm{ug} / \mathrm{kg}$ & 1.93 & 1.97 & 1.95 & 1.95 & 0.0282843 \\
\hline K25C1101517 & PPCB & Dieldrin & 2 & 0 & $\mathrm{ug} / \mathrm{kg}$ & 3.86 & 3.93 & 3.895 & 3.895 & 0.0494975 \\
\hline K25C1101517 & PPCB & Endosulfan I & 2 & 0 & $\mathrm{ug} / \mathrm{kg}$ & 1.93 & 1.97 & 1.95 & 1.95 & 0.0282843 \\
\hline K25C1101517 & PPCB & Endosulfan II & 2 & 0 & $\mathrm{ug} / \mathrm{kg}$ & 3.86 & 3.93 & 3.895 & 3.895 & 0.0494975 \\
\hline K25C1101517 & PPCB & Endosulfan sulfate & 2 & 0 & $\mathrm{ug} / \mathrm{kg}$ & 3.86 & 3.93 & 3.895 & 3.895 & 0.0494975 \\
\hline K25C1101517 & РPCB & Endrin & 4 & 0 & $\mathrm{ug} / \mathrm{kg}$ & 0.0004 & 3.93 & 1.9477 & 1.9302 & 2.2487299 \\
\hline K25C1101517 & РPCB & Endrin aldehyde & 2 & 0 & $\mathrm{ug} / \mathrm{kg}$ & 3.86 & 3.93 & 3.895 & 3.895 & 0.0494975 \\
\hline K25C1101517 & PPCB & Endrin ketone & 2 & 0 & $\mathrm{ug} / \mathrm{kg}$ & 3.86 & 3.93 & 3.895 & 3.895 & 0.0494975 \\
\hline K25C1101517 & PPCB & gamma-Chlordane & 2 & 0 & $\mathrm{ug} / \mathrm{kg}$ & 1.93 & 1.97 & 1.95 & 1.95 & 0.0282843 \\
\hline K25C1101517 & PPCB & Heptachlor & 4 & 0 & $\mathrm{ug} / \mathrm{kg}$ & 0.0002 & 1.97 & 0.9751 & 0.9651 & 1.125836 \\
\hline K25C1101517 & PPCB & Heptachlor epoxide & 4 & 0 & $\mathrm{ug} / \mathrm{kg}$ & 0.0002 & 1.97 & 0.9751 & 0.9651 & 1.125836 \\
\hline K25C1101517 & РPCB & Lindane & 4 & 0 & $\mathrm{ug} / \mathrm{kg}$ & 0.0002 & 1.97 & 0.9751 & 0.9651 & 1.125836 \\
\hline K25C1101517 & PPCB & Methoxychlor & 4 & 0 & $\mathrm{ug} / \mathrm{kg}$ & 0.002 & 19.7 & 9.751 & 9.651 & 11.25836 \\
\hline K25C1101517 & PPCB & Toxaphene & 4 & 0 & $\mathrm{ug} / \mathrm{kg}$ & 0.005 & 49.2 & 24.3775 & 24.1525 & 28.145337 \\
\hline K25C1101517 & RADS & Americium-241 & 2 & 0 & $\mathrm{pCi} / \mathrm{g}$ & 0.0112 & 0.0363 & 0.02375 & 0.02375 & 0.0177484 \\
\hline K25C1101517 & RADS & Carbon-14 & 2 & 0 & $\mathrm{pCi} / \mathrm{g}$ & -3.14 & -1.89 & -2.515 & -2.515 & 0.8838835 \\
\hline K25C1101517 & RADS & Cesium-137 & 2 & 1 & $\mathrm{pCi} / \mathrm{g}$ & -0.0035 & 0.405 & 0.20075 & 0.20075 & 0.2888531 \\
\hline K25C1101517 & RADS & Cobalt- 60 & 2 & 0 & $\mathrm{pCi} / \mathrm{g}$ & 0.044 & 0.0668 & 0.0554 & 0.0554 & 0.016122 \\
\hline K25C1101517 & RADS & Curium-243/244 & 2 & 0 & $\mathrm{pCi} / \mathrm{g}$ & -0.0127 & 0.0656 & 0.02645 & 0.02645 & 0.0553665 \\
\hline K25C1101517 & RADS & Iodine-129 & 2 & 0 & $\mathrm{pCi} / \mathrm{g}$ & -0.0703 & 0.0331 & -0.0186 & -0.0186 & 0.0731148 \\
\hline K25C1101517 & RADS & Neptunium-237 & 2 & 0 & $\mathrm{pCi} / \mathrm{g}$ & -0.0314 & 0.118 & 0.0433 & 0.0433 & 0.1056418 \\
\hline K25C1101517 & RADS & Plutonium-238 & 2 & 0 & $\mathrm{pCi} / \mathrm{g}$ & -0.00464 & 0.0159 & 0.00563 & 0.00563 & 0.014524 \\
\hline K25C1101517 & RADS & Plutonium-239/240 & 2 & 0 & $\mathrm{pCi} / \mathrm{g}$ & -0.00928 & -0.00502 & -0.00715 & -0.00715 & 0.0030123 \\
\hline K25C1101517 & RADS & Radium-226 & 2 & 0 & $\mathrm{pCi} / \mathrm{g}$ & -1.34 & 0.0768 & -0.6316 & -0.6316 & 1.0018289 \\
\hline K25C1101517 & RADS & Strontium-90 & 2 & 0 & $\mathrm{pCi} / \mathrm{g}$ & -1.42 & -0.991 & -1.2055 & -1.2055 & 0.3033488 \\
\hline K25C1101517 & RADS & Technetium-99 & 2 & 0 & $\mathrm{pCi} / \mathrm{g}$ & 0.308 & 0.688 & 0.498 & 0.498 & 0.2687006 \\
\hline K25C1101517 & RADS & Thorium-230 & 2 & 0 & $\mathrm{pCi} / \mathrm{g}$ & -12.6 & 20.7 & 4.05 & 4.05 & 23.546656 \\
\hline K25C1101517 & RADS & Thorium-232 & 2 & 0 & $\mathrm{pCi} / \mathrm{g}$ & 11.4 & 23.7 & 17.55 & 17.55 & 8.6974134 \\
\hline K25C1101517 & RADS & Tritium & 2 & 0 & $\mathrm{pCi} / \mathrm{g}$ & -53.1 & 39.1 & -7 & -7 & 65.195245 \\
\hline K25C1101517 & RADS & Uranium-233/234 & 2 & 0 & $\mathrm{pCi} / \mathrm{g}$ & -0.00179 & 0.00712 & 0.002665 & 0.002665 & 0.0063003 \\
\hline K25C1101517 & RADS & Uranium-235/236 & 2 & 0 & $\mathrm{pCi} / \mathrm{g}$ & 0.0116 & 0.012 & 0.0118 & 0.0118 & 0.0002828 \\
\hline K25C1101517 & RADS & Uranium-238 & 2 & 0 & $\mathrm{pCi} / \mathrm{g}$ & 0.00623 & 0.034 & 0.020115 & 0.020115 & 0.0196364 \\
\hline K25C1101517 & SVOA & 1,2,4-Trichlorobenzene & 2 & 0 & $\mathrm{ug} / \mathrm{kg}$ & 98 & 459 & 278.5 & 278.5 & 255.26555 \\
\hline K25C1101517 & SVOA & 1,2-Dichlorobenzene & 2 & 0 & $\mathrm{ug} / \mathrm{kg}$ & 98 & 459 & 278.5 & 278.5 & 255.26555 \\
\hline K25C1101517 & SVOA & 1,3-Dichlorobenzene & 2 & 0 & $\mathrm{ug} / \mathrm{kg}$ & 98 & 459 & 278.5 & 278.5 & 255.26555 \\
\hline K25C1101517 & SVOA & 1,4-Dichlorobenzene & 4 & 0 & $\mathrm{ug} / \mathrm{kg}$ & 0.01 & 459 & 139.255 & 49.005 & 218.11097 \\
\hline K25C1101517 & SVOA & 2,2'-Dichlorodiisopropyl ether & 2 & 0 & $\mathrm{ug} / \mathrm{kg}$ & 377 & 982 & 679.5 & 679.5 & 427.7996 \\
\hline K25C1101517 & SVOA & 2,3,4,6-Tetrachlorophenol & 2 & 0 & $\mathrm{ug} / \mathrm{kg}$ & 377 & 982 & 679.5 & 679.5 & 427.7996 \\
\hline
\end{tabular}




\begin{tabular}{|c|c|c|c|c|c|c|c|c|c|c|}
\hline K25C1101517 & SVOA & 2,4,5-Trichlorophenol & 4 & 0 & $\mathrm{ug} / \mathrm{kg}$ & 0.05 & 982 & 339.775 & 188.525 & 463.56042 \\
\hline K25C1101517 & SVOA & 2,4,6-Trichlorophenol & 4 & 0 & $\mathrm{ug} / \mathrm{kg}$ & 0.05 & 982 & 339.775 & 188.525 & 463.56042 \\
\hline K25C1101517 & SVOA & 2,4-Dichlorophenol & 2 & 0 & $\mathrm{ug} / \mathrm{kg}$ & 377 & 982 & 679.5 & 679.5 & 427.7996 \\
\hline K25C1101517 & SVOA & 2,4-Dimethylphenol & 2 & 0 & $\mathrm{ug} / \mathrm{kg}$ & 377 & 982 & 679.5 & 679.5 & 427.7996 \\
\hline K25C1101517 & SVOA & 2,4-Dinitrophenol & 2 & 0 & $\mathrm{ug} / \mathrm{kg}$ & 754 & 1960 & 1357 & 1357 & 852.77078 \\
\hline K25C1101517 & SVOA & 2,4-Dinitrotoluene & 4 & 0 & $\mathrm{ug} / \mathrm{kg}$ & 0.05 & 982 & 339.775 & 188.525 & 463.56042 \\
\hline K25C1101517 & SVOA & 2,6-Dinitrotoluene & 2 & 0 & $\mathrm{ug} / \mathrm{kg}$ & 377 & 982 & 679.5 & 679.5 & 427.7996 \\
\hline K25C1101517 & SVOA & 2-Chloronaphthalene & 2 & 0 & $\mathrm{ug} / \mathrm{kg}$ & 37.7 & 98.2 & 67.95 & 67.95 & 42.77996 \\
\hline K25C1101517 & SVOA & 2-Chlorophenol & 2 & 0 & $\mathrm{ug} / \mathrm{kg}$ & 377 & 982 & 679.5 & 679.5 & 427.7996 \\
\hline K25C1101517 & SVOA & 2-Methyl-4,6-dinitrophenol & 2 & 0 & $\mathrm{ug} / \mathrm{kg}$ & 377 & 982 & 679.5 & 679.5 & 427.7996 \\
\hline K25C1101517 & SVOA & 2-Methylnaphthalene & 2 & 0 & $\mathrm{ug} / \mathrm{kg}$ & 37.7 & 98.2 & 67.95 & 67.95 & 42.77996 \\
\hline K25C1101517 & SVOA & 2-Methylphenol & 4 & 0 & $\mathrm{ug} / \mathrm{kg}$ & 0.05 & 982 & 339.775 & 188.525 & 463.56042 \\
\hline K25C1101517 & SVOA & 2-Nitrobenzenamine & 2 & 0 & $\mathrm{ug} / \mathrm{kg}$ & 377 & 982 & 679.5 & 679.5 & 427.7996 \\
\hline K25C1101517 & SVOA & 2-Nitrophenol & 2 & 0 & $\mathrm{ug} / \mathrm{kg}$ & 377 & 982 & 679.5 & 679.5 & 427.7996 \\
\hline K25C1101517 & SVOA & 3,3'-Dichlorobenzidine & 2 & 0 & $\mathrm{ug} / \mathrm{kg}$ & 377 & 982 & 679.5 & 679.5 & 427.7996 \\
\hline K25C1101517 & SVOA & 3-Nitrobenzenamine & 2 & 0 & $\mathrm{ug} / \mathrm{kg}$ & 377 & 982 & 679.5 & 679.5 & 427.7996 \\
\hline K25C1101517 & SVOA & 4-Bromophenyl phenyl ether & 2 & 0 & $\mathrm{ug} / \mathrm{kg}$ & 377 & 982 & 679.5 & 679.5 & 427.7996 \\
\hline K25C1101517 & SVOA & 4-Chloro-3-methylphenol & 2 & 0 & $\mathrm{ug} / \mathrm{kg}$ & 377 & 982 & 679.5 & 679.5 & 427.7996 \\
\hline K25C1101517 & SVOA & 4-Chlorobenzenamine & 2 & 0 & $\mathrm{ug} / \mathrm{kg}$ & 377 & 982 & 679.5 & 679.5 & 427.7996 \\
\hline K25C1101517 & SVOA & 4-Chlorophenyl phenyl ether & 2 & 0 & $\mathrm{ug} / \mathrm{kg}$ & 377 & 982 & 679.5 & 679.5 & 427.7996 \\
\hline K25C1101517 & SVOA & 4-Nitrobenzenamine & 2 & 0 & $\mathrm{ug} / \mathrm{kg}$ & 377 & 982 & 679.5 & 679.5 & 427.7996 \\
\hline K25C1101517 & SVOA & 4-Nitrophenol & 2 & 0 & $\mathrm{ug} / \mathrm{kg}$ & 377 & 982 & 679.5 & 679.5 & 427.7996 \\
\hline K25C1101517 & SVOA & Acenaphthene & 2 & 0 & $\mathrm{ug} / \mathrm{kg}$ & 37.7 & 98.2 & 67.95 & 67.95 & 42.77996 \\
\hline K25C1101517 & SVOA & Acenaphthylene & 2 & 0 & $\mathrm{ug} / \mathrm{kg}$ & 37.7 & 98.2 & 67.95 & 67.95 & 42.77996 \\
\hline K25C1101517 & SVOA & Acetophenone & 2 & 0 & $\mathrm{ug} / \mathrm{kg}$ & 377 & 982 & 679.5 & 679.5 & 427.7996 \\
\hline K25C1101517 & SVOA & Anthracene & 2 & 0 & $\mathrm{ug} / \mathrm{kg}$ & 37.7 & 98.2 & 67.95 & 67.95 & 42.77996 \\
\hline K25C1101517 & SVOA & Benz(a)anthracene & 2 & 0 & $\mathrm{ug} / \mathrm{kg}$ & 37.7 & 98.2 & 67.95 & 67.95 & 42.77996 \\
\hline K25C1101517 & SVOA & Benzenemethanol & 2 & 0 & $\mathrm{ug} / \mathrm{kg}$ & 377 & 982 & 679.5 & 679.5 & 427.7996 \\
\hline K25C1101517 & SVOA & Benzidine & 2 & 0 & $\mathrm{ug} / \mathrm{kg}$ & 377 & 982 & 679.5 & 679.5 & 427.7996 \\
\hline K25C1101517 & SVOA & Benzo(a)pyrene & 2 & 0 & $\mathrm{ug} / \mathrm{kg}$ & 37.7 & 98.2 & 67.95 & 67.95 & 42.77996 \\
\hline K25C1101517 & SVOA & Benzo(b)fluoranthene & 2 & 0 & $\mathrm{ug} / \mathrm{kg}$ & 37.7 & 98.2 & 67.95 & 67.95 & 42.77996 \\
\hline K25C1101517 & SVOA & Benzo(ghi)perylene & 2 & 0 & $\mathrm{ug} / \mathrm{kg}$ & 37.7 & 98.2 & 67.95 & 67.95 & 42.77996 \\
\hline K25C1101517 & SVOA & Benzo(k)fluoranthene & 2 & 0 & $\mathrm{ug} / \mathrm{kg}$ & 37.7 & 98.2 & 67.95 & 67.95 & 42.77996 \\
\hline K25C1101517 & SVOA & Benzoic acid & 2 & 0 & $\mathrm{ug} / \mathrm{kg}$ & 754 & 1960 & 1357 & 1357 & 852.77078 \\
\hline K25C1101517 & SVOA & Bis(2-chloroethoxy)methane & 2 & 0 & $\mathrm{ug} / \mathrm{kg}$ & 377 & 982 & 679.5 & 679.5 & 427.7996 \\
\hline K25C1101517 & SVOA & Bis(2-chloroethyl) ether & 2 & 0 & $\mathrm{ug} / \mathrm{kg}$ & 377 & 982 & 679.5 & 679.5 & 427.7996 \\
\hline K25C1101517 & SVOA & Bis(2-ethylhexyl)phthalate & 2 & 1 & $\mathrm{ug} / \mathrm{kg}$ & 459 & 982 & 720.5 & 720.5 & 369.81685 \\
\hline K25C1101517 & SVOA & Butyl benzyl phthalate & 2 & 0 & $\mathrm{ug} / \mathrm{kg}$ & 377 & 982 & 679.5 & 679.5 & 427.7996 \\
\hline K25C1101517 & SVOA & Carbazole & 2 & 0 & $\mathrm{ug} / \mathrm{kg}$ & 37.7 & 98.2 & 67.95 & 67.95 & 42.77996 \\
\hline K25C1101517 & SVOA & Chrysene & 2 & 0 & $\mathrm{ug} / \mathrm{kg}$ & 37.7 & 98.2 & 67.95 & 67.95 & 42.77996 \\
\hline K25C1101517 & SVOA & Dibenz(a,h)anthracene & 2 & 0 & $\mathrm{ug} / \mathrm{kg}$ & 37.7 & 98.2 & 67.95 & 67.95 & 42.77996 \\
\hline K25C1101517 & SVOA & Dibenzofuran & 2 & 0 & $\mathrm{ug} / \mathrm{kg}$ & 377 & 982 & 679.5 & 679.5 & 427.7996 \\
\hline K25C1101517 & SVOA & Diethyl phthalate & 2 & 0 & $\mathrm{ug} / \mathrm{kg}$ & 377 & 982 & 679.5 & 679.5 & 427.7996 \\
\hline K25C1101517 & SVOA & Dimethyl phthalate & 2 & 0 & $\mathrm{ug} / \mathrm{kg}$ & 377 & 982 & 679.5 & 679.5 & 427.7996 \\
\hline K25C1101517 & SVOA & Di-n-butyl phthalate & 2 & 0 & $\mathrm{ug} / \mathrm{kg}$ & 377 & 982 & 679.5 & 679.5 & 427.7996 \\
\hline K25C1101517 & SVOA & Di-n-octylphthalate & 2 & 0 & $\mathrm{ug} / \mathrm{kg}$ & 377 & 982 & 679.5 & 679.5 & 427.7996 \\
\hline K25C1101517 & SVOA & Diphenylamine & 2 & 0 & $\mathrm{ug} / \mathrm{kg}$ & 377 & 982 & 679.5 & 679.5 & 427.7996 \\
\hline K25C1101517 & SVOA & Fluoranthene & 2 & 0 & $\mathrm{ug} / \mathrm{kg}$ & 37.7 & 98.2 & 67.95 & 67.95 & 42.77996 \\
\hline K25C1101517 & SVOA & Fluorene & 2 & 0 & $\mathrm{ug} / \mathrm{kg}$ & 37.7 & 98.2 & 67.95 & 67.95 & 42.77996 \\
\hline K25C1101517 & SVOA & Hexachlorobenzene & 4 & 0 & $\mathrm{ug} / \mathrm{kg}$ & 0.05 & 982 & 339.775 & 188.525 & 463.56042 \\
\hline K25C1101517 & SVOA & Hexachlorobutadiene & 4 & 0 & $\mathrm{ug} / \mathrm{kg}$ & 0.05 & 982 & 339.775 & 188.525 & 463.56042 \\
\hline K25C1101517 & SVOA & Hexachlorocyclopentadiene & 2 & 0 & $\mathrm{ug} / \mathrm{kg}$ & 377 & 982 & 679.5 & 679.5 & 427.7996 \\
\hline K25C1101517 & SVOA & Hexachloroethane & 4 & 0 & $\mathrm{ug} / \mathrm{kg}$ & 0.05 & 982 & 339.775 & 188.525 & 463.56042 \\
\hline K25C1101517 & SVOA & Indeno(1,2,3-cd)pyrene & 2 & 0 & $\mathrm{ug} / \mathrm{kg}$ & 37.7 & 98.2 & 67.95 & 67.95 & 42.77996 \\
\hline K25C1101517 & SVOA & Isophorone & 2 & 0 & $\mathrm{ug} / \mathrm{kg}$ & 377 & 982 & 679.5 & 679.5 & 427.7996 \\
\hline K25C1101517 & SVOA & $\mathrm{m}+\mathrm{p}$ Methylphenol & 4 & 0 & $\mathrm{ug} / \mathrm{kg}$ & 0.05 & 982 & 339.775 & 188.525 & 463.56042 \\
\hline K25C1101517 & SVOA & Naphthalene & 2 & 0 & $\mathrm{ug} / \mathrm{kg}$ & 37.7 & 98.2 & 67.95 & 67.95 & 42.77996 \\
\hline K25C1101517 & SVOA & Nitrobenzene & 4 & 0 & $\mathrm{ug} / \mathrm{kg}$ & 0.05 & 982 & 339.775 & 188.525 & 463.56042 \\
\hline K25C1101517 & SVOA & N-Nitroso-di-n-propylamine & 2 & 0 & $\mathrm{ug} / \mathrm{kg}$ & 377 & 982 & 679.5 & 679.5 & 427.7996 \\
\hline K25C1101517 & SVOA & Pentachlorophenol & 4 & 0 & $\mathrm{ug} / \mathrm{kg}$ & 0.05 & 982 & 339.775 & 188.525 & 463.56042 \\
\hline K25C1101517 & SVOA & Phenanthrene & 2 & 0 & $\mathrm{ug} / \mathrm{kg}$ & 37.7 & 98.2 & 67.95 & 67.95 & 42.77996 \\
\hline K25C1101517 & SVOA & Phenol & 2 & 0 & $\mathrm{ug} / \mathrm{kg}$ & 377 & 982 & 679.5 & 679.5 & 427.7996 \\
\hline K25C1101517 & SVOA & Pyridine & 4 & 0 & $\mathrm{ug} / \mathrm{kg}$ & 0.05 & 982 & 339.775 & 188.525 & 463.56042 \\
\hline K25C1101517 & VOA & (1,1-Dimethylethyl)benzene & 2 & 0 & $\mathrm{ug} / \mathrm{kg}$ & 98 & 459 & 278.5 & 278.5 & 255.26555 \\
\hline K25C1101517 & VOA & (1-Methylpropyl)benzene & 2 & 0 & $\mathrm{ug} / \mathrm{kg}$ & 98 & 459 & 278.5 & 278.5 & 255.26555 \\
\hline K25C1101517 & VOA & 1,1,1,2-Tetrachloroethane & 2 & 0 & $\mathrm{ug} / \mathrm{kg}$ & 98 & 459 & 278.5 & 278.5 & 255.26555 \\
\hline K25C1101517 & VOA & 1,1,1-Trichloroethane & 2 & 0 & $\mathrm{ug} / \mathrm{kg}$ & 98 & 459 & 278.5 & 278.5 & 255.26555 \\
\hline K25C1101517 & VOA & 1,1,2,2-Tetrachloroethane & 2 & 0 & $\mathrm{ug} / \mathrm{kg}$ & 98 & 459 & 278.5 & 278.5 & 255.26555 \\
\hline K25C1101517 & VOA & 1,1,2-Trichloroethane & 2 & 0 & $\mathrm{ug} / \mathrm{kg}$ & 98 & 459 & 278.5 & 278.5 & 255.26555 \\
\hline K25C1101517 & VOA & 1,1-Dichloroethane & 2 & 0 & $\mathrm{ug} / \mathrm{kg}$ & 98 & 459 & 278.5 & 278.5 & 255.26555 \\
\hline
\end{tabular}




\begin{tabular}{|c|c|c|c|c|c|c|c|c|c|c|}
\hline K25C1101517 & VOA & 1,1-Dichloroethene & 4 & 0 & $\mathrm{ug} / \mathrm{kg}$ & 0.01 & 459 & 139.255 & 49.005 & 218.11097 \\
\hline K25C1101517 & VOA & 1,1-Dichloropropene & 2 & 0 & $\mathrm{ug} / \mathrm{kg}$ & 98 & 459 & 278.5 & 278.5 & 255.26555 \\
\hline K25C1101517 & VOA & 1,2,3-Trichlorobenzene & 2 & 0 & $\mathrm{ug} / \mathrm{kg}$ & 98 & 459 & 278.5 & 278.5 & 255.26555 \\
\hline K25C1101517 & VOA & 1,2,3-Trichloropropane & 2 & 0 & $\mathrm{ug} / \mathrm{kg}$ & 98 & 459 & 278.5 & 278.5 & 255.26555 \\
\hline K25C1101517 & VOA & 1,2,4-Trimethylbenzene & 2 & 0 & $\mathrm{ug} / \mathrm{kg}$ & 98 & 459 & 278.5 & 278.5 & 255.26555 \\
\hline K25C1101517 & VOA & 1,2-Dibromo-3-chloropropane & 2 & 0 & $\mathrm{ug} / \mathrm{kg}$ & 98 & 459 & 278.5 & 278.5 & 255.26555 \\
\hline K25C1101517 & VOA & 1,2-Dibromoethane & 2 & 0 & $\mathrm{ug} / \mathrm{kg}$ & 98 & 459 & 278.5 & 278.5 & 255.26555 \\
\hline K25C1101517 & VOA & 1,2-Dichloroethane & 4 & 0 & $\mathrm{ug} / \mathrm{kg}$ & 0.01 & 459 & 139.255 & 49.005 & 218.11097 \\
\hline K25C1101517 & VOA & 1,2-Dichloroethene & 2 & 0 & $\mathrm{ug} / \mathrm{kg}$ & 196 & 917 & 556.5 & 556.5 & 509.82399 \\
\hline K25C1101517 & VOA & 1,2-Dichloropropane & 2 & 0 & $\mathrm{ug} / \mathrm{kg}$ & 98 & 459 & 278.5 & 278.5 & 255.26555 \\
\hline K25C1101517 & VOA & 1,2-Dimethylbenzene & 2 & 0 & $\mathrm{ug} / \mathrm{kg}$ & 98 & 459 & 278.5 & 278.5 & 255.26555 \\
\hline K25C1101517 & VOA & 1,3,5-Trimethylbenzene & 2 & 0 & $\mathrm{ug} / \mathrm{kg}$ & 98 & 459 & 278.5 & 278.5 & 255.26555 \\
\hline K25C1101517 & VOA & 1,3-Dichloropropane & 2 & 0 & $\mathrm{ug} / \mathrm{kg}$ & 98 & 459 & 278.5 & 278.5 & 255.26555 \\
\hline K25C1101517 & VOA & 1-Chloro-4-methylbenzene & 2 & 0 & $\mathrm{ug} / \mathrm{kg}$ & 98 & 459 & 278.5 & 278.5 & 255.26555 \\
\hline K25C1101517 & VOA & 1-Methyl-4-(1-methylethyl)benzene & 2 & 0 & $\mathrm{ug} / \mathrm{kg}$ & 98 & 459 & 278.5 & 278.5 & 255.26555 \\
\hline K25C1101517 & VOA & 2,2-Dichloropropane & 2 & 0 & $\mathrm{ug} / \mathrm{kg}$ & 98 & 459 & 278.5 & 278.5 & 255.26555 \\
\hline K25C1101517 & VOA & 2-Butanone & 4 & 0 & $\mathrm{ug} / \mathrm{kg}$ & 0.05 & 2290 & 695.025 & 245.025 & 1088.1117 \\
\hline K25C1101517 & VOA & 2-Hexanone & 2 & 0 & $\mathrm{ug} / \mathrm{kg}$ & 490 & 2290 & 1390 & 1390 & 1272.7922 \\
\hline K25C1101517 & VOA & 4-Methyl-2-pentanone & 2 & 0 & $\mathrm{ug} / \mathrm{kg}$ & 490 & 2290 & 1390 & 1390 & 1272.7922 \\
\hline K25C1101517 & VOA & Acetone & 2 & 0 & $\mathrm{ug} / \mathrm{kg}$ & 490 & 2290 & 1390 & 1390 & 1272.7922 \\
\hline K25C1101517 & VOA & Acetonitrile & 2 & 0 & $\mathrm{ug} / \mathrm{kg}$ & 2450 & 11500 & 6975 & 6975 & 6399.3164 \\
\hline K25C1101517 & VOA & Acrolein & 2 & 0 & $\mathrm{ug} / \mathrm{kg}$ & 490 & 2290 & 1390 & 1390 & 1272.7922 \\
\hline K25C1101517 & VOA & Acrylonitrile & 2 & 0 & $\mathrm{ug} / \mathrm{kg}$ & 490 & 2290 & 1390 & 1390 & 1272.7922 \\
\hline K25C1101517 & VOA & Benzene & 4 & 0 & $\mathrm{ug} / \mathrm{kg}$ & 0.01 & 459 & 139.255 & 49.005 & 218.11097 \\
\hline K25C1101517 & VOA & Bromobenzene & 2 & 0 & $\mathrm{ug} / \mathrm{kg}$ & 98 & 459 & 278.5 & 278.5 & 255.26555 \\
\hline K25C1101517 & VOA & Bromochloromethane & 2 & 0 & $\mathrm{ug} / \mathrm{kg}$ & 98 & 459 & 278.5 & 278.5 & 255.26555 \\
\hline K25C1101517 & VOA & Bromodichloromethane & 2 & 0 & $\mathrm{ug} / \mathrm{kg}$ & 98 & 459 & 278.5 & 278.5 & 255.26555 \\
\hline K25C1101517 & VOA & Bromoform & 2 & 0 & $\mathrm{ug} / \mathrm{kg}$ & 98 & 459 & 278.5 & 278.5 & 255.26555 \\
\hline K25C1101517 & VOA & Bromomethane & 2 & 0 & $\mathrm{ug} / \mathrm{kg}$ & 98 & 459 & 278.5 & 278.5 & 255.26555 \\
\hline K25C1101517 & VOA & Butylbenzene & 2 & 0 & $\mathrm{ug} / \mathrm{kg}$ & 98 & 459 & 278.5 & 278.5 & 255.26555 \\
\hline K25C1101517 & VOA & Carbon disulfide & 2 & 0 & $\mathrm{ug} / \mathrm{kg}$ & 490 & 2290 & 1390 & 1390 & 1272.7922 \\
\hline K25C1101517 & VOA & Carbon tetrachloride & 4 & 0 & $\mathrm{ug} / \mathrm{kg}$ & 0.01 & 459 & 139.255 & 49.005 & 218.11097 \\
\hline K25C1101517 & VOA & Chlorobenzene & 4 & 0 & $\mathrm{ug} / \mathrm{kg}$ & 0.01 & 459 & 139.255 & 49.005 & 218.11097 \\
\hline K25C1101517 & VOA & Chloroethane & 2 & 0 & $\mathrm{ug} / \mathrm{kg}$ & 98 & 459 & 278.5 & 278.5 & 255.26555 \\
\hline K25C1101517 & VOA & Chloroform & 4 & 0 & $\mathrm{ug} / \mathrm{kg}$ & 0.01 & 459 & 139.255 & 49.005 & 218.11097 \\
\hline K25C1101517 & VOA & Chloromethane & 2 & 0 & $\mathrm{ug} / \mathrm{kg}$ & 98 & 459 & 278.5 & 278.5 & 255.26555 \\
\hline K25C1101517 & VOA & cis-1,2-Dichloroethene & 2 & 0 & $\mathrm{ug} / \mathrm{kg}$ & 98 & 459 & 278.5 & 278.5 & 255.26555 \\
\hline K25C1101517 & VOA & cis-1,3-Dichloropropene & 2 & 0 & $\mathrm{ug} / \mathrm{kg}$ & 98 & 459 & 278.5 & 278.5 & 255.26555 \\
\hline K25C1101517 & VOA & Cumene & 2 & 0 & $\mathrm{ug} / \mathrm{kg}$ & 98 & 459 & 278.5 & 278.5 & 255.26555 \\
\hline K25C1101517 & VOA & Dibromochloromethane & 2 & 0 & $\mathrm{ug} / \mathrm{kg}$ & 98 & 459 & 278.5 & 278.5 & 255.26555 \\
\hline K25C1101517 & VOA & Dibromomethane & 2 & 0 & $\mathrm{ug} / \mathrm{kg}$ & 98 & 459 & 278.5 & 278.5 & 255.26555 \\
\hline K25C1101517 & VOA & Dichlorodifluoromethane & 2 & 0 & $\mathrm{ug} / \mathrm{kg}$ & 98 & 459 & 278.5 & 278.5 & 255.26555 \\
\hline K25C1101517 & VOA & Ethylbenzene & 2 & 0 & $\mathrm{ug} / \mathrm{kg}$ & 98 & 459 & 278.5 & 278.5 & 255.26555 \\
\hline K25C1101517 & VOA & Hexane & 2 & 0 & $\mathrm{ug} / \mathrm{kg}$ & 490 & 2290 & 1390 & 1390 & 1272.7922 \\
\hline K25C1101517 & VOA & M + P Xylene & 2 & 0 & $\mathrm{ug} / \mathrm{kg}$ & 196 & 917 & 556.5 & 556.5 & 509.82399 \\
\hline K25C1101517 & VOA & Methanol & 2 & 0 & $\mathrm{ug} / \mathrm{kg}$ & 4670 & 10000 & 7335 & 7335 & 3768.8791 \\
\hline K25C1101517 & VOA & Methyl methacrylate & 2 & 0 & $\mathrm{ug} / \mathrm{kg}$ & 490 & 2290 & 1390 & 1390 & 1272.7922 \\
\hline K25C1101517 & VOA & Methylcyclohexane & 2 & 0 & $\mathrm{ug} / \mathrm{kg}$ & 98 & 459 & 278.5 & 278.5 & 255.26555 \\
\hline K25C1101517 & VOA & Methylene chloride & 2 & 0 & $\mathrm{ug} / \mathrm{kg}$ & 490 & 2290 & 1390 & 1390 & 1272.7922 \\
\hline K25C1101517 & VOA & o-Chlorotoluene & 2 & 0 & $\mathrm{ug} / \mathrm{kg}$ & 98 & 459 & 278.5 & 278.5 & 255.26555 \\
\hline K25C1101517 & VOA & Propylbenzene & 2 & 0 & $\mathrm{ug} / \mathrm{kg}$ & 98 & 459 & 278.5 & 278.5 & 255.26555 \\
\hline K25C1101517 & VOA & Propylene glycol & 2 & 0 & $\mathrm{ug} / \mathrm{kg}$ & 46700 & 100000 & 73350 & 73350 & 37688.791 \\
\hline K25C1101517 & VOA & Styrene & 2 & 0 & $\mathrm{ug} / \mathrm{kg}$ & 98 & 459 & 278.5 & 278.5 & 255.26555 \\
\hline K25C1101517 & VOA & Tetrachloroethene & 4 & 0 & $\mathrm{ug} / \mathrm{kg}$ & 0.01 & 459 & 139.255 & 49.005 & 218.11097 \\
\hline K25C1101517 & VOA & Toluene & 2 & 0 & $\mathrm{ug} / \mathrm{kg}$ & 98 & 459 & 278.5 & 278.5 & 255.26555 \\
\hline K25C1101517 & VOA & Total Xylene & 2 & 0 & $\mathrm{ug} / \mathrm{kg}$ & 294 & 1380 & 837 & 837 & 767.91796 \\
\hline K25C1101517 & VOA & trans-1,2-Dichloroethene & 2 & 0 & $\mathrm{ug} / \mathrm{kg}$ & 98 & 459 & 278.5 & 278.5 & 255.26555 \\
\hline K25C1101517 & VOA & trans-1,3-Dichloropropene & 2 & 0 & $\mathrm{ug} / \mathrm{kg}$ & 98 & 459 & 278.5 & 278.5 & 255.26555 \\
\hline K25C1101517 & VOA & Trichloroethene & 4 & 0 & $\mathrm{ug} / \mathrm{kg}$ & 0.01 & 459 & 139.255 & 49.005 & 218.11097 \\
\hline K25C1101517 & VOA & Trichlorofluoromethane & 2 & 0 & $\mathrm{ug} / \mathrm{kg}$ & 98 & 459 & 278.5 & 278.5 & 255.26555 \\
\hline K25C1101517 & VOA & Vinyl chloride & 4 & 0 & $\mathrm{ug} / \mathrm{kg}$ & 0.01 & 459 & 139.255 & 49.005 & 218.11097 \\
\hline K25C1101518 & HERB & $2,4-\mathrm{D}$ & 2 & 0 & $\mathrm{ug} / \mathrm{kg}$ & 0.05 & 123 & 61.525 & 61.525 & 86.938779 \\
\hline K25C1101518 & HERB & Silvex & 2 & 0 & $\mathrm{ug} / \mathrm{kg}$ & 0.05 & 123 & 61.525 & 61.525 & 86.938779 \\
\hline K25C1101518 & METAL & Arsenic & 2 & 0 & $\mathrm{mg} / \mathrm{L}$ & 0.3 & 144 & 72.15 & 72.15 & 101.61124 \\
\hline K25C1101518 & METAL & Barium & 2 & 1 & $\mathrm{mg} / \mathrm{L}$ & 0.0552 & 24 & 12.0276 & 12.0276 & 16.93153 \\
\hline K25C1101518 & METAL & Cadmium & 2 & 0 & $\mathrm{mg} / \mathrm{L}$ & 0.05 & 24 & 12.025 & 12.025 & 16.935207 \\
\hline K25C1101518 & METAL & Chromium & 2 & 1 & $\mathrm{mg} / \mathrm{L}$ & 0.05 & 147 & 73.525 & 73.525 & 103.90934 \\
\hline K25C1101518 & METAL & Lead & 2 & 0 & $\mathrm{mg} / \mathrm{L}$ & 0.1 & 48.1 & 24.1 & 24.1 & 33.941125 \\
\hline K25C1101518 & METAL & Mercury & 2 & 0 & $\mathrm{mg} / \mathrm{L}$ & 0.002 & 0.4 & 0.201 & 0.201 & 0.2814285 \\
\hline K25C1101518 & METAL & Selenium & 2 & 0 & $\mathrm{mg} / \mathrm{L}$ & 0.3 & 144 & 72.15 & 72.15 & 101.61124 \\
\hline
\end{tabular}




\begin{tabular}{|c|c|c|c|c|c|c|c|c|c|c|}
\hline K25C1101518 & METAL & Silver & 2 & 0 & $\mathrm{mg} / \mathrm{L}$ & 0.05 & 24 & 12.025 & 12.025 & 16.935207 \\
\hline K25C1101518 & METAL & Total Uranium & 1 & 0 & $\mathrm{pCi} / \mathrm{g}$ & 0.0281 & 0.0281 & 0.0281 & & \\
\hline K25C1101518 & PPCB & 4,4 '-DDD & 1 & 0 & $\mathrm{ug} / \mathrm{kg}$ & 3.96 & 3.96 & 3.96 & & \\
\hline K25C1101518 & PPCB & 4,4'-DDE & 1 & 0 & $\mathrm{ug} / \mathrm{kg}$ & 3.96 & 3.96 & 3.96 & & \\
\hline K25C1101518 & РPCB & 4,4'-DDT & 1 & 0 & $\mathrm{ug} / \mathrm{kg}$ & 3.96 & 3.96 & 3.96 & & \\
\hline K25C1101518 & PPCB & Aldrin & 1 & 0 & $\mathrm{ug} / \mathrm{kg}$ & 1.98 & 1.98 & 1.98 & & \\
\hline K25C1101518 & PPCB & alpha-BHC & 1 & 0 & $\mathrm{ug} / \mathrm{kg}$ & 1.98 & 1.98 & 1.98 & & \\
\hline K25C1101518 & РPCB & alpha-Chlordane & 1 & 0 & $\mathrm{ug} / \mathrm{kg}$ & 1.98 & 1.98 & 1.98 & & \\
\hline K25C1101518 & РPCB & beta-BHC & 1 & 0 & $\mathrm{ug} / \mathrm{kg}$ & 1.98 & 1.98 & 1.98 & & \\
\hline K25C1101518 & PPCB & Chlordane & 2 & 0 & $\mathrm{ug} / \mathrm{kg}$ & 0.0025 & 24.8 & 12.40125 & 12.40125 & 17.53448 \\
\hline K25C1101518 & PPCB & delta-BHC & 1 & 0 & $\mathrm{ug} / \mathrm{kg}$ & 1.98 & 1.98 & 1.98 & & \\
\hline K25C1101518 & РPCB & Dieldrin & 1 & 0 & ug $/ \mathrm{kg}$ & 3.96 & 3.96 & 3.96 & & \\
\hline K25C1101518 & РPCB & Endosulfan I & 1 & 0 & $\mathrm{ug} / \mathrm{kg}$ & 1.98 & 1.98 & 1.98 & & \\
\hline K25C1101518 & PPCB & Endosulfan II & 1 & 0 & $\mathrm{ug} / \mathrm{kg}$ & 3.96 & 3.96 & 3.96 & & \\
\hline K25C1101518 & PPCB & Endosulfan sulfate & 1 & 0 & $\mathrm{ug} / \mathrm{kg}$ & 3.96 & 3.96 & 3.96 & & \\
\hline K25C1101518 & PРCB & Endrin & 2 & 0 & $\mathrm{ug} / \mathrm{kg}$ & 0.0004 & 3.96 & 1.9802 & 1.9802 & 2.79986 \\
\hline K25C1101518 & РPCB & Endrin aldehyde & 1 & 0 & $\mathrm{ug} / \mathrm{kg}$ & 3.96 & 3.96 & 3.96 & & \\
\hline K25C1101518 & PPCB & Endrin ketone & 1 & 0 & $\mathrm{ug} / \mathrm{kg}$ & 3.96 & 3.96 & 3.96 & & \\
\hline K25C1101518 & PPCB & gamma-Chlordane & 1 & 0 & $\mathrm{ug} / \mathrm{kg}$ & 1.98 & 1.98 & 1.98 & & \\
\hline K25C1101518 & РPCB & Heptachlor & 2 & 0 & $\mathrm{ug} / \mathrm{kg}$ & 0.0002 & 1.98 & 0.9901 & 0.9901 & 1.39993 \\
\hline K25C1101518 & РPCB & Heptachlor epoxide & 2 & 0 & $\mathrm{ug} / \mathrm{kg}$ & 0.0002 & 1.98 & 0.9901 & 0.9901 & 1.39993 \\
\hline K25C1101518 & PPCB & Lindane & 2 & 0 & $\mathrm{ug} / \mathrm{kg}$ & 0.0002 & 1.98 & 0.9901 & 0.9901 & 1.39993 \\
\hline K25C1101518 & PPCB & Methoxychlor & 2 & 0 & $\mathrm{ug} / \mathrm{kg}$ & 0.002 & 19.8 & 9.901 & 9.901 & 13.9993 \\
\hline K25C1101518 & РPCB & Toxaphene & 2 & 0 & $\mathrm{ug} / \mathrm{kg}$ & 0.005 & 49.6 & 24.8025 & 24.8025 & 35.068961 \\
\hline K25C1101518 & RADS & Americium-241 & 1 & 0 & $\mathrm{pCi} / \mathrm{g}$ & -0.00681 & -0.00681 & -0.00681 & & \\
\hline K25C1101518 & RADS & Carbon-14 & 1 & 0 & $\mathrm{pCi} / \mathrm{g}$ & -1.98 & -1.98 & -1.98 & & \\
\hline K25C1101518 & RADS & Cesium-137 & 1 & 0 & $\mathrm{pCi} / \mathrm{g}$ & 0.011 & 0.011 & 0.011 & & \\
\hline K25C1101518 & RADS & Cobalt- 60 & 1 & 0 & $\mathrm{pCi} / \mathrm{g}$ & -0.00603 & -0.00603 & -0.00603 & & \\
\hline K25C1101518 & RADS & Curium-243/244 & 1 & 0 & $\mathrm{pCi} / \mathrm{g}$ & 0.0122 & 0.0122 & 0.0122 & & \\
\hline K25C1101518 & RADS & Iodine-129 & 1 & 0 & $\mathrm{pCi} / \mathrm{g}$ & 0.201 & 0.201 & 0.201 & & \\
\hline K25C1101518 & RADS & Neptunium-237 & 1 & 0 & $\mathrm{pCi} / \mathrm{g}$ & 0.0125 & 0.0125 & 0.0125 & & \\
\hline K25C1101518 & RADS & Plutonium-238 & 1 & 0 & $\mathrm{pCi} / \mathrm{g}$ & -0.00899 & -0.00899 & -0.00899 & & \\
\hline K25C1101518 & RADS & Plutonium-239/240 & 1 & 0 & $\mathrm{pCi} / \mathrm{g}$ & -0.0245 & -0.0245 & -0.0245 & & \\
\hline K25C1101518 & RADS & Radium-226 & 1 & 0 & $\mathrm{pCi} / \mathrm{g}$ & 0.344 & 0.344 & 0.344 & & \\
\hline K25C1101518 & RADS & Strontium-90 & 1 & 0 & $\mathrm{pCi} / \mathrm{g}$ & -0.495 & -0.495 & -0.495 & & \\
\hline K25C1101518 & RADS & Technetium-99 & 1 & 0 & $\mathrm{pCi} / \mathrm{g}$ & 0.411 & 0.411 & 0.411 & & \\
\hline K25C1101518 & RADS & Thorium-230 & 1 & 0 & $\mathrm{pCi} / \mathrm{g}$ & -5.59 & -5.59 & -5.59 & & \\
\hline K25C1101518 & RADS & Thorium-232 & 1 & 0 & $\mathrm{pCi} / \mathrm{g}$ & 4.04 & 4.04 & 4.04 & & \\
\hline K25C1101518 & RADS & Tritium & 1 & 0 & $\mathrm{pCi} / \mathrm{g}$ & 63.2 & 63.2 & 63.2 & & \\
\hline K25C1101518 & RADS & Uranium-233/234 & 1 & 0 & $\mathrm{pCi} / \mathrm{g}$ & 0.0347 & 0.0347 & 0.0347 & & \\
\hline K25C1101518 & RADS & Uranium-235/236 & 1 & 0 & $\mathrm{pCi} / \mathrm{g}$ & 0 & 0 & 0 & & \\
\hline K25C1101518 & RADS & Uranium-238 & 1 & 0 & $\mathrm{pCi} / \mathrm{g}$ & -0.00661 & -0.00661 & -0.00661 & & \\
\hline K25C1101518 & SVOA & 1,2,4-Trichlorobenzene & 1 & 0 & $\mathrm{ug} / \mathrm{kg}$ & 100 & 100 & 100 & & \\
\hline K25C1101518 & SVOA & 1,2-Dichlorobenzene & 1 & 0 & $\mathrm{ug} / \mathrm{kg}$ & 100 & 100 & 100 & & \\
\hline K25C1101518 & SVOA & 1,3-Dichlorobenzene & 1 & 0 & $\mathrm{ug} / \mathrm{kg}$ & 100 & 100 & 100 & & \\
\hline K25C1101518 & SVOA & 1,4-Dichlorobenzene & 2 & 0 & $\mathrm{ug} / \mathrm{kg}$ & 0.01 & 100 & 50.005 & 50.005 & 70.703607 \\
\hline K25C1101518 & SVOA & 2,2'-Dichlorodiisopropyl ether & 1 & 0 & $\mathrm{ug} / \mathrm{kg}$ & 1000 & 1000 & 1000 & & \\
\hline K25C1101518 & SVOA & 2,3,4,6-Tetrachlorophenol & 1 & 0 & $\mathrm{ug} / \mathrm{kg}$ & 1000 & 1000 & 1000 & & \\
\hline K25C1101518 & SVOA & 2,4,5-Trichlorophenol & 2 & 0 & $\mathrm{ug} / \mathrm{kg}$ & 0.05 & 1000 & 500.025 & 500.025 & 707.07143 \\
\hline K25C1101518 & SVOA & 2,4,6-Trichlorophenol & 2 & 0 & $\mathrm{ug} / \mathrm{kg}$ & 0.05 & 1000 & 500.025 & 500.025 & 707.07143 \\
\hline K25C1101518 & SVOA & 2,4-Dichlorophenol & 1 & 0 & $\mathrm{ug} / \mathrm{kg}$ & 1000 & 1000 & 1000 & & \\
\hline K25C1101518 & SVOA & 2,4-Dimethylphenol & 1 & 0 & $\mathrm{ug} / \mathrm{kg}$ & 1000 & 1000 & 1000 & & \\
\hline K25C1101518 & SVOA & 2,4-Dinitrophenol & 1 & 0 & $\mathrm{ug} / \mathrm{kg}$ & 2000 & 2000 & 2000 & & \\
\hline K25C1101518 & SVOA & 2,4-Dinitrotoluene & 2 & 0 & $\mathrm{ug} / \mathrm{kg}$ & 0.05 & 1000 & 500.025 & 500.025 & 707.07143 \\
\hline K25C1101518 & SVOA & 2,6-Dinitrotoluene & 1 & 0 & $\mathrm{ug} / \mathrm{kg}$ & 1000 & 1000 & 1000 & & \\
\hline K25C1101518 & SVOA & 2-Chloronaphthalene & 1 & 0 & $\mathrm{ug} / \mathrm{kg}$ & 100 & 100 & 100 & & \\
\hline K25C1101518 & SVOA & 2-Chlorophenol & 1 & 0 & $\mathrm{ug} / \mathrm{kg}$ & 1000 & 1000 & 1000 & & \\
\hline K25C1101518 & SVOA & 2-Methyl-4,6-dinitrophenol & 1 & 0 & $\mathrm{ug} / \mathrm{kg}$ & 1000 & 1000 & 1000 & & \\
\hline K25C1101518 & SVOA & 2-Methylnaphthalene & 1 & 0 & $\mathrm{ug} / \mathrm{kg}$ & 100 & 100 & 100 & & \\
\hline K25C1101518 & SVOA & 2-Methylphenol & 2 & 0 & $\mathrm{ug} / \mathrm{kg}$ & 0.05 & 1000 & 500.025 & 500.025 & 707.07143 \\
\hline K25C1101518 & SVOA & 2-Nitrobenzenamine & 1 & 0 & $\mathrm{ug} / \mathrm{kg}$ & 1000 & 1000 & 1000 & & \\
\hline K25C1101518 & SVOA & 2-Nitrophenol & 1 & 0 & $\mathrm{ug} / \mathrm{kg}$ & 1000 & 1000 & 1000 & & \\
\hline K25C1101518 & SVOA & 3,3'-Dichlorobenzidine & 1 & 0 & $\mathrm{ug} / \mathrm{kg}$ & 1000 & 1000 & 1000 & & \\
\hline K25C1101518 & SVOA & 3-Nitrobenzenamine & 1 & 0 & $\mathrm{ug} / \mathrm{kg}$ & 1000 & 1000 & 1000 & & \\
\hline K25C1101518 & SVOA & 4-Bromophenyl phenyl ether & 1 & 0 & $\mathrm{ug} / \mathrm{kg}$ & 1000 & 1000 & 1000 & & \\
\hline K25C1101518 & SVOA & 4-Chloro-3-methylphenol & 1 & 0 & $\mathrm{ug} / \mathrm{kg}$ & 1000 & 1000 & 1000 & & \\
\hline K25C1101518 & SVOA & 4-Chlorobenzenamine & 1 & 0 & $\mathrm{ug} / \mathrm{kg}$ & 1000 & 1000 & 1000 & & \\
\hline K25C1101518 & SVOA & 4-Chlorophenyl phenyl ether & 1 & 0 & $\mathrm{ug} / \mathrm{kg}$ & 1000 & 1000 & 1000 & & \\
\hline K25C1101518 & SVOA & 4-Nitrobenzenamine & 1 & 0 & $\mathrm{ug} / \mathrm{kg}$ & 1000 & 1000 & 1000 & & \\
\hline K25C1101518 & SVOA & 4-Nitrophenol & 1 & 0 & $\mathrm{ug} / \mathrm{kg}$ & 1000 & 1000 & 1000 & & \\
\hline
\end{tabular}




\begin{tabular}{|c|c|c|c|c|c|c|c|c|c|c|}
\hline K25C1101518 & SVOA & Acenaphthene & 1 & 0 & $\mathrm{ug} / \mathrm{kg}$ & 100 & 100 & 100 & & \\
\hline K25C1101518 & SVOA & Acenaphthylene & 1 & 0 & $\mathrm{ug} / \mathrm{kg}$ & 100 & 100 & 100 & & \\
\hline K25C1101518 & SVOA & Acetophenone & 1 & 0 & $\mathrm{ug} / \mathrm{kg}$ & 1000 & 1000 & 1000 & & \\
\hline K25C1101518 & SVOA & Anthracene & 1 & 0 & $\mathrm{ug} / \mathrm{kg}$ & 100 & 100 & 100 & & \\
\hline K25C1101518 & SVOA & Benz(a)anthracene & 1 & 0 & $\mathrm{ug} / \mathrm{kg}$ & 100 & 100 & 100 & & \\
\hline K25C1101518 & SVOA & Benzenemethanol & 1 & 0 & $\mathrm{ug} / \mathrm{kg}$ & 1000 & 1000 & 1000 & & \\
\hline K25C1101518 & SVOA & Benzidine & 1 & 0 & $\mathrm{ug} / \mathrm{kg}$ & 1000 & 1000 & 1000 & & \\
\hline K25C1101518 & SVOA & Benzo(a)pyrene & 1 & 0 & $\mathrm{ug} / \mathrm{kg}$ & 100 & 100 & 100 & & \\
\hline K25C1101518 & SVOA & Benzo(b)fluoranthene & 1 & 0 & $\mathrm{ug} / \mathrm{kg}$ & 100 & 100 & 100 & & \\
\hline K25C1101518 & SVOA & Benzo(ghi)perylene & 1 & 0 & $\mathrm{ug} / \mathrm{kg}$ & 100 & 100 & 100 & & \\
\hline K25C1101518 & SVOA & Benzo(k)fluoranthene & 1 & 0 & $\mathrm{ug} / \mathrm{kg}$ & 100 & 100 & 100 & & \\
\hline K25C1101518 & SVOA & Benzoic acid & 1 & 0 & $\mathrm{ug} / \mathrm{kg}$ & 2000 & 2000 & 2000 & & \\
\hline K25C1101518 & SVOA & Bis(2-chloroethoxy)methane & 1 & 0 & $\mathrm{ug} / \mathrm{kg}$ & 1000 & 1000 & 1000 & & \\
\hline K25C1101518 & SVOA & Bis(2-chloroethyl) ether & 1 & 0 & $\mathrm{ug} / \mathrm{kg}$ & 1000 & 1000 & 1000 & & \\
\hline K25C1101518 & SVOA & Bis(2-ethylhexyl)phthalate & 1 & 0 & $\mathrm{ug} / \mathrm{kg}$ & 1000 & 1000 & 1000 & & \\
\hline K25C1101518 & SVOA & Butyl benzyl phthalate & 1 & 0 & $\mathrm{ug} / \mathrm{kg}$ & 1000 & 1000 & 1000 & & \\
\hline K25C1101518 & SVOA & Carbazole & 1 & 0 & $\mathrm{ug} / \mathrm{kg}$ & 100 & 100 & 100 & & \\
\hline K25C1101518 & SVOA & Chrysene & 1 & 0 & $\mathrm{ug} / \mathrm{kg}$ & 100 & 100 & 100 & & \\
\hline K25C1101518 & SVOA & Dibenz $(\mathrm{a}, \mathrm{h})$ anthracene & 1 & 0 & $\mathrm{ug} / \mathrm{kg}$ & 100 & 100 & 100 & & \\
\hline K25C1101518 & SVOA & Dibenzofuran & 1 & 0 & $\mathrm{ug} / \mathrm{kg}$ & 1000 & 1000 & 1000 & & \\
\hline K25C1101518 & SVOA & Diethyl phthalate & 1 & 0 & $\mathrm{ug} / \mathrm{kg}$ & 1000 & 1000 & 1000 & & \\
\hline K25C1101518 & SVOA & Dimethyl phthalate & 1 & 0 & $\mathrm{ug} / \mathrm{kg}$ & 1000 & 1000 & 1000 & & \\
\hline K25C1101518 & SVOA & Di-n-butyl phthalate & 1 & 0 & $\mathrm{ug} / \mathrm{kg}$ & 1000 & 1000 & 1000 & & \\
\hline K25C1101518 & SVOA & Di-n-octylphthalate & 1 & 0 & $\mathrm{ug} / \mathrm{kg}$ & 1000 & 1000 & 1000 & & \\
\hline K25C1101518 & SVOA & Diphenylamine & 1 & 0 & $\mathrm{ug} / \mathrm{kg}$ & 1000 & 1000 & 1000 & & \\
\hline K25C1101518 & SVOA & Fluoranthene & 1 & 0 & $\mathrm{ug} / \mathrm{kg}$ & 100 & 100 & 100 & & \\
\hline K25C1101518 & SVOA & Fluorene & 1 & 0 & $\mathrm{ug} / \mathrm{kg}$ & 100 & 100 & 100 & & \\
\hline K25C1101518 & SVOA & Hexachlorobenzene & 2 & 0 & $\mathrm{ug} / \mathrm{kg}$ & 0.05 & 1000 & 500.025 & 500.025 & 707.07143 \\
\hline K25C1101518 & SVOA & Hexachlorobutadiene & 2 & 0 & $\mathrm{ug} / \mathrm{kg}$ & 0.05 & 1000 & 500.025 & 500.025 & 707.07143 \\
\hline K25C1101518 & SVOA & Hexachlorocyclopentadiene & 1 & 0 & $\mathrm{ug} / \mathrm{kg}$ & 1000 & 1000 & 1000 & & \\
\hline K25C1101518 & SVOA & Hexachloroethane & 2 & 0 & $\mathrm{ug} / \mathrm{kg}$ & 0.05 & 1000 & 500.025 & 500.025 & 707.07143 \\
\hline K25C1101518 & SVOA & Indeno(1,2,3-cd)pyrene & 1 & 0 & $\mathrm{ug} / \mathrm{kg}$ & 100 & 100 & 100 & & \\
\hline K25C1101518 & SVOA & Isophorone & 1 & 0 & $\mathrm{ug} / \mathrm{kg}$ & 1000 & 1000 & 1000 & & \\
\hline K25C1101518 & SVOA & $\mathrm{m}+\mathrm{p}$ Methylphenol & 2 & 0 & $\mathrm{ug} / \mathrm{kg}$ & 0.05 & 1000 & 500.025 & 500.025 & 707.07143 \\
\hline K25C1101518 & SVOA & Naphthalene & 1 & 0 & $\mathrm{ug} / \mathrm{kg}$ & 100 & 100 & 100 & & \\
\hline K25C1101518 & SVOA & Nitrobenzene & 2 & 0 & $\mathrm{ug} / \mathrm{kg}$ & 0.05 & 1000 & 500.025 & 500.025 & 707.07143 \\
\hline K25C1101518 & SVOA & N-Nitroso-di-n-propylamine & 1 & 0 & $\mathrm{ug} / \mathrm{kg}$ & 1000 & 1000 & 1000 & & \\
\hline K25C1101518 & SVOA & Pentachlorophenol & 2 & 0 & $\mathrm{ug} / \mathrm{kg}$ & 0.05 & 1000 & 500.025 & 500.025 & 707.07143 \\
\hline K25C1101518 & SVOA & Phenanthrene & 1 & 0 & $\mathrm{ug} / \mathrm{kg}$ & 100 & 100 & 100 & & \\
\hline K25C1101518 & SVOA & Phenol & 1 & 0 & $\mathrm{ug} / \mathrm{kg}$ & 1000 & 1000 & 1000 & & \\
\hline K25C1101518 & SVOA & Pyridine & 2 & 0 & $\mathrm{ug} / \mathrm{kg}$ & 0.05 & 1000 & 500.025 & 500.025 & 707.07143 \\
\hline K25C1101518 & $\mathrm{VOA}$ & (1,1-Dimethylethyl)benzene & 1 & 0 & $\mathrm{ug} / \mathrm{kg}$ & 100 & 100 & 100 & & \\
\hline K25C1101518 & VOA & (1-Methylpropyl)benzene & 1 & 0 & $\mathrm{ug} / \mathrm{kg}$ & 100 & 100 & 100 & & \\
\hline K25C1101518 & VOA & 1,1,1,2-Tetrachloroethane & 1 & 0 & $\mathrm{ug} / \mathrm{kg}$ & 100 & 100 & 100 & & \\
\hline K25C1101518 & VOA & 1,1,1-Trichloroethane & 1 & 0 & $\mathrm{ug} / \mathrm{kg}$ & 100 & 100 & 100 & & \\
\hline K25C1101518 & $\mathrm{VOA}$ & 1,1,2,2-Tetrachloroethane & 1 & 0 & $\mathrm{ug} / \mathrm{kg}$ & 100 & 100 & 100 & & \\
\hline K25C1101518 & VOA & 1,1,2-Trichloroethane & 1 & 0 & $\mathrm{ug} / \mathrm{kg}$ & 100 & 100 & 100 & & \\
\hline K25C1101518 & VOA & 1,1-Dichloroethane & 1 & 0 & $\mathrm{ug} / \mathrm{kg}$ & 100 & 100 & 100 & & \\
\hline K25C1101518 & VOA & 1,1-Dichloroethene & 2 & 0 & $\mathrm{ug} / \mathrm{kg}$ & 0.01 & 100 & 50.005 & 50.005 & 70.703607 \\
\hline K25C1101518 & VOA & 1,1-Dichloropropene & 1 & 0 & $\mathrm{ug} / \mathrm{kg}$ & 100 & 100 & 100 & & \\
\hline K25C1101518 & VOA & 1,2,3-Trichlorobenzene & 1 & 0 & $\mathrm{ug} / \mathrm{kg}$ & 100 & 100 & 100 & & \\
\hline K25C1101518 & VOA & 1,2,3-Trichloropropane & 1 & 0 & $\mathrm{ug} / \mathrm{kg}$ & 100 & 100 & 100 & & \\
\hline K25C1101518 & VOA & 1,2,4-Trimethylbenzene & 1 & 0 & $\mathrm{ug} / \mathrm{kg}$ & 100 & 100 & 100 & & \\
\hline K25C1101518 & VOA & 1,2-Dibromo-3-chloropropane & 1 & 0 & $\mathrm{ug} / \mathrm{kg}$ & 100 & 100 & 100 & & \\
\hline K25C1101518 & VOA & 1,2-Dibromoethane & 1 & 0 & $\mathrm{ug} / \mathrm{kg}$ & 100 & 100 & 100 & & \\
\hline K25C1101518 & VOA & 1,2-Dichloroethane & 2 & 0 & $\mathrm{ug} / \mathrm{kg}$ & 0.01 & 100 & 50.005 & 50.005 & 70.703607 \\
\hline K25C1101518 & VOA & 1,2-Dichloroethene & 1 & 0 & $\mathrm{ug} / \mathrm{kg}$ & 200 & 200 & 200 & & \\
\hline K25C1101518 & VOA & 1,2-Dichloropropane & 1 & 0 & $\mathrm{ug} / \mathrm{kg}$ & 100 & 100 & 100 & & \\
\hline K25C1101518 & VOA & 1,2-Dimethylbenzene & 1 & 0 & $\mathrm{ug} / \mathrm{kg}$ & 100 & 100 & 100 & & \\
\hline K25C1101518 & VOA & 1,3,5-Trimethylbenzene & 1 & 0 & $\mathrm{ug} / \mathrm{kg}$ & 100 & 100 & 100 & & \\
\hline K25C1101518 & VOA & 1,3-Dichloropropane & 1 & 0 & $\mathrm{ug} / \mathrm{kg}$ & 100 & 100 & 100 & & \\
\hline K25C1101518 & VOA & 1-Chloro-4-methylbenzene & 1 & 0 & $\mathrm{ug} / \mathrm{kg}$ & 100 & 100 & 100 & & \\
\hline K25C1101518 & VOA & 1-Methyl-4-(1-methylethyl)benzene & 1 & 0 & $\mathrm{ug} / \mathrm{kg}$ & 100 & 100 & 100 & & \\
\hline K25C1101518 & VOA & 2,2-Dichloropropane & 1 & 0 & $\mathrm{ug} / \mathrm{kg}$ & 100 & 100 & 100 & & \\
\hline K25C1101518 & VOA & 2-Butanone & 2 & 0 & $\mathrm{ug} / \mathrm{kg}$ & 0.05 & 500 & 250.025 & 250.025 & 353.51804 \\
\hline K25C1101518 & VOA & 2-Hexanone & 1 & 0 & $\mathrm{ug} / \mathrm{kg}$ & 500 & 500 & 500 & & \\
\hline K25C1101518 & $\mathrm{VOA}$ & 4-Methyl-2-pentanone & 1 & 0 & $\mathrm{ug} / \mathrm{kg}$ & 500 & 500 & 500 & & \\
\hline K25C1101518 & VOA & Acetone & 1 & 0 & $\mathrm{ug} / \mathrm{kg}$ & 500 & 500 & 500 & & \\
\hline K25C1101518 & VOA & Acetonitrile & 1 & 0 & $\mathrm{ug} / \mathrm{kg}$ & 2500 & 2500 & 2500 & & \\
\hline K25C1101518 & VOA & Acrolein & 1 & 0 & $\mathrm{ug} / \mathrm{kg}$ & 500 & 500 & 500 & & \\
\hline
\end{tabular}




\begin{tabular}{|c|c|c|c|c|c|c|c|c|c|c|}
\hline K25C1101518 & VOA & Acrylonitrile & 1 & 0 & $\mathrm{ug} / \mathrm{kg}$ & 500 & 500 & 500 & & \\
\hline K25C1101518 & $\mathrm{VOA}$ & Benzene & 2 & 0 & $\mathrm{ug} / \mathrm{kg}$ & 0.01 & 100 & 50.005 & 50.005 & 70.703607 \\
\hline K25C1101518 & VOA & Bromobenzene & 1 & 0 & $\mathrm{ug} / \mathrm{kg}$ & 100 & 100 & 100 & & \\
\hline K25C1101518 & VOA & Bromochloromethane & 1 & 0 & $\mathrm{ug} / \mathrm{kg}$ & 100 & 100 & 100 & & \\
\hline K25C1101518 & VOA & Bromodichloromethane & 1 & 0 & $\mathrm{ug} / \mathrm{kg}$ & 100 & 100 & 100 & & \\
\hline K25C1101518 & VOA & Bromoform & 1 & 0 & $\mathrm{ug} / \mathrm{kg}$ & 100 & 100 & 100 & & \\
\hline K25C1101518 & VOA & Bromomethane & 1 & 0 & $\mathrm{ug} / \mathrm{kg}$ & 100 & 100 & 100 & & \\
\hline K25C1101518 & VOA & Butylbenzene & 1 & 0 & $\mathrm{ug} / \mathrm{kg}$ & 100 & 100 & 100 & & \\
\hline K25C1101518 & VOA & Carbon disulfide & 1 & 0 & $\mathrm{ug} / \mathrm{kg}$ & 500 & 500 & 500 & & \\
\hline K25C1101518 & VOA & Carbon tetrachloride & 2 & 0 & $\mathrm{ug} / \mathrm{kg}$ & 0.01 & 100 & 50.005 & 50.005 & 70.703607 \\
\hline K25C1101518 & VOA & Chlorobenzene & 2 & 0 & $\mathrm{ug} / \mathrm{kg}$ & 0.01 & 100 & 50.005 & 50.005 & 70.703607 \\
\hline K25C1101518 & VOA & Chloroethane & 1 & 0 & $\mathrm{ug} / \mathrm{kg}$ & 100 & 100 & 100 & & \\
\hline K25C1101518 & VOA & Chloroform & 2 & 0 & $\mathrm{ug} / \mathrm{kg}$ & 0.01 & 100 & 50.005 & 50.005 & 70.703607 \\
\hline K25C1101518 & VOA & Chloromethane & 1 & 0 & $\mathrm{ug} / \mathrm{kg}$ & 100 & 100 & 100 & & \\
\hline K25C1101518 & VOA & cis-1,2-Dichloroethene & 1 & 0 & $\mathrm{ug} / \mathrm{kg}$ & 100 & 100 & 100 & & \\
\hline K25C1101518 & VOA & cis-1,3-Dichloropropene & 1 & 0 & $\mathrm{ug} / \mathrm{kg}$ & 100 & 100 & 100 & & \\
\hline K25C1101518 & $\mathrm{VOA}$ & Cumene & 1 & 0 & $\mathrm{ug} / \mathrm{kg}$ & 100 & 100 & 100 & & \\
\hline K25C1101518 & VOA & Dibromochloromethane & 1 & 0 & $\mathrm{ug} / \mathrm{kg}$ & 100 & 100 & 100 & & \\
\hline K25C1101518 & VOA & Dibromomethane & 1 & 0 & $\mathrm{ug} / \mathrm{kg}$ & 100 & 100 & 100 & & \\
\hline K25C1101518 & VOA & Dichlorodifluoromethane & 1 & 0 & $\mathrm{ug} / \mathrm{kg}$ & 100 & 100 & 100 & & \\
\hline K25C1101518 & VOA & Ethylbenzene & 1 & 0 & $\mathrm{ug} / \mathrm{kg}$ & 100 & 100 & 100 & & \\
\hline K25C1101518 & VOA & Hexane & 1 & 0 & $\mathrm{ug} / \mathrm{kg}$ & 500 & 500 & 500 & & \\
\hline K25C1101518 & $\mathrm{VOA}$ & M + P Xylene & 1 & 0 & $\mathrm{ug} / \mathrm{kg}$ & 200 & 200 & 200 & & \\
\hline K25C1101518 & VOA & Methanol & 1 & 0 & $\mathrm{ug} / \mathrm{kg}$ & 5000 & 5000 & 5000 & & \\
\hline K25C1101518 & VOA & Methyl methacrylate & 1 & 0 & $\mathrm{ug} / \mathrm{kg}$ & 500 & 500 & 500 & & \\
\hline K25C1101518 & VOA & Methylcyclohexane & 1 & 0 & $\mathrm{ug} / \mathrm{kg}$ & 100 & 100 & 100 & & \\
\hline K25C1101518 & VOA & Methylene chloride & 1 & 0 & $\mathrm{ug} / \mathrm{kg}$ & 500 & 500 & 500 & & \\
\hline K25C1101518 & VOA & o-Chlorotoluene & 1 & 0 & $\mathrm{ug} / \mathrm{kg}$ & 100 & 100 & 100 & & \\
\hline K25C1101518 & VOA & Propylbenzene & 1 & 0 & $\mathrm{ug} / \mathrm{kg}$ & 100 & 100 & 100 & & \\
\hline K25C1101518 & VOA & Propylene glycol & 1 & 0 & $\mathrm{ug} / \mathrm{kg}$ & 50000 & 50000 & 50000 & & \\
\hline K25C1101518 & VOA & Styrene & 1 & 0 & $\mathrm{ug} / \mathrm{kg}$ & 100 & 100 & 100 & & \\
\hline K25C1101518 & $\mathrm{VOA}$ & Tetrachloroethene & 2 & 0 & $\mathrm{ug} / \mathrm{kg}$ & 0.01 & 100 & 50.005 & 50.005 & 70.703607 \\
\hline K25C1101518 & VOA & Toluene & 1 & 0 & $\mathrm{ug} / \mathrm{kg}$ & 100 & 100 & 100 & & \\
\hline K25C1101518 & VOA & Total Xylene & 1 & 0 & $\mathrm{ug} / \mathrm{kg}$ & 300 & 300 & 300 & & \\
\hline K25C1101518 & VOA & trans-1,2-Dichloroethene & 1 & 0 & $\mathrm{ug} / \mathrm{kg}$ & 100 & 100 & 100 & & \\
\hline K25C1101518 & VOA & trans-1,3-Dichloropropene & 1 & 0 & $\mathrm{ug} / \mathrm{kg}$ & 100 & 100 & 100 & & \\
\hline K25C1101518 & VOA & Trichloroethene & 2 & 0 & $\mathrm{ug} / \mathrm{kg}$ & 0.01 & 100 & 50.005 & 50.005 & 70.703607 \\
\hline K25C1101518 & VOA & Trichlorofluoromethane & 1 & 0 & $\mathrm{ug} / \mathrm{kg}$ & 100 & 100 & 100 & & \\
\hline K25C1101518 & VOA & Vinyl chloride & 2 & 0 & $\mathrm{ug} / \mathrm{kg}$ & 0.01 & 100 & 50.005 & 50.005 & 70.703607 \\
\hline Pallet with Misc Items & HERB & $2,4-\mathrm{D}$ & 2 & 0 & $\mathrm{ug} / \mathrm{kg}$ & 0.05 & 23.6 & 11.825 & 11.825 & 16.652365 \\
\hline Pallet with Misc Items & HERB & Silvex & 2 & 0 & $\mathrm{ug} / \mathrm{kg}$ & 0.05 & 23.6 & 11.825 & 11.825 & 16.652365 \\
\hline Pallet with Misc Items & METAL & Arsenic & 2 & 0 & $\mathrm{mg} / \mathrm{L}$ & 0.3 & 4.43 & 2.365 & 2.365 & 2.920351 \\
\hline Pallet with Misc Items & METAL & Barium & 1 & 1 & $\mathrm{mg} / \mathrm{L}$ & 0.269 & 0.269 & 0.269 & 0.269 & \\
\hline Pallet with Misc Items & METAL & Cadmium & 2 & 0 & $\mathrm{mg} / \mathrm{L}$ & 0.05 & 0.484 & 0.267 & 0.267 & 0.3068843 \\
\hline Pallet with Misc Items & METAL & Chromium & 2 & 1 & $\mathrm{mg} / \mathrm{L}$ & 0.05 & 4.78 & 2.415 & 2.415 & 3.3446151 \\
\hline Pallet with Misc Items & METAL & Lead & 2 & 1 & $\mathrm{mg} / \mathrm{L}$ & 0.1 & 42 & 21.05 & 21.05 & 29.627774 \\
\hline Pallet with Misc Items & METAL & Mercury & 2 & 0 & $\mathrm{mg} / \mathrm{L}$ & 0.002 & 0.4 & 0.201 & 0.201 & 0.2814285 \\
\hline Pallet with Misc Items & METAL & Selenium & 2 & 0 & $\mathrm{mg} / \mathrm{L}$ & 0.3 & 2.91 & 1.605 & 1.605 & 1.8455487 \\
\hline Pallet with Misc Items & METAL & Silver & 2 & 0 & $\mathrm{mg} / \mathrm{L}$ & 0.05 & 4.84 & 2.445 & 2.445 & 3.3870415 \\
\hline Pallet with Misc Items & METAL & Total Uranium & 1 & 0 & $\mathrm{pCi} / \mathrm{g}$ & 0.251 & 0.251 & 0.251 & & \\
\hline Pallet with Misc Items & PPCB & 4,4'-DDD & 1 & 0 & $\mathrm{ug} / \mathrm{kg}$ & 3.61 & 3.61 & 3.61 & & \\
\hline Pallet with Misc Items & PPCB & 4,4'-DDE & 1 & 0 & $\mathrm{ug} / \mathrm{kg}$ & 3.61 & 3.61 & 3.61 & & \\
\hline Pallet with Misc Items & РPCB & 4,4'-DDT & 1 & 0 & $\mathrm{ug} / \mathrm{kg}$ & 3.61 & 3.61 & 3.61 & & \\
\hline Pallet with Misc Items & РPCB & Aldrin & 1 & 0 & $\mathrm{ug} / \mathrm{kg}$ & 1.8 & 1.8 & 1.8 & & \\
\hline Pallet with Misc Items & РPCB & alpha-BHC & 1 & 0 & $\mathrm{ug} / \mathrm{kg}$ & 1.8 & 1.8 & 1.8 & & \\
\hline Pallet with Misc Items & РPCB & alpha-Chlordane & 1 & 0 & $\mathrm{ug} / \mathrm{kg}$ & 1.8 & 1.8 & 1.8 & & \\
\hline Pallet with Misc Items & PPCB & beta-BHC & 1 & 0 & $\mathrm{ug} / \mathrm{kg}$ & 1.8 & 1.8 & 1.8 & & \\
\hline Pallet with Misc Items & PPCB & Chlordane & 2 & 0 & $\mathrm{ug} / \mathrm{kg}$ & 0.0025 & 22.5 & 11.25125 & 11.25125 & 15.908135 \\
\hline Pallet with Misc Items & PPCB & delta-BHC & 1 & 0 & $\mathrm{ug} / \mathrm{kg}$ & 1.8 & 1.8 & 1.8 & & \\
\hline Pallet with Misc Items & PPCB & Dieldrin & 1 & 0 & $\mathrm{ug} / \mathrm{kg}$ & 3.61 & 3.61 & 3.61 & & \\
\hline Pallet with Misc Items & PPCB & Endosulfan I & 1 & 0 & $\mathrm{ug} / \mathrm{kg}$ & 1.8 & 1.8 & 1.8 & & \\
\hline Pallet with Misc Items & PPCB & Endosulfan II & 1 & 0 & $\mathrm{ug} / \mathrm{kg}$ & 3.61 & 3.61 & 3.61 & & \\
\hline Pallet with Misc Items & РPCB & Endosulfan sulfate & 1 & 0 & $\mathrm{ug} / \mathrm{kg}$ & 3.61 & 3.61 & 3.61 & & \\
\hline Pallet with Misc Items & PPCB & Endrin & 2 & 0 & $\mathrm{ug} / \mathrm{kg}$ & 0.0004 & 3.61 & 1.8052 & 1.8052 & 2.5523726 \\
\hline Pallet with Misc Items & PPCB & Endrin aldehyde & 1 & 0 & $\mathrm{ug} / \mathrm{kg}$ & 3.61 & 3.61 & 3.61 & & \\
\hline Pallet with Misc Items & PPCB & Endrin ketone & 1 & 0 & $\mathrm{ug} / \mathrm{kg}$ & 3.61 & 3.61 & 3.61 & & \\
\hline Pallet with Misc Items & РPCB & gamma-Chlordane & 1 & 0 & $\mathrm{ug} / \mathrm{kg}$ & 1.8 & 1.8 & 1.8 & & \\
\hline Pallet with Misc Items & PPCB & Heptachlor & 2 & 0 & $\mathrm{ug} / \mathrm{kg}$ & 0.0002 & 1.8 & 0.9001 & 0.9001 & 1.2726508 \\
\hline Pallet with Misc Items & PPCB & Heptachlor epoxide & 2 & 0 & $\mathrm{ug} / \mathrm{kg}$ & 0.0002 & 1.8 & 0.9001 & 0.9001 & 1.2726508 \\
\hline Pallet with Misc Items & PPCB & Lindane & 2 & 0 & $\mathrm{ug} / \mathrm{kg}$ & 0.0002 & 1.8 & 0.9001 & 0.9001 & 1.2726508 \\
\hline
\end{tabular}




\begin{tabular}{|c|c|c|c|c|c|c|c|c|c|c|}
\hline Pallet with Misc Items & РPCB & Methoxychlor & 2 & 0 & $\mathrm{ug} / \mathrm{kg}$ & 0.002 & 18 & 9.001 & 9.001 & 12.726508 \\
\hline Pallet with Misc Items & РPCB & Toxaphene & 2 & 0 & $\mathrm{ug} / \mathrm{kg}$ & 0.005 & 45.1 & 22.5525 & 22.5525 & 31.88698 \\
\hline Pallet with Misc Items & RADS & Americium-241 & 1 & 0 & $\mathrm{pCi} / \mathrm{g}$ & 0.129 & 0.129 & 0.129 & & \\
\hline Pallet with Misc Items & RADS & Carbon-14 & 1 & 0 & $\mathrm{pCi} / \mathrm{g}$ & 10.4 & 10.4 & 10.4 & & \\
\hline Pallet with Misc Items & RADS & Cesium-137 & 1 & 0 & $\mathrm{pCi} / \mathrm{g}$ & 0.0235 & 0.0235 & 0.0235 & & \\
\hline Pallet with Misc Items & RADS & Cobalt- 60 & 1 & 0 & $\mathrm{pCi} / \mathrm{g}$ & -0.00561 & -0.00561 & -0.00561 & & \\
\hline Pallet with Misc Items & RADS & Curium-243/244 & 1 & 0 & $\mathrm{pCi} / \mathrm{g}$ & 0.127 & 0.127 & 0.127 & & \\
\hline Pallet with Misc Items & RADS & Iodine-129 & 1 & 0 & $\mathrm{pCi} / \mathrm{g}$ & 0.334 & 0.334 & 0.334 & & \\
\hline Pallet with Misc Items & RADS & Neptunium-237 & 1 & 0 & $\mathrm{pCi} / \mathrm{g}$ & 0.0354 & 0.0354 & 0.0354 & & \\
\hline Pallet with Misc Items & RADS & Plutonium-238 & 1 & 0 & $\mathrm{pCi} / \mathrm{g}$ & -0.0119 & -0.0119 & -0.0119 & & \\
\hline Pallet with Misc Items & RADS & Plutonium-239/240 & 1 & 0 & $\mathrm{pCi} / \mathrm{g}$ & 0.0126 & 0.0126 & 0.0126 & & \\
\hline Pallet with Misc Items & RADS & Radium-226 & 1 & 1 & $\mathrm{pCi} / \mathrm{g}$ & 0.0744 & 0.0744 & 0.0744 & 0.0744 & \\
\hline Pallet with Misc Items & RADS & Strontium-90 & 1 & 0 & $\mathrm{pCi} / \mathrm{g}$ & -0.328 & -0.328 & -0.328 & & \\
\hline Pallet with Misc Items & RADS & Technetium-99 & 1 & 0 & $\mathrm{pCi} / \mathrm{g}$ & -0.23 & -0.23 & -0.23 & & \\
\hline Pallet with Misc Items & RADS & Thorium-230 & 1 & 1 & $\mathrm{pCi} / \mathrm{g}$ & 0.0744 & 0.0744 & 0.0744 & 0.0744 & \\
\hline Pallet with Misc Items & RADS & Thorium-232 & 1 & 0 & $\mathrm{pCi} / \mathrm{g}$ & 0.125 & 0.125 & 0.125 & & \\
\hline Pallet with Misc Items & RADS & Tritium & 1 & 0 & $\mathrm{pCi} / \mathrm{g}$ & 136 & 136 & 136 & & \\
\hline Pallet with Misc Items & RADS & Uranium-232 & 1 & 1 & $\%$ & 90 & 90 & 90 & 90 & \\
\hline Pallet with Misc Items & RADS & Uranium-233/234 & 1 & 0 & $\mathrm{pCi} / \mathrm{g}$ & 0.116 & 0.116 & 0.116 & & \\
\hline Pallet with Misc Items & RADS & Uranium-235/236 & 1 & 0 & $\mathrm{pCi} / \mathrm{g}$ & 0.00456 & 0.00456 & 0.00456 & & \\
\hline Pallet with Misc Items & RADS & Uranium-238 & 1 & 1 & $\mathrm{pCi} / \mathrm{g}$ & 0.131 & 0.131 & 0.131 & 0.131 & \\
\hline Pallet with Misc Items & SVOA & 1,2,4-Trichlorobenzene & 1 & 0 & $\mathrm{ug} / \mathrm{kg}$ & 98 & 98 & 98 & & \\
\hline Pallet with Misc Items & SVOA & 1,2-Dichlorobenzene & 1 & 0 & $\mathrm{ug} / \mathrm{kg}$ & 98 & 98 & 98 & & \\
\hline Pallet with Misc Items & SVOA & 1,3-Dichlorobenzene & 1 & 0 & $\mathrm{ug} / \mathrm{kg}$ & 98 & 98 & 98 & & \\
\hline Pallet with Misc Items & SVOA & 1,4-Dichlorobenzene & 2 & 0 & $\mathrm{ug} / \mathrm{kg}$ & 0.01 & 98 & 49.005 & 49.005 & 69.289393 \\
\hline Pallet with Misc Items & SVOA & 2,2'-Dichlorodiisopropyl ether & 1 & 0 & $\mathrm{ug} / \mathrm{kg}$ & 992 & 992 & 992 & & \\
\hline Pallet with Misc Items & SVOA & 2,3,4,6-Tetrachlorophenol & 1 & 0 & $\mathrm{ug} / \mathrm{kg}$ & 992 & 992 & 992 & & \\
\hline Pallet with Misc Items & SVOA & 2,4,5-Trichlorophenol & 2 & 0 & $\mathrm{ug} / \mathrm{kg}$ & 0.05 & 992 & 496.025 & 496.025 & 701.41457 \\
\hline Pallet with Misc Items & SVOA & 2,4,6-Trichlorophenol & 2 & 0 & $\mathrm{ug} / \mathrm{kg}$ & 0.05 & 992 & 496.025 & 496.025 & 701.41457 \\
\hline Pallet with Misc Items & SVOA & 2,4-Dichlorophenol & 1 & 0 & $\mathrm{ug} / \mathrm{kg}$ & 992 & 992 & 992 & & \\
\hline Pallet with Misc Items & SVOA & 2,4-Dimethylphenol & 1 & 0 & $\mathrm{ug} / \mathrm{kg}$ & 992 & 992 & 992 & & \\
\hline Pallet with Misc Items & SVOA & 2,4-Dinitrophenol & 1 & 0 & $\mathrm{ug} / \mathrm{kg}$ & 1980 & 1980 & 1980 & & \\
\hline Pallet with Misc Items & SVOA & 2,4-Dinitrotoluene & 2 & 0 & $\mathrm{ug} / \mathrm{kg}$ & 0.05 & 992 & 496.025 & 496.025 & 701.41457 \\
\hline Pallet with Misc Items & SVOA & 2,6-Dinitrotoluene & 1 & 0 & $\mathrm{ug} / \mathrm{kg}$ & 992 & 992 & 992 & & \\
\hline Pallet with Misc Items & SVOA & 2-Chloronaphthalene & 1 & 0 & $\mathrm{ug} / \mathrm{kg}$ & 99.2 & 99.2 & 99.2 & & \\
\hline Pallet with Misc Items & SVOA & 2-Chlorophenol & 1 & 0 & $\mathrm{ug} / \mathrm{kg}$ & 992 & 992 & 992 & & \\
\hline Pallet with Misc Items & SVOA & 2-Methyl-4,6-dinitrophenol & 1 & 0 & $\mathrm{ug} / \mathrm{kg}$ & 992 & 992 & 992 & & \\
\hline Pallet with Misc Items & SVOA & 2-Methylnaphthalene & 1 & 0 & $\mathrm{ug} / \mathrm{kg}$ & 99.2 & 99.2 & 99.2 & & \\
\hline Pallet with Misc Items & SVOA & 2-Methylphenol & 2 & 0 & $\mathrm{ug} / \mathrm{kg}$ & 0.05 & 992 & 496.025 & 496.025 & 701.41457 \\
\hline Pallet with Misc Items & SVOA & 2-Nitrobenzenamine & 1 & 0 & $\mathrm{ug} / \mathrm{kg}$ & 992 & 992 & 992 & & \\
\hline Pallet with Misc Items & SVOA & 2-Nitrophenol & 1 & 0 & $\mathrm{ug} / \mathrm{kg}$ & 992 & 992 & 992 & & \\
\hline Pallet with Misc Items & SVOA & 3,3'-Dichlorobenzidine & 1 & 0 & $\mathrm{ug} / \mathrm{kg}$ & 992 & 992 & 992 & & \\
\hline Pallet with Misc Items & SVOA & 3-Nitrobenzenamine & 1 & 0 & $\mathrm{ug} / \mathrm{kg}$ & 992 & 992 & 992 & & \\
\hline Pallet with Misc Items & SVOA & 4-Bromophenyl phenyl ether & 1 & 0 & $\mathrm{ug} / \mathrm{kg}$ & 992 & 992 & 992 & & \\
\hline Pallet with Misc Items & SVOA & 4-Chloro-3-methylphenol & 1 & 0 & $\mathrm{ug} / \mathrm{kg}$ & 992 & 992 & 992 & & \\
\hline Pallet with Misc Items & SVOA & 4-Chlorobenzenamine & 1 & 0 & $\mathrm{ug} / \mathrm{kg}$ & 992 & 992 & 992 & & \\
\hline Pallet with Misc Items & SVOA & 4-Chlorophenyl phenyl ether & 1 & 0 & $\mathrm{ug} / \mathrm{kg}$ & 992 & 992 & 992 & & \\
\hline Pallet with Misc Items & SVOA & 4-Nitrobenzenamine & 1 & 0 & $\mathrm{ug} / \mathrm{kg}$ & 992 & 992 & 992 & & \\
\hline Pallet with Misc Items & SVOA & 4-Nitrophenol & 1 & 0 & $\mathrm{ug} / \mathrm{kg}$ & 992 & 992 & 992 & & \\
\hline Pallet with Misc Items & SVOA & Acenaphthene & 1 & 0 & $\mathrm{ug} / \mathrm{kg}$ & 99.2 & 99.2 & 99.2 & & \\
\hline Pallet with Misc Items & SVOA & Acenaphthylene & 1 & 0 & $\mathrm{ug} / \mathrm{kg}$ & 99.2 & 99.2 & 99.2 & & \\
\hline Pallet with Misc Items & SVOA & Acetophenone & 1 & 0 & $\mathrm{ug} / \mathrm{kg}$ & 992 & 992 & 992 & & \\
\hline Pallet with Misc Items & SVOA & Anthracene & 1 & 0 & $\mathrm{ug} / \mathrm{kg}$ & 99.2 & 99.2 & 99.2 & & \\
\hline Pallet with Misc Items & SVOA & Benz(a)anthracene & 1 & 0 & $\mathrm{ug} / \mathrm{kg}$ & 99.2 & 99.2 & 99.2 & & \\
\hline Pallet with Misc Items & SVOA & Benzenemethanol & 1 & 0 & $\mathrm{ug} / \mathrm{kg}$ & 992 & 992 & 992 & & \\
\hline Pallet with Misc Items & SVOA & Benzidine & 1 & 0 & $\mathrm{ug} / \mathrm{kg}$ & 992 & 992 & 992 & & \\
\hline Pallet with Misc Items & SVOA & Benzo(a)pyrene & 1 & 0 & $\mathrm{ug} / \mathrm{kg}$ & 99.2 & 99.2 & 99.2 & & \\
\hline Pallet with Misc Items & SVOA & Benzo(b)fluoranthene & 1 & 0 & $\mathrm{ug} / \mathrm{kg}$ & 99.2 & 99.2 & 99.2 & & \\
\hline Pallet with Misc Items & SVOA & Benzo(ghi)perylene & 1 & 0 & $\mathrm{ug} / \mathrm{kg}$ & 99.2 & 99.2 & 99.2 & & \\
\hline Pallet with Misc Items & SVOA & Benzo(k)fluoranthene & 1 & 0 & $\mathrm{ug} / \mathrm{kg}$ & 99.2 & 99.2 & 99.2 & & \\
\hline Pallet with Misc Items & SVOA & Benzoic acid & 1 & 0 & $\mathrm{ug} / \mathrm{kg}$ & 1980 & 1980 & 1980 & & \\
\hline Pallet with Misc Items & SVOA & Bis(2-chloroethoxy)methane & 1 & 0 & $\mathrm{ug} / \mathrm{kg}$ & 992 & 992 & 992 & & \\
\hline Pallet with Misc Items & SVOA & Bis(2-chloroethyl) ether & 1 & 0 & $\mathrm{ug} / \mathrm{kg}$ & 992 & 992 & 992 & & \\
\hline Pallet with Misc Items & SVOA & Bis(2-ethylhexyl)phthalate & 1 & 0 & $\mathrm{ug} / \mathrm{kg}$ & 992 & 992 & 992 & & \\
\hline Pallet with Misc Items & SVOA & Butyl benzyl phthalate & 1 & 0 & $\mathrm{ug} / \mathrm{kg}$ & 992 & 992 & 992 & & \\
\hline Pallet with Misc Items & SVOA & Carbazole & 1 & 0 & $\mathrm{ug} / \mathrm{kg}$ & 99.2 & 99.2 & 99.2 & & \\
\hline Pallet with Misc Items & SVOA & Chrysene & 1 & 0 & $\mathrm{ug} / \mathrm{kg}$ & 99.2 & 99.2 & 99.2 & & \\
\hline Pallet with Misc Items & SVOA & $\operatorname{Dibenz}(\mathrm{a}, \mathrm{h})$ anthracene & 1 & 0 & $\mathrm{ug} / \mathrm{kg}$ & 99.2 & 99.2 & 99.2 & & \\
\hline Pallet with Misc Items & SVOA & Dibenzofuran & 1 & 0 & $\mathrm{ug} / \mathrm{kg}$ & 992 & 992 & 992 & & \\
\hline Pallet with Misc Items & SVOA & Diethyl phthalate & 1 & 0 & $\mathrm{ug} / \mathrm{kg}$ & 992 & 992 & 992 & & \\
\hline
\end{tabular}




\begin{tabular}{|c|c|c|c|c|c|c|c|c|c|c|}
\hline Pallet with Misc Items & SVOA & Dimethyl phthalate & 1 & 0 & $\mathrm{ug} / \mathrm{kg}$ & 992 & 992 & 992 & & \\
\hline Pallet with Misc Items & SVOA & Di-n-butyl phthalate & 1 & 0 & $\mathrm{ug} / \mathrm{kg}$ & 992 & 992 & 992 & & \\
\hline Pallet with Misc Items & SVOA & Di-n-octylphthalate & 1 & 0 & $\mathrm{ug} / \mathrm{kg}$ & 992 & 992 & 992 & & \\
\hline Pallet with Misc Items & SVOA & Diphenylamine & 1 & 0 & $\mathrm{ug} / \mathrm{kg}$ & 992 & 992 & 992 & & \\
\hline Pallet with Misc Items & SVOA & Fluoranthene & 1 & 0 & $\mathrm{ug} / \mathrm{kg}$ & 99.2 & 99.2 & 99.2 & & \\
\hline Pallet with Misc Items & SVOA & Fluorene & 1 & 0 & $\mathrm{ug} / \mathrm{kg}$ & 99.2 & 99.2 & 99.2 & & \\
\hline Pallet with Misc Items & SVOA & Hexachlorobenzene & 2 & 0 & $\mathrm{ug} / \mathrm{kg}$ & 0.05 & 992 & 496.025 & 496.025 & 701.41457 \\
\hline Pallet with Misc Items & SVOA & Hexachlorobutadiene & 2 & 0 & $\mathrm{ug} / \mathrm{kg}$ & 0.05 & 992 & 496.025 & 496.025 & 701.41457 \\
\hline Pallet with Misc Items & SVOA & Hexachlorocyclopentadiene & 1 & 0 & $\mathrm{ug} / \mathrm{kg}$ & 992 & 992 & 992 & & \\
\hline Pallet with Misc Items & SVOA & Hexachloroethane & 2 & 0 & $\mathrm{ug} / \mathrm{kg}$ & 0.05 & 992 & 496.025 & 496.025 & 701.41457 \\
\hline Pallet with Misc Items & SVOA & Indeno(1,2,3-cd)pyrene & 1 & 0 & $\mathrm{ug} / \mathrm{kg}$ & 99.2 & 99.2 & 99.2 & & \\
\hline Pallet with Misc Items & SVOA & Isophorone & 1 & 0 & $\mathrm{ug} / \mathrm{kg}$ & 992 & 992 & 992 & & \\
\hline Pallet with Misc Items & SVOA & $\mathrm{m}+\mathrm{p}$ Methylphenol & 2 & 0 & $\mathrm{ug} / \mathrm{kg}$ & 0.05 & 992 & 496.025 & 496.025 & 701.41457 \\
\hline Pallet with Misc Items & SVOA & Naphthalene & 1 & 0 & $\mathrm{ug} / \mathrm{kg}$ & 99.2 & 99.2 & 99.2 & & \\
\hline Pallet with Misc Items & SVOA & Nitrobenzene & 2 & 0 & $\mathrm{ug} / \mathrm{kg}$ & 0.05 & 992 & 496.025 & 496.025 & 701.41457 \\
\hline Pallet with Misc Items & SVOA & N-Nitroso-di-n-propylamine & 1 & 0 & $\mathrm{ug} / \mathrm{kg}$ & 992 & 992 & 992 & & \\
\hline Pallet with Misc Items & SVOA & Pentachlorophenol & 2 & 0 & $\mathrm{ug} / \mathrm{kg}$ & 0.05 & 992 & 496.025 & 496.025 & 701.41457 \\
\hline Pallet with Misc Items & SVOA & Phenanthrene & 1 & 0 & $\mathrm{ug} / \mathrm{kg}$ & 99.2 & 99.2 & 99.2 & & \\
\hline Pallet with Misc Items & SVOA & Phenol & 1 & 0 & $\mathrm{ug} / \mathrm{kg}$ & 992 & 992 & 992 & & \\
\hline Pallet with Misc Items & SVOA & Pyridine & 2 & 0 & $\mathrm{ug} / \mathrm{kg}$ & 0.05 & 992 & 496.025 & 496.025 & 701.41457 \\
\hline Pallet with Misc Items & VOA & (1,1-Dimethylethyl)benzene & 1 & 0 & $\mathrm{ug} / \mathrm{kg}$ & 98 & 98 & 98 & & \\
\hline Pallet with Misc Items & VOA & (1-Methylpropyl)benzene & 1 & 0 & $\mathrm{ug} / \mathrm{kg}$ & 98 & 98 & 98 & & \\
\hline Pallet with Misc Items & VOA & 1,1,1,2-Tetrachloroethane & 1 & 0 & $\mathrm{ug} / \mathrm{kg}$ & 98 & 98 & 98 & & \\
\hline Pallet with Misc Items & VOA & 1,1,1-Trichloroethane & 1 & 0 & $\mathrm{ug} / \mathrm{kg}$ & 98 & 98 & 98 & & \\
\hline Pallet with Misc Items & VOA & 1,1,2,2-Tetrachloroethane & 1 & 0 & $\mathrm{ug} / \mathrm{kg}$ & 98 & 98 & 98 & & \\
\hline Pallet with Misc Items & VOA & 1,1,2-Trichloroethane & 1 & 0 & $\mathrm{ug} / \mathrm{kg}$ & 98 & 98 & 98 & & \\
\hline Pallet with Misc Items & VOA & 1,1-Dichloroethane & 1 & 0 & $\mathrm{ug} / \mathrm{kg}$ & 98 & 98 & 98 & & \\
\hline Pallet with Misc Items & VOA & 1,1-Dichloroethene & 2 & 0 & $\mathrm{ug} / \mathrm{kg}$ & 0.01 & 98 & 49.005 & 49.005 & 69.289393 \\
\hline Pallet with Misc Items & VOA & 1,1-Dichloropropene & 1 & 0 & $\mathrm{ug} / \mathrm{kg}$ & 98 & 98 & 98 & & \\
\hline Pallet with Misc Items & VOA & 1,2,3-Trichlorobenzene & 1 & 0 & $\mathrm{ug} / \mathrm{kg}$ & 98 & 98 & 98 & & \\
\hline Pallet with Misc Items & VOA & 1,2,3-Trichloropropane & 1 & 0 & $\mathrm{ug} / \mathrm{kg}$ & 98 & 98 & 98 & & \\
\hline Pallet with Misc Items & VOA & 1,2,4-Trimethylbenzene & 1 & 0 & $\mathrm{ug} / \mathrm{kg}$ & 98 & 98 & 98 & & \\
\hline Pallet with Misc Items & VOA & 1,2-Dibromo-3-chloropropane & 1 & 0 & $\mathrm{ug} / \mathrm{kg}$ & 98 & 98 & 98 & & \\
\hline Pallet with Misc Items & VOA & 1,2-Dibromoethane & 1 & 0 & $\mathrm{ug} / \mathrm{kg}$ & 98 & 98 & 98 & & \\
\hline Pallet with Misc Items & VOA & 1,2-Dichloroethane & 2 & 0 & $\mathrm{ug} / \mathrm{kg}$ & 0.01 & 98 & 49.005 & 49.005 & 69.289393 \\
\hline Pallet with Misc Items & VOA & 1,2-Dichloroethene & 1 & 0 & $\mathrm{ug} / \mathrm{kg}$ & 196 & 196 & 196 & & \\
\hline Pallet with Misc Items & VOA & 1,2-Dichloropropane & 1 & 0 & $\mathrm{ug} / \mathrm{kg}$ & 98 & 98 & 98 & & \\
\hline Pallet with Misc Items & VOA & 1,2-Dimethylbenzene & 1 & 0 & $\mathrm{ug} / \mathrm{kg}$ & 98 & 98 & 98 & & \\
\hline Pallet with Misc Items & VOA & 1,3,5-Trimethylbenzene & 1 & 0 & $\mathrm{ug} / \mathrm{kg}$ & 98 & 98 & 98 & & \\
\hline Pallet with Misc Items & VOA & 1,3-Dichloropropane & 1 & 0 & $\mathrm{ug} / \mathrm{kg}$ & 98 & 98 & 98 & & \\
\hline Pallet with Misc Items & VOA & 1-Chloro-4-methylbenzene & 1 & 0 & $\mathrm{ug} / \mathrm{kg}$ & 98 & 98 & 98 & & \\
\hline Pallet with Misc Items & VOA & 1-Methyl-4-(1-methylethyl)benzene & 1 & 0 & $\mathrm{ug} / \mathrm{kg}$ & 98 & 98 & 98 & & \\
\hline Pallet with Misc Items & VOA & 2,2-Dichloropropane & 1 & 0 & $\mathrm{ug} / \mathrm{kg}$ & 98 & 98 & 98 & & \\
\hline Pallet with Misc Items & VOA & 2-Butanone & 2 & 0 & $\mathrm{ug} / \mathrm{kg}$ & 0.05 & 490 & 245.025 & 245.025 & 346.44697 \\
\hline Pallet with Misc Items & VOA & 2-Hexanone & 1 & 0 & $\mathrm{ug} / \mathrm{kg}$ & 490 & 490 & 490 & & \\
\hline Pallet with Misc Items & VOA & 4-Methyl-2-pentanone & 1 & 0 & $\mathrm{ug} / \mathrm{kg}$ & 490 & 490 & 490 & & \\
\hline Pallet with Misc Items & VOA & Acetone & 1 & 0 & $\mathrm{ug} / \mathrm{kg}$ & 490 & 490 & 490 & & \\
\hline Pallet with Misc Items & VOA & Acetonitrile & 1 & 0 & $\mathrm{ug} / \mathrm{kg}$ & 2450 & 2450 & 2450 & & \\
\hline Pallet with Misc Items & VOA & Acrolein & 1 & 0 & $\mathrm{ug} / \mathrm{kg}$ & 490 & 490 & 490 & & \\
\hline Pallet with Misc Items & VOA & Acrylonitrile & 1 & 0 & $\mathrm{ug} / \mathrm{kg}$ & 490 & 490 & 490 & & \\
\hline Pallet with Misc Items & VOA & Benzene & 2 & 0 & $\mathrm{ug} / \mathrm{kg}$ & 0.01 & 98 & 49.005 & 49.005 & 69.289393 \\
\hline Pallet with Misc Items & VOA & Bromobenzene & 1 & 0 & $\mathrm{ug} / \mathrm{kg}$ & 98 & 98 & 98 & & \\
\hline Pallet with Misc Items & VOA & Bromochloromethane & 1 & 0 & $\mathrm{ug} / \mathrm{kg}$ & 98 & 98 & 98 & & \\
\hline Pallet with Misc Items & VOA & Bromodichloromethane & 1 & 0 & $\mathrm{ug} / \mathrm{kg}$ & 98 & 98 & 98 & & \\
\hline Pallet with Misc Items & VOA & Bromoform & 1 & 0 & $\mathrm{ug} / \mathrm{kg}$ & 98 & 98 & 98 & & \\
\hline Pallet with Misc Items & VOA & Bromomethane & 1 & 0 & $\mathrm{ug} / \mathrm{kg}$ & 98 & 98 & 98 & & \\
\hline Pallet with Misc Items & VOA & Butylbenzene & 1 & 0 & $\mathrm{ug} / \mathrm{kg}$ & 98 & 98 & 98 & & \\
\hline Pallet with Misc Items & VOA & Carbon disulfide & 1 & 0 & $\mathrm{ug} / \mathrm{kg}$ & 490 & 490 & 490 & & \\
\hline Pallet with Misc Items & VOA & Carbon tetrachloride & 2 & 0 & $\mathrm{ug} / \mathrm{kg}$ & 0.01 & 98 & 49.005 & 49.005 & 69.289393 \\
\hline Pallet with Misc Items & VOA & Chlorobenzene & 2 & 0 & $\mathrm{ug} / \mathrm{kg}$ & 0.01 & 98 & 49.005 & 49.005 & 69.289393 \\
\hline Pallet with Misc Items & VOA & Chloroethane & 1 & 0 & $\mathrm{ug} / \mathrm{kg}$ & 98 & 98 & 98 & & \\
\hline Pallet with Misc Items & VOA & Chloroform & 2 & 0 & $\mathrm{ug} / \mathrm{kg}$ & 0.01 & 98 & 49.005 & 49.005 & 69.289393 \\
\hline Pallet with Misc Items & VOA & Chloromethane & 1 & 0 & $\mathrm{ug} / \mathrm{kg}$ & 98 & 98 & 98 & & \\
\hline Pallet with Misc Items & VOA & cis-1,2-Dichloroethene & 1 & 0 & $\mathrm{ug} / \mathrm{kg}$ & 98 & 98 & 98 & & \\
\hline Pallet with Misc Items & VOA & cis-1,3-Dichloropropene & 1 & 0 & $\mathrm{ug} / \mathrm{kg}$ & 98 & 98 & 98 & & \\
\hline Pallet with Misc Items & VOA & Cumene & 1 & 0 & $\mathrm{ug} / \mathrm{kg}$ & 98 & 98 & 98 & & \\
\hline Pallet with Misc Items & VOA & Dibromochloromethane & 1 & 0 & $\mathrm{ug} / \mathrm{kg}$ & 98 & 98 & 98 & & \\
\hline Pallet with Misc Items & VOA & Dibromomethane & 1 & 0 & $\mathrm{ug} / \mathrm{kg}$ & 98 & 98 & 98 & & \\
\hline Pallet with Misc Items & VOA & Dichlorodifluoromethane & 1 & 0 & $\mathrm{ug} / \mathrm{kg}$ & 98 & 98 & 98 & & \\
\hline Pallet with Misc Items & VOA & Ethylbenzene & 1 & 0 & $\mathrm{ug} / \mathrm{kg}$ & 98 & 98 & 98 & & \\
\hline
\end{tabular}




\begin{tabular}{|c|c|c|c|c|c|c|c|c|c|c|}
\hline Pallet with Misc Items & VOA & Hexane & 1 & 0 & $\mathrm{ug} / \mathrm{kg}$ & 490 & 490 & 490 & & \\
\hline Pallet with Misc Items & VOA & $\mathrm{M}+\mathrm{P}$ Xylene & 1 & 0 & $\mathrm{ug} / \mathrm{kg}$ & 196 & 196 & 196 & & \\
\hline Pallet with Misc Items & VOA & Methanol & 1 & 0 & $\mathrm{ug} / \mathrm{kg}$ & 5000 & 5000 & 5000 & & \\
\hline Pallet with Misc Items & VOA & Methyl methacrylate & 1 & 0 & $\mathrm{ug} / \mathrm{kg}$ & 490 & 490 & 490 & & \\
\hline Pallet with Misc Items & VOA & Methylcyclohexane & 1 & 0 & $\mathrm{ug} / \mathrm{kg}$ & 98 & 98 & 98 & & \\
\hline Pallet with Misc Items & VOA & Methylene chloride & 1 & 0 & $\mathrm{ug} / \mathrm{kg}$ & 490 & 490 & 490 & & \\
\hline Pallet with Misc Items & VOA & o-Chlorotoluene & 1 & 0 & $\mathrm{ug} / \mathrm{kg}$ & 98 & 98 & 98 & & \\
\hline Pallet with Misc Items & VOA & Propylbenzene & 1 & 0 & $\mathrm{ug} / \mathrm{kg}$ & 98 & 98 & 98 & & \\
\hline Pallet with Misc Items & VOA & Propylene glycol & 1 & 0 & $\mathrm{ug} / \mathrm{kg}$ & 50000 & 50000 & 50000 & & \\
\hline Pallet with Misc Items & VOA & Styrene & 1 & 0 & $\mathrm{ug} / \mathrm{kg}$ & 98 & 98 & 98 & & \\
\hline Pallet with Misc Items & VOA & Tetrachloroethene & 2 & 0 & $\mathrm{ug} / \mathrm{kg}$ & 0.01 & 98 & 49.005 & 49.005 & 69.289393 \\
\hline Pallet with Misc Items & VOA & Toluene & 1 & 0 & $\mathrm{ug} / \mathrm{kg}$ & 98 & 98 & 98 & & \\
\hline Pallet with Misc Items & VOA & Total Xylene & 1 & 0 & $\mathrm{ug} / \mathrm{kg}$ & 294 & 294 & 294 & & \\
\hline Pallet with Misc Items & VOA & trans-1,2-Dichloroethene & 1 & 0 & $\mathrm{ug} / \mathrm{kg}$ & 98 & 98 & 98 & & \\
\hline Pallet with Misc Items & VOA & trans-1,3-Dichloropropene & 1 & 0 & $\mathrm{ug} / \mathrm{kg}$ & 98 & 98 & 98 & & \\
\hline Pallet with Misc Items & VOA & Trichloroethene & 2 & 0 & $\mathrm{ug} / \mathrm{kg}$ & 0.01 & 98 & 49.005 & 49.005 & 69.289393 \\
\hline Pallet with Misc Items & VOA & Trichlorofluoromethane & 1 & 0 & $\mathrm{ug} / \mathrm{kg}$ & 98 & 98 & 98 & & \\
\hline Pallet with Misc Items & $\mathrm{VOA}$ & Vinyl chloride & 2 & 0 & $\mathrm{ug} / \mathrm{kg}$ & 0.01 & 98 & 49.005 & 49.005 & 69.289393 \\
\hline US-DOE-X134120 & HERB & $2,4-\mathrm{D}$ & 2 & 0 & $\mathrm{ug} / \mathrm{kg}$ & 0.05 & 250 & 125.025 & 125.025 & 176.74134 \\
\hline US-DOE-X134120 & HERB & Silvex & 2 & 0 & $\mathrm{ug} / \mathrm{kg}$ & 0.05 & 250 & 125.025 & 125.025 & 176.74134 \\
\hline US-DOE-X134120 & METAL & Arsenic & 2 & 0 & $\mathrm{mg} / \mathrm{L}$ & 0.3 & 144 & 72.15 & 72.15 & 101.61124 \\
\hline US-DOE-X134120 & METAL & Barium & 2 & 2 & $\mathrm{mg} / \mathrm{L}$ & 0.0792 & 24.9 & 12.4896 & 12.4896 & 17.550956 \\
\hline US-DOE-X134120 & METAL & Cadmium & 2 & 1 & $\mathrm{mg} / \mathrm{L}$ & 0.109 & 24 & 12.0545 & 12.0545 & 16.893488 \\
\hline US-DOE-X134120 & METAL & Chromium & 2 & 1 & $\mathrm{mg} / \mathrm{L}$ & 0.05 & 93.9 & 46.975 & 46.975 & 66.361971 \\
\hline US-DOE-X134120 & METAL & Lead & 2 & 2 & $\mathrm{mg} / \mathrm{L}$ & 1.09 & 5870 & 2935.545 & 2935.545 & 4149.9461 \\
\hline US-DOE-X134120 & METAL & Mercury & 2 & 0 & $\mathrm{mg} / \mathrm{L}$ & 0.002 & 0.4 & 0.201 & 0.201 & 0.2814285 \\
\hline US-DOE-X134120 & METAL & Selenium & 2 & 0 & $\mathrm{mg} / \mathrm{L}$ & 0.3 & 144 & 72.15 & 72.15 & 101.61124 \\
\hline US-DOE-X134120 & METAL & Silver & 2 & 1 & $\mathrm{mg} / \mathrm{L}$ & 0.05 & 86.2 & 43.125 & 43.125 & 60.917249 \\
\hline US-DOE-X134120 & METAL & Total Uranium & 1 & 0 & $\mathrm{pCi} / \mathrm{g}$ & 0.0485 & 0.0485 & 0.0485 & & \\
\hline US-DOE-X134120 & PPCB & 4,4'-DDD & 1 & 0 & $\mathrm{ug} / \mathrm{kg}$ & 40 & 40 & 40 & & \\
\hline US-DOE-X134120 & РPCB & 4,4'-DDE & 1 & 0 & $\mathrm{ug} / \mathrm{kg}$ & 40 & 40 & 40 & & \\
\hline US-DOE-X134120 & РPCB & 4,4'-DDT & 1 & 0 & $\mathrm{ug} / \mathrm{kg}$ & 40 & 40 & 40 & & \\
\hline US-DOE-X134120 & РРCВ & Aldrin & 1 & 0 & $\mathrm{ug} / \mathrm{kg}$ & 20 & 20 & 20 & & \\
\hline US-DOE-X134120 & PPCB & alpha-BHC & 1 & 0 & $\mathrm{ug} / \mathrm{kg}$ & 20 & 20 & 20 & & \\
\hline US-DOE-X134120 & РPCB & alpha-Chlordane & 1 & 0 & $\mathrm{ug} / \mathrm{kg}$ & 20 & 20 & 20 & & \\
\hline US-DOE-X134120 & РPCB & beta-BHC & 1 & 0 & $\mathrm{ug} / \mathrm{kg}$ & 20 & 20 & 20 & & \\
\hline US-DOE-X134120 & РPCB & Chlordane & 2 & 0 & $\mathrm{ug} / \mathrm{kg}$ & 0.0025 & 250 & 125.00125 & 125.00125 & 176.77493 \\
\hline US-DOE-X134120 & РРСВ & delta-BHC & 1 & 0 & $\mathrm{ug} / \mathrm{kg}$ & 20 & 20 & 20 & & \\
\hline US-DOE-X134120 & РPCB & Dieldrin & 1 & 0 & $\mathrm{ug} / \mathrm{kg}$ & 40 & 40 & 40 & & \\
\hline US-DOE-X134120 & PPCB & Endosulfan I & 1 & 0 & $\mathrm{ug} / \mathrm{kg}$ & 20 & 20 & 20 & & \\
\hline US-DOE-X134120 & РPCB & Endosulfan II & 1 & 0 & $\mathrm{ug} / \mathrm{kg}$ & 40 & 40 & 40 & & \\
\hline US-DOE-X134120 & РPCB & Endosulfan sulfate & 1 & 0 & $\mathrm{ug} / \mathrm{kg}$ & 40 & 40 & 40 & & \\
\hline US-DOE-X134120 & РPCB & Endrin & 2 & 0 & $\mathrm{ug} / \mathrm{kg}$ & 0.0004 & 40 & 20.0002 & 20.0002 & 28.283988 \\
\hline US-DOE-X134120 & PPCB & Endrin aldehyde & 1 & 0 & $\mathrm{ug} / \mathrm{kg}$ & 40 & 40 & 40 & & \\
\hline US-DOE-X134120 & PPCB & Endrin ketone & 1 & 0 & $\mathrm{ug} / \mathrm{kg}$ & 40 & 40 & 40 & & \\
\hline US-DOE-X134120 & РPCB & gamma-Chlordane & 1 & 0 & $\mathrm{ug} / \mathrm{kg}$ & 20 & 20 & 20 & & \\
\hline US-DOE-X134120 & РPCB & Heptachlor & 2 & 0 & $\mathrm{ug} / \mathrm{kg}$ & 0.0002 & 20 & 10.0001 & 10.0001 & 14.141994 \\
\hline US-DOE-X134120 & РPCB & Heptachlor epoxide & 2 & 0 & $\mathrm{ug} / \mathrm{kg}$ & 0.0002 & 20 & 10.0001 & 10.0001 & 14.141994 \\
\hline US-DOE-X134120 & PPCB & Lindane & 2 & 0 & $\mathrm{ug} / \mathrm{kg}$ & 0.0002 & 20 & 10.0001 & 10.0001 & 14.141994 \\
\hline US-DOE-X134120 & РPCB & Methoxychlor & 2 & 0 & $\mathrm{ug} / \mathrm{kg}$ & 0.002 & 200 & 100.001 & 100.001 & 141.41994 \\
\hline US-DOE-X134120 & РPCB & Toxaphene & 2 & 0 & $\mathrm{ug} / \mathrm{kg}$ & 0.005 & 500 & 250.0025 & 250.0025 & 353.54986 \\
\hline US-DOE-X134120 & RADS & Americium-241 & 1 & 0 & $\mathrm{pCi} / \mathrm{g}$ & -0.0133 & -0.0133 & -0.0133 & & \\
\hline US-DOE-X134120 & RADS & Carbon-14 & 1 & 0 & $\mathrm{pCi} / \mathrm{g}$ & -1.78 & -1.78 & -1.78 & & \\
\hline US-DOE-X134120 & RADS & Cesium-137 & 1 & 0 & $\mathrm{pCi} / \mathrm{g}$ & 0.0271 & 0.0271 & 0.0271 & & \\
\hline US-DOE-X134120 & RADS & Cobalt- 60 & 1 & 0 & $\mathrm{pCi} / \mathrm{g}$ & 0.0066 & 0.0066 & 0.0066 & & \\
\hline US-DOE-X134120 & RADS & Curium-243/244 & 1 & 0 & $\mathrm{pCi} / \mathrm{g}$ & -0.0239 & -0.0239 & -0.0239 & & \\
\hline US-DOE-X134120 & RADS & Iodine-129 & 1 & 0 & $\mathrm{pCi} / \mathrm{g}$ & -0.0863 & -0.0863 & -0.0863 & & \\
\hline US-DOE-X134120 & RADS & Neptunium-237 & 1 & 0 & $\mathrm{pCi} / \mathrm{g}$ & -0.0657 & -0.0657 & -0.0657 & & \\
\hline US-DOE-X134120 & RADS & Plutonium-238 & 1 & 0 & $\mathrm{pCi} / \mathrm{g}$ & -0.00808 & -0.00808 & -0.00808 & & \\
\hline US-DOE-X134120 & RADS & Plutonium-239/240 & 1 & 0 & $\mathrm{pCi} / \mathrm{g}$ & -0.0194 & -0.0194 & -0.0194 & & \\
\hline US-DOE-X134120 & RADS & Radium-226 & 1 & 0 & $\mathrm{pCi} / \mathrm{g}$ & 0.582 & 0.582 & 0.582 & & \\
\hline US-DOE-X134120 & RADS & Strontium-90 & 1 & 0 & $\mathrm{pCi} / \mathrm{g}$ & 0.633 & 0.633 & 0.633 & & \\
\hline US-DOE-X134120 & RADS & Technetium-99 & 1 & 0 & $\mathrm{pCi} / \mathrm{g}$ & -0.24 & -0.24 & -0.24 & & \\
\hline US-DOE-X134120 & RADS & Thorium-230 & 1 & 0 & $\mathrm{pCi} / \mathrm{g}$ & -9.92 & -9.92 & -9.92 & & \\
\hline US-DOE-X134120 & RADS & Thorium-232 & 1 & 0 & $\mathrm{pCi} / \mathrm{g}$ & -24.2 & -24.2 & -24.2 & & \\
\hline US-DOE-X134120 & RADS & Tritium & 1 & 0 & $\mathrm{pCi} / \mathrm{g}$ & 24.4 & 24.4 & 24.4 & & \\
\hline US-DOE-X134120 & RADS & Uranium-233/234 & 1 & 0 & $\mathrm{pCi} / \mathrm{g}$ & 0.0576 & 0.0576 & 0.0576 & & \\
\hline US-DOE-X134120 & RADS & Uranium-235/236 & 1 & 0 & $\mathrm{pCi} / \mathrm{g}$ & 0.00054 & 0.00054 & 0.00054 & & \\
\hline US-DOE-X134120 & RADS & Uranium-238 & 1 & 0 & $\mathrm{pCi} / \mathrm{g}$ & -0.0097 & -0.0097 & -0.0097 & & \\
\hline
\end{tabular}




\begin{tabular}{|c|c|c|c|c|c|c|c|c|c|c|}
\hline US-DOE-X134120 & SVOA & 1,2-Dichlorobenzene & 1 & 0 & $\mathrm{ug} / \mathrm{kg}$ & 96.2 & 96.2 & 96.2 & & \\
\hline US-DOE-X134120 & SVOA & 1,3-Dichlorobenzene & 1 & 0 & $\mathrm{ug} / \mathrm{kg}$ & 96.2 & 96.2 & 96.2 & & \\
\hline US-DOE-X134120 & SVOA & 1,4-Dichlorobenzene & 2 & 0 & $\mathrm{ug} / \mathrm{kg}$ & 0.01 & 96.2 & 48.105 & 48.105 & 68.016601 \\
\hline US-DOE-X134120 & SVOA & 2,2'-Dichlorodiisopropyl ether & 1 & 0 & $\mathrm{ug} / \mathrm{kg}$ & 9940 & 9940 & 9940 & & \\
\hline US-DOE-X134120 & SVOA & 2,3,4,6-Tetrachlorophenol & 1 & 0 & $\mathrm{ug} / \mathrm{kg}$ & 9940 & 9940 & 9940 & & \\
\hline US-DOE-X134120 & SVOA & 2,4,5-Trichlorophenol & 2 & 0 & $\mathrm{ug} / \mathrm{kg}$ & 0.05 & 9940 & 4970.025 & 4970.025 & 7028.606 \\
\hline US-DOE-X134120 & SVOA & 2,4,6-Trichlorophenol & 2 & 0 & $\mathrm{ug} / \mathrm{kg}$ & 0.05 & 9940 & 4970.025 & 4970.025 & 7028.606 \\
\hline US-DOE-X134120 & SVOA & 2,4-Dichlorophenol & 1 & 0 & $\mathrm{ug} / \mathrm{kg}$ & 9940 & 9940 & 9940 & & \\
\hline US-DOE-X134120 & SVOA & 2,4-Dimethylphenol & 1 & 0 & $\mathrm{ug} / \mathrm{kg}$ & 9940 & 9940 & 9940 & & \\
\hline US-DOE-X134120 & SVOA & 2,4-Dinitrophenol & 1 & 0 & $\mathrm{ug} / \mathrm{kg}$ & 19900 & 19900 & 19900 & & \\
\hline US-DOE-X134120 & SVOA & 2,4-Dinitrotoluene & 2 & 0 & $\mathrm{ug} / \mathrm{kg}$ & 0.05 & 9940 & 4970.025 & 4970.025 & 7028.606 \\
\hline US-DOE-X134120 & SVOA & 2,6-Dinitrotoluene & 1 & 0 & $\mathrm{ug} / \mathrm{kg}$ & 9940 & 9940 & 9940 & & \\
\hline US-DOE-X134120 & SVOA & 2-Chloronaphthalene & 1 & 0 & $\mathrm{ug} / \mathrm{kg}$ & 994 & 994 & 994 & & \\
\hline US-DOE-X134120 & SVOA & 2-Chlorophenol & 1 & 0 & $\mathrm{ug} / \mathrm{kg}$ & 9940 & 9940 & 9940 & & \\
\hline US-DOE-X134120 & SVOA & 2-Methyl-4,6-dinitrophenol & 1 & 0 & $\mathrm{ug} / \mathrm{kg}$ & 9940 & 9940 & 9940 & & \\
\hline US-DOE-X134120 & SVOA & 2-Methylnaphthalene & 1 & 0 & $\mathrm{ug} / \mathrm{kg}$ & 994 & 994 & 994 & & \\
\hline US-DOE-X134120 & SVOA & 2-Methylphenol & 2 & 0 & $\mathrm{ug} / \mathrm{kg}$ & 0.05 & 9940 & 4970.025 & 4970.025 & 7028.606 \\
\hline US-DOE-X134120 & SVOA & 2-Nitrobenzenamine & 1 & 0 & $\mathrm{ug} / \mathrm{kg}$ & 9940 & 9940 & 9940 & & \\
\hline US-DOE-X134120 & SVOA & 2-Nitrophenol & 1 & 0 & $\mathrm{ug} / \mathrm{kg}$ & 9940 & 9940 & 9940 & & \\
\hline US-DOE-X134120 & SVOA & 3,3'-Dichlorobenzidine & 1 & 0 & $\mathrm{ug} / \mathrm{kg}$ & 9940 & 9940 & 9940 & & \\
\hline US-DOE-X134120 & SVOA & 3-Nitrobenzenamine & 1 & 0 & $\mathrm{ug} / \mathrm{kg}$ & 9940 & 9940 & 9940 & & \\
\hline US-DOE-X134120 & SVOA & 4-Bromophenyl phenyl ether & 1 & 0 & $\mathrm{ug} / \mathrm{kg}$ & 9940 & 9940 & 9940 & & \\
\hline US-DOE-X134120 & SVOA & 4-Chloro-3-methylphenol & 1 & 0 & $\mathrm{ug} / \mathrm{kg}$ & 9940 & 9940 & 9940 & & \\
\hline US-DOE-X134120 & SVOA & 4-Chlorobenzenamine & 1 & 0 & $\mathrm{ug} / \mathrm{kg}$ & 9940 & 9940 & 9940 & & \\
\hline US-DOE-X134120 & SVOA & 4-Chlorophenyl phenyl ether & 1 & 0 & $\mathrm{ug} / \mathrm{kg}$ & 9940 & 9940 & 9940 & & \\
\hline US-DOE-X134120 & SVOA & 4-Nitrobenzenamine & 1 & 0 & $\mathrm{ug} / \mathrm{kg}$ & 9940 & 9940 & 9940 & & \\
\hline US-DOE-X134120 & SVOA & 4-Nitrophenol & 1 & 0 & $\mathrm{ug} / \mathrm{kg}$ & 9940 & 9940 & 9940 & & \\
\hline US-DOE-X134120 & SVOA & Acenaphthene & 1 & 0 & $\mathrm{ug} / \mathrm{kg}$ & 994 & 994 & 994 & & \\
\hline US-DOE-X134120 & SVOA & Acenaphthylene & 1 & 0 & $\mathrm{ug} / \mathrm{kg}$ & 994 & 994 & 994 & & \\
\hline US-DOE-X134120 & SVOA & Acetophenone & 1 & 0 & $\mathrm{ug} / \mathrm{kg}$ & 9940 & 9940 & 9940 & & \\
\hline US-DOE-X134120 & SVOA & Anthracene & 1 & 0 & $\mathrm{ug} / \mathrm{kg}$ & 994 & 994 & 994 & & \\
\hline US-DOE-X134120 & SVOA & Benz(a)anthracene & 1 & 0 & $\mathrm{ug} / \mathrm{kg}$ & 994 & 994 & 994 & & \\
\hline US-DOE-X134120 & SVOA & Benzenemethanol & 1 & 0 & $\mathrm{ug} / \mathrm{kg}$ & 9940 & 9940 & 9940 & & \\
\hline US-DOE-X134120 & SVOA & Benzidine & 1 & 0 & $\mathrm{ug} / \mathrm{kg}$ & 9940 & 9940 & 9940 & & \\
\hline US-DOE-X134120 & SVOA & Benzo(a)pyrene & 1 & 0 & $\mathrm{ug} / \mathrm{kg}$ & 994 & 994 & 994 & & \\
\hline US-DOE-X134120 & SVOA & Benzo(b)fluoranthene & 1 & 0 & $\mathrm{ug} / \mathrm{kg}$ & 994 & 994 & 994 & & \\
\hline US-DOE-X134120 & SVOA & Benzo(ghi)perylene & 1 & 0 & $\mathrm{ug} / \mathrm{kg}$ & 994 & 994 & 994 & & \\
\hline US-DOE-X134120 & SVOA & Benzo(k)fluoranthene & 1 & 0 & $\mathrm{ug} / \mathrm{kg}$ & 994 & 994 & 994 & & \\
\hline US-DOE-X134120 & SVOA & Benzoic acid & 1 & 0 & $\mathrm{ug} / \mathrm{kg}$ & 19900 & 19900 & 19900 & & \\
\hline US-DOE-X134120 & SVOA & Bis(2-chloroethoxy)methane & 1 & 0 & $\mathrm{ug} / \mathrm{kg}$ & 9940 & 9940 & 9940 & & \\
\hline US-DOE-X134120 & SVOA & Bis(2-chloroethyl) ether & 1 & 0 & $\mathrm{ug} / \mathrm{kg}$ & 9940 & 9940 & 9940 & & \\
\hline US-DOE-X134120 & SVOA & Bis(2-ethylhexyl)phthalate & 1 & 0 & $\mathrm{ug} / \mathrm{kg}$ & 9940 & 9940 & 9940 & & \\
\hline US-DOE-X134120 & SVOA & Butyl benzyl phthalate & 1 & 0 & $\mathrm{ug} / \mathrm{kg}$ & 9940 & 9940 & 9940 & & \\
\hline US-DOE-X134120 & SVOA & Carbazole & 1 & 0 & $\mathrm{ug} / \mathrm{kg}$ & 994 & 994 & 994 & & \\
\hline US-DOE-X134120 & SVOA & Chrysene & 1 & 0 & $\mathrm{ug} / \mathrm{kg}$ & 994 & 994 & 994 & & \\
\hline US-DOE-X134120 & SVOA & $\operatorname{Dibenz}(\mathrm{a}, \mathrm{h})$ anthracene & 1 & 0 & $\mathrm{ug} / \mathrm{kg}$ & 994 & 994 & 994 & & \\
\hline US-DOE-X134120 & SVOA & Dibenzofuran & 1 & 0 & $\mathrm{ug} / \mathrm{kg}$ & 9940 & 9940 & 9940 & & \\
\hline US-DOE-X134120 & SVOA & Diethyl phthalate & 1 & 0 & $\mathrm{ug} / \mathrm{kg}$ & 9940 & 9940 & 9940 & & \\
\hline US-DOE-X134120 & SVOA & Dimethyl phthalate & 1 & 0 & $\mathrm{ug} / \mathrm{kg}$ & 9940 & 9940 & 9940 & & \\
\hline US-DOE-X134120 & SVOA & Di-n-butyl phthalate & 1 & 0 & $\mathrm{ug} / \mathrm{kg}$ & 9940 & 9940 & 9940 & & \\
\hline US-DOE-X134120 & SVOA & Di-n-octylphthalate & 1 & 0 & $\mathrm{ug} / \mathrm{kg}$ & 9940 & 9940 & 9940 & & \\
\hline US-DOE-X134120 & SVOA & Diphenylamine & 1 & 0 & $\mathrm{ug} / \mathrm{kg}$ & 9940 & 9940 & 9940 & & \\
\hline US-DOE-X134120 & SVOA & Fluoranthene & 1 & 0 & $\mathrm{ug} / \mathrm{kg}$ & 994 & 994 & 994 & & \\
\hline US-DOE-X134120 & SVOA & Fluorene & 1 & 0 & $\mathrm{ug} / \mathrm{kg}$ & 994 & 994 & 994 & & \\
\hline US-DOE-X134120 & SVOA & Hexachlorobenzene & 2 & 0 & $\mathrm{ug} / \mathrm{kg}$ & 0.05 & 9940 & 4970.025 & 4970.025 & 7028.606 \\
\hline US-DOE-X134120 & SVOA & Hexachlorobutadiene & 2 & 0 & $\mathrm{ug} / \mathrm{kg}$ & 0.05 & 9940 & 4970.025 & 4970.025 & 7028.606 \\
\hline US-DOE-X134120 & SVOA & Hexachlorocyclopentadiene & 1 & 0 & $\mathrm{ug} / \mathrm{kg}$ & 9940 & 9940 & 9940 & & \\
\hline US-DOE-X134120 & SVOA & Hexachloroethane & 2 & 0 & $\mathrm{ug} / \mathrm{kg}$ & 0.05 & 9940 & 4970.025 & 4970.025 & 7028.606 \\
\hline US-DOE-X134120 & SVOA & Indeno(1,2,3-cd)pyrene & 1 & 0 & $\mathrm{ug} / \mathrm{kg}$ & 994 & 994 & 994 & & \\
\hline US-DOE-X134120 & SVOA & Isophorone & 1 & 0 & $\mathrm{ug} / \mathrm{kg}$ & 9940 & 9940 & 9940 & & \\
\hline US-DOE-X134120 & SVOA & $\mathrm{m}+\mathrm{p}$ Methylphenol & 2 & 0 & $\mathrm{ug} / \mathrm{kg}$ & 0.05 & 9940 & 4970.025 & 4970.025 & 7028.606 \\
\hline US-DOE-X134120 & SVOA & Naphthalene & 1 & 0 & $\mathrm{ug} / \mathrm{kg}$ & 994 & 994 & 994 & & \\
\hline US-DOE-X134120 & SVOA & Nitrobenzene & 2 & 0 & $\mathrm{ug} / \mathrm{kg}$ & 0.05 & 9940 & 4970.025 & 4970.025 & 7028.606 \\
\hline US-DOE-X134120 & SVOA & N-Nitroso-di-n-propylamine & 1 & 0 & $\mathrm{ug} / \mathrm{kg}$ & 9940 & 9940 & 9940 & & \\
\hline US-DOE-X134120 & SVOA & Pentachlorophenol & 2 & 0 & $\mathrm{ug} / \mathrm{kg}$ & 0.05 & 9940 & 4970.025 & 4970.025 & 7028.606 \\
\hline US-DOE-X134120 & SVOA & Phenanthrene & 1 & 0 & $\mathrm{ug} / \mathrm{kg}$ & 994 & 994 & 994 & & \\
\hline US-DOE-X134120 & SVOA & Phenol & 1 & 0 & $\mathrm{ug} / \mathrm{kg}$ & 9940 & 9940 & 9940 & & \\
\hline US-DOE-X134120 & SVOA & Pyridine & 2 & 0 & $\mathrm{ug} / \mathrm{kg}$ & 0.05 & 9940 & 4970.025 & 4970.025 & 7028.606 \\
\hline US-DOE-X134120 & VOA & (1,1-Dimethylethyl)benzene & 1 & 0 & $\mathrm{ug} / \mathrm{kg}$ & 96.2 & 96.2 & 96.2 & & \\
\hline US-DOE-X134120 & VOA & (1-Methylpropyl)benzene & 1 & 0 & $\mathrm{ug} / \mathrm{kg}$ & 96.2 & 96.2 & 96.2 & & \\
\hline
\end{tabular}




\begin{tabular}{|c|c|c|c|c|c|c|c|c|c|c|}
\hline US-DOE-X134120 & VOA & 1,1,1,2-Tetrachloroethane & 1 & 0 & $\mathrm{ug} / \mathrm{kg}$ & 96.2 & 96.2 & 96.2 & & \\
\hline US-DOE-X134120 & VOA & 1,1,1-Trichloroethane & 1 & 0 & $\mathrm{ug} / \mathrm{kg}$ & 96.2 & 96.2 & 96.2 & & \\
\hline US-DOE-X134120 & VOA & 1,1,2,2-Tetrachloroethane & 1 & 0 & $\mathrm{ug} / \mathrm{kg}$ & 96.2 & 96.2 & 96.2 & & \\
\hline US-DOE-X134120 & VOA & 1,1,2-Trichloroethane & 1 & 0 & $\mathrm{ug} / \mathrm{kg}$ & 96.2 & 96.2 & 96.2 & & \\
\hline US-DOE-X134120 & VOA & 1,1-Dichloroethane & 1 & 0 & $\mathrm{ug} / \mathrm{kg}$ & 96.2 & 96.2 & 96.2 & & \\
\hline US-DOE-X134120 & VOA & 1,1-Dichloroethene & 2 & 0 & $\mathrm{ug} / \mathrm{kg}$ & 0.01 & 96.2 & 48.105 & 48.105 & 68.016601 \\
\hline US-DOE-X134120 & VOA & 1,1-Dichloropropene & 1 & 0 & $\mathrm{ug} / \mathrm{kg}$ & 96.2 & 96.2 & 96.2 & & \\
\hline US-DOE-X134120 & VOA & 1,2,3-Trichlorobenzene & 1 & 0 & $\mathrm{ug} / \mathrm{kg}$ & 96.2 & 96.2 & 96.2 & & \\
\hline US-DOE-X134120 & VOA & 1,2,3-Trichloropropane & 1 & 0 & $\mathrm{ug} / \mathrm{kg}$ & 96.2 & 96.2 & 96.2 & & \\
\hline US-DOE-X134120 & VOA & 1,2,4-Trimethylbenzene & 1 & 0 & $\mathrm{ug} / \mathrm{kg}$ & 96.2 & 96.2 & 96.2 & & \\
\hline US-DOE-X134120 & VOA & 1,2-Dibromo-3-chloropropane & 1 & 0 & $\mathrm{ug} / \mathrm{kg}$ & 96.2 & 96.2 & 96.2 & & \\
\hline US-DOE-X134120 & VOA & 1,2-Dibromoethane & 1 & 0 & $\mathrm{ug} / \mathrm{kg}$ & 96.2 & 96.2 & 96.2 & & \\
\hline US-DOE-X134120 & VOA & 1,2-Dichloroethane & 2 & 0 & $\mathrm{ug} / \mathrm{kg}$ & 0.01 & 96.2 & 48.105 & 48.105 & 68.016601 \\
\hline US-DOE-X134120 & VOA & 1,2-Dichloroethene & 1 & 0 & $\mathrm{ug} / \mathrm{kg}$ & 192 & 192 & 192 & & \\
\hline US-DOE-X134120 & VOA & 1,2-Dichloropropane & 1 & 0 & $\mathrm{ug} / \mathrm{kg}$ & 96.2 & 96.2 & 96.2 & & \\
\hline US-DOE-X134120 & VOA & 1,2-Dimethylbenzene & 1 & 0 & $\mathrm{ug} / \mathrm{kg}$ & 96.2 & 96.2 & 96.2 & & \\
\hline US-DOE-X134120 & VOA & 1,3,5-Trimethylbenzene & 1 & 0 & $\mathrm{ug} / \mathrm{kg}$ & 96.2 & 96.2 & 96.2 & & \\
\hline US-DOE-X134120 & VOA & 1,3-Dichloropropane & 1 & 0 & $\mathrm{ug} / \mathrm{kg}$ & 96.2 & 96.2 & 96.2 & & \\
\hline US-DOE-X134120 & VOA & 1-Chloro-4-methylbenzene & 1 & 0 & $\mathrm{ug} / \mathrm{kg}$ & 96.2 & 96.2 & 96.2 & & \\
\hline US-DOE-X134120 & VOA & 1-Methyl-4-(1-methylethyl)benzene & 1 & 0 & $\mathrm{ug} / \mathrm{kg}$ & 96.2 & 96.2 & 96.2 & & \\
\hline US-DOE-X134120 & VOA & 2,2-Dichloropropane & 1 & 0 & $\mathrm{ug} / \mathrm{kg}$ & 96.2 & 96.2 & 96.2 & & \\
\hline US-DOE-X134120 & VOA & 2-Butanone & 2 & 0 & $\mathrm{ug} / \mathrm{kg}$ & 0.05 & 481 & 240.525 & 240.525 & 340.08301 \\
\hline US-DOE-X134120 & VOA & 2-Hexanone & 1 & 0 & $\mathrm{ug} / \mathrm{kg}$ & 481 & 481 & 481 & & \\
\hline US-DOE-X134120 & VOA & 4-Methyl-2-pentanone & 1 & 0 & $\mathrm{ug} / \mathrm{kg}$ & 481 & 481 & 481 & & \\
\hline US-DOE-X134120 & VOA & Acetone & 1 & 0 & $\mathrm{ug} / \mathrm{kg}$ & 481 & 481 & 481 & & \\
\hline US-DOE-X134120 & VOA & Acetonitrile & 1 & 0 & $\mathrm{ug} / \mathrm{kg}$ & 2400 & 2400 & 2400 & & \\
\hline US-DOE-X134120 & VOA & Acrolein & 1 & 0 & $\mathrm{ug} / \mathrm{kg}$ & 481 & 481 & 481 & & \\
\hline US-DOE-X134120 & VOA & Acrylonitrile & 1 & 0 & $\mathrm{ug} / \mathrm{kg}$ & 481 & 481 & 481 & & \\
\hline US-DOE-X134120 & VOA & Benzene & 2 & 0 & $\mathrm{ug} / \mathrm{kg}$ & 0.01 & 96.2 & 48.105 & 48.105 & 68.016601 \\
\hline US-DOE-X134120 & VOA & Bromobenzene & 1 & 0 & $\mathrm{ug} / \mathrm{kg}$ & 96.2 & 96.2 & 96.2 & & \\
\hline US-DOE-X134120 & VOA & Bromochloromethane & 1 & 0 & $\mathrm{ug} / \mathrm{kg}$ & 96.2 & 96.2 & 96.2 & & \\
\hline US-DOE-X134120 & VOA & Bromodichloromethane & 1 & 0 & $\mathrm{ug} / \mathrm{kg}$ & 96.2 & 96.2 & 96.2 & & \\
\hline US-DOE-X134120 & VOA & Bromoform & 1 & 0 & $\mathrm{ug} / \mathrm{kg}$ & 96.2 & 96.2 & 96.2 & & \\
\hline US-DOE-X134120 & VOA & Bromomethane & 1 & 0 & $\mathrm{ug} / \mathrm{kg}$ & 96.2 & 96.2 & 96.2 & & \\
\hline US-DOE-X134120 & VOA & Butylbenzene & 1 & 0 & $\mathrm{ug} / \mathrm{kg}$ & 96.2 & 96.2 & 96.2 & & \\
\hline US-DOE-X134120 & VOA & Carbon disulfide & 1 & 0 & $\mathrm{ug} / \mathrm{kg}$ & 481 & 481 & 481 & & \\
\hline US-DOE-X134120 & VOA & Carbon tetrachloride & 2 & 0 & $\mathrm{ug} / \mathrm{kg}$ & 0.01 & 96.2 & 48.105 & 48.105 & 68.016601 \\
\hline US-DOE-X134120 & VOA & Chlorobenzene & 2 & 0 & $\mathrm{ug} / \mathrm{kg}$ & 0.01 & 96.2 & 48.105 & 48.105 & 68.016601 \\
\hline US-DOE-X134120 & VOA & Chloroethane & 1 & 0 & $\mathrm{ug} / \mathrm{kg}$ & 96.2 & 96.2 & 96.2 & & \\
\hline US-DOE-X134120 & VOA & Chloroform & 2 & 0 & $\mathrm{ug} / \mathrm{kg}$ & 0.01 & 96.2 & 48.105 & 48.105 & 68.016601 \\
\hline US-DOE-X134120 & VOA & Chloromethane & 1 & 0 & $\mathrm{ug} / \mathrm{kg}$ & 96.2 & 96.2 & 96.2 & & \\
\hline US-DOE-X134120 & VOA & cis-1,2-Dichloroethene & 1 & 0 & $\mathrm{ug} / \mathrm{kg}$ & 96.2 & 96.2 & 96.2 & & \\
\hline US-DOE-X134120 & VOA & cis-1,3-Dichloropropene & 1 & 0 & $\mathrm{ug} / \mathrm{kg}$ & 96.2 & 96.2 & 96.2 & & \\
\hline US-DOE-X134120 & VOA & Cumene & 1 & 0 & $\mathrm{ug} / \mathrm{kg}$ & 96.2 & 96.2 & 96.2 & & \\
\hline US-DOE-X134120 & VOA & Dibromochloromethane & 1 & 0 & $\mathrm{ug} / \mathrm{kg}$ & 96.2 & 96.2 & 96.2 & & \\
\hline US-DOE-X134120 & VOA & Dibromomethane & 1 & 0 & $\mathrm{ug} / \mathrm{kg}$ & 96.2 & 96.2 & 96.2 & & \\
\hline US-DOE-X134120 & VOA & Dichlorodifluoromethane & 1 & 0 & $\mathrm{ug} / \mathrm{kg}$ & 96.2 & 96.2 & 96.2 & & \\
\hline US-DOE-X134120 & VOA & Ethylbenzene & 1 & 0 & $\mathrm{ug} / \mathrm{kg}$ & 96.2 & 96.2 & 96.2 & & \\
\hline US-DOE-X134120 & VOA & Hexane & 1 & 0 & $\mathrm{ug} / \mathrm{kg}$ & 481 & 481 & 481 & & \\
\hline US-DOE-X134120 & VOA & M + P Xylene & 1 & 0 & $\mathrm{ug} / \mathrm{kg}$ & 192 & 192 & 192 & & \\
\hline US-DOE-X134120 & VOA & Methanol & 1 & 0 & $\mathrm{ug} / \mathrm{kg}$ & 4240 & 4240 & 4240 & & \\
\hline US-DOE-X134120 & VOA & Methyl methacrylate & 1 & 0 & $\mathrm{ug} / \mathrm{kg}$ & 481 & 481 & 481 & & \\
\hline US-DOE-X134120 & VOA & Methylcyclohexane & 1 & 0 & $\mathrm{ug} / \mathrm{kg}$ & 96.2 & 96.2 & 96.2 & & \\
\hline US-DOE-X134120 & VOA & Methylene chloride & 1 & 0 & $\mathrm{ug} / \mathrm{kg}$ & 481 & 481 & 481 & & \\
\hline US-DOE-X134120 & VOA & o-Chlorotoluene & 1 & 0 & $\mathrm{ug} / \mathrm{kg}$ & 96.2 & 96.2 & 96.2 & & \\
\hline US-DOE-X134120 & VOA & Propylbenzene & 1 & 0 & $\mathrm{ug} / \mathrm{kg}$ & 96.2 & 96.2 & 96.2 & & \\
\hline US-DOE-X134120 & VOA & Propylene glycol & 1 & 0 & $\mathrm{ug} / \mathrm{kg}$ & 42400 & 42400 & 42400 & & \\
\hline US-DOE-X134120 & VOA & Styrene & 1 & 0 & $\mathrm{ug} / \mathrm{kg}$ & 96.2 & 96.2 & 96.2 & & \\
\hline US-DOE-X134120 & VOA & Tetrachloroethene & 2 & 0 & $\mathrm{ug} / \mathrm{kg}$ & 0.01 & 96.2 & 48.105 & 48.105 & 68.016601 \\
\hline US-DOE-X134120 & VOA & Toluene & 1 & 0 & $\mathrm{ug} / \mathrm{kg}$ & 96.2 & 96.2 & 96.2 & & \\
\hline US-DOE-X134120 & VOA & Total Xylene & 1 & 0 & $\mathrm{ug} / \mathrm{kg}$ & 288 & 288 & 288 & & \\
\hline US-DOE-X134120 & VOA & trans-1,2-Dichloroethene & 1 & 0 & $\mathrm{ug} / \mathrm{kg}$ & 96.2 & 96.2 & 96.2 & & \\
\hline US-DOE-X134120 & VOA & trans-1,3-Dichloropropene & 1 & 0 & $\mathrm{ug} / \mathrm{kg}$ & 96.2 & 96.2 & 96.2 & & \\
\hline US-DOE-X134120 & VOA & Trichloroethene & 2 & 0 & $\mathrm{ug} / \mathrm{kg}$ & 0.01 & 96.2 & 48.105 & 48.105 & 68.016601 \\
\hline US-DOE-X134120 & VOA & Trichlorofluoromethane & 1 & 0 & $\mathrm{ug} / \mathrm{kg}$ & 96.2 & 96.2 & 96.2 & & \\
\hline US-DOE-X134120 & VOA & Vinyl chloride & 2 & 0 & $\mathrm{ug} / \mathrm{kg}$ & 0.01 & 96.2 & 48.105 & 48.105 & 68.016601 \\
\hline $\mathrm{X} 10 \mathrm{C} 0400325$ & HERB & $2,4-\mathrm{D}$ & 11 & 0 & $\mathrm{ug} / \mathrm{kg}$ & 0.05 & 246 & 108.84545 & 0.05 & 125.08891 \\
\hline X10C0400325 & HERB & Silvex & 11 & 0 & $\mathrm{ug} / \mathrm{kg}$ & 0.05 & 246 & 108.84545 & 0.05 & 125.08891 \\
\hline X10C0400325 & METAL & Arsenic & 10 & 0 & $\mathrm{mg} / \mathrm{L}$ & 0.3 & 13.7 & 2.652 & 1.51 & 4.0808055 \\
\hline X10C0400325 & METAL & Barium & 10 & 6 & $\mathrm{mg} / \mathrm{L}$ & 0.05 & 19.3 & 2.7119 & 0.9445 & 5.8932578 \\
\hline
\end{tabular}




\begin{tabular}{|c|c|c|c|c|c|c|c|c|c|c|}
\hline X10C0400325 & METAL & Cadmium & 10 & 1 & $\mathrm{mg} / \mathrm{L}$ & 0.05 & 77.9 & 8.0038 & 0.252 & 24.559918 \\
\hline X10C0400325 & METAL & Chromium & 10 & 4 & $\mathrm{mg} / \mathrm{L}$ & 0.05 & 134 & 15.3764 & 0.26 & 41.946042 \\
\hline X10C0400325 & METAL & Lead & 10 & 4 & $\mathrm{mg} / \mathrm{L}$ & 0.1 & 44 & 5.786 & 0.52 & 13.572792 \\
\hline X10C0400325 & METAL & Mercury & 10 & 0 & $\mathrm{mg} / \mathrm{L}$ & 0.002 & 0.4 & 0.201 & 0.201 & 0.2097644 \\
\hline X10C0400325 & METAL & Selenium & 10 & 0 & $\mathrm{mg} / \mathrm{L}$ & 0.3 & 13.7 & 2.652 & 1.51 & 4.0808055 \\
\hline X10C0400325 & METAL & Silver & 10 & 0 & $\mathrm{mg} / \mathrm{L}$ & 0.05 & 2.29 & 0.4428 & 0.252 & 0.682144 \\
\hline $\mathrm{X} 10 \mathrm{C} 0400325$ & METAL & Total Uranium & 5 & 3 & $\mathrm{pCi} / \mathrm{g}$ & 0.00138 & 8.36 & 2.582194 & 0.278 & 3.703416 \\
\hline X10C0400325 & PPCB & $4,4^{\prime}-\mathrm{DDD}$ & 5 & 0 & $\mathrm{ug} / \mathrm{kg}$ & 37.1 & 80.1 & 55.02 & 39.5 & 22.7291 \\
\hline X10C0400325 & РPCB & 4,4'-DDE & 5 & 0 & $\mathrm{ug} / \mathrm{kg}$ & 37.1 & 80.1 & 55.02 & 39.5 & 22.7291 \\
\hline X10C0400325 & PPCB & 4,4'-DDT & 5 & 0 & $\mathrm{ug} / \mathrm{kg}$ & 37.1 & 80.1 & 55.02 & 39.5 & 22.7291 \\
\hline X10C0400325 & РPCB & Aldrin & 5 & 0 & $\mathrm{ug} / \mathrm{kg}$ & 18.5 & 40 & 27.5 & 19.8 & 11.329607 \\
\hline X10C0400325 & РPCB & alpha-BHC & 5 & 0 & $\mathrm{ug} / \mathrm{kg}$ & 18.5 & 40 & 27.5 & 19.8 & 11.329607 \\
\hline X10C0400325 & РPCB & alpha-Chlordane & 5 & 0 & $\mathrm{ug} / \mathrm{kg}$ & 18.5 & 40 & 27.5 & 19.8 & 11.329607 \\
\hline $\mathrm{X} 10 \mathrm{C} 0400325$ & РPCB & beta-BHC & 5 & 0 & $\mathrm{ug} / \mathrm{kg}$ & 18.5 & 40 & 27.5 & 19.8 & 11.329607 \\
\hline X10C0400325 & PPCB & Chlordane & 10 & 0 & $\mathrm{ug} / \mathrm{kg}$ & 0.0025 & 501 & 172.00125 & 116.00125 & 204.54766 \\
\hline X10C0400325 & РPCB & delta-BHC & 5 & 0 & $\mathrm{ug} / \mathrm{kg}$ & 18.5 & 40 & 27.5 & 19.8 & 11.329607 \\
\hline X10C0400325 & РPCB & Dieldrin & 5 & 0 & $\mathrm{ug} / \mathrm{kg}$ & 37.1 & 80.1 & 55.02 & 39.5 & 22.7291 \\
\hline X10C0400325 & РPCB & Endosulfan I & 5 & 0 & $\mathrm{ug} / \mathrm{kg}$ & 18.5 & 40 & 27.5 & 19.8 & 11.329607 \\
\hline X10C0400325 & PPCB & Endosulfan II & 5 & 0 & $\mathrm{ug} / \mathrm{kg}$ & 37.1 & 80.1 & 55.02 & 39.5 & 22.7291 \\
\hline X10C0400325 & РPCB & Endosulfan sulfate & 5 & 0 & $\mathrm{ug} / \mathrm{kg}$ & 37.1 & 80.1 & 55.02 & 39.5 & 22.7291 \\
\hline X10C0400325 & РPCB & Endrin & 10 & 0 & $\mathrm{ug} / \mathrm{kg}$ & 0.0004 & 80.1 & 27.5102 & 18.5502 & 32.718223 \\
\hline X10C0400325 & РPCB & Endrin aldehyde & 5 & 0 & $\mathrm{ug} / \mathrm{kg}$ & 37.1 & 80.1 & 55.02 & 39.5 & 22.7291 \\
\hline X10C0400325 & PPCB & Endrin ketone & 5 & 0 & $\mathrm{ug} / \mathrm{kg}$ & 37.1 & 80.1 & 55.02 & 39.5 & 22.7291 \\
\hline X10C0400325 & РPCB & gamma-Chlordane & 5 & 0 & $\mathrm{ug} / \mathrm{kg}$ & 18.5 & 40 & 27.5 & 19.8 & 11.329607 \\
\hline X10C0400325 & PPCB & Heptachlor & 10 & 0 & $\mathrm{ug} / \mathrm{kg}$ & 0.0002 & 40 & 13.7501 & 9.2501 & 16.343662 \\
\hline X10C0400325 & PPCB & Heptachlor epoxide & 10 & 0 & $\mathrm{ug} / \mathrm{kg}$ & 0.0002 & 40 & 13.7501 & 9.2501 & 16.343662 \\
\hline X10C0400325 & PPCB & Lindane & 10 & 0 & $\mathrm{ug} / \mathrm{kg}$ & 0.0002 & 40 & 13.7501 & 9.2501 & 16.343662 \\
\hline X10C0400325 & РPCB & Methoxychlor & 10 & 0 & $\mathrm{ug} / \mathrm{kg}$ & 0.002 & 400 & 137.501 & 92.501 & 163.43662 \\
\hline $\mathrm{X} 10 \mathrm{C} 0400325$ & РPCB & Toxaphene & 10 & 0 & $\mathrm{ug} / \mathrm{kg}$ & 0.005 & 1000 & 343.7025 & 231.5025 & 408.70567 \\
\hline $\mathrm{X} 10 \mathrm{C} 0400325$ & RADS & Americium-241 & 5 & 4 & $\mathrm{pCi} / \mathrm{g}$ & 0.067 & 151 & 59.898 & 15 & 75.459147 \\
\hline X10C0400325 & RADS & Carbon-14 & 5 & 1 & $\mathrm{pCi} / \mathrm{g}$ & -8.08 & 20.2 & 2.38204 & -0.0298 & 11.026989 \\
\hline X10C0400325 & RADS & Cesium-137 & 5 & 5 & $\mathrm{pCi} / \mathrm{g}$ & 1.02 & 2020 & 648.664 & 260 & 840.43879 \\
\hline X10C0400325 & RADS & Cobalt- 60 & 5 & 1 & $\mathrm{pCi} / \mathrm{g}$ & -0.0285 & 0.804 & 0.14776 & -0.025 & 0.3672578 \\
\hline X10C0400325 & RADS & Curium-243/244 & 5 & 4 & $\mathrm{pCi} / \mathrm{g}$ & -0.00845 & 21.9 & 8.88431 & 6.41 & 8.0946827 \\
\hline X10C0400325 & RADS & Iodine-129 & 4 & 0 & $\mathrm{pCi} / \mathrm{g}$ & -0.517 & 0.459 & -0.01625 & -0.0035 & 0.4809985 \\
\hline X10C0400325 & RADS & Neptunium-237 & 5 & 0 & $\mathrm{pCi} / \mathrm{g}$ & -1.89 & 0.0707 & -0.463864 & -0.00612 & 0.8305946 \\
\hline X10C0400325 & RADS & Plutonium-238 & 5 & 4 & $\mathrm{pCi} / \mathrm{g}$ & -0.00729 & 56.8 & 25.838542 & 12.4 & 25.621909 \\
\hline X10C0400325 & RADS & Plutonium-239/240 & 5 & 4 & $\mathrm{pCi} / \mathrm{g}$ & 0 & 186 & 72.8668 & 10.4 & 94.929157 \\
\hline X10C0400325 & RADS & Radium-226 & 5 & 0 & $\mathrm{pCi} / \mathrm{g}$ & 0.318 & 6.3 & 1.7856 & 0.739 & 2.5315691 \\
\hline X10C0400325 & RADS & Strontium-90 & 5 & 3 & $\mathrm{pCi} / \mathrm{g}$ & 0.768 & 1700 & 632.1826 & 39.2 & 852.88685 \\
\hline X10C0400325 & RADS & Technetium-99 & 5 & 1 & $\mathrm{pCi} / \mathrm{g}$ & -0.268 & 1.73 & 0.606 & 0.785 & 0.8173943 \\
\hline X10C0400325 & RADS & Thorium-230 & 5 & 0 & $\mathrm{pCi} / \mathrm{g}$ & -3.4 & 43.3 & 8.3592 & 0.738 & 19.61135 \\
\hline X10C0400325 & RADS & Thorium-232 & 5 & 0 & $\mathrm{pCi} / \mathrm{g}$ & -56.8 & 64 & 1.4676 & 0.199 & 42.755884 \\
\hline $\mathrm{X} 10 \mathrm{C} 0400325$ & RADS & Tritium & 5 & 0 & $\mathrm{pCi} / \mathrm{g}$ & -153 & 101 & -45.38 & -84.9 & 119.86376 \\
\hline $\mathrm{X} 10 \mathrm{C} 0400325$ & RADS & Uranium-233/234 & 5 & 3 & $\mathrm{pCi} / \mathrm{g}$ & 0.0181 & 8.38 & 2.68484 & 0.305 & 3.753877 \\
\hline X10C0400325 & RADS & Uranium-235/236 & 5 & 0 & $\mathrm{pCi} / \mathrm{g}$ & -0.238 & 0 & -0.052588 & -0.00975 & 0.1037426 \\
\hline $\mathrm{X} 10 \mathrm{C} 0400325$ & RADS & Uranium-238 & 5 & 0 & $\mathrm{pCi} / \mathrm{g}$ & -0.197 & -0.00972 & -0.050384 & -0.0162 & 0.0820087 \\
\hline X10C0400325 & SVOA & 1,2,4-Trichlorobenzene & 5 & 0 & $\mathrm{ug} / \mathrm{kg}$ & 95.9 & 476 & 246.04 & 99.4 & 204.04691 \\
\hline X10C0400325 & SVOA & 1,2-Dichlorobenzene & 5 & 0 & $\mathrm{ug} / \mathrm{kg}$ & 95.9 & 476 & 246.04 & 99.4 & 204.04691 \\
\hline X10C0400325 & SVOA & 1,3-Dichlorobenzene & 5 & 0 & $\mathrm{ug} / \mathrm{kg}$ & 95.9 & 476 & 246.04 & 99.4 & 204.04691 \\
\hline X10C0400325 & SVOA & 1,4-Dichlorobenzene & 10 & 0 & $\mathrm{ug} / \mathrm{kg}$ & 0.01 & 476 & 123.025 & 47.955 & 187.93246 \\
\hline X10C0400325 & SVOA & 2,2'-Dichlorodiisopropyl ether & 5 & 0 & $\mathrm{ug} / \mathrm{kg}$ & 930 & 148000 & 57426 & 19800 & 63280.047 \\
\hline $\mathrm{X} 10 \mathrm{C} 0400325$ & SVOA & 2,3,4,6-Tetrachlorophenol & 5 & 0 & $\mathrm{ug} / \mathrm{kg}$ & 930 & 148000 & 57426 & 19800 & 63280.047 \\
\hline X10C0400325 & SVOA & 2,4,5-Trichlorophenol & 10 & 0 & $\mathrm{ug} / \mathrm{kg}$ & 0.05 & 148000 & 28713.025 & 465.025 & 51920.673 \\
\hline X10C0400325 & SVOA & 2,4,6-Trichlorophenol & 10 & 0 & $\mathrm{ug} / \mathrm{kg}$ & 0.05 & 148000 & 28713.025 & 465.025 & 51920.673 \\
\hline X10C0400325 & SVOA & 2,4-Dichlorophenol & 5 & 0 & $\mathrm{ug} / \mathrm{kg}$ & 930 & 148000 & 57426 & 19800 & 63280.047 \\
\hline $\mathrm{X} 10 \mathrm{C} 0400325$ & SVOA & 2,4-Dimethylphenol & 5 & 0 & $\mathrm{ug} / \mathrm{kg}$ & 930 & 148000 & 57426 & 19800 & 63280.047 \\
\hline X10C0400325 & SVOA & 2,4-Dinitrophenol & 5 & 0 & $\mathrm{ug} / \mathrm{kg}$ & 1860 & 296000 & 114812 & 39700 & 126464.25 \\
\hline X10C0400325 & SVOA & 2,4-Dinitrotoluene & 10 & 0 & $\mathrm{ug} / \mathrm{kg}$ & 0.05 & 148000 & 28713.025 & 465.025 & 51920.673 \\
\hline X10C0400325 & SVOA & 2,6-Dinitrotoluene & 5 & 0 & $\mathrm{ug} / \mathrm{kg}$ & 930 & 148000 & 57426 & 19800 & 63280.047 \\
\hline X10C0400325 & SVOA & 2-Chloronaphthalene & 5 & 0 & $\mathrm{ug} / \mathrm{kg}$ & 93 & 14800 & 5742.6 & 1980 & 6328.0047 \\
\hline X10C0400325 & SVOA & 2-Chlorophenol & 5 & 0 & $\mathrm{ug} / \mathrm{kg}$ & 930 & 148000 & 57426 & 19800 & 63280.047 \\
\hline X10C0400325 & SVOA & 2-Methyl-4,6-dinitrophenol & 5 & 0 & $\mathrm{ug} / \mathrm{kg}$ & 930 & 148000 & 57426 & 19800 & 63280.047 \\
\hline X10C0400325 & SVOA & 2-Methylnaphthalene & 5 & 0 & $\mathrm{ug} / \mathrm{kg}$ & 93 & 14800 & 5742.6 & 1980 & 6328.0047 \\
\hline X10C0400325 & SVOA & 2-Methylphenol & 10 & 0 & $\mathrm{ug} / \mathrm{kg}$ & 0.05 & 148000 & 28713.025 & 465.025 & 51920.673 \\
\hline $\mathrm{X} 10 \mathrm{C} 0400325$ & SVOA & 2-Nitrobenzenamine & 5 & 0 & $\mathrm{ug} / \mathrm{kg}$ & 930 & 148000 & 57426 & 19800 & 63280.047 \\
\hline X10C0400325 & SVOA & 2-Nitrophenol & 5 & 0 & $\mathrm{ug} / \mathrm{kg}$ & 930 & 148000 & 57426 & 19800 & 63280.047 \\
\hline X10C0400325 & SVOA & 3,3'-Dichlorobenzidine & 5 & 0 & $\mathrm{ug} / \mathrm{kg}$ & 930 & 148000 & 57426 & 19800 & 63280.047 \\
\hline X10C0400325 & SVOA & 3-Nitrobenzenamine & 5 & 0 & $\mathrm{ug} / \mathrm{kg}$ & 930 & 148000 & 57426 & 19800 & 63280.047 \\
\hline X10C0400325 & SVOA & 4-Bromophenyl phenyl ether & 5 & 0 & $\mathrm{ug} / \mathrm{kg}$ & 930 & 148000 & 57426 & 19800 & 63280.047 \\
\hline
\end{tabular}




\begin{tabular}{|c|c|c|c|c|c|c|c|c|c|c|}
\hline $\mathrm{X} 10 \mathrm{C} 0400325$ & SVOA & 4-Chloro-3-methylphenol & 5 & 0 & $\mathrm{ug} / \mathrm{kg}$ & 930 & 148000 & 57426 & 19800 & 63280.047 \\
\hline X10C0400325 & SVOA & 4-Chlorobenzenamine & 5 & 0 & $\mathrm{ug} / \mathrm{kg}$ & 930 & 148000 & 57426 & 19800 & 63280.047 \\
\hline X10C0400325 & SVOA & 4-Chlorophenyl phenyl ether & 5 & 0 & $\mathrm{ug} / \mathrm{kg}$ & 930 & 148000 & 57426 & 19800 & 63280.047 \\
\hline X10C0400325 & SVOA & 4-Nitrobenzenamine & 5 & 0 & $\mathrm{ug} / \mathrm{kg}$ & 930 & 148000 & 57426 & 19800 & 63280.047 \\
\hline X10C0400325 & SVOA & 4-Nitrophenol & 5 & 0 & $\mathrm{ug} / \mathrm{kg}$ & 930 & 148000 & 57426 & 19800 & 63280.047 \\
\hline X10C0400325 & SVOA & Acenaphthene & 5 & 0 & $\mathrm{ug} / \mathrm{kg}$ & 93 & 14800 & 5742.6 & 1980 & 6328.0047 \\
\hline X10C0400325 & SVOA & Acenaphthylene & 5 & 0 & $\mathrm{ug} / \mathrm{kg}$ & 93 & 14800 & 5742.6 & 1980 & 6328.0047 \\
\hline X10C0400325 & SVOA & Acetophenone & 5 & 0 & $\mathrm{ug} / \mathrm{kg}$ & 930 & 148000 & 57426 & 19800 & 63280.047 \\
\hline X10C0400325 & SVOA & Anthracene & 5 & 0 & $\mathrm{ug} / \mathrm{kg}$ & 93 & 14800 & 5742.6 & 1980 & 6328.0047 \\
\hline X10C0400325 & SVOA & Benz(a)anthracene & 5 & 0 & $\mathrm{ug} / \mathrm{kg}$ & 93 & 14800 & 5742.6 & 1980 & 6328.0047 \\
\hline X10C0400325 & SVOA & Benzenemethanol & 5 & 0 & $\mathrm{ug} / \mathrm{kg}$ & 930 & 148000 & 57426 & 19800 & 63280.047 \\
\hline X10C0400325 & SVOA & Benzidine & 5 & 0 & $\mathrm{ug} / \mathrm{kg}$ & 930 & 148000 & 57426 & 19800 & 63280.047 \\
\hline X10C0400325 & SVOA & Benzo(a)pyrene & 5 & 0 & $\mathrm{ug} / \mathrm{kg}$ & 93 & 14800 & 5742.6 & 1980 & 6328.0047 \\
\hline X10C0400325 & SVOA & Benzo(b)fluoranthene & 5 & 0 & $\mathrm{ug} / \mathrm{kg}$ & 93 & 14800 & 5742.6 & 1980 & 6328.0047 \\
\hline X10C0400325 & SVOA & Benzo(ghi)perylene & 5 & 0 & $\mathrm{ug} / \mathrm{kg}$ & 93 & 14800 & 5742.6 & 1980 & 6328.0047 \\
\hline X10C0400325 & SVOA & Benzo(k)fluoranthene & 5 & 0 & $\mathrm{ug} / \mathrm{kg}$ & 93 & 14800 & 5742.6 & 1980 & 6328.0047 \\
\hline X10C0400325 & SVOA & Benzoic acid & 5 & 1 & $\mathrm{ug} / \mathrm{kg}$ & 2450 & 296000 & 114930 & 39700 & 126332.71 \\
\hline X10C0400325 & SVOA & $\operatorname{Bis}(2$-chloroethoxy)methane & 5 & 0 & $\mathrm{ug} / \mathrm{kg}$ & 930 & 148000 & 57426 & 19800 & 63280.047 \\
\hline X10C0400325 & SVOA & Bis(2-chloroethyl) ether & 5 & 0 & $\mathrm{ug} / \mathrm{kg}$ & 930 & 148000 & 57426 & 19800 & 63280.047 \\
\hline X10C0400325 & SVOA & Bis(2-ethylhexyl)phthalate & 5 & 2 & $\mathrm{ug} / \mathrm{kg}$ & 19200 & 148000 & 63320 & 29600 & 57807.197 \\
\hline X10C0400325 & SVOA & Butyl benzyl phthalate & 5 & 0 & $\mathrm{ug} / \mathrm{kg}$ & 930 & 148000 & 57426 & 19800 & 63280.047 \\
\hline X10C0400325 & SVOA & Carbazole & 5 & 0 & $\mathrm{ug} / \mathrm{kg}$ & 93 & 14800 & 5742.6 & 1980 & 6328.0047 \\
\hline X10C0400325 & SVOA & Chrysene & 5 & 0 & $\mathrm{ug} / \mathrm{kg}$ & 93 & 14800 & 5742.6 & 1980 & 6328.0047 \\
\hline X10C0400325 & SVOA & Dibenz(a,h)anthracene & 5 & 0 & $\mathrm{ug} / \mathrm{kg}$ & 93 & 14800 & 5742.6 & 1980 & 6328.0047 \\
\hline X10C0400325 & SVOA & Dibenzofuran & 5 & 0 & $\mathrm{ug} / \mathrm{kg}$ & 930 & 148000 & 57426 & 19800 & 63280.047 \\
\hline X10C0400325 & SVOA & Diethyl phthalate & 5 & 0 & $\mathrm{ug} / \mathrm{kg}$ & 930 & 148000 & 57426 & 19800 & 63280.047 \\
\hline X10C0400325 & SVOA & Dimethyl phthalate & 5 & 0 & $\mathrm{ug} / \mathrm{kg}$ & 930 & 148000 & 57426 & 19800 & 63280.047 \\
\hline X10C0400325 & SVOA & Di-n-butyl phthalate & 5 & 0 & $\mathrm{ug} / \mathrm{kg}$ & 930 & 148000 & 57426 & 19800 & 63280.047 \\
\hline X10C0400325 & SVOA & Di-n-octylphthalate & 5 & 0 & $\mathrm{ug} / \mathrm{kg}$ & 930 & 148000 & 57426 & 19800 & 63280.047 \\
\hline X10C0400325 & SVOA & Diphenylamine & 5 & 0 & $\mathrm{ug} / \mathrm{kg}$ & 930 & 148000 & 57426 & 19800 & 63280.047 \\
\hline X10C0400325 & SVOA & Fluoranthene & 5 & 0 & $\mathrm{ug} / \mathrm{kg}$ & 93 & 14800 & 5742.6 & 1980 & 6328.0047 \\
\hline X10C0400325 & SVOA & Fluorene & 5 & 0 & $\mathrm{ug} / \mathrm{kg}$ & 93 & 14800 & 5742.6 & 1980 & 6328.0047 \\
\hline X10C0400325 & SVOA & Hexachlorobenzene & 10 & 0 & $\mathrm{ug} / \mathrm{kg}$ & 0.05 & 148000 & 28713.025 & 465.025 & 51920.673 \\
\hline X10C0400325 & SVOA & Hexachlorobutadiene & 10 & 0 & $\mathrm{ug} / \mathrm{kg}$ & 0.05 & 148000 & 28713.025 & 465.025 & 51920.673 \\
\hline X10C0400325 & SVOA & Hexachlorocyclopentadiene & 5 & 0 & $\mathrm{ug} / \mathrm{kg}$ & 930 & 148000 & 57426 & 19800 & 63280.047 \\
\hline X10C0400325 & SVOA & Hexachloroethane & 10 & 0 & $\mathrm{ug} / \mathrm{kg}$ & 0.05 & 148000 & 28713.025 & 465.025 & 51920.673 \\
\hline X10C0400325 & SVOA & Indeno(1,2,3-cd)pyrene & 5 & 0 & $\mathrm{ug} / \mathrm{kg}$ & 93 & 14800 & 5742.6 & 1980 & 6328.0047 \\
\hline X10C0400325 & SVOA & Isophorone & 5 & 0 & $\mathrm{ug} / \mathrm{kg}$ & 930 & 148000 & 57426 & 19800 & 63280.047 \\
\hline X10C0400325 & SVOA & $\mathrm{m}+\mathrm{p}$ Methylphenol & 10 & 0 & $\mathrm{ug} / \mathrm{kg}$ & 0.05 & 148000 & 28713.025 & 465.025 & 51920.673 \\
\hline X10C0400325 & SVOA & Naphthalene & 5 & 0 & $\mathrm{ug} / \mathrm{kg}$ & 93 & 14800 & 5742.6 & 1980 & 6328.0047 \\
\hline X10C0400325 & SVOA & Nitrobenzene & 10 & 0 & $\mathrm{ug} / \mathrm{kg}$ & 0.05 & 148000 & 28713.025 & 465.025 & 51920.673 \\
\hline X10C0400325 & SVOA & N-Nitroso-di-n-propylamine & 5 & 0 & $\mathrm{ug} / \mathrm{kg}$ & 930 & 148000 & 57426 & 19800 & 63280.047 \\
\hline X10C0400325 & SVOA & Pentachlorophenol & 10 & 0 & $\mathrm{ug} / \mathrm{kg}$ & 0.05 & 148000 & 28713.025 & 465.025 & 51920.673 \\
\hline X10C0400325 & SVOA & Phenanthrene & 5 & 0 & $\mathrm{ug} / \mathrm{kg}$ & 93 & 14800 & 5742.6 & 1980 & 6328.0047 \\
\hline X10C0400325 & SVOA & Phenol & 5 & 0 & $\mathrm{ug} / \mathrm{kg}$ & 930 & 148000 & 57426 & 19800 & 63280.047 \\
\hline X10C0400325 & SVOA & Pyridine & 10 & 0 & $\mathrm{ug} / \mathrm{kg}$ & 0.05 & 148000 & 28713.025 & 465.025 & 51920.673 \\
\hline X10C0400325 & $\mathrm{VOA}$ & (1,1-Dimethylethyl)benzene & 5 & 0 & $\mathrm{ug} / \mathrm{kg}$ & 95.9 & 476 & 246.04 & 99.4 & 204.04691 \\
\hline X10C0400325 & VOA & (1-Methylpropyl)benzene & 5 & 0 & $\mathrm{ug} / \mathrm{kg}$ & 95.9 & 476 & 246.04 & 99.4 & 204.04691 \\
\hline X10C0400325 & VOA & 1,1,1,2-Tetrachloroethane & 5 & 0 & $\mathrm{ug} / \mathrm{kg}$ & 95.9 & 476 & 246.04 & 99.4 & 204.04691 \\
\hline X10C0400325 & VOA & 1,1,1-Trichloroethane & 5 & 0 & $\mathrm{ug} / \mathrm{kg}$ & 95.9 & 476 & 246.04 & 99.4 & 204.04691 \\
\hline X10C0400325 & VOA & 1,1,2,2-Tetrachloroethane & 5 & 0 & $\mathrm{ug} / \mathrm{kg}$ & 95.9 & 476 & 246.04 & 99.4 & 204.04691 \\
\hline X10C0400325 & VOA & 1,1,2-Trichloroethane & 5 & 0 & $\mathrm{ug} / \mathrm{kg}$ & 95.9 & 476 & 246.04 & 99.4 & 204.04691 \\
\hline X10C0400325 & VOA & 1,1-Dichloroethane & 5 & 0 & $\mathrm{ug} / \mathrm{kg}$ & 95.9 & 476 & 246.04 & 99.4 & 204.04691 \\
\hline X10C0400325 & VOA & 1,1-Dichloroethene & 10 & 0 & $\mathrm{ug} / \mathrm{kg}$ & 0.01 & 476 & 123.025 & 47.955 & 187.93246 \\
\hline X10C0400325 & VOA & 1,1-Dichloropropene & 5 & 0 & $\mathrm{ug} / \mathrm{kg}$ & 95.9 & 476 & 246.04 & 99.4 & 204.04691 \\
\hline X10C0400325 & VOA & 1,2,3-Trichlorobenzene & 5 & 0 & $\mathrm{ug} / \mathrm{kg}$ & 95.9 & 476 & 246.04 & 99.4 & 204.04691 \\
\hline X10C0400325 & VOA & 1,2,3-Trichloropropane & 5 & 0 & $\mathrm{ug} / \mathrm{kg}$ & 95.9 & 476 & 246.04 & 99.4 & 204.04691 \\
\hline X10C0400325 & VOA & 1,2,4-Trimethylbenzene & 5 & 3 & $\mathrm{ug} / \mathrm{kg}$ & 99.4 & 1690 & 513.88 & 180 & 672.89581 \\
\hline X10C0400325 & VOA & 1,2-Dibromo-3-chloropropane & 5 & 0 & $\mathrm{ug} / \mathrm{kg}$ & 95.9 & 476 & 246.04 & 99.4 & 204.04691 \\
\hline X10C0400325 & VOA & 1,2-Dibromoethane & 5 & 0 & $\mathrm{ug} / \mathrm{kg}$ & 95.9 & 476 & 246.04 & 99.4 & 204.04691 \\
\hline X10C0400325 & VOA & 1,2-Dichloroethane & 10 & 0 & $\mathrm{ug} / \mathrm{kg}$ & 0.01 & 476 & 123.025 & 47.955 & 187.93246 \\
\hline X10C0400325 & VOA & 1,2-Dichloroethene & 5 & 0 & $\mathrm{ug} / \mathrm{kg}$ & 192 & 952 & 492.2 & 199 & 407.98431 \\
\hline X10C0400325 & VOA & 1,2-Dichloropropane & 5 & 0 & $\mathrm{ug} / \mathrm{kg}$ & 95.9 & 476 & 246.04 & 99.4 & 204.04691 \\
\hline X10C0400325 & VOA & 1,2-Dimethylbenzene & 5 & 0 & $\mathrm{ug} / \mathrm{kg}$ & 99.4 & 1670 & 610.48 & 463 & 610.86991 \\
\hline X10C0400325 & VOA & 1,3,5-Trimethylbenzene & 5 & 1 & $\mathrm{ug} / \mathrm{kg}$ & 95.9 & 514 & 253.64 & 99.4 & 215.15851 \\
\hline X10C0400325 & VOA & 1,3-Dichloropropane & 5 & 0 & $\mathrm{ug} / \mathrm{kg}$ & 95.9 & 476 & 246.04 & 99.4 & 204.04691 \\
\hline X10C0400325 & VOA & 1-Chloro-4-methylbenzene & 5 & 0 & $\mathrm{ug} / \mathrm{kg}$ & 95.9 & 476 & 246.04 & 99.4 & 204.04691 \\
\hline X10C0400325 & VOA & 1-Methyl-4-(1-methylethyl)benzene & 5 & 0 & $\mathrm{ug} / \mathrm{kg}$ & 95.9 & 476 & 246.04 & 99.4 & 204.04691 \\
\hline X10C0400325 & VOA & 2,2-Dichloropropane & 5 & 0 & $\mathrm{ug} / \mathrm{kg}$ & 95.9 & 476 & 246.04 & 99.4 & 204.04691 \\
\hline X10C0400325 & $\mathrm{VOA}$ & 2-Butanone & 10 & 0 & $\mathrm{ug} / \mathrm{kg}$ & 0.05 & 2380 & 614.525 & 239.525 & 938.67408 \\
\hline
\end{tabular}




\begin{tabular}{|c|c|c|c|c|c|c|c|c|c|c|}
\hline X10C0400325 & VOA & 2-Hexanone & 5 & 0 & $\mathrm{ug} / \mathrm{kg}$ & 479 & 2380 & 1229 & 497 & 1019.091 \\
\hline X10C0400325 & VOA & 4-Methyl-2-pentanone & 5 & 0 & $\mathrm{ug} / \mathrm{kg}$ & 479 & 2380 & 1229 & 497 & 1019.091 \\
\hline X10C0400325 & VOA & Acetone & 5 & 0 & $\mathrm{ug} / \mathrm{kg}$ & 479 & 2380 & 1229 & 497 & 1019.091 \\
\hline X10C0400325 & VOA & Acetonitrile & 5 & 0 & $\mathrm{ug} / \mathrm{kg}$ & 2400 & 11900 & 6158 & 2490 & 5106.0082 \\
\hline X10C0400325 & VOA & Acrolein & 5 & 0 & $\mathrm{ug} / \mathrm{kg}$ & 479 & 2380 & 1229 & 497 & 1019.091 \\
\hline X10C0400325 & VOA & Acrylonitrile & 5 & 0 & $\mathrm{ug} / \mathrm{kg}$ & 479 & 2380 & 1229 & 497 & 1019.091 \\
\hline X10C0400325 & VOA & Benzene & 10 & 1 & $\mathrm{ug} / \mathrm{kg}$ & 0.01 & 1810 & 294.0903 & 47.9815 & 564.75635 \\
\hline X10C0400325 & VOA & Bromobenzene & 5 & 0 & $\mathrm{ug} / \mathrm{kg}$ & 95.9 & 476 & 246.04 & 99.4 & 204.04691 \\
\hline X10C0400325 & VOA & Bromochloromethane & 5 & 0 & $\mathrm{ug} / \mathrm{kg}$ & 95.9 & 476 & 246.04 & 99.4 & 204.04691 \\
\hline X10C0400325 & VOA & Bromodichloromethane & 5 & 0 & $\mathrm{ug} / \mathrm{kg}$ & 95.9 & 476 & 246.04 & 99.4 & 204.04691 \\
\hline X10C0400325 & VOA & Bromoform & 5 & 0 & $\mathrm{ug} / \mathrm{kg}$ & 95.9 & 476 & 246.04 & 99.4 & 204.04691 \\
\hline $\mathrm{X} 10 \mathrm{C} 0400325$ & VOA & Bromomethane & 5 & 0 & $\mathrm{ug} / \mathrm{kg}$ & 95.9 & 476 & 246.04 & 99.4 & 204.04691 \\
\hline X10C0400325 & VOA & Butylbenzene & 5 & 0 & $\mathrm{ug} / \mathrm{kg}$ & 95.9 & 476 & 246.04 & 99.4 & 204.04691 \\
\hline X10C0400325 & VOA & Carbon disulfide & 5 & 1 & $\mathrm{ug} / \mathrm{kg}$ & 479 & 2380 & 1403.2 & 1350 & 929.32755 \\
\hline X10C0400325 & VOA & Carbon tetrachloride & 10 & 0 & $\mathrm{ug} / \mathrm{kg}$ & 0.01 & 476 & 123.025 & 47.955 & 187.93246 \\
\hline X10C0400325 & VOA & Chlorobenzene & 10 & 0 & $\mathrm{ug} / \mathrm{kg}$ & 0.01 & 476 & 123.025 & 47.955 & 187.93246 \\
\hline X10C0400325 & VOA & Chloroethane & 5 & 0 & $\mathrm{ug} / \mathrm{kg}$ & 95.9 & 476 & 246.04 & 99.4 & 204.04691 \\
\hline X10C0400325 & VOA & Chloroform & 10 & 0 & $\mathrm{ug} / \mathrm{kg}$ & 0.01 & 476 & 123.025 & 47.955 & 187.93246 \\
\hline X10C0400325 & VOA & Chloromethane & 5 & 0 & $\mathrm{ug} / \mathrm{kg}$ & 95.9 & 476 & 246.04 & 99.4 & 204.04691 \\
\hline X10C0400325 & VOA & cis-1,2-Dichloroethene & 5 & 0 & $\mathrm{ug} / \mathrm{kg}$ & 95.9 & 476 & 246.04 & 99.4 & 204.04691 \\
\hline X10C0400325 & VOA & cis-1,3-Dichloropropene & 5 & 0 & $\mathrm{ug} / \mathrm{kg}$ & 95.9 & 476 & 246.04 & 99.4 & 204.04691 \\
\hline X10C0400325 & VOA & Cumene & 5 & 0 & $\mathrm{ug} / \mathrm{kg}$ & 95.9 & 476 & 291.76 & 328 & 187.95154 \\
\hline X10C0400325 & VOA & Dibromochloromethane & 5 & 0 & $\mathrm{ug} / \mathrm{kg}$ & 95.9 & 476 & 246.04 & 99.4 & 204.04691 \\
\hline X10C0400325 & VOA & Dibromomethane & 5 & 0 & $\mathrm{ug} / \mathrm{kg}$ & 95.9 & 476 & 246.04 & 99.4 & 204.04691 \\
\hline X10C0400325 & VOA & Dichlorodifluoromethane & 5 & 0 & $\mathrm{ug} / \mathrm{kg}$ & 95.9 & 476 & 246.04 & 99.4 & 204.04691 \\
\hline X10C0400325 & VOA & Ethylbenzene & 5 & 1 & $\mathrm{ug} / \mathrm{kg}$ & 95.9 & 1700 & 566.16 & 463 & 660.80498 \\
\hline X10C0400325 & VOA & Hexane & 5 & 0 & $\mathrm{ug} / \mathrm{kg}$ & 479 & 2380 & 1229 & 497 & 1019.091 \\
\hline X10C0400325 & VOA & M + P Xylene & 5 & 0 & $\mathrm{ug} / \mathrm{kg}$ & 192 & 952 & 492.2 & 199 & 407.98431 \\
\hline X10C0400325 & VOA & Methanol & 4 & 0 & $\mathrm{ug} / \mathrm{kg}$ & 4550 & 10000 & 7782.5 & 8290 & 2305.0145 \\
\hline X10C0400325 & VOA & Methyl methacrylate & 5 & 0 & $\mathrm{ug} / \mathrm{kg}$ & 479 & 2380 & 1229 & 497 & 1019.091 \\
\hline X10C0400325 & VOA & Methylcyclohexane & 5 & 0 & $\mathrm{ug} / \mathrm{kg}$ & 95.9 & 476 & 246.04 & 99.4 & 204.04691 \\
\hline X10C0400325 & VOA & Methylene chloride & 5 & 0 & $\mathrm{ug} / \mathrm{kg}$ & 479 & 2380 & 1229 & 497 & 1019.091 \\
\hline X10C0400325 & VOA & o-Chlorotoluene & 5 & 0 & $\mathrm{ug} / \mathrm{kg}$ & 95.9 & 476 & 246.04 & 99.4 & 204.04691 \\
\hline X10C0400325 & VOA & Propylbenzene & 5 & 1 & $\mathrm{ug} / \mathrm{kg}$ & 95.9 & 476 & 288.56 & 312 & 187.31541 \\
\hline X10C0400325 & VOA & Propylene glycol & 4 & 0 & $\mathrm{ug} / \mathrm{kg}$ & 45500 & 100000 & 77825 & 82900 & 23050.145 \\
\hline X10C0400325 & VOA & Styrene & 5 & 1 & $\mathrm{ug} / \mathrm{kg}$ & 95.9 & 707 & 367.56 & 463 & 266.31084 \\
\hline X10C0400325 & VOA & Tetrachloroethene & 10 & 0 & $\mathrm{ug} / \mathrm{kg}$ & 0.01 & 476 & 123.025 & 47.955 & 187.93246 \\
\hline X10C0400325 & VOA & Toluene & 5 & 1 & $\mathrm{ug} / \mathrm{kg}$ & 95.9 & 476 & 255.96 & 149 & 196.18997 \\
\hline X10C0400325 & $\mathrm{VOA}$ & Total Xylene & 5 & 3 & $\mathrm{ug} / \mathrm{kg}$ & 298 & 2040 & 930.2 & 523 & 756.58324 \\
\hline X10C0400325 & VOA & trans-1,2-Dichloroethene & 5 & 0 & $\mathrm{ug} / \mathrm{kg}$ & 95.9 & 476 & 246.04 & 99.4 & 204.04691 \\
\hline X10C0400325 & VOA & trans-1,3-Dichloropropene & 5 & 0 & $\mathrm{ug} / \mathrm{kg}$ & 95.9 & 476 & 246.04 & 99.4 & 204.04691 \\
\hline X10C0400325 & VOA & Trichloroethene & 10 & 0 & $\mathrm{ug} / \mathrm{kg}$ & 0.01 & 476 & 123.025 & 47.955 & 187.93246 \\
\hline X10C0400325 & VOA & Trichlorofluoromethane & 5 & 0 & $\mathrm{ug} / \mathrm{kg}$ & 95.9 & 476 & 246.04 & 99.4 & 204.04691 \\
\hline $\mathrm{X} 10 \mathrm{C} 0400325$ & VOA & Vinyl chloride & 10 & 0 & $\mathrm{ug} / \mathrm{kg}$ & 0.01 & 476 & 123.025 & 47.955 & 187.93246 \\
\hline X10C0404149 & HERB & $2,4-\mathrm{D}$ & 2 & 0 & $\mathrm{ug} / \mathrm{kg}$ & 0.05 & 59 & 29.525 & 29.525 & 41.683945 \\
\hline $\mathrm{X} 10 \mathrm{C} 0404149$ & HERB & Silvex & 2 & 0 & $\mathrm{ug} / \mathrm{kg}$ & 0.05 & 59 & 29.525 & 29.525 & 41.683945 \\
\hline X10C0404149 & METAL & Arsenic & 2 & 0 & $\mathrm{mg} / \mathrm{L}$ & 0.3 & 146 & 73.15 & 73.15 & 103.02546 \\
\hline X10C0404149 & METAL & Barium & 2 & 1 & $\mathrm{mg} / \mathrm{L}$ & 0.05 & 8.81 & 4.43 & 4.43 & 6.1942554 \\
\hline X10C0404149 & METAL & Cadmium & 2 & 0 & $\mathrm{mg} / \mathrm{L}$ & 0.05 & 24.4 & 12.225 & 12.225 & 17.21805 \\
\hline X10C0404149 & METAL & Chromium & 2 & 1 & $\mathrm{mg} / \mathrm{L}$ & 0.05 & 13800 & 6900.025 & 6900.025 & 9758.0382 \\
\hline X10C0404149 & METAL & Lead & 2 & 1 & $\mathrm{mg} / \mathrm{L}$ & 0.1 & 161 & 80.55 & 80.55 & 113.77348 \\
\hline X10C0404149 & METAL & Mercury & 2 & 1 & $\mathrm{mg} / \mathrm{L}$ & 0.002 & 0.4 & 0.201 & 0.201 & 0.2814285 \\
\hline X10C0404149 & METAL & Selenium & 2 & 0 & $\mathrm{mg} / \mathrm{L}$ & 0.3 & 146 & 73.15 & 73.15 & 103.02546 \\
\hline X10C0404149 & METAL & Silver & 2 & 0 & $\mathrm{mg} / \mathrm{L}$ & 0.05 & 24.4 & 12.225 & 12.225 & 17.21805 \\
\hline X10C0404149 & METAL & Total Uranium & 1 & 0 & $\mathrm{pCi} / \mathrm{g}$ & -0.0168 & -0.0168 & -0.0168 & & \\
\hline X10C0404149 & PPCB & 4,4'-DDD & 1 & 0 & $\mathrm{ug} / \mathrm{kg}$ & 133 & 133 & 133 & & \\
\hline X10C0404149 & РPCB & 4,4'-DDE & 1 & 0 & $\mathrm{ug} / \mathrm{kg}$ & 133 & 133 & 133 & & \\
\hline X10C0404149 & PPCB & 4,4'-DDT & 1 & 0 & $\mathrm{ug} / \mathrm{kg}$ & 133 & 133 & 133 & & \\
\hline X10C0404149 & РPCB & Aldrin & 1 & 0 & $\mathrm{ug} / \mathrm{kg}$ & 66.4 & 66.4 & 66.4 & & \\
\hline X10C0404149 & РPCB & alpha-BHC & 1 & 0 & $\mathrm{ug} / \mathrm{kg}$ & 66.4 & 66.4 & 66.4 & & \\
\hline X10C0404149 & PPCB & alpha-Chlordane & 1 & 0 & $\mathrm{ug} / \mathrm{kg}$ & 66.4 & 66.4 & 66.4 & & \\
\hline X10C0404149 & PPCB & beta-BHC & 1 & 0 & $\mathrm{ug} / \mathrm{kg}$ & 66.4 & 66.4 & 66.4 & & \\
\hline X10C0404149 & РPCB & Chlordane & 2 & 0 & $\mathrm{ug} / \mathrm{kg}$ & 0.0025 & 830 & 415.00125 & 415.00125 & 586.89686 \\
\hline X10C0404149 & РPCB & delta-BHC & 1 & 0 & $\mathrm{ug} / \mathrm{kg}$ & 66.4 & 66.4 & 66.4 & & \\
\hline X10C0404149 & PPCB & Dieldrin & 1 & 0 & $\mathrm{ug} / \mathrm{kg}$ & 133 & 133 & 133 & & \\
\hline X10C0404149 & РPCB & Endosulfan I & 1 & 0 & $\mathrm{ug} / \mathrm{kg}$ & 66.4 & 66.4 & 66.4 & & \\
\hline X10C0404149 & РPCB & Endosulfan II & 1 & 0 & $\mathrm{ug} / \mathrm{kg}$ & 133 & 133 & 133 & & \\
\hline X10C0404149 & РPCB & Endosulfan sulfate & 1 & 0 & $\mathrm{ug} / \mathrm{kg}$ & 133 & 133 & 133 & & \\
\hline X10C0404149 & РPCB & Endrin & 2 & 0 & $\mathrm{ug} / \mathrm{kg}$ & 0.0004 & 133 & 66.5002 & 66.5002 & 94.044919 \\
\hline X10C0404149 & РPCB & Endrin aldehyde & 1 & 0 & $\mathrm{ug} / \mathrm{kg}$ & 133 & 133 & 133 & & \\
\hline
\end{tabular}




\begin{tabular}{|c|c|c|c|c|c|c|c|c|c|c|}
\hline X10C0404149 & PPCB & Endrin ketone & 1 & 0 & $\mathrm{ug} / \mathrm{kg}$ & 133 & 133 & 133 & & \\
\hline X10C0404149 & РPCB & gamma-Chlordane & 1 & 0 & $\mathrm{ug} / \mathrm{kg}$ & 66.4 & 66.4 & 66.4 & & \\
\hline X10C0404149 & РPCB & Heptachlor & 2 & 0 & $\mathrm{ug} / \mathrm{kg}$ & 0.0002 & 66.4 & 33.2001 & 33.2001 & 46.951749 \\
\hline X10C0404149 & РPCB & Heptachlor epoxide & 2 & 0 & $\mathrm{ug} / \mathrm{kg}$ & 0.0002 & 66.4 & 33.2001 & 33.2001 & 46.951749 \\
\hline X10C0404149 & PPCB & Lindane & 2 & 0 & $\mathrm{ug} / \mathrm{kg}$ & 0.0002 & 66.4 & 33.2001 & 33.2001 & 46.951749 \\
\hline X10C0404149 & PPCB & Methoxychlor & 2 & 0 & $\mathrm{ug} / \mathrm{kg}$ & 0.002 & 664 & 332.001 & 332.001 & 469.51749 \\
\hline X10C0404149 & PPCB & Toxaphene & 2 & 0 & $\mathrm{ug} / \mathrm{kg}$ & 0.005 & 1660 & 830.0025 & 830.0025 & 1173.7937 \\
\hline X10C0404149 & RADS & Americium-241 & 1 & 0 & $\mathrm{pCi} / \mathrm{g}$ & 0.056 & 0.056 & 0.056 & & \\
\hline X10C0404149 & RADS & Carbon-14 & 1 & 0 & $\mathrm{pCi} / \mathrm{g}$ & -0.973 & -0.973 & -0.973 & & \\
\hline X10C0404149 & RADS & Cesium-137 & 1 & 0 & $\mathrm{pCi} / \mathrm{g}$ & -0.0129 & -0.0129 & -0.0129 & & \\
\hline X10C0404149 & RADS & Cobalt- 60 & 1 & 0 & $\mathrm{pCi} / \mathrm{g}$ & 0.0163 & 0.0163 & 0.0163 & & \\
\hline X10C0404149 & RADS & Curium-243/244 & 1 & 0 & $\mathrm{pCi} / \mathrm{g}$ & -0.0265 & -0.0265 & -0.0265 & & \\
\hline X10C0404149 & RADS & Iodine-129 & 1 & 0 & $\mathrm{pCi} / \mathrm{g}$ & 0.0444 & 0.0444 & 0.0444 & & \\
\hline X10C0404149 & RADS & Neptunium-237 & 1 & 0 & $\mathrm{pCi} / \mathrm{g}$ & 0.0514 & 0.0514 & 0.0514 & & \\
\hline X10C0404149 & RADS & Plutonium-238 & 1 & 0 & $\mathrm{pCi} / \mathrm{g}$ & -0.0148 & -0.0148 & -0.0148 & & \\
\hline X10C0404149 & RADS & Plutonium-239/240 & 1 & 0 & $\mathrm{pCi} / \mathrm{g}$ & -0.000293 & -0.000293 & -0.000293 & & \\
\hline X10C0404149 & RADS & Radium-226 & 1 & 0 & $\mathrm{pCi} / \mathrm{g}$ & 0.354 & 0.354 & 0.354 & & \\
\hline X10C0404149 & RADS & Strontium-90 & 1 & 0 & $\mathrm{pCi} / \mathrm{g}$ & -0.731 & -0.731 & -0.731 & & \\
\hline X10C0404149 & RADS & Technetium-99 & 1 & 0 & $\mathrm{pCi} / \mathrm{g}$ & -0.292 & -0.292 & -0.292 & & \\
\hline X10C0404149 & RADS & Thorium-230 & 1 & 0 & $\mathrm{pCi} / \mathrm{g}$ & -1.32 & -1.32 & -1.32 & & \\
\hline X10C0404149 & RADS & Thorium-232 & 1 & 0 & $\mathrm{pCi} / \mathrm{g}$ & 0.369 & 0.369 & 0.369 & & \\
\hline X10C0404149 & RADS & Tritium & 1 & 0 & $\mathrm{pCi} / \mathrm{g}$ & 135 & 135 & 135 & & \\
\hline X10C0404149 & RADS & Uranium-232 & 1 & 0 & $\%$ & 88.5 & 88.5 & 88.5 & & \\
\hline X10C0404149 & RADS & Uranium-233/234 & 1 & 0 & $\mathrm{pCi} / \mathrm{g}$ & -0.0462 & -0.0462 & -0.0462 & & \\
\hline X10C0404149 & RADS & Uranium-235/236 & 1 & 0 & $\mathrm{pCi} / \mathrm{g}$ & 0.0386 & 0.0386 & 0.0386 & & \\
\hline X10C0404149 & RADS & Uranium-238 & 1 & 0 & $\mathrm{pCi} / \mathrm{g}$ & -0.00925 & -0.00925 & -0.00925 & & \\
\hline X10C0404149 & SVOA & 1,2,4-Trichlorobenzene & 1 & 0 & $\mathrm{ug} / \mathrm{kg}$ & 109 & 109 & 109 & & \\
\hline X10C0404149 & SVOA & 1,2-Dichlorobenzene & 1 & 0 & $\mathrm{ug} / \mathrm{kg}$ & 109 & 109 & 109 & & \\
\hline X10C0404149 & SVOA & 1,3-Dichlorobenzene & 1 & 0 & $\mathrm{ug} / \mathrm{kg}$ & 109 & 109 & 109 & & \\
\hline X10C0404149 & SVOA & 1,4-Dichlorobenzene & 2 & 0 & $\mathrm{ug} / \mathrm{kg}$ & 0.01 & 109 & 54.505 & 54.505 & 77.067568 \\
\hline X10C0404149 & SVOA & 2,2'-Dichlorodiisopropyl ether & 1 & 0 & $\mathrm{ug} / \mathrm{kg}$ & 6600 & 6600 & 6600 & & \\
\hline X10C0404149 & SVOA & 2,3,4,6-Tetrachlorophenol & 1 & 0 & $\mathrm{ug} / \mathrm{kg}$ & 6600 & 6600 & 6600 & & \\
\hline X10C0404149 & SVOA & 2,4,5-Trichlorophenol & 2 & 0 & $\mathrm{ug} / \mathrm{kg}$ & 0.05 & 6600 & 3300.025 & 3300.025 & 4666.8694 \\
\hline X10C0404149 & SVOA & 2,4,6-Trichlorophenol & 2 & 0 & $\mathrm{ug} / \mathrm{kg}$ & 0.05 & 6600 & 3300.025 & 3300.025 & 4666.8694 \\
\hline X10C0404149 & SVOA & 2,4-Dichlorophenol & 1 & 0 & $\mathrm{ug} / \mathrm{kg}$ & 6600 & 6600 & 6600 & & \\
\hline X10C0404149 & SVOA & 2,4-Dimethylphenol & 1 & 0 & $\mathrm{ug} / \mathrm{kg}$ & 6600 & 6600 & 6600 & & \\
\hline X10C0404149 & SVOA & 2,4-Dinitrophenol & 1 & 0 & $\mathrm{ug} / \mathrm{kg}$ & 13200 & 13200 & 13200 & & \\
\hline X10C0404149 & SVOA & 2,4-Dinitrotoluene & 2 & 0 & $\mathrm{ug} / \mathrm{kg}$ & 0.05 & 6600 & 3300.025 & 3300.025 & 4666.8694 \\
\hline X10C0404149 & SVOA & 2,6-Dinitrotoluene & 1 & 0 & $\mathrm{ug} / \mathrm{kg}$ & 6600 & 6600 & 6600 & & \\
\hline X10C0404149 & SVOA & 2-Chloronaphthalene & 1 & 0 & $\mathrm{ug} / \mathrm{kg}$ & 660 & 660 & 660 & & \\
\hline X10C0404149 & SVOA & 2-Chlorophenol & 1 & 0 & $\mathrm{ug} / \mathrm{kg}$ & 6600 & 6600 & 6600 & & \\
\hline X10C0404149 & SVOA & 2-Methyl-4,6-dinitrophenol & 1 & 0 & $\mathrm{ug} / \mathrm{kg}$ & 6600 & 6600 & 6600 & & \\
\hline X10C0404149 & SVOA & 2-Methylnaphthalene & 1 & 0 & $\mathrm{ug} / \mathrm{kg}$ & 660 & 660 & 660 & & \\
\hline X10C0404149 & SVOA & 2-Methylphenol & 2 & 0 & $\mathrm{ug} / \mathrm{kg}$ & 0.05 & 6600 & 3300.025 & 3300.025 & 4666.8694 \\
\hline X10C0404149 & SVOA & 2-Nitrobenzenamine & 1 & 0 & $\mathrm{ug} / \mathrm{kg}$ & 6600 & 6600 & 6600 & & \\
\hline X10C0404149 & SVOA & 2-Nitrophenol & 1 & 0 & $\mathrm{ug} / \mathrm{kg}$ & 6600 & 6600 & 6600 & & \\
\hline X10C0404149 & SVOA & 3,3'-Dichlorobenzidine & 1 & 0 & $\mathrm{ug} / \mathrm{kg}$ & 6600 & 6600 & 6600 & & \\
\hline X10C0404149 & SVOA & 3-Nitrobenzenamine & 1 & 0 & $\mathrm{ug} / \mathrm{kg}$ & 6600 & 6600 & 6600 & & \\
\hline X10C0404149 & SVOA & 4-Bromophenyl phenyl ether & 1 & 0 & $\mathrm{ug} / \mathrm{kg}$ & 6600 & 6600 & 6600 & & \\
\hline X10C0404149 & SVOA & 4-Chloro-3-methylphenol & 1 & 0 & $\mathrm{ug} / \mathrm{kg}$ & 6600 & 6600 & 6600 & & \\
\hline X10C0404149 & SVOA & 4-Chlorobenzenamine & 1 & 0 & $\mathrm{ug} / \mathrm{kg}$ & 6600 & 6600 & 6600 & & \\
\hline X10C0404149 & SVOA & 4-Chlorophenyl phenyl ether & 1 & 0 & $\mathrm{ug} / \mathrm{kg}$ & 6600 & 6600 & 6600 & & \\
\hline X10C0404149 & SVOA & 4-Nitrobenzenamine & 1 & 0 & $\mathrm{ug} / \mathrm{kg}$ & 6600 & 6600 & 6600 & & \\
\hline X10C0404149 & SVOA & 4-Nitrophenol & 1 & 0 & $\mathrm{ug} / \mathrm{kg}$ & 6600 & 6600 & 6600 & & \\
\hline X10C0404149 & SVOA & Acenaphthene & 1 & 0 & $\mathrm{ug} / \mathrm{kg}$ & 660 & 660 & 660 & & \\
\hline X10C0404149 & SVOA & Acenaphthylene & 1 & 0 & $\mathrm{ug} / \mathrm{kg}$ & 660 & 660 & 660 & & \\
\hline X10C0404149 & SVOA & Acetophenone & 1 & 0 & $\mathrm{ug} / \mathrm{kg}$ & 6600 & 6600 & 6600 & & \\
\hline X10C0404149 & SVOA & Anthracene & 1 & 0 & $\mathrm{ug} / \mathrm{kg}$ & 660 & 660 & 660 & & \\
\hline X10C0404149 & SVOA & Benz(a)anthracene & 1 & 0 & $\mathrm{ug} / \mathrm{kg}$ & 660 & 660 & 660 & & \\
\hline X10C0404149 & SVOA & Benzenemethanol & 1 & 0 & $\mathrm{ug} / \mathrm{kg}$ & 6600 & 6600 & 6600 & & \\
\hline X10C0404149 & SVOA & Benzidine & 1 & 0 & $\mathrm{ug} / \mathrm{kg}$ & 6600 & 6600 & 6600 & & \\
\hline X10C0404149 & SVOA & Benzo(a)pyrene & 1 & 0 & $\mathrm{ug} / \mathrm{kg}$ & 660 & 660 & 660 & & \\
\hline X10C0404149 & SVOA & Benzo(b)fluoranthene & 1 & 0 & $\mathrm{ug} / \mathrm{kg}$ & 660 & 660 & 660 & & \\
\hline X10C0404149 & SVOA & Benzo(ghi)perylene & 1 & 0 & $\mathrm{ug} / \mathrm{kg}$ & 660 & 660 & 660 & & \\
\hline X10C0404149 & SVOA & Benzo(k)fluoranthene & 1 & 0 & $\mathrm{ug} / \mathrm{kg}$ & 660 & 660 & 660 & & \\
\hline X10C0404149 & SVOA & Benzoic acid & 1 & 0 & $\mathrm{ug} / \mathrm{kg}$ & 13200 & 13200 & 13200 & & \\
\hline X10C0404149 & SVOA & Bis(2-chloroethoxy)methane & 1 & 0 & $\mathrm{ug} / \mathrm{kg}$ & 6600 & 6600 & 6600 & & \\
\hline X10C0404149 & SVOA & Bis(2-chloroethyl) ether & 1 & 0 & $\mathrm{ug} / \mathrm{kg}$ & 6600 & 6600 & 6600 & & \\
\hline X10C0404149 & SVOA & Bis(2-ethylhexyl)phthalate & 1 & 1 & $\mathrm{ug} / \mathrm{kg}$ & 18500000 & 18500000 & 18500000 & 18500000 & \\
\hline X10C0404149 & SVOA & Butyl benzyl phthalate & 1 & 0 & $\mathrm{ug} / \mathrm{kg}$ & 6600 & 6600 & 6600 & & \\
\hline
\end{tabular}




\begin{tabular}{|c|c|c|c|c|c|c|c|c|c|c|}
\hline X10C0404149 & SVOA & Carbazole & 1 & 0 & $\mathrm{ug} / \mathrm{kg}$ & 660 & 660 & 660 & & \\
\hline X10C0404149 & SVOA & Chrysene & 1 & 0 & $\mathrm{ug} / \mathrm{kg}$ & 660 & 660 & 660 & & \\
\hline X10C0404149 & SVOA & Dibenz $(\mathrm{a}, \mathrm{h})$ anthracene & 1 & 0 & $\mathrm{ug} / \mathrm{kg}$ & 660 & 660 & 660 & & \\
\hline X10C0404149 & SVOA & Dibenzofuran & 1 & 0 & $\mathrm{ug} / \mathrm{kg}$ & 6600 & 6600 & 6600 & & \\
\hline X10C0404149 & SVOA & Diethyl phthalate & 1 & 0 & $\mathrm{ug} / \mathrm{kg}$ & 6600 & 6600 & 6600 & & \\
\hline X10C0404149 & SVOA & Dimethyl phthalate & 1 & 0 & $\mathrm{ug} / \mathrm{kg}$ & 6600 & 6600 & 6600 & & \\
\hline X10C0404149 & SVOA & Di-n-butyl phthalate & 1 & 0 & $\mathrm{ug} / \mathrm{kg}$ & 6600 & 6600 & 6600 & & \\
\hline X10C0404149 & SVOA & Di-n-octylphthalate & 1 & 1 & $\mathrm{ug} / \mathrm{kg}$ & 12100 & 12100 & 12100 & 12100 & \\
\hline X10C0404149 & SVOA & Diphenylamine & 1 & 0 & $\mathrm{ug} / \mathrm{kg}$ & 6600 & 6600 & 6600 & & \\
\hline X10C0404149 & SVOA & Fluoranthene & 1 & 0 & $\mathrm{ug} / \mathrm{kg}$ & 660 & 660 & 660 & & \\
\hline X10C0404149 & SVOA & Fluorene & 1 & 0 & $\mathrm{ug} / \mathrm{kg}$ & 660 & 660 & 660 & & \\
\hline X10C0404149 & SVOA & Hexachlorobenzene & 2 & 0 & $\mathrm{ug} / \mathrm{kg}$ & 0.05 & 6600 & 3300.025 & 3300.025 & 4666.8694 \\
\hline X10C0404149 & SVOA & Hexachlorobutadiene & 2 & 0 & $\mathrm{ug} / \mathrm{kg}$ & 0.05 & 6600 & 3300.025 & 3300.025 & 4666.8694 \\
\hline X10C0404149 & SVOA & Hexachlorocyclopentadiene & 1 & 0 & $\mathrm{ug} / \mathrm{kg}$ & 6600 & 6600 & 6600 & & \\
\hline X10C0404149 & SVOA & Hexachloroethane & 2 & 0 & $\mathrm{ug} / \mathrm{kg}$ & 0.05 & 6600 & 3300.025 & 3300.025 & 4666.8694 \\
\hline X10C0404149 & SVOA & Indeno(1,2,3-cd)pyrene & 1 & 0 & $\mathrm{ug} / \mathrm{kg}$ & 660 & 660 & 660 & & \\
\hline X10C0404149 & SVOA & Isophorone & 1 & 0 & $\mathrm{ug} / \mathrm{kg}$ & 6600 & 6600 & 6600 & & \\
\hline X10C0404149 & SVOA & $\mathrm{m}+\mathrm{p}$ Methylphenol & 2 & 0 & $\mathrm{ug} / \mathrm{kg}$ & 0.05 & 6600 & 3300.025 & 3300.025 & 4666.8694 \\
\hline X10C0404149 & SVOA & Naphthalene & 1 & 0 & $\mathrm{ug} / \mathrm{kg}$ & 660 & 660 & 660 & & \\
\hline X10C0404149 & SVOA & Nitrobenzene & 2 & 0 & $\mathrm{ug} / \mathrm{kg}$ & 0.05 & 6600 & 3300.025 & 3300.025 & 4666.8694 \\
\hline X10C0404149 & SVOA & N-Nitroso-di-n-propylamine & 1 & 0 & $\mathrm{ug} / \mathrm{kg}$ & 6600 & 6600 & 6600 & & \\
\hline X10C0404149 & SVOA & Pentachlorophenol & 2 & 0 & $\mathrm{ug} / \mathrm{kg}$ & 0.05 & 6600 & 3300.025 & 3300.025 & 4666.8694 \\
\hline X10C0404149 & SVOA & Phenanthrene & 1 & 0 & $\mathrm{ug} / \mathrm{kg}$ & 660 & 660 & 660 & & \\
\hline X10C0404149 & SVOA & Phenol & 1 & 0 & $\mathrm{ug} / \mathrm{kg}$ & 6600 & 6600 & 6600 & & \\
\hline X10C0404149 & SVOA & Pyridine & 2 & 0 & $\mathrm{ug} / \mathrm{kg}$ & 0.05 & 6600 & 3300.025 & 3300.025 & 4666.8694 \\
\hline $\mathrm{X} 10 \mathrm{C} 0404149$ & $\mathrm{VOA}$ & (1,1-Dimethylethyl)benzene & 1 & 0 & $\mathrm{ug} / \mathrm{kg}$ & 109 & 109 & 109 & & \\
\hline X10C0404149 & VOA & (1-Methylpropyl)benzene & 1 & 0 & $\mathrm{ug} / \mathrm{kg}$ & 109 & 109 & 109 & & \\
\hline X10C0404149 & VOA & 1,1,1,2-Tetrachloroethane & 1 & 0 & $\mathrm{ug} / \mathrm{kg}$ & 109 & 109 & 109 & & \\
\hline X10C0404149 & VOA & 1,1,1-Trichloroethane & 1 & 0 & $\mathrm{ug} / \mathrm{kg}$ & 109 & 109 & 109 & & \\
\hline X10C0404149 & VOA & 1,1,2,2-Tetrachloroethane & 1 & 0 & $\mathrm{ug} / \mathrm{kg}$ & 109 & 109 & 109 & & \\
\hline X10C0404149 & VOA & 1,1,2-Trichloroethane & 1 & 0 & $\mathrm{ug} / \mathrm{kg}$ & 109 & 109 & 109 & & \\
\hline X10C0404149 & VOA & 1,1-Dichloroethane & 1 & 0 & $\mathrm{ug} / \mathrm{kg}$ & 109 & 109 & 109 & & \\
\hline X10C0404149 & VOA & 1,1-Dichloroethene & 2 & 0 & $\mathrm{ug} / \mathrm{kg}$ & 0.01 & 109 & 54.505 & 54.505 & 77.067568 \\
\hline X10C0404149 & VOA & 1,1-Dichloropropene & 1 & 0 & $\mathrm{ug} / \mathrm{kg}$ & 109 & 109 & 109 & & \\
\hline X10C0404149 & VOA & 1,2,3-Trichlorobenzene & 1 & 0 & $\mathrm{ug} / \mathrm{kg}$ & 109 & 109 & 109 & & \\
\hline X10C0404149 & VOA & 1,2,3-Trichloropropane & 1 & 0 & $\mathrm{ug} / \mathrm{kg}$ & 109 & 109 & 109 & & \\
\hline X10C0404149 & $\mathrm{VOA}$ & 1,2,4-Trimethylbenzene & 1 & 0 & $\mathrm{ug} / \mathrm{kg}$ & 109 & 109 & 109 & & \\
\hline X10C0404149 & VOA & 1,2-Dibromo-3-chloropropane & 1 & 0 & $\mathrm{ug} / \mathrm{kg}$ & 109 & 109 & 109 & & \\
\hline X10C0404149 & VOA & 1,2-Dibromoethane & 1 & 0 & $\mathrm{ug} / \mathrm{kg}$ & 109 & 109 & 109 & & \\
\hline X10C0404149 & VOA & 1,2-Dichloroethane & 2 & 0 & $\mathrm{ug} / \mathrm{kg}$ & 0.01 & 109 & 54.505 & 54.505 & 77.067568 \\
\hline X10C0404149 & VOA & 1,2-Dichloroethene & 1 & 0 & $\mathrm{ug} / \mathrm{kg}$ & 217 & 217 & 217 & & \\
\hline X10C0404149 & VOA & 1,2-Dichloropropane & 1 & 0 & $\mathrm{ug} / \mathrm{kg}$ & 109 & 109 & 109 & & \\
\hline X10C0404149 & $\mathrm{VOA}$ & 1,2-Dimethylbenzene & 1 & 0 & $\mathrm{ug} / \mathrm{kg}$ & 109 & 109 & 109 & & \\
\hline X10C0404149 & $\mathrm{VOA}$ & 1,3,5-Trimethylbenzene & 1 & 0 & $\mathrm{ug} / \mathrm{kg}$ & 109 & 109 & 109 & & \\
\hline X10C0404149 & VOA & 1,3-Dichloropropane & 1 & 0 & $\mathrm{ug} / \mathrm{kg}$ & 109 & 109 & 109 & & \\
\hline X10C0404149 & VOA & 1-Chloro-4-methylbenzene & 1 & 0 & $\mathrm{ug} / \mathrm{kg}$ & 109 & 109 & 109 & & \\
\hline X10C0404149 & VOA & 1-Methyl-4-(1-methylethyl)benzene & 1 & 0 & $\mathrm{ug} / \mathrm{kg}$ & 109 & 109 & 109 & & \\
\hline X10C0404149 & VOA & 2,2-Dichloropropane & 1 & 0 & $\mathrm{ug} / \mathrm{kg}$ & 109 & 109 & 109 & & \\
\hline X10C0404149 & VOA & 2-Butanone & 2 & 0 & $\mathrm{ug} / \mathrm{kg}$ & 0.05 & 543 & 271.525 & 271.525 & 383.92363 \\
\hline X10C0404149 & VOA & 2-Hexanone & 1 & 0 & $\mathrm{ug} / \mathrm{kg}$ & 543 & 543 & 543 & & \\
\hline X10C0404149 & VOA & 4-Methyl-2-pentanone & 1 & 0 & $\mathrm{ug} / \mathrm{kg}$ & 543 & 543 & 543 & & \\
\hline X10C0404149 & VOA & Acetone & 1 & 0 & $\mathrm{ug} / \mathrm{kg}$ & 543 & 543 & 543 & & \\
\hline X10C0404149 & VOA & Acetonitrile & 1 & 0 & $\mathrm{ug} / \mathrm{kg}$ & 2720 & 2720 & 2720 & & \\
\hline X10C0404149 & VOA & Acrolein & 1 & 0 & $\mathrm{ug} / \mathrm{kg}$ & 543 & 543 & 543 & & \\
\hline X10C0404149 & VOA & Acrylonitrile & 1 & 0 & $\mathrm{ug} / \mathrm{kg}$ & 543 & 543 & 543 & & \\
\hline X10C0404149 & VOA & Benzene & 2 & 0 & $\mathrm{ug} / \mathrm{kg}$ & 0.01 & 109 & 54.505 & 54.505 & 77.067568 \\
\hline X10C0404149 & $\mathrm{VOA}$ & Bromobenzene & 1 & 0 & $\mathrm{ug} / \mathrm{kg}$ & 109 & 109 & 109 & & \\
\hline X10C0404149 & VOA & Bromochloromethane & 1 & 0 & $\mathrm{ug} / \mathrm{kg}$ & 109 & 109 & 109 & & \\
\hline X10C0404149 & VOA & Bromodichloromethane & 1 & 0 & $\mathrm{ug} / \mathrm{kg}$ & 109 & 109 & 109 & & \\
\hline X10C0404149 & VOA & Bromoform & 1 & 0 & $\mathrm{ug} / \mathrm{kg}$ & 109 & 109 & 109 & & \\
\hline X10C0404149 & VOA & Bromomethane & 1 & 0 & $\mathrm{ug} / \mathrm{kg}$ & 109 & 109 & 109 & & \\
\hline X10C0404149 & VOA & Butylbenzene & 1 & 0 & $\mathrm{ug} / \mathrm{kg}$ & 109 & 109 & 109 & & \\
\hline X10C0404149 & VOA & Carbon disulfide & 1 & 0 & $\mathrm{ug} / \mathrm{kg}$ & 543 & 543 & 543 & & \\
\hline X10C0404149 & VOA & Carbon tetrachloride & 2 & 0 & $\mathrm{ug} / \mathrm{kg}$ & 0.01 & 109 & 54.505 & 54.505 & 77.067568 \\
\hline X10C0404149 & VOA & Chlorobenzene & 2 & 0 & $\mathrm{ug} / \mathrm{kg}$ & 0.01 & 109 & 54.505 & 54.505 & 77.067568 \\
\hline X10C0404149 & VOA & Chloroethane & 1 & 0 & $\mathrm{ug} / \mathrm{kg}$ & 109 & 109 & 109 & & \\
\hline X10C0404149 & VOA & Chloroform & 2 & 0 & $\mathrm{ug} / \mathrm{kg}$ & 0.01 & 109 & 54.505 & 54.505 & 77.067568 \\
\hline X10C0404149 & VOA & Chloromethane & 1 & 0 & $\mathrm{ug} / \mathrm{kg}$ & 109 & 109 & 109 & & \\
\hline X10C0404149 & VOA & cis-1,2-Dichloroethene & 1 & 0 & $\mathrm{ug} / \mathrm{kg}$ & 109 & 109 & 109 & & \\
\hline X10C0404149 & VOA & cis-1,3-Dichloropropene & 1 & 0 & $\mathrm{ug} / \mathrm{kg}$ & 109 & 109 & 109 & & \\
\hline
\end{tabular}




\begin{tabular}{|c|c|c|c|c|c|c|c|c|c|c|}
\hline X10C0404149 & VOA & Cumene & 1 & 0 & $\mathrm{ug} / \mathrm{kg}$ & 109 & 109 & 109 & & \\
\hline X10C0404149 & VOA & Dibromochloromethane & 1 & 0 & $\mathrm{ug} / \mathrm{kg}$ & 109 & 109 & 109 & & \\
\hline X10C0404149 & VOA & Dibromomethane & 1 & 0 & $\mathrm{ug} / \mathrm{kg}$ & 109 & 109 & 109 & & \\
\hline X10C0404149 & VOA & Dichlorodifluoromethane & 1 & 0 & $\mathrm{ug} / \mathrm{kg}$ & 109 & 109 & 109 & & \\
\hline X10C0404149 & VOA & Ethylbenzene & 1 & 0 & $\mathrm{ug} / \mathrm{kg}$ & 109 & 109 & 109 & & \\
\hline X10C0404149 & VOA & Hexane & 1 & 0 & $\mathrm{ug} / \mathrm{kg}$ & 543 & 543 & 543 & & \\
\hline X10C0404149 & $\mathrm{VOA}$ & M + P Xylene & 1 & 0 & $\mathrm{ug} / \mathrm{kg}$ & 217 & 217 & 217 & & \\
\hline X10C0404149 & VOA & Methanol & 1 & 0 & $\mathrm{ug} / \mathrm{kg}$ & 4250 & 4250 & 4250 & & \\
\hline X10C0404149 & VOA & Methyl methacrylate & 1 & 0 & $\mathrm{ug} / \mathrm{kg}$ & 543 & 543 & 543 & & \\
\hline X10C0404149 & VOA & Methylcyclohexane & 1 & 0 & $\mathrm{ug} / \mathrm{kg}$ & 109 & 109 & 109 & & \\
\hline X10C0404149 & VOA & Methylene chloride & 1 & 0 & $\mathrm{ug} / \mathrm{kg}$ & 543 & 543 & 543 & & \\
\hline X10C0404149 & VOA & o-Chlorotoluene & 1 & 0 & $\mathrm{ug} / \mathrm{kg}$ & 109 & 109 & 109 & & \\
\hline X10C0404149 & VOA & Propylbenzene & 1 & 0 & $\mathrm{ug} / \mathrm{kg}$ & 109 & 109 & 109 & & \\
\hline X10C0404149 & VOA & Propylene glycol & 1 & 0 & $\mathrm{ug} / \mathrm{kg}$ & 42500 & 42500 & 42500 & & \\
\hline X10C0404149 & VOA & Styrene & 1 & 0 & $\mathrm{ug} / \mathrm{kg}$ & 109 & 109 & 109 & & \\
\hline X10C0404149 & VOA & Tetrachloroethene & 2 & 0 & $\mathrm{ug} / \mathrm{kg}$ & 0.01 & 109 & 54.505 & 54.505 & 77.067568 \\
\hline X10C0404149 & VOA & Toluene & 1 & 0 & $\mathrm{ug} / \mathrm{kg}$ & 109 & 109 & 109 & & \\
\hline X10C0404149 & VOA & Total Xylene & 1 & 0 & $\mathrm{ug} / \mathrm{kg}$ & 326 & 326 & 326 & & \\
\hline X10C0404149 & VOA & trans-1,2-Dichloroethene & 1 & 0 & $\mathrm{ug} / \mathrm{kg}$ & 109 & 109 & 109 & & \\
\hline X10C0404149 & VOA & trans-1,3-Dichloropropene & 1 & 0 & $\mathrm{ug} / \mathrm{kg}$ & 109 & 109 & 109 & & \\
\hline X10C0404149 & VOA & Trichloroethene & 2 & 0 & $\mathrm{ug} / \mathrm{kg}$ & 0.01 & 109 & 54.505 & 54.505 & 77.067568 \\
\hline X10C0404149 & $\mathrm{VOA}$ & Trichlorofluoromethane & 1 & 0 & $\mathrm{ug} / \mathrm{kg}$ & 109 & 109 & 109 & & \\
\hline X10C0404149 & VOA & Vinyl chloride & 2 & 0 & $\mathrm{ug} / \mathrm{kg}$ & 0.01 & 109 & 54.505 & 54.505 & 77.067568 \\
\hline X10C0404151 & HERB & $2,4-\mathrm{D}$ & 2 & 0 & $\mathrm{ug} / \mathrm{kg}$ & 0.05 & 24.4 & 12.225 & 12.225 & 17.21805 \\
\hline $\mathrm{X} 10 \mathrm{C} 0404151$ & HERB & Silvex & 2 & 0 & $\mathrm{ug} / \mathrm{kg}$ & 0.05 & 24.4 & 12.225 & 12.225 & 17.21805 \\
\hline X10C0404151 & METAL & Arsenic & 2 & 0 & $\mathrm{mg} / \mathrm{L}$ & 0.3 & 136 & 68.15 & 68.15 & 95.95439 \\
\hline X10C0404151 & METAL & Barium & 2 & 0 & $\mathrm{mg} / \mathrm{L}$ & 0.05 & 22.7 & 11.375 & 11.375 & 16.015969 \\
\hline X10C0404151 & METAL & Cadmium & 2 & 0 & $\mathrm{mg} / \mathrm{L}$ & 0.05 & 22.7 & 11.375 & 11.375 & 16.015969 \\
\hline X10C0404151 & METAL & Chromium & 2 & 1 & $\mathrm{mg} / \mathrm{L}$ & 0.05 & 279 & 139.525 & 139.525 & 197.24744 \\
\hline X10C0404151 & METAL & Lead & 2 & 0 & $\mathrm{mg} / \mathrm{L}$ & 0.1 & 45.5 & 22.8 & 22.8 & 32.102648 \\
\hline X10C0404151 & METAL & Mercury & 2 & 0 & $\mathrm{mg} / \mathrm{L}$ & 0.002 & 0.4 & 0.201 & 0.201 & 0.2814285 \\
\hline X10C0404151 & METAL & Selenium & 2 & 0 & $\mathrm{mg} / \mathrm{L}$ & 0.3 & 136 & 68.15 & 68.15 & 95.95439 \\
\hline X10C0404151 & METAL & Silver & 2 & 1 & $\mathrm{mg} / \mathrm{L}$ & 0.05 & 22.9 & 11.475 & 11.475 & 16.15739 \\
\hline $\mathrm{X} 10 \mathrm{C} 0404151$ & METAL & Total Uranium & 1 & 0 & $\mathrm{pCi} / \mathrm{g}$ & 0.0609 & 0.0609 & 0.0609 & & \\
\hline X10C0404151 & PPCB & $4,4^{\prime}-\mathrm{DDD}$ & 1 & 0 & $\mathrm{ug} / \mathrm{kg}$ & 3.96 & 3.96 & 3.96 & & \\
\hline X10C0404151 & РPCB & 4,4'-DDE & 1 & 0 & $\mathrm{ug} / \mathrm{kg}$ & 3.96 & 3.96 & 3.96 & & \\
\hline X10C0404151 & PPCB & 4,4'-DDT & 1 & 0 & $\mathrm{ug} / \mathrm{kg}$ & 3.96 & 3.96 & 3.96 & & \\
\hline X10C0404151 & PPCB & Aldrin & 1 & 0 & $\mathrm{ug} / \mathrm{kg}$ & 1.98 & 1.98 & 1.98 & & \\
\hline X10C0404151 & РPCB & alpha-BHC & 1 & 0 & $\mathrm{ug} / \mathrm{kg}$ & 1.98 & 1.98 & 1.98 & & \\
\hline X10C0404151 & PPCB & alpha-Chlordane & 1 & 0 & $\mathrm{ug} / \mathrm{kg}$ & 1.98 & 1.98 & 1.98 & & \\
\hline X10C0404151 & PPCB & beta-BHC & 1 & 0 & $\mathrm{ug} / \mathrm{kg}$ & 1.98 & 1.98 & 1.98 & & \\
\hline X10C0404151 & PPCB & Chlordane & 2 & 0 & $\mathrm{ug} / \mathrm{kg}$ & 0.0025 & 24.7 & 12.35125 & 12.35125 & 17.46377 \\
\hline X10C0404151 & РPCB & delta-BHC & 1 & 0 & $\mathrm{ug} / \mathrm{kg}$ & 1.98 & 1.98 & 1.98 & & \\
\hline X10C0404151 & PPCB & Dieldrin & 1 & 0 & $\mathrm{ug} / \mathrm{kg}$ & 3.96 & 3.96 & 3.96 & & \\
\hline X10C0404151 & PPCB & Endosulfan I & 1 & 0 & $\mathrm{ug} / \mathrm{kg}$ & 1.98 & 1.98 & 1.98 & & \\
\hline X10C0404151 & РPCB & Endosulfan II & 1 & 0 & $\mathrm{ug} / \mathrm{kg}$ & 3.96 & 3.96 & 3.96 & & \\
\hline X10C0404151 & PPCB & Endosulfan sulfate & 1 & 0 & $\mathrm{ug} / \mathrm{kg}$ & 3.96 & 3.96 & 3.96 & & \\
\hline X10C0404151 & PPCB & Endrin & 2 & 0 & $\mathrm{ug} / \mathrm{kg}$ & 0.0004 & 3.96 & 1.9802 & 1.9802 & 2.79986 \\
\hline X10C0404151 & PPCB & Endrin aldehyde & 1 & 0 & $\mathrm{ug} / \mathrm{kg}$ & 3.96 & 3.96 & 3.96 & & \\
\hline X10C0404151 & PPCB & Endrin ketone & 1 & 0 & $\mathrm{ug} / \mathrm{kg}$ & 3.96 & 3.96 & 3.96 & & \\
\hline X10C0404151 & РPCB & gamma-Chlordane & 1 & 0 & $\mathrm{ug} / \mathrm{kg}$ & 1.98 & 1.98 & 1.98 & & \\
\hline X10C0404151 & PPCB & Heptachlor & 2 & 0 & $\mathrm{ug} / \mathrm{kg}$ & 0.0002 & 1.98 & 0.9901 & 0.9901 & 1.39993 \\
\hline X10C0404151 & РPCB & Heptachlor epoxide & 2 & 0 & $\mathrm{ug} / \mathrm{kg}$ & 0.0002 & 1.98 & 0.9901 & 0.9901 & 1.39993 \\
\hline X10C0404151 & РPCB & Lindane & 2 & 0 & $\mathrm{ug} / \mathrm{kg}$ & 0.0002 & 1.98 & 0.9901 & 0.9901 & 1.39993 \\
\hline X10C0404151 & РPCB & Methoxychlor & 2 & 0 & $\mathrm{ug} / \mathrm{kg}$ & 0.002 & 19.8 & 9.901 & 9.901 & 13.9993 \\
\hline X10C0404151 & РPCB & Toxaphene & 2 & 0 & $\mathrm{ug} / \mathrm{kg}$ & 0.005 & 49.5 & 24.7525 & 24.7525 & 34.99825 \\
\hline X10C0404151 & RADS & Americium-241 & 1 & 0 & $\mathrm{pCi} / \mathrm{g}$ & 0.0224 & 0.0224 & 0.0224 & & \\
\hline X10C0404151 & RADS & Carbon-14 & 1 & 0 & $\mathrm{pCi} / \mathrm{g}$ & -1.59 & -1.59 & -1.59 & & \\
\hline X10C0404151 & RADS & Cesium-137 & 1 & 1 & $\mathrm{pCi} / \mathrm{g}$ & 0.359 & 0.359 & 0.359 & 0.359 & \\
\hline X10C0404151 & RADS & Cobalt- 60 & 1 & 0 & $\mathrm{pCi} / \mathrm{g}$ & -0.0185 & -0.0185 & -0.0185 & & \\
\hline X10C0404151 & RADS & Curium-243/244 & 1 & 0 & $\mathrm{pCi} / \mathrm{g}$ & -0.00698 & -0.00698 & -0.00698 & & \\
\hline X10C0404151 & RADS & Iodine-129 & 1 & 0 & $\mathrm{pCi} / \mathrm{g}$ & -0.00878 & -0.00878 & -0.00878 & & \\
\hline X10C0404151 & RADS & Neptunium-237 & 1 & 0 & $\mathrm{pCi} / \mathrm{g}$ & -0.0245 & -0.0245 & -0.0245 & & \\
\hline X10C0404151 & RADS & Plutonium-238 & 1 & 0 & $\mathrm{pCi} / \mathrm{g}$ & -0.0136 & -0.0136 & -0.0136 & & \\
\hline X10C0404151 & RADS & Plutonium-239/240 & 1 & 0 & $\mathrm{pCi} / \mathrm{g}$ & 0.0392 & 0.0392 & 0.0392 & & \\
\hline X10C0404151 & RADS & Radium-226 & 1 & 0 & $\mathrm{pCi} / \mathrm{g}$ & 0.778 & 0.778 & 0.778 & & \\
\hline X10C0404151 & RADS & Strontium-90 & 1 & 0 & $\mathrm{pCi} / \mathrm{g}$ & 0.37 & 0.37 & 0.37 & & \\
\hline X10C0404151 & RADS & Technetium-99 & 1 & 0 & $\mathrm{pCi} / \mathrm{g}$ & 0.89 & 0.89 & 0.89 & & \\
\hline X10C0404151 & RADS & Thorium-230 & 1 & 0 & $\mathrm{pCi} / \mathrm{g}$ & -5.33 & -5.33 & -5.33 & & \\
\hline X10C0404151 & RADS & Thorium-232 & 1 & 0 & $\mathrm{pCi} / \mathrm{g}$ & -9.43 & -9.43 & -9.43 & & \\
\hline
\end{tabular}




\begin{tabular}{|c|c|c|c|c|c|c|c|c|c|c|}
\hline X10C0404151 & RADS & Tritium & 1 & 0 & $\mathrm{pCi} / \mathrm{g}$ & -25.8 & -25.8 & -25.8 & & \\
\hline X10C0404151 & RADS & Uranium-233/234 & 1 & 0 & $\mathrm{pCi} / \mathrm{g}$ & 0.0442 & 0.0442 & 0.0442 & & \\
\hline X10C0404151 & RADS & Uranium-235/236 & 1 & 0 & $\mathrm{pCi} / \mathrm{g}$ & 0.0283 & 0.0283 & 0.0283 & & \\
\hline X10C0404151 & RADS & Uranium-238 & 1 & 0 & $\mathrm{pCi} / \mathrm{g}$ & -0.0115 & -0.0115 & -0.0115 & & \\
\hline X10C0404151 & SVOA & 1,2,4-Trichlorobenzene & 1 & 0 & $\mathrm{ug} / \mathrm{kg}$ & 100 & 100 & 100 & & \\
\hline X10C0404151 & SVOA & 1,2-Dichlorobenzene & 1 & 0 & $\mathrm{ug} / \mathrm{kg}$ & 100 & 100 & 100 & & \\
\hline X10C0404151 & SVOA & 1,3-Dichlorobenzene & 1 & 0 & $\mathrm{ug} / \mathrm{kg}$ & 100 & 100 & 100 & & \\
\hline X10C0404151 & SVOA & 1,4-Dichlorobenzene & 2 & 0 & $\mathrm{ug} / \mathrm{kg}$ & 0.01 & 100 & 50.005 & 50.005 & 70.703607 \\
\hline X10C0404151 & SVOA & 2,2'-Dichlorodiisopropyl ether & 1 & 0 & $\mathrm{ug} / \mathrm{kg}$ & 961 & 961 & 961 & & \\
\hline X10C0404151 & SVOA & 2,3,4,6-Tetrachlorophenol & 1 & 0 & $\mathrm{ug} / \mathrm{kg}$ & 961 & 961 & 961 & & \\
\hline X10C0404151 & SVOA & 2,4,5-Trichlorophenol & 2 & 0 & $\mathrm{ug} / \mathrm{kg}$ & 0.05 & 961 & 480.525 & 480.525 & 679.49426 \\
\hline X10C0404151 & SVOA & 2,4,6-Trichlorophenol & 2 & 0 & $\mathrm{ug} / \mathrm{kg}$ & 0.05 & 961 & 480.525 & 480.525 & 679.49426 \\
\hline X10C0404151 & SVOA & 2,4-Dichlorophenol & 1 & 0 & $\mathrm{ug} / \mathrm{kg}$ & 961 & 961 & 961 & & \\
\hline X10C0404151 & SVOA & 2,4-Dimethylphenol & 1 & 0 & $\mathrm{ug} / \mathrm{kg}$ & 961 & 961 & 961 & & \\
\hline X10C0404151 & SVOA & 2,4-Dinitrophenol & 1 & 0 & $\mathrm{ug} / \mathrm{kg}$ & 1920 & 1920 & 1920 & & \\
\hline X10C0404151 & SVOA & 2,4-Dinitrotoluene & 2 & 0 & $\mathrm{ug} / \mathrm{kg}$ & 0.05 & 961 & 480.525 & 480.525 & 679.49426 \\
\hline X10C0404151 & SVOA & 2,6-Dinitrotoluene & 1 & 0 & $\mathrm{ug} / \mathrm{kg}$ & 961 & 961 & 961 & & \\
\hline X10C0404151 & SVOA & 2-Chloronaphthalene & 1 & 0 & $\mathrm{ug} / \mathrm{kg}$ & 96.1 & 96.1 & 96.1 & & \\
\hline X10C0404151 & SVOA & 2-Chlorophenol & 1 & 0 & $\mathrm{ug} / \mathrm{kg}$ & 961 & 961 & 961 & & \\
\hline X10C0404151 & SVOA & 2-Methyl-4,6-dinitrophenol & 1 & 0 & $\mathrm{ug} / \mathrm{kg}$ & 961 & 961 & 961 & & \\
\hline X10C0404151 & SVOA & 2-Methylnaphthalene & 1 & 0 & $\mathrm{ug} / \mathrm{kg}$ & 96.1 & 96.1 & 96.1 & & \\
\hline X10C0404151 & SVOA & 2-Methylphenol & 2 & 0 & $\mathrm{ug} / \mathrm{kg}$ & 0.05 & 961 & 480.525 & 480.525 & 679.49426 \\
\hline X10C0404151 & SVOA & 2-Nitrobenzenamine & 1 & 0 & $\mathrm{ug} / \mathrm{kg}$ & 961 & 961 & 961 & & \\
\hline X10C0404151 & SVOA & 2-Nitrophenol & 1 & 0 & $\mathrm{ug} / \mathrm{kg}$ & 961 & 961 & 961 & & \\
\hline X10C0404151 & SVOA & 3,3'-Dichlorobenzidine & 1 & 0 & $\mathrm{ug} / \mathrm{kg}$ & 961 & 961 & 961 & & \\
\hline X10C0404151 & SVOA & 3-Nitrobenzenamine & 1 & 0 & $\mathrm{ug} / \mathrm{kg}$ & 961 & 961 & 961 & & \\
\hline X10C0404151 & SVOA & 4-Bromophenyl phenyl ether & 1 & 0 & $\mathrm{ug} / \mathrm{kg}$ & 961 & 961 & 961 & & \\
\hline X10C0404151 & SVOA & 4-Chloro-3-methylphenol & 1 & 0 & $\mathrm{ug} / \mathrm{kg}$ & 961 & 961 & 961 & & \\
\hline X10C0404151 & SVOA & 4-Chlorobenzenamine & 1 & 0 & $\mathrm{ug} / \mathrm{kg}$ & 961 & 961 & 961 & & \\
\hline X10C0404151 & SVOA & 4-Chlorophenyl phenyl ether & 1 & 0 & $\mathrm{ug} / \mathrm{kg}$ & 961 & 961 & 961 & & \\
\hline X10C0404151 & SVOA & 4-Nitrobenzenamine & 1 & 0 & $\mathrm{ug} / \mathrm{kg}$ & 961 & 961 & 961 & & \\
\hline X10C0404151 & SVOA & 4-Nitrophenol & 1 & 0 & $\mathrm{ug} / \mathrm{kg}$ & 961 & 961 & 961 & & \\
\hline X10C0404151 & SVOA & Acenaphthene & 1 & 0 & $\mathrm{ug} / \mathrm{kg}$ & 96.1 & 96.1 & 96.1 & & \\
\hline X10C0404151 & SVOA & Acenaphthylene & 1 & 0 & $\mathrm{ug} / \mathrm{kg}$ & 96.1 & 96.1 & 96.1 & & \\
\hline X10C0404151 & SVOA & Acetophenone & 1 & 0 & $\mathrm{ug} / \mathrm{kg}$ & 961 & 961 & 961 & & \\
\hline X10C0404151 & SVOA & Anthracene & 1 & 0 & $\mathrm{ug} / \mathrm{kg}$ & 96.1 & 96.1 & 96.1 & & \\
\hline X10C0404151 & SVOA & Benz(a)anthracene & 1 & 0 & $\mathrm{ug} / \mathrm{kg}$ & 96.1 & 96.1 & 96.1 & & \\
\hline X10C0404151 & SVOA & Benzenemethanol & 1 & 0 & $\mathrm{ug} / \mathrm{kg}$ & 961 & 961 & 961 & & \\
\hline X10C0404151 & SVOA & Benzidine & 1 & 0 & $\mathrm{ug} / \mathrm{kg}$ & 961 & 961 & 961 & & \\
\hline X10C0404151 & SVOA & Benzo(a)pyrene & 1 & 0 & $\mathrm{ug} / \mathrm{kg}$ & 96.1 & 96.1 & 96.1 & & \\
\hline X10C0404151 & SVOA & Benzo(b)fluoranthene & 1 & 0 & $\mathrm{ug} / \mathrm{kg}$ & 96.1 & 96.1 & 96.1 & & \\
\hline X10C0404151 & SVOA & Benzo(ghi)perylene & 1 & 0 & $\mathrm{ug} / \mathrm{kg}$ & 96.1 & 96.1 & 96.1 & & \\
\hline X10C0404151 & SVOA & Benzo(k)fluoranthene & 1 & 0 & $\mathrm{ug} / \mathrm{kg}$ & 96.1 & 96.1 & 96.1 & & \\
\hline X10C0404151 & SVOA & Benzoic acid & 1 & 0 & $\mathrm{ug} / \mathrm{kg}$ & 1920 & 1920 & 1920 & & \\
\hline X10C0404151 & SVOA & Bis(2-chloroethoxy)methane & 1 & 0 & $\mathrm{ug} / \mathrm{kg}$ & 961 & 961 & 961 & & \\
\hline X10C0404151 & SVOA & $\operatorname{Bis}(2$-chloroethyl) ether & 1 & 0 & $\mathrm{ug} / \mathrm{kg}$ & 961 & 961 & 961 & & \\
\hline X10C0404151 & SVOA & Bis(2-ethylhexyl)phthalate & 1 & 0 & $\mathrm{ug} / \mathrm{kg}$ & 961 & 961 & 961 & & \\
\hline X10C0404151 & SVOA & Butyl benzyl phthalate & 1 & 0 & $\mathrm{ug} / \mathrm{kg}$ & 961 & 961 & 961 & & \\
\hline X10C0404151 & SVOA & Carbazole & 1 & 0 & $\mathrm{ug} / \mathrm{kg}$ & 96.1 & 96.1 & 96.1 & & \\
\hline X10C0404151 & SVOA & Chrysene & 1 & 0 & $\mathrm{ug} / \mathrm{kg}$ & 96.1 & 96.1 & 96.1 & & \\
\hline X10C0404151 & SVOA & $\operatorname{Dibenz}(\mathrm{a}, \mathrm{h})$ anthracene & 1 & 0 & $\mathrm{ug} / \mathrm{kg}$ & 96.1 & 96.1 & 96.1 & & \\
\hline X10C0404151 & SVOA & Dibenzofuran & 1 & 0 & $\mathrm{ug} / \mathrm{kg}$ & 961 & 961 & 961 & & \\
\hline X10C0404151 & SVOA & Diethyl phthalate & 1 & 0 & $\mathrm{ug} / \mathrm{kg}$ & 961 & 961 & 961 & & \\
\hline X10C0404151 & SVOA & Dimethyl phthalate & 1 & 0 & $\mathrm{ug} / \mathrm{kg}$ & 961 & 961 & 961 & & \\
\hline X10C0404151 & SVOA & Di-n-butyl phthalate & 1 & 0 & $\mathrm{ug} / \mathrm{kg}$ & 961 & 961 & 961 & & \\
\hline X10C0404151 & SVOA & Di-n-octylphthalate & 1 & 0 & $\mathrm{ug} / \mathrm{kg}$ & 961 & 961 & 961 & & \\
\hline X10C0404151 & SVOA & Diphenylamine & 1 & 0 & $\mathrm{ug} / \mathrm{kg}$ & 961 & 961 & 961 & & \\
\hline X10C0404151 & SVOA & Fluoranthene & 1 & 0 & $\mathrm{ug} / \mathrm{kg}$ & 96.1 & 96.1 & 96.1 & & \\
\hline X10C0404151 & SVOA & Fluorene & 1 & 0 & $\mathrm{ug} / \mathrm{kg}$ & 96.1 & 96.1 & 96.1 & & \\
\hline X10C0404151 & SVOA & Hexachlorobenzene & 2 & 0 & $\mathrm{ug} / \mathrm{kg}$ & 0.05 & 961 & 480.525 & 480.525 & 679.49426 \\
\hline X10C0404151 & SVOA & Hexachlorobutadiene & 2 & 0 & $\mathrm{ug} / \mathrm{kg}$ & 0.05 & 961 & 480.525 & 480.525 & 679.49426 \\
\hline X10C0404151 & SVOA & Hexachlorocyclopentadiene & 1 & 0 & $\mathrm{ug} / \mathrm{kg}$ & 961 & 961 & 961 & & \\
\hline X10C0404151 & SVOA & Hexachloroethane & 2 & 0 & $\mathrm{ug} / \mathrm{kg}$ & 0.05 & 961 & 480.525 & 480.525 & 679.49426 \\
\hline X10C0404151 & SVOA & Indeno(1,2,3-cd)pyrene & 1 & 0 & $\mathrm{ug} / \mathrm{kg}$ & 96.1 & 96.1 & 96.1 & & \\
\hline X10C0404151 & SVOA & Isophorone & 1 & 0 & $\mathrm{ug} / \mathrm{kg}$ & 961 & 961 & 961 & & \\
\hline X10C0404151 & SVOA & $\mathrm{m}+\mathrm{p}$ Methylphenol & 2 & 0 & $\mathrm{ug} / \mathrm{kg}$ & 0.05 & 961 & 480.525 & 480.525 & 679.49426 \\
\hline X10C0404151 & SVOA & Naphthalene & 1 & 0 & $\mathrm{ug} / \mathrm{kg}$ & 96.1 & 96.1 & 96.1 & & \\
\hline X10C0404151 & SVOA & Nitrobenzene & 2 & 0 & $\mathrm{ug} / \mathrm{kg}$ & 0.05 & 961 & 480.525 & 480.525 & 679.49426 \\
\hline X10C0404151 & SVOA & N-Nitroso-di-n-propylamine & 1 & 0 & $\mathrm{ug} / \mathrm{kg}$ & 961 & 961 & 961 & & \\
\hline X10C0404151 & SVOA & Pentachlorophenol & 2 & 0 & $\mathrm{ug} / \mathrm{kg}$ & 0.05 & 961 & 480.525 & 480.525 & 679.49426 \\
\hline
\end{tabular}




\begin{tabular}{|c|c|c|c|c|c|c|c|c|c|c|}
\hline X10C0404151 & SVOA & Phenanthrene & 1 & 0 & $\mathrm{ug} / \mathrm{kg}$ & 96.1 & 96.1 & 96.1 & & \\
\hline X10C0404151 & SVOA & Phenol & 1 & 0 & $\mathrm{ug} / \mathrm{kg}$ & 961 & 961 & 961 & & \\
\hline $\mathrm{X} 10 \mathrm{C} 0404151$ & SVOA & Pyridine & 2 & 0 & $\mathrm{ug} / \mathrm{kg}$ & 0.05 & 961 & 480.525 & 480.525 & 679.49426 \\
\hline X10C0404151 & VOA & (1,1-Dimethylethyl)benzene & 1 & 0 & $\mathrm{ug} / \mathrm{kg}$ & 100 & 100 & 100 & & \\
\hline X10C0404151 & VOA & (1-Methylpropyl)benzene & 1 & 0 & $\mathrm{ug} / \mathrm{kg}$ & 100 & 100 & 100 & & \\
\hline X10C0404151 & VOA & 1,1,1,2-Tetrachloroethane & 1 & 0 & $\mathrm{ug} / \mathrm{kg}$ & 100 & 100 & 100 & & \\
\hline X10C0404151 & VOA & 1,1,1-Trichloroethane & 1 & 0 & $\mathrm{ug} / \mathrm{kg}$ & 100 & 100 & 100 & & \\
\hline X10C0404151 & $\mathrm{VOA}$ & 1,1,2,2-Tetrachloroethane & 1 & 0 & $\mathrm{ug} / \mathrm{kg}$ & 100 & 100 & 100 & & \\
\hline X10C0404151 & VOA & 1,1,2-Trichloroethane & 1 & 0 & $\mathrm{ug} / \mathrm{kg}$ & 100 & 100 & 100 & & \\
\hline X10C0404151 & VOA & 1,1-Dichloroethane & 1 & 0 & $\mathrm{ug} / \mathrm{kg}$ & 100 & 100 & 100 & & \\
\hline X10C0404151 & VOA & 1,1-Dichloroethene & 2 & 0 & $\mathrm{ug} / \mathrm{kg}$ & 0.01 & 100 & 50.005 & 50.005 & 70.703607 \\
\hline X10C0404151 & VOA & 1,1-Dichloropropene & 1 & 0 & $\mathrm{ug} / \mathrm{kg}$ & 100 & 100 & 100 & & \\
\hline X10C0404151 & VOA & 1,2,3-Trichlorobenzene & 1 & 0 & $\mathrm{ug} / \mathrm{kg}$ & 100 & 100 & 100 & & \\
\hline X10C0404151 & VOA & 1,2,3-Trichloropropane & 1 & 0 & $\mathrm{ug} / \mathrm{kg}$ & 100 & 100 & 100 & & \\
\hline X10C0404151 & VOA & 1,2,4-Trimethylbenzene & 1 & 0 & $\mathrm{ug} / \mathrm{kg}$ & 100 & 100 & 100 & & \\
\hline X10C0404151 & VOA & 1,2-Dibromo-3-chloropropane & 1 & 0 & $\mathrm{ug} / \mathrm{kg}$ & 100 & 100 & 100 & & \\
\hline X10C0404151 & VOA & 1,2-Dibromoethane & 1 & 0 & $\mathrm{ug} / \mathrm{kg}$ & 100 & 100 & 100 & & \\
\hline X10C0404151 & VOA & 1,2-Dichloroethane & 2 & 0 & $\mathrm{ug} / \mathrm{kg}$ & 0.01 & 100 & 50.005 & 50.005 & 70.703607 \\
\hline X10C0404151 & VOA & 1,2-Dichloroethene & 1 & 0 & $\mathrm{ug} / \mathrm{kg}$ & 200 & 200 & 200 & & \\
\hline X10C0404151 & VOA & 1,2-Dichloropropane & 1 & 0 & $\mathrm{ug} / \mathrm{kg}$ & 100 & 100 & 100 & & \\
\hline X10C0404151 & VOA & 1,2-Dimethylbenzene & 1 & 0 & $\mathrm{ug} / \mathrm{kg}$ & 100 & 100 & 100 & & \\
\hline X10C0404151 & VOA & 1,3,5-Trimethylbenzene & 1 & 0 & $\mathrm{ug} / \mathrm{kg}$ & 100 & 100 & 100 & & \\
\hline X10C0404151 & VOA & 1,3-Dichloropropane & 1 & 0 & $\mathrm{ug} / \mathrm{kg}$ & 100 & 100 & 100 & & \\
\hline X10C0404151 & VOA & 1-Chloro-4-methylbenzene & 1 & 0 & $\mathrm{ug} / \mathrm{kg}$ & 100 & 100 & 100 & & \\
\hline X10C0404151 & VOA & 1-Methyl-4-(1-methylethyl)benzene & 1 & 0 & $\mathrm{ug} / \mathrm{kg}$ & 100 & 100 & 100 & & \\
\hline X10C0404151 & VOA & 2,2-Dichloropropane & 1 & 0 & $\mathrm{ug} / \mathrm{kg}$ & 100 & 100 & 100 & & \\
\hline X10C0404151 & VOA & 2-Butanone & 2 & 0 & $\mathrm{ug} / \mathrm{kg}$ & 0.05 & 500 & 250.025 & 250.025 & 353.51804 \\
\hline X10C0404151 & VOA & 2-Hexanone & 1 & 0 & $\mathrm{ug} / \mathrm{kg}$ & 500 & 500 & 500 & & \\
\hline X10C0404151 & VOA & 4-Methyl-2-pentanone & 1 & 0 & $\mathrm{ug} / \mathrm{kg}$ & 500 & 500 & 500 & & \\
\hline X10C0404151 & VOA & Acetone & 1 & 0 & $\mathrm{ug} / \mathrm{kg}$ & 500 & 500 & 500 & & \\
\hline X10C0404151 & VOA & Acetonitrile & 1 & 0 & $\mathrm{ug} / \mathrm{kg}$ & 2500 & 2500 & 2500 & & \\
\hline X10C0404151 & VOA & Acrolein & 1 & 0 & $\mathrm{ug} / \mathrm{kg}$ & 500 & 500 & 500 & & \\
\hline X10C0404151 & VOA & Acrylonitrile & 1 & 0 & $\mathrm{ug} / \mathrm{kg}$ & 500 & 500 & 500 & & \\
\hline X10C0404151 & VOA & Benzene & 2 & 0 & $\mathrm{ug} / \mathrm{kg}$ & 0.01 & 100 & 50.005 & 50.005 & 70.703607 \\
\hline X10C0404151 & VOA & Bromobenzene & 1 & 0 & $\mathrm{ug} / \mathrm{kg}$ & 100 & 100 & 100 & & \\
\hline X10C0404151 & VOA & Bromochloromethane & 1 & 0 & $\mathrm{ug} / \mathrm{kg}$ & 100 & 100 & 100 & & \\
\hline X10C0404151 & VOA & Bromodichloromethane & 1 & 0 & $\mathrm{ug} / \mathrm{kg}$ & 100 & 100 & 100 & & \\
\hline X10C0404151 & VOA & Bromoform & 1 & 0 & $\mathrm{ug} / \mathrm{kg}$ & 100 & 100 & 100 & & \\
\hline X10C0404151 & VOA & Bromomethane & 1 & 0 & $\mathrm{ug} / \mathrm{kg}$ & 100 & 100 & 100 & & \\
\hline X10C0404151 & VOA & Butylbenzene & 1 & 0 & $\mathrm{ug} / \mathrm{kg}$ & 100 & 100 & 100 & & \\
\hline X10C0404151 & VOA & Carbon disulfide & 1 & 0 & $\mathrm{ug} / \mathrm{kg}$ & 500 & 500 & 500 & & \\
\hline X10C0404151 & VOA & Carbon tetrachloride & 2 & 0 & $\mathrm{ug} / \mathrm{kg}$ & 0.01 & 100 & 50.005 & 50.005 & 70.703607 \\
\hline X10C0404151 & VOA & Chlorobenzene & 2 & 0 & $\mathrm{ug} / \mathrm{kg}$ & 0.01 & 100 & 50.005 & 50.005 & 70.703607 \\
\hline X10C0404151 & VOA & Chloroethane & 1 & 0 & $\mathrm{ug} / \mathrm{kg}$ & 100 & 100 & 100 & & \\
\hline X10C0404151 & VOA & Chloroform & 2 & 0 & $\mathrm{ug} / \mathrm{kg}$ & 0.01 & 100 & 50.005 & 50.005 & 70.703607 \\
\hline X10C0404151 & VOA & Chloromethane & 1 & 0 & $\mathrm{ug} / \mathrm{kg}$ & 100 & 100 & 100 & & \\
\hline X10C0404151 & VOA & cis-1,2-Dichloroethene & 1 & 0 & $\mathrm{ug} / \mathrm{kg}$ & 100 & 100 & 100 & & \\
\hline X10C0404151 & VOA & cis-1,3-Dichloropropene & 1 & 0 & $\mathrm{ug} / \mathrm{kg}$ & 100 & 100 & 100 & & \\
\hline X10C0404151 & VOA & Cumene & 1 & 0 & $\mathrm{ug} / \mathrm{kg}$ & 100 & 100 & 100 & & \\
\hline X10C0404151 & VOA & Dibromochloromethane & 1 & 0 & $\mathrm{ug} / \mathrm{kg}$ & 100 & 100 & 100 & & \\
\hline X10C0404151 & VOA & Dibromomethane & 1 & 0 & $\mathrm{ug} / \mathrm{kg}$ & 100 & 100 & 100 & & \\
\hline X10C0404151 & VOA & Dichlorodifluoromethane & 1 & 0 & $\mathrm{ug} / \mathrm{kg}$ & 100 & 100 & 100 & & \\
\hline X10C0404151 & VOA & Ethylbenzene & 1 & 0 & $\mathrm{ug} / \mathrm{kg}$ & 100 & 100 & 100 & & \\
\hline X10C0404151 & VOA & Hexane & 1 & 0 & $\mathrm{ug} / \mathrm{kg}$ & 500 & 500 & 500 & & \\
\hline X10C0404151 & VOA & M + P Xylene & 1 & 0 & $\mathrm{ug} / \mathrm{kg}$ & 200 & 200 & 200 & & \\
\hline X10C0404151 & VOA & Methanol & 1 & 0 & $\mathrm{ug} / \mathrm{kg}$ & 5000 & 5000 & 5000 & & \\
\hline X10C0404151 & VOA & Methyl methacrylate & 1 & 0 & $\mathrm{ug} / \mathrm{kg}$ & 500 & 500 & 500 & & \\
\hline X10C0404151 & VOA & Methylcyclohexane & 1 & 0 & $\mathrm{ug} / \mathrm{kg}$ & 100 & 100 & 100 & & \\
\hline X10C0404151 & VOA & Methylene chloride & 1 & 0 & $\mathrm{ug} / \mathrm{kg}$ & 500 & 500 & 500 & & \\
\hline X10C0404151 & VOA & o-Chlorotoluene & 1 & 0 & $\mathrm{ug} / \mathrm{kg}$ & 100 & 100 & 100 & & \\
\hline X10C0404151 & VOA & Propylbenzene & 1 & 0 & $\mathrm{ug} / \mathrm{kg}$ & 100 & 100 & 100 & & \\
\hline X10C0404151 & VOA & Propylene glycol & 1 & 0 & $\mathrm{ug} / \mathrm{kg}$ & 50000 & 50000 & 50000 & & \\
\hline X10C0404151 & VOA & Styrene & 1 & 0 & $\mathrm{ug} / \mathrm{kg}$ & 100 & 100 & 100 & & \\
\hline X10C0404151 & VOA & Tetrachloroethene & 2 & 0 & $\mathrm{ug} / \mathrm{kg}$ & 0.01 & 100 & 50.005 & 50.005 & 70.703607 \\
\hline X10C0404151 & VOA & Toluene & 1 & 0 & $\mathrm{ug} / \mathrm{kg}$ & 100 & 100 & 100 & & \\
\hline X10C0404151 & VOA & Total Xylene & 1 & 0 & $\mathrm{ug} / \mathrm{kg}$ & 300 & 300 & 300 & & \\
\hline X10C0404151 & VOA & trans-1,2-Dichloroethene & 1 & 0 & $\mathrm{ug} / \mathrm{kg}$ & 100 & 100 & 100 & & \\
\hline X10C0404151 & VOA & trans-1,3-Dichloropropene & 1 & 0 & $\mathrm{ug} / \mathrm{kg}$ & 100 & 100 & 100 & & \\
\hline X10C0404151 & VOA & Trichloroethene & 2 & 0 & $\mathrm{ug} / \mathrm{kg}$ & 0.01 & 100 & 50.005 & 50.005 & 70.703607 \\
\hline X10C0404151 & VOA & Trichlorofluoromethane & 1 & 0 & $\mathrm{ug} / \mathrm{kg}$ & 100 & 100 & 100 & & \\
\hline
\end{tabular}




\begin{tabular}{|c|c|c|c|c|c|c|c|c|c|c|}
\hline X10C0404151 & VOA & Vinyl chloride & 2 & 0 & $\mathrm{ug} / \mathrm{kg}$ & 0.01 & 100 & 50.005 & 50.005 & 70.703607 \\
\hline X10C0404159 & HERB & $2,4-\mathrm{D}$ & 2 & 0 & $\mathrm{ug} / \mathrm{kg}$ & 0.05 & 116 & 58.025 & 58.025 & 81.989031 \\
\hline X10C0404159 & HERB & Silvex & 2 & 0 & $\mathrm{ug} / \mathrm{kg}$ & 0.05 & 116 & 58.025 & 58.025 & 81.989031 \\
\hline X10C0404159 & METAL & Arsenic & 2 & 0 & $\mathrm{mg} / \mathrm{L}$ & 0.3 & 2.76 & 1.53 & 1.53 & 1.7394827 \\
\hline X10C0404159 & METAL & Barium & 2 & 2 & $\mathrm{mg} / \mathrm{L}$ & 0.108 & 49.1 & 24.604 & 24.604 & 34.642575 \\
\hline X10C0404159 & METAL & Cadmium & 2 & 0 & $\mathrm{mg} / \mathrm{L}$ & 0.05 & 0.46 & 0.255 & 0.255 & 0.2899138 \\
\hline X10C0404159 & METAL & Chromium & 2 & 2 & $\mathrm{mg} / \mathrm{L}$ & 0.0506 & 0.821 & 0.4358 & 0.4358 & 0.5447551 \\
\hline X10C0404159 & METAL & Lead & 2 & 1 & $\mathrm{mg} / \mathrm{L}$ & 0.1 & 1.52 & 0.81 & 0.81 & 1.0040916 \\
\hline X10C0404159 & METAL & Mercury & 2 & 0 & $\mathrm{mg} / \mathrm{L}$ & 0.002 & 0.4 & 0.201 & 0.201 & 0.2814285 \\
\hline X10C0404159 & METAL & Selenium & 2 & 0 & $\mathrm{mg} / \mathrm{L}$ & 0.3 & 2.76 & 1.53 & 1.53 & 1.7394827 \\
\hline X10C0404159 & METAL & Silver & 2 & 0 & $\mathrm{mg} / \mathrm{L}$ & 0.05 & 0.46 & 0.255 & 0.255 & 0.2899138 \\
\hline X10C0404159 & METAL & Total Uranium & 1 & 1 & $\mathrm{pCi} / \mathrm{g}$ & 2.9 & 2.9 & 2.9 & 2.9 & \\
\hline X10C0404159 & PPCB & $4,4^{\prime}-\mathrm{DDD}$ & 1 & 0 & $\mathrm{ug} / \mathrm{kg}$ & 39.9 & 39.9 & 39.9 & & \\
\hline X10C0404159 & PPCB & 4,4'-DDE & 1 & 0 & $\mathrm{ug} / \mathrm{kg}$ & 39.9 & 39.9 & 39.9 & & \\
\hline X10C0404159 & PPCB & 4,4'-DDT & 1 & 0 & $\mathrm{ug} / \mathrm{kg}$ & 39.9 & 39.9 & 39.9 & & \\
\hline X10C0404159 & РPCB & Aldrin & 1 & 0 & $\mathrm{ug} / \mathrm{kg}$ & 20 & 20 & 20 & & \\
\hline X10C0404159 & РPCB & alpha-BHC & 1 & 0 & $\mathrm{ug} / \mathrm{kg}$ & 20 & 20 & 20 & & \\
\hline X10C0404159 & PPCB & alpha-Chlordane & 1 & 0 & $\mathrm{ug} / \mathrm{kg}$ & 20 & 20 & 20 & & \\
\hline X10C0404159 & PPCB & beta-BHC & 1 & 0 & $\mathrm{ug} / \mathrm{kg}$ & 20 & 20 & 20 & & \\
\hline X10C0404159 & PPCB & Chlordane & 2 & 0 & $\mathrm{ug} / \mathrm{kg}$ & 0.0025 & 250 & 125.00125 & 125.00125 & 176.77493 \\
\hline X10C0404159 & РPCB & delta-BHC & 1 & 0 & $\mathrm{ug} / \mathrm{kg}$ & 20 & 20 & 20 & & \\
\hline X10C0404159 & РPCB & Dieldrin & 1 & 0 & $\mathrm{ug} / \mathrm{kg}$ & 39.9 & 39.9 & 39.9 & & \\
\hline X10C0404159 & PPCB & Endosulfan I & 1 & 0 & $\mathrm{ug} / \mathrm{kg}$ & 20 & 20 & 20 & & \\
\hline X10C0404159 & PPCB & Endosulfan II & 1 & 0 & $\mathrm{ug} / \mathrm{kg}$ & 39.9 & 39.9 & 39.9 & & \\
\hline X10C0404159 & РPCB & Endosulfan sulfate & 1 & 0 & $\mathrm{ug} / \mathrm{kg}$ & 39.9 & 39.9 & 39.9 & & \\
\hline X10C0404159 & PPCB & Endrin & 2 & 0 & $\mathrm{ug} / \mathrm{kg}$ & 0.0004 & 39.9 & 19.9502 & 19.9502 & 28.213278 \\
\hline X10C0404159 & PPCB & Endrin aldehyde & 1 & 0 & $\mathrm{ug} / \mathrm{kg}$ & 39.9 & 39.9 & 39.9 & & \\
\hline X10C0404159 & PPCB & Endrin ketone & 1 & 0 & $\mathrm{ug} / \mathrm{kg}$ & 39.9 & 39.9 & 39.9 & & \\
\hline X10C0404159 & PPCB & gamma-Chlordane & 1 & 0 & $\mathrm{ug} / \mathrm{kg}$ & 20 & 20 & 20 & & \\
\hline X10C0404159 & PPCB & Heptachlor & 2 & 0 & $\mathrm{ug} / \mathrm{kg}$ & 0.0002 & 20 & 10.0001 & 10.0001 & 14.141994 \\
\hline X10C0404159 & РPCB & Heptachlor epoxide & 2 & 0 & $\mathrm{ug} / \mathrm{kg}$ & 0.0002 & 20 & 10.0001 & 10.0001 & 14.141994 \\
\hline X10C0404159 & РPCB & Lindane & 2 & 0 & $\mathrm{ug} / \mathrm{kg}$ & 0.0002 & 20 & 10.0001 & 10.0001 & 14.141994 \\
\hline X10C0404159 & РPCB & Methoxychlor & 2 & 0 & $\mathrm{ug} / \mathrm{kg}$ & 0.002 & 200 & 100.001 & 100.001 & 141.41994 \\
\hline $\mathrm{X} 10 \mathrm{C} 0404159$ & PPCB & Toxaphene & 2 & 0 & $\mathrm{ug} / \mathrm{kg}$ & 0.005 & 499 & 249.5025 & 249.5025 & 352.84275 \\
\hline X10C0404159 & RADS & Americium-241 & 1 & 1 & $\mathrm{pCi} / \mathrm{g}$ & 1.57 & 1.57 & 1.57 & 1.57 & \\
\hline X10C0404159 & RADS & Carbon-14 & 1 & 0 & $\mathrm{pCi} / \mathrm{g}$ & -4.36 & -4.36 & -4.36 & & \\
\hline X10C0404159 & RADS & Cesium-137 & 1 & 1 & $\mathrm{pCi} / \mathrm{g}$ & 3.56 & 3.56 & 3.56 & 3.56 & \\
\hline X10C0404159 & RADS & Cobalt- 60 & 1 & 1 & $\mathrm{pCi} / \mathrm{g}$ & 13.1 & 13.1 & 13.1 & 13.1 & \\
\hline X10C0404159 & RADS & Curium-243/244 & 1 & 1 & $\mathrm{pCi} / \mathrm{g}$ & 1.16 & 1.16 & 1.16 & 1.16 & \\
\hline X10C0404159 & RADS & Iodine-129 & 1 & 0 & $\mathrm{pCi} / \mathrm{g}$ & 0.105 & 0.105 & 0.105 & & \\
\hline X10C0404159 & RADS & Neptunium-237 & 1 & 0 & $\mathrm{pCi} / \mathrm{g}$ & 0.0463 & 0.0463 & 0.0463 & & \\
\hline X10C0404159 & RADS & Plutonium-238 & 1 & 1 & $\mathrm{pCi} / \mathrm{g}$ & 0.361 & 0.361 & 0.361 & 0.361 & \\
\hline X10C0404159 & RADS & Plutonium-239/240 & 1 & 1 & $\mathrm{pCi} / \mathrm{g}$ & 0.375 & 0.375 & 0.375 & 0.375 & \\
\hline X10C0404159 & RADS & Radium-226 & 1 & 0 & $\mathrm{pCi} / \mathrm{g}$ & 0.372 & 0.372 & 0.372 & & \\
\hline X10C0404159 & RADS & Strontium-90 & 1 & 0 & $\mathrm{pCi} / \mathrm{g}$ & 0.426 & 0.426 & 0.426 & & \\
\hline X10C0404159 & RADS & Technetium-99 & 1 & 0 & $\mathrm{pCi} / \mathrm{g}$ & -0.204 & -0.204 & -0.204 & & \\
\hline X10C0404159 & RADS & Thorium-230 & 1 & 0 & $\mathrm{pCi} / \mathrm{g}$ & 0.372 & 0.372 & 0.372 & & \\
\hline X10C0404159 & RADS & Thorium-232 & 1 & 0 & $\mathrm{pCi} / \mathrm{g}$ & 0.234 & 0.234 & 0.234 & & \\
\hline X10C0404159 & RADS & Tritium & 1 & 0 & $\mathrm{pCi} / \mathrm{g}$ & -113 & -113 & -113 & & \\
\hline X10C0404159 & RADS & Uranium-233/234 & 1 & 1 & $\mathrm{pCi} / \mathrm{g}$ & 1.75 & 1.75 & 1.75 & 1.75 & \\
\hline X10C0404159 & RADS & Uranium-235/236 & 1 & 0 & $\mathrm{pCi} / \mathrm{g}$ & -0.024 & -0.024 & -0.024 & & \\
\hline X10C0404159 & RADS & Uranium-238 & 1 & 1 & $\mathrm{pCi} / \mathrm{g}$ & 1.18 & 1.18 & 1.18 & 1.18 & \\
\hline $\mathrm{X} 10 \mathrm{C} 0404159$ & SVOA & 1,2,4-Trichlorobenzene & 1 & 0 & $\mathrm{ug} / \mathrm{kg}$ & 446 & 446 & 446 & & \\
\hline X10C0404159 & SVOA & 1,2-Dichlorobenzene & 1 & 0 & $\mathrm{ug} / \mathrm{kg}$ & 446 & 446 & 446 & & \\
\hline X10C0404159 & SVOA & 1,3-Dichlorobenzene & 1 & 0 & $\mathrm{ug} / \mathrm{kg}$ & 446 & 446 & 446 & & \\
\hline X10C0404159 & SVOA & 1,4-Dichlorobenzene & 2 & 0 & $\mathrm{ug} / \mathrm{kg}$ & 0.01 & 446 & 223.005 & 223.005 & 315.36255 \\
\hline X10C0404159 & SVOA & 2,2'-Dichlorodiisopropyl ether & 1 & 0 & $\mathrm{ug} / \mathrm{kg}$ & 20000 & 20000 & 20000 & & \\
\hline X10C0404159 & SVOA & 2,3,4,6-Tetrachlorophenol & 1 & 0 & $\mathrm{ug} / \mathrm{kg}$ & 20000 & 20000 & 20000 & & \\
\hline X10C0404159 & SVOA & 2,4,5-Trichlorophenol & 2 & 0 & $\mathrm{ug} / \mathrm{kg}$ & 0.05 & 20000 & 10000.025 & 10000.025 & 14142.1 \\
\hline X10C0404159 & SVOA & 2,4,6-Trichlorophenol & 2 & 0 & $\mathrm{ug} / \mathrm{kg}$ & 0.05 & 20000 & 10000.025 & 10000.025 & 14142.1 \\
\hline X10C0404159 & SVOA & 2,4-Dichlorophenol & 1 & 0 & $\mathrm{ug} / \mathrm{kg}$ & 20000 & 20000 & 20000 & & \\
\hline X10C0404159 & SVOA & 2,4-Dimethylphenol & 1 & 0 & $\mathrm{ug} / \mathrm{kg}$ & 20000 & 20000 & 20000 & & \\
\hline X10C0404159 & SVOA & 2,4-Dinitrophenol & 1 & 0 & $\mathrm{ug} / \mathrm{kg}$ & 40000 & 40000 & 40000 & & \\
\hline X10C0404159 & SVOA & 2,4-Dinitrotoluene & 2 & 0 & $\mathrm{ug} / \mathrm{kg}$ & 0.05 & 20000 & 10000.025 & 10000.025 & 14142.1 \\
\hline X10C0404159 & SVOA & 2,6-Dinitrotoluene & 1 & 0 & $\mathrm{ug} / \mathrm{kg}$ & 20000 & 20000 & 20000 & & \\
\hline X10C0404159 & SVOA & 2-Chloronaphthalene & 1 & 0 & $\mathrm{ug} / \mathrm{kg}$ & 2000 & 2000 & 2000 & & \\
\hline X10C0404159 & SVOA & 2-Chlorophenol & 1 & 0 & $\mathrm{ug} / \mathrm{kg}$ & 20000 & 20000 & 20000 & & \\
\hline X10C0404159 & SVOA & 2-Methyl-4,6-dinitrophenol & 1 & 0 & $\mathrm{ug} / \mathrm{kg}$ & 20000 & 20000 & 20000 & & \\
\hline X10C0404159 & SVOA & 2-Methylnaphthalene & 1 & 0 & $\mathrm{ug} / \mathrm{kg}$ & 2000 & 2000 & 2000 & & \\
\hline X10C0404159 & SVOA & 2-Methylphenol & 2 & 0 & $\mathrm{ug} / \mathrm{kg}$ & 0.05 & 20000 & 10000.025 & 10000.025 & 14142.1 \\
\hline
\end{tabular}




\begin{tabular}{|c|c|c|c|c|c|c|c|c|c|c|}
\hline X10C0404159 & SVOA & 2-Nitrobenzenamine & 1 & 0 & $\mathrm{ug} / \mathrm{kg}$ & 20000 & 20000 & 20000 & & \\
\hline X10C0404159 & SVOA & 2-Nitrophenol & 1 & 0 & $\mathrm{ug} / \mathrm{kg}$ & 20000 & 20000 & 20000 & & \\
\hline X10C0404159 & SVOA & 3,3'-Dichlorobenzidine & 1 & 0 & $\mathrm{ug} / \mathrm{kg}$ & 20000 & 20000 & 20000 & & \\
\hline X10C0404159 & SVOA & 3-Nitrobenzenamine & 1 & 0 & $\mathrm{ug} / \mathrm{kg}$ & 20000 & 20000 & 20000 & & \\
\hline X10C0404159 & SVOA & 4-Bromophenyl phenyl ether & 1 & 0 & $\mathrm{ug} / \mathrm{kg}$ & 20000 & 20000 & 20000 & & \\
\hline X10C0404159 & SVOA & 4-Chloro-3-methylphenol & 1 & 0 & $\mathrm{ug} / \mathrm{kg}$ & 20000 & 20000 & 20000 & & \\
\hline X10C0404159 & SVOA & 4-Chlorobenzenamine & 1 & 0 & $\mathrm{ug} / \mathrm{kg}$ & 20000 & 20000 & 20000 & & \\
\hline X10C0404159 & SVOA & 4-Chlorophenyl phenyl ether & 1 & 0 & $\mathrm{ug} / \mathrm{kg}$ & 20000 & 20000 & 20000 & & \\
\hline X10C0404159 & SVOA & 4-Nitrobenzenamine & 1 & 0 & $\mathrm{ug} / \mathrm{kg}$ & 20000 & 20000 & 20000 & & \\
\hline X10C0404159 & SVOA & 4-Nitrophenol & 1 & 0 & $\mathrm{ug} / \mathrm{kg}$ & 20000 & 20000 & 20000 & & \\
\hline X10C0404159 & SVOA & Acenaphthene & 1 & 0 & $\mathrm{ug} / \mathrm{kg}$ & 2000 & 2000 & 2000 & & \\
\hline X10C0404159 & SVOA & Acenaphthylene & 1 & 0 & $\mathrm{ug} / \mathrm{kg}$ & 2000 & 2000 & 2000 & & \\
\hline X10C0404159 & SVOA & Acetophenone & 1 & 0 & $\mathrm{ug} / \mathrm{kg}$ & 20000 & 20000 & 20000 & & \\
\hline X10C0404159 & SVOA & Anthracene & 1 & 0 & $\mathrm{ug} / \mathrm{kg}$ & 2000 & 2000 & 2000 & & \\
\hline X10C0404159 & SVOA & Benz(a)anthracene & 1 & 0 & $\mathrm{ug} / \mathrm{kg}$ & 2000 & 2000 & 2000 & & \\
\hline X10C0404159 & SVOA & Benzenemethanol & 1 & 0 & $\mathrm{ug} / \mathrm{kg}$ & 20000 & 20000 & 20000 & & \\
\hline X10C0404159 & SVOA & Benzidine & 1 & 0 & $\mathrm{ug} / \mathrm{kg}$ & 20000 & 20000 & 20000 & & \\
\hline X10C0404159 & SVOA & Benzo(a)pyrene & 1 & 0 & $\mathrm{ug} / \mathrm{kg}$ & 2000 & 2000 & 2000 & & \\
\hline X10C0404159 & SVOA & Benzo(b)fluoranthene & 1 & 0 & $\mathrm{ug} / \mathrm{kg}$ & 2000 & 2000 & 2000 & & \\
\hline X10C0404159 & SVOA & Benzo(ghi)perylene & 1 & 0 & $\mathrm{ug} / \mathrm{kg}$ & 2000 & 2000 & 2000 & & \\
\hline X10C0404159 & SVOA & Benzo(k)fluoranthene & 1 & 0 & $\mathrm{ug} / \mathrm{kg}$ & 2000 & 2000 & 2000 & & \\
\hline X10C0404159 & SVOA & Benzoic acid & 1 & 0 & $\mathrm{ug} / \mathrm{kg}$ & 40000 & 40000 & 40000 & & \\
\hline X10C0404159 & SVOA & Bis(2-chloroethoxy)methane & 1 & 0 & $\mathrm{ug} / \mathrm{kg}$ & 20000 & 20000 & 20000 & & \\
\hline X10C0404159 & SVOA & Bis(2-chloroethyl) ether & 1 & 0 & $\mathrm{ug} / \mathrm{kg}$ & 20000 & 20000 & 20000 & & \\
\hline X10C0404159 & SVOA & Bis(2-ethylhexyl)phthalate & 1 & 0 & $\mathrm{ug} / \mathrm{kg}$ & 20000 & 20000 & 20000 & & \\
\hline X10C0404159 & SVOA & Butyl benzyl phthalate & 1 & 0 & $\mathrm{ug} / \mathrm{kg}$ & 20000 & 20000 & 20000 & & \\
\hline X10C0404159 & SVOA & Carbazole & 1 & 0 & $\mathrm{ug} / \mathrm{kg}$ & 2000 & 2000 & 2000 & & \\
\hline X10C0404159 & SVOA & Chrysene & 1 & 0 & $\mathrm{ug} / \mathrm{kg}$ & 2000 & 2000 & 2000 & & \\
\hline X10C0404159 & SVOA & Dibenz(a,h)anthracene & 1 & 0 & $\mathrm{ug} / \mathrm{kg}$ & 2000 & 2000 & 2000 & & \\
\hline X10C0404159 & SVOA & Dibenzofuran & 1 & 0 & $\mathrm{ug} / \mathrm{kg}$ & 20000 & 20000 & 20000 & & \\
\hline X10C0404159 & SVOA & Diethyl phthalate & 1 & 0 & $\mathrm{ug} / \mathrm{kg}$ & 20000 & 20000 & 20000 & & \\
\hline X10C0404159 & SVOA & Dimethyl phthalate & 1 & 0 & $\mathrm{ug} / \mathrm{kg}$ & 20000 & 20000 & 20000 & & \\
\hline X10C0404159 & SVOA & Di-n-butyl phthalate & 1 & 0 & $\mathrm{ug} / \mathrm{kg}$ & 20000 & 20000 & 20000 & & \\
\hline X10C0404159 & SVOA & Di-n-octylphthalate & 1 & 0 & $\mathrm{ug} / \mathrm{kg}$ & 20000 & 20000 & 20000 & & \\
\hline X10C0404159 & SVOA & Diphenylamine & 1 & 0 & $\mathrm{ug} / \mathrm{kg}$ & 20000 & 20000 & 20000 & & \\
\hline X10C0404159 & SVOA & Fluoranthene & 1 & 0 & $\mathrm{ug} / \mathrm{kg}$ & 2000 & 2000 & 2000 & & \\
\hline X10C0404159 & SVOA & Fluorene & 1 & 0 & $\mathrm{ug} / \mathrm{kg}$ & 2000 & 2000 & 2000 & & \\
\hline X10C0404159 & SVOA & Hexachlorobenzene & 2 & 0 & $\mathrm{ug} / \mathrm{kg}$ & 0.05 & 20000 & 10000.025 & 10000.025 & 14142.1 \\
\hline X10C0404159 & SVOA & Hexachlorobutadiene & 2 & 0 & $\mathrm{ug} / \mathrm{kg}$ & 0.05 & 20000 & 10000.025 & 10000.025 & 14142.1 \\
\hline X10C0404159 & SVOA & Hexachlorocyclopentadiene & 1 & 0 & $\mathrm{ug} / \mathrm{kg}$ & 20000 & 20000 & 20000 & & \\
\hline X10C0404159 & SVOA & Hexachloroethane & 2 & 0 & $\mathrm{ug} / \mathrm{kg}$ & 0.05 & 20000 & 10000.025 & 10000.025 & 14142.1 \\
\hline X10C0404159 & SVOA & Indeno(1,2,3-cd)pyrene & 1 & 0 & $\mathrm{ug} / \mathrm{kg}$ & 2000 & 2000 & 2000 & & \\
\hline X10C0404159 & SVOA & Isophorone & 1 & 0 & $\mathrm{ug} / \mathrm{kg}$ & 20000 & 20000 & 20000 & & \\
\hline X10C0404159 & SVOA & $\mathrm{m}+\mathrm{p}$ Methylphenol & 2 & 0 & $\mathrm{ug} / \mathrm{kg}$ & 0.05 & 20000 & 10000.025 & 10000.025 & 14142.1 \\
\hline X10C0404159 & SVOA & Naphthalene & 1 & 0 & $\mathrm{ug} / \mathrm{kg}$ & 2000 & 2000 & 2000 & & \\
\hline X10C0404159 & SVOA & Nitrobenzene & 2 & 0 & $\mathrm{ug} / \mathrm{kg}$ & 0.05 & 20000 & 10000.025 & 10000.025 & 14142.1 \\
\hline X10C0404159 & SVOA & N-Nitroso-di-n-propylamine & 1 & 0 & $\mathrm{ug} / \mathrm{kg}$ & 20000 & 20000 & 20000 & & \\
\hline X10C0404159 & SVOA & Pentachlorophenol & 2 & 0 & $\mathrm{ug} / \mathrm{kg}$ & 0.05 & 20000 & 10000.025 & 10000.025 & 14142.1 \\
\hline X10C0404159 & SVOA & Phenanthrene & 1 & 0 & $\mathrm{ug} / \mathrm{kg}$ & 2000 & 2000 & 2000 & & \\
\hline X10C0404159 & SVOA & Phenol & 1 & 0 & $\mathrm{ug} / \mathrm{kg}$ & 20000 & 20000 & 20000 & & \\
\hline X10C0404159 & SVOA & Pyridine & 2 & 0 & $\mathrm{ug} / \mathrm{kg}$ & 0.05 & 20000 & 10000.025 & 10000.025 & 14142.1 \\
\hline $\mathrm{X} 10 \mathrm{C} 0404159$ & $\mathrm{VOA}$ & (1,1-Dimethylethyl)benzene & 1 & 0 & $\mathrm{ug} / \mathrm{kg}$ & 446 & 446 & 446 & & \\
\hline X10C0404159 & VOA & (1-Methylpropyl)benzene & 1 & 0 & $\mathrm{ug} / \mathrm{kg}$ & 446 & 446 & 446 & & \\
\hline X10C0404159 & VOA & 1,1,1,2-Tetrachloroethane & 1 & 0 & $\mathrm{ug} / \mathrm{kg}$ & 446 & 446 & 446 & & \\
\hline X10C0404159 & VOA & 1,1,1-Trichloroethane & 1 & 0 & $\mathrm{ug} / \mathrm{kg}$ & 446 & 446 & 446 & & \\
\hline X10C0404159 & VOA & 1,1,2,2-Tetrachloroethane & 1 & 0 & $\mathrm{ug} / \mathrm{kg}$ & 446 & 446 & 446 & & \\
\hline X10C0404159 & VOA & 1,1,2-Trichloroethane & 1 & 0 & $\mathrm{ug} / \mathrm{kg}$ & 446 & 446 & 446 & & \\
\hline X10C0404159 & VOA & 1,1-Dichloroethane & 1 & 0 & $\mathrm{ug} / \mathrm{kg}$ & 446 & 446 & 446 & & \\
\hline X10C0404159 & VOA & 1,1-Dichloroethene & 2 & 0 & $\mathrm{ug} / \mathrm{kg}$ & 0.01 & 446 & 223.005 & 223.005 & 315.36255 \\
\hline X10C0404159 & VOA & 1,1-Dichloropropene & 1 & 0 & $\mathrm{ug} / \mathrm{kg}$ & 446 & 446 & 446 & & \\
\hline X10C0404159 & VOA & 1,2,3-Trichlorobenzene & 1 & 0 & $\mathrm{ug} / \mathrm{kg}$ & 446 & 446 & 446 & & \\
\hline X10C0404159 & VOA & 1,2,3-Trichloropropane & 1 & 0 & $\mathrm{ug} / \mathrm{kg}$ & 446 & 446 & 446 & & \\
\hline X10C0404159 & VOA & 1,2,4-Trimethylbenzene & 1 & 0 & $\mathrm{ug} / \mathrm{kg}$ & 446 & 446 & 446 & & \\
\hline X10C0404159 & VOA & 1,2-Dibromo-3-chloropropane & 1 & 0 & $\mathrm{ug} / \mathrm{kg}$ & 446 & 446 & 446 & & \\
\hline X10C0404159 & VOA & 1,2-Dibromoethane & 1 & 0 & $\mathrm{ug} / \mathrm{kg}$ & 446 & 446 & 446 & & \\
\hline X10C0404159 & VOA & 1,2-Dichloroethane & 2 & 0 & $\mathrm{ug} / \mathrm{kg}$ & 0.01 & 446 & 223.005 & 223.005 & 315.36255 \\
\hline X10C0404159 & VOA & 1,2-Dichloroethene & 1 & 0 & $\mathrm{ug} / \mathrm{kg}$ & 893 & 893 & 893 & & \\
\hline X10C0404159 & VOA & 1,2-Dichloropropane & 1 & 0 & $\mathrm{ug} / \mathrm{kg}$ & 446 & 446 & 446 & & \\
\hline X10C0404159 & VOA & 1,2-Dimethylbenzene & 1 & 0 & $\mathrm{ug} / \mathrm{kg}$ & 446 & 446 & 446 & & \\
\hline X10C0404159 & VOA & 1,3,5-Trimethylbenzene & 1 & 0 & $\mathrm{ug} / \mathrm{kg}$ & 446 & 446 & 446 & & \\
\hline
\end{tabular}




\begin{tabular}{|c|c|c|c|c|c|c|c|c|c|c|}
\hline X10C0404159 & VOA & 1,3-Dichloropropane & 1 & 0 & $\mathrm{ug} / \mathrm{kg}$ & 446 & 446 & 446 & & \\
\hline X10C0404159 & $\mathrm{VOA}$ & 1-Chloro-4-methylbenzene & 1 & 0 & $\mathrm{ug} / \mathrm{kg}$ & 446 & 446 & 446 & & \\
\hline X10C0404159 & VOA & 1-Methyl-4-(1-methylethyl)benzene & 1 & 1 & $\mathrm{ug} / \mathrm{kg}$ & 2140 & 2140 & 2140 & 2140 & \\
\hline X10C0404159 & VOA & 2,2-Dichloropropane & 1 & 0 & $\mathrm{ug} / \mathrm{kg}$ & 446 & 446 & 446 & & \\
\hline X10C0404159 & VOA & 2-Butanone & 2 & 0 & $\mathrm{ug} / \mathrm{kg}$ & 0.05 & 2230 & 1115.025 & 1115.025 & 1576.8128 \\
\hline X10C0404159 & VOA & 2-Hexanone & 1 & 0 & $\mathrm{ug} / \mathrm{kg}$ & 2230 & 2230 & 2230 & & \\
\hline X10C0404159 & VOA & 4-Methyl-2-pentanone & 1 & 0 & $\mathrm{ug} / \mathrm{kg}$ & 2230 & 2230 & 2230 & & \\
\hline X10C0404159 & VOA & Acetone & 1 & 0 & $\mathrm{ug} / \mathrm{kg}$ & 2230 & 2230 & 2230 & & \\
\hline X10C0404159 & VOA & Acetonitrile & 1 & 0 & $\mathrm{ug} / \mathrm{kg}$ & 11200 & 11200 & 11200 & & \\
\hline X10C0404159 & VOA & Acrolein & 1 & 0 & $\mathrm{ug} / \mathrm{kg}$ & 2230 & 2230 & 2230 & & \\
\hline X10C0404159 & VOA & Acrylonitrile & 1 & 0 & $\mathrm{ug} / \mathrm{kg}$ & 2230 & 2230 & 2230 & & \\
\hline X10C0404159 & VOA & Benzene & 2 & 0 & $\mathrm{ug} / \mathrm{kg}$ & 0.01 & 446 & 223.005 & 223.005 & 315.36255 \\
\hline X10C0404159 & VOA & Bromobenzene & 1 & 0 & $\mathrm{ug} / \mathrm{kg}$ & 446 & 446 & 446 & & \\
\hline X10C0404159 & VOA & Bromochloromethane & 1 & 0 & $\mathrm{ug} / \mathrm{kg}$ & 446 & 446 & 446 & & \\
\hline X10C0404159 & VOA & Bromodichloromethane & 1 & 0 & $\mathrm{ug} / \mathrm{kg}$ & 446 & 446 & 446 & & \\
\hline X10C0404159 & VOA & Bromoform & 1 & 0 & $\mathrm{ug} / \mathrm{kg}$ & 446 & 446 & 446 & & \\
\hline X10C0404159 & VOA & Bromomethane & 1 & 0 & $\mathrm{ug} / \mathrm{kg}$ & 446 & 446 & 446 & & \\
\hline X10C0404159 & VOA & Butylbenzene & 1 & 0 & $\mathrm{ug} / \mathrm{kg}$ & 446 & 446 & 446 & & \\
\hline X10C0404159 & VOA & Carbon disulfide & 1 & 0 & $\mathrm{ug} / \mathrm{kg}$ & 2230 & 2230 & 2230 & & \\
\hline X10C0404159 & VOA & Carbon tetrachloride & 2 & 0 & $\mathrm{ug} / \mathrm{kg}$ & 0.01 & 446 & 223.005 & 223.005 & 315.36255 \\
\hline X10C0404159 & VOA & Chlorobenzene & 2 & 0 & $\mathrm{ug} / \mathrm{kg}$ & 0.01 & 446 & 223.005 & 223.005 & 315.36255 \\
\hline X10C0404159 & VOA & Chloroethane & 1 & 0 & $\mathrm{ug} / \mathrm{kg}$ & 446 & 446 & 446 & & \\
\hline X10C0404159 & VOA & Chloroform & 2 & 0 & $\mathrm{ug} / \mathrm{kg}$ & 0.01 & 446 & 223.005 & 223.005 & 315.36255 \\
\hline X10C0404159 & VOA & Chloromethane & 1 & 0 & $\mathrm{ug} / \mathrm{kg}$ & 446 & 446 & 446 & & \\
\hline X10C0404159 & VOA & cis-1,2-Dichloroethene & 1 & 0 & $\mathrm{ug} / \mathrm{kg}$ & 446 & 446 & 446 & & \\
\hline X10C0404159 & VOA & cis-1,3-Dichloropropene & 1 & 0 & $\mathrm{ug} / \mathrm{kg}$ & 446 & 446 & 446 & & \\
\hline X10C0404159 & VOA & Cumene & 1 & 0 & $\mathrm{ug} / \mathrm{kg}$ & 446 & 446 & 446 & & \\
\hline X10C0404159 & VOA & Dibromochloromethane & 1 & 0 & $\mathrm{ug} / \mathrm{kg}$ & 446 & 446 & 446 & & \\
\hline X10C0404159 & VOA & Dibromomethane & 1 & 0 & $\mathrm{ug} / \mathrm{kg}$ & 446 & 446 & 446 & & \\
\hline X10C0404159 & VOA & Dichlorodifluoromethane & 1 & 0 & $\mathrm{ug} / \mathrm{kg}$ & 446 & 446 & 446 & & \\
\hline X10C0404159 & VOA & Ethylbenzene & 1 & 0 & $\mathrm{ug} / \mathrm{kg}$ & 446 & 446 & 446 & & \\
\hline X10C0404159 & VOA & Hexane & 1 & 0 & $\mathrm{ug} / \mathrm{kg}$ & 2230 & 2230 & 2230 & & \\
\hline X10C0404159 & VOA & $\mathrm{M}+\mathrm{P}$ Xylene & 1 & 0 & $\mathrm{ug} / \mathrm{kg}$ & 893 & 893 & 893 & & \\
\hline X10C0404159 & VOA & Methanol & 1 & 0 & $\mathrm{ug} / \mathrm{kg}$ & 9700 & 9700 & 9700 & & \\
\hline X10C0404159 & VOA & Methyl methacrylate & 1 & 0 & $\mathrm{ug} / \mathrm{kg}$ & 2230 & 2230 & 2230 & & \\
\hline X10C0404159 & VOA & Methylcyclohexane & 1 & 0 & $\mathrm{ug} / \mathrm{kg}$ & 446 & 446 & 446 & & \\
\hline X10C0404159 & VOA & Methylene chloride & 1 & 0 & $\mathrm{ug} / \mathrm{kg}$ & 2230 & 2230 & 2230 & & \\
\hline X10C0404159 & VOA & o-Chlorotoluene & 1 & 0 & $\mathrm{ug} / \mathrm{kg}$ & 446 & 446 & 446 & & \\
\hline X10C0404159 & VOA & Propylbenzene & 1 & 0 & $\mathrm{ug} / \mathrm{kg}$ & 446 & 446 & 446 & & \\
\hline X10C0404159 & VOA & Propylene glycol & 1 & 0 & $\mathrm{ug} / \mathrm{kg}$ & 50000 & 50000 & 50000 & & \\
\hline X10C0404159 & VOA & Styrene & 1 & 0 & $\mathrm{ug} / \mathrm{kg}$ & 446 & 446 & 446 & & \\
\hline X10C0404159 & VOA & Tetrachloroethene & 2 & 0 & $\mathrm{ug} / \mathrm{kg}$ & 0.01 & 446 & 223.005 & 223.005 & 315.36255 \\
\hline X10C0404159 & VOA & Toluene & 1 & 1 & $\mathrm{ug} / \mathrm{kg}$ & 2300 & 2300 & 2300 & 2300 & \\
\hline X10C0404159 & VOA & Total Xylene & 1 & 0 & $\mathrm{ug} / \mathrm{kg}$ & 1340 & 1340 & 1340 & & \\
\hline X10C0404159 & VOA & trans-1,2-Dichloroethene & 1 & 0 & $\mathrm{ug} / \mathrm{kg}$ & 446 & 446 & 446 & & \\
\hline X10C0404159 & VOA & trans-1,3-Dichloropropene & 1 & 0 & $\mathrm{ug} / \mathrm{kg}$ & 446 & 446 & 446 & & \\
\hline X10C0404159 & $\mathrm{VOA}$ & Trichloroethene & 2 & 0 & $\mathrm{ug} / \mathrm{kg}$ & 0.01 & 446 & 223.005 & 223.005 & 315.36255 \\
\hline X10C0404159 & VOA & Trichlorofluoromethane & 1 & 0 & $\mathrm{ug} / \mathrm{kg}$ & 446 & 446 & 446 & & \\
\hline X10C0404159 & VOA & Vinyl chloride & 2 & 0 & $\mathrm{ug} / \mathrm{kg}$ & 0.01 & 446 & 223.005 & 223.005 & 315.36255 \\
\hline $\mathrm{X} 10 \mathrm{C} 0500911$ & HERB & $2,4-\mathrm{D}$ & 10 & $\overline{0}$ & $\mathrm{ug} / \mathrm{kg}$ & 0.05 & 249 & 98.025 & 61.025 & 112.57344 \\
\hline $\mathrm{X} 10 \mathrm{C} 0500911$ & HERB & Silvex & 10 & 0 & $\mathrm{ug} / \mathrm{kg}$ & 0.05 & 249 & 98.025 & 61.025 & 112.57344 \\
\hline X10C0500911 & METAL & Arsenic & 10 & 0 & $\mathrm{mg} / \mathrm{L}$ & 0.3 & 2.99 & 1.545 & 1.41 & 1.3172636 \\
\hline X10C0500911 & METAL & Barium & 10 & 10 & $\mathrm{mg} / \mathrm{L}$ & 0.0685 & 121 & 17.88145 & 0.6165 & 38.178537 \\
\hline X10C0500911 & METAL & Cadmium & 10 & 1 & $\mathrm{mg} / \mathrm{L}$ & 0.05 & 5.7 & 0.7803 & 0.2345 & 1.7408645 \\
\hline X10C0500911 & METAL & Chromium & 10 & 5 & $\mathrm{mg} / \mathrm{L}$ & 0.05 & 14.6 & 1.9635 & 0.362 & 4.4920173 \\
\hline X10C0500911 & METAL & Lead & 10 & 6 & $\mathrm{mg} / \mathrm{L}$ & 0.1 & 30.7 & 5.0073 & 0.5055 & 9.6468478 \\
\hline X10C0500911 & METAL & Mercury & 10 & 0 & $\mathrm{mg} / \mathrm{L}$ & 0.002 & 0.4 & 0.201 & 0.201 & 0.2097644 \\
\hline X10C0500911 & METAL & Selenium & 10 & 0 & $\mathrm{mg} / \mathrm{L}$ & 0.3 & 2.99 & 1.545 & 1.41 & 1.3172636 \\
\hline X10C0500911 & METAL & Silver & 10 & 0 & $\mathrm{mg} / \mathrm{L}$ & 0.05 & 0.498 & 0.2574 & 0.2345 & 0.219458 \\
\hline X10C0500911 & METAL & Total Uranium & 5 & 0 & $\mathrm{pCi} / \mathrm{g}$ & -0.045 & 0.528 & 0.19464 & 0.0555 & 0.2769051 \\
\hline X10C0500911 & PPCB & 4,4 '-DDD $^{\prime}$ & 5 & 0 & $\mathrm{ug} / \mathrm{kg}$ & 38.5 & 78.9 & 47.24 & 39.8 & 17.707569 \\
\hline X10C0500911 & PPCB & 4,4'-DDE & 5 & 0 & $\mathrm{ug} / \mathrm{kg}$ & 38.5 & 78.9 & 47.24 & 39.8 & 17.707569 \\
\hline X10C0500911 & PPCB & 4,4'-DDT & 5 & 0 & $\mathrm{ug} / \mathrm{kg}$ & 38.5 & 78.9 & 47.24 & 39.8 & 17.707569 \\
\hline X10C0500911 & РPCB & Aldrin & 5 & 0 & $\mathrm{ug} / \mathrm{kg}$ & 19.2 & 39.4 & 23.58 & 19.9 & 8.8485592 \\
\hline X10C0500911 & PPCB & alpha-BHC & 5 & 0 & $\mathrm{ug} / \mathrm{kg}$ & 19.2 & 39.4 & 23.58 & 19.9 & 8.8485592 \\
\hline X10C0500911 & РPCB & alpha-Chlordane & 5 & 0 & $\mathrm{ug} / \mathrm{kg}$ & 19.2 & 39.4 & 23.58 & 19.9 & 8.8485592 \\
\hline X10C0500911 & PPCB & beta-BHC & 5 & 0 & $\mathrm{ug} / \mathrm{kg}$ & 19.2 & 39.4 & 23.58 & 19.9 & 8.8485592 \\
\hline X10C0500911 & РPCB & Chlordane & 10 & 0 & $\mathrm{ug} / \mathrm{kg}$ & 0.0025 & 493 & 147.50125 & 120.00125 & 172.11779 \\
\hline X10C0500911 & РPCB & delta-BHC & 5 & 0 & $\mathrm{ug} / \mathrm{kg}$ & 19.2 & 39.4 & 23.58 & 19.9 & 8.8485592 \\
\hline X10C0500911 & PPCB & Dieldrin & 5 & 0 & $\mathrm{ug} / \mathrm{kg}$ & 38.5 & 78.9 & 47.24 & 39.8 & 17.707569 \\
\hline
\end{tabular}




\begin{tabular}{|c|c|c|c|c|c|c|c|c|c|c|}
\hline X10C0500911 & PPCB & Endosulfan I & 5 & 0 & $\mathrm{ug} / \mathrm{kg}$ & 19.2 & 39.4 & 23.58 & 19.9 & 8.8485592 \\
\hline X10C0500911 & РPCB & Endosulfan II & 5 & 0 & $\mathrm{ug} / \mathrm{kg}$ & 38.5 & 78.9 & 47.24 & 39.8 & 17.707569 \\
\hline X10C0500911 & PPCB & Endosulfan sulfate & 5 & 0 & $\mathrm{ug} / \mathrm{kg}$ & 38.5 & 78.9 & 47.24 & 39.8 & 17.707569 \\
\hline X10C0500911 & PPCB & Endrin & 10 & 0 & $\mathrm{ug} / \mathrm{kg}$ & 0.0004 & 78.9 & 23.6202 & 19.2502 & 27.554353 \\
\hline X10C0500911 & PPCB & Endrin aldehyde & 5 & 0 & $\mathrm{ug} / \mathrm{kg}$ & 38.5 & 78.9 & 47.24 & 39.8 & 17.707569 \\
\hline X10C0500911 & РPCB & Endrin ketone & 5 & 0 & $\mathrm{ug} / \mathrm{kg}$ & 38.5 & 78.9 & 47.24 & 39.8 & 17.707569 \\
\hline X10C0500911 & PPCB & gamma-Chlordane & 5 & 0 & $\mathrm{ug} / \mathrm{kg}$ & 19.2 & 39.4 & 23.58 & 19.9 & 8.8485592 \\
\hline X10C0500911 & PPCB & Heptachlor & 10 & 0 & $\mathrm{ug} / \mathrm{kg}$ & 0.0002 & 39.4 & 11.7901 & 9.6001 & 13.756636 \\
\hline X10C0500911 & PPCB & Heptachlor epoxide & 10 & 0 & $\mathrm{ug} / \mathrm{kg}$ & 0.0002 & 39.4 & 11.7901 & 9.6001 & 13.756636 \\
\hline X10C0500911 & PPCB & Lindane & 10 & 0 & $\mathrm{ug} / \mathrm{kg}$ & 0.0002 & 39.4 & 11.7901 & 9.6001 & 13.756636 \\
\hline X10C0500911 & РPCB & Methoxychlor & 10 & 0 & $\mathrm{ug} / \mathrm{kg}$ & 0.002 & 394 & 117.901 & 96.001 & 137.56636 \\
\hline $\mathrm{X} 10 \mathrm{C} 0500911$ & PPCB & Toxaphene & 10 & 0 & $\mathrm{ug} / \mathrm{kg}$ & 0.005 & 986 & 295.3025 & 240.5025 & 344.4234 \\
\hline X10C0500911 & RADS & Americium-241 & 5 & 0 & $\mathrm{pCi} / \mathrm{g}$ & -0.00735 & 0.159 & 0.06085 & 0.0116 & 0.08191 \\
\hline X10C0500911 & RADS & Carbon-14 & 5 & 0 & $\mathrm{pCi} / \mathrm{g}$ & -8.81 & 2.43 & -2.0064 & 0.448 & 4.9807303 \\
\hline X10C0500911 & RADS & Cesium-137 & 5 & 5 & $\mathrm{pCi} / \mathrm{g}$ & 325 & 68000 & 17292.6 & 470 & 29277.047 \\
\hline X10C0500911 & RADS & Cobalt-60 & 5 & 2 & $\mathrm{pCi} / \mathrm{g}$ & 0.0386 & 18.6 & 5.70152 & 0.718 & 8.0673804 \\
\hline X10C0500911 & RADS & Curium-243/244 & 5 & 0 & $\mathrm{pCi} / \mathrm{g}$ & 0 & 0.0942 & 0.032354 & 0.00787 & 0.0426197 \\
\hline X10C0500911 & RADS & Iodine-129 & 5 & 0 & $\mathrm{pCi} / \mathrm{g}$ & -0.171 & 0.366 & 0.06508 & 0.0764 & 0.2177401 \\
\hline X10C0500911 & RADS & Neptunium-237 & 5 & 0 & $\mathrm{pCi} / \mathrm{g}$ & -1.62 & 5.04 & 0.3778 & -0.619 & 2.6539273 \\
\hline X10C0500911 & RADS & Plutonium-238 & 5 & 0 & $\mathrm{pCi} / \mathrm{g}$ & -0.0089 & 0.121 & 0.037242 & 0 & 0.0594447 \\
\hline X10C0500911 & RADS & Plutonium-239/240 & 5 & 0 & $\mathrm{pCi} / \mathrm{g}$ & -0.0393 & 0.0436 & 0.010662 & 0.0198 & 0.0335235 \\
\hline X10C0500911 & RADS & Radium-226 & 5 & 0 & $\mathrm{pCi} / \mathrm{g}$ & -0.947 & 5.91 & 2.15836 & 0.257 & 3.2981854 \\
\hline X10C0500911 & RADS & Strontium-90 & 5 & 4 & $\mathrm{pCi} / \mathrm{g}$ & 2.22 & 27700 & 5670.348 & 16.9 & 12317.903 \\
\hline X10C0500911 & RADS & Technetium-99 & 5 & 0 & $\mathrm{pCi} / \mathrm{g}$ & 0.44 & 3.06 & 1.696 & 1.84 & 0.956389 \\
\hline X10C0500911 & RADS & Thorium-230 & 5 & 0 & $\mathrm{pCi} / \mathrm{g}$ & -91.1 & 5.91 & -21.496 & -0.947 & 40.284212 \\
\hline X10C0500911 & RADS & Thorium-232 & 5 & 0 & $\mathrm{pCi} / \mathrm{g}$ & -266 & 176 & -16.9822 & 1.21 & 158.35845 \\
\hline X10C0500911 & RADS & Tritium & 5 & 0 & $\mathrm{pCi} / \mathrm{g}$ & -50.2 & 194 & 23.892 & -2.54 & 98.84401 \\
\hline X10C0500911 & RADS & Uranium-233/234 & 5 & 0 & $\mathrm{pCi} / \mathrm{g}$ & -0.0265 & 0.314 & 0.12018 & 0.0416 & 0.1664409 \\
\hline X10C0500911 & RADS & Uranium-235/236 & 5 & 0 & $\mathrm{pCi} / \mathrm{g}$ & -0.0271 & 0.192 & 0.040358 & -0.00371 & 0.0887591 \\
\hline $\mathrm{X} 10 \mathrm{C} 0500911$ & RADS & Uranium-238 & 5 & 0 & $\mathrm{pCi} / \mathrm{g}$ & -0.0459 & 0.197 & 0.034128 & 0.00863 & 0.0942817 \\
\hline X10C0500911 & SVOA & 1,2,4-Trichlorobenzene & 5 & 0 & $\mathrm{ug} / \mathrm{kg}$ & 94.7 & 500 & 325.2 & 463 & 210.13066 \\
\hline X10C0500911 & SVOA & 1,2-Dichlorobenzene & 5 & 0 & $\mathrm{ug} / \mathrm{kg}$ & 94.7 & 500 & 325.2 & 463 & 210.13066 \\
\hline X10C0500911 & SVOA & 1,3-Dichlorobenzene & 5 & 0 & $\mathrm{ug} / \mathrm{kg}$ & 94.7 & 500 & 325.2 & 463 & 210.13066 \\
\hline X10C0500911 & SVOA & 1,4-Dichlorobenzene & 10 & 0 & $\mathrm{ug} / \mathrm{kg}$ & 0.01 & 500 & 162.605 & 47.355 & 221.35716 \\
\hline X10C0500911 & SVOA & 2,2'-Dichlorodiisopropyl ether & 5 & 0 & $\mathrm{ug} / \mathrm{kg}$ & 35500 & 839000 & 270660 & 125000 & 327728.13 \\
\hline X10C0500911 & SVOA & 2,3,4,6-Tetrachlorophenol & 5 & 0 & $\mathrm{ug} / \mathrm{kg}$ & 35500 & 839000 & 270660 & 125000 & 327728.13 \\
\hline X10C0500911 & SVOA & 2,4,5-Trichlorophenol & 10 & 0 & $\mathrm{ug} / \mathrm{kg}$ & 0.05 & 839000 & 135330.03 & 17750.025 & 260931.01 \\
\hline X10C0500911 & SVOA & 2,4,6-Trichlorophenol & 10 & 0 & $\mathrm{ug} / \mathrm{kg}$ & 0.05 & 839000 & 135330.03 & 17750.025 & 260931.01 \\
\hline X10C0500911 & SVOA & 2,4-Dichlorophenol & 5 & 0 & $\mathrm{ug} / \mathrm{kg}$ & 35500 & 839000 & 270660 & 125000 & 327728.13 \\
\hline X10C0500911 & SVOA & 2,4-Dimethylphenol & 5 & 0 & $\mathrm{ug} / \mathrm{kg}$ & 35500 & 839000 & 270660 & 125000 & 327728.13 \\
\hline X10C0500911 & SVOA & 2,4-Dinitrophenol & 5 & 0 & $\mathrm{ug} / \mathrm{kg}$ & 71100 & 1680000 & 542020 & 251000 & 656141.72 \\
\hline X10C0500911 & SVOA & 2,4-Dinitrotoluene & 10 & 0 & $\mathrm{ug} / \mathrm{kg}$ & 0.05 & 839000 & 135330.03 & 17750.025 & 260931.01 \\
\hline X10C0500911 & SVOA & 2,6-Dinitrotoluene & 5 & 0 & $\mathrm{ug} / \mathrm{kg}$ & 35500 & 839000 & 270660 & 125000 & 327728.13 \\
\hline X10C0500911 & SVOA & 2-Chloronaphthalene & 5 & 0 & $\mathrm{ug} / \mathrm{kg}$ & 3550 & 83900 & 27066 & 12500 & 32772.813 \\
\hline X10C0500911 & SVOA & 2-Chlorophenol & 5 & 0 & $\mathrm{ug} / \mathrm{kg}$ & 35500 & 839000 & 270660 & 125000 & 327728.13 \\
\hline X10C0500911 & SVOA & 2-Methyl-4,6-dinitrophenol & 5 & 0 & $\mathrm{ug} / \mathrm{kg}$ & 35500 & 839000 & 270660 & 125000 & 327728.13 \\
\hline X10C0500911 & SVOA & 2-Methylnaphthalene & 5 & 0 & $\mathrm{ug} / \mathrm{kg}$ & 3550 & 83900 & 27066 & 12500 & 32772.813 \\
\hline X10C0500911 & SVOA & 2-Methylphenol & 10 & 0 & $\mathrm{ug} / \mathrm{kg}$ & 0.05 & 839000 & 135330.03 & 17750.025 & 260931.01 \\
\hline X10C0500911 & SVOA & 2-Nitrobenzenamine & 5 & 0 & $\mathrm{ug} / \mathrm{kg}$ & 35500 & 839000 & 270660 & 125000 & 327728.13 \\
\hline X10C0500911 & SVOA & 2-Nitrophenol & 5 & 0 & $\mathrm{ug} / \mathrm{kg}$ & 35500 & 839000 & 270660 & 125000 & 327728.13 \\
\hline X10C0500911 & SVOA & 3,3'-Dichlorobenzidine & 5 & 0 & $\mathrm{ug} / \mathrm{kg}$ & 35500 & 839000 & 270660 & 125000 & 327728.13 \\
\hline X10C0500911 & SVOA & 3-Nitrobenzenamine & 5 & 0 & $\mathrm{ug} / \mathrm{kg}$ & 35500 & 839000 & 270660 & 125000 & 327728.13 \\
\hline X10C0500911 & SVOA & 4-Bromophenyl phenyl ether & 5 & 0 & $\mathrm{ug} / \mathrm{kg}$ & 35500 & 839000 & 270660 & 125000 & 327728.13 \\
\hline X10C0500911 & SVOA & 4-Chloro-3-methylphenol & 5 & 0 & $\mathrm{ug} / \mathrm{kg}$ & 35500 & 839000 & 270660 & 125000 & 327728.13 \\
\hline X10C0500911 & SVOA & 4-Chlorobenzenamine & 5 & 0 & $\mathrm{ug} / \mathrm{kg}$ & 35500 & 839000 & 270660 & 125000 & 327728.13 \\
\hline X10C0500911 & SVOA & 4-Chlorophenyl phenyl ether & 5 & 0 & $\mathrm{ug} / \mathrm{kg}$ & 35500 & 839000 & 270660 & 125000 & 327728.13 \\
\hline X10C0500911 & SVOA & 4-Nitrobenzenamine & 5 & 0 & $\mathrm{ug} / \mathrm{kg}$ & 35500 & 839000 & 270660 & 125000 & 327728.13 \\
\hline X10C0500911 & SVOA & 4-Nitrophenol & 5 & 0 & $\mathrm{ug} / \mathrm{kg}$ & 35500 & 839000 & 270660 & 125000 & 327728.13 \\
\hline X10C0500911 & SVOA & Acenaphthene & 5 & 0 & $\mathrm{ug} / \mathrm{kg}$ & 3550 & 83900 & 27066 & 12500 & 32772.813 \\
\hline X10C0500911 & SVOA & Acenaphthylene & 5 & 0 & $\mathrm{ug} / \mathrm{kg}$ & 3550 & 83900 & 27066 & 12500 & 32772.813 \\
\hline X10C0500911 & SVOA & Acetophenone & 5 & 0 & $\mathrm{ug} / \mathrm{kg}$ & 35500 & 839000 & 270660 & 125000 & 327728.13 \\
\hline X10C0500911 & SVOA & Anthracene & 5 & 0 & $\mathrm{ug} / \mathrm{kg}$ & 3550 & 83900 & 27066 & 12500 & 32772.813 \\
\hline X10C0500911 & SVOA & Benz(a)anthracene & 5 & 0 & $\mathrm{ug} / \mathrm{kg}$ & 3550 & 83900 & 27066 & 12500 & 32772.813 \\
\hline X10C0500911 & SVOA & Benzenemethanol & 5 & 0 & $\mathrm{ug} / \mathrm{kg}$ & 35500 & 839000 & 270660 & 125000 & 327728.13 \\
\hline X10C0500911 & SVOA & Benzidine & 5 & 0 & $\mathrm{ug} / \mathrm{kg}$ & 35500 & 839000 & 270660 & 125000 & 327728.13 \\
\hline X10C0500911 & SVOA & Benzo(a)pyrene & 5 & 0 & $\mathrm{ug} / \mathrm{kg}$ & 3550 & 83900 & 27066 & 12500 & 32772.813 \\
\hline X10C0500911 & SVOA & Benzo(b)fluoranthene & 5 & 0 & $\mathrm{ug} / \mathrm{kg}$ & 3550 & 83900 & 27066 & 12500 & 32772.813 \\
\hline X10C0500911 & SVOA & Benzo(ghi)perylene & 5 & 0 & $\mathrm{ug} / \mathrm{kg}$ & 3550 & 83900 & 27066 & 12500 & 32772.813 \\
\hline X10C0500911 & SVOA & Benzo(k)fluoranthene & 5 & 0 & $\mathrm{ug} / \mathrm{kg}$ & 3550 & 83900 & 27066 & 12500 & 32772.813 \\
\hline X10C0500911 & SVOA & Benzoic acid & 5 & 0 & $\mathrm{ug} / \mathrm{kg}$ & 71100 & 1680000 & 542020 & 251000 & 656141.72 \\
\hline
\end{tabular}




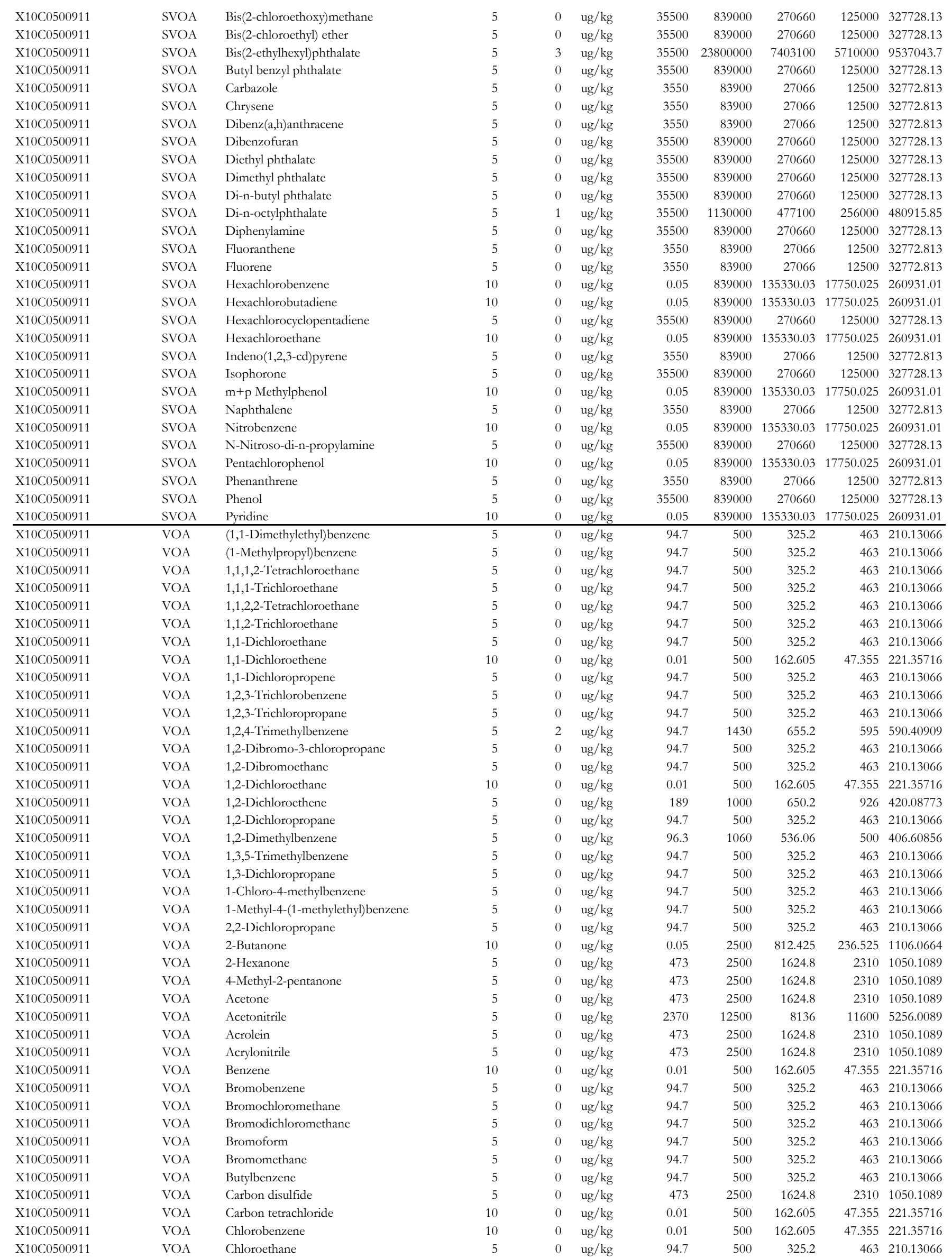




\begin{tabular}{|c|c|c|c|c|c|c|c|c|c|c|}
\hline X10C0500911 & VOA & Chloroform & 10 & 0 & $\mathrm{ug} / \mathrm{kg}$ & 0.01 & 500 & 162.605 & 47.355 & 221.35716 \\
\hline X10C0500911 & VOA & Chloromethane & 5 & 0 & $\mathrm{ug} / \mathrm{kg}$ & 94.7 & 500 & 325.2 & 463 & 210.13066 \\
\hline X10C0500911 & VOA & cis-1,2-Dichloroethene & 5 & 0 & $\mathrm{ug} / \mathrm{kg}$ & 94.7 & 500 & 325.2 & 463 & 210.13066 \\
\hline X10C0500911 & VOA & cis-1,3-Dichloropropene & 5 & 0 & $\mathrm{ug} / \mathrm{kg}$ & 94.7 & 500 & 325.2 & 463 & 210.13066 \\
\hline X10C0500911 & VOA & Cumene & 5 & 0 & $\mathrm{ug} / \mathrm{kg}$ & 94.7 & 500 & 325.2 & 463 & 210.13066 \\
\hline X10C0500911 & VOA & Dibromochloromethane & 5 & 0 & $\mathrm{ug} / \mathrm{kg}$ & 94.7 & 500 & 325.2 & 463 & 210.13066 \\
\hline X10C0500911 & $\mathrm{VOA}$ & Dibromomethane & 5 & 0 & $\mathrm{ug} / \mathrm{kg}$ & 94.7 & 500 & 325.2 & 463 & 210.13066 \\
\hline X10C0500911 & VOA & Dichlorodifluoromethane & 5 & 0 & $\mathrm{ug} / \mathrm{kg}$ & 94.7 & 500 & 325.2 & 463 & 210.13066 \\
\hline X10C0500911 & VOA & Ethylbenzene & 5 & 2 & $\mathrm{ug} / \mathrm{kg}$ & 96.3 & 500 & 326.66 & 463 & 208.14473 \\
\hline X10C0500911 & VOA & Hexane & 5 & 1 & $\mathrm{ug} / \mathrm{kg}$ & 473 & 2500 & 1624.8 & 2310 & 1050.1089 \\
\hline X10C0500911 & VOA & M + P Xylene & 5 & 1 & $\mathrm{ug} / \mathrm{kg}$ & 189 & 1060 & 673.6 & 926 & 443.10304 \\
\hline X10C0500911 & VOA & Methanol & 5 & 1 & $\mathrm{ug} / \mathrm{kg}$ & 4240 & 9920 & 6664 & 4720 & 2963.6346 \\
\hline X10C0500911 & VOA & Methyl methacrylate & 5 & 1 & $\mathrm{ug} / \mathrm{kg}$ & 473 & 2500 & 1624.8 & 2310 & 1050.1089 \\
\hline X10C0500911 & VOA & Methylcyclohexane & 5 & 1 & $\mathrm{ug} / \mathrm{kg}$ & 94.7 & 500 & 325.2 & 463 & 210.13066 \\
\hline X10C0500911 & VOA & Methylene chloride & 5 & 1 & $\mathrm{ug} / \mathrm{kg}$ & 473 & 2500 & 1624.8 & 2310 & 1050.1089 \\
\hline X10C0500911 & VOA & o-Chlorotoluene & 5 & 1 & $\mathrm{ug} / \mathrm{kg}$ & 94.7 & 500 & 325.2 & 463 & 210.13066 \\
\hline X10C0500911 & VOA & Propylbenzene & 5 & 1 & $\mathrm{ug} / \mathrm{kg}$ & 94.7 & 500 & 325.2 & 463 & 210.13066 \\
\hline X10C0500911 & VOA & Propylene glycol & 5 & 1 & $\mathrm{ug} / \mathrm{kg}$ & 42400 & 99200 & 65880 & 47200 & 28634.018 \\
\hline X10C0500911 & VOA & Styrene & 5 & 1 & $\mathrm{ug} / \mathrm{kg}$ & 94.7 & 500 & 325.2 & 463 & 210.13066 \\
\hline X10C0500911 & VOA & Tetrachloroethene & 10 & 1 & $\mathrm{ug} / \mathrm{kg}$ & 0.01 & 500 & 162.605 & 47.355 & 221.35716 \\
\hline X10C0500911 & VOA & Toluene & 5 & 1 & $\mathrm{ug} / \mathrm{kg}$ & 94.7 & 500 & 325.2 & 463 & 210.13066 \\
\hline X10C0500911 & $\mathrm{VOA}$ & Total Xylene & 5 & 3 & $\mathrm{ug} / \mathrm{kg}$ & 284 & 1880 & 1140.6 & 1500 & 791.55467 \\
\hline X10C0500911 & VOA & trans-1,2-Dichloroethene & 5 & 1 & $\mathrm{ug} / \mathrm{kg}$ & 94.7 & 500 & 325.2 & 463 & 210.13066 \\
\hline X10C0500911 & VOA & trans-1,3-Dichloropropene & 5 & 1 & $\mathrm{ug} / \mathrm{kg}$ & 94.7 & 500 & 325.2 & 463 & 210.13066 \\
\hline X10C0500911 & VOA & Trichloroethene & 10 & 1 & $\mathrm{ug} / \mathrm{kg}$ & 0.01 & 500 & 162.605 & 47.355 & 221.35716 \\
\hline X10C0500911 & $\mathrm{VOA}$ & Trichlorofluoromethane & 5 & 1 & $\mathrm{ug} / \mathrm{kg}$ & 94.7 & 500 & 325.2 & 463 & 210.13066 \\
\hline $\mathrm{X} 10 \mathrm{C} 0500911$ & $\mathrm{VOA}$ & Vinyl chloride & 10 & 1 & $\mathrm{ug} / \mathrm{kg}$ & 0.01 & 500 & 162.605 & 47.355 & 221.35716 \\
\hline $\mathrm{X} 10 \mathrm{C} 0700129$ & HERB & $2,4-\mathrm{D}$ & 4 & 0 & $\mathrm{ug} / \mathrm{kg}$ & 0.05 & 24.6 & 12.325 & 12.325 & 14.173949 \\
\hline X10C0700129 & HERB & Silvex & 4 & 0 & $\mathrm{ug} / \mathrm{kg}$ & 0.05 & 24.6 & 12.325 & 12.325 & 14.173949 \\
\hline X10C0700129 & METAL & Arsenic & 4 & 1 & $\mathrm{mg} / \mathrm{L}$ & 0.3 & 148 & 71.9 & 69.65 & 82.758162 \\
\hline X10C0700129 & METAL & Barium & 4 & 4 & $\mathrm{mg} / \mathrm{L}$ & 3.19 & 114 & 49.4475 & 40.3 & 53.950765 \\
\hline X10C0700129 & METAL & Cadmium & 4 & 0 & $\mathrm{mg} / \mathrm{L}$ & 0.05 & 24.5 & 11.925 & 11.575 & 13.723975 \\
\hline X10C0700129 & METAL & Chromium & 4 & 3 & $\mathrm{mg} / \mathrm{L}$ & 0.05 & 514 & 254.03123 & 251.03745 & 293.2988 \\
\hline X10C0700129 & METAL & Lead & 4 & 0 & $\mathrm{mg} / \mathrm{L}$ & 0.1 & 49 & 23.875 & 23.2 & 27.475125 \\
\hline X10C0700129 & METAL & Mercury & 4 & 0 & $\mathrm{mg} / \mathrm{L}$ & 0.002 & 0.4 & 0.201 & 0.201 & 0.2297854 \\
\hline X10C0700129 & METAL & Selenium & 4 & 0 & $\mathrm{mg} / \mathrm{L}$ & 0.3 & 147 & 71.65 & 69.65 & 82.452592 \\
\hline X10C0700129 & METAL & Silver & 4 & 1 & $\mathrm{mg} / \mathrm{L}$ & 0.05 & 24.5 & 12.125 & 11.975 & 13.94516 \\
\hline $\mathrm{X} 10 \mathrm{C} 0700129$ & METAL & Total Uranium & 2 & 0 & $\mathrm{pCi} / \mathrm{g}$ & -0.255 & 0.00127 & -0.126865 & -0.126865 & 0.1812103 \\
\hline X10C0700129 & PPCB & 4,4'-DDD & 2 & 0 & $\mathrm{ug} / \mathrm{kg}$ & 3.89 & 3.95 & 3.92 & 3.92 & 0.0424264 \\
\hline X10C0700129 & РPCB & 4,4'-DDE & 2 & 0 & $\mathrm{ug} / \mathrm{kg}$ & 3.89 & 3.95 & 3.92 & 3.92 & 0.0424264 \\
\hline X10C0700129 & PPCB & 4,4'-DDT & 2 & 0 & $\mathrm{ug} / \mathrm{kg}$ & 3.89 & 3.95 & 3.92 & 3.92 & 0.0424264 \\
\hline X10C0700129 & РPCB & Aldrin & 2 & 0 & $\mathrm{ug} / \mathrm{kg}$ & 1.95 & 1.97 & 1.96 & 1.96 & 0.0141421 \\
\hline X10C0700129 & РPCB & alpha-BHC & 2 & 0 & $\mathrm{ug} / \mathrm{kg}$ & 1.95 & 1.97 & 1.96 & 1.96 & 0.0141421 \\
\hline X10C0700129 & PPCB & alpha-Chlordane & 2 & 0 & $\mathrm{ug} / \mathrm{kg}$ & 1.95 & 1.97 & 1.96 & 1.96 & 0.0141421 \\
\hline X10C0700129 & PPCB & beta-BHC & 2 & 0 & $\mathrm{ug} / \mathrm{kg}$ & 1.95 & 1.97 & 1.96 & 1.96 & 0.0141421 \\
\hline X10C0700129 & РPCB & Chlordane & 4 & 0 & $\mathrm{ug} / \mathrm{kg}$ & 0.0025 & 24.7 & 12.25125 & 12.15125 & 14.144581 \\
\hline X10C0700129 & PPCB & delta-BHC & 2 & 0 & $\mathrm{ug} / \mathrm{kg}$ & 1.95 & 1.97 & 1.96 & 1.96 & 0.0141421 \\
\hline X10C0700129 & РPCB & Dieldrin & 2 & 0 & $\mathrm{ug} / \mathrm{kg}$ & 3.89 & 3.95 & 3.92 & 3.92 & 0.0424264 \\
\hline X10C0700129 & PРCB & Endosulfan I & 2 & 0 & $\mathrm{ug} / \mathrm{kg}$ & 1.95 & 1.97 & 1.96 & 1.96 & 0.0141421 \\
\hline X10C0700129 & РPCB & Endosulfan II & 2 & 0 & $\mathrm{ug} / \mathrm{kg}$ & 3.89 & 3.95 & 3.92 & 3.92 & 0.0424264 \\
\hline X10C0700129 & PPCB & Endosulfan sulfate & 2 & 0 & $\mathrm{ug} / \mathrm{kg}$ & 3.89 & 3.95 & 3.92 & 3.92 & 0.0424264 \\
\hline X10C0700129 & PPCB & Endrin & 4 & 0 & $\mathrm{ug} / \mathrm{kg}$ & 0.0004 & 3.95 & 1.9602 & 1.9452 & 2.2631147 \\
\hline X10C0700129 & РPCB & Endrin aldehyde & 2 & 0 & $\mathrm{ug} / \mathrm{kg}$ & 3.89 & 3.95 & 3.92 & 3.92 & 0.0424264 \\
\hline X10C0700129 & РPCB & Endrin ketone & 2 & 0 & $\mathrm{ug} / \mathrm{kg}$ & 3.89 & 3.95 & 3.92 & 3.92 & 0.0424264 \\
\hline X10C0700129 & РPCB & gamma-Chlordane & 2 & 0 & $\mathrm{ug} / \mathrm{kg}$ & 1.95 & 1.97 & 1.96 & 1.96 & 0.0141421 \\
\hline X10C0700129 & РPCB & Heptachlor & 4 & 0 & $\mathrm{ug} / \mathrm{kg}$ & 0.0002 & 1.97 & 0.9801 & 0.9751 & 1.1315205 \\
\hline X10C0700129 & PPCB & Heptachlor epoxide & 4 & 0 & $\mathrm{ug} / \mathrm{kg}$ & 0.0002 & 1.97 & 0.9801 & 0.9751 & 1.1315205 \\
\hline X10C0700129 & РPCB & Lindane & 4 & 0 & $\mathrm{ug} / \mathrm{kg}$ & 0.0002 & 1.97 & 0.9801 & 0.9751 & 1.1315205 \\
\hline X10C0700129 & РPCB & Methoxychlor & 4 & 0 & $\mathrm{ug} / \mathrm{kg}$ & 0.002 & 19.7 & 9.801 & 9.751 & 11.315205 \\
\hline $\mathrm{X} 10 \mathrm{C} 0700129$ & PPCB & Toxaphene & 4 & 0 & $\mathrm{ug} / \mathrm{kg}$ & 0.005 & 49.4 & 24.5025 & 24.3025 & 28.289162 \\
\hline $\mathrm{X} 10 \mathrm{C} 0700129$ & RADS & Americium-241 & 2 & 2 & $\mathrm{pCi} / \mathrm{g}$ & 0.129 & 0.404 & 0.2665 & 0.2665 & 0.1944544 \\
\hline X10C0700129 & RADS & Carbon-14 & 2 & 0 & $\mathrm{pCi} / \mathrm{g}$ & -5.1 & -2.88 & -3.99 & -3.99 & 1.5697771 \\
\hline X10C0700129 & RADS & Cesium-137 & 2 & 2 & $\mathrm{pCi} / \mathrm{g}$ & 1 & 1.29 & 1.145 & 1.145 & 0.205061 \\
\hline X10C0700129 & RADS & Cobalt- 60 & 2 & 0 & $\mathrm{pCi} / \mathrm{g}$ & -0.0276 & 0.023 & -0.0023 & -0.0023 & 0.0357796 \\
\hline X10C0700129 & RADS & Curium-243/244 & 2 & 0 & $\mathrm{pCi} / \mathrm{g}$ & 0.0595 & 0.0963 & 0.0779 & 0.0779 & 0.0260215 \\
\hline X10C0700129 & RADS & Iodine-129 & 2 & 0 & $\mathrm{pCi} / \mathrm{g}$ & -0.175 & 0.492 & 0.1585 & 0.1585 & 0.4716402 \\
\hline X10C0700129 & RADS & Neptunium-237 & 2 & 0 & $\mathrm{pCi} / \mathrm{g}$ & -0.0424 & 0.0404 & -0.001 & -0.001 & 0.0585484 \\
\hline X10C0700129 & RADS & Plutonium-238 & 2 & 0 & $\mathrm{pCi} / \mathrm{g}$ & -0.0191 & 0.0353 & 0.0081 & 0.0081 & 0.0384666 \\
\hline X10C0700129 & RADS & Plutonium-239/240 & 2 & 2 & $\mathrm{pCi} / \mathrm{g}$ & 0.286 & 0.296 & 0.291 & 0.291 & 0.0070711 \\
\hline X10C0700129 & RADS & Radium-226 & 2 & 0 & $\mathrm{pCi} / \mathrm{g}$ & -0.252 & 1.55 & 0.649 & 0.649 & 1.2742064 \\
\hline
\end{tabular}




\begin{tabular}{|c|c|c|c|c|c|c|c|c|c|c|}
\hline X10C0700129 & RADS & Strontium-90 & 2 & 0 & $\mathrm{pCi} / \mathrm{g}$ & -0.32 & 1.18 & 0.43 & 0.43 & 1.0606602 \\
\hline X10C0700129 & RADS & Technetium-99 & 2 & 0 & $\mathrm{pCi} / \mathrm{g}$ & -0.965 & -0.546 & -0.7555 & -0.7555 & 0.2962777 \\
\hline X10C0700129 & RADS & Thorium-232 & 2 & 0 & $\mathrm{pCi} / \mathrm{g}$ & -11.8 & 21.8 & 5 & 5 & 23.758788 \\
\hline X10C0700129 & RADS & Uranium-233/234 & 2 & 0 & $\mathrm{pCi} / \mathrm{g}$ & -0.0711 & -0.0187 & -0.0449 & -0.0449 & 0.0370524 \\
\hline X10C0700129 & RADS & Uranium-235/236 & 2 & 0 & $\mathrm{pCi} / \mathrm{g}$ & -0.0885 & 0.00814 & -0.04018 & -0.04018 & 0.0683348 \\
\hline X10C0700129 & RADS & Uranium-238 & 2 & 0 & $\mathrm{pCi} / \mathrm{g}$ & -0.0956 & 0.0119 & -0.04185 & -0.04185 & 0.076014 \\
\hline $\mathrm{X} 10 \mathrm{C} 0700129$ & SVOA & 1,2,4-Trichlorobenzene & 2 & 0 & $\mathrm{ug} / \mathrm{kg}$ & 96.2 & 98 & 97.1 & 97.1 & 1.2727922 \\
\hline X10C0700129 & SVOA & 1,4-Dichlorobenzene & 4 & 0 & $\mathrm{ug} / \mathrm{kg}$ & 0.01 & 98 & 48.555 & 48.105 & 56.059754 \\
\hline X10C0700129 & SVOA & 2,2'-Dichlorodiisopropyl ether & 2 & 0 & $\mathrm{ug} / \mathrm{kg}$ & 987 & 1000 & 993.5 & 993.5 & 9.1923882 \\
\hline X10C0700129 & SVOA & 2,3,4,6-Tetrachlorophenol & 2 & 0 & $\mathrm{ug} / \mathrm{kg}$ & 987 & 1000 & 993.5 & 993.5 & 9.1923882 \\
\hline X10C0700129 & SVOA & 2,4,5-Trichlorophenol & 4 & 0 & $\mathrm{ug} / \mathrm{kg}$ & 0.05 & 1000 & 496.775 & 493.525 & 573.59318 \\
\hline X10C0700129 & SVOA & 2,4,6-Trichlorophenol & 4 & 0 & $\mathrm{ug} / \mathrm{kg}$ & 0.05 & 1000 & 496.775 & 493.525 & 573.59318 \\
\hline X10C0700129 & SVOA & 2,4-Dichlorophenol & 2 & 0 & $\mathrm{ug} / \mathrm{kg}$ & 987 & 1000 & 993.5 & 993.5 & 9.1923882 \\
\hline X10C0700129 & SVOA & 2-Chloronaphthalene & 2 & 0 & $\mathrm{ug} / \mathrm{kg}$ & 98.7 & 100 & 99.35 & 99.35 & 0.9192388 \\
\hline X10C0700129 & SVOA & 2-Chlorophenol & 2 & 0 & $\mathrm{ug} / \mathrm{kg}$ & 987 & 1000 & 993.5 & 993.5 & 9.1923882 \\
\hline X10C0700129 & SVOA & 2-Methyl-4,6-dinitrophenol & 2 & 0 & $\mathrm{ug} / \mathrm{kg}$ & 987 & 1000 & 993.5 & 993.5 & 9.1923882 \\
\hline X10C0700129 & SVOA & 2-Methylnaphthalene & 2 & 0 & $\mathrm{ug} / \mathrm{kg}$ & 98.7 & 100 & 99.35 & 99.35 & 0.9192388 \\
\hline X10C0700129 & SVOA & 2-Methylphenol & 4 & 0 & $\mathrm{ug} / \mathrm{kg}$ & 0.05 & 1000 & 496.775 & 493.525 & 573.59318 \\
\hline X10C0700129 & SVOA & 2-Nitrobenzenamine & 2 & 0 & $\mathrm{ug} / \mathrm{kg}$ & 987 & 1000 & 993.5 & 993.5 & 9.1923882 \\
\hline X10C0700129 & SVOA & 2-Nitrophenol & 2 & 0 & $\mathrm{ug} / \mathrm{kg}$ & 987 & 1000 & 993.5 & 993.5 & 9.1923882 \\
\hline X10C0700129 & SVOA & 3,3'-Dichlorobenzidine & 2 & 0 & $\mathrm{ug} / \mathrm{kg}$ & 987 & 1000 & 993.5 & 993.5 & 9.1923882 \\
\hline X10C0700129 & SVOA & 3-Nitrobenzenamine & 2 & 0 & $\mathrm{ug} / \mathrm{kg}$ & 987 & 1000 & 993.5 & 993.5 & 9.1923882 \\
\hline X10C0700129 & SVOA & 4-Bromophenyl phenyl ether & 2 & 0 & $\mathrm{ug} / \mathrm{kg}$ & 987 & 1000 & 993.5 & 993.5 & 9.1923882 \\
\hline X10C0700129 & SVOA & 4-Chloro-3-methylphenol & 2 & 0 & $\mathrm{ug} / \mathrm{kg}$ & 987 & 1000 & 993.5 & 993.5 & 9.1923882 \\
\hline X10C0700129 & SVOA & 4-Chlorobenzenamine & 2 & 0 & $\mathrm{ug} / \mathrm{kg}$ & 987 & 1000 & 993.5 & 993.5 & 9.1923882 \\
\hline X10C0700129 & SVOA & 4-Chlorophenyl phenyl ether & 2 & 0 & $\mathrm{ug} / \mathrm{kg}$ & 987 & 1000 & 993.5 & 993.5 & 9.1923882 \\
\hline X10C0700129 & SVOA & Benzo(a)pyrene & 2 & 0 & $\mathrm{ug} / \mathrm{kg}$ & 98.7 & 100 & 99.35 & 99.35 & 0.9192388 \\
\hline X10C0700129 & SVOA & Benzo(b)fluoranthene & 2 & 0 & $\mathrm{ug} / \mathrm{kg}$ & 98.7 & 100 & 99.35 & 99.35 & 0.9192388 \\
\hline X10C0700129 & SVOA & Benzo(ghi)perylene & 2 & 0 & $\mathrm{ug} / \mathrm{kg}$ & 98.7 & 100 & 99.35 & 99.35 & 0.9192388 \\
\hline X10C0700129 & SVOA & Benzo(k)fluoranthene & 2 & 0 & $\mathrm{ug} / \mathrm{kg}$ & 98.7 & 100 & 99.35 & 99.35 & 0.9192388 \\
\hline X10C0700129 & SVOA & Benzoic acid & 2 & 0 & $\mathrm{ug} / \mathrm{kg}$ & 1970 & 2000 & 1985 & 1985 & 21.213203 \\
\hline X10C0700129 & SVOA & Bis(2-chloroethoxy)methane & 2 & 0 & $\mathrm{ug} / \mathrm{kg}$ & 987 & 1000 & 993.5 & 993.5 & 9.1923882 \\
\hline X10C0700129 & SVOA & Bis(2-chloroethyl) ether & 2 & 0 & $\mathrm{ug} / \mathrm{kg}$ & 987 & 1000 & 993.5 & 993.5 & 9.1923882 \\
\hline X10C0700129 & SVOA & $\operatorname{Bis}(2$-ethylhexyl)phthalate & 2 & 0 & $\mathrm{ug} / \mathrm{kg}$ & 987 & 1000 & 993.5 & 993.5 & 9.1923882 \\
\hline X10C0700129 & SVOA & Butyl benzyl phthalate & 2 & 0 & $\mathrm{ug} / \mathrm{kg}$ & 987 & 1000 & 993.5 & 993.5 & 9.1923882 \\
\hline X10C0700129 & SVOA & Carbazole & 2 & 0 & $\mathrm{ug} / \mathrm{kg}$ & 98.7 & 100 & 99.35 & 99.35 & 0.9192388 \\
\hline X10C0700129 & SVOA & Chrysene & 2 & 0 & $\mathrm{ug} / \mathrm{kg}$ & 98.7 & 100 & 99.35 & 99.35 & 0.9192388 \\
\hline X10C0700129 & SVOA & Dibenz(a,h)anthracene & 2 & 0 & $\mathrm{ug} / \mathrm{kg}$ & 98.7 & 100 & 99.35 & 99.35 & 0.9192388 \\
\hline X10C0700129 & SVOA & Dibenzofuran & 2 & 0 & $\mathrm{ug} / \mathrm{kg}$ & 987 & 1000 & 993.5 & 993.5 & 9.1923882 \\
\hline X10C0700129 & SVOA & Diethyl phthalate & 2 & 0 & $\mathrm{ug} / \mathrm{kg}$ & 987 & 1000 & 993.5 & 993.5 & 9.1923882 \\
\hline X10C0700129 & SVOA & Dimethyl phthalate & 2 & 0 & $\mathrm{ug} / \mathrm{kg}$ & 987 & 1000 & 993.5 & 993.5 & 9.1923882 \\
\hline X10C0700129 & SVOA & Di-n-butyl phthalate & 2 & 0 & $\mathrm{ug} / \mathrm{kg}$ & 987 & 1000 & 993.5 & 993.5 & 9.1923882 \\
\hline X10C0700129 & SVOA & Di-n-octylphthalate & 2 & 0 & $\mathrm{ug} / \mathrm{kg}$ & 987 & 1000 & 993.5 & 993.5 & 9.1923882 \\
\hline X10C0700129 & SVOA & Diphenylamine & 2 & 0 & $\mathrm{ug} / \mathrm{kg}$ & 987 & 1000 & 993.5 & 993.5 & 9.1923882 \\
\hline X10C0700129 & SVOA & Fluoranthene & 2 & 0 & $\mathrm{ug} / \mathrm{kg}$ & 98.7 & 100 & 99.35 & 99.35 & 0.9192388 \\
\hline X10C0700129 & SVOA & Fluorene & 2 & 0 & $\mathrm{ug} / \mathrm{kg}$ & 98.7 & 100 & 99.35 & 99.35 & 0.9192388 \\
\hline X10C0700129 & SVOA & Hexachlorobenzene & 4 & 0 & $\mathrm{ug} / \mathrm{kg}$ & 0.05 & 1000 & 496.775 & 493.525 & 573.59318 \\
\hline X10C0700129 & SVOA & Hexachlorobutadiene & 4 & 0 & $\mathrm{ug} / \mathrm{kg}$ & 0.05 & 1000 & 496.775 & 493.525 & 573.59318 \\
\hline X10C0700129 & SVOA & Hexachlorocyclopentadiene & 2 & 0 & $\mathrm{ug} / \mathrm{kg}$ & 987 & 1000 & 993.5 & 993.5 & 9.1923882 \\
\hline X10C0700129 & SVOA & Hexachloroethane & 4 & 0 & $\mathrm{ug} / \mathrm{kg}$ & 0.05 & 1000 & 496.775 & 493.525 & 573.59318 \\
\hline X10C0700129 & SVOA & Indeno(1,2,3-cd)pyrene & 2 & 0 & $\mathrm{ug} / \mathrm{kg}$ & 98.7 & 100 & 99.35 & 99.35 & 0.9192388 \\
\hline
\end{tabular}




\begin{tabular}{|c|c|c|c|c|c|c|c|c|c|c|}
\hline X10C0700129 & SVOA & Naphthalene & 2 & 0 & $\mathrm{ug} / \mathrm{kg}$ & 98.7 & 100 & 99.35 & 99.35 & 0.9192388 \\
\hline X10C0700129 & SVOA & Nitrobenzene & 4 & 0 & $\mathrm{ug} / \mathrm{kg}$ & 0.05 & 1000 & 496.775 & 493.525 & 573.59318 \\
\hline X10C0700129 & SVOA & N-Nitroso-di-n-propylamine & 2 & 0 & $\mathrm{ug} / \mathrm{kg}$ & 987 & 1000 & 993.5 & 993.5 & 9.1923882 \\
\hline X10C0700129 & SVOA & Pentachlorophenol & 4 & 0 & $\mathrm{ug} / \mathrm{kg}$ & 0.05 & 1000 & 496.775 & 493.525 & 573.59318 \\
\hline X10C0700129 & SVOA & Phenanthrene & 2 & 0 & $\mathrm{ug} / \mathrm{kg}$ & 98.7 & 100 & 99.35 & 99.35 & 0.9192388 \\
\hline X10C0700129 & SVOA & Phenol & 2 & 0 & $\mathrm{ug} / \mathrm{kg}$ & 987 & 1000 & 993.5 & 993.5 & 9.1923882 \\
\hline $\mathrm{X} 10 \mathrm{C} 0700129$ & SVOA & Pyridine & 4 & 0 & $\mathrm{ug} / \mathrm{kg}$ & 0.05 & 1000 & 496.775 & 493.525 & 573.59318 \\
\hline X10C0700129 & VOA & (1,1-Dimethylethyl)benzene & 2 & 0 & $\mathrm{ug} / \mathrm{kg}$ & 96.2 & 98 & 97.1 & 97.1 & 1.2727922 \\
\hline X10C0700129 & VOA & (1-Methylpropyl)benzene & 2 & 0 & $\mathrm{ug} / \mathrm{kg}$ & 96.2 & 98 & 97.1 & 97.1 & 1.2727922 \\
\hline X10C0700129 & VOA & 1,1,1,2-Tetrachloroethane & 2 & 0 & $\mathrm{ug} / \mathrm{kg}$ & 96.2 & 98 & 97.1 & 97.1 & 1.2727922 \\
\hline X10C0700129 & VOA & 1,1,1-Trichloroethane & 2 & 0 & $\mathrm{ug} / \mathrm{kg}$ & 96.2 & 98 & 97.1 & 97.1 & 1.2727922 \\
\hline X10C0700129 & VOA & 1,1,2,2-Tetrachloroethane & 2 & 0 & $\mathrm{ug} / \mathrm{kg}$ & 96.2 & 98 & 97.1 & 97.1 & 1.2727922 \\
\hline X10C0700129 & VOA & 1,1,2-Trichloroethane & 2 & 0 & $\mathrm{ug} / \mathrm{kg}$ & 96.2 & 98 & 97.1 & 97.1 & 1.2727922 \\
\hline X10C0700129 & VOA & 1,1-Dichloroethane & 2 & 0 & $\mathrm{ug} / \mathrm{kg}$ & 96.2 & 98 & 97.1 & 97.1 & 1.2727922 \\
\hline X10C0700129 & VOA & 1,1-Dichloroethene & 4 & 0 & $\mathrm{ug} / \mathrm{kg}$ & 0.01 & 98 & 48.555 & 48.105 & 56.059754 \\
\hline X10C0700129 & VOA & 1,1-Dichloropropene & 2 & 0 & $\mathrm{ug} / \mathrm{kg}$ & 96.2 & 98 & 97.1 & 97.1 & 1.2727922 \\
\hline X10C0700129 & VOA & 1,2,3-Trichlorobenzene & 2 & 0 & $\mathrm{ug} / \mathrm{kg}$ & 96.2 & 98 & 97.1 & 97.1 & 1.2727922 \\
\hline X10C0700129 & VOA & 1,2,3-Trichloropropane & 2 & 0 & $\mathrm{ug} / \mathrm{kg}$ & 96.2 & 98 & 97.1 & 97.1 & 1.2727922 \\
\hline X10C0700129 & VOA & 1,2,4-Trimethylbenzene & 2 & 0 & $\mathrm{ug} / \mathrm{kg}$ & 96.2 & 98 & 97.1 & 97.1 & 1.2727922 \\
\hline X10C0700129 & VOA & 1,2-Dibromo-3-chloropropane & 2 & 0 & $\mathrm{ug} / \mathrm{kg}$ & 96.2 & 98 & 97.1 & 97.1 & 1.2727922 \\
\hline X10C0700129 & VOA & 1,2-Dibromoethane & 2 & 0 & $\mathrm{ug} / \mathrm{kg}$ & 96.2 & 98 & 97.1 & 97.1 & 1.2727922 \\
\hline X10C0700129 & VOA & 1,2-Dichloroethane & 4 & 0 & $\mathrm{ug} / \mathrm{kg}$ & 0.01 & 98 & 48.555 & 48.105 & 56.059754 \\
\hline X10C0700129 & VOA & 1,2-Dichloroethene & 2 & 0 & $\mathrm{ug} / \mathrm{kg}$ & 192 & 196 & 194 & 194 & 2.8284271 \\
\hline X10C0700129 & VOA & 1,2-Dichloropropane & 2 & 0 & $\mathrm{ug} / \mathrm{kg}$ & 96.2 & 98 & 97.1 & 97.1 & 1.2727922 \\
\hline X10C0700129 & VOA & 1,2-Dimethylbenzene & 2 & 0 & $\mathrm{ug} / \mathrm{kg}$ & 96.2 & 98 & 97.1 & 97.1 & 1.2727922 \\
\hline X10C0700129 & VOA & 1,3,5-Trimethylbenzene & 2 & 0 & $\mathrm{ug} / \mathrm{kg}$ & 96.2 & 98 & 97.1 & 97.1 & 1.2727922 \\
\hline X10C0700129 & VOA & 1,3-Dichloropropane & 2 & 0 & $\mathrm{ug} / \mathrm{kg}$ & 96.2 & 98 & 97.1 & 97.1 & 1.2727922 \\
\hline X10C0700129 & VOA & 1-Chloro-4-methylbenzene & 2 & 0 & $\mathrm{ug} / \mathrm{kg}$ & 96.2 & 98 & 97.1 & 97.1 & 1.2727922 \\
\hline X10C0700129 & VOA & 1-Methyl-4-(1-methylethyl)benzene & 2 & 0 & $\mathrm{ug} / \mathrm{kg}$ & 96.2 & 98 & 97.1 & 97.1 & 1.2727922 \\
\hline X10C0700129 & VOA & 2,2-Dichloropropane & 2 & 0 & $\mathrm{ug} / \mathrm{kg}$ & 96.2 & 98 & 97.1 & 97.1 & 1.2727922 \\
\hline X10C0700129 & VOA & 2-Butanone & 4 & 0 & $\mathrm{ug} / \mathrm{kg}$ & 0.05 & 490 & 242.775 & 240.525 & 280.29877 \\
\hline X10C0700129 & VOA & 2-Hexanone & 2 & 0 & $\mathrm{ug} / \mathrm{kg}$ & 481 & 490 & 485.5 & 485.5 & 6.363961 \\
\hline X10C0700129 & VOA & 4-Methyl-2-pentanone & 2 & 0 & $\mathrm{ug} / \mathrm{kg}$ & 481 & 490 & 485.5 & 485.5 & 6.363961 \\
\hline X10C0700129 & VOA & Acetone & 2 & 0 & $\mathrm{ug} / \mathrm{kg}$ & 481 & 490 & 485.5 & 485.5 & 6.363961 \\
\hline X10C0700129 & VOA & Acetonitrile & 2 & 0 & $\mathrm{ug} / \mathrm{kg}$ & 2400 & 2450 & 2425 & 2425 & 35.355339 \\
\hline X10C0700129 & VOA & Acrolein & 2 & 0 & $\mathrm{ug} / \mathrm{kg}$ & 481 & 490 & 485.5 & 485.5 & 6.363961 \\
\hline X10C0700129 & VOA & Acrylonitrile & 2 & 0 & $\mathrm{ug} / \mathrm{kg}$ & 481 & 490 & 485.5 & 485.5 & 6.363961 \\
\hline X10C0700129 & VOA & Benzene & 4 & 0 & $\mathrm{ug} / \mathrm{kg}$ & 0.01 & 98 & 48.555 & 48.105 & 56.059754 \\
\hline X10C0700129 & VOA & Bromobenzene & 2 & 0 & $\mathrm{ug} / \mathrm{kg}$ & 96.2 & 98 & 97.1 & 97.1 & 1.2727922 \\
\hline X10C0700129 & VOA & Bromochloromethane & 2 & 0 & $\mathrm{ug} / \mathrm{kg}$ & 96.2 & 98 & 97.1 & 97.1 & 1.2727922 \\
\hline X10C0700129 & VOA & Bromodichloromethane & 2 & 0 & $\mathrm{ug} / \mathrm{kg}$ & 96.2 & 98 & 97.1 & 97.1 & 1.2727922 \\
\hline X10C0700129 & VOA & Bromoform & 2 & 0 & $\mathrm{ug} / \mathrm{kg}$ & 96.2 & 98 & 97.1 & 97.1 & 1.2727922 \\
\hline $\mathrm{X} 10 \mathrm{C} 0700129$ & VOA & Bromomethane & 2 & 0 & $\mathrm{ug} / \mathrm{kg}$ & 96.2 & 98 & 97.1 & 97.1 & 1.2727922 \\
\hline X10C0700129 & VOA & Butylbenzene & 2 & 0 & $\mathrm{ug} / \mathrm{kg}$ & 96.2 & 98 & 97.1 & 97.1 & 1.2727922 \\
\hline X10C0700129 & VOA & Carbon disulfide & 2 & 0 & $\mathrm{ug} / \mathrm{kg}$ & 481 & 490 & 485.5 & 485.5 & 6.363961 \\
\hline X10C0700129 & VOA & Carbon tetrachloride & 4 & 0 & $\mathrm{ug} / \mathrm{kg}$ & 0.01 & 98 & 48.555 & 48.105 & 56.059754 \\
\hline X10C0700129 & VOA & Chlorobenzene & 4 & 0 & $\mathrm{ug} / \mathrm{kg}$ & 0.01 & 98 & 48.555 & 48.105 & 56.059754 \\
\hline X10C0700129 & VOA & Chloroethane & 2 & 0 & $\mathrm{ug} / \mathrm{kg}$ & 96.2 & 98 & 97.1 & 97.1 & 1.2727922 \\
\hline $\mathrm{X} 10 \mathrm{C} 0700129$ & VOA & Chloroform & 4 & 0 & $\mathrm{ug} / \mathrm{kg}$ & 0.01 & 98 & 48.555 & 48.105 & 56.059754 \\
\hline X10C0700129 & VOA & Chloromethane & 2 & 0 & $\mathrm{ug} / \mathrm{kg}$ & 96.2 & 98 & 97.1 & 97.1 & 1.2727922 \\
\hline X10C0700129 & VOA & cis-1,2-Dichloroethene & 2 & 0 & $\mathrm{ug} / \mathrm{kg}$ & 96.2 & 98 & 97.1 & 97.1 & 1.2727922 \\
\hline X10C0700129 & VOA & cis-1,3-Dichloropropene & 2 & 0 & $\mathrm{ug} / \mathrm{kg}$ & 96.2 & 98 & 97.1 & 97.1 & 1.2727922 \\
\hline X10C0700129 & VOA & Cumene & 2 & 0 & $\mathrm{ug} / \mathrm{kg}$ & 96.2 & 98 & 97.1 & 97.1 & 1.2727922 \\
\hline X10C0700129 & VOA & Dibromochloromethane & 2 & 0 & $\mathrm{ug} / \mathrm{kg}$ & 96.2 & 98 & 97.1 & 97.1 & 1.2727922 \\
\hline X10C0700129 & VOA & Dibromomethane & 2 & 0 & $\mathrm{ug} / \mathrm{kg}$ & 96.2 & 98 & 97.1 & 97.1 & 1.2727922 \\
\hline X10C0700129 & VOA & Dichlorodifluoromethane & 2 & 0 & $\mathrm{ug} / \mathrm{kg}$ & 96.2 & 98 & 97.1 & 97.1 & 1.2727922 \\
\hline X10C0700129 & VOA & Ethylbenzene & 2 & 0 & $\mathrm{ug} / \mathrm{kg}$ & 96.2 & 98 & 97.1 & 97.1 & 1.2727922 \\
\hline X10C0700129 & VOA & Hexane & 2 & 0 & $\mathrm{ug} / \mathrm{kg}$ & 481 & 490 & 485.5 & 485.5 & 6.363961 \\
\hline X10C0700129 & VOA & M + P Xylene & 2 & 0 & $\mathrm{ug} / \mathrm{kg}$ & 192 & 196 & 194 & 194 & 2.8284271 \\
\hline X10C0700129 & VOA & Methanol & 2 & 0 & $\mathrm{ug} / \mathrm{kg}$ & 4460 & 4950 & 4705 & 4705 & 346.48232 \\
\hline X10C0700129 & VOA & Methyl methacrylate & 2 & 0 & $\mathrm{ug} / \mathrm{kg}$ & 481 & 490 & 485.5 & 485.5 & 6.363961 \\
\hline X10C0700129 & VOA & Methylcyclohexane & 2 & 0 & $\mathrm{ug} / \mathrm{kg}$ & 96.2 & 98 & 97.1 & 97.1 & 1.2727922 \\
\hline X10C0700129 & VOA & Methylene chloride & 2 & 0 & $\mathrm{ug} / \mathrm{kg}$ & 481 & 490 & 485.5 & 485.5 & 6.363961 \\
\hline X10C0700129 & VOA & o-Chlorotoluene & 2 & 0 & $\mathrm{ug} / \mathrm{kg}$ & 96.2 & 98 & 97.1 & 97.1 & 1.2727922 \\
\hline X10C0700129 & VOA & Propylbenzene & 2 & 0 & $\mathrm{ug} / \mathrm{kg}$ & 96.2 & 98 & 97.1 & 97.1 & 1.2727922 \\
\hline X10C0700129 & VOA & Propylene glycol & 2 & 0 & $\mathrm{ug} / \mathrm{kg}$ & 44600 & 49500 & 47050 & 47050 & 3464.8232 \\
\hline X10C0700129 & VOA & Styrene & 2 & 0 & $\mathrm{ug} / \mathrm{kg}$ & 96.2 & 98 & 97.1 & 97.1 & 1.2727922 \\
\hline X10C0700129 & VOA & Tetrachloroethene & 4 & 0 & $\mathrm{ug} / \mathrm{kg}$ & 0.01 & 98 & 48.555 & 48.105 & 56.059754 \\
\hline X10C0700129 & VOA & Toluene & 2 & 0 & $\mathrm{ug} / \mathrm{kg}$ & 96.2 & 98 & 97.1 & 97.1 & 1.2727922 \\
\hline X10C0700129 & VOA & Total Xylene & 2 & 0 & $\mathrm{ug} / \mathrm{kg}$ & 288 & 294 & 291 & 291 & 4.2426407 \\
\hline
\end{tabular}




\begin{tabular}{|c|c|c|c|c|c|c|c|c|c|c|}
\hline X10C0700129 & VOA & trans-1,2-Dichloroethene & 2 & 0 & $\mathrm{ug} / \mathrm{kg}$ & 96.2 & 98 & 97.1 & 97.1 & 1.2727922 \\
\hline X10C0700129 & VOA & trans-1,3-Dichloropropene & 2 & 0 & $\mathrm{ug} / \mathrm{kg}$ & 96.2 & 98 & 97.1 & 97.1 & 1.2727922 \\
\hline X10C0700129 & VOA & Trichloroethene & 4 & 0 & $\mathrm{ug} / \mathrm{kg}$ & 0.01 & 98 & 48.555 & 48.105 & 56.059754 \\
\hline X10C0700129 & VOA & Trichlorofluoromethane & 2 & 0 & $\mathrm{ug} / \mathrm{kg}$ & 96.2 & 98 & 97.1 & 97.1 & 1.2727922 \\
\hline X10C0700129 & $\mathrm{VOA}$ & Vinyl chloride & 4 & 0 & $\mathrm{ug} / \mathrm{kg}$ & 0.01 & 98 & 48.555 & 48.105 & 56.059754 \\
\hline X10C1101242 & HERB & $2,4-\mathrm{D}$ & 2 & 0 & $\mathrm{ug} / \mathrm{kg}$ & 0.05 & 24.8 & 12.425 & 12.425 & 17.500893 \\
\hline X10C1101242 & HERB & Silvex & 2 & 0 & $\mathrm{ug} / \mathrm{kg}$ & 0.05 & 24.8 & 12.425 & 12.425 & 17.500893 \\
\hline X10C1101242 & METAL & Arsenic & 2 & 0 & $\mathrm{mg} / \mathrm{L}$ & 0.3 & 3 & 1.65 & 1.65 & 1.9091883 \\
\hline X10C1101242 & METAL & Barium & 2 & 0 & $\mathrm{mg} / \mathrm{L}$ & 0.05 & 0.5 & 0.275 & 0.275 & 0.3181981 \\
\hline X10C1101242 & METAL & Cadmium & 2 & 0 & $\mathrm{mg} / \mathrm{L}$ & 0.05 & 0.5 & 0.275 & 0.275 & 0.3181981 \\
\hline X10C1101242 & METAL & Chromium & 2 & 1 & $\mathrm{mg} / \mathrm{L}$ & 0.05 & 1.18 & 0.615 & 0.615 & 0.7990307 \\
\hline X10C1101242 & METAL & Lead & 2 & 0 & $\mathrm{mg} / \mathrm{L}$ & 0.1 & 1 & 0.55 & 0.55 & 0.6363961 \\
\hline X10C1101242 & METAL & Mercury & 2 & 0 & $\mathrm{mg} / \mathrm{L}$ & 0.002 & 0.4 & 0.201 & 0.201 & 0.2814285 \\
\hline X10C1101242 & METAL & Selenium & 2 & 0 & $\mathrm{mg} / \mathrm{L}$ & 0.3 & 3 & 1.65 & 1.65 & 1.9091883 \\
\hline X10C1101242 & METAL & Silver & 2 & 0 & $\mathrm{mg} / \mathrm{L}$ & 0.05 & 0.5 & 0.275 & 0.275 & 0.3181981 \\
\hline X10C1101242 & METAL & Total Uranium & 1 & 0 & $\mathrm{pCi} / \mathrm{g}$ & 0.0576 & 0.0576 & 0.0576 & & \\
\hline X10C1101242 & РPCB & 4,4'-DDD & 1 & 0 & $\mathrm{ug} / \mathrm{kg}$ & 3.68 & 3.68 & 3.68 & & \\
\hline X10C1101242 & РPCB & 4,4'-DDE & 1 & 0 & $\mathrm{ug} / \mathrm{kg}$ & 3.68 & 3.68 & 3.68 & & \\
\hline X10C1101242 & РPCB & 4,4'-DDT & 1 & 0 & $\mathrm{ug} / \mathrm{kg}$ & 3.68 & 3.68 & 3.68 & & \\
\hline X10C1101242 & РPCB & Aldrin & 1 & 0 & $\mathrm{ug} / \mathrm{kg}$ & 1.84 & 1.84 & 1.84 & & \\
\hline X10C1101242 & РPCB & alpha-BHC & 1 & 0 & $\mathrm{ug} / \mathrm{kg}$ & 1.84 & 1.84 & 1.84 & & \\
\hline X10C1101242 & РPCB & alpha-Chlordane & 1 & 0 & $\mathrm{ug} / \mathrm{kg}$ & 1.84 & 1.84 & 1.84 & & \\
\hline X10C1101242 & РPCB & beta-BHC & 1 & 0 & $\mathrm{ug} / \mathrm{kg}$ & 1.84 & 1.84 & 1.84 & & \\
\hline X10C1101242 & РPCB & Chlordane & 2 & 0 & $\mathrm{ug} / \mathrm{kg}$ & 0.0025 & 23 & 11.50125 & 11.50125 & 16.261688 \\
\hline X10C1101242 & РРСВ & delta-BHC & 1 & 0 & $\mathrm{ug} / \mathrm{kg}$ & 1.84 & 1.84 & 1.84 & & \\
\hline X10C1101242 & РPCB & Dieldrin & 1 & 0 & $\mathrm{ug} / \mathrm{kg}$ & 3.68 & 3.68 & 3.68 & & \\
\hline X10C1101242 & РPCB & Endosulfan I & 1 & 0 & $\mathrm{ug} / \mathrm{kg}$ & 1.84 & 1.84 & 1.84 & & \\
\hline X10C1101242 & РPCB & Endosulfan II & 1 & 0 & $\mathrm{ug} / \mathrm{kg}$ & 3.68 & 3.68 & 3.68 & & \\
\hline X10C1101242 & РPCB & Endosulfan sulfate & 1 & 0 & $\mathrm{ug} / \mathrm{kg}$ & 3.68 & 3.68 & 3.68 & & \\
\hline X10C1101242 & РPCB & Endrin & 2 & 0 & $\mathrm{ug} / \mathrm{kg}$ & 0.0004 & 3.68 & 1.8402 & 1.8402 & 2.6018701 \\
\hline X10C1101242 & РPCB & Endrin aldehyde & 1 & 0 & $\mathrm{ug} / \mathrm{kg}$ & 3.68 & 3.68 & 3.68 & & \\
\hline X10C1101242 & РPCB & Endrin ketone & 1 & 0 & $\mathrm{ug} / \mathrm{kg}$ & 3.68 & 3.68 & 3.68 & & \\
\hline X10C1101242 & РPCB & gamma-Chlordane & 1 & 0 & $\mathrm{ug} / \mathrm{kg}$ & 1.84 & 1.84 & 1.84 & & \\
\hline X10C1101242 & РPCB & Heptachlor & 2 & 0 & $\mathrm{ug} / \mathrm{kg}$ & 0.0002 & 1.84 & 0.9201 & 0.9201 & 1.3009351 \\
\hline X10C1101242 & РPCB & Heptachlor epoxide & 2 & 0 & $\mathrm{ug} / \mathrm{kg}$ & 0.0002 & 1.84 & 0.9201 & 0.9201 & 1.3009351 \\
\hline X10C1101242 & РPCB & Lindane & 2 & 0 & $\mathrm{ug} / \mathrm{kg}$ & 0.0002 & 1.84 & 0.9201 & 0.9201 & 1.3009351 \\
\hline X10C1101242 & PPCB & Methoxychlor & 2 & 0 & $\mathrm{ug} / \mathrm{kg}$ & 0.002 & 18.4 & 9.201 & 9.201 & 13.009351 \\
\hline X10C1101242 & РPCB & Toxaphene & 2 & 0 & $\mathrm{ug} / \mathrm{kg}$ & 0.005 & 46 & 23.0025 & 23.0025 & 32.523376 \\
\hline X10C1101242 & RADS & Americium-241 & 1 & 0 & $\mathrm{pCi} / \mathrm{g}$ & -0.00427 & -0.00427 & -0.00427 & & \\
\hline X10C1101242 & RADS & Carbon-14 & 1 & 0 & $\mathrm{pCi} / \mathrm{g}$ & 0.58 & 0.58 & 0.58 & & \\
\hline X10C1101242 & RADS & Cesium-137 & 1 & 0 & $\mathrm{pCi} / \mathrm{g}$ & 0.0217 & 0.0217 & 0.0217 & & \\
\hline X10C1101242 & RADS & Cobalt- 60 & 1 & 0 & $\mathrm{pCi} / \mathrm{g}$ & -0.0906 & -0.0906 & -0.0906 & & \\
\hline X10C1101242 & RADS & Curium-243/244 & 1 & 0 & $\mathrm{pCi} / \mathrm{g}$ & -0.0126 & -0.0126 & -0.0126 & & \\
\hline X10C1101242 & RADS & Neptunium-237 & 1 & 0 & $\mathrm{pCi} / \mathrm{g}$ & 0.108 & 0.108 & 0.108 & & \\
\hline X10C1101242 & RADS & Plutonium-238 & 1 & 0 & $\mathrm{pCi} / \mathrm{g}$ & -0.021 & -0.021 & -0.021 & & \\
\hline X10C1101242 & RADS & Plutonium-239/240 & 1 & 0 & $\mathrm{pCi} / \mathrm{g}$ & -0.0265 & -0.0265 & -0.0265 & & \\
\hline X10C1101242 & RADS & Radium-226 & 1 & 0 & $\mathrm{pCi} / \mathrm{g}$ & 0.239 & 0.239 & 0.239 & & \\
\hline X10C1101242 & RADS & Strontium-90 & 1 & 0 & $\mathrm{pCi} / \mathrm{g}$ & 1.07 & 1.07 & 1.07 & & \\
\hline X10C1101242 & RADS & Technetium-99 & 1 & 0 & $\mathrm{pCi} / \mathrm{g}$ & 0.347 & 0.347 & 0.347 & & \\
\hline X10C1101242 & RADS & Thorium-230 & 1 & 0 & $\mathrm{pCi} / \mathrm{g}$ & 49.7 & 49.7 & 49.7 & & \\
\hline X10C1101242 & RADS & Thorium-232 & 1 & 0 & $\mathrm{pCi} / \mathrm{g}$ & 3.12 & 3.12 & 3.12 & & \\
\hline X10C1101242 & RADS & Tritium & 1 & 0 & $\mathrm{pCi} / \mathrm{g}$ & -36.4 & -36.4 & -36.4 & & \\
\hline X10C1101242 & RADS & Uranium-233/234 & 1 & 0 & $\mathrm{pCi} / \mathrm{g}$ & 0.0442 & 0.0442 & 0.0442 & & \\
\hline X10C1101242 & RADS & Uranium-235/236 & 1 & 0 & $\mathrm{pCi} / \mathrm{g}$ & 0 & 0 & 0 & & \\
\hline X10C1101242 & RADS & Uranium-238 & 1 & 0 & $\mathrm{pCi} / \mathrm{g}$ & 0.0133 & 0.0133 & 0.0133 & & \\
\hline X10C1101242 & SVOA & 1,2,4-Trichlorobenzene & 1 & 0 & $\mathrm{ug} / \mathrm{kg}$ & 490 & 490 & 490 & & \\
\hline X10C1101242 & SVOA & 1,2-Dichlorobenzene & 1 & 0 & $\mathrm{ug} / \mathrm{kg}$ & 490 & 490 & 490 & & \\
\hline X10C1101242 & SVOA & 1,3-Dichlorobenzene & 1 & 0 & $\mathrm{ug} / \mathrm{kg}$ & 490 & 490 & 490 & & \\
\hline X10C1101242 & SVOA & 1,4-Dichlorobenzene & 1 & 0 & $\mathrm{ug} / \mathrm{kg}$ & 490 & 490 & 490 & & \\
\hline X10C1101242 & SVOA & 2,2'-Dichlorodiisopropyl ether & 1 & 0 & $\mathrm{ug} / \mathrm{kg}$ & 997 & 997 & 997 & & \\
\hline X10C1101242 & SVOA & 2,3,4,6-Tetrachlorophenol & 1 & 0 & $\mathrm{ug} / \mathrm{kg}$ & 997 & 997 & 997 & & \\
\hline X10C1101242 & SVOA & 2,4,5-Trichlorophenol & 2 & 0 & $\mathrm{ug} / \mathrm{kg}$ & 0.05 & 997 & 498.525 & 498.525 & 704.95011 \\
\hline X10C1101242 & SVOA & 2,4,6-Trichlorophenol & 2 & 0 & $\mathrm{ug} / \mathrm{kg}$ & 0.05 & 997 & 498.525 & 498.525 & 704.95011 \\
\hline X10C1101242 & SVOA & 2,4-Dichlorophenol & 1 & 0 & $\mathrm{ug} / \mathrm{kg}$ & 997 & 997 & 997 & & \\
\hline X10C1101242 & SVOA & 2,4-Dimethylphenol & 1 & 0 & $\mathrm{ug} / \mathrm{kg}$ & 997 & 997 & 997 & & \\
\hline X10C1101242 & SVOA & 2,4-Dinitrophenol & 1 & 0 & $\mathrm{ug} / \mathrm{kg}$ & 1990 & 1990 & 1990 & & \\
\hline X10C1101242 & SVOA & 2,4-Dinitrotoluene & 2 & 0 & $\mathrm{ug} / \mathrm{kg}$ & 0.05 & 997 & 498.525 & 498.525 & 704.95011 \\
\hline X10C1101242 & SVOA & 2,6-Dinitrotoluene & 1 & 0 & $\mathrm{ug} / \mathrm{kg}$ & 997 & 997 & 997 & & \\
\hline X10C1101242 & SVOA & 2-Chloronaphthalene & 1 & 0 & $\mathrm{ug} / \mathrm{kg}$ & 99.7 & 99.7 & 99.7 & & \\
\hline X10C1101242 & SVOA & 2-Chlorophenol & 1 & 0 & $\mathrm{ug} / \mathrm{kg}$ & 997 & 997 & 997 & & \\
\hline
\end{tabular}




\begin{tabular}{|c|c|c|c|c|c|c|c|c|c|c|}
\hline X10C1101242 & SVOA & 2-Methyl-4,6-dinitrophenol & 1 & 0 & $\mathrm{ug} / \mathrm{kg}$ & 997 & 997 & 997 & & \\
\hline X10C1101242 & SVOA & 2-Methylnaphthalene & 1 & 0 & $\mathrm{ug} / \mathrm{kg}$ & 99.7 & 99.7 & 99.7 & & \\
\hline X10C1101242 & SVOA & 2-Methylphenol & 2 & 0 & $\mathrm{ug} / \mathrm{kg}$ & 0.05 & 997 & 498.525 & 498.525 & 704.95011 \\
\hline X10C1101242 & SVOA & 2-Nitrobenzenamine & 1 & 0 & $\mathrm{ug} / \mathrm{kg}$ & 997 & 997 & 997 & & \\
\hline X10C1101242 & SVOA & 2-Nitrophenol & 1 & 0 & $\mathrm{ug} / \mathrm{kg}$ & 997 & 997 & 997 & & \\
\hline X10C1101242 & SVOA & 3,3'-Dichlorobenzidine & 1 & 0 & $\mathrm{ug} / \mathrm{kg}$ & 997 & 997 & 997 & & \\
\hline X10C1101242 & SVOA & 3-Nitrobenzenamine & 1 & 0 & $\mathrm{ug} / \mathrm{kg}$ & 997 & 997 & 997 & & \\
\hline X10C1101242 & SVOA & 4-Bromophenyl phenyl ether & 1 & 0 & $\mathrm{ug} / \mathrm{kg}$ & 997 & 997 & 997 & & \\
\hline X10C1101242 & SVOA & 4-Chloro-3-methylphenol & 1 & 0 & $\mathrm{ug} / \mathrm{kg}$ & 997 & 997 & 997 & & \\
\hline X10C1101242 & SVOA & 4-Chlorobenzenamine & 1 & 0 & $\mathrm{ug} / \mathrm{kg}$ & 997 & 997 & 997 & & \\
\hline X10C1101242 & SVOA & 4-Chlorophenyl phenyl ether & 1 & 0 & $\mathrm{ug} / \mathrm{kg}$ & 997 & 997 & 997 & & \\
\hline X10C1101242 & SVOA & 4-Nitrobenzenamine & 1 & 0 & $\mathrm{ug} / \mathrm{kg}$ & 997 & 997 & 997 & & \\
\hline X10C1101242 & SVOA & 4-Nitrophenol & 1 & 0 & $\mathrm{ug} / \mathrm{kg}$ & 997 & 997 & 997 & & \\
\hline X10C1101242 & SVOA & Acenaphthene & 1 & 0 & $\mathrm{ug} / \mathrm{kg}$ & 99.7 & 99.7 & 99.7 & & \\
\hline X10C1101242 & SVOA & Acenaphthylene & 1 & 0 & $\mathrm{ug} / \mathrm{kg}$ & 99.7 & 99.7 & 99.7 & & \\
\hline X10C1101242 & SVOA & Acetophenone & 1 & 0 & $\mathrm{ug} / \mathrm{kg}$ & 997 & 997 & 997 & & \\
\hline X10C1101242 & SVOA & Anthracene & 1 & 0 & $\mathrm{ug} / \mathrm{kg}$ & 99.7 & 99.7 & 99.7 & & \\
\hline X10C1101242 & SVOA & Benz(a)anthracene & 1 & 0 & $\mathrm{ug} / \mathrm{kg}$ & 99.7 & 99.7 & 99.7 & & \\
\hline X10C1101242 & SVOA & Benzenemethanol & 1 & 0 & $\mathrm{ug} / \mathrm{kg}$ & 997 & 997 & 997 & & \\
\hline X10C1101242 & SVOA & Benzidine & 1 & 0 & $\mathrm{ug} / \mathrm{kg}$ & 997 & 997 & 997 & & \\
\hline X10C1101242 & SVOA & Benzo(a)pyrene & 1 & 0 & $\mathrm{ug} / \mathrm{kg}$ & 99.7 & 99.7 & 99.7 & & \\
\hline X10C1101242 & SVOA & Benzo(b)fluoranthene & 1 & 0 & $\mathrm{ug} / \mathrm{kg}$ & 99.7 & 99.7 & 99.7 & & \\
\hline X10C1101242 & SVOA & Benzo(ghi)perylene & 1 & 0 & $\mathrm{ug} / \mathrm{kg}$ & 99.7 & 99.7 & 99.7 & & \\
\hline X10C1101242 & SVOA & Benzo(k)fluoranthene & 1 & 0 & $\mathrm{ug} / \mathrm{kg}$ & 99.7 & 99.7 & 99.7 & & \\
\hline X10C1101242 & SVOA & Benzoic acid & 1 & 0 & $\mathrm{ug} / \mathrm{kg}$ & 1990 & 1990 & 1990 & & \\
\hline X10C1101242 & SVOA & Bis(2-chloroethoxy)methane & 1 & 0 & $\mathrm{ug} / \mathrm{kg}$ & 997 & 997 & 997 & & \\
\hline X10C1101242 & SVOA & Bis(2-chloroethyl) ether & 1 & 0 & $\mathrm{ug} / \mathrm{kg}$ & 997 & 997 & 997 & & \\
\hline X10C1101242 & SVOA & Bis(2-ethylhexyl)phthalate & 1 & 0 & $\mathrm{ug} / \mathrm{kg}$ & 997 & 997 & 997 & & \\
\hline X10C1101242 & SVOA & Butyl benzyl phthalate & 1 & 0 & $\mathrm{ug} / \mathrm{kg}$ & 997 & 997 & 997 & & \\
\hline X10C1101242 & SVOA & Carbazole & 1 & 0 & $\mathrm{ug} / \mathrm{kg}$ & 99.7 & 99.7 & 99.7 & & \\
\hline X10C1101242 & SVOA & Chrysene & 1 & 0 & $\mathrm{ug} / \mathrm{kg}$ & 99.7 & 99.7 & 99.7 & & \\
\hline X10C1101242 & SVOA & Dibenz(a,h)anthracene & 1 & 0 & $\mathrm{ug} / \mathrm{kg}$ & 99.7 & 99.7 & 99.7 & & \\
\hline X10C1101242 & SVOA & Dibenzofuran & 1 & 0 & $\mathrm{ug} / \mathrm{kg}$ & 997 & 997 & 997 & & \\
\hline X10C1101242 & SVOA & Diethyl phthalate & 1 & 0 & $\mathrm{ug} / \mathrm{kg}$ & 997 & 997 & 997 & & \\
\hline X10C1101242 & SVOA & Dimethyl phthalate & 1 & 0 & $\mathrm{ug} / \mathrm{kg}$ & 997 & 997 & 997 & & \\
\hline X10C1101242 & SVOA & Di-n-butyl phthalate & 1 & 0 & $\mathrm{ug} / \mathrm{kg}$ & 997 & 997 & 997 & & \\
\hline X10C1101242 & SVOA & Di-n-octylphthalate & 1 & 0 & $\mathrm{ug} / \mathrm{kg}$ & 997 & 997 & 997 & & \\
\hline X10C1101242 & SVOA & Diphenylamine & 1 & 0 & $\mathrm{ug} / \mathrm{kg}$ & 997 & 997 & 997 & & \\
\hline X10C1101242 & SVOA & Fluoranthene & 1 & 0 & $\mathrm{ug} / \mathrm{kg}$ & 99.7 & 99.7 & 99.7 & & \\
\hline X10C1101242 & SVOA & Fluorene & 1 & 0 & $\mathrm{ug} / \mathrm{kg}$ & 99.7 & 99.7 & 99.7 & & \\
\hline X10C1101242 & SVOA & Hexachlorobenzene & 2 & 0 & $\mathrm{ug} / \mathrm{kg}$ & 0.05 & 997 & 498.525 & 498.525 & 704.95011 \\
\hline X10C1101242 & SVOA & Hexachlorobutadiene & 2 & 0 & $\mathrm{ug} / \mathrm{kg}$ & 0.05 & 997 & 498.525 & 498.525 & 704.95011 \\
\hline X10C1101242 & SVOA & Hexachlorocyclopentadiene & 1 & 0 & $\mathrm{ug} / \mathrm{kg}$ & 997 & 997 & 997 & & \\
\hline X10C1101242 & SVOA & Hexachloroethane & 2 & 0 & $\mathrm{ug} / \mathrm{kg}$ & 0.05 & 997 & 498.525 & 498.525 & 704.95011 \\
\hline X10C1101242 & SVOA & Indeno(1,2,3-cd)pyrene & 1 & 0 & $\mathrm{ug} / \mathrm{kg}$ & 99.7 & 99.7 & 99.7 & & \\
\hline X10C1101242 & SVOA & Isophorone & 1 & 0 & $\mathrm{ug} / \mathrm{kg}$ & 997 & 997 & 997 & & \\
\hline X10C1101242 & SVOA & $\mathrm{m}+\mathrm{p}$ Methylphenol & 2 & 0 & $\mathrm{ug} / \mathrm{kg}$ & 0.05 & 997 & 498.525 & 498.525 & 704.95011 \\
\hline X10C1101242 & SVOA & Naphthalene & 1 & 0 & $\mathrm{ug} / \mathrm{kg}$ & 99.7 & 99.7 & 99.7 & & \\
\hline X10C1101242 & SVOA & Nitrobenzene & 2 & 0 & $\mathrm{ug} / \mathrm{kg}$ & 0.05 & 997 & 498.525 & 498.525 & 704.95011 \\
\hline X10C1101242 & SVOA & N-Nitroso-di-n-propylamine & 1 & 0 & $\mathrm{ug} / \mathrm{kg}$ & 997 & 997 & 997 & & \\
\hline X10C1101242 & SVOA & Pentachlorophenol & 2 & 0 & $\mathrm{ug} / \mathrm{kg}$ & 0.05 & 997 & 498.525 & 498.525 & 704.95011 \\
\hline X10C1101242 & SVOA & Phenanthrene & 1 & 0 & $\mathrm{ug} / \mathrm{kg}$ & 99.7 & 99.7 & 99.7 & & \\
\hline X10C1101242 & SVOA & Phenol & 1 & 0 & $\mathrm{ug} / \mathrm{kg}$ & 997 & 997 & 997 & & \\
\hline X10C1101242 & SVOA & Pyridine & 2 & 0 & $\mathrm{ug} / \mathrm{kg}$ & 0.05 & 997 & 498.525 & 498.525 & 704.95011 \\
\hline X10C1101242 & VOA & (1,1-Dimethylethyl)benzene & 1 & 0 & $\mathrm{ug} / \mathrm{kg}$ & 490 & 490 & 490 & & \\
\hline X10C1101242 & VOA & (1-Methylpropyl)benzene & 1 & 0 & $\mathrm{ug} / \mathrm{kg}$ & 490 & 490 & 490 & & \\
\hline X10C1101242 & VOA & 1,1,1,2-Tetrachloroethane & 1 & 0 & $\mathrm{ug} / \mathrm{kg}$ & 490 & 490 & 490 & & \\
\hline X10C1101242 & VOA & 1,1,1-Trichloroethane & 1 & 0 & $\mathrm{ug} / \mathrm{kg}$ & 490 & 490 & 490 & & \\
\hline X10C1101242 & VOA & 1,1,2,2-Tetrachloroethane & 1 & 0 & $\mathrm{ug} / \mathrm{kg}$ & 490 & 490 & 490 & & \\
\hline X10C1101242 & VOA & 1,1,2-Trichloroethane & 1 & 0 & $\mathrm{ug} / \mathrm{kg}$ & 490 & 490 & 490 & & \\
\hline X10C1101242 & VOA & 1,1-Dichloroethane & 1 & 0 & $\mathrm{ug} / \mathrm{kg}$ & 490 & 490 & 490 & & \\
\hline X10C1101242 & VOA & 1,1-Dichloroethene & 1 & 0 & $\mathrm{ug} / \mathrm{kg}$ & 490 & 490 & 490 & & \\
\hline X10C1101242 & VOA & 1,1-Dichloropropene & 1 & 0 & $\mathrm{ug} / \mathrm{kg}$ & 490 & 490 & 490 & & \\
\hline X10C1101242 & VOA & 1,2,3-Trichlorobenzene & 1 & 0 & $\mathrm{ug} / \mathrm{kg}$ & 490 & 490 & 490 & & \\
\hline X10C1101242 & VOA & 1,2,3-Trichloropropane & 1 & 0 & $\mathrm{ug} / \mathrm{kg}$ & 490 & 490 & 490 & & \\
\hline X10C1101242 & VOA & 1,2,4-Trimethylbenzene & 1 & 0 & $\mathrm{ug} / \mathrm{kg}$ & 490 & 490 & 490 & & \\
\hline X10C1101242 & VOA & 1,2-Dibromo-3-chloropropane & 1 & 0 & $\mathrm{ug} / \mathrm{kg}$ & 490 & 490 & 490 & & \\
\hline X10C1101242 & VOA & 1,2-Dibromoethane & 1 & 0 & $\mathrm{ug} / \mathrm{kg}$ & 490 & 490 & 490 & & \\
\hline X10C1101242 & VOA & 1,2-Dichloroethane & 1 & 0 & $\mathrm{ug} / \mathrm{kg}$ & 490 & 490 & 490 & & \\
\hline X10C1101242 & VOA & 1,2-Dichloroethene & 1 & 0 & $\mathrm{ug} / \mathrm{kg}$ & 980 & 980 & 980 & & \\
\hline
\end{tabular}




\begin{tabular}{|c|c|c|c|c|c|c|c|c|c|c|}
\hline X10C1101242 & VOA & 1,2-Dichloropropane & 1 & 0 & $\mathrm{ug} / \mathrm{kg}$ & 490 & 490 & 490 & & \\
\hline X10C1101242 & $\mathrm{VOA}$ & 1,2-Dimethylbenzene & 1 & 0 & $\mathrm{ug} / \mathrm{kg}$ & 490 & 490 & 490 & & \\
\hline X10C1101242 & VOA & 1,3,5-Trimethylbenzene & 1 & 0 & $\mathrm{ug} / \mathrm{kg}$ & 490 & 490 & 490 & & \\
\hline X10C1101242 & VOA & 1,3-Dichloropropane & 1 & 0 & $\mathrm{ug} / \mathrm{kg}$ & 490 & 490 & 490 & & \\
\hline X10C1101242 & VOA & 1-Chloro-4-methylbenzene & 1 & 0 & $\mathrm{ug} / \mathrm{kg}$ & 490 & 490 & 490 & & \\
\hline X10C1101242 & VOA & 1-Methyl-4-(1-methylethyl)benzene & 1 & 0 & $\mathrm{ug} / \mathrm{kg}$ & 490 & 490 & 490 & & \\
\hline X10C1101242 & VOA & 2,2-Dichloropropane & 1 & 0 & $\mathrm{ug} / \mathrm{kg}$ & 490 & 490 & 490 & & \\
\hline X10C1101242 & VOA & 2-Butanone & 1 & 0 & $\mathrm{ug} / \mathrm{kg}$ & 2450 & 2450 & 2450 & & \\
\hline X10C1101242 & VOA & 2-Hexanone & 1 & 0 & $\mathrm{ug} / \mathrm{kg}$ & 2450 & 2450 & 2450 & & \\
\hline X10C1101242 & VOA & 4-Methyl-2-pentanone & 1 & 0 & $\mathrm{ug} / \mathrm{kg}$ & 2450 & 2450 & 2450 & & \\
\hline X10C1101242 & VOA & Acetone & 1 & 0 & $\mathrm{ug} / \mathrm{kg}$ & 2450 & 2450 & 2450 & & \\
\hline X10C1101242 & VOA & Acetonitrile & 1 & 0 & $\mathrm{ug} / \mathrm{kg}$ & 12300 & 12300 & 12300 & & \\
\hline X10C1101242 & VOA & Acrolein & 1 & 0 & $\mathrm{ug} / \mathrm{kg}$ & 2450 & 2450 & 2450 & & \\
\hline X10C1101242 & VOA & Acrylonitrile & 1 & 0 & $\mathrm{ug} / \mathrm{kg}$ & 2450 & 2450 & 2450 & & \\
\hline X10C1101242 & VOA & Benzene & 1 & 0 & $\mathrm{ug} / \mathrm{kg}$ & 490 & 490 & 490 & & \\
\hline X10C1101242 & VOA & Bromobenzene & 1 & 0 & $\mathrm{ug} / \mathrm{kg}$ & 490 & 490 & 490 & & \\
\hline X10C1101242 & VOA & Bromochloromethane & 1 & 0 & $\mathrm{ug} / \mathrm{kg}$ & 490 & 490 & 490 & & \\
\hline X10C1101242 & VOA & Bromodichloromethane & 1 & 0 & $\mathrm{ug} / \mathrm{kg}$ & 490 & 490 & 490 & & \\
\hline X10C1101242 & VOA & Bromoform & 1 & 0 & $\mathrm{ug} / \mathrm{kg}$ & 490 & 490 & 490 & & \\
\hline X10C1101242 & $\mathrm{VOA}$ & Bromomethane & 1 & 0 & $\mathrm{ug} / \mathrm{kg}$ & 490 & 490 & 490 & & \\
\hline X10C1101242 & VOA & Butylbenzene & 1 & 0 & $\mathrm{ug} / \mathrm{kg}$ & 490 & 490 & 490 & & \\
\hline X10C1101242 & $\mathrm{VOA}$ & Carbon disulfide & 1 & 0 & $\mathrm{ug} / \mathrm{kg}$ & 2450 & 2450 & 2450 & & \\
\hline X10C1101242 & VOA & Carbon tetrachloride & 1 & 0 & $\mathrm{ug} / \mathrm{kg}$ & 490 & 490 & 490 & & \\
\hline X10C1101242 & VOA & Chlorobenzene & 1 & 0 & $\mathrm{ug} / \mathrm{kg}$ & 490 & 490 & 490 & & \\
\hline X10C1101242 & VOA & Chloroethane & 1 & 0 & $\mathrm{ug} / \mathrm{kg}$ & 490 & 490 & 490 & & \\
\hline X10C1101242 & VOA & Chloroform & 1 & 0 & $\mathrm{ug} / \mathrm{kg}$ & 490 & 490 & 490 & & \\
\hline X10C1101242 & VOA & Chloromethane & 1 & 0 & $\mathrm{ug} / \mathrm{kg}$ & 490 & 490 & 490 & & \\
\hline X10C1101242 & VOA & cis-1,2-Dichloroethene & 1 & 0 & $\mathrm{ug} / \mathrm{kg}$ & 490 & 490 & 490 & & \\
\hline X10C1101242 & VOA & cis-1,3-Dichloropropene & 1 & 0 & $\mathrm{ug} / \mathrm{kg}$ & 490 & 490 & 490 & & \\
\hline X10C1101242 & VOA & Cumene & 1 & 0 & $\mathrm{ug} / \mathrm{kg}$ & 490 & 490 & 490 & & \\
\hline X10C1101242 & VOA & Dibromochloromethane & 1 & 0 & $\mathrm{ug} / \mathrm{kg}$ & 490 & 490 & 490 & & \\
\hline X10C1101242 & VOA & Dibromomethane & 1 & 0 & $\mathrm{ug} / \mathrm{kg}$ & 490 & 490 & 490 & & \\
\hline X10C1101242 & VOA & Dichlorodifluoromethane & 1 & 0 & $\mathrm{ug} / \mathrm{kg}$ & 490 & 490 & 490 & & \\
\hline X10C1101242 & VOA & Ethylbenzene & 1 & 0 & $\mathrm{ug} / \mathrm{kg}$ & 490 & 490 & 490 & & \\
\hline X10C1101242 & VOA & Hexane & 1 & 0 & $\mathrm{ug} / \mathrm{kg}$ & 2450 & 2450 & 2450 & & \\
\hline X10C1101242 & VOA & M + P Xylene & 1 & 0 & $\mathrm{ug} / \mathrm{kg}$ & 980 & 980 & 980 & & \\
\hline X10C1101242 & VOA & Methyl methacrylate & 1 & 0 & $\mathrm{ug} / \mathrm{kg}$ & 2450 & 2450 & 2450 & & \\
\hline X10C1101242 & VOA & Methylcyclohexane & 1 & 0 & $\mathrm{ug} / \mathrm{kg}$ & 490 & 490 & 490 & & \\
\hline X10C1101242 & VOA & Methylene chloride & 1 & 0 & $\mathrm{ug} / \mathrm{kg}$ & 2450 & 2450 & 2450 & & \\
\hline X10C1101242 & VOA & o-Chlorotoluene & 1 & 0 & $\mathrm{ug} / \mathrm{kg}$ & 490 & 490 & 490 & & \\
\hline X10C1101242 & VOA & Propylbenzene & 1 & 0 & $\mathrm{ug} / \mathrm{kg}$ & 490 & 490 & 490 & & \\
\hline X10C1101242 & VOA & Styrene & 1 & 0 & $\mathrm{ug} / \mathrm{kg}$ & 490 & 490 & 490 & & \\
\hline X10C1101242 & VOA & Tetrachloroethene & 1 & 0 & $\mathrm{ug} / \mathrm{kg}$ & 490 & 490 & 490 & & \\
\hline X10C1101242 & VOA & Toluene & 1 & 0 & $\mathrm{ug} / \mathrm{kg}$ & 490 & 490 & 490 & & \\
\hline X10C1101242 & VOA & Total Xylene & 1 & 0 & $\mathrm{ug} / \mathrm{kg}$ & 1470 & 1470 & 1470 & & \\
\hline X10C1101242 & VOA & trans-1,2-Dichloroethene & 1 & 0 & $\mathrm{ug} / \mathrm{kg}$ & 490 & 490 & 490 & & \\
\hline X10C1101242 & $\mathrm{VOA}$ & trans-1,3-Dichloropropene & 1 & 0 & $\mathrm{ug} / \mathrm{kg}$ & 490 & 490 & 490 & & \\
\hline X10C1101242 & VOA & Trichloroethene & 1 & 0 & $\mathrm{ug} / \mathrm{kg}$ & 490 & 490 & 490 & & \\
\hline X10C1101242 & VOA & Trichlorofluoromethane & 1 & 0 & $\mathrm{ug} / \mathrm{kg}$ & 490 & 490 & 490 & & \\
\hline X10C1101242 & VOA & Vinyl chloride & 1 & 0 & $\mathrm{ug} / \mathrm{kg}$ & 490 & 490 & 490 & & \\
\hline X10C9601883 & HERB & $2,4-\mathrm{D}$ & 2 & 0 & $\mathrm{ug} / \mathrm{kg}$ & 0.05 & 23.4 & 11.725 & 11.725 & 16.510943 \\
\hline $\mathrm{X} 10 \mathrm{C} 9601883$ & HERB & Silvex & 2 & 0 & $\mathrm{ug} / \mathrm{kg}$ & 0.05 & 23.4 & 11.725 & 11.725 & 16.510943 \\
\hline X10C9601883 & METAL & Arsenic & 2 & 1 & $\mathrm{mg} / \mathrm{L}$ & 0.3 & 4.64 & 2.47 & 2.47 & 3.0688434 \\
\hline X10C9601883 & METAL & Barium & 2 & 1 & $\mathrm{mg} / \mathrm{L}$ & 0.0669 & 0.449 & 0.25795 & 0.25795 & 0.2701855 \\
\hline X10C9601883 & METAL & Cadmium & 2 & 0 & $\mathrm{mg} / \mathrm{L}$ & 0.05 & 44.9 & 22.475 & 22.475 & 31.713739 \\
\hline X10C9601883 & METAL & Chromium & 2 & 0 & $\mathrm{mg} / \mathrm{L}$ & 0.05 & 0.449 & 0.2495 & 0.2495 & 0.2821356 \\
\hline X10C9601883 & METAL & Lead & 2 & 1 & $\mathrm{mg} / \mathrm{L}$ & 0.767 & 898 & 449.3835 & 449.3835 & 634.43954 \\
\hline X10C9601883 & METAL & Mercury & 2 & 0 & $\mathrm{mg} / \mathrm{L}$ & 0.002 & 0.4 & 0.201 & 0.201 & 0.2814285 \\
\hline X10C9601883 & METAL & Selenium & 2 & 0 & $\mathrm{mg} / \mathrm{L}$ & 0.3 & 2.69 & 1.495 & 1.495 & 1.6899852 \\
\hline X10C9601883 & METAL & Silver & 2 & 1 & $\mathrm{mg} / \mathrm{L}$ & 0.05 & 32.2 & 16.125 & 16.125 & 22.733483 \\
\hline X10C9601883 & METAL & Total Uranium & 1 & 0 & $\mathrm{pCi} / \mathrm{g}$ & 0.0964 & 0.0964 & 0.0964 & & \\
\hline X10C9601883 & PPCB & 4,4'-DDD & 1 & 0 & $\mathrm{ug} / \mathrm{kg}$ & 2.76 & 2.76 & 2.76 & & \\
\hline X10C9601883 & PPCB & 4,4'-DDE & 1 & 0 & $\mathrm{ug} / \mathrm{kg}$ & 2.76 & 2.76 & 2.76 & & \\
\hline X10C9601883 & РPCB & 4,4'-DDT & 1 & 0 & $\mathrm{ug} / \mathrm{kg}$ & 2.76 & 2.76 & 2.76 & & \\
\hline X10C9601883 & PPCB & Aldrin & 1 & 0 & $\mathrm{ug} / \mathrm{kg}$ & 1.38 & 1.38 & 1.38 & & \\
\hline X10C9601883 & РPCB & alpha-BHC & 1 & 0 & $\mathrm{ug} / \mathrm{kg}$ & 1.38 & 1.38 & 1.38 & & \\
\hline X10C9601883 & РPCB & alpha-Chlordane & 1 & 0 & $\mathrm{ug} / \mathrm{kg}$ & 1.38 & 1.38 & 1.38 & & \\
\hline X10C9601883 & РPCB & beta-BHC & 1 & 0 & $\mathrm{ug} / \mathrm{kg}$ & 1.38 & 1.38 & 1.38 & & \\
\hline X10C9601883 & РPCB & Chlordane & 2 & 0 & $\mathrm{ug} / \mathrm{kg}$ & 0.0025 & 17.3 & 8.65125 & 8.65125 & 12.23118 \\
\hline X10C9601883 & PPCB & delta-BHC & 1 & 0 & $\mathrm{ug} / \mathrm{kg}$ & 1.38 & 1.38 & 1.38 & & \\
\hline
\end{tabular}




\begin{tabular}{|c|c|c|c|c|c|c|c|c|c|c|}
\hline X10C9601883 & PPCB & Dieldrin & 1 & 0 & $\mathrm{ug} / \mathrm{kg}$ & 2.76 & 2.76 & 2.76 & & \\
\hline X10C9601883 & PPCB & Endosulfan I & 1 & 0 & $\mathrm{ug} / \mathrm{kg}$ & 1.38 & 1.38 & 1.38 & & \\
\hline X10C9601883 & РPCB & Endosulfan II & 1 & 0 & $\mathrm{ug} / \mathrm{kg}$ & 2.76 & 2.76 & 2.76 & & \\
\hline X10C9601883 & PPCB & Endosulfan sulfate & 1 & 0 & $\mathrm{ug} / \mathrm{kg}$ & 2.76 & 2.76 & 2.76 & & \\
\hline X10C9601883 & РPCB & Endrin & 2 & 0 & $\mathrm{ug} / \mathrm{kg}$ & 0.0004 & 2.76 & 1.3802 & 1.3802 & 1.9513319 \\
\hline X10C9601883 & РPCB & Endrin aldehyde & 1 & 0 & $\mathrm{ug} / \mathrm{kg}$ & 2.76 & 2.76 & 2.76 & & \\
\hline X10C9601883 & PPCB & Endrin ketone & 1 & 0 & $\mathrm{ug} / \mathrm{kg}$ & 2.76 & 2.76 & 2.76 & & \\
\hline X10C9601883 & PPCB & gamma-Chlordane & 1 & 0 & $\mathrm{ug} / \mathrm{kg}$ & 1.38 & 1.38 & 1.38 & & \\
\hline X10C9601883 & PPCB & Heptachlor & 2 & 0 & $\mathrm{ug} / \mathrm{kg}$ & 0.0002 & 1.38 & 0.6901 & 0.6901 & 0.9756659 \\
\hline X10C9601883 & PPCB & Heptachlor epoxide & 2 & 0 & $\mathrm{ug} / \mathrm{kg}$ & 0.0002 & 1.38 & 0.6901 & 0.6901 & 0.9756659 \\
\hline X10C9601883 & PPCB & Lindane & 2 & 0 & $\mathrm{ug} / \mathrm{kg}$ & 0.0002 & 1.38 & 0.6901 & 0.6901 & 0.9756659 \\
\hline X10C9601883 & PPCB & Methoxychlor & 2 & 0 & $\mathrm{ug} / \mathrm{kg}$ & 0.002 & 13.8 & 6.901 & 6.901 & 9.7566594 \\
\hline X10C9601883 & PPCB & Toxaphene & 2 & 0 & $\mathrm{ug} / \mathrm{kg}$ & 0.005 & 34.6 & 17.3025 & 17.3025 & 24.462359 \\
\hline X10C9601883 & RADS & Americium-241 & 1 & 0 & $\mathrm{pCi} / \mathrm{g}$ & 0.0775 & 0.0775 & 0.0775 & & \\
\hline X10C9601883 & RADS & Cesium-137 & 1 & 0 & $\mathrm{pCi} / \mathrm{g}$ & 0.055 & 0.055 & 0.055 & & \\
\hline X10C9601883 & RADS & Cobalt-60 & 1 & 0 & $\mathrm{pCi} / \mathrm{g}$ & 0.092 & 0.092 & 0.092 & & \\
\hline X10C9601883 & RADS & Curium-243/244 & 1 & 0 & $\mathrm{pCi} / \mathrm{g}$ & 0.0199 & 0.0199 & 0.0199 & & \\
\hline X10C9601883 & RADS & Neptunium-237 & 1 & 0 & $\mathrm{pCi} / \mathrm{g}$ & 0.0731 & 0.0731 & 0.0731 & & \\
\hline X10C9601883 & RADS & Plutonium-238 & 1 & 0 & $\mathrm{pCi} / \mathrm{g}$ & 0.0184 & 0.0184 & 0.0184 & & \\
\hline X10C9601883 & RADS & Plutonium-239/240 & 1 & 0 & $\mathrm{pCi} / \mathrm{g}$ & 0 & 0 & 0 & & \\
\hline X10C9601883 & RADS & Radium-226 & 1 & 0 & $\mathrm{pCi} / \mathrm{g}$ & 0.774 & 0.774 & 0.774 & & \\
\hline X10C9601883 & RADS & Strontium-90 & 1 & 0 & $\mathrm{pCi} / \mathrm{g}$ & -0.172 & -0.172 & -0.172 & & \\
\hline X10C9601883 & RADS & Technetium-99 & 1 & 0 & $\mathrm{pCi} / \mathrm{g}$ & 0.623 & 0.623 & 0.623 & & \\
\hline X10C9601883 & RADS & Thorium-230 & 1 & 0 & $\mathrm{pCi} / \mathrm{g}$ & -4.25 & -4.25 & -4.25 & & \\
\hline X10C9601883 & RADS & Thorium-232 & 1 & 0 & $\mathrm{pCi} / \mathrm{g}$ & -7.78 & -7.78 & -7.78 & & \\
\hline X10C9601883 & RADS & Uranium-232 & 1 & 0 & $\%$ & 103 & 103 & 103 & & \\
\hline X10C9601883 & RADS & Uranium-233/234 & 1 & 0 & $\mathrm{pCi} / \mathrm{g}$ & 0.0572 & 0.0572 & 0.0572 & & \\
\hline X10C9601883 & RADS & Uranium-235/236 & 1 & 0 & $\mathrm{pCi} / \mathrm{g}$ & 0.025 & 0.025 & 0.025 & & \\
\hline $\mathrm{X} 10 \mathrm{C} 9601883$ & RADS & Uranium-238 & 1 & 0 & $\mathrm{pCi} / \mathrm{g}$ & 0.0142 & 0.0142 & 0.0142 & & \\
\hline X10C9601883 & SVOA & 1,2,4-Trichlorobenzene & 1 & 0 & $\mathrm{ug} / \mathrm{kg}$ & 455 & 455 & 455 & & \\
\hline X10C9601883 & SVOA & 1,2-Dichlorobenzene & 1 & 0 & $\mathrm{ug} / \mathrm{kg}$ & 455 & 455 & 455 & & \\
\hline X10C9601883 & SVOA & 1,3-Dichlorobenzene & 1 & 0 & $\mathrm{ug} / \mathrm{kg}$ & 455 & 455 & 455 & & \\
\hline X10C9601883 & SVOA & 1,4-Dichlorobenzene & 2 & 0 & $\mathrm{ug} / \mathrm{kg}$ & 0.01 & 455 & 227.505 & 227.505 & 321.72651 \\
\hline X10C9601883 & SVOA & 2,2'-Dichlorodiisopropyl ether & 1 & 0 & $\mathrm{ug} / \mathrm{kg}$ & 917 & 917 & 917 & & \\
\hline X10C9601883 & SVOA & 2,3,4,6-Tetrachlorophenol & 1 & 0 & $\mathrm{ug} / \mathrm{kg}$ & 917 & 917 & 917 & & \\
\hline X10C9601883 & SVOA & 2,4,5-Trichlorophenol & 2 & 0 & $\mathrm{ug} / \mathrm{kg}$ & 0.05 & 917 & 458.525 & 458.525 & 648.38156 \\
\hline X10C9601883 & SVOA & 2,4,6-Trichlorophenol & 2 & 0 & $\mathrm{ug} / \mathrm{kg}$ & 0.05 & 917 & 458.525 & 458.525 & 648.38156 \\
\hline X10C9601883 & SVOA & 2,4-Dichlorophenol & 1 & 0 & $\mathrm{ug} / \mathrm{kg}$ & 917 & 917 & 917 & & \\
\hline X10C9601883 & SVOA & 2,4-Dimethylphenol & 1 & 0 & $\mathrm{ug} / \mathrm{kg}$ & 917 & 917 & 917 & & \\
\hline X10C9601883 & SVOA & 2,4-Dinitrophenol & 1 & 0 & $\mathrm{ug} / \mathrm{kg}$ & 1830 & 1830 & 1830 & & \\
\hline X10C9601883 & SVOA & 2,4-Dinitrotoluene & 2 & 0 & $\mathrm{ug} / \mathrm{kg}$ & 0.05 & 917 & 458.525 & 458.525 & 648.38156 \\
\hline X10C9601883 & SVOA & 2,6-Dinitrotoluene & 1 & 0 & $\mathrm{ug} / \mathrm{kg}$ & 917 & 917 & 917 & & \\
\hline X10C9601883 & SVOA & 2-Chloronaphthalene & 1 & 0 & $\mathrm{ug} / \mathrm{kg}$ & 91.7 & 91.7 & 91.7 & & \\
\hline X10C9601883 & SVOA & 2-Chlorophenol & 1 & 0 & $\mathrm{ug} / \mathrm{kg}$ & 917 & 917 & 917 & & \\
\hline X10C9601883 & SVOA & 2-Methyl-4,6-dinitrophenol & 1 & 0 & $\mathrm{ug} / \mathrm{kg}$ & 917 & 917 & 917 & & \\
\hline X10C9601883 & SVOA & 2-Methylnaphthalene & 1 & 0 & $\mathrm{ug} / \mathrm{kg}$ & 91.7 & 91.7 & 91.7 & & \\
\hline X10C9601883 & SVOA & 2-Methylphenol & 2 & 0 & $\mathrm{ug} / \mathrm{kg}$ & 0.05 & 917 & 458.525 & 458.525 & 648.38156 \\
\hline X10C9601883 & SVOA & 2-Nitrobenzenamine & 1 & 0 & $\mathrm{ug} / \mathrm{kg}$ & 917 & 917 & 917 & & \\
\hline X10C9601883 & SVOA & 2-Nitrophenol & 1 & 0 & $\mathrm{ug} / \mathrm{kg}$ & 917 & 917 & 917 & & \\
\hline X10C9601883 & SVOA & 3,3'-Dichlorobenzidine & 1 & 0 & $\mathrm{ug} / \mathrm{kg}$ & 917 & 917 & 917 & & \\
\hline X10C9601883 & SVOA & 3-Nitrobenzenamine & 1 & 0 & $\mathrm{ug} / \mathrm{kg}$ & 917 & 917 & 917 & & \\
\hline X10C9601883 & SVOA & 4-Bromophenyl phenyl ether & 1 & 0 & $\mathrm{ug} / \mathrm{kg}$ & 917 & 917 & 917 & & \\
\hline X10C9601883 & SVOA & 4-Chloro-3-methylphenol & 1 & 0 & $\mathrm{ug} / \mathrm{kg}$ & 917 & 917 & 917 & & \\
\hline X10C9601883 & SVOA & 4-Chlorobenzenamine & 1 & 0 & $\mathrm{ug} / \mathrm{kg}$ & 917 & 917 & 917 & & \\
\hline X10C9601883 & SVOA & 4-Chlorophenyl phenyl ether & 1 & 0 & $\mathrm{ug} / \mathrm{kg}$ & 917 & 917 & 917 & & \\
\hline X10C9601883 & SVOA & 4-Nitrobenzenamine & 1 & 0 & $\mathrm{ug} / \mathrm{kg}$ & 917 & 917 & 917 & & \\
\hline X10C9601883 & SVOA & 4-Nitrophenol & 1 & 0 & $\mathrm{ug} / \mathrm{kg}$ & 917 & 917 & 917 & & \\
\hline X10C9601883 & SVOA & Acenaphthene & 1 & 0 & $\mathrm{ug} / \mathrm{kg}$ & 91.7 & 91.7 & 91.7 & & \\
\hline X10C9601883 & SVOA & Acenaphthylene & 1 & 0 & $\mathrm{ug} / \mathrm{kg}$ & 91.7 & 91.7 & 91.7 & & \\
\hline X10C9601883 & SVOA & Acetophenone & 1 & 0 & $\mathrm{ug} / \mathrm{kg}$ & 917 & 917 & 917 & & \\
\hline X10C9601883 & SVOA & Anthracene & 1 & 0 & $\mathrm{ug} / \mathrm{kg}$ & 91.7 & 91.7 & 91.7 & & \\
\hline X10C9601883 & SVOA & Benz(a)anthracene & 1 & 0 & $\mathrm{ug} / \mathrm{kg}$ & 91.7 & 91.7 & 91.7 & & \\
\hline X10C9601883 & SVOA & Benzenemethanol & 1 & 0 & $\mathrm{ug} / \mathrm{kg}$ & 917 & 917 & 917 & & \\
\hline X10C9601883 & SVOA & Benzidine & 1 & 0 & $\mathrm{ug} / \mathrm{kg}$ & 917 & 917 & 917 & & \\
\hline X10C9601883 & SVOA & Benzo(a)pyrene & 1 & 0 & $\mathrm{ug} / \mathrm{kg}$ & 91.7 & 91.7 & 91.7 & & \\
\hline X10C9601883 & SVOA & Benzo(b)fluoranthene & 1 & 0 & $\mathrm{ug} / \mathrm{kg}$ & 91.7 & 91.7 & 91.7 & & \\
\hline X10C9601883 & SVOA & Benzo(ghi)perylene & 1 & 0 & $\mathrm{ug} / \mathrm{kg}$ & 91.7 & 91.7 & 91.7 & & \\
\hline X10C9601883 & SVOA & Benzo(k)fluoranthene & 1 & 0 & $\mathrm{ug} / \mathrm{kg}$ & 91.7 & 91.7 & 91.7 & & \\
\hline X10C9601883 & SVOA & Benzoic acid & 1 & 0 & $\mathrm{ug} / \mathrm{kg}$ & 1830 & 1830 & 1830 & & \\
\hline X10C9601883 & SVOA & Bis(2-chloroethoxy)methane & 1 & 0 & $\mathrm{ug} / \mathrm{kg}$ & 917 & 917 & 917 & & \\
\hline
\end{tabular}




\begin{tabular}{|c|c|c|c|c|c|c|c|c|c|c|}
\hline X10C9601883 & SVOA & Bis(2-chloroethyl) ether & 1 & 0 & $\mathrm{ug} / \mathrm{kg}$ & 917 & 917 & 917 & & \\
\hline X10C9601883 & SVOA & Bis(2-ethylhexyl)phthalate & 1 & 0 & $\mathrm{ug} / \mathrm{kg}$ & 1090 & 1090 & 1090 & & \\
\hline X10C9601883 & SVOA & Butyl benzyl phthalate & 1 & 0 & $\mathrm{ug} / \mathrm{kg}$ & 917 & 917 & 917 & & \\
\hline X10C9601883 & SVOA & Carbazole & 1 & 0 & $\mathrm{ug} / \mathrm{kg}$ & 91.7 & 91.7 & 91.7 & & \\
\hline X10C9601883 & SVOA & Chrysene & 1 & 0 & $\mathrm{ug} / \mathrm{kg}$ & 91.7 & 91.7 & 91.7 & & \\
\hline X10C9601883 & SVOA & $\operatorname{Dibenz}(\mathrm{a}, \mathrm{h})$ anthracene & 1 & 0 & $\mathrm{ug} / \mathrm{kg}$ & 91.7 & 91.7 & 91.7 & & \\
\hline X10C9601883 & SVOA & Dibenzofuran & 1 & 0 & $\mathrm{ug} / \mathrm{kg}$ & 917 & 917 & 917 & & \\
\hline X10C9601883 & SVOA & Diethyl phthalate & 1 & 0 & $\mathrm{ug} / \mathrm{kg}$ & 917 & 917 & 917 & & \\
\hline X10C9601883 & SVOA & Dimethyl phthalate & 1 & 0 & $\mathrm{ug} / \mathrm{kg}$ & 917 & 917 & 917 & & \\
\hline X10C9601883 & SVOA & Di-n-butyl phthalate & 1 & 0 & $\mathrm{ug} / \mathrm{kg}$ & 917 & 917 & 917 & & \\
\hline X10C9601883 & SVOA & Di-n-octylphthalate & 1 & 0 & $\mathrm{ug} / \mathrm{kg}$ & 917 & 917 & 917 & & \\
\hline X10C9601883 & SVOA & Diphenylamine & 1 & 0 & $\mathrm{ug} / \mathrm{kg}$ & 917 & 917 & 917 & & \\
\hline X10C9601883 & SVOA & Fluoranthene & 1 & 0 & $\mathrm{ug} / \mathrm{kg}$ & 91.7 & 91.7 & 91.7 & & \\
\hline X10C9601883 & SVOA & Fluorene & 1 & 0 & $\mathrm{ug} / \mathrm{kg}$ & 91.7 & 91.7 & 91.7 & & \\
\hline X10C9601883 & SVOA & Hexachlorobenzene & 2 & 0 & $\mathrm{ug} / \mathrm{kg}$ & 0.05 & 917 & 458.525 & 458.525 & 648.38156 \\
\hline X10C9601883 & SVOA & Hexachlorobutadiene & 2 & 0 & $\mathrm{ug} / \mathrm{kg}$ & 0.05 & 917 & 458.525 & 458.525 & 648.38156 \\
\hline X10C9601883 & SVOA & Hexachlorocyclopentadiene & 1 & 0 & $\mathrm{ug} / \mathrm{kg}$ & 917 & 917 & 917 & & \\
\hline X10C9601883 & SVOA & Hexachloroethane & 2 & 0 & $\mathrm{ug} / \mathrm{kg}$ & 0.05 & 917 & 458.525 & 458.525 & 648.38156 \\
\hline X10C9601883 & SVOA & Indeno(1,2,3-cd)pyrene & 1 & 0 & $\mathrm{ug} / \mathrm{kg}$ & 91.7 & 91.7 & 91.7 & & \\
\hline X10C9601883 & SVOA & Isophorone & 1 & 0 & $\mathrm{ug} / \mathrm{kg}$ & 917 & 917 & 917 & & \\
\hline X10C9601883 & SVOA & $\mathrm{m}+\mathrm{p}$ Methylphenol & 2 & 0 & $\mathrm{ug} / \mathrm{kg}$ & 0.05 & 917 & 458.525 & 458.525 & 648.38156 \\
\hline X10C9601883 & SVOA & Naphthalene & 1 & 0 & $\mathrm{ug} / \mathrm{kg}$ & 91.7 & 91.7 & 91.7 & & \\
\hline X10C9601883 & SVOA & Nitrobenzene & 2 & 0 & $\mathrm{ug} / \mathrm{kg}$ & 0.05 & 917 & 458.525 & 458.525 & 648.38156 \\
\hline X10C9601883 & SVOA & N-Nitroso-di-n-propylamine & 1 & 0 & $\mathrm{ug} / \mathrm{kg}$ & 917 & 917 & 917 & & \\
\hline X10C9601883 & SVOA & Pentachlorophenol & 2 & 0 & $\mathrm{ug} / \mathrm{kg}$ & 0.05 & 917 & 458.525 & 458.525 & 648.38156 \\
\hline X10C9601883 & SVOA & Phenanthrene & 1 & 0 & $\mathrm{ug} / \mathrm{kg}$ & 91.7 & 91.7 & 91.7 & & \\
\hline X10C9601883 & SVOA & Phenol & 1 & 0 & $\mathrm{ug} / \mathrm{kg}$ & 917 & 917 & 917 & & \\
\hline X10C9601883 & SVOA & Pyridine & 2 & 0 & $\mathrm{ug} / \mathrm{kg}$ & 0.05 & 917 & 458.525 & 458.525 & 648.38156 \\
\hline X10C9601883 & VOA & (1,1-Dimethylethyl)benzene & 1 & 0 & $\mathrm{ug} / \mathrm{kg}$ & 455 & 455 & 455 & & \\
\hline X10C9601883 & VOA & (1-Methylpropyl)benzene & 1 & 0 & $\mathrm{ug} / \mathrm{kg}$ & 455 & 455 & 455 & & \\
\hline X10C9601883 & VOA & 1,1,1,2-Tetrachloroethane & 1 & 0 & $\mathrm{ug} / \mathrm{kg}$ & 455 & 455 & 455 & & \\
\hline X10C9601883 & VOA & 1,1,1-Trichloroethane & 1 & 0 & $\mathrm{ug} / \mathrm{kg}$ & 455 & 455 & 455 & & \\
\hline X10C9601883 & VOA & 1,1,2,2-Tetrachloroethane & 1 & 0 & $\mathrm{ug} / \mathrm{kg}$ & 455 & 455 & 455 & & \\
\hline X10C9601883 & VOA & 1,1,2-Trichloroethane & 1 & 0 & $\mathrm{ug} / \mathrm{kg}$ & 455 & 455 & 455 & & \\
\hline X10C9601883 & VOA & 1,1-Dichloroethane & 1 & 0 & $\mathrm{ug} / \mathrm{kg}$ & 455 & 455 & 455 & & \\
\hline X10C9601883 & VOA & 1,1-Dichloroethene & 2 & 0 & $\mathrm{ug} / \mathrm{kg}$ & 0.01 & 455 & 227.505 & 227.505 & 321.72651 \\
\hline X10C9601883 & VOA & 1,1-Dichloropropene & 1 & 0 & $\mathrm{ug} / \mathrm{kg}$ & 455 & 455 & 455 & & \\
\hline X10C9601883 & VOA & 1,2,3-Trichlorobenzene & 1 & 0 & $\mathrm{ug} / \mathrm{kg}$ & 455 & 455 & 455 & & \\
\hline X10C9601883 & VOA & 1,2,3-Trichloropropane & 1 & 0 & $\mathrm{ug} / \mathrm{kg}$ & 455 & 455 & 455 & & \\
\hline X10C9601883 & VOA & 1,2,4-Trimethylbenzene & 1 & 0 & $\mathrm{ug} / \mathrm{kg}$ & 455 & 455 & 455 & & \\
\hline X10C9601883 & VOA & 1,2-Dibromo-3-chloropropane & 1 & 0 & $\mathrm{ug} / \mathrm{kg}$ & 455 & 455 & 455 & & \\
\hline X10C9601883 & VOA & 1,2-Dibromoethane & 1 & 0 & $\mathrm{ug} / \mathrm{kg}$ & 455 & 455 & 455 & & \\
\hline X10C9601883 & VOA & 1,2-Dichloroethane & 2 & 0 & $\mathrm{ug} / \mathrm{kg}$ & 0.01 & 455 & 227.505 & 227.505 & 321.72651 \\
\hline X10C9601883 & VOA & 1,2-Dichloroethene & 1 & 0 & $\mathrm{ug} / \mathrm{kg}$ & 909 & 909 & 909 & & \\
\hline X10C9601883 & VOA & 1,2-Dichloropropane & 1 & 0 & $\mathrm{ug} / \mathrm{kg}$ & 455 & 455 & 455 & & \\
\hline X10C9601883 & VOA & 1,2-Dimethylbenzene & 1 & 0 & $\mathrm{ug} / \mathrm{kg}$ & 455 & 455 & 455 & & \\
\hline X10C9601883 & VOA & 1,3,5-Trimethylbenzene & 1 & 0 & $\mathrm{ug} / \mathrm{kg}$ & 455 & 455 & 455 & & \\
\hline X10C9601883 & VOA & 1,3-Dichloropropane & 1 & 0 & $\mathrm{ug} / \mathrm{kg}$ & 455 & 455 & 455 & & \\
\hline X10C9601883 & VOA & 1-Chloro-4-methylbenzene & 1 & 0 & $\mathrm{ug} / \mathrm{kg}$ & 455 & 455 & 455 & & \\
\hline X10C9601883 & VOA & 1-Methyl-4-(1-methylethyl)benzene & 1 & 0 & $\mathrm{ug} / \mathrm{kg}$ & 455 & 455 & 455 & & \\
\hline X10C9601883 & VOA & 2,2-Dichloropropane & 1 & 0 & $\mathrm{ug} / \mathrm{kg}$ & 455 & 455 & 455 & & \\
\hline X10C9601883 & VOA & 2-Butanone & 2 & 0 & $\mathrm{ug} / \mathrm{kg}$ & 0.05 & 2270 & 1135.025 & 1135.025 & 1605.097 \\
\hline X10C9601883 & VOA & 2-Hexanone & 1 & 0 & $\mathrm{ug} / \mathrm{kg}$ & 2270 & 2270 & 2270 & & \\
\hline X10C9601883 & VOA & 4-Methyl-2-pentanone & 1 & 0 & $\mathrm{ug} / \mathrm{kg}$ & 2270 & 2270 & 2270 & & \\
\hline X10C9601883 & VOA & Acetone & 1 & 0 & $\mathrm{ug} / \mathrm{kg}$ & 2270 & 2270 & 2270 & & \\
\hline X10C9601883 & VOA & Acetonitrile & 1 & 0 & $\mathrm{ug} / \mathrm{kg}$ & 11400 & 11400 & 11400 & & \\
\hline X10C9601883 & VOA & Acrolein & 1 & 0 & $\mathrm{ug} / \mathrm{kg}$ & 2270 & 2270 & 2270 & & \\
\hline X10C9601883 & VOA & Acrylonitrile & 1 & 0 & $\mathrm{ug} / \mathrm{kg}$ & 2270 & 2270 & 2270 & & \\
\hline X10C9601883 & VOA & Benzene & 2 & 0 & $\mathrm{ug} / \mathrm{kg}$ & 0.01 & 455 & 227.505 & 227.505 & 321.72651 \\
\hline X10C9601883 & VOA & Bromobenzene & 1 & 0 & $\mathrm{ug} / \mathrm{kg}$ & 455 & 455 & 455 & & \\
\hline X10C9601883 & VOA & Bromochloromethane & 1 & 0 & $\mathrm{ug} / \mathrm{kg}$ & 455 & 455 & 455 & & \\
\hline X10C9601883 & VOA & Bromodichloromethane & 1 & 0 & $\mathrm{ug} / \mathrm{kg}$ & 455 & 455 & 455 & & \\
\hline X10C9601883 & VOA & Bromoform & 1 & 0 & $\mathrm{ug} / \mathrm{kg}$ & 455 & 455 & 455 & & \\
\hline X10C9601883 & VOA & Bromomethane & 1 & 0 & $\mathrm{ug} / \mathrm{kg}$ & 455 & 455 & 455 & & \\
\hline X10C9601883 & VOA & Butylbenzene & 1 & 0 & $\mathrm{ug} / \mathrm{kg}$ & 455 & 455 & 455 & & \\
\hline X10C9601883 & VOA & Carbon disulfide & 1 & 0 & $\mathrm{ug} / \mathrm{kg}$ & 2270 & 2270 & 2270 & & \\
\hline X10C9601883 & VOA & Carbon tetrachloride & 2 & 0 & $\mathrm{ug} / \mathrm{kg}$ & 0.01 & 455 & 227.505 & 227.505 & 321.72651 \\
\hline X10C9601883 & VOA & Chlorobenzene & 2 & 0 & $\mathrm{ug} / \mathrm{kg}$ & 0.01 & 455 & 227.505 & 227.505 & 321.72651 \\
\hline X10C9601883 & VOA & Chloroethane & 1 & 0 & $\mathrm{ug} / \mathrm{kg}$ & 455 & 455 & 455 & & \\
\hline X10C9601883 & VOA & Chloroform & 2 & 0 & $\mathrm{ug} / \mathrm{kg}$ & 0.01 & 455 & 227.505 & 227.505 & 321.72651 \\
\hline
\end{tabular}




\begin{tabular}{|c|c|c|c|c|c|c|c|c|c|c|}
\hline X10C9601883 & VOA & Chloromethane & 1 & 0 & $\mathrm{ug} / \mathrm{kg}$ & 455 & 455 & 455 & & \\
\hline X10C9601883 & $\mathrm{VOA}$ & cis-1,2-Dichloroethene & 1 & 0 & $\mathrm{ug} / \mathrm{kg}$ & 455 & 455 & 455 & & \\
\hline X10C9601883 & VOA & cis-1,3-Dichloropropene & 1 & 0 & $\mathrm{ug} / \mathrm{kg}$ & 455 & 455 & 455 & & \\
\hline X10C9601883 & VOA & Cumene & 1 & 0 & $\mathrm{ug} / \mathrm{kg}$ & 455 & 455 & 455 & & \\
\hline X10C9601883 & VOA & Dibromochloromethane & 1 & 0 & $\mathrm{ug} / \mathrm{kg}$ & 455 & 455 & 455 & & \\
\hline X10C9601883 & VOA & Dibromomethane & 1 & 0 & $\mathrm{ug} / \mathrm{kg}$ & 455 & 455 & 455 & & \\
\hline X10C9601883 & VOA & Dichlorodifluoromethane & 1 & 0 & $\mathrm{ug} / \mathrm{kg}$ & 455 & 455 & 455 & & \\
\hline X10C9601883 & VOA & Ethylbenzene & 1 & 0 & $\mathrm{ug} / \mathrm{kg}$ & 455 & 455 & 455 & & \\
\hline X10C9601883 & VOA & Hexane & 1 & 0 & $\mathrm{ug} / \mathrm{kg}$ & 2270 & 2270 & 2270 & & \\
\hline X10C9601883 & VOA & M + P Xylene & 1 & 0 & $\mathrm{ug} / \mathrm{kg}$ & 909 & 909 & 909 & & \\
\hline X10C9601883 & VOA & Methanol & 1 & 0 & $\mathrm{ug} / \mathrm{kg}$ & 4850 & 4850 & 4850 & & \\
\hline X10C9601883 & VOA & Methyl methacrylate & 1 & 0 & $\mathrm{ug} / \mathrm{kg}$ & 2270 & 2270 & 2270 & & \\
\hline X10C9601883 & VOA & Methylcyclohexane & 1 & 0 & $\mathrm{ug} / \mathrm{kg}$ & 455 & 455 & 455 & & \\
\hline X10C9601883 & VOA & Methylene chloride & 1 & 0 & $\mathrm{ug} / \mathrm{kg}$ & 2270 & 2270 & 2270 & & \\
\hline X10C9601883 & VOA & o-Chlorotoluene & 1 & 0 & $\mathrm{ug} / \mathrm{kg}$ & 455 & 455 & 455 & & \\
\hline X10C9601883 & VOA & Propylbenzene & 1 & 0 & $\mathrm{ug} / \mathrm{kg}$ & 455 & 455 & 455 & & \\
\hline X10C9601883 & VOA & Propylene glycol & 1 & 0 & $\mathrm{ug} / \mathrm{kg}$ & 48500 & 48500 & 48500 & & \\
\hline X10C9601883 & VOA & Styrene & 1 & 0 & $\mathrm{ug} / \mathrm{kg}$ & 455 & 455 & 455 & & \\
\hline X10C9601883 & VOA & Tetrachloroethene & 2 & 0 & $\mathrm{ug} / \mathrm{kg}$ & 0.01 & 455 & 227.505 & 227.505 & 321.72651 \\
\hline X10C9601883 & $\mathrm{VOA}$ & Toluene & 1 & 0 & $\mathrm{ug} / \mathrm{kg}$ & 455 & 455 & 455 & & \\
\hline X10C9601883 & VOA & Total Xylene & 1 & 0 & $\mathrm{ug} / \mathrm{kg}$ & 1360 & 1360 & 1360 & & \\
\hline X10C9601883 & $\mathrm{VOA}$ & trans-1,2-Dichloroethene & 1 & 0 & $\mathrm{ug} / \mathrm{kg}$ & 455 & 455 & 455 & & \\
\hline X10C9601883 & VOA & trans-1,3-Dichloropropene & 1 & 0 & $\mathrm{ug} / \mathrm{kg}$ & 455 & 455 & 455 & & \\
\hline X10C9601883 & VOA & Trichloroethene & 2 & 0 & $\mathrm{ug} / \mathrm{kg}$ & 0.01 & 455 & 227.505 & 227.505 & 321.72651 \\
\hline X10C9601883 & VOA & Trichlorofluoromethane & 1 & 0 & $\mathrm{ug} / \mathrm{kg}$ & 455 & 455 & 455 & & \\
\hline X10C9601883 & VOA & Vinyl chloride & 2 & 0 & $\mathrm{ug} / \mathrm{kg}$ & 0.01 & 455 & 227.505 & 227.505 & 321.72651 \\
\hline X10I0700534 & HERB & $2,4-\mathrm{D}$ & 2 & 0 & $\mathrm{ug} / \mathrm{kg}$ & 0.05 & 24.8 & 12.425 & 12.425 & 17.500893 \\
\hline $\mathrm{X} 10 \mathrm{I} 0700534$ & HERB & Silvex & 2 & 0 & $\mathrm{ug} / \mathrm{kg}$ & 0.05 & 24.8 & 12.425 & 12.425 & 17.500893 \\
\hline X10I0700534 & METAL & Arsenic & 2 & 0 & $\mathrm{mg} / \mathrm{L}$ & 0.3 & 150 & 75.15 & 75.15 & 105.85389 \\
\hline X10I0700534 & METAL & Barium & 2 & 1 & $\mathrm{mg} / \mathrm{L}$ & 0.05 & 30.8 & 15.425 & 15.425 & 21.743534 \\
\hline X10I0700534 & METAL & Cadmium & 2 & 0 & $\mathrm{mg} / \mathrm{L}$ & 0.05 & 25 & 12.525 & 12.525 & 17.642314 \\
\hline X10I0700534 & METAL & Chromium & 2 & 2 & $\mathrm{mg} / \mathrm{L}$ & 0.0623 & 56900 & 28450.031 & 28450.031 & 40234.332 \\
\hline X10I0700534 & METAL & Lead & 2 & 0 & $\mathrm{mg} / \mathrm{L}$ & 0.1 & 50 & 25.05 & 25.05 & 35.284628 \\
\hline X10I0700534 & METAL & Mercury & 2 & 0 & $\mathrm{mg} / \mathrm{L}$ & 0.002 & 0.4 & 0.201 & 0.201 & 0.2814285 \\
\hline X10I0700534 & METAL & Selenium & 2 & 0 & $\mathrm{mg} / \mathrm{L}$ & 0.3 & 150 & 75.15 & 75.15 & 105.85389 \\
\hline X10I0700534 & METAL & Silver & 2 & 0 & $\mathrm{mg} / \mathrm{L}$ & 0.05 & 25 & 12.525 & 12.525 & 17.642314 \\
\hline X10I0700534 & METAL & Total Uranium & 1 & 0 & $\mathrm{pCi} / \mathrm{g}$ & 0.038 & 0.038 & 0.038 & & \\
\hline X10I0700534 & PPCB & 4,4'-DDD & 1 & 0 & $\mathrm{ug} / \mathrm{kg}$ & 19.5 & 19.5 & 19.5 & & \\
\hline X10I0700534 & РPCB & 4,4'-DDE & 1 & 0 & $\mathrm{ug} / \mathrm{kg}$ & 19.5 & 19.5 & 19.5 & & \\
\hline X10I0700534 & PPCB & 4,4'-DDT & 1 & 0 & $\mathrm{ug} / \mathrm{kg}$ & 19.5 & 19.5 & 19.5 & & \\
\hline X10I0700534 & РPCB & Aldrin & 1 & 0 & $\mathrm{ug} / \mathrm{kg}$ & 9.74 & 9.74 & 9.74 & & \\
\hline X10I0700534 & PPCB & alpha-BHC & 1 & 0 & $\mathrm{ug} / \mathrm{kg}$ & 9.74 & 9.74 & 9.74 & & \\
\hline X10I0700534 & РPCB & alpha-Chlordane & 1 & 0 & $\mathrm{ug} / \mathrm{kg}$ & 9.74 & 9.74 & 9.74 & & \\
\hline X10I0700534 & PPCB & beta-BHC & 1 & 0 & $\mathrm{ug} / \mathrm{kg}$ & 9.74 & 9.74 & 9.74 & & \\
\hline X10I0700534 & PPCB & Chlordane & 3 & 0 & $\mathrm{ug} / \mathrm{kg}$ & 0.0025 & 122 & 40.668333 & 0.0025 & 70.435289 \\
\hline X10I0700534 & PPCB & delta-BHC & 1 & 0 & $\mathrm{ug} / \mathrm{kg}$ & 9.74 & 9.74 & 9.74 & & \\
\hline X10I0700534 & PPCB & Dieldrin & 1 & 0 & $\mathrm{ug} / \mathrm{kg}$ & 19.5 & 19.5 & 19.5 & & \\
\hline X10I0700534 & PPCB & Endosulfan I & 1 & 0 & $\mathrm{ug} / \mathrm{kg}$ & 9.74 & 9.74 & 9.74 & & \\
\hline X10I0700534 & РPCB & Endosulfan II & 1 & 0 & $\mathrm{ug} / \mathrm{kg}$ & 19.5 & 19.5 & 19.5 & & \\
\hline X10I0700534 & PPCB & Endosulfan sulfate & 1 & 0 & $\mathrm{ug} / \mathrm{kg}$ & 19.5 & 19.5 & 19.5 & & \\
\hline X10I0700534 & РPCB & Endrin & 3 & 0 & $\mathrm{ug} / \mathrm{kg}$ & 0.0004 & 19.5 & 6.5002667 & 0.0004 & 11.258099 \\
\hline X10I0700534 & PPCB & Endrin aldehyde & 1 & 0 & $\mathrm{ug} / \mathrm{kg}$ & 19.5 & 19.5 & 19.5 & & \\
\hline X10I0700534 & PPCB & Endrin ketone & 1 & 0 & $\mathrm{ug} / \mathrm{kg}$ & 19.5 & 19.5 & 19.5 & & \\
\hline X10I0700534 & PPCB & gamma-Chlordane & 1 & 0 & $\mathrm{ug} / \mathrm{kg}$ & 9.74 & 9.74 & 9.74 & & \\
\hline X10I0700534 & PPCB & Heptachlor & 3 & 0 & $\mathrm{ug} / \mathrm{kg}$ & 0.0002 & 9.74 & 3.2468 & 0.0002 & 5.6232762 \\
\hline X10I0700534 & РPCB & Heptachlor epoxide & 3 & 0 & $\mathrm{ug} / \mathrm{kg}$ & 0.0002 & 9.74 & 3.2468 & 0.0002 & 5.6232762 \\
\hline X10I0700534 & РPCB & Lindane & 3 & 0 & $\mathrm{ug} / \mathrm{kg}$ & 0.0002 & 9.74 & 3.2468 & 0.0002 & 5.6232762 \\
\hline X10I0700534 & PPCB & Methoxychlor & 3 & 0 & $\mathrm{ug} / \mathrm{kg}$ & 0.002 & 97.4 & 32.468 & 0.002 & 56.232762 \\
\hline $\mathrm{X} 10 \mathrm{I} 0700534$ & PPCB & Toxaphene & 3 & 0 & $\mathrm{ug} / \mathrm{kg}$ & 0.005 & 243 & 81.003333 & 0.005 & 140.29323 \\
\hline X10I0700534 & RADS & Americium-241 & 1 & 0 & $\mathrm{pCi} / \mathrm{g}$ & -0.014 & -0.014 & -0.014 & & \\
\hline X10I0700534 & RADS & Carbon-14 & 1 & 0 & $\mathrm{pCi} / \mathrm{g}$ & -2.08 & -2.08 & -2.08 & & \\
\hline X10I0700534 & RADS & Cesium-137 & 1 & 0 & $\mathrm{pCi} / \mathrm{g}$ & -0.0203 & -0.0203 & -0.0203 & & \\
\hline X10I0700534 & RADS & Cobalt- 60 & 1 & 0 & $\mathrm{pCi} / \mathrm{g}$ & -0.00519 & -0.00519 & -0.00519 & & \\
\hline X10I0700534 & RADS & Curium-243/244 & 1 & 0 & $\mathrm{pCi} / \mathrm{g}$ & -0.00344 & -0.00344 & -0.00344 & & \\
\hline X10I0700534 & RADS & Iodine-129 & 1 & 0 & $\mathrm{pCi} / \mathrm{g}$ & -0.104 & -0.104 & -0.104 & & \\
\hline X10I0700534 & RADS & Neptunium-237 & 1 & 0 & $\mathrm{pCi} / \mathrm{g}$ & -0.0356 & -0.0356 & -0.0356 & & \\
\hline X10I0700534 & RADS & Plutonium-238 & 1 & 0 & $\mathrm{pCi} / \mathrm{g}$ & -0.00277 & -0.00277 & -0.00277 & & \\
\hline X10I0700534 & RADS & Plutonium-239/240 & 1 & 0 & $\mathrm{pCi} / \mathrm{g}$ & 0.000461 & 0.000461 & 0.000461 & & \\
\hline X10I0700534 & RADS & Radium-226 & 1 & 0 & $\mathrm{pCi} / \mathrm{g}$ & -0.348 & -0.348 & -0.348 & & \\
\hline X10I0700534 & RADS & Strontium-90 & 1 & 0 & $\mathrm{pCi} / \mathrm{g}$ & 1.66 & 1.66 & 1.66 & & \\
\hline
\end{tabular}




\begin{tabular}{|c|c|c|c|c|c|c|c|c|c|c|}
\hline X10I0700534 & RADS & Technetium-99 & 1 & 0 & $\mathrm{pCi} / \mathrm{g}$ & -0.868 & -0.868 & -0.868 & & \\
\hline X10I0700534 & RADS & Thorium-230 & 1 & 0 & $\mathrm{pCi} / \mathrm{g}$ & 5.87 & 5.87 & 5.87 & & \\
\hline X10I0700534 & RADS & Thorium-232 & 1 & 0 & $\mathrm{pCi} / \mathrm{g}$ & -9.88 & -9.88 & -9.88 & & \\
\hline X10I0700534 & RADS & Tritium & 1 & 0 & $\mathrm{pCi} / \mathrm{g}$ & 25.1 & 25.1 & 25.1 & & \\
\hline X10I0700534 & RADS & Uranium-233/234 & 1 & 0 & $\mathrm{pCi} / \mathrm{g}$ & 0.0117 & 0.0117 & 0.0117 & & \\
\hline X10I0700534 & RADS & Uranium-235/236 & 1 & 0 & $\mathrm{pCi} / \mathrm{g}$ & 0.0041 & 0.0041 & 0.0041 & & \\
\hline X10I0700534 & RADS & Uranium-238 & 1 & 0 & $\mathrm{pCi} / \mathrm{g}$ & 0.0222 & 0.0222 & 0.0222 & & \\
\hline X10I0700534 & SVOA & 1,2,4-Trichlorobenzene & 1 & 0 & $\mathrm{ug} / \mathrm{kg}$ & 96.2 & 96.2 & 96.2 & & \\
\hline X10I0700534 & SVOA & 1,2-Dichlorobenzene & 1 & 0 & $\mathrm{ug} / \mathrm{kg}$ & 96.2 & 96.2 & 96.2 & & \\
\hline X10I0700534 & SVOA & 1,3-Dichlorobenzene & 1 & 0 & $\mathrm{ug} / \mathrm{kg}$ & 96.2 & 96.2 & 96.2 & & \\
\hline X10I0700534 & SVOA & 1,4-Dichlorobenzene & 2 & 0 & $\mathrm{ug} / \mathrm{kg}$ & 0.01 & 96.2 & 48.105 & 48.105 & 68.016601 \\
\hline $\mathrm{X} 10 \mathrm{I} 0700534$ & SVOA & 2,2'-Dichlorodiisopropyl ether & 1 & 0 & $\mathrm{ug} / \mathrm{kg}$ & 80000 & 80000 & 80000 & & \\
\hline X10I0700534 & SVOA & 2,3,4,6-Tetrachlorophenol & 1 & 0 & $\mathrm{ug} / \mathrm{kg}$ & 80000 & 80000 & 80000 & & \\
\hline X10I0700534 & SVOA & 2,4,5-Trichlorophenol & 2 & 0 & $\mathrm{ug} / \mathrm{kg}$ & 0.05 & 80000 & 40000.025 & 40000.025 & 56568.507 \\
\hline X10I0700534 & SVOA & 2,4,6-Trichlorophenol & 2 & 0 & $\mathrm{ug} / \mathrm{kg}$ & 0.05 & 80000 & 40000.025 & 40000.025 & 56568.507 \\
\hline X10I0700534 & SVOA & 2,4-Dichlorophenol & 1 & 0 & $\mathrm{ug} / \mathrm{kg}$ & 80000 & 80000 & 80000 & & \\
\hline X10I0700534 & SVOA & 2,4-Dimethylphenol & 1 & 0 & $\mathrm{ug} / \mathrm{kg}$ & 80000 & 80000 & 80000 & & \\
\hline X10I0700534 & SVOA & 2,4-Dinitrophenol & 1 & 0 & $\mathrm{ug} / \mathrm{kg}$ & 160000 & 160000 & 160000 & & \\
\hline X10I0700534 & SVOA & 2,4-Dinitrotoluene & 2 & 0 & $\mathrm{ug} / \mathrm{kg}$ & 0.05 & 80000 & 40000.025 & 40000.025 & 56568.507 \\
\hline $\mathrm{X} 10 \mathrm{I} 0700534$ & SVOA & 2,6-Dinitrotoluene & 1 & 0 & $\mathrm{ug} / \mathrm{kg}$ & 80000 & 80000 & 80000 & & \\
\hline X10I0700534 & SVOA & 2-Chloronaphthalene & 1 & 0 & $\mathrm{ug} / \mathrm{kg}$ & 8000 & 8000 & 8000 & & \\
\hline X10I0700534 & SVOA & 2-Chlorophenol & 1 & 0 & $\mathrm{ug} / \mathrm{kg}$ & 80000 & 80000 & 80000 & & \\
\hline X10I0700534 & SVOA & 2-Methyl-4,6-dinitrophenol & 1 & 0 & $\mathrm{ug} / \mathrm{kg}$ & 80000 & 80000 & 80000 & & \\
\hline X10I0700534 & SVOA & 2-Methylnaphthalene & 1 & 0 & $\mathrm{ug} / \mathrm{kg}$ & 8000 & 8000 & 8000 & & \\
\hline X10I0700534 & SVOA & 2-Methylphenol & 2 & 0 & $\mathrm{ug} / \mathrm{kg}$ & 0.05 & 80000 & 40000.025 & 40000.025 & 56568.507 \\
\hline X10I0700534 & SVOA & 2-Nitrobenzenamine & 1 & 0 & $\mathrm{ug} / \mathrm{kg}$ & 80000 & 80000 & 80000 & & \\
\hline X10I0700534 & SVOA & 2-Nitrophenol & 1 & 0 & $\mathrm{ug} / \mathrm{kg}$ & 80000 & 80000 & 80000 & & \\
\hline X10I0700534 & SVOA & 3,3'-Dichlorobenzidine & 1 & 0 & $\mathrm{ug} / \mathrm{kg}$ & 80000 & 80000 & 80000 & & \\
\hline X10I0700534 & SVOA & 3-Nitrobenzenamine & 1 & 0 & $\mathrm{ug} / \mathrm{kg}$ & 80000 & 80000 & 80000 & & \\
\hline X10I0700534 & SVOA & 4-Bromophenyl phenyl ether & 1 & 0 & $\mathrm{ug} / \mathrm{kg}$ & 80000 & 80000 & 80000 & & \\
\hline X10I0700534 & SVOA & 4-Chloro-3-methylphenol & 1 & 0 & $\mathrm{ug} / \mathrm{kg}$ & 80000 & 80000 & 80000 & & \\
\hline X10I0700534 & SVOA & 4-Chlorobenzenamine & 1 & 0 & $\mathrm{ug} / \mathrm{kg}$ & 80000 & 80000 & 80000 & & \\
\hline X10I0700534 & SVOA & 4-Chlorophenyl phenyl ether & 1 & 0 & $\mathrm{ug} / \mathrm{kg}$ & 80000 & 80000 & 80000 & & \\
\hline X10I0700534 & SVOA & 4-Nitrobenzenamine & 1 & 0 & $\mathrm{ug} / \mathrm{kg}$ & 80000 & 80000 & 80000 & & \\
\hline X10I0700534 & SVOA & 4-Nitrophenol & 1 & 0 & $\mathrm{ug} / \mathrm{kg}$ & 80000 & 80000 & 80000 & & \\
\hline X10I0700534 & SVOA & Acenaphthene & 1 & 0 & $\mathrm{ug} / \mathrm{kg}$ & 8000 & 8000 & 8000 & & \\
\hline X10I0700534 & SVOA & Acenaphthylene & 1 & 0 & $\mathrm{ug} / \mathrm{kg}$ & 8000 & 8000 & 8000 & & \\
\hline X10I0700534 & SVOA & Acetophenone & 1 & 0 & $\mathrm{ug} / \mathrm{kg}$ & 80000 & 80000 & 80000 & & \\
\hline X10I0700534 & SVOA & Anthracene & 1 & 0 & $\mathrm{ug} / \mathrm{kg}$ & 8000 & 8000 & 8000 & & \\
\hline X10I0700534 & SVOA & Benz(a)anthracene & 1 & 0 & $\mathrm{ug} / \mathrm{kg}$ & 8000 & 8000 & 8000 & & \\
\hline X10I0700534 & SVOA & Benzenemethanol & 1 & 0 & $\mathrm{ug} / \mathrm{kg}$ & 80000 & 80000 & 80000 & & \\
\hline X10I0700534 & SVOA & Benzidine & 1 & 0 & $\mathrm{ug} / \mathrm{kg}$ & 80000 & 80000 & 80000 & & \\
\hline X10I0700534 & SVOA & Benzo(a)pyrene & 1 & 0 & $\mathrm{ug} / \mathrm{kg}$ & 8000 & 8000 & 8000 & & \\
\hline X10I0700534 & SVOA & Benzo(b)fluoranthene & 1 & 0 & $\mathrm{ug} / \mathrm{kg}$ & 8000 & 8000 & 8000 & & \\
\hline X10I0700534 & SVOA & Benzo(ghi)perylene & 1 & 0 & $\mathrm{ug} / \mathrm{kg}$ & 8000 & 8000 & 8000 & & \\
\hline X10I0700534 & SVOA & Benzo(k)fluoranthene & 1 & 0 & $\mathrm{ug} / \mathrm{kg}$ & 8000 & 8000 & 8000 & & \\
\hline X10I0700534 & SVOA & Benzoic acid & 1 & 0 & $\mathrm{ug} / \mathrm{kg}$ & 160000 & 160000 & 160000 & & \\
\hline X10I0700534 & SVOA & Bis(2-chloroethoxy)methane & 1 & 0 & $\mathrm{ug} / \mathrm{kg}$ & 80000 & 80000 & 80000 & & \\
\hline X10I0700534 & SVOA & Bis(2-chloroethyl) ether & 1 & 0 & $\mathrm{ug} / \mathrm{kg}$ & 80000 & 80000 & 80000 & & \\
\hline X10I0700534 & SVOA & Bis(2-ethylhexyl)phthalate & 1 & 0 & $\mathrm{ug} / \mathrm{kg}$ & 80000 & 80000 & 80000 & & \\
\hline X10I0700534 & SVOA & Butyl benzyl phthalate & 1 & 0 & $\mathrm{ug} / \mathrm{kg}$ & 80000 & 80000 & 80000 & & \\
\hline X10I0700534 & SVOA & Carbazole & 1 & 0 & $\mathrm{ug} / \mathrm{kg}$ & 8000 & 8000 & 8000 & & \\
\hline X10I0700534 & SVOA & Chrysene & 1 & 0 & $\mathrm{ug} / \mathrm{kg}$ & 8000 & 8000 & 8000 & & \\
\hline X10I0700534 & SVOA & Dibenz(a,h)anthracene & 1 & 0 & $\mathrm{ug} / \mathrm{kg}$ & 8000 & 8000 & 8000 & & \\
\hline X10I0700534 & SVOA & Dibenzofuran & 1 & 0 & $\mathrm{ug} / \mathrm{kg}$ & 80000 & 80000 & 80000 & & \\
\hline X10I0700534 & SVOA & Diethyl phthalate & 1 & 0 & $\mathrm{ug} / \mathrm{kg}$ & 80000 & 80000 & 80000 & & \\
\hline X10I0700534 & SVOA & Dimethyl phthalate & 1 & 0 & $\mathrm{ug} / \mathrm{kg}$ & 80000 & 80000 & 80000 & & \\
\hline X10I0700534 & SVOA & Di-n-butyl phthalate & 1 & 0 & $\mathrm{ug} / \mathrm{kg}$ & 80000 & 80000 & 80000 & & \\
\hline X10I0700534 & SVOA & Di-n-octylphthalate & 1 & 0 & $\mathrm{ug} / \mathrm{kg}$ & 80000 & 80000 & 80000 & & \\
\hline X10I0700534 & SVOA & Diphenylamine & 1 & 0 & $\mathrm{ug} / \mathrm{kg}$ & 80000 & 80000 & 80000 & & \\
\hline X10I0700534 & SVOA & Fluoranthene & 1 & 0 & $\mathrm{ug} / \mathrm{kg}$ & 8000 & 8000 & 8000 & & \\
\hline X10I0700534 & SVOA & Fluorene & 1 & 0 & $\mathrm{ug} / \mathrm{kg}$ & 8000 & 8000 & 8000 & & \\
\hline X10I0700534 & SVOA & Hexachlorobenzene & 2 & 0 & $\mathrm{ug} / \mathrm{kg}$ & 0.05 & 80000 & 40000.025 & 40000.025 & 56568.507 \\
\hline X10I0700534 & SVOA & Hexachlorobutadiene & 2 & 0 & $\mathrm{ug} / \mathrm{kg}$ & 0.05 & 80000 & 40000.025 & 40000.025 & 56568.507 \\
\hline X10I0700534 & SVOA & Hexachlorocyclopentadiene & 1 & 0 & $\mathrm{ug} / \mathrm{kg}$ & 80000 & 80000 & 80000 & & \\
\hline X10I0700534 & SVOA & Hexachloroethane & 2 & 0 & $\mathrm{ug} / \mathrm{kg}$ & 0.05 & 80000 & 40000.025 & 40000.025 & 56568.507 \\
\hline X10I0700534 & SVOA & Indeno(1,2,3-cd)pyrene & 1 & 0 & $\mathrm{ug} / \mathrm{kg}$ & 8000 & 8000 & 8000 & & \\
\hline X10I0700534 & SVOA & Isophorone & 1 & 0 & $\mathrm{ug} / \mathrm{kg}$ & 80000 & 80000 & 80000 & & \\
\hline X10I0700534 & SVOA & $\mathrm{m}+\mathrm{p}$ Methylphenol & 2 & 0 & $\mathrm{ug} / \mathrm{kg}$ & 0.05 & 80000 & 40000.025 & 40000.025 & 56568.507 \\
\hline X10I0700534 & SVOA & Naphthalene & 1 & 0 & $\mathrm{ug} / \mathrm{kg}$ & 8000 & 8000 & 8000 & & \\
\hline
\end{tabular}




\begin{tabular}{|c|c|c|c|c|c|c|c|c|c|c|}
\hline X10I0700534 & SVOA & Nitrobenzene & 2 & 0 & $\mathrm{ug} / \mathrm{kg}$ & 0.05 & 80000 & 40000.025 & 40000.025 & 56568.507 \\
\hline X10I0700534 & SVOA & N-Nitroso-di-n-propylamine & 1 & 0 & $\mathrm{ug} / \mathrm{kg}$ & 80000 & 80000 & 80000 & & \\
\hline X10I0700534 & SVOA & Pentachlorophenol & 2 & 0 & $\mathrm{ug} / \mathrm{kg}$ & 0.05 & 80000 & 40000.025 & 40000.025 & 56568.507 \\
\hline X10I0700534 & SVOA & Phenanthrene & 1 & 0 & $\mathrm{ug} / \mathrm{kg}$ & 8000 & 8000 & 8000 & & \\
\hline X10I0700534 & SVOA & Phenol & 1 & 0 & $\mathrm{ug} / \mathrm{kg}$ & 80000 & 80000 & 80000 & & \\
\hline $\mathrm{X} 10 \mathrm{I} 0700534$ & SVOA & Pyridine & 2 & 0 & $\mathrm{ug} / \mathrm{kg}$ & 0.05 & 80000 & 40000.025 & 40000.025 & 56568.507 \\
\hline X10I0700534 & VOA & (1,1-Dimethylethyl)benzene & 1 & 0 & $\mathrm{ug} / \mathrm{kg}$ & 96.2 & 96.2 & 96.2 & & \\
\hline X10I0700534 & VOA & (1-Methylpropyl)benzene & 1 & 0 & $\mathrm{ug} / \mathrm{kg}$ & 96.2 & 96.2 & 96.2 & & \\
\hline X10I0700534 & VOA & 1,1,1,2-Tetrachloroethane & 1 & 0 & $\mathrm{ug} / \mathrm{kg}$ & 96.2 & 96.2 & 96.2 & & \\
\hline X10I0700534 & VOA & 1,1,1-Trichloroethane & 1 & 0 & $\mathrm{ug} / \mathrm{kg}$ & 96.2 & 96.2 & 96.2 & & \\
\hline X10I0700534 & VOA & 1,1,2,2-Tetrachloroethane & 1 & 0 & $\mathrm{ug} / \mathrm{kg}$ & 96.2 & 96.2 & 96.2 & & \\
\hline X10I0700534 & VOA & 1,1,2-Trichloroethane & 1 & 0 & $\mathrm{ug} / \mathrm{kg}$ & 96.2 & 96.2 & 96.2 & & \\
\hline X10I0700534 & VOA & 1,1-Dichloroethane & 1 & 0 & $\mathrm{ug} / \mathrm{kg}$ & 96.2 & 96.2 & 96.2 & & \\
\hline X10I0700534 & VOA & 1,1-Dichloroethene & 2 & 0 & $\mathrm{ug} / \mathrm{kg}$ & 0.01 & 96.2 & 48.105 & 48.105 & 68.016601 \\
\hline X10I0700534 & VOA & 1,1-Dichloropropene & 1 & 0 & $\mathrm{ug} / \mathrm{kg}$ & 96.2 & 96.2 & 96.2 & & \\
\hline X10I0700534 & VOA & 1,2,3-Trichlorobenzene & 1 & 0 & $\mathrm{ug} / \mathrm{kg}$ & 96.2 & 96.2 & 96.2 & & \\
\hline X10I0700534 & VOA & 1,2,3-Trichloropropane & 1 & 0 & $\mathrm{ug} / \mathrm{kg}$ & 96.2 & 96.2 & 96.2 & & \\
\hline X10I0700534 & VOA & 1,2,4-Trimethylbenzene & 1 & 0 & $\mathrm{ug} / \mathrm{kg}$ & 96.2 & 96.2 & 96.2 & & \\
\hline X10I0700534 & VOA & 1,2-Dibromo-3-chloropropane & 1 & 0 & $\mathrm{ug} / \mathrm{kg}$ & 96.2 & 96.2 & 96.2 & & \\
\hline X10I0700534 & VOA & 1,2-Dibromoethane & 1 & 0 & $\mathrm{ug} / \mathrm{kg}$ & 96.2 & 96.2 & 96.2 & & \\
\hline X10I0700534 & VOA & 1,2-Dichloroethane & 2 & 0 & $\mathrm{ug} / \mathrm{kg}$ & 0.01 & 96.2 & 48.105 & 48.105 & 68.016601 \\
\hline X10I0700534 & VOA & 1,2-Dichloroethene & 1 & 0 & $\mathrm{ug} / \mathrm{kg}$ & 192 & 192 & 192 & & \\
\hline X10I0700534 & VOA & 1,2-Dichloropropane & 1 & 0 & $\mathrm{ug} / \mathrm{kg}$ & 96.2 & 96.2 & 96.2 & & \\
\hline X10I0700534 & VOA & 1,2-Dimethylbenzene & 1 & 0 & $\mathrm{ug} / \mathrm{kg}$ & 96.2 & 96.2 & 96.2 & & \\
\hline X10I0700534 & VOA & 1,3,5-Trimethylbenzene & 1 & 0 & $\mathrm{ug} / \mathrm{kg}$ & 96.2 & 96.2 & 96.2 & & \\
\hline X10I0700534 & VOA & 1,3-Dichloropropane & 1 & 0 & $\mathrm{ug} / \mathrm{kg}$ & 96.2 & 96.2 & 96.2 & & \\
\hline X10I0700534 & VOA & 1-Chloro-4-methylbenzene & 1 & 0 & $\mathrm{ug} / \mathrm{kg}$ & 96.2 & 96.2 & 96.2 & & \\
\hline X10I0700534 & VOA & 1-Methyl-4-(1-methylethyl)benzene & 1 & 0 & $\mathrm{ug} / \mathrm{kg}$ & 96.2 & 96.2 & 96.2 & & \\
\hline X10I0700534 & VOA & 2,2-Dichloropropane & 1 & 0 & $\mathrm{ug} / \mathrm{kg}$ & 96.2 & 96.2 & 96.2 & & \\
\hline X10I0700534 & VOA & 2-Butanone & 2 & 0 & $\mathrm{ug} / \mathrm{kg}$ & 0.05 & 481 & 240.525 & 240.525 & 340.08301 \\
\hline X10I0700534 & VOA & 2-Hexanone & 1 & 0 & $\mathrm{ug} / \mathrm{kg}$ & 481 & 481 & 481 & & \\
\hline X10I0700534 & VOA & 4-Methyl-2-pentanone & 1 & 0 & $\mathrm{ug} / \mathrm{kg}$ & 481 & 481 & 481 & & \\
\hline X10I0700534 & VOA & Acetone & 1 & 0 & $\mathrm{ug} / \mathrm{kg}$ & 481 & 481 & 481 & & \\
\hline X10I0700534 & VOA & Acetonitrile & 1 & 0 & $\mathrm{ug} / \mathrm{kg}$ & 2400 & 2400 & 2400 & & \\
\hline X10I0700534 & VOA & Acrolein & 1 & 0 & $\mathrm{ug} / \mathrm{kg}$ & 481 & 481 & 481 & & \\
\hline X10I0700534 & VOA & Acrylonitrile & 1 & 0 & $\mathrm{ug} / \mathrm{kg}$ & 481 & 481 & 481 & & \\
\hline X10I0700534 & VOA & Benzene & 2 & 0 & $\mathrm{ug} / \mathrm{kg}$ & 0.01 & 96.2 & 48.105 & 48.105 & 68.016601 \\
\hline X10I0700534 & VOA & Bromobenzene & 1 & 0 & $\mathrm{ug} / \mathrm{kg}$ & 96.2 & 96.2 & 96.2 & & \\
\hline X10I0700534 & VOA & Bromochloromethane & 1 & 0 & $\mathrm{ug} / \mathrm{kg}$ & 96.2 & 96.2 & 96.2 & & \\
\hline X10I0700534 & VOA & Bromodichloromethane & 1 & 0 & $\mathrm{ug} / \mathrm{kg}$ & 96.2 & 96.2 & 96.2 & & \\
\hline X10I0700534 & VOA & Bromoform & 1 & 0 & $\mathrm{ug} / \mathrm{kg}$ & 96.2 & 96.2 & 96.2 & & \\
\hline X10I0700534 & VOA & Bromomethane & 1 & 0 & $\mathrm{ug} / \mathrm{kg}$ & 96.2 & 96.2 & 96.2 & & \\
\hline X10I0700534 & VOA & Butylbenzene & 1 & 0 & $\mathrm{ug} / \mathrm{kg}$ & 96.2 & 96.2 & 96.2 & & \\
\hline X10I0700534 & VOA & Carbon disulfide & 1 & 0 & $\mathrm{ug} / \mathrm{kg}$ & 481 & 481 & 481 & & \\
\hline X10I0700534 & VOA & Carbon tetrachloride & 2 & 0 & $\mathrm{ug} / \mathrm{kg}$ & 0.01 & 96.2 & 48.105 & 48.105 & 68.016601 \\
\hline X10I0700534 & VOA & Chlorobenzene & 2 & 0 & $\mathrm{ug} / \mathrm{kg}$ & 0.01 & 96.2 & 48.105 & 48.105 & 68.016601 \\
\hline X10I0700534 & VOA & Chloroethane & 1 & 0 & $\mathrm{ug} / \mathrm{kg}$ & 96.2 & 96.2 & 96.2 & & \\
\hline X10I0700534 & VOA & Chloroform & 2 & 0 & $\mathrm{ug} / \mathrm{kg}$ & 0.01 & 96.2 & 48.105 & 48.105 & 68.016601 \\
\hline X10I0700534 & VOA & Chloromethane & 1 & 0 & $\mathrm{ug} / \mathrm{kg}$ & 96.2 & 96.2 & 96.2 & & \\
\hline X10I0700534 & VOA & cis-1,2-Dichloroethene & 1 & 0 & $\mathrm{ug} / \mathrm{kg}$ & 96.2 & 96.2 & 96.2 & & \\
\hline X10I0700534 & VOA & cis-1,3-Dichloropropene & 1 & 0 & $\mathrm{ug} / \mathrm{kg}$ & 96.2 & 96.2 & 96.2 & & \\
\hline $\mathrm{X} 10 \mathrm{I} 0700534$ & VOA & Cumene & 1 & 0 & $\mathrm{ug} / \mathrm{kg}$ & 96.2 & 96.2 & 96.2 & & \\
\hline X10I0700534 & VOA & Dibromochloromethane & 1 & 0 & $\mathrm{ug} / \mathrm{kg}$ & 96.2 & 96.2 & 96.2 & & \\
\hline X10I0700534 & VOA & Dibromomethane & 1 & 0 & $\mathrm{ug} / \mathrm{kg}$ & 96.2 & 96.2 & 96.2 & & \\
\hline X10I0700534 & VOA & Dichlorodifluoromethane & 1 & 0 & $\mathrm{ug} / \mathrm{kg}$ & 96.2 & 96.2 & 96.2 & & \\
\hline X10I0700534 & VOA & Ethylbenzene & 1 & 0 & $\mathrm{ug} / \mathrm{kg}$ & 96.2 & 96.2 & 96.2 & & \\
\hline X10I0700534 & VOA & Hexane & 1 & 0 & $\mathrm{ug} / \mathrm{kg}$ & 481 & 481 & 481 & & \\
\hline X10I0700534 & VOA & M + P Xylene & 1 & 0 & $\mathrm{ug} / \mathrm{kg}$ & 192 & 192 & 192 & & \\
\hline X10I0700534 & VOA & Methanol & 1 & 0 & $\mathrm{ug} / \mathrm{kg}$ & 4850 & 4850 & 4850 & & \\
\hline X10I0700534 & VOA & Methyl methacrylate & 1 & 0 & $\mathrm{ug} / \mathrm{kg}$ & 481 & 481 & 481 & & \\
\hline X10I0700534 & $\mathrm{VOA}$ & Methylcyclohexane & 1 & 0 & $\mathrm{ug} / \mathrm{kg}$ & 96.2 & 96.2 & 96.2 & & \\
\hline X10I0700534 & $\mathrm{VOA}$ & Methylene chloride & 1 & 0 & $\mathrm{ug} / \mathrm{kg}$ & 481 & 481 & 481 & & \\
\hline X10I0700534 & VOA & o-Chlorotoluene & 1 & 0 & $\mathrm{ug} / \mathrm{kg}$ & 96.2 & 96.2 & 96.2 & & \\
\hline X10I0700534 & VOA & Propylbenzene & 1 & 0 & $\mathrm{ug} / \mathrm{kg}$ & 96.2 & 96.2 & 96.2 & & \\
\hline X10I0700534 & VOA & Propylene glycol & 1 & 0 & $\mathrm{ug} / \mathrm{kg}$ & 48500 & 48500 & 48500 & & \\
\hline X10I0700534 & VOA & Styrene & 1 & 1 & $\mathrm{ug} / \mathrm{kg}$ & 387 & 387 & 387 & 387 & \\
\hline X10I0700534 & VOA & Tetrachloroethene & 2 & 0 & $\mathrm{ug} / \mathrm{kg}$ & 0.01 & 96.2 & 48.105 & 48.105 & 68.016601 \\
\hline X10I0700534 & VOA & Toluene & 1 & 0 & $\mathrm{ug} / \mathrm{kg}$ & 96.2 & 96.2 & 96.2 & & \\
\hline X10I0700534 & VOA & Total Xylene & 1 & 0 & $\mathrm{ug} / \mathrm{kg}$ & 288 & 288 & 288 & & \\
\hline X10I0700534 & $\mathrm{VOA}$ & trans-1,2-Dichloroethene & 1 & 0 & $\mathrm{ug} / \mathrm{kg}$ & 96.2 & 96.2 & 96.2 & & \\
\hline
\end{tabular}




\begin{tabular}{|c|c|c|c|c|c|c|c|c|c|c|}
\hline X10I0700534 & VOA & trans-1,3-Dichloropropene & 1 & 0 & $\mathrm{ug} / \mathrm{kg}$ & 96.2 & 96.2 & 96.2 & & \\
\hline X10I0700534 & VOA & Trichloroethene & 2 & 0 & $\mathrm{ug} / \mathrm{kg}$ & 0.01 & 96.2 & 48.105 & 48.105 & 68.016601 \\
\hline X10I0700534 & VOA & Trichlorofluoromethane & 1 & 0 & $\mathrm{ug} / \mathrm{kg}$ & 96.2 & 96.2 & 96.2 & & \\
\hline X10I0700534 & VOA & Vinyl chloride & 2 & 0 & $\mathrm{ug} / \mathrm{kg}$ & 0.01 & 96.2 & 48.105 & 48.105 & 68.016601 \\
\hline X10I1020445 & HERB & $2,4-\mathrm{D}$ & 8 & 0 & $\mathrm{ug} / \mathrm{kg}$ & 0.05 & 498 & 98.9625 & 12.325 & 181.42947 \\
\hline X10I1020445 & HERB & Silvex & 8 & 0 & $\mathrm{ug} / \mathrm{kg}$ & 0.05 & 498 & 98.9625 & 12.325 & 181.42947 \\
\hline X10I1020445 & METAL & Arsenic & 8 & 1 & $\mathrm{mg} / \mathrm{L}$ & 0.3 & 150 & 54.91875 & 3.725 & 73.667137 \\
\hline X10I1020445 & METAL & Barium & 8 & 5 & $\mathrm{mg} / \mathrm{L}$ & 0.05 & 310 & 72.84725 & 11.4415 & 122.44253 \\
\hline X10I1020445 & METAL & Cadmium & 8 & 0 & $\mathrm{mg} / \mathrm{L}$ & 0.05 & 25 & 9.6175 & 2.495 & 11.993946 \\
\hline X10I1020445 & METAL & Chromium & 8 & 6 & $\mathrm{mg} / \mathrm{L}$ & 0.05 & 154000 & 19959.042 & 8.569 & 54194.16 \\
\hline X10I1020445 & METAL & Lead & 8 & 2 & $\mathrm{mg} / \mathrm{L}$ & 0.1 & 6460 & 870.80475 & 5.069 & 2263.5846 \\
\hline X10I1020445 & METAL & Mercury & 8 & 0 & $\mathrm{mg} / \mathrm{L}$ & 0.002 & 0.4 & 0.201 & 0.201 & 0.2127399 \\
\hline X10I1020445 & METAL & Selenium & 8 & 0 & $\mathrm{mg} / \mathrm{L}$ & 0.3 & 150 & 54.395 & 1.63 & 74.069073 \\
\hline X10I1020445 & METAL & Silver & 8 & 2 & $\mathrm{mg} / \mathrm{L}$ & 0.05 & 45.1 & 14.06175 & 0.272 & 20.0581 \\
\hline X10I1020445 & METAL & Total Uranium & 4 & 0 & $\mathrm{pCi} / \mathrm{g}$ & 0.0196 & 0.0737 & 0.04705 & 0.04745 & 0.0236523 \\
\hline X10I1020445 & РPCB & $4,4^{\prime}-\mathrm{DDD}$ & 4 & 0 & $\mathrm{ug} / \mathrm{kg}$ & 3.86 & 39.3 & 21.4625 & 21.345 & 20.252112 \\
\hline X10I1020445 & PPCB & 4,4'-DDE & 4 & 0 & $\mathrm{ug} / \mathrm{kg}$ & 3.86 & 39.3 & 21.4625 & 21.345 & 20.252112 \\
\hline X10I1020445 & РPCB & 4,4'-DDT & 4 & 0 & $\mathrm{ug} / \mathrm{kg}$ & 3.86 & 39.3 & 21.4625 & 21.345 & 20.252112 \\
\hline X10I1020445 & PPCB & Aldrin & 4 & 0 & $\mathrm{ug} / \mathrm{kg}$ & 1.93 & 19.6 & 10.7325 & 10.7 & 10.124207 \\
\hline X10I1020445 & PPCB & alpha-BHC & 4 & 0 & $\mathrm{ug} / \mathrm{kg}$ & 1.93 & 19.6 & 10.7325 & 10.7 & 10.124207 \\
\hline X10I1020445 & PPCB & alpha-Chlordane & 4 & 0 & $\mathrm{ug} / \mathrm{kg}$ & 1.93 & 19.6 & 10.7325 & 10.7 & 10.124207 \\
\hline X10I1020445 & РPCB & beta-BHC & 4 & 0 & $\mathrm{ug} / \mathrm{kg}$ & 1.93 & 19.6 & 10.7325 & 10.7 & 10.124207 \\
\hline X10I1020445 & PPCB & Chlordane & 8 & 0 & $\mathrm{ug} / \mathrm{kg}$ & 0.0025 & 246 & 67.13875 & 12.05125 & 109.69107 \\
\hline X10I1020445 & PPCB & delta-BHC & 4 & 0 & $\mathrm{ug} / \mathrm{kg}$ & 1.93 & 19.6 & 10.7325 & 10.7 & 10.124207 \\
\hline X10I1020445 & PPCB & Dieldrin & 4 & 0 & $\mathrm{ug} / \mathrm{kg}$ & 3.86 & 39.3 & 21.4625 & 21.345 & 20.252112 \\
\hline X10I1020445 & PPCB & Endosulfan I & 4 & 0 & $\mathrm{ug} / \mathrm{kg}$ & 1.93 & 19.6 & 10.7325 & 10.7 & 10.124207 \\
\hline X10I1020445 & PPCB & Endosulfan II & 4 & 0 & $\mathrm{ug} / \mathrm{kg}$ & 3.86 & 39.3 & 21.4625 & 21.345 & 20.252112 \\
\hline X10I1020445 & PPCB & Endosulfan sulfate & 4 & 0 & $\mathrm{ug} / \mathrm{kg}$ & 3.86 & 39.3 & 21.4625 & 21.345 & 20.252112 \\
\hline X10I1020445 & PPCB & Endrin & 8 & 0 & $\mathrm{ug} / \mathrm{kg}$ & 0.0004 & 39.3 & 10.73145 & 1.9302 & 17.532368 \\
\hline X10I1020445 & PPCB & Endrin aldehyde & 4 & 0 & $\mathrm{ug} / \mathrm{kg}$ & 3.86 & 39.3 & 21.4625 & 21.345 & 20.252112 \\
\hline X10I1020445 & PPCB & Endrin ketone & 4 & 0 & $\mathrm{ug} / \mathrm{kg}$ & 3.86 & 39.3 & 21.4625 & 21.345 & 20.252112 \\
\hline X10I1020445 & РPCB & gamma-Chlordane & 4 & 0 & $\mathrm{ug} / \mathrm{kg}$ & 1.93 & 19.6 & 10.7325 & 10.7 & 10.124207 \\
\hline X10I1020445 & PPCB & Heptachlor & 8 & 0 & $\mathrm{ug} / \mathrm{kg}$ & 0.0002 & 19.6 & 5.36635 & 0.9651 & 8.7657059 \\
\hline X10I1020445 & РPCB & Heptachlor epoxide & 8 & 0 & $\mathrm{ug} / \mathrm{kg}$ & 0.0002 & 19.6 & 5.36635 & 0.9651 & 8.7657059 \\
\hline X10I1020445 & PPCB & Lindane & 8 & 0 & $\mathrm{ug} / \mathrm{kg}$ & 0.0002 & 19.6 & 5.36635 & 0.9651 & 8.7657059 \\
\hline X10I1020445 & РPCB & Methoxychlor & 8 & 0 & $\mathrm{ug} / \mathrm{kg}$ & 0.002 & 196 & 53.6635 & 9.651 & 87.657059 \\
\hline X10I1020445 & РPCB & Toxaphene & 8 & 0 & $\mathrm{ug} / \mathrm{kg}$ & 0.005 & 491 & 134.14 & 24.1025 & 219.15485 \\
\hline X10I1020445 & RADS & Americium-241 & 4 & 0 & $\mathrm{pCi} / \mathrm{g}$ & -0.0411 & 0.0506 & 0.005825 & 0.0069 & 0.0378783 \\
\hline X10I1020445 & RADS & Carbon-14 & 4 & 0 & $\mathrm{pCi} / \mathrm{g}$ & -4.64 & -0.232 & -1.39125 & -0.3465 & 2.1668981 \\
\hline X10I1020445 & RADS & Cesium-137 & 4 & 0 & $\mathrm{pCi} / \mathrm{g}$ & -0.0223 & 0.063 & 0.0217375 & 0.023125 & 0.0425004 \\
\hline X10I1020445 & RADS & Cobalt- 60 & 4 & 0 & $\mathrm{pCi} / \mathrm{g}$ & -0.0194 & 0.0922 & 0.0260175 & 0.015635 & 0.0500143 \\
\hline X10I1020445 & RADS & Curium-243/244 & 4 & 0 & $\mathrm{pCi} / \mathrm{g}$ & -0.0102 & 0.0592 & 0.0177308 & 0.0109615 & 0.0306017 \\
\hline X10I1020445 & RADS & Iodine-129 & 4 & 0 & $\mathrm{pCi} / \mathrm{g}$ & -0.51 & 0.349 & -0.015625 & 0.04925 & 0.3698228 \\
\hline X10I1020445 & RADS & Neptunium-237 & 4 & 0 & $\mathrm{pCi} / \mathrm{g}$ & -0.286 & 0.0271 & -0.098075 & -0.0667 & 0.1431567 \\
\hline X10I1020445 & RADS & Plutonium-238 & 4 & 0 & $\mathrm{pCi} / \mathrm{g}$ & -0.0302 & 0.0375 & -0.0006 & -0.00485 & 0.0320132 \\
\hline X10I1020445 & RADS & Plutonium-239/240 & 4 & 0 & $\mathrm{pCi} / \mathrm{g}$ & -0.0315 & 0.014 & -0.0073 & -0.00585 & 0.0236698 \\
\hline X10I1020445 & RADS & Radium-226 & 4 & 0 & $\mathrm{pCi} / \mathrm{g}$ & -0.266 & 1.76 & 0.7465 & 0.746 & 0.9588206 \\
\hline X10I1020445 & RADS & Strontium-90 & 4 & 0 & $\mathrm{pCi} / \mathrm{g}$ & -0.407 & 1.08 & 0.388 & 0.4395 & 0.6100202 \\
\hline X10I1020445 & RADS & Technetium-99 & 4 & 0 & $\mathrm{pCi} / \mathrm{g}$ & -0.716 & 1.54 & 0.21075 & 0.0095 & 1.0302166 \\
\hline X10I1020445 & RADS & Thorium-230 & 4 & 0 & $\mathrm{pCi} / \mathrm{g}$ & -9.49 & 2.74 & -3.00725 & -2.6395 & 5.7326675 \\
\hline X10I1020445 & RADS & Thorium-232 & 4 & 0 & $\mathrm{pCi} / \mathrm{g}$ & 4.27 & 55.6 & 24.43 & 18.925 & 24.655194 \\
\hline X10I1020445 & RADS & Tritium & 4 & 0 & $\mathrm{pCi} / \mathrm{g}$ & -76.1 & 49.8 & -1.835 & 9.48 & 53.094227 \\
\hline X10I1020445 & RADS & Uranium-233/234 & 4 & 0 & $\mathrm{pCi} / \mathrm{g}$ & 0.004 & 0.0431 & 0.026375 & 0.0292 & 0.0173485 \\
\hline X10I1020445 & RADS & Uranium-235/236 & 4 & 0 & $\mathrm{pCi} / \mathrm{g}$ & -0.00636 & 0.0182 & 0.007575 & 0.00923 & 0.0102977 \\
\hline X10I1020445 & RADS & Uranium-238 & 4 & 0 & $\mathrm{pCi} / \mathrm{g}$ & -0.00696 & 0.0429 & 0.013095 & 0.00822 & 0.0213933 \\
\hline X10I1020445 & SVOA & 1,2,4-Trichlorobenzene & 4 & 0 & $\mathrm{ug} / \mathrm{kg}$ & 96.2 & 1000 & 423.55 & 299 & 428.67238 \\
\hline X10I1020445 & SVOA & 1,2-Dichlorobenzene & 4 & 0 & $\mathrm{ug} / \mathrm{kg}$ & 96.2 & 1000 & 423.55 & 299 & 428.67238 \\
\hline X10I1020445 & SVOA & 1,3-Dichlorobenzene & 4 & 0 & $\mathrm{ug} / \mathrm{kg}$ & 96.2 & 1000 & 423.55 & 299 & 428.67238 \\
\hline X10I1020445 & SVOA & 1,4-Dichlorobenzene & 8 & 0 & $\mathrm{ug} / \mathrm{kg}$ & 0.01 & 1000 & 211.78 & 48.105 & 360.56549 \\
\hline X10I1020445 & SVOA & 2,2'-Dichlorodiisopropyl ether & 4 & 0 & $\mathrm{ug} / \mathrm{kg}$ & 990 & 999 & 993.25 & 992 & 4.2720019 \\
\hline X10I1020445 & SVOA & 2,3,4,6-Tetrachlorophenol & 4 & 0 & $\mathrm{ug} / \mathrm{kg}$ & 990 & 999 & 993.25 & 992 & 4.2720019 \\
\hline X10I1020445 & SVOA & 2,4,5-Trichlorophenol & 8 & 0 & $\mathrm{ug} / \mathrm{kg}$ & 0.05 & 999 & 496.65 & 495.025 & 530.8951 \\
\hline X10I1020445 & SVOA & 2,4,6-Trichlorophenol & 8 & 0 & $\mathrm{ug} / \mathrm{kg}$ & 0.05 & 999 & 496.65 & 495.025 & 530.8951 \\
\hline X10I1020445 & SVOA & 2,4-Dichlorophenol & 4 & 0 & $\mathrm{ug} / \mathrm{kg}$ & 990 & 999 & 993.25 & 992 & 4.2720019 \\
\hline X10I1020445 & SVOA & 2,4-Dimethylphenol & 4 & 0 & $\mathrm{ug} / \mathrm{kg}$ & 990 & 999 & 993.25 & 992 & 4.2720019 \\
\hline X10I1020445 & SVOA & 2,4-Dinitrophenol & 4 & 0 & $\mathrm{ug} / \mathrm{kg}$ & 1980 & 2000 & 1987.5 & 1985 & 9.5742711 \\
\hline X10I1020445 & SVOA & 2,4-Dinitrotoluene & 8 & 0 & $\mathrm{ug} / \mathrm{kg}$ & 0.05 & 999 & 496.65 & 495.025 & 530.8951 \\
\hline X10I1020445 & SVOA & 2,6-Dinitrotoluene & 4 & 0 & $\mathrm{ug} / \mathrm{kg}$ & 990 & 999 & 993.25 & 992 & 4.2720019 \\
\hline X10I1020445 & SVOA & 2-Chloronaphthalene & 4 & 0 & $\mathrm{ug} / \mathrm{kg}$ & 99 & 99.9 & 99.325 & 99.2 & 0.4272002 \\
\hline X10I1020445 & SVOA & 2-Chlorophenol & 4 & 0 & $\mathrm{ug} / \mathrm{kg}$ & 990 & 999 & 993.25 & 992 & 4.2720019 \\
\hline
\end{tabular}




\begin{tabular}{|c|c|c|c|c|c|c|c|c|c|c|}
\hline X10I1020445 & SVOA & 2-Methyl-4,6-dinitrophenol & 4 & 0 & $\mathrm{ug} / \mathrm{kg}$ & 990 & 999 & 993.25 & 992 & 4.2720019 \\
\hline X10I1020445 & SVOA & 2-Methylnaphthalene & 4 & 0 & $\mathrm{ug} / \mathrm{kg}$ & 99 & 99.9 & 99.325 & 99.2 & 0.4272002 \\
\hline X10I1020445 & SVOA & 2-Methylphenol & 8 & 0 & $\mathrm{ug} / \mathrm{kg}$ & 0.05 & 999 & 496.65 & 495.025 & 530.8951 \\
\hline X10I1020445 & SVOA & 2-Nitrobenzenamine & 4 & 0 & $\mathrm{ug} / \mathrm{kg}$ & 990 & 999 & 993.25 & 992 & 4.2720019 \\
\hline X10I1020445 & SVOA & 2-Nitrophenol & 4 & 0 & $\mathrm{ug} / \mathrm{kg}$ & 990 & 999 & 993.25 & 992 & 4.2720019 \\
\hline X10I1020445 & SVOA & 3,3'-Dichlorobenzidine & 4 & 0 & $\mathrm{ug} / \mathrm{kg}$ & 990 & 999 & 993.25 & 992 & 4.2720019 \\
\hline X10I1020445 & SVOA & 3-Nitrobenzenamine & 4 & 0 & $\mathrm{ug} / \mathrm{kg}$ & 990 & 999 & 993.25 & 992 & 4.2720019 \\
\hline X10I1020445 & SVOA & 4-Bromophenyl phenyl ether & 4 & 0 & $\mathrm{ug} / \mathrm{kg}$ & 990 & 999 & 993.25 & 992 & 4.2720019 \\
\hline X10I1020445 & SVOA & 4-Chloro-3-methylphenol & 4 & 0 & $\mathrm{ug} / \mathrm{kg}$ & 990 & 999 & 993.25 & 992 & 4.2720019 \\
\hline X10I1020445 & SVOA & 4-Chlorobenzenamine & 4 & 0 & $\mathrm{ug} / \mathrm{kg}$ & 990 & 999 & 993.25 & 992 & 4.2720019 \\
\hline X10I1020445 & SVOA & 4-Chlorophenyl phenyl ether & 4 & 0 & $\mathrm{ug} / \mathrm{kg}$ & 990 & 999 & 993.25 & 992 & 4.2720019 \\
\hline X10I1020445 & SVOA & 4-Nitrobenzenamine & 4 & 0 & $\mathrm{ug} / \mathrm{kg}$ & 990 & 999 & 993.25 & 992 & 4.2720019 \\
\hline X10I1020445 & SVOA & 4-Nitrophenol & 4 & 0 & $\mathrm{ug} / \mathrm{kg}$ & 990 & 999 & 993.25 & 992 & 4.2720019 \\
\hline X10I1020445 & SVOA & Acenaphthene & 4 & 0 & $\mathrm{ug} / \mathrm{kg}$ & 99 & 99.9 & 99.325 & 99.2 & 0.4272002 \\
\hline X10I1020445 & SVOA & Acenaphthylene & 4 & 0 & $\mathrm{ug} / \mathrm{kg}$ & 99 & 99.9 & 99.325 & 99.2 & 0.4272002 \\
\hline X10I1020445 & SVOA & Acetophenone & 4 & 0 & $\mathrm{ug} / \mathrm{kg}$ & 990 & 999 & 993.25 & 992 & 4.2720019 \\
\hline X10I1020445 & SVOA & Anthracene & 4 & 0 & $\mathrm{ug} / \mathrm{kg}$ & 99 & 99.9 & 99.325 & 99.2 & 0.4272002 \\
\hline X10I1020445 & SVOA & Benz(a)anthracene & 4 & 0 & $\mathrm{ug} / \mathrm{kg}$ & 99 & 99.9 & 99.325 & 99.2 & 0.4272002 \\
\hline X10I1020445 & SVOA & Benzenemethanol & 4 & 0 & $\mathrm{ug} / \mathrm{kg}$ & 990 & 999 & 993.25 & 992 & 4.2720019 \\
\hline X10I1020445 & SVOA & Benzidine & 4 & 0 & $\mathrm{ug} / \mathrm{kg}$ & 990 & 999 & 993.25 & 992 & 4.2720019 \\
\hline X10I1020445 & SVOA & Benzo(a)pyrene & 4 & 0 & $\mathrm{ug} / \mathrm{kg}$ & 99 & 99.9 & 99.325 & 99.2 & 0.4272002 \\
\hline X10I1020445 & SVOA & Benzo(b)fluoranthene & 4 & 0 & $\mathrm{ug} / \mathrm{kg}$ & 99 & 99.9 & 99.325 & 99.2 & 0.4272002 \\
\hline X10I1020445 & SVOA & Benzo(ghi)perylene & 4 & 0 & $\mathrm{ug} / \mathrm{kg}$ & 99 & 99.9 & 99.325 & 99.2 & 0.4272002 \\
\hline X10I1020445 & SVOA & Benzo(k)fluoranthene & 4 & 0 & $\mathrm{ug} / \mathrm{kg}$ & 99 & 99.9 & 99.325 & 99.2 & 0.4272002 \\
\hline X10I1020445 & SVOA & Benzoic acid & 4 & 0 & $\mathrm{ug} / \mathrm{kg}$ & 1980 & 2000 & 1987.5 & 1985 & 9.5742711 \\
\hline X10I1020445 & SVOA & Bis(2-chloroethoxy)methane & 4 & 0 & $\mathrm{ug} / \mathrm{kg}$ & 990 & 999 & 993.25 & 992 & 4.2720019 \\
\hline X10I1020445 & SVOA & Bis(2-chloroethyl) ether & 4 & 0 & $\mathrm{ug} / \mathrm{kg}$ & 990 & 999 & 993.25 & 992 & 4.2720019 \\
\hline X10I1020445 & SVOA & Bis(2-ethylhexyl)phthalate & 4 & 2 & $\mathrm{ug} / \mathrm{kg}$ & 990 & 85200 & 29846 & 16597 & 39727.041 \\
\hline X10I1020445 & SVOA & Butyl benzyl phthalate & 4 & 0 & $\mathrm{ug} / \mathrm{kg}$ & 990 & 19800 & 5695.75 & 996.5 & 9402.8341 \\
\hline X10I1020445 & SVOA & Carbazole & 4 & 0 & $\mathrm{ug} / \mathrm{kg}$ & 99 & 99.9 & 99.325 & 99.2 & 0.4272002 \\
\hline X10I1020445 & SVOA & Chrysene & 4 & 0 & $\mathrm{ug} / \mathrm{kg}$ & 99 & 99.9 & 99.325 & 99.2 & 0.4272002 \\
\hline X10I1020445 & SVOA & Dibenz(a,h)anthracene & 4 & 0 & $\mathrm{ug} / \mathrm{kg}$ & 99 & 99.9 & 99.325 & 99.2 & 0.4272002 \\
\hline X10I1020445 & SVOA & Dibenzofuran & 4 & 0 & $\mathrm{ug} / \mathrm{kg}$ & 990 & 999 & 993.25 & 992 & 4.2720019 \\
\hline X10I1020445 & SVOA & Diethyl phthalate & 4 & 0 & $\mathrm{ug} / \mathrm{kg}$ & 990 & 999 & 993.25 & 992 & 4.2720019 \\
\hline X10I1020445 & SVOA & Dimethyl phthalate & 4 & 0 & $\mathrm{ug} / \mathrm{kg}$ & 990 & 999 & 993.25 & 992 & 4.2720019 \\
\hline X10I1020445 & SVOA & Di-n-butyl phthalate & 4 & 0 & $\mathrm{ug} / \mathrm{kg}$ & 990 & 1350 & 1083.25 & 996.5 & 177.87144 \\
\hline X10I1020445 & SVOA & Di-n-octylphthalate & 4 & 0 & $\mathrm{ug} / \mathrm{kg}$ & 990 & 999 & 993.25 & 992 & 4.2720019 \\
\hline X10I1020445 & SVOA & Diphenylamine & 4 & 0 & $\mathrm{ug} / \mathrm{kg}$ & 990 & 999 & 993.25 & 992 & 4.2720019 \\
\hline X10I1020445 & SVOA & Fluoranthene & 4 & 0 & $\mathrm{ug} / \mathrm{kg}$ & 99 & 99.9 & 99.325 & 99.2 & 0.4272002 \\
\hline X10I1020445 & SVOA & Fluorene & 4 & 0 & $\mathrm{ug} / \mathrm{kg}$ & 99 & 99.9 & 99.325 & 99.2 & 0.4272002 \\
\hline X10I1020445 & SVOA & Hexachlorobenzene & 8 & 0 & $\mathrm{ug} / \mathrm{kg}$ & 0.05 & 999 & 496.65 & 495.025 & 530.8951 \\
\hline X10I1020445 & SVOA & Hexachlorobutadiene & 8 & 0 & $\mathrm{ug} / \mathrm{kg}$ & 0.05 & 999 & 496.65 & 495.025 & 530.8951 \\
\hline X10I1020445 & SVOA & Hexachlorocyclopentadiene & 4 & 0 & $\mathrm{ug} / \mathrm{kg}$ & 990 & 999 & 993.25 & 992 & 4.2720019 \\
\hline X10I1020445 & SVOA & Hexachloroethane & 8 & 0 & $\mathrm{ug} / \mathrm{kg}$ & 0.05 & 999 & 496.65 & 495.025 & 530.8951 \\
\hline X10I1020445 & SVOA & Indeno(1,2,3-cd)pyrene & 4 & 0 & $\mathrm{ug} / \mathrm{kg}$ & 99 & 99.9 & 99.325 & 99.2 & 0.4272002 \\
\hline X10I1020445 & SVOA & Isophorone & 4 & 0 & $\mathrm{ug} / \mathrm{kg}$ & 990 & 999 & 993.25 & 992 & 4.2720019 \\
\hline X10I1020445 & SVOA & $\mathrm{m}+\mathrm{p}$ Methylphenol & 8 & 0 & $\mathrm{ug} / \mathrm{kg}$ & 0.05 & 999 & 496.65 & 495.025 & 530.8951 \\
\hline X10I1020445 & SVOA & Naphthalene & 4 & 0 & $\mathrm{ug} / \mathrm{kg}$ & 99 & 99.9 & 99.325 & 99.2 & 0.4272002 \\
\hline X10I1020445 & SVOA & Nitrobenzene & 8 & 0 & $\mathrm{ug} / \mathrm{kg}$ & 0.05 & 999 & 496.65 & 495.025 & 530.8951 \\
\hline X10I1020445 & SVOA & N-Nitroso-di-n-propylamine & 4 & 0 & $\mathrm{ug} / \mathrm{kg}$ & 990 & 999 & 993.25 & 992 & 4.2720019 \\
\hline X10I1020445 & SVOA & Pentachlorophenol & 8 & 0 & $\mathrm{ug} / \mathrm{kg}$ & 0.05 & 999 & 496.65 & 495.025 & 530.8951 \\
\hline X10I1020445 & SVOA & Phenanthrene & 4 & 0 & $\mathrm{ug} / \mathrm{kg}$ & 99 & 99.9 & 99.325 & 99.2 & 0.4272002 \\
\hline X10I1020445 & SVOA & Phenol & 4 & 0 & $\mathrm{ug} / \mathrm{kg}$ & 990 & 999 & 993.25 & 992 & 4.2720019 \\
\hline X10I1020445 & SVOA & Pyridine & 8 & 0 & $\mathrm{ug} / \mathrm{kg}$ & 0.05 & 999 & 496.65 & 495.025 & 530.8951 \\
\hline X10I1020445 & VOA & (1,1-Dimethylethyl)benzene & 4 & 0 & $\mathrm{ug} / \mathrm{kg}$ & 96.2 & 1000 & 423.55 & 299 & 428.67238 \\
\hline X10I1020445 & VOA & (1-Methylpropyl)benzene & 4 & 0 & $\mathrm{ug} / \mathrm{kg}$ & 96.2 & 1000 & 423.55 & 299 & 428.67238 \\
\hline X10I1020445 & VOA & 1,1,1,2-Tetrachloroethane & 4 & 0 & $\mathrm{ug} / \mathrm{kg}$ & 96.2 & 1000 & 423.55 & 299 & 428.67238 \\
\hline X10I1020445 & VOA & 1,1,1-Trichloroethane & 4 & 0 & $\mathrm{ug} / \mathrm{kg}$ & 96.2 & 1000 & 423.55 & 299 & 428.67238 \\
\hline X10I1020445 & VOA & 1,1,2,2-Tetrachloroethane & 4 & 0 & $\mathrm{ug} / \mathrm{kg}$ & 96.2 & 1000 & 423.55 & 299 & 428.67238 \\
\hline X10I1020445 & VOA & 1,1,2-Trichloroethane & 4 & 0 & $\mathrm{ug} / \mathrm{kg}$ & 96.2 & 1000 & 423.55 & 299 & 428.67238 \\
\hline X10I1020445 & VOA & 1,1-Dichloroethane & 4 & 0 & $\mathrm{ug} / \mathrm{kg}$ & 96.2 & 1000 & 423.55 & 299 & 428.67238 \\
\hline X10I1020445 & VOA & 1,1-Dichloroethene & 8 & 0 & $\mathrm{ug} / \mathrm{kg}$ & 0.01 & 1000 & 211.78 & 48.105 & 360.56549 \\
\hline X10I1020445 & VOA & 1,1-Dichloropropene & 4 & 0 & $\mathrm{ug} / \mathrm{kg}$ & 96.2 & 1000 & 423.55 & 299 & 428.67238 \\
\hline X10I1020445 & VOA & 1,2,3-Trichlorobenzene & 4 & 0 & $\mathrm{ug} / \mathrm{kg}$ & 96.2 & 1000 & 423.55 & 299 & 428.67238 \\
\hline X10I1020445 & VOA & 1,2,3-Trichloropropane & 4 & 0 & $\mathrm{ug} / \mathrm{kg}$ & 96.2 & 1000 & 423.55 & 299 & 428.67238 \\
\hline X10I1020445 & VOA & 1,2,4-Trimethylbenzene & 4 & 0 & $\mathrm{ug} / \mathrm{kg}$ & 96.2 & 1000 & 423.55 & 299 & 428.67238 \\
\hline X10I1020445 & $\mathrm{VOA}$ & 1,2-Dibromo-3-chloropropane & 4 & 0 & $\mathrm{ug} / \mathrm{kg}$ & 96.2 & 1000 & 423.55 & 299 & 428.67238 \\
\hline X10I1020445 & VOA & 1,2-Dibromoethane & 4 & 0 & $\mathrm{ug} / \mathrm{kg}$ & 96.2 & 1000 & 423.55 & 299 & 428.67238 \\
\hline X10I1020445 & VOA & 1,2-Dichloroethane & 8 & 0 & $\mathrm{ug} / \mathrm{kg}$ & 0.01 & 1000 & 211.78 & 48.105 & 360.56549 \\
\hline X10I1020445 & VOA & 1,2-Dichloroethene & 4 & 0 & $\mathrm{ug} / \mathrm{kg}$ & 192 & 2000 & 847 & 598 & 857.4466 \\
\hline
\end{tabular}




\begin{tabular}{|c|c|c|c|c|c|c|c|c|c|c|}
\hline X10I1020445 & VOA & 1,2-Dichloropropane & 4 & 0 & $\mathrm{ug} / \mathrm{kg}$ & 96.2 & 1000 & 423.55 & 299 & 428.67238 \\
\hline X10I1020445 & VOA & 1,2-Dimethylbenzene & 4 & 0 & $\mathrm{ug} / \mathrm{kg}$ & 96.2 & 1000 & 531.05 & 514 & 501.89708 \\
\hline X10I1020445 & VOA & 1,3,5-Trimethylbenzene & 4 & 1 & $\mathrm{ug} / \mathrm{kg}$ & 96.2 & 1000 & 544.8 & 541.5 & 516.99622 \\
\hline X10I1020445 & VOA & 1,3-Dichloropropane & 4 & 0 & $\mathrm{ug} / \mathrm{kg}$ & 96.2 & 1000 & 423.55 & 299 & 428.67238 \\
\hline X10I1020445 & VOA & 1-Chloro-4-methylbenzene & 4 & 0 & $\mathrm{ug} / \mathrm{kg}$ & 96.2 & 1000 & 423.55 & 299 & 428.67238 \\
\hline X10I1020445 & $\mathrm{VOA}$ & 1-Methyl-4-(1-methylethyl)benzene & 4 & 0 & $\mathrm{ug} / \mathrm{kg}$ & 96.2 & 1000 & 423.55 & 299 & 428.67238 \\
\hline X10I1020445 & $\mathrm{VOA}$ & 2,2-Dichloropropane & 4 & 0 & $\mathrm{ug} / \mathrm{kg}$ & 96.2 & 1000 & 423.55 & 299 & 428.67238 \\
\hline X10I1020445 & VOA & 2-Butanone & 8 & 0 & $\mathrm{ug} / \mathrm{kg}$ & 0.05 & 5000 & 1058.9 & 240.525 & 1802.8274 \\
\hline X10I1020445 & VOA & 2-Hexanone & 4 & 0 & $\mathrm{ug} / \mathrm{kg}$ & 481 & 5000 & 2117.75 & 1495 & 2143.3619 \\
\hline X10I1020445 & VOA & 4-Methyl-2-pentanone & 4 & 1 & $\mathrm{ug} / \mathrm{kg}$ & 481 & 5000 & 2502.75 & 2265 & 2362.0634 \\
\hline X10I1020445 & $\mathrm{VOA}$ & Acetone & 4 & 0 & $\mathrm{ug} / \mathrm{kg}$ & 481 & 5000 & 2117.75 & 1495 & 2143.3619 \\
\hline X10I1020445 & $\mathrm{VOA}$ & Acetonitrile & 4 & 0 & $\mathrm{ug} / \mathrm{kg}$ & 2400 & 25000 & 10587.5 & 7475 & 10718.082 \\
\hline X10I1020445 & VOA & Acrolein & 4 & 0 & $\mathrm{ug} / \mathrm{kg}$ & 481 & 5000 & 2117.75 & 1495 & 2143.3619 \\
\hline X10I1020445 & $\mathrm{VOA}$ & Acrylonitrile & 4 & 0 & $\mathrm{ug} / \mathrm{kg}$ & 481 & 5000 & 2117.75 & 1495 & 2143.3619 \\
\hline X10I1020445 & $\mathrm{VOA}$ & Benzene & 8 & 0 & $\mathrm{ug} / \mathrm{kg}$ & 0.01 & 1000 & 211.78 & 48.105 & 360.56549 \\
\hline X10I1020445 & VOA & Bromobenzene & 4 & 0 & $\mathrm{ug} / \mathrm{kg}$ & 96.2 & 1000 & 423.55 & 299 & 428.67238 \\
\hline X10I1020445 & VOA & Bromochloromethane & 4 & 0 & $\mathrm{ug} / \mathrm{kg}$ & 96.2 & 1000 & 423.55 & 299 & 428.67238 \\
\hline X10I1020445 & VOA & Bromodichloromethane & 4 & 0 & $\mathrm{ug} / \mathrm{kg}$ & 96.2 & 1000 & 423.55 & 299 & 428.67238 \\
\hline X10I1020445 & VOA & Bromoform & 4 & 0 & $\mathrm{ug} / \mathrm{kg}$ & 96.2 & 1000 & 423.55 & 299 & 428.67238 \\
\hline X10I1020445 & VOA & Bromomethane & 4 & 0 & $\mathrm{ug} / \mathrm{kg}$ & 96.2 & 1000 & 423.55 & 299 & 428.67238 \\
\hline X10I1020445 & VOA & Butylbenzene & 4 & 0 & $\mathrm{ug} / \mathrm{kg}$ & 96.2 & 1000 & 423.55 & 299 & 428.67238 \\
\hline X10I1020445 & $\mathrm{VOA}$ & Carbon disulfide & 4 & 0 & $\mathrm{ug} / \mathrm{kg}$ & 481 & 5000 & 2117.75 & 1495 & 2143.3619 \\
\hline X10I1020445 & $\mathrm{VOA}$ & Carbon tetrachloride & 8 & 0 & $\mathrm{ug} / \mathrm{kg}$ & 0.01 & 1000 & 211.78 & 48.105 & 360.56549 \\
\hline X10I1020445 & VOA & Chlorobenzene & 8 & 0 & $\mathrm{ug} / \mathrm{kg}$ & 0.01 & 1000 & 211.78 & 48.105 & 360.56549 \\
\hline X10I1020445 & $\mathrm{VOA}$ & Chloroethane & 4 & 0 & $\mathrm{ug} / \mathrm{kg}$ & 96.2 & 1000 & 423.55 & 299 & 428.67238 \\
\hline X10I1020445 & VOA & Chloroform & 8 & 0 & $\mathrm{ug} / \mathrm{kg}$ & 0.01 & 1000 & 211.78 & 48.105 & 360.56549 \\
\hline X10I1020445 & VOA & Chloromethane & 4 & 0 & $\mathrm{ug} / \mathrm{kg}$ & 96.2 & 1000 & 423.55 & 299 & 428.67238 \\
\hline X10I1020445 & VOA & cis-1,2-Dichloroethene & 4 & 0 & $\mathrm{ug} / \mathrm{kg}$ & 96.2 & 1000 & 423.55 & 299 & 428.67238 \\
\hline X10I1020445 & VOA & cis-1,3-Dichloropropene & 4 & 0 & $\mathrm{ug} / \mathrm{kg}$ & 96.2 & 1000 & 423.55 & 299 & 428.67238 \\
\hline X10I1020445 & $\mathrm{VOA}$ & Cumene & 4 & 0 & $\mathrm{ug} / \mathrm{kg}$ & 96.2 & 1000 & 423.55 & 299 & 428.67238 \\
\hline X10I1020445 & VOA & Dibromochloromethane & 4 & 0 & $\mathrm{ug} / \mathrm{kg}$ & 96.2 & 1000 & 423.55 & 299 & 428.67238 \\
\hline X10I1020445 & VOA & Dibromomethane & 4 & 0 & $\mathrm{ug} / \mathrm{kg}$ & 96.2 & 1000 & 423.55 & 299 & 428.67238 \\
\hline X10I1020445 & VOA & Dichlorodifluoromethane & 4 & 0 & $\mathrm{ug} / \mathrm{kg}$ & 96.2 & 1000 & 423.55 & 299 & 428.67238 \\
\hline X10I1020445 & $\mathrm{VOA}$ & Ethylbenzene & 4 & 0 & $\mathrm{ug} / \mathrm{kg}$ & 96.2 & 1000 & 423.55 & 299 & 428.67238 \\
\hline X10I1020445 & VOA & Hexane & 4 & 0 & $\mathrm{ug} / \mathrm{kg}$ & 481 & 5000 & 2117.75 & 1495 & 2143.3619 \\
\hline X10I1020445 & VOA & $\mathrm{M}+\mathrm{P}$ Xylene & 4 & 0 & $\mathrm{ug} / \mathrm{kg}$ & 192 & 2000 & 847 & 598 & 857.4466 \\
\hline X10I1020445 & $\mathrm{VOA}$ & Methanol & 4 & 0 & $\mathrm{ug} / \mathrm{kg}$ & 4240 & 10000 & 5940 & 4760 & 2718.7375 \\
\hline X10I1020445 & VOA & Methyl methacrylate & 4 & 0 & $\mathrm{ug} / \mathrm{kg}$ & 481 & 5000 & 2117.75 & 1495 & 2143.3619 \\
\hline X10I1020445 & VOA & Methylcyclohexane & 4 & 0 & $\mathrm{ug} / \mathrm{kg}$ & 96.2 & 1660 & 713.55 & 549 & 761.10534 \\
\hline X10I1020445 & $\mathrm{VOA}$ & Methylene chloride & 4 & 0 & $\mathrm{ug} / \mathrm{kg}$ & 481 & 5000 & 2117.75 & 1495 & 2143.3619 \\
\hline X10I1020445 & VOA & o-Chlorotoluene & 4 & 0 & $\mathrm{ug} / \mathrm{kg}$ & 96.2 & 1000 & 423.55 & 299 & 428.67238 \\
\hline X10I1020445 & VOA & Propylbenzene & 4 & 0 & $\mathrm{ug} / \mathrm{kg}$ & 96.2 & 1000 & 423.55 & 299 & 428.67238 \\
\hline X10I1020445 & $\mathrm{VOA}$ & Propylene glycol & 4 & 0 & $\mathrm{ug} / \mathrm{kg}$ & 42400 & 100000 & 59400 & 47600 & 27187.375 \\
\hline X10I1020445 & VOA & Styrene & 4 & 0 & $\mathrm{ug} / \mathrm{kg}$ & 96.2 & 1000 & 423.55 & 299 & 428.67238 \\
\hline X10I1020445 & $\mathrm{VOA}$ & Tetrachloroethene & 8 & 0 & $\mathrm{ug} / \mathrm{kg}$ & 0.01 & 1000 & 211.78 & 48.105 & 360.56549 \\
\hline X10I1020445 & VOA & Toluene & 4 & 0 & $\mathrm{ug} / \mathrm{kg}$ & 96.2 & 1000 & 423.55 & 299 & 428.67238 \\
\hline X10I1020445 & $\mathrm{VOA}$ & Total Xylene & 4 & 0 & $\mathrm{ug} / \mathrm{kg}$ & 288 & 3000 & 1270.5 & 897 & 1286.1699 \\
\hline X10I1020445 & $\mathrm{VOA}$ & trans-1,2-Dichloroethene & 4 & 0 & $\mathrm{ug} / \mathrm{kg}$ & 96.2 & 1000 & 423.55 & 299 & 428.67238 \\
\hline X10I1020445 & VOA & trans-1,3-Dichloropropene & 4 & 0 & $\mathrm{ug} / \mathrm{kg}$ & 96.2 & 1000 & 423.55 & 299 & 428.67238 \\
\hline X10I1020445 & VOA & Trichloroethene & 8 & 0 & $\mathrm{ug} / \mathrm{kg}$ & 0.01 & 1000 & 211.78 & 48.105 & 360.56549 \\
\hline X10I1020445 & VOA & Trichlorofluoromethane & 4 & 0 & $\mathrm{ug} / \mathrm{kg}$ & 96.2 & 1000 & 423.55 & 299 & 428.67238 \\
\hline X10I1020445 & $\mathrm{VOA}$ & Vinyl chloride & 8 & 0 & $\mathrm{ug} / \mathrm{kg}$ & 0.01 & 1000 & 211.78 & 48.105 & 360.56549 \\
\hline X19C0700324 & HERB & $2,4-\mathrm{D}$ & 8 & 0 & $\mathrm{ug} / \mathrm{kg}$ & 0.05 & 24.7 & 12.3125 & 12.275 & 13.109314 \\
\hline $\mathrm{X} 19 \mathrm{C} 0700324$ & HERB & Silvex & 8 & 0 & $\mathrm{ug} / \mathrm{kg}$ & 0.05 & 24.7 & 12.3125 & 12.275 & 13.109314 \\
\hline $\mathrm{X} 19 \mathrm{C} 0700324$ & METAL & Arsenic & 8 & 2 & $\mathrm{mg} / \mathrm{L}$ & 0.3 & 142 & 37.7125 & 3.45 & 62.768132 \\
\hline X19C0700324 & METAL & Barium & 8 & 6 & $\mathrm{mg} / \mathrm{L}$ & 0.05 & 243 & 35.525975 & 1.365 & 84.286203 \\
\hline X19C0700324 & METAL & Cadmium & 8 & 0 & $\mathrm{mg} / \mathrm{L}$ & 0.05 & 23.6 & 9.14 & 2.385 & 11.375611 \\
\hline X19C0700324 & METAL & Chromium & 8 & 4 & $\mathrm{mg} / \mathrm{L}$ & 0.05 & 53600 & 6719.5276 & 10.9855 & 18942.609 \\
\hline X19C0700324 & METAL & Lead & 8 & 2 & $\mathrm{mg} / \mathrm{L}$ & 0.1 & 472 & 120.4035 & 22.214 & 196.9796 \\
\hline X19C0700324 & METAL & Mercury & 8 & 0 & $\mathrm{mg} / \mathrm{L}$ & 0.002 & 0.4 & 0.201 & 0.201 & 0.2127399 \\
\hline X19C0700324 & METAL & Selenium & 8 & 1 & $\mathrm{mg} / \mathrm{L}$ & 0.3 & 142 & 40.0625 & 6.65 & 61.864159 \\
\hline X19C0700324 & METAL & Silver & 8 & 3 & $\mathrm{mg} / \mathrm{L}$ & 0.05 & 62.4 & 14.42625 & 0.43 & 22.688174 \\
\hline $\mathrm{X} 19 \mathrm{C} 0700324$ & METAL & Total Uranium & 4 & 0 & $\mathrm{pCi} / \mathrm{g}$ & 0.0249 & 0.685 & 0.206 & 0.05705 & 0.3200361 \\
\hline X19C0700324 & PPCB & 4,4'-DDD & 4 & 0 & $\mathrm{ug} / \mathrm{kg}$ & 3.89 & 19.9 & 7.92 & 3.945 & 7.9867181 \\
\hline X19C0700324 & PPCB & 4,4'-DDE & 4 & 0 & $\mathrm{ug} / \mathrm{kg}$ & 3.89 & 19.9 & 7.92 & 3.945 & 7.9867181 \\
\hline X19C0700324 & PPCB & 4,4'-DDT & 4 & 0 & $\mathrm{ug} / \mathrm{kg}$ & 3.89 & 19.9 & 7.92 & 3.945 & 7.9867181 \\
\hline X19C0700324 & PPCB & Aldrin & 4 & 0 & $\mathrm{ug} / \mathrm{kg}$ & 1.95 & 9.94 & 3.9575 & 1.97 & 3.9883528 \\
\hline X19C0700324 & РPCB & alpha-BHC & 4 & 0 & $\mathrm{ug} / \mathrm{kg}$ & 1.95 & 9.94 & 3.9575 & 1.97 & 3.9883528 \\
\hline X19C0700324 & РРCB & alpha-Chlordane & 4 & 0 & $\mathrm{ug} / \mathrm{kg}$ & 1.95 & 9.94 & 3.9575 & 1.97 & 3.9883528 \\
\hline X19C0700324 & PPCB & beta-BHC & 4 & 1 & $\mathrm{ug} / \mathrm{kg}$ & 1.96 & 9.94 & 4.1925 & 2.435 & 3.8561412 \\
\hline
\end{tabular}




\begin{tabular}{|c|c|c|c|c|c|c|c|c|c|c|}
\hline X19C0700324 & РPCB & Chlordane & 8 & 0 & $\mathrm{ug} / \mathrm{kg}$ & 0.0025 & 124 & 24.68875 & 12.15125 & 41.919234 \\
\hline X19C0700324 & РPCB & delta-BHC & 4 & 0 & $\mathrm{ug} / \mathrm{kg}$ & 1.95 & 9.94 & 3.9575 & 1.97 & 3.9883528 \\
\hline X19C0700324 & РPCB & Endosulfan I & 4 & 0 & $\mathrm{ug} / \mathrm{kg}$ & 1.95 & 9.94 & 3.9575 & 1.97 & 3.9883528 \\
\hline X19C0700324 & РРСВ & Endosulfan sulfate & 4 & 0 & $\mathrm{ug} / \mathrm{kg}$ & 3.89 & 19.9 & 7.92 & 3.945 & 7.9867181 \\
\hline X19C0700324 & РРСВ & Endrin & 8 & 0 & $\mathrm{ug} / \mathrm{kg}$ & 0.0004 & 19.9 & 3.9602 & 1.9452 & 6.7273761 \\
\hline X19C0700324 & РРСВ & Endrin aldehyde & 4 & 0 & $\mathrm{ug} / \mathrm{kg}$ & 3.89 & 19.9 & 7.92 & 3.945 & 7.9867181 \\
\hline X19C0700324 & РРСВ & Endrin ketone & 4 & 0 & $\mathrm{ug} / \mathrm{kg}$ & 3.89 & 19.9 & 7.92 & 3.945 & 7.9867181 \\
\hline X19C0700324 & РРСВ & Heptachlor epoxide & 8 & 0 & $\mathrm{ug} / \mathrm{kg}$ & 0.0002 & 9.94 & 1.97885 & 0.9751 & 3.3603002 \\
\hline X19C0700324 & РРСВ & Lindane & 8 & 0 & $\mathrm{ug} / \mathrm{kg}$ & 0.0002 & 9.94 & 1.97885 & 0.9751 & 3.3603002 \\
\hline X19C0700324 & РРСВ & Methoxychlor & 8 & 0 & $\mathrm{ug} / \mathrm{kg}$ & 0.002 & 99.4 & 19.7885 & 9.751 & 33.603002 \\
\hline $\mathrm{X} 19 \mathrm{C} 0700324$ & РРСВ & Toxaphene & 8 & 0 & $\mathrm{ug} / \mathrm{kg}$ & 0.005 & 249 & 49.5275 & 24.3025 & 84.176884 \\
\hline X19C0700324 & RADS & Americium-241 & 4 & 0 & $\mathrm{pCi} / \mathrm{g}$ & 0.0158 & 0.0813 & 0.03855 & 0.02855 & 0.029314 \\
\hline X19C0700324 & RADS & Carbon-14 & 4 & 0 & $\mathrm{pCi} / \mathrm{g}$ & -3.93 & 0.677 & -1.94325 & -2.26 & 2.0463257 \\
\hline X19C0700324 & RADS & Neptunium-237 & 4 & 0 & $\mathrm{pCi} / \mathrm{g}$ & -0.0489 & 0.0216 & -0.012121 & -0.010592 & 0.0301557 \\
\hline X19C0700324 & RADS & Plutonium-238 & 4 & 0 & $\mathrm{pCi} / \mathrm{g}$ & -0.023 & 0.0225 & 0.002975 & 0.0062 & 0.0196096 \\
\hline X19C0700324 & RADS & Plutonium-239/240 & 4 & 0 & $\mathrm{pCi} / \mathrm{g}$ & 0 & 0.0621 & 0.0269 & 0.02275 & 0.02705 \\
\hline X19C0700324 & RADS & Radium-226 & 4 & 0 & $\mathrm{pCi} / \mathrm{g}$ & -0.52 & 1.08 & 0.0855 & -0.109 & 0.7609387 \\
\hline X19C0700324 & RADS & Strontium-90 & 4 & 0 & $\mathrm{pCi} / \mathrm{g}$ & -0.201 & 1.32 & 0.273775 & -0.01195 & 0.7066552 \\
\hline X19C0700324 & RADS & Technetium-99 & 4 & 0 & $\mathrm{pCi} / \mathrm{g}$ & -1.26 & 0.762 & -0.3445 & -0.44 & 1.0634728 \\
\hline X19C0700324 & RADS & Thorium-230 & 4 & 0 & $\mathrm{pCi} / \mathrm{g}$ & -6.51 & 14.1 & 1.495 & -0.805 & 9.7547271 \\
\hline X19C0700324 & RADS & Thorium-232 & 4 & 0 & $\mathrm{pCi} / \mathrm{g}$ & -11.1 & 12.4 & 2.4825 & 4.315 & 11.359837 \\
\hline X19C0700324 & RADS & Tritium & 4 & 0 & $\mathrm{pCi} / \mathrm{g}$ & -97 & 16.2 & -24.78 & -9.16 & 49.931235 \\
\hline X19C0700324 & RADS & Uranium-233/234 & 4 & 0 & $\mathrm{pCi} / \mathrm{g}$ & 0.0178 & 0.363 & 0.110675 & 0.03095 & 0.1684184 \\
\hline X19C0700324 & RADS & Uranium-235/236 & 4 & 0 & $\mathrm{pCi} / \mathrm{g}$ & -0.00769 & 0.19 & 0.046695 & 0.002235 & 0.0956686 \\
\hline $\mathrm{X} 19 \mathrm{C} 0700324$ & RADS & Uranium-238 & 4 & 0 & $\mathrm{pCi} / \mathrm{g}$ & 0.0083 & 0.132 & 0.048625 & 0.0271 & 0.0569346 \\
\hline X19C0700324 & SVOA & 1,2,4-Trichlorobenzene & 4 & 0 & $\mathrm{ug} / \mathrm{kg}$ & 96.2 & 1000 & 423.55 & 299 & 428.67238 \\
\hline X19C0700324 & SVOA & 2,4-Dinitrophenol & 4 & 0 & $\mathrm{ug} / \mathrm{kg}$ & 1950 & 1990 & 1967.5 & 1965 & 17.078251 \\
\hline X19C0700324 & SVOA & 2,4-Dinitrotoluene & 8 & 0 & $\mathrm{ug} / \mathrm{kg}$ & 0.05 & 997 & 492.775 & 488.025 & 526.77732 \\
\hline X19C0700324 & SVOA & 2,6-Dinitrotoluene & 4 & 0 & $\mathrm{ug} / \mathrm{kg}$ & 976 & 997 & 985.5 & 984.5 & 8.8881944 \\
\hline X19C0700324 & SVOA & 2-Chloronaphthalene & 4 & 0 & $\mathrm{ug} / \mathrm{kg}$ & 97.6 & 99.7 & 98.55 & 98.45 & 0.8888194 \\
\hline X19C0700324 & SVOA & 2-Chlorophenol & 4 & 0 & $\mathrm{ug} / \mathrm{kg}$ & 976 & 997 & 985.5 & 984.5 & 8.8881944 \\
\hline X19C0700324 & SVOA & 2-Methyl-4,6-dinitrophenol & 4 & 0 & $\mathrm{ug} / \mathrm{kg}$ & 976 & 997 & 985.5 & 984.5 & 8.8881944 \\
\hline X19C0700324 & SVOA & 2-Methylnaphthalene & 4 & 0 & $\mathrm{ug} / \mathrm{kg}$ & 97.6 & 99.7 & 98.55 & 98.45 & 0.8888194 \\
\hline X19C0700324 & SVOA & 2-Methylphenol & 8 & 0 & $\mathrm{ug} / \mathrm{kg}$ & 0.05 & 997 & 492.775 & 488.025 & 526.77732 \\
\hline X19C0700324 & SVOA & 2-Nitrobenzenamine & 4 & 0 & $\mathrm{ug} / \mathrm{kg}$ & 976 & 997 & 985.5 & 984.5 & 8.8881944 \\
\hline X19C0700324 & SVOA & 2-Nitrophenol & 4 & 0 & $\mathrm{ug} / \mathrm{kg}$ & 976 & 997 & 985.5 & 984.5 & 8.8881944 \\
\hline X19C0700324 & SVOA & 3,3'-Dichlorobenzidine & 4 & 0 & $\mathrm{ug} / \mathrm{kg}$ & 976 & 997 & 985.5 & 984.5 & 8.8881944 \\
\hline X19C0700324 & SVOA & 3-Nitrobenzenamine & 4 & 0 & $\mathrm{ug} / \mathrm{kg}$ & 976 & 997 & 985.5 & 984.5 & 8.8881944 \\
\hline X19C0700324 & SVOA & 4-Bromophenyl phenyl ether & 4 & 0 & $\mathrm{ug} / \mathrm{kg}$ & 976 & 997 & 985.5 & 984.5 & 8.8881944 \\
\hline X19C0700324 & SVOA & 4-Chloro-3-methylphenol & 4 & 0 & $\mathrm{ug} / \mathrm{kg}$ & 976 & 997 & 985.5 & 984.5 & 8.8881944 \\
\hline X19C0700324 & SVOA & 4-Chlorobenzenamine & 4 & 0 & $\mathrm{ug} / \mathrm{kg}$ & 976 & 997 & 985.5 & 984.5 & 8.8881944 \\
\hline X19C0700324 & SVOA & 4-Chlorophenyl phenyl ether & 4 & 0 & $\mathrm{ug} / \mathrm{kg}$ & 976 & 997 & 985.5 & 984.5 & 8.8881944 \\
\hline X19C0700324 & SVOA & 4-Nitrobenzenamine & 4 & 0 & $\mathrm{ug} / \mathrm{kg}$ & 976 & 997 & 985.5 & 984.5 & 8.8881944 \\
\hline X19C0700324 & SVOA & 4-Nitrophenol & 4 & 0 & $\mathrm{ug} / \mathrm{kg}$ & 976 & 997 & 985.5 & 984.5 & 8.8881944 \\
\hline X19C0700324 & SVOA & Acenaphthene & 4 & 0 & $\mathrm{ug} / \mathrm{kg}$ & 97.6 & 99.7 & 98.55 & 98.45 & 0.8888194 \\
\hline X19C0700324 & SVOA & Acenaphthylene & 4 & 0 & $\mathrm{ug} / \mathrm{kg}$ & 97.6 & 99.7 & 98.55 & 98.45 & 0.8888194 \\
\hline X19C0700324 & SVOA & Acetophenone & 4 & 0 & $\mathrm{ug} / \mathrm{kg}$ & 976 & 997 & 985.5 & 984.5 & 8.8881944 \\
\hline X19C0700324 & SVOA & Anthracene & 4 & 0 & $\mathrm{ug} / \mathrm{kg}$ & 97.6 & 99.7 & 98.55 & 98.45 & 0.8888194 \\
\hline X19C0700324 & SVOA & Benz(a)anthracene & 4 & 0 & $\mathrm{ug} / \mathrm{kg}$ & 97.6 & 99.7 & 98.55 & 98.45 & 0.8888194 \\
\hline X19C0700324 & SVOA & Benzenemethanol & 4 & 0 & $\mathrm{ug} / \mathrm{kg}$ & 976 & 997 & 985.5 & 984.5 & 8.8881944 \\
\hline X19C0700324 & SVOA & Benzidine & 4 & 0 & $\mathrm{ug} / \mathrm{kg}$ & 976 & 997 & 985.5 & 984.5 & 8.8881944 \\
\hline
\end{tabular}




\begin{tabular}{|c|c|c|c|c|c|c|c|c|c|c|}
\hline X19C0700324 & SVOA & Benzo(ghi)perylene & 4 & 0 & $\mathrm{ug} / \mathrm{kg}$ & 97.6 & 99.7 & 98.55 & 98.45 & 0.8888194 \\
\hline X19C0700324 & SVOA & Benzo(k)fluoranthene & 4 & 0 & $\mathrm{ug} / \mathrm{kg}$ & 97.6 & 99.7 & 98.55 & 98.45 & 0.8888194 \\
\hline X19C0700324 & SVOA & Bis(2-chloroethoxy)methane & 4 & 0 & $\mathrm{ug} / \mathrm{kg}$ & 976 & 997 & 985.5 & 984.5 & 8.8881944 \\
\hline X19C0700324 & SVOA & Bis(2-ethylhexyl)phthalate & 4 & 1 & $\mathrm{ug} / \mathrm{kg}$ & 976 & 2420 & 1345 & 992 & 716.71798 \\
\hline X19C0700324 & SVOA & Butyl benzyl phthalate & 4 & 0 & $\mathrm{ug} / \mathrm{kg}$ & 976 & 997 & 985.5 & 984.5 & 8.8881944 \\
\hline X19C0700324 & SVOA & Carbazole & 4 & 0 & $\mathrm{ug} / \mathrm{kg}$ & 97.6 & 99.7 & 98.55 & 98.45 & 0.8888194 \\
\hline X19C0700324 & SVOA & Chrysene & 4 & 0 & $\mathrm{ug} / \mathrm{kg}$ & 97.6 & 99.7 & 98.55 & 98.45 & 0.8888194 \\
\hline X19C0700324 & SVOA & Diethyl phthalate & 4 & 0 & $\mathrm{ug} / \mathrm{kg}$ & 976 & 997 & 985.5 & 984.5 & 8.8881944 \\
\hline X19C0700324 & SVOA & Dimethyl phthalate & 4 & 0 & $\mathrm{ug} / \mathrm{kg}$ & 976 & 997 & 985.5 & 984.5 & 8.8881944 \\
\hline X19C0700324 & SVOA & Di-n-butyl phthalate & 4 & 0 & $\mathrm{ug} / \mathrm{kg}$ & 976 & 997 & 985.5 & 984.5 & 8.8881944 \\
\hline X19C0700324 & SVOA & Di-n-octylphthalate & 4 & 0 & $\mathrm{ug} / \mathrm{kg}$ & 976 & 997 & 985.5 & 984.5 & 8.8881944 \\
\hline X19C0700324 & SVOA & Diphenylamine & 4 & 0 & $\mathrm{ug} / \mathrm{kg}$ & 976 & 997 & 985.5 & 984.5 & 8.8881944 \\
\hline X19C0700324 & SVOA & Fluoranthene & 4 & 0 & $\mathrm{ug} / \mathrm{kg}$ & 97.6 & 99.7 & 98.55 & 98.45 & 0.8888194 \\
\hline X19C0700324 & SVOA & Hexachloroethane & 8 & 0 & $\mathrm{ug} / \mathrm{kg}$ & 0.05 & 997 & 492.775 & 488.025 & 526.77732 \\
\hline X19C0700324 & SVOA & Indeno(1,2,3-cd)pyrene & 4 & 0 & $\mathrm{ug} / \mathrm{kg}$ & 97.6 & 99.7 & 98.55 & 98.45 & 0.8888194 \\
\hline X19C0700324 & SVOA & Isophorone & 4 & 0 & $\mathrm{ug} / \mathrm{kg}$ & 976 & 997 & 985.5 & 984.5 & 8.8881944 \\
\hline X19C0700324 & SVOA & $\mathrm{m}+\mathrm{p}$ Methylphenol & 8 & 0 & $\mathrm{ug} / \mathrm{kg}$ & 0.05 & 997 & 492.775 & 488.025 & 526.77732 \\
\hline X19C0700324 & SVOA & Naphthalene & 4 & 0 & $\mathrm{ug} / \mathrm{kg}$ & 97.6 & 99.7 & 98.55 & 98.45 & 0.8888194 \\
\hline X19C0700324 & SVOA & Nitrobenzene & 8 & 0 & $\mathrm{ug} / \mathrm{kg}$ & 0.05 & 997 & 492.775 & 488.025 & 526.77732 \\
\hline X19C0700324 & SVOA & N-Nitroso-di-n-propylamine & 4 & 0 & $\mathrm{ug} / \mathrm{kg}$ & 976 & 997 & 985.5 & 984.5 & 8.8881944 \\
\hline X19C0700324 & SVOA & Pentachlorophenol & 8 & 0 & $\mathrm{ug} / \mathrm{kg}$ & 0.05 & 997 & 492.775 & 488.025 & 526.77732 \\
\hline X19C0700324 & SVOA & Phenanthrene & 4 & 0 & $\mathrm{ug} / \mathrm{kg}$ & 97.6 & 99.7 & 98.55 & 98.45 & 0.8888194 \\
\hline X19C0700324 & SVOA & Phenol & 4 & 0 & $\mathrm{ug} / \mathrm{kg}$ & 976 & 997 & 985.5 & 984.5 & 8.8881944 \\
\hline $\mathrm{X} 19 \mathrm{C} 0700324$ & SVOA & Pyridine & 8 & 0 & $\mathrm{ug} / \mathrm{kg}$ & 0.05 & 997 & 492.775 & 488.025 & 526.77732 \\
\hline X19C0700324 & $\mathrm{VOA}$ & (1,1-Dimethylethyl)benzene & 4 & 0 & $\mathrm{ug} / \mathrm{kg}$ & 96.2 & 1000 & 423.55 & 299 & 428.67238 \\
\hline X19C0700324 & VOA & (1-Methylpropyl)benzene & 4 & 0 & $\mathrm{ug} / \mathrm{kg}$ & 96.2 & 1000 & 423.55 & 299 & 428.67238 \\
\hline X19C0700324 & VOA & 1,2,4-Trimethylbenzene & 4 & 0 & $\mathrm{ug} / \mathrm{kg}$ & 96.2 & 1000 & 423.55 & 299 & 428.67238 \\
\hline X19C0700324 & VOA & 1,2-Dibromo-3-chloropropane & 4 & 0 & $\mathrm{ug} / \mathrm{kg}$ & 96.2 & 1000 & 423.55 & 299 & 428.67238 \\
\hline X19C0700324 & VOA & 1,2-Dibromoethane & 4 & 0 & $\mathrm{ug} / \mathrm{kg}$ & 96.2 & 1000 & 423.55 & 299 & 428.67238 \\
\hline X19C0700324 & VOA & 1,2-Dichloroethane & 8 & 0 & $\mathrm{ug} / \mathrm{kg}$ & 0.01 & 1000 & 211.78 & 48.105 & 360.56549 \\
\hline X19C0700324 & VOA & 1,2-Dichloroethene & 4 & 0 & $\mathrm{ug} / \mathrm{kg}$ & 192 & 2000 & 847 & 598 & 857.4466 \\
\hline X19C0700324 & VOA & 1,2-Dichloropropane & 4 & 0 & $\mathrm{ug} / \mathrm{kg}$ & 96.2 & 1000 & 423.55 & 299 & 428.67238 \\
\hline X19C0700324 & VOA & 1,2-Dimethylbenzene & 4 & 0 & $\mathrm{ug} / \mathrm{kg}$ & 96.2 & 1000 & 423.55 & 299 & 428.67238 \\
\hline X19C0700324 & VOA & 1,3,5-Trimethylbenzene & 4 & 0 & $\mathrm{ug} / \mathrm{kg}$ & 96.2 & 1000 & 423.55 & 299 & 428.67238 \\
\hline X19C0700324 & VOA & 1,3-Dichloropropane & 4 & 0 & $\mathrm{ug} / \mathrm{kg}$ & 96.2 & 1000 & 423.55 & 299 & 428.67238 \\
\hline X19C0700324 & VOA & 1-Chloro-4-methylbenzene & 4 & 0 & $\mathrm{ug} / \mathrm{kg}$ & 96.2 & 1000 & 423.55 & 299 & 428.67238 \\
\hline X19C0700324 & $\mathrm{VOA}$ & 1-Methyl-4-(1-methylethyl)benzene & 4 & 0 & $\mathrm{ug} / \mathrm{kg}$ & 96.2 & 1000 & 423.55 & 299 & 428.67238 \\
\hline X19C0700324 & VOA & 2,2-Dichloropropane & 4 & 0 & $\mathrm{ug} / \mathrm{kg}$ & 96.2 & 1000 & 423.55 & 299 & 428.67238 \\
\hline X19C0700324 & VOA & 2-Butanone & 8 & 0 & $\mathrm{ug} / \mathrm{kg}$ & 0.05 & 5000 & 1058.9 & 240.525 & 1802.8274 \\
\hline X19C0700324 & VOA & 2-Hexanone & 4 & 0 & $\mathrm{ug} / \mathrm{kg}$ & 481 & 5000 & 2117.75 & 1495 & 2143.3619 \\
\hline X19C0700324 & VOA & 4-Methyl-2-pentanone & 4 & 0 & $\mathrm{ug} / \mathrm{kg}$ & 481 & 5000 & 2117.75 & 1495 & 2143.3619 \\
\hline X19C0700324 & $\mathrm{VOA}$ & Acetone & 4 & 0 & $\mathrm{ug} / \mathrm{kg}$ & 481 & 5000 & 2117.75 & 1495 & 2143.3619 \\
\hline X19C0700324 & $\mathrm{VOA}$ & Acetonitrile & 4 & 0 & $\mathrm{ug} / \mathrm{kg}$ & 2400 & 25000 & 10587.5 & 7475 & 10718.082 \\
\hline X19C0700324 & $\mathrm{VOA}$ & Acrolein & 4 & 0 & $\mathrm{ug} / \mathrm{kg}$ & 481 & 5000 & 2117.75 & 1495 & 2143.3619 \\
\hline X19C0700324 & VOA & Acrylonitrile & 4 & 0 & $\mathrm{ug} / \mathrm{kg}$ & 481 & 5000 & 2117.75 & 1495 & 2143.3619 \\
\hline X19C0700324 & $\mathrm{VOA}$ & Benzene & 8 & 0 & $\mathrm{ug} / \mathrm{kg}$ & 0.01 & 1000 & 211.78 & 48.105 & 360.56549 \\
\hline X19C0700324 & $\mathrm{VOA}$ & Bromobenzene & 4 & 0 & $\mathrm{ug} / \mathrm{kg}$ & 96.2 & 1000 & 423.55 & 299 & 428.67238 \\
\hline X19C0700324 & $\mathrm{VOA}$ & Bromochloromethane & 4 & 0 & $\mathrm{ug} / \mathrm{kg}$ & 96.2 & 1000 & 423.55 & 299 & 428.67238 \\
\hline X19C0700324 & $\mathrm{VOA}$ & Bromodichloromethane & 4 & 0 & $\mathrm{ug} / \mathrm{kg}$ & 96.2 & 1000 & 423.55 & 299 & 428.67238 \\
\hline X19C0700324 & $\mathrm{VOA}$ & Bromoform & 4 & 0 & $\mathrm{ug} / \mathrm{kg}$ & 96.2 & 1000 & 423.55 & 299 & 428.67238 \\
\hline X19C0700324 & $\mathrm{VOA}$ & Bromomethane & 4 & 0 & $\mathrm{ug} / \mathrm{kg}$ & 96.2 & 1000 & 423.55 & 299 & 428.67238 \\
\hline
\end{tabular}




\begin{tabular}{|c|c|c|c|c|c|c|c|c|c|c|}
\hline $\mathrm{X} 19 \mathrm{C} 0700324$ & VOA & Carbon tetrachloride & 8 & 0 & $\mathrm{ug} / \mathrm{kg}$ & 0.01 & 1000 & 211.78 & 48.105 & 360.56549 \\
\hline X19C0700324 & VOA & Chlorobenzene & 8 & 0 & ug/kg & 0.01 & 1000 & 211.78 & 48.105 & 360.56549 \\
\hline X19C0700324 & VOA & Chloroethane & 4 & 0 & $\mathrm{ug} / \mathrm{kg}$ & 96.2 & 1000 & 423.55 & 299 & 428.67238 \\
\hline X19C0700324 & VOA & Chloroform & 8 & 0 & $\mathrm{ug} / \mathrm{kg}$ & 0.01 & 1000 & 211.78 & 48.105 & 360.56549 \\
\hline X19C0700324 & VOA & Chloromethane & 4 & 0 & $\mathrm{ug} / \mathrm{kg}$ & 96.2 & 1000 & 423.55 & 299 & 428.67238 \\
\hline $\mathrm{X} 19 \mathrm{C} 0700324$ & VOA & cis-1,2-Dichloroethene & 4 & 0 & $\mathrm{ug} / \mathrm{kg}$ & 96.2 & 1000 & 423.55 & 299 & 428.67238 \\
\hline X19C0700324 & VOA & cis-1,3-Dichloropropene & 4 & 0 & $\mathrm{ug} / \mathrm{kg}$ & 96.2 & 1000 & 423.55 & 299 & 428.67238 \\
\hline X19C0700324 & VOA & Cumene & 4 & 0 & $\mathrm{ug} / \mathrm{kg}$ & 96.2 & 1000 & 423.55 & 299 & 428.67238 \\
\hline X19C0700324 & VOA & Dibromochloromethane & 4 & 0 & $\mathrm{ug} / \mathrm{kg}$ & 96.2 & 1000 & 423.55 & 299 & 428.67238 \\
\hline X19C0700324 & VOA & Dibromomethane & 4 & 0 & $\mathrm{ug} / \mathrm{kg}$ & 96.2 & 1000 & 423.55 & 299 & 428.67238 \\
\hline X19C0700324 & VOA & Dichlorodifluoromethane & 4 & 0 & $\mathrm{ug} / \mathrm{kg}$ & 96.2 & 1000 & 423.55 & 299 & 428.67238 \\
\hline X19C0700324 & VOA & Ethylbenzene & 4 & 0 & $\mathrm{ug} / \mathrm{kg}$ & 96.2 & 1000 & 423.55 & 299 & 428.67238 \\
\hline X19C0700324 & VOA & Hexane & 4 & 0 & $\mathrm{ug} / \mathrm{kg}$ & 481 & 5000 & 2117.75 & 1495 & 2143.3619 \\
\hline $\mathrm{X} 19 \mathrm{C} 0700324$ & VOA & M + P Xylene & 4 & 0 & $\mathrm{ug} / \mathrm{kg}$ & 192 & 2000 & 847 & 598 & 857.4466 \\
\hline $\mathrm{X} 19 \mathrm{C} 0700324$ & VOA & Methanol & 4 & 0 & $\mathrm{ug} / \mathrm{kg}$ & 4500 & 4720 & 4620 & 4630 & 96.263527 \\
\hline $\mathrm{X} 19 \mathrm{C} 0700324$ & VOA & Methyl methacrylate & 4 & 0 & $\mathrm{ug} / \mathrm{kg}$ & 481 & 5000 & 2117.75 & 1495 & 2143.3619 \\
\hline X19C0700324 & VOA & Methylcyclohexane & 4 & 0 & $\mathrm{ug} / \mathrm{kg}$ & 96.2 & 1000 & 423.55 & 299 & 428.67238 \\
\hline X19C0700324 & VOA & Methylene chloride & 4 & 0 & $\mathrm{ug} / \mathrm{kg}$ & 481 & 5000 & 2117.75 & 1495 & 2143.3619 \\
\hline $\mathrm{X} 19 \mathrm{C} 0700324$ & VOA & o-Chlorotoluene & 4 & 0 & $\mathrm{ug} / \mathrm{kg}$ & 96.2 & 1000 & 423.55 & 299 & 428.67238 \\
\hline $\mathrm{X} 19 \mathrm{C} 0700324$ & VOA & Propylbenzene & 4 & 0 & $\mathrm{ug} / \mathrm{kg}$ & 96.2 & 1000 & 423.55 & 299 & 428.67238 \\
\hline $\mathrm{X} 19 \mathrm{C} 0700324$ & VOA & Propylene glycol & 4 & 0 & $\mathrm{ug} / \mathrm{kg}$ & 45000 & 47200 & 46200 & 46300 & 962.63527 \\
\hline X19C0700324 & VOA & Styrene & 4 & 0 & $\mathrm{ug} / \mathrm{kg}$ & 96.2 & 1000 & 423.55 & 299 & 428.67238 \\
\hline $\mathrm{X} 19 \mathrm{C} 0700324$ & VOA & Tetrachloroethene & 8 & 0 & $\mathrm{ug} / \mathrm{kg}$ & 0.01 & 1000 & 211.78 & 48.105 & 360.56549 \\
\hline $\mathrm{X} 19 \mathrm{C} 0700324$ & VOA & Toluene & 4 & 0 & $\mathrm{ug} / \mathrm{kg}$ & 96.2 & 1000 & 423.55 & 299 & 428.67238 \\
\hline $\mathrm{X} 19 \mathrm{C} 0700324$ & VOA & Total Xylene & 4 & 0 & $\mathrm{ug} / \mathrm{kg}$ & 288 & 3000 & 1270.5 & 897 & 1286.1699 \\
\hline X19C0700324 & VOA & trans-1,2-Dichloroethene & 4 & 0 & $\mathrm{ug} / \mathrm{kg}$ & 96.2 & 1000 & 423.55 & 299 & 428.67238 \\
\hline X19C0700324 & VOA & trans-1,3-Dichloropropene & 4 & 0 & $\mathrm{ug} / \mathrm{kg}$ & 96.2 & 1000 & 423.55 & 299 & 428.67238 \\
\hline X19C0700324 & VOA & Trichloroethene & 8 & 0 & $\mathrm{ug} / \mathrm{kg}$ & 0.01 & 1000 & 211.78 & 48.105 & 360.56549 \\
\hline $\mathrm{X} 19 \mathrm{C} 0700324$ & VOA & Trichlorofluoromethane & 4 & 0 & $\mathrm{ug} / \mathrm{kg}$ & 96.2 & 1000 & 423.55 & 299 & 428.67238 \\
\hline $\mathrm{X} 19 \mathrm{C} 0700324$ & VOA & Vinyl chloride & 8 & 0 & $\mathrm{ug} / \mathrm{kg}$ & 0.01 & 1000 & 211.78 & 48.105 & 360.56549 \\
\hline
\end{tabular}

